SAND89-2100 $\cdot \mathrm{UC}-610$

Unlimited Release

Printed June 1990

\title{
Accident Progression Event Tree Analysis for Postulated Severe Accidents at $\mathbf{N}$ Reactor
}

Gregory D. Wyss, Allen L. Camp, LeAnn A. Miller, Susan E. Dingman, David M. Kunsman, Gordon T. Medford

\section{Prepared by}

Sandia National Laboratories

Albuquerque, New Mexico 87185 and Livermore, California 94550

for the United States Department of Energy

under Contract DE-AC04-76DP00789 


\section{DISCLAIMER}

This report was prepared as an account of work sponsored by an agency of the United States Government. Neither the United States Government nor any agency Thereof, nor any of their employees, makes any warranty, express or implied, or assumes any legal liability or responsibility for the accuracy, completeness, or usefulness of any information, apparatus, product, or process disclosed, or represents that its use would not infringe privately owned rights. Reference herein to any specific commercial product, process, or service by trade name, trademark, manufacturer, or otherwise does not necessarily constitute or imply its endorsement, recommendation, or favoring by the United States Government or any agency thereof. The views and opinions of authors expressed herein do not necessarily state or reflect those of the United States Government or any agency thereof. 


\section{DISCLAIMER}

Portions of this document may be illegible in electronic image products. Images are produced from the best available original document. 
Issued by Sandia National Laboratories, operated for the United States Department of Energy by Sandia Corporation.

NOTICE: This report was prepared as an account of work sponsored by an agency of the United States Government. Neither the United States Government nor any agency thereof, nor any of their employees, nor any of their contractors, subcontractors, or their employees, makes any warranty, express or implied, or assumes any legal liability or responsibility for the accuracy, completeness, or usefulness of any information, apparatus, product, or process disclosed, or represents that its use would not infringe privately owned rights. Reference herein to any specific commercial product, process, or service by trade name, trademark, manufacturer, or otherwise, does not necessarily constitute or imply its endorsement, recommendation, or favoring by the United States Government, any agency thereof or any of their contractors or subcontractors. The views and opinions expressed herein do not necessarily state or reflect those of the United States Government, any agency thereof or any of their contractors.

Printed in the United States of America. This report has been reproduced directly from the best available copy.

Available to DOE and DOE contractors from

Office of Scientific and Technical Information

PO Box 62

Oak Ridge, TN 37831

Prices available from (615) 576-8401, FTS 626-8401

Available to the public from

National Technical Information Service

US Department of Commerce

5285 Port Royal Rd

Springfield, VA 22161

NTIS price codes

Printed copy: A24

Microfiche copy: A01

\section{DO NOT MICROFLLM THIS PAGE}




\section{PAGES $\mathrm{i}$ to $\mathrm{ii}$ WERE INTENTIONALLY LEFT BLANK}


SAND- -89-2100

DE9 0013558

Distribution

Category UC-610

ACCIDENT PROGRESSION EVENT TREE ANALYSIS

FOR POSTULATED SEVERE ACCIDENTS AT N REACTOR

Gregory D. Wyss, Allen L. Camp, LeAnn A. Miller, Susan E. Dingman and David M. Kunsman

Reactor Systems Safety Analysis Division Sandia National Laboratories

Albuquerque, NM $\mathbf{8 7 1 8 5}$

Gordon T. Medford

Science Applications International Corporation Albuquerque, NM 87106

\section{ABSTRACT}

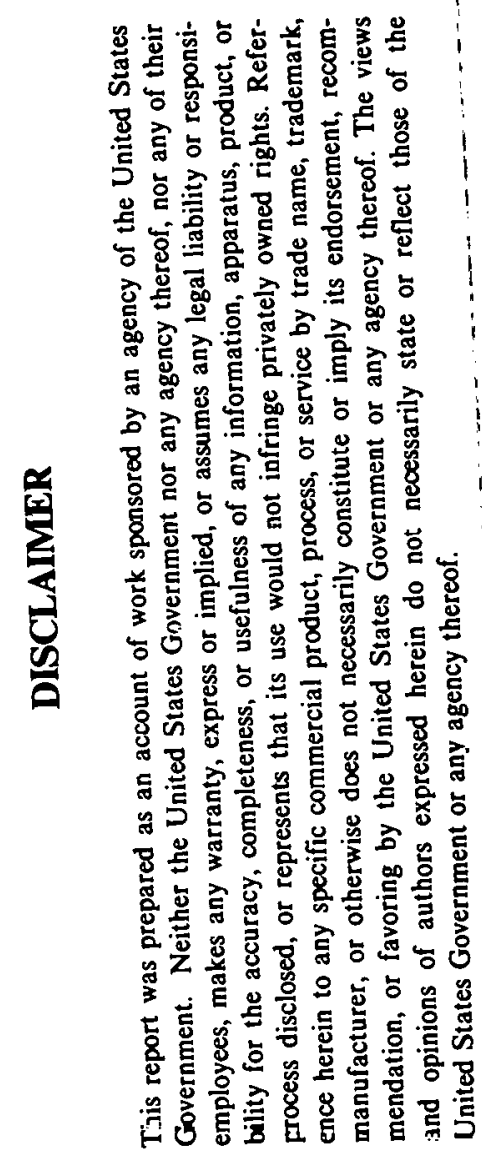

A Level II/III probabilistic risk assessment (PRA) has been performed for $N$ Reactor, a Department of Energy (DOE) production reactor located on the Hanford reservation in Washington. The accident progression analysis documented in this reporl determines how core damage accidents identified in the Level I PRA progress from fuel damage to confinement response and potential releases to the environment. The objectives of the study are to generate accident progression data for the Level II/III PRA source term model and to identify changes that could improve plant response under accident conditions. The scope of the analysis is comprehensive, excluding only sabotage and operator errors of commission. State-of-the-art methodology is employed based largely on the methods developed by Sandia for the U.S. Nuclear Regulatory Commission in support of the NUREG-1150 study. The accident progression model allows complex interactions and dependencies between systems to be explicitly considered. Latin Hypercube sampling was used to assess the phenomenological and systemic uncertainties associated with the primary and confinement system responses to the core damage accident. The results of the analysis show that the $N$ Reactor confinement concept provides significant radiological protection for most of the accident progression pathways studied. 


\section{Acknowledgments}

During the course of this accident progression analysis, we have been assisted in our work by many people. At Sandia, Tim Wheeler conducted many of the expert judgment elicitations and performed the LHS and TEMAC analyses. In the latter effort, he was assisted by Sharon Daniel and Teresa Sype. Mike Bohn and John Lambright guided us through their Level I PRA external events work while Ken Murata provided his expertise concerning the behavior of filters. Emily Preston provided valuable contributions to the compilation and typing of this report.

Several subcontractors have helped us as well. Lanny Smith of SAIC actually executed the final APET analysis runs. Alan Kolaczkowski, Jim Clark, and Walter Ferrell, all of SAIC, assisted with earlier versions of the event tree. Clint Shaffer of SEA was a member of a number of the phenomenological issues panels.

WHC personnel have been of valuable service over the past few years. Garill Coles performed the confinement systems analyses, explained the internal events Level I PRA, and was our main point of contact concerning plant systems and operations. Suzanne Lindberg conducted the systems analysis for the Hydrogen Mitigation System. John Baxter and Dong Nguyen also were excellent conduits of information for us. The analysis could not have been performed without the many discussions with personnel familiar with plant operations and phenomenological response such such as Gary Smith, John Long, Scott Heaberlin, Robert Moffitt and Don Ogden and his staff. We would also like to thank oliver Wang and Tom Rainey for their support and guidance in this effort. 
CONTENTS

$\underline{\text { Section }}$

Page

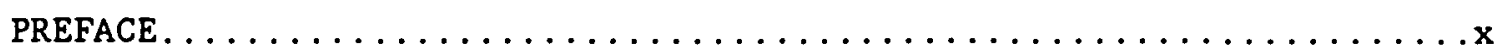

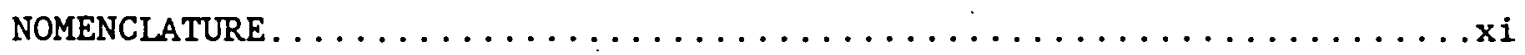

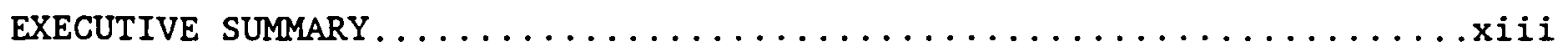

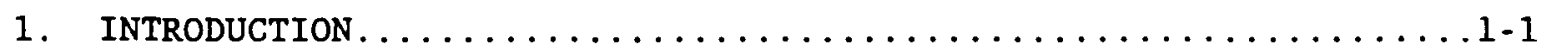

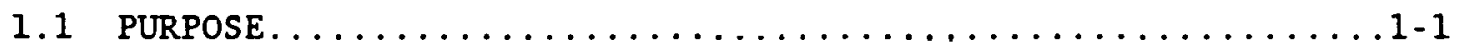

1.2 RELATIONSHIP TO THE OVERALL PRA ANALYSIS ..............

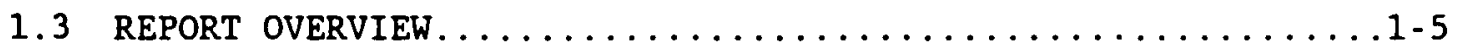

1.4 REFERENCES ........................

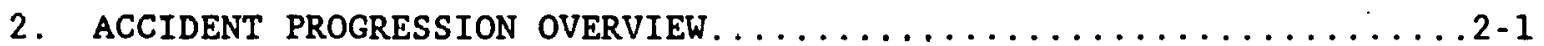

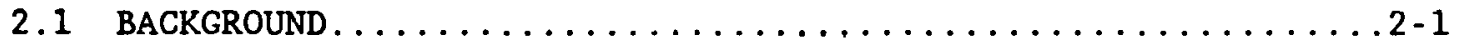

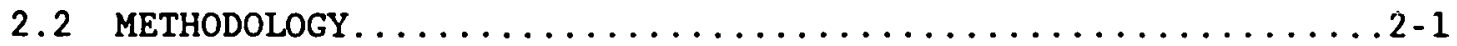

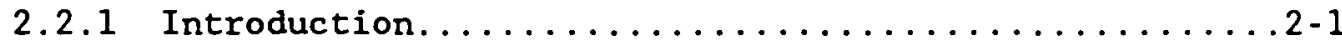

2.2.2 Principal Subtasks.......................

2.2.3 Incorporation of Phenomenological Calculations......2-4

2.2.4 Accident Progression Uncertainty Analysis......... 2-4

2.2.5 Accident Progression Analysis Tool............. 2-6

2.3 ACCIDENT PROGRESSION ANALYSIS ASSUMPTIONS AND

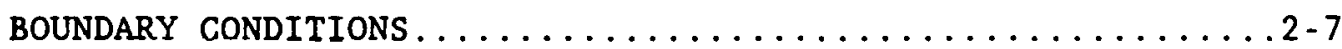

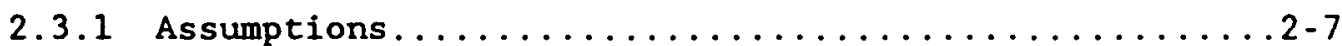

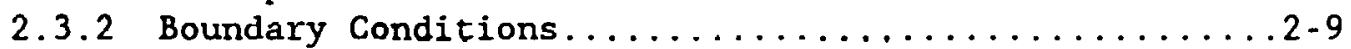

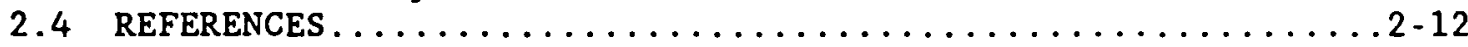

3. DESCRIPTION OF THE ACCIDENT PROGRESSION EVENT TREE..........

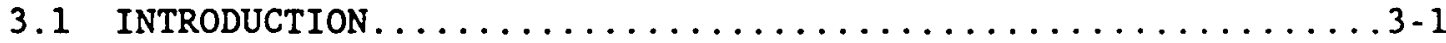

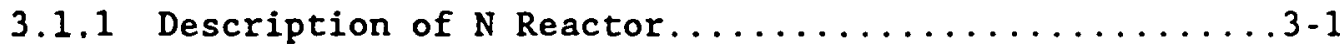

3.1.2 Accident Progression Event Tree Structure...........3-8

3.1 .3 Notation..............................

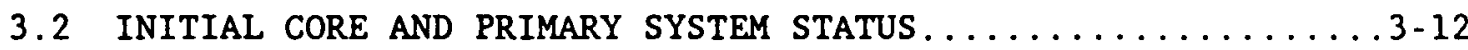

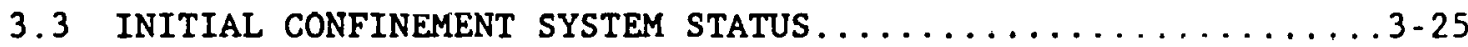

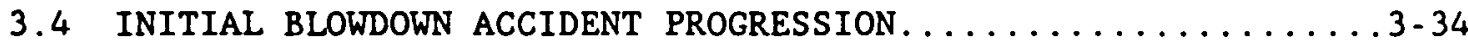

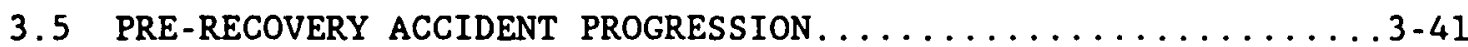

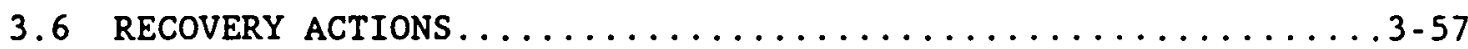

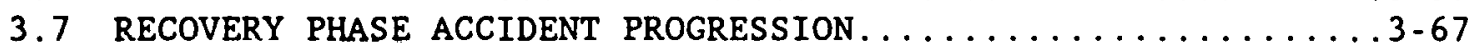

3.8 POST-RECOVERY STATUS AND SOURCE TERM ISSUES $\ldots \ldots \ldots \ldots \ldots \ldots 3-82$

3.9 BINNING OF ACCIDENT PROGRESSION EVENT TREE OUTPUT ........

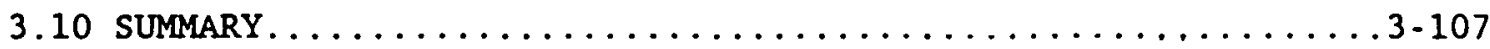

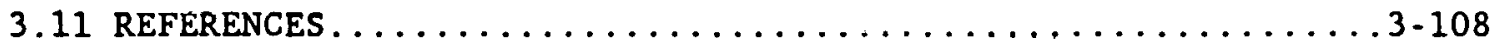


CONTENTS (continued)

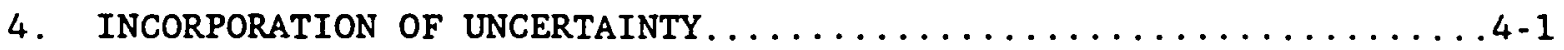

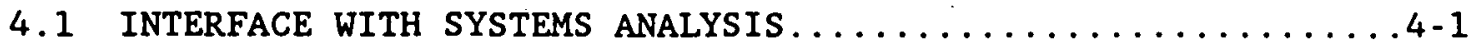

4.1 .1 Introduction......................

4.1.2 Level I - Internal Event Systems Analysis.........4-2

4.1.3 Level I - External Event Analysis..............4-2

4.1 .4 Level I - Confinement Systems..............4-9

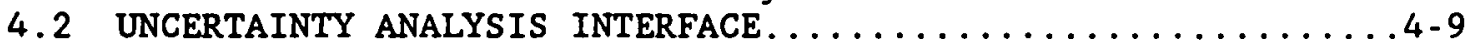

4.2.1 Internal Event Uncertainty Analysis............. 4-9

4.2.2 Propagation of Sequence Frequencies and

Split Fractions......................

4.3 INCORPORATION OF PHENOMENOLOGICAL UNCERTAINTY ..........

4.3.1 Hydrogen Phenomenology................... 4-13

4.3.2 Confinement Building Failure...............

4.3.3 Fuel Damage for Partial ECCS Accidents...........4-15

4.3.4 Fuel Damage for Late ECCS Failure............. 4-16

4.3.5 Fuel Damage Propagation.................. 4-17

4.3.6 Confinement Response to Late ECCS Actuation........4-18

4.3.7 Pressure Tube Failure.................... 4-19

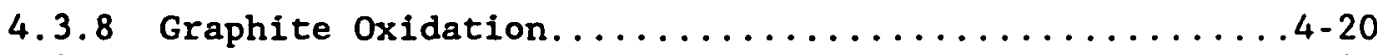

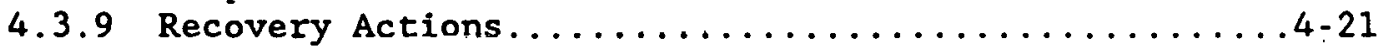

4.3.10 Filter Path Reisolation................. 4-23

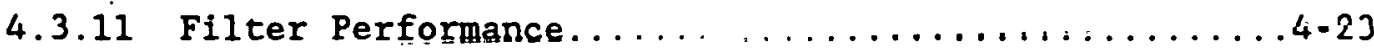

4.3.12 LERF Plastic Liner Failure.................. 4-26

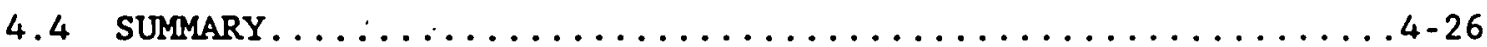

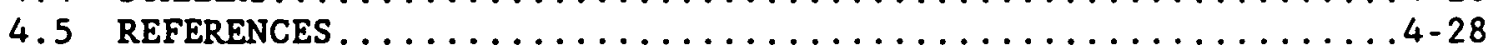

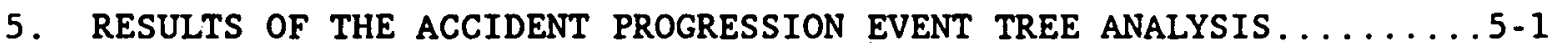

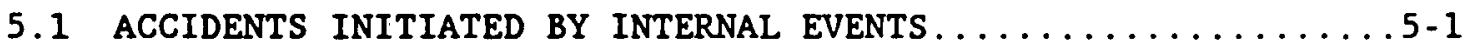

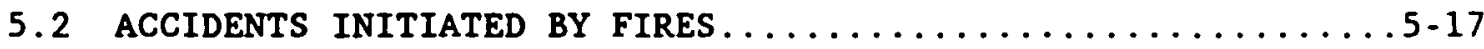

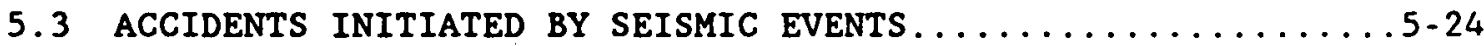

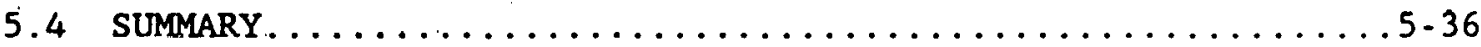

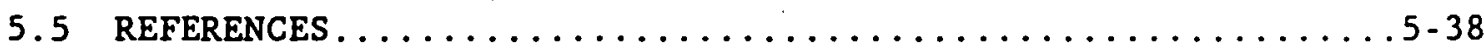

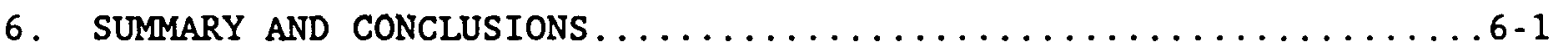

6.1 ACCIDENT PROGRESSION INSIGHTS ......................

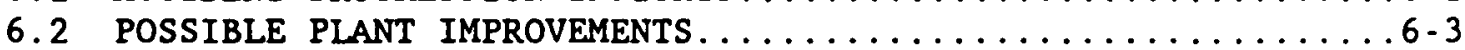

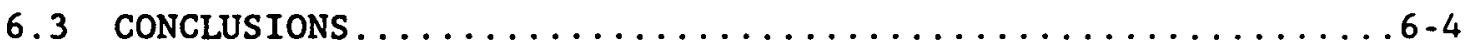

6.4 REFERENCES .............................. 6.4

APPENDIX A: INTERFACE WITH INTERNAL EVENTS UNCERTAINTY ANALYSIS . . . A-1

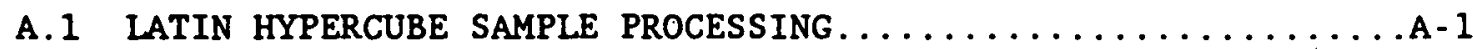

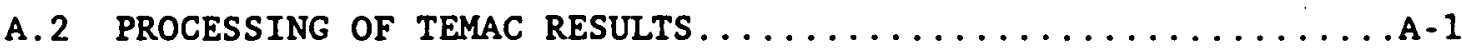

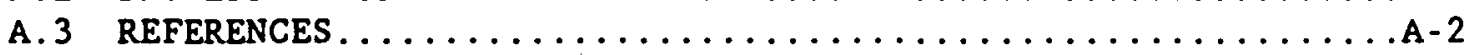


CONTENTS (continued)

Section

Page

APPENDIX B: INTERFACE WITH EXTERNAL EVENTS UNCERTAINTY ANALYSIS . . . B-1

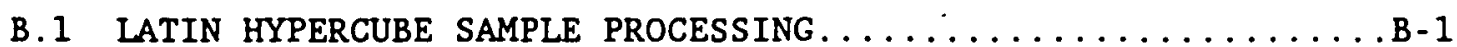

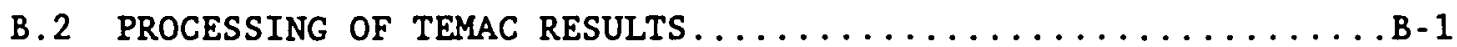

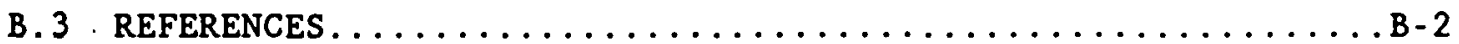

APPENDIX $c$ : SUPPORTING INFORMATION FOR THE N REACTOR APET $\ldots \ldots \ldots \ldots c-1$

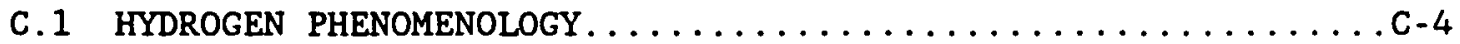

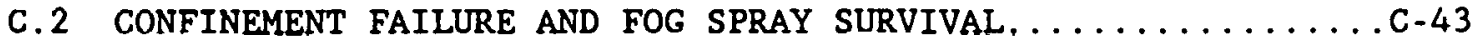

C. 3 FUEL DAMAGE FOR PARTIAL ECCS AND PARTIAL CORE ACCIDENTS.....C-5I

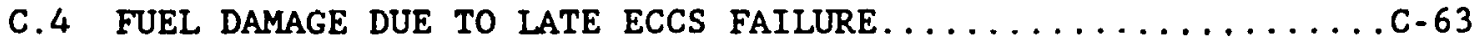

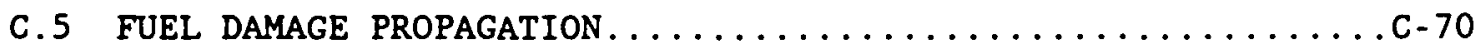

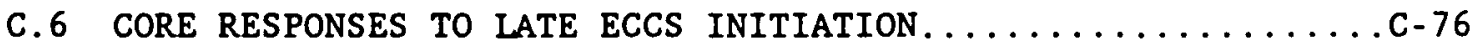

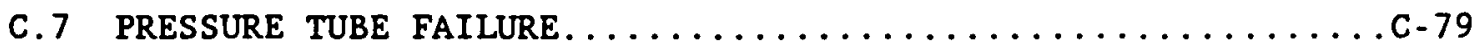

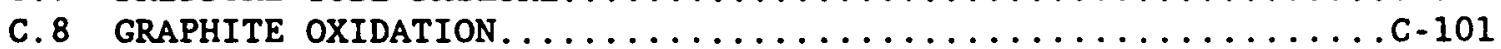

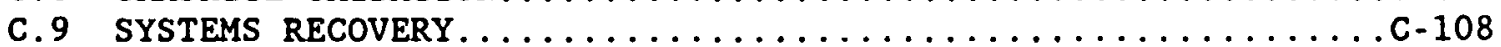

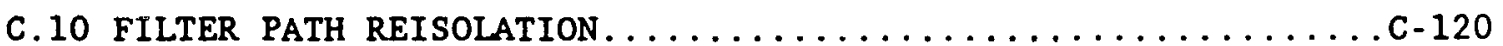

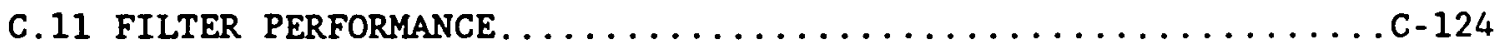

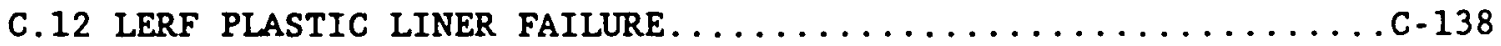

C.13 SEISMIC ASSESSMENT OF CONFINEMENT STRUCTURES ........... 143

APPENDIX $\mathrm{D}$ : SOURCES OF MISCELLANEOUS EVENT TREE DATA..........

D.1 INTRODUCTION . . . . . . . . . . . . . . . . . . . . D - 1

D. 2 PRIMARY SYSTEM SPLIT FRACTIONS FOR LARGE LOCAS $\ldots \ldots \ldots \ldots \ldots$ D- 2

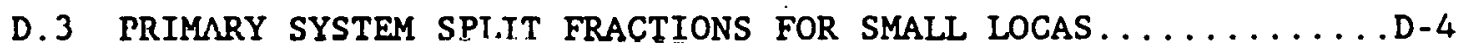

D.4. PRIMARY SYSTEM SPLIT FRACTIONS FOR TRANSIENTS ...........

D.5. REFERENCES . . . . . . . . . . . . . . . . . . . . . 6

APPENDIX E: INPUT TO THE EVNTRE CODE PACKAGE $\ldots \ldots \ldots \ldots \ldots \ldots \ldots \ldots \ldots$

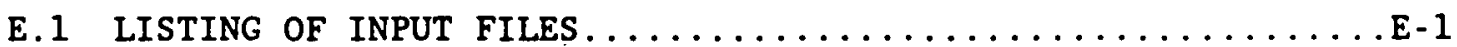

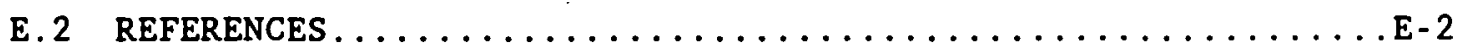

APPENDIX $\mathrm{F}$ : EVNTRE EXECUTION AND SELECTED OUTPUT $\ldots \ldots \ldots \ldots \ldots \ldots \ldots \ldots-1$

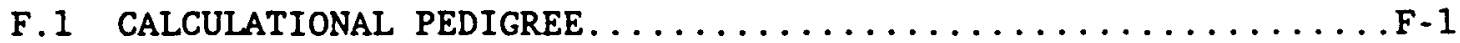

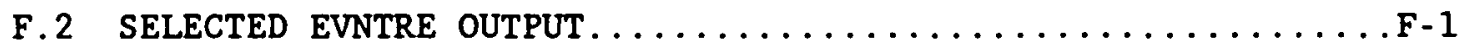

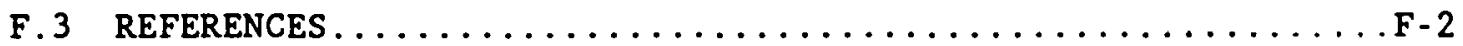




\section{LIST OF TABLES}

Table

3.1 N Reactor ground acceleration categories and mean annual frequency of exceedence.......................

3.2 Summary of the $\mathrm{N}$ Reactor binning structure by comparison to important source term phenomena................. 3-106

4.1 N Reactor Level I Dominant Sequences - Internal Events......4-3

4.2 N Reactor Dominant Fire Area Core Damage Sequences and Uncertainties............................ 4-4

4.3 N Reactor Dominant Seismic Core Damage Sequences...........45

4.4 N Reactor Dominant Seismic Core Damage Frequencies and Uncertainties.............................4-7

5.1 Accident initiator and reactor protection system (RPS) success fractions for the internal events analysis.........5-2

5.2 Average fuel damage fractions and quantiles for the accident initiators for the internal events analysis........5-3

5.3 Confinement fallure and isolation failure fractions and quantiles for the accident initiators for the internal

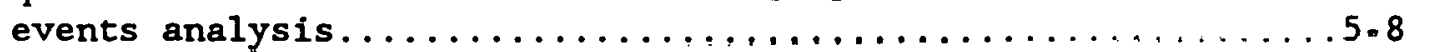

5.4 Confinement and dump tank mean failure fractions for accident initiators for the internal events analysis........5-11

5.5 Filtration failure mean fractions and quantiles for accident initiators and core damage amounts for the internal events analysis..................... 14

5.6 Core damage uncertainty for fire-initiated accidents.......5-18

5.7 Confinement survival uncertainty for fire-initiated accidents................................ 19

5.8 Filtration success uncertainty for fire-initiated accidents..5-23

5.9 N Reactor ground acceleration categories and mean annual frequency of exceedence..................... 5-25

5.10 Accident initiator and fuel damage fraction means and quantiles for the seismic APET analysis..............

5.11 Tank failure fractions by primary system configuration for the seismic APET analysis........................

5.12 Confinement failure fractions by primary system configuration for the seismic APET analysis............5-31

5.13 Graphite oxidation fractions by primary system configuration for the seismic APÉT analysis.............5-33

5.14 Filtration success fractions by primary system configuration and core damage amount for the seismic APET analysis.......5-35 


\section{LIST OF FIGURES}

Figure

Page

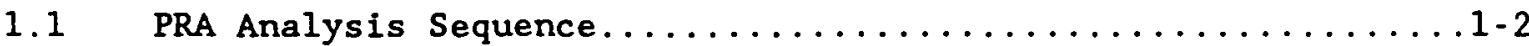

3.1 Simplified Flow Diagram of the $\mathrm{N}$ Reactor Coolant System......3-3

3.2

Simplified Top View of the $N$ Reactor Confinement Buildings....3-4

3.3

Key Components in the $\mathbf{N}$ Reactor Confinement System.......... 3-5

3.4 Elevation Drawing of the $N$ Reactor Banana Wall............

5.1 Total Fuel Damage for Internal Initiators.............

5.2 Confinement Failure for Internal Initiators.............

5.3 Confinement Failure for Internal Initiators...............

5.4 Emergency Dump Tank Status for Internal Initiators.........

5.5 Filter Performance for Internal Initiators.............5-16

5.6 Confinement Failure for Fire Initiators...............

5.7 Emergency Dump Tank Status for Fire Initiators...........

5.8 Total Fuel Damage for Seismic Initiators.................

5.9 Emergency Dump Tank Status for Seismic Initiators..........5-30

5.10 Confinement Failure for Seismic Initiators.............. 


\section{PREFACE}

This report was prepared for Westinghouse Hanford Company (WHC) as a part of the overall Level II/III PRA effort for $N$ Reactor. WHC has provided extensive technical guidance throughout the accident progression event tree analysis in particular and the $N$ Reactor PRA effort in general. WHC probabilistic safety analysis staff members have reviewed the models and results found in this report and are in substantial agreement with them. 
NOMENCIATURE

\begin{tabular}{|c|c|}
\hline APET & Accident Progression Event Tree \\
\hline ATWS & Anticipated Transient Without SCRAM \\
\hline BWR & Boiling Water Reactor \\
\hline CET & Containment Event Tree \\
\hline DDT & Deflagration to Detonation Transition \\
\hline DF & Decontamination Factor \\
\hline DOE & U.S. Department of Energy \\
\hline ECCS & Emergency Core Cooling System \\
\hline EDT & Emergency Dump Tank \\
\hline ERG & Emergency Response Guide \\
\hline GSCS & Graphite and Shield Cooling System \\
\hline HECTR & Hydrogen Events Containment Transient Response code \\
\hline HEPA & High-Efficiency Particle Absorber \\
\hline HPI & High Pressure Injection \\
\hline LERF & Liquid Effluent Retention Facility \\
\hline LHS & Latin Hypercube Sampling \\
\hline LOCA & Loss of Coolant Accident \\
\hline LPI & Low Pressure Injection \\
\hline LWDF & Liquid Waste Disposal Facility \\
\hline LWR & Light Water Reactor \\
\hline MFCI & Molten Fuel-Coolant Interactions \\
\hline MPTR & Multiple Pressure Tube Rupture \\
\hline MWt & Megawatts, thermal \\
\hline NAS & National Academy of Sciences \\
\hline NRC & U.S. Nuclear Regulatory Commission \\
\hline PCS & Primary Coolant System \\
\hline PRA & Probabilistic Risk Assessment \\
\hline psid & pounds per square inch differential \\
\hline psig & pounds per square inch gauge \\
\hline PWR & Pressurized Water Reactor \\
\hline RPS & Reactor Protection System \\
\hline SNL & Sandia National Laboratories \\
\hline SSE & Safe Shutdown Earthquake \\
\hline TEMAC & Top Event Matrix Analysis Code \\
\hline TMI & Three Mile Island \\
\hline UNC & UNC Nuclear Industries, Inc. \\
\hline WHC & Westinghouse Hanford Company \\
\hline
\end{tabular}




\section{ACCIDENT PROGRESSION EVENT TREE ANALYSIS \\ FOR POSTULATED SEVERE ACCIDENTS AT N REACTOR}

\section{EXECUTIVE SUMMARY}

A Level II/III probabilistic risk assessment (PRA) has been performed for $N$ Reactor, a Department of Energy (DOE) production reactor located on the Hanford reservation in Washington. The Level II and III portions of the PRA effort have been performed by Sandia National Laboratories (SNL) for the reservation contractor, Westinghouse Hanford Company (WHC), with their technical assistance. The Level I portion of the PRA for internal events was performed by another WHC contractor and for external events by SNL but documented elsewhere.

The accident progression event tree (APET) portion of the Level II/III PRA analysis is documented in this report. The APET analysis models how core damage accidents identified in the Level I PRA progress from fuel damage to confinement response and potential releases to the environment. The objectives of the APET study are to generate accident progression data for the Level II/III PRA radioactive source term model and to identify changes that could improve plant response under accident conditions.

The state-of-the-art methodology being employed is based largely on the methods developed by SNL for the U.S. Nuclear Regulatory Commission (NRC) and used in the massive five plant PRA study known as NUREG-1150, although the unique design of $N$ Reactor required adapting some of the tools. In addition, analyzing the progression of potential severe accidents for a reactor type not encountered in other PRAs results in unique analytic considerations and calculated responses.

The scope of the APET model is comprehensive, excluding only sabotage and operator errors of commission. The APET analysis takes as its input the results of the Level I PRA. The Level I PRA analyses considered all known internal initiating events (those due to hardware failures and human errors within the plant) and external initiating events (those due to outside hazards, such as earthquakes and high winds, along with internal floods and fires). In the accident progression analysis discussed in this report, all known accident progression phenomena were considered. Many phenomena were ruled out on physical or probabilistic grounds; those decisions are clearly documented in the report.

A highly complex accident progression model was used in order to allow for the effects of many highly unlikely beyond design basis accident phenomena. The structure of the models allowed interactions and dependencies between systems and phenomena to be explicitly considered. The method incorporates detailed information about the plant design, 
operating characteristics, phenomenological calculations, experiments, severe accident research, and expert judgment. Latin Hypercube sampling was used to assess the phenomenological and systemic uncertainties associated with the primary and confinement system responses to the core damage accident. The use of this method and the supporting information yield results and uncertainties that are technically defensible.

Several significant technical insights were gained in the course of the $\mathrm{N}$ Reactor accident progression analysis. These include:

- More than 958 of the internally initiated accident sequences are expected to result in damage to less than the full core, while many of the fire and seismic sequences involve full core damage as a result of combined Emergency Core Cooling System (ECCS) and Graphite Shield Cooling System (GSCS) failure.

- The confinement maintains its integrity in a large majority of the internally initiated accidents and almost two-thirds of the fireinitiated core damage accidents.

- Should the confinement boundary be compromised during an internally initiated accident, it is likely (up to 508) that the failure will occur initially with the failure of the confinement isolation system rather than a phenomenologically induced confinement failure.

- Induced pressure tube rupture events caused by the delayed actuation of the ECCS after significant core heatup has occurred is a major contributor to confinement failure for the internal events and fire scenarios.

- Many of the phenomenologically induced confinement failures are caused by hydrogen combustion events inside the emergency dump tank.

- The hydrogen mitigation was found to significantly reduce the frequency of hydrogen combustion events in most areas of the confinement that might otherwise lead to confinement failure.

- Confinement integrity depends largely on the success or failure of the hydrogen mitigation system. This system succeeds for most internal and fire events, but fails in many severe, low frequency, high ground acceleration $(\geq 0.5 \mathrm{~g})$ seismic sequences.

- Graphite oxidation was not seen in any of the internal events accident progression pathways modeled, but was seen in a few of the fire and seismic analyses.

- Approximately 4.48 of all core damage accidents in which the fog spray system functions (regardless of initiator) result in liquid releases from the plant that could enter the Columbia River.

- The multiple pressure tube rupture event is important to the seismic analysis because it fails all cooling to the core, and can open up a1r ingress pathways into the graphite stack and allow graphite 
oxidation to occur. This is only seen in the very rarest highest ground acceleration (as much as three times the $N$ Reactor SSE) seismic scenarios.

- The filter system is expected to operate successfully in about $80 \%$ of the internally initiated accidents, and, for many accidents where it fails, it fails only because the filters are bypassed because of a confinement failure.

Current operating procedures and features that tend to prevent or mitigate severe accidents are already included in the analysis. Because the final risk results (as documented in the $N$ Reactor Level II/III PRA Final Report) are so low, no specific recommendations are made for plant improvements, but some plant changes are described in the main report that could improve plant response under accident conditions. It should be noted that these conclusions are based on an analysis of plutonium production operations at $\mathrm{N}$ Reactor with reactor core characteristics as they were at the time of the administrative stand down. Other operational modes were not analyzed.

The $N$ Reactor PRA has been a major effort to evaluate the $N$ Reactor risk using state-of-the-art PRA technology. While there are limitations in all PRAs, we have taken particular care to clearly identify the assumptions and limitations specific to this study. Every effort was made to be comprehensive in the consideration of systems and phenomena, resulting in highly complex accident progression models. Whenever possible, the results are based on definitive experiments, data, and calculations; expert judgments are clearly identified when they are used. While some aspects of this study tend to be slightly conservative, overall we believe that this work has resulted in realistic, as opposed to unduly conservative or nonconservative results. The uncertainty ranges depict the region within which the true answer should be expected to lie for the accidents considered in this study. Considering the uncertainties, we believe that the basic conclusions of this study are very robust. These results show that the $\mathrm{N}$ Reactor confinement concept provides significant radiological protection for most of the accident progression pathways studied. 
ACCIDENT PROGRESSION EVENT TREE ANALYSIS

FOR POSTULATED SEVERE ACCIDENTS AT N REACTOR

\section{INTRODUCTION}

\subsection{PURPOSE}

The 1982 Triennial Review of the UNC Nuclear Industries, Inc. (UNC) Nuclear Safety Review System [1-1] recommended that a Probabilistic Risk Assessment (PRA) be performed for the $N$ Reactor, a defense production reactor owned by the U.S. Department of Energy (DOE). N Reactor is the only large water-cooled, graphite moderated reactor in operation in the United States. The 1986 accident at the Chernobyl nuclear power plant in the Soviet Union provided added urgency to the PRA project because, while all experts agreed that the Chernobyl accident could not occur at $N$ Reactor, the superficial similarities between $N$ Reactor and the Chernobyl plant caused many to take a new look at safety issues at all of the DOE production reactors.

In 1986, UNC set out to perform a full scope PRA for $N$ Reactor to include all phases of the PRA process up to and including the calculation of radiological consequences to the offsite population and to workers at co-located DOE facilities on the Hanford reservation. A full scope PRA consisls of three lovels, as depicted in Figure 1.1. The first level is the identification of accident sequence frequencies. The second level analyzes the progression of those accidents through all of the potential phases to the release of radionuclides from the $\mathrm{N}$ Reactor confinement, and the third level estimates the consequences of those releases and integrates the entire PRA process into a risk perspective. The second and third levels, as shown in Figure 1.1, are each composed of two parts. Level II consists of the accident progression and source term analyses, and Level III is both the consequence analysis and the risk integration.

Under the PRA program undertaken for $N$ Reactor, UNC and its successor Westinghouse Hanford (WHC) completed a Level I PRA analysis for internally initiated events in 1988 [1-2]. At that time, the Level I analysis for externally initiated events as well as the Level II and Level III analyses for both internal and external events were still in progress. These efforts have now been completed $[1-3,4]$.

This report documents one portion of the $N$ Reactor Level II PRA: the Accident Progression Event Tree (APET) analysis. As such, it is a part of a larger research effort and should be viewed in that light. The relationship between the APET analysis and the overall PRA process is discussed in Section 1.2 below. 


\section{PRA ANALYSIS SEQUENCE}

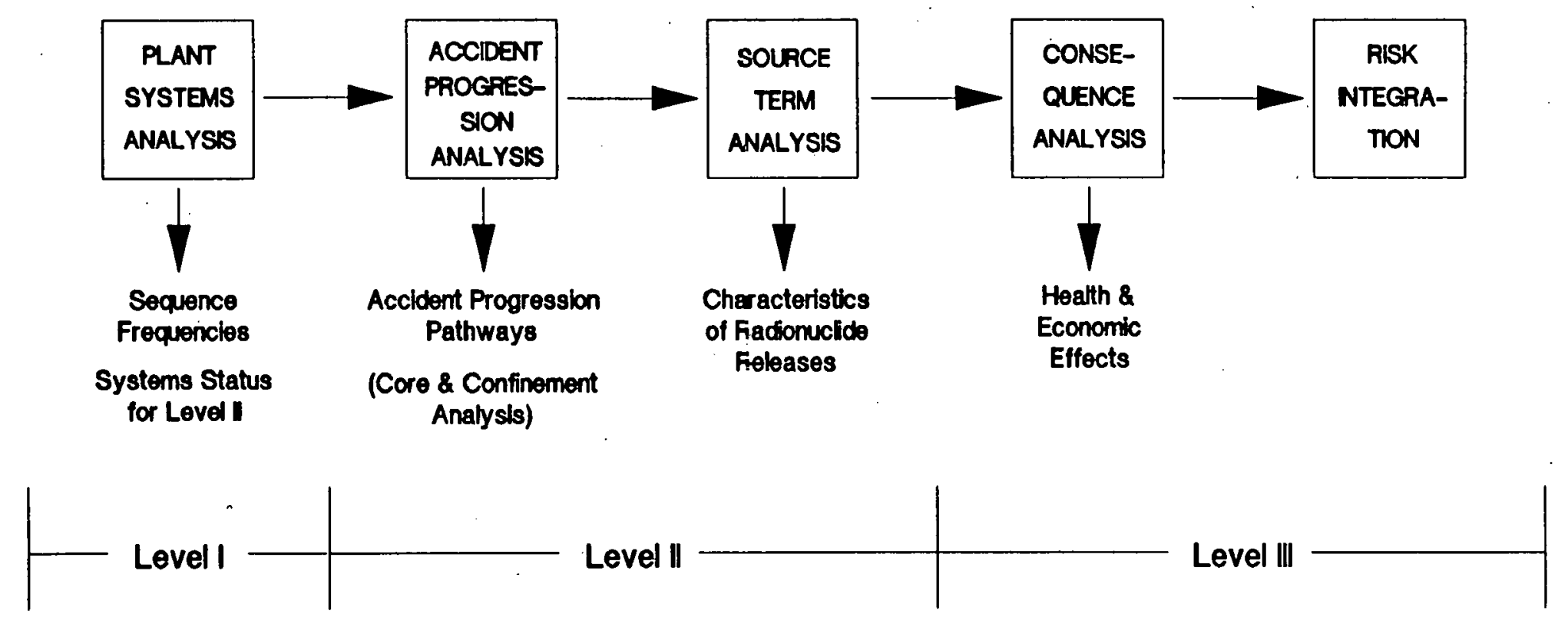

Figure 1.1. PRA Analysis Sequence 


\subsection{RELATIONSHIP TO THE OVERALL PRA ANALYSIS}

As mentioned briefly above, nuclear reactor PRA analyses are conducted in three stages. The first stage, called a Level I PRA, considers only the frequency of occurrence of core damage and its causes. A Level II PRA determines the radiological releases associated with the core damage accidents postulated in the Level I analysis. A Level III PRA computes offsite consequences from the releases described in the Level II analysis, and uses this information to make an overall assessment of risk. These stages, and the relationship of the APET analysis to them, are discussed in more detail below.

A Level I PRA consists of a probabilistic analysis of those plant systems whose failure could lead to damage to the reactor core. The events that demand the operation of the safety systems can be initiated either internal to the plant systems or external to the plant systems. An example of the former is a pipe break in the primary coolant system of the reactor, and an example of the latter is an earthquake. In the Level I analysis, success criteria are determined for the various front line core cooling systems which must respond to the plant status caused by the initiating event, and fault trees are constructed for those systems and their related plant support systems. The fault trees are solved to obtain boolean expressions representing the minimum equipment failures and operator errors required for core damage to occur. Each such minimal combination is called a cut set. The frequency of a cut set is the product of the frequencies of each of the constituent events because all of the events must happen together for core damage to occur. The final product of the level I PRA is the total core damage frequency. This is obtained by summing the frequencies of all cut sets because each cut set represents a different core damage scenario. Examination of the individual cut sets is also instructive because they reveal the most likely causes of core damage and, often, the most cost effective ways to reduce the frequency of such an outcome.

A Level II PRA analysis takes the core damage scenarios found in the Level I analysis and determines the radioactive source terms that are possible for each one. A "source term" is a full description of the release of radionuclides to the environment during a particular core damage accident scenario. Such a description would include the form of the release (airborne, liquid, etc.), the magnitude and composition of the release (the amount of each radionuclide), and other characteristics of the release such as the altitudes and times over which the release occurs.

The process of determining the possible source terms for each Level I core damage sequence involves determining the responses of the containment or confinement structures and systems since these responses can have a significant mitigative effect on the magnitude of the release. It also involves predicting the size of the radioactive release from the fuel and tracking it through the plant and out to the environment. In addition, it must consider the mechanisms available within the plant for retention and mitigation of the release and estimate the timing and energy of the 
potential release. The end result of the Level II analysis is a set of possible radioactive source terms and their associated frequencies.

The Level III PRA analysis takes the set of possible source terms generated in the Level II analysis and determines the expected radiological consequences for them. The consequences are usually presented in terms of a number of cancers, early deaths, and land interdiction costs. The costs are then weighted by the source term frequencies to arrive at an overall estimate of the risks (defined as the expected consequences per year) associated with operating the plant. The risk estimate is the end product of the overall PRA project.

The APET analysis documented in this report is the first part of the Level II PRA. Specifically, the APET models the possible responses of the confinement to the accident scenarios predicted by the Level I PRA. Thus, it uses the results of the Level I PRA [1-2,3] for input to determine which accident sequences are to be analyzed. It also draws input from fault tree analyses that have been performed on the confinement systems $[1-5,6]$ that were analyzed separately from the Level I PRA. It must also consider various phenomenological possibilities, such as hydrogen combustion, that can have a profound effect on the amount of the radioactive release. The result of the APET analysis is a list of accident progression pathway descriptions. These descriptions include those features of the each accident progression that are relevant to the calculation of the source term by the NSOR code [1-4] such as the Level I core damage event, the confinement system status, and all relevant confinement phenomena that occur during the accident. The frequency associated with each pathway is also calculated in this analysis. A more complete description of the APET methodology and structure are found in Chapters 2 and 3 , respectively.

The results of the APET analysis are used as input to the source term model. The source term model screens the accident progression pathways for events that will make a difference in the overall radioactive release and, from these events, determines the source term for the accident progression pathway defined by the APET analysis. The output of the source term model is a list of source terms and their associated accident progression pathways and frequencies. This is the final product of the Level II PRA. The Level II portion of the PRA, as well as the Level III portion, is presented in Reference 1-4.

The analysis of the accident progression necessitated having knowledge of the phenomena which could potentially occur at $\mathrm{N}$ Reactor during severe accidents. We relied heavily on phenomenological analyses conducted by WHC and its subcontractors, but we also adapted and applied phenomenological computer codes ourselves during the course of this project. The adaptations were necessary because the codes had originally been developed for application to severe accidents occurring in commercial reactors, and the adaptations are documented in References 1-7, 1-8, 1-9, and 1-10. The calculations which we did in support of the PRA in general and the APET in particular are presented in References 1-11 and 1-12.

Finally, an important part of a PRA is the incorporation of uncertainties into the analysis. As discussed in Chapter 2, we used the 
PRA methodology developed for the recently published NUREG-1150 study, which performed PRAs for five commercial reactors for the U.S. Nuclear Regulatory Commission [1-13]. This methodology explicitly treats systemic and phenomenological uncertainties.

\subsection{REPORT OVERVIEW}

This report is written in six chapters. Chapters 1 and 2 provide an introduction to the purpose and scope of the accident progression event tree analysis project. Chapter 2 provides more detailed background information about the methodology used, assumptions made, and boundary conditions of the APET analysis. Chapter 3 contains a detailed description of the questions asked and logic structure used in the construction of the APET. Chapter 4 details the methods by which the uncertainties from the Level I PRA, confinement system fault tree analysis, and Level II phenomenological issues were incorporated into and propagated through the APET analysis. Chapter 5 presents the detailed results of the APET analysis for the various classes of accidents analyzed. Finally, Chapter 6 presents conclusions, insights and recommendations gained from the APET analysis.

It is the intention of the $N$ Reactor Level II/III analysis team that all relevant data and calculations be reproducible from the information presented in these final reports. For this reason, there are numerous appendices following the body of this report. They include reference calculations for specific data, documentation of the uncertainty issues, and input and output from the various computer code packages. The reader is encouraged to refer to these appendices to gain a clearer understanding of the details of the analytical method used in the generation of these results. 


\subsection{REFERENCES}

1-1. H. J. Kouts and M. C. Leverett, Report of Three-Year Review of UNC Nuclear Safety Review System, Brookhaven National Laboratory, 1982

1-2. M. D. Zentner, et al, N Reactor Level I Probabilistic Risk Assessment: Final Report, WHC-EP-0322, Westinghouse Hanford, August 1988

1-3. J. A. Lambright, et al., Analysis of Core Damage Frequency due to External Events at the DOE $N$ Reactor, SAND89-1147, Sandia National Laboratories, 1990.

1-4. D. M. Kunsman, et al., Level III Probabilistic Risk Assessment for $N$ Reactor, WHC-MR-0045, SAND89-2102, Prepared by Sandia National Laboratories for Westinghouse Hanford, April 1990.

1-5. G. A. Coles, N Reactor Confinement System Sequence Analysis Including Recovery, WHC-SD-NR-RA-001, Westinghouse Hanford, 1989.

1-6. G. A. Coles, Westinghouse Hanford, Letter to D. M. Kunsman and G. D. Wyss, Sandia National Laboratories, "Effect of Fire Sequences on Confinement System for $N$ Reactor Level 2 Probabilistic Risk Assessment," Westinghouse Hanford Correspondence \#8953739, June 21, 1989.

1-7. G. D. Wyss, "N-Reactor Core (CRN) Package Users' Guide," Draft Version 1.8XA, Sandia National Laboratories, March 17, 1989.

1-8. A. L. Camp and S. E. Dingman, HECTR Version 1.5N--A Modification of HECTR Version 1.5 for Application to N Reactor, SAND87-0027, Sandia National Laboratories, May 1987.

1-9. L. A. Miller, Sandia National Laboratories, letter to Sam Wood, Westinghouse Hanford, "HECTR Version 1.51N," February 17, 1988.

1-10. J. L. Tills, The CONTAIN Code Severe Accident Confinement Analysis for the N-Reactor: Phase 1 and 2, SAND88-7010, Sandia National Laboratories, November 1988.

1-11. L. A. Miller, et al., N Reactor Probabilistic Risk Assessment Supporting Calculations, SAND89-2101, Sandia National Laboratories, 1990.

1-12. A. C. Payne, Jr. and A. L. Camp, Parametric HECTR Calculations of Hydrogen Transport and Combustion at N Reactor, SAND86-2630, Sandia National Laboratories, June 1987.

1-13. Severe Accident Risks: An Assessment for Five U,S. Nuclear Power Plants, NUREG-1150, Second Draft for Peer Review, U.S. Nuclear Regulatory Commission, June 1989. 


\section{ACCIDENT PROGRESSION OVERVIEW}

\subsection{BACKGROUND}

This chapter briefly presents the methodology used in analyzing the progression of postulated severe accidents at $\mathrm{N}$ Reactor. This methodology follows closely that developed in the recently published NUREG-1150 document [2-1], which is a state-of-the-art probabilistic risk assessment (PRA) for five commercial reactors. The method we employed for the Level II/III PRA of $N$ Reactor [2-2] is basically that presented in that U.S. Nuclear Regulatory Commission document. The methodology section below describes briefly the tasks necessary to perform an analysis of the accident progression, discusses in general how phenomenological calculations are incorporated into the analysis, presents the reasons and the means for including uncertainties in the study, and describes the computational tool we used in performing the accident progression analysis.

This chapter also contains a list of the important assumptions, both systemic and phenomenological, used during the course of the analysis. If these assumptions are wrong, then the results discussed later should be revisited as any wrong assumptions could cause those results to change. In this same vein, important boundary conditions of our analysis are also listed. These include important procedural steps that, if changed, would also potentially change the results.

\subsection{METHODOLOGY}

\subsubsection{Introduction}

The analysis of the accident progression is the second major portion of a full Level III PRA. The purpose of the accident progression analysis is to track the physical progression of the accldent from the initiating event to the conclusion of all releases of radioactive material (if any) from the confinement building or liquid retention facilities. Because of the unique $N$ Reactor design where loss of core cooling would not always, or even usually, be an all or nothing situation, much effort has been expended in describing the levels of core cooling possible for each group of cut sets identified by the Level $I$ analysis as well as the effect of these different levels of cooling on the fuel damage process. At the same time, the accident progression analysis tracks the impact of the accident on the confinement building and the confinement system response.

For each general type of postulated accident, the analysis considers the important characteristics of the fuel damage process, the challenges to the confinement building, and the response of the confinement to those challenges. To organize and quantify the large amounts of information used in this analysis, an accident progression event tree (APET) has been 
constructed. The APET combines information from many sources, e.g., detailed mechanistic computer simulations, experimental results, and expert judgment when necessary, and it is the principal tool used for the delineation and characterization of the possible scenarios in this study. Computer processing of this event tree allows the construction of as large and complex a tree as necessary to describe the progression of severe accidents at $\mathbf{N}$ Reactor. Such a tree has a large number of possible outcomes. The outcomes can be grouped according to the potential effect on the source term and then passed to the source term analysts for further analysis.

\subsubsection{Principal Subtasks}

There are five principal subtasks for the accident progression analysis.

2.2.2.1 Interface with the Level I Core Damage Analysis. The Level II accident progression and source term analyses build on the results of the Level I core damage analysis. Thus, considerable effort is required to ensure that the interface between those analyses is logical and consistent. The cut sets generated in the Level $I$ analysis define the "initial conditions" of the plant for the accident progression analysis. The effects of the system failures that occur in a given cut set must be propagated through the accident progression analysis in order for the results of that analysis to be reasonable. For example, the state of the plant's electrical system can be very important to the accident progression analysis. This information is frequently contained in each cut set from The Level 1 analysis. Thus, it is the job of the accident progression analyst to extract that information from the cut sets and use it in the accident progression analysis.

Extracting and using systems information from the thousands of Level I PRA cut sets can be very time consuming--especially when uncertainty is also to be considered in the analysis. There is no real substitute for a cut set by cut set analysis of this information where the analyst groups the cut sets according to the systems that are operating. Once this initial grouping is done, however, the fraction of the core damage frequency that each group of cut sets represents can be determined automatically by the TEMAC code as discussed in Chapter 3 of Reference 2-2. This is eventually done by TEMAC for Monte Carlo sample member for the uncertainty analysis, with the results being fed directly to the event tree analysis program.

2.2.2.2 Development of Accident Progression Event Tree. An accident progression event tree is used to identify, to order sequentially, and to quantify probabilistically the important events associated with the progression of a hypothetical severe accident at $N$ Reactor. The development of the APET consists of identifying potentially important issues, determining possible values of each parameter (including the dependencies on previous parameters in the event tree), ordering the events chronologically, and defining the information needed to determine each parameter. The information base consists of accident experimental data and 
results from accident simulation computer codes. Many safety studies have occurred at $\mathrm{N}$ Reactor in recent years, and the results from them have been used as appropriate in this PRA.

While the event tree development process used in this study is conceptually similar to that used by other PRA practitioners, the complexity of the tree (the number of parameters and possible outcomes) is, in general, substantially greater than those done prior to NUREG-1150. This permits more explicit use of the data base of severe accident experimental and calculational information, and allows the complexities and dependencies of the unique reactor design to be explicitly represented.

2.2.2.3 Structural Analysis. Full utilization has been made of the existing structural analysis and experimental efforts at $N$ Reactor to determine the building responses to accldent loadings. In addition, as described in Reference 2-3, the relief capacity characteristics of the banana wall have been studied as part of this PRA. In addition, we have used recently performed analyses, as well as previously completed work, concerning the integrity of the pressure tubes.

2.2.2.4 Probabilistic Quantification of Event Tree Issues. With the use of the APET logic structure and information base developed in the previous steps, probability distributions of those uncertain with the greatest capacity to influence the accident progression event tree results have been generated. These issues are usually phenomenological in nature. As is typical of any PRA, this assignment of parameter values is subjective, based on the interpretation of the data base by the risk analyst. For a number of uncertainty issues, however, people knowledgeable about the issues were elicited using formal expert judgment techniques. (The technique is documented in Reference 2-4.) Also for this study, the documentation of the rationale for the distributions used is presented in Chapter 4 and in more detail in Appendix $C$.

Events other than those concerned with the uncertainty issues also appear in the APET, and they are usually systemic in nature. Examples of such events are the initial conditions established by the Level I accident sequeluc frequeney cvaluation. These values are not constant because there is uncertainty about the actual fallure rate of plant components as expressed in the Level I PRA analysis. Thus, the APET analysis also accounts for this type of uncertainty. The treatment of the systemic events is discussed in Chapter 4 and in more detail in Reference 2-2.

2.2.2.5 Grouping of Event Tree Outcomes. An accident progression event tree produces a large set of alternative outcomes. (A tree with eighty binary questions can have more than Avogadro's number of outcomes.) As is typically done in PRAs, the outcomes are grouped into a smaller set of "accident progression bins," where all the outcomes which are grouped into a given bin are estimated to have the same effect on the analysis of the radioactive material transport. The definition of the bins is a joint effort of the accident progression and source term analysts. The product of the accident progression analysis then is a set of accident progression bins with the frequenoy of occurrence of each bin. 


\subsubsection{Incorporation of Phenomenological Calculations}

To gain phenomenological insights for the formulation of the APET logic and the quantification of its branch probabilities, we modified and applied several deterministic computer codes to the geometry, physics, chemistry, and systems of the unique $N$ Reactor design. The writing and application of these computer codes were originally sponsored by the U.S. Nuclear Regulatory Commission so that they could be used to study severe accident phenomena at commercial nuclear power plants, and the differences in design between them and $N$ Reactor necessitated the modifications. These modified codes were then applied to specific problems identified during the course of the accident progression analysis in order to gain understanding of how the underlying phenomena could affect the accident progression. Other analyses performed by either WHC personnel or other wHC contractors were also used to gain this understanding. The calculations we performed are documented in Reference 2-3, and the other analyses are referenced as appropriate throughout the remainder of this report.

It is vitally important to realize that rarely in performing the accident progression analysis are the numerical results of a specific phenomenological code calculation used directly. A single, detailed phenomenological calculation is relevant to a very limited set of the plethora of possibilities for the accident progression. For other possibilities, the systemic or thermal-hydraulic conditions would be different, and the actual numerical results could change as well. No one funding a PRA can afford to expend the resources necessary to perform detailed calculations for each and every possibility, and even if that could be done, there would still be uncertainty in the results due to the approximate nature of computational representation of physical processes and due to some necessary coarseness in the model inputs (every molecule cannot be modeled individually).

Hence, in this PRA as in others, the insights gained from the phenomenological calculations of a limited set of accident possibilities are applied to a broader spectrum of accidents. The usages in the accident progression analysis to which the code calculations were put will become apparent in Chapter 3 when the APET is described and in Chapter 4 when the uncertainty issues are discussed.

\subsubsection{Accident Progression Uncertainty Analysis}

As stated above, an important characteristic of this probabilistic risk assessment in general and this accident progression analysis in particular is the explicit inclusion of an estimation of the uncertainties in the analysis. These uncertainties exist because of incomplete understanding of both systemic and phenomenological parameters and inadequate capability of modeling the current state of understanding. This does not mean that this PRA was incompetently performed. Rather, it is an acknowledgement that there are many areas where there is a lack of definitive knowledge and that computations are just approximations of reality and not reality itself. 
The total number of parameters that could be varied to produce an estimate of the uncertainty in the accident progression is large, but the number of parameters that can be varied is limited by the computer capacity required to execute the uncertainty analyses. Thus, only the most important sources of uncertainty were included in the analysis. The uncertainty issues that were used were selected because each represents a possibility for either a significant additional release of radionuclides from the fuel, a release of radionuclides to the environment, or a major change in the progression of the accident. The method used for selection of APET issues is not glamorous or profound, but rather practical. Many candidate issues were determined based on an examination of all phenomena modeled in the APET. An examination of the candidate issues was done to find the issues that had both the potential to significantly change the accident progression and significant uncertainties associated with them. This was done by examining the results of point estimate APET analyses and selecting issues that were both uncertain and significant to the results of the point estimate analysis.

Subjective probability distributions for the parameters identified as uncertainty issues were estimated in one of two ways. First, if another PRA had thoroughly examined the uncertainties of a particular parameter and the situation concerning that parameter at the uniquely designed $N$ Reactor was similar to a situation concerning that parameter at another reactor with a different design, then the information from the other PRA was used. Second, if no such thorough examination could be found or the situation at $\mathrm{N}$ Reactor was dissimilar, then formal elicitations of the project staff and knowledgeable personnel at WHC were made. These are presented briefly in Chapter 4 of this report and in detail in Appendix $C$.

In order for uncertainties in accident phenomena to be included in the PRA, they had to be expressed in terms of uncertainties in the parameters that were actually used in the analysis. Each analysis part, however, did not calculate the characteristics of the accidents in as much detail as would a detailed mechanistic computer "code. In the accident progression analysis, the uncertain input parameters are "high level," and the relationships between fundamental physical parameters and the high level parameters may not always be clear. This lack of understanding can lead to a form of modeling uncertainty. In addition, the values of some important physical or chemical parameters are not precisely known and can result in uncertainties in the high level parameters. These uncertainties can be referred to as data uncertainties. Both types of uncertainties--modeling and data--are included in this analysis, and no attempt is made to differentiate between them.

As to the differences between what is a fundamental parameter and what is its high level representation, consider the generation of hydrogen within the pressure tubes when steam is flowing through them, but the steam is insufficient to cool the fuel. The fundamental physical parameters involved in the amount of hydrogen generated may be the temperatures of the steam and zirconium molecules and the mobility the steam molecule has within the pressure tube. The sampled variable, however, could be the 
amount of coolant flow through the core as that might be determined, in a somewhat crude way, to indicate the block temperatures seen within the pressure tube.

Probability distributions for input parameters were developed by a number of methods. As stated above, some were taken from previous studies if the relevance was determined, and others were developed by the elicitees. Those developed subjectively followed the method described in Reference 2-1 and were based on the interpolation and extrapolation of detailed code calculations and existing experimental data. The development of the probability distributions and the reasoning behind the distributions are described in detail later in this report.

A specialized Monte Carlo method, Latin Hypercube sampling, was used to sample the probability distributions defined for the many input parameters. The sample observations were propagated through the analysis of the accident progression event tree to produce a set of accident progression bins and their frequencies of occurrence for each sample member. The calculation was repeated for each sample member, each of which can be thought of as being one view of the accident progression reality. In the PRA, the accident progression bins produced by the analysis of each sample member were passed to the source term analysis (see Reference 2-2 for a complete description of the process).

Monte Carlo methods easily treat distributions with wide ranges and can incorporate correlations between variables. Latin hypercube sampling provides for a more efficient sampling technique than straight-forward Monte Carlo sampling while retaining the benefits of the technique. It has been shown to be an effective technique when compared to other, more costly, methods [2-5]. Since some of the probability distributions used in this accident progression analysis are subjective distributions, the composite probability distribution for APET outcomes must also be considered subjective.

\subsubsection{Accident Progression Analysis Tool}

The APET was constructed and analyzed using the EVNTRE code [2-6]. EVNTRE is a generalized event tree processor and has been developed at Sandia National Laboratories. Although it can be used to analyze any event tree, the code was specifically developed to process complex event trees that systematically follow the progression of severe accidents in nuclear power plants. The code treats event trees which can be very complicated in structure. They can have multiple branches at each question and the branch probabilities at each question can be dependent on the exact path taken through the tree to arrive at that question. EVNTRE accommodates continuous processes by allowing branch probabilities that are functions of path dependent parameters. Furthermore, the capacity of the code is large so that time dependencies can be analyzed by dividing the tree structure into several time regimes. In addition, it can process multiple input sets so that it is compatible with Monte Carlo sampling for uncertainty analyses. 
Previous event tree analyses often oversimplified the problems being considered because they were incapable of processing large and complex trees. In this analysis, much of this restriction has been removed because of the capabilities of EVNTRE.

\subsection{ACCIDENT PROGRESSION ANALYSIS ASSUMPTIONS AND BOUNDARY CONDITIONS}

In conducting the accident progression analysis portion of this PRA, we found it necessary to make several assumptions which, if changed, could change the results. It is thus incumbent upon us to list these assumptions. In addition, the analysis was performed with certain factors in mind that are more boundary conditions than assumptions. Altering those conditions could also change the results, and thus the reader should be apprised of those conditions as well.

\subsubsection{Assumptions}

The assumptions we have made are of two types: systemic and phenomenological. The systemic assumptions are:

1. We have assumed that the hydrogen mitigation system is completely installed and can operate when called upon to do so. It effectively mitigates many potential problems within the buildings which could be caused by combustible gas generation. In addition, we have assumed that the hydrogen mitigation system emergency operating procedures are written as they have been discussed with us by WHC personnel. These procedures are discussed in more detail in Chapter 3.

2. The cabling for the nitrogen subsystem of the hydrogen mitigation system is assumed to be routed as currently designed. The current design calls for the nitrogen subsystem cables to leave Room 6 near where the $V-3$ and $V-4$ valve cables leave that same room, and proceed along a route that does not go through any of the identified core damage fire areas. Any rerouting of these cables through core damage fire areas would significantly change the results of this accident progression analysis.

3. For those seismic accident sequences in which the silo is failed but the ECCS and primary system piping is intact, we do not give any credit for using alternate water supplies, such as those from connections to other sites on the Hanford Reservation. Procedures do not exist for doing so, and we believe that there is a strong likelihood that the alternate supplies would be unavailable anyway due to the earthquake.

4. We assume that the filter path and filters will not fail due to vacuum as long as the vacuum breakers operate successfully. This question arose during the course of this analysis, and wilC personnel and we had some doubt as to whether the entire filter path was qualified adequately for vacuum, particularly as the path 
begins at the 105 building. We were assured by plant personnel that prior to restart it would be examined and, if it could not maintain its integrity in a vacuum, it would be fixed. If it is not qualified for vacuum and is not fixed, then any accident in which fog sprays operate leads directly to confinement failure.

5. We have analyzed the plant with the emergency dump tank return line venting at the top of the pipe gallery although there has been some discussion about cutting the pipe so that the returned gases enter the confinement at the bottom of the pipe gallery. If the pipe height was cut, this would change the mixing of any hydrogen returning from the dump tank with the confinement atmosphere, be it undergoing nitrogen injection from the hydrogen mitigation system or not.

6. In almost all cases, we have assumed that the operators will follow the procedures as written, with failing to do so being modeled as a human error. Thus for example, the operators are assumed to leave the fog spray system operating once it is actuated for the duration of the accident since they are not instructed to turn it off. This action (or "non-action") can, in some scenarios, lead to undesired results as discussed in Chapter 3.

7. An exception to systemic assumption 6 above is that we have assumed that in one situation, the operators may not follow procedures. That situation involves reflooding the core after a time specified by procedures has elapsed. We treated this error of commission as an uncertainty variable, and our reasons for even considering it are discussed in detail in Chapter 3.

8. There is no procedural guidance for the operators when the helium blanket around the graphite stack is lost. As such a loss degrades the GSCS heat removal capability, we assumed that the operators will treat that loss as a loss of GSCS function for which there is procedural guidance.

The phenomenological assumptions we have made are:

1. There is a possibility that an additional accident sequence exists at $\mathrm{N}$ Reactor which is not currently included in this PRA. It is not included because that possibility is undergoing intense analysis at WHC to ascertain whether or not it would in fact cause core damage, and if so, what the system response and accident frequency would be. The hypothetical sequence is initiated by a large break LOCA ( $4^{n}$ or greater in diameter) in one of the inlet risers which is postulated to defeat both primary cooling and the emergency core cooling systems. A riser break between the CV-2R and $C V-5$ valving could both be an initiating event and be the cause of all cooling failure to the 1/16th of the core which is fed by that riser. The internal events Level I PRA [2-7], on the other hand, assessed that there were no break locations which 
would defeat both primary and ECCS cooling to any region of the core. The possibility of this scenario was first raised in Reference 2-8, but it appears as of this writing that only a very limited set of all the possible break locations and sizes which could occur in the inlet riser area can possibly defeat the cooling to the riser. As this issue is highly uncertain at this time and is being investigated further to ascertain even if it is credible let alone what the effect of it is, the accident progression analysis presented herein omits the scenario from further scrutiny.

2. We assume that ECCS functions properly in all ATWS scenarios because, as discussed in Chapter 3 , current analyses indicate that ATWS with ECCS success can cause fuel damage. On the other hand, ATWS with ECCS failure does not return to power and, thus, would not be significantly different from a non-ATWS ECCS failure accident. In addition, ECCS failure combined with ATWS is less than 28 of all ATWS sequences, and ATWS itself is a low frequency event. Thus, ATWS with ECCS failure is a trivial frequency contributor to similar, non-ATWS core damage scenarios for which consequence analyses are already being performed. For this reason, ATWS events with ECCS failure are ignored.

3. As is the case for the external events Level I PRA analysis [2-9], we assume that a fire in an area instantaneously affects all cables in that area. Thus, if ECCS and confinement cables both go through an area, all are assumed to fail simultaneously:

4. Early in this project, we could detect no difference in confinement response to different initiating events whether one cross-vent was open or more to the resolution with which we nodalized the building. As one cross-vent was always open, we have assumed a cross-vent reliability of 1.0 .

\subsubsection{Boundary Conditions}

In addition to the above assumptions, the analysis was performed with other boundary conditions. These conditions arose because they either previously existed or were a result of investigations we performed during the course of the study. As to this latter kind, often in work such as this, the documentation includes only that portion of the analysis which remains and neglects those items which were explored but assessed to be irrelevant for the particular study. Below, we briefly mention these boundary conditions. They are:

1. We are using systems analysis done by other research efforts, and we assume that the internal and external events Level I PRA analyses $[2-7,9]$ are correct as is the confinement systems fault tree analysis $[2-10]$. 
2. The operating status of the V36 valve in our analysis is open which was the normal configuration of the valve when $N$ Reactor last operated. If the valve would be closed, flow into the two outlet riser flush lines would no longer be connected. The supporting calculations to this analysis [2-3] have shown that this communication is important in the event of failures of v3 or V4 valves on one side of the core in that some cooling is provided to the uncooled side through this open connection.

3. We have not analyzed the deliberate causing of accident sequences. Acts of sabotage are not within the scope of the analysis.

4. The treatment of design and construction errors in PRAs is the subject of methodology development activities. For this effort, we have analyzed the plant with its systems being capable of performing their design functions, and have assumed that the systems have been constructed as designed or documented in "as built" specifications.

5. Our analysis presumes that the procedures for initiating the nitrogen injection portion of the hydrogen mitigation system are such that the operators will do so during any accident which involves the entire core. Cooling of the filters by the nitrogen flow in many such scenarios ensues from this presumption.

6. The cooling of the filters is either adequate or they burn. There is no intermediate state in our analysis for "undercooled" filters.

7. Several phenomena which can arise in the progression of severe accidents at commercial reactors have been judged by us to be not credible at $N$ Reactor due to the core design which employs individual pressure tubes instead of one pressure vessel for the fuel. These include core-concrete interactions, direct heating of the building atmosphere, and in-core steam explosions. For the first of these, we are unable to identify any means by which gross core relocation could occur so that molten fuel would flow to the exterior of the graphite stack and subsequently attack the concrete floor. For the latter two phenomena, we cannot conceive of any series of events which are not incredible by which a sufficient amount of molten fuel could be co-located so as to effect the phenomena. (As to steam "explosions" in general, as explained in Chapter 3, molten fuel interactions with water, which are smaller than the "classic" molten fuel-coolant interaction, are possible and analyzed as leading to steam spikes.)

8. The environmental qualification of the equipment within the confinement is not an issue in this study. First, there are very few components which are used to respond to accidents that are actually inside of the confinement. Second, if the steam vents are demanded and work, the confinement atmosphere is relatively dry and cool. Third, if the steam vents are demanded and do not work, the confinement will either fail or the hot moisture will 
relieve through the banana wall. Fourth, if the steam vents are not demanded, the confinement atmosphere is relatively dry and cool.

9. The flow from the V4 valves will go to the dump tank and not to the radwaste system. During shutdown, the V23 valves can be aligned such that the flow through the V4 valves goes to the radwaste system. At startup, the position of the V23 valves are double-checked and mechanically gagged to insure that the flow would go to the dump tank. They are not accessible during reactor operations, and the indication of the valves are verified frequently in the main control room so that we dismissed the misalignment on probabilistic grounds. Further, we examined the details of the internal design of the valves and judged that the impulse loading of them resulting from the opening of the V4 valves at system pressure would not fail them.

10. The failure of the helium blanket rupture disk from overpressurization within the graphite stack is included explicitly in the APET. Such failure will occur at 3 psid. Failure of the disk from overpressurization in the opposite direction is deemed to be negligible probabilistically except when challenged by a local hydrogen detonation. The disk is convex and can withstand a greater differential pressure from the "outside in" direction (at least 10 psid), and, except for hydrogen detonations, we have not seen such pressures in the vicinity of the disk in our analyses.

11. This analysis was conducted using the $\mathbf{N}$ Reactor mission and core design (fuel loading) as they were at the beginning of the administrative stand down. Specifically, $N$ Reactor was analyzed in its plutonium producing mode of operation where fuel enrichments do not exceed 1.258 235U. This study and its conclusions would have to be revisited and, possibly, significantly revised if $N$ Reactor were to be configured for a different mission with different core characteristics or operating procedures.

12. Based on commitments from WHC, we have analyzed the plant as if the identified Corridor 19 fire protection improvements had been installed. This is consistent with the external events core damage analysis [2-9]. 


\subsection{REFERENCES}

2-1. Severe Accident Risks: An Assessment for Five U.S. Nuclear Power Plants, NUREG-1150, Second Draft for Peer Review, U.S. Nuclear Regulatory Commission, June 1989.

2-2. D. M. Kunsman, et al., Level III Probabilistic Risk Assessment for $N$ Reactor, WHC-MR-0045, SAND89-2102, Prepared by Sandia National Laboratories for Westinghouse Hanford, April 1990.

2-3. L. A. Miller, et al., N Reactor Probabilistic Risk Assessment Supporting Calculations, SAND89-2101, Sandia National Laboratories, 1990.

2-4. T. A. Wheeler, et al., Analysis of Core Damage Frequency from Internal Events: Expert Judgment Elicitation, NUREG/CR-4550, Vo1. 2, SAND86-2084, Sandia National Laboratories, April 1989.

2-5. R. L. Iman and J. C. Helton, A Comparison of Uncertainty and Sensitivity Analysis Techniques for Computer Models, NUREG/CR-3904, SAND84-1461, Sandia National Laboratories, May 1985.

2-6. J. M. Griesmeyer and L. N. Smith, A Reference Manual for the Event Progression and Analysis Code (EVNTRE), NUREG/CR-5174, SAND88-1607, Sandia National Laboratories, September 1989.

2-7. M. D. Zentner, et al., N Reactor Level 1 Probabilistic Risk Assessment: Final Report, WHC-EP-0322, Westinghouse Hanford Co., Auguse 1988 .

2-8. M. A. Bolander and C. D. Fletcher, Simulation of Inlet and Outlet Riser Break Sequences in the $\mathrm{N}$ Reactor, EGG-TFM-7930, Idaho National Engineering Laboratory, February 1988.

2-9. J. A. Lambright, et al., Analysis of Core Damage Frequency Due to External Events at the DOE N-Reactor, SAND89-1147, Sandia National Laboratories, 1990.

2-10. G. A. Coles, $N$ Reactor Confinement Systems Sequence Analysis Including Recovery, WHC-SD-NR-RA-001, Westinghouse Hanford, March 1990. 


\section{DESCRIPTION OF THE ACCIDENT PROGRESSION EVENT TREE}

\subsection{INTRODUCTION}

The reasons for and methodology behind the development of the $N$ Reactor Accident Progression Event Tree (APET) were discussed in Chapters 1 and 2. The purpose of this chapter is to describe the APET events, branches and logic in detail. Section 3.1 provides a brief description of $\mathrm{N}$ Reactor, an introduction to the structure of the $\mathrm{N}$ Reactor APET and the notation used in the detailed APET description. Sections 3.2 through 3.8 are detailed descriptions of the seven sections of the APET. Section 3.9 provides a discussion of the method by which the event tree output is grouped for the source term analysis. The chapter is summarized in Section 3.10 .

\subsubsection{Description of $\mathbf{N}$ Reactor}

$\mathrm{N}$ Reactor is a pressurized water reactor, designed by General Electric and located on the Columbia River within the Hanford Reservation in the south-central part of the state of Washington. (Most of the information contained in this subsection comes from the "N Reactor Updated Safety Analysis Report" or NUSAR for short [3-1].) It has a safety analysis design basis power limit of $4080 \mathrm{MWt}$. Although steam can be supplied to turbine generators owned by the Washington Public Power Supply System, the primary purpose of $\mathrm{N}$ Reactor is the production of special nuclear material for the DOE. The reactor began operation in 1963 and was put into standby status in 1988 .

The zircaloy-clad, low enrichment (less than 1.38235 ) metallic alloy uranium fuel is arrayed in 1003 horizontal pressure tubes, through which flows the water coolant. The zircaloy tubes are supported by a Lincoln-log assemblagc of graphite moderator hlnrks. The twelve steam generators are horizontal, and ten of these are normally in operation. Two diverse reactor protection systems provide for rapid reactor shutdown. They are a system of 84 horizontal boron-carbide control rods and a system of boroncarbide balls held in hoppers above the core. The pumps of the emergency core cooling system (ECCS) are individually diesel-driven, and the injection into the fuel tubes occurs through sixteen headers, each containing separate valving. Thus, it is possible to fail cooling to a portion of the core while maintaining flow to the rest. The ECCS provides initial coolant to the core at 330 psi; therefore, the primary coolant system is provided with a depressurization capability into an emergency dump tank (EDT) filled with subcooled water. After the ECCS pumps have exhausted the onsite stored water, suction for the pumps will be supplied from a silo that is kept filled with water by low-lift pumps pumping from 
the Columbia river. The low-lift pumps are also individually dieseldriven. Figure 3.1 shows the above features graphically in a simplified primary and emergency cooling system flow model for the various modes of $\mathrm{N}$ Reactor operation examined in this analysis.

Liquid overflow from the EDT egresses to either the Liquid Effluent Retention Facility (LERF), the current design preference, or the Liquid Waste Disposal Facility (LWDF), formerly the designed receptor and now the backup to the LERF. Gas discharged to the EDT or generated within it is vented back to the reactor building. A graphite and shield cooling system (GSCS) removes heat from the moderator during normal operation. The GSCS heat removal capacity is sufficient to prevent core damage without additional assistance from the ECCS after the reactor has been shut down and cooled for approximately two hours. A high pressure injection (HPI) system provides about $600 \mathrm{gpm}$ of makeup during normal operation.

N Reactor does not have a containment building but rather is designed with a confinement. The confinement principle' is to allow the steam released in a rapid primary system blowdown to escape the building through vents opened by the pressure rise. These vents then reclose to prevent the subsequent release of radioactive material. Subsequent pressurization is relieved through a filtered stack. The buildings and components associated with confinement operation are shown in Figures 3.2 and 3.3 .

There is an additional connection between the reactor building and the refueling bay through a water-filled refueling canal. A "banana" wall extends downward into the water in the refueling canal to provide isolation from the remainder of the plant. In some scenarios in which the confinement pressurizes, the pressure will relieve through the water under this banana wall. A diagram of the banana wall is shown in Figure 3.4 .

The confinement design pressure is $5 \mathrm{psig}$, and two interconnected buildings comprise its pressure boundary: the reactor building (the 105 building) and the primary coolant system building (the 109 building). After any initial pressure rise is relieved, the pathway to the filter/stack building (the 117 building), which has a design pressure of 2 psig, forms the remainder of the pressure boundary. The EDT is exterior to any of these buildings. Also provided for the confinement are fog sprays whose pumps are independently diesel-driven. (The fog spray pumps, pumping through different piping, can provide a diverse and redundant means of pumping water through the GSCS tubes in the graphite moderator.) Spray actuation is location dependent. The confinement is provided with vacuum breakers should the spray actuation result in a vacuum. Laterally, much of the confinement abuts other building volumes.

A hydrogen mitigation capability has recently been designed, and some of it was built prior to the stand down of the reactor. The system is a post-accident nitrogen inerting/purge system and an internal fan recirculation system, of which the latter has already been installed. 


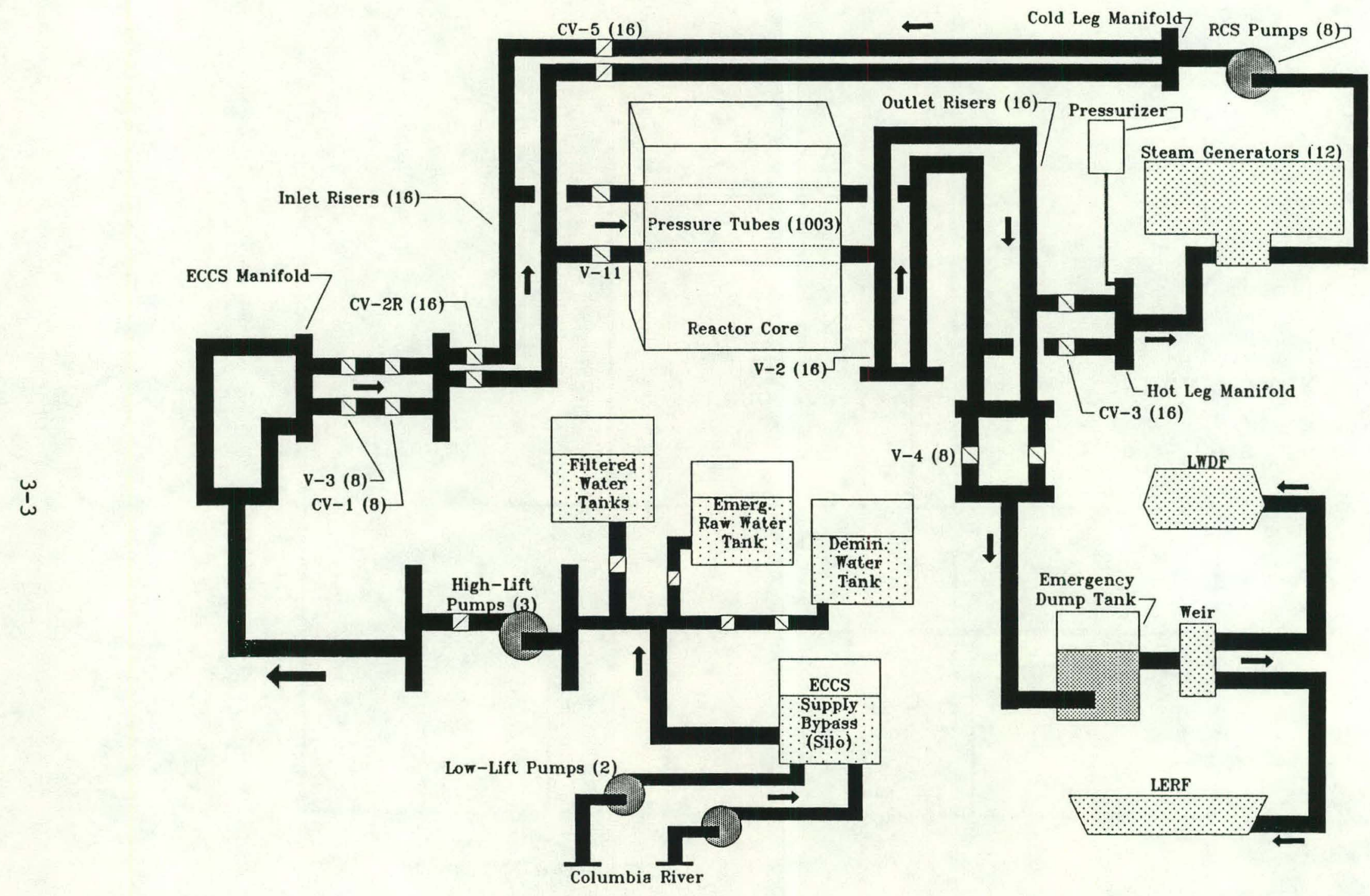

Figure 3.1. Simplified Flow Diagram of the $\mathrm{N}$ Reactor Coolant System 


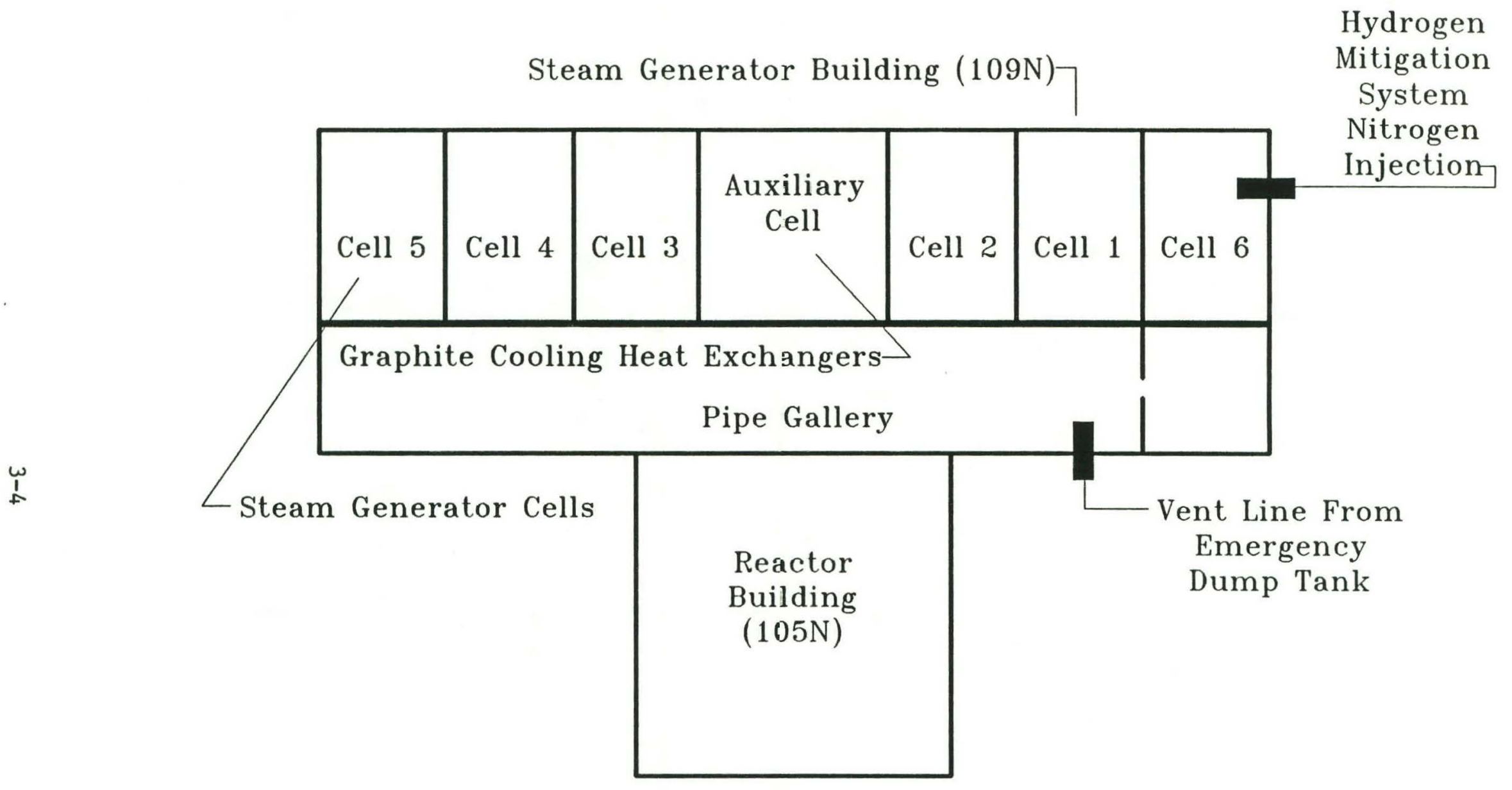

Figure 3.2. Simplified Top View of the N Reactor Confinement Buildings 


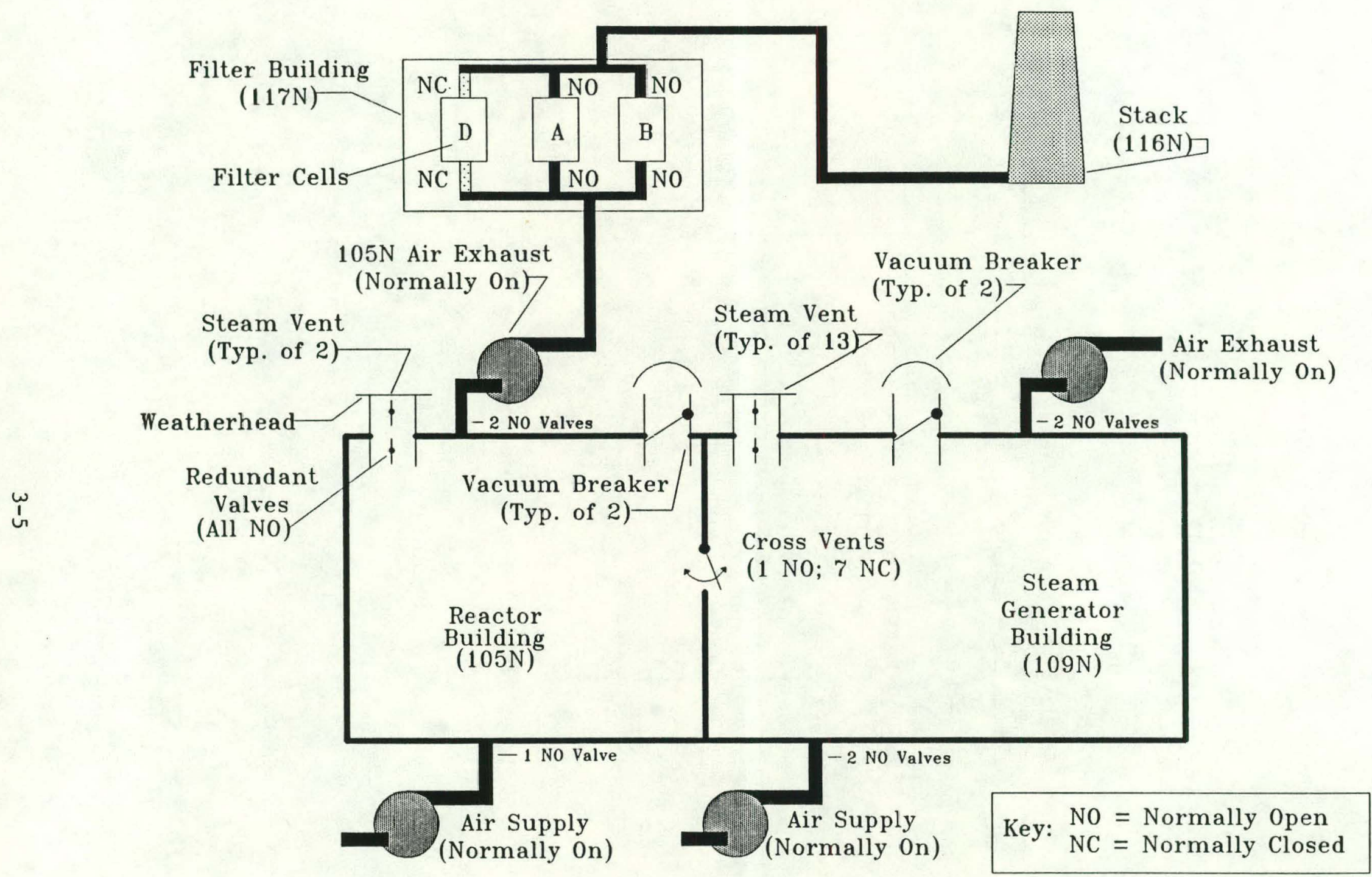

Figure 3.3. Key Components in the

N Reactor Confinement System 


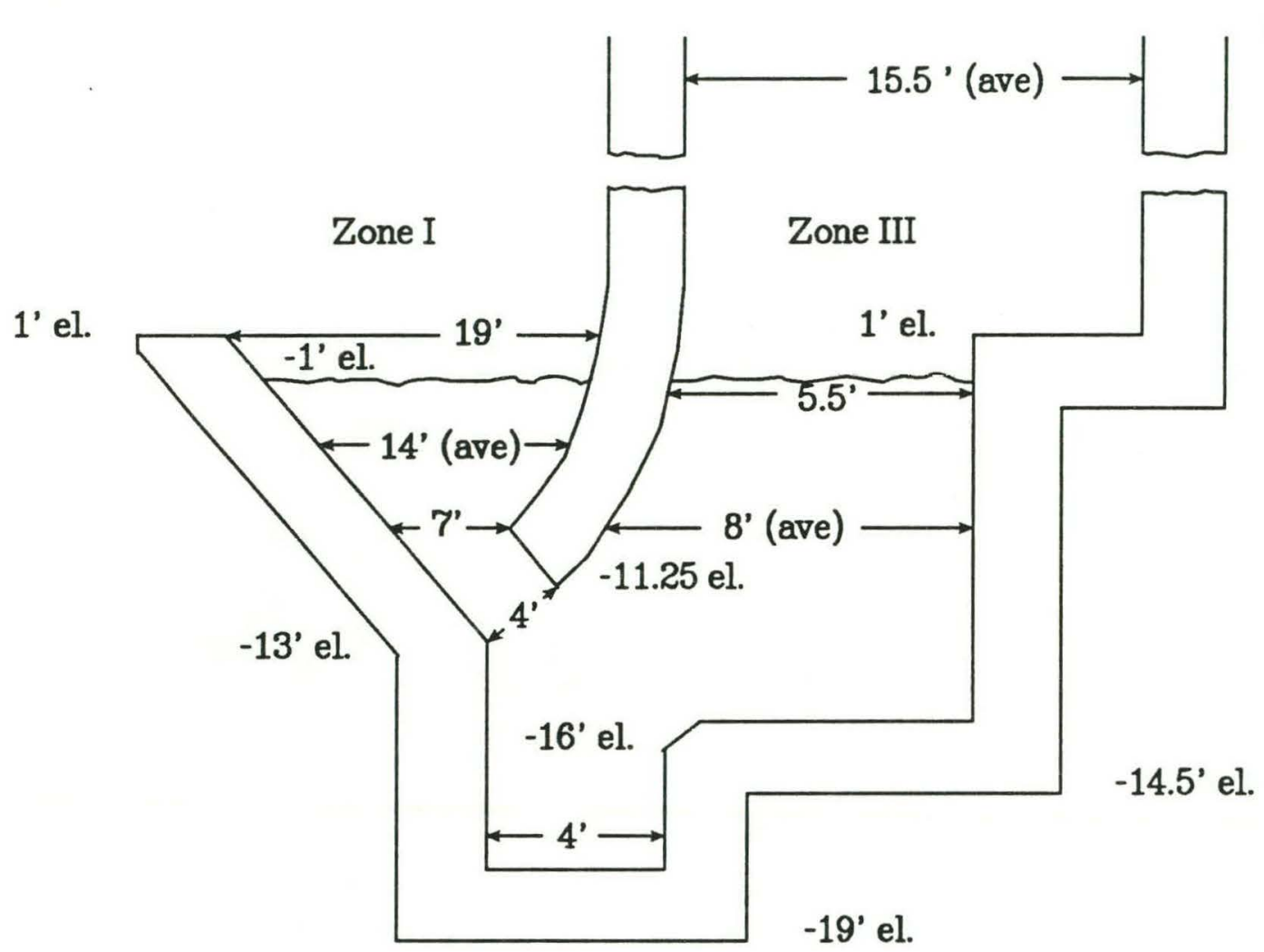

Figure 3.4. Elevation Drawing of the $\mathrm{N}$ Reactor Banana Wall 
Liquid effluent accumulating on the confinement floor is pumped to either the LERF (preferred) or the LWDF. If this pumping capability fails, the water inside the confinement will eventually overflow from the building sumps through the banana wall connection to the refueling bay and thence to the environment where it could flow to the Columbia River. There is a spill drain line which, if it opened, could take about half of the flow to the IWDF prior to its leaving the confinement and flowing under the banana wall.

As can be readily discerned from this brief description of $\mathrm{N}$ Reactor, its design presents analytic challenges for a PRA. There is little relevant literature concerning the possible paths by which accidents could progress for a reactor with the physics, chemistry, geometry, and systems encountered in this design. For example, experiments and calculations concerning the removal of radioisotopes by sprays from building atmospheres have been for hot, humid conditions. Here, at $\mathrm{N}$ Reactor, with the proper operation of the confinement vents, the release of fission products from the fuel into the building will occur in a relatively cool, and often dry atmosphere. Likewise, the commercial industry experience has been with oxide fuel, whereas the metallic fuel of $N$ Reactor melts at lower temperatures. One result of this is that the correlations used for hydrogen generation in LWRs can be inappropriate here. Furthermore, if fuel damage occurs, it will happen in horizontal tubes, each surrounded by graphite and not in a vertical vessel. Hence, the possible slumping and relocation of entire LWR cores is absent from this design because the graphite acts like a crucible for each fuel assembly. Finally, as noted above, accidents involving loss of cooling to portions of the core are possible at $\mathbf{N}$ Reactor. This possibility requires the examination of whether initial core damage can cause further degradation.

The preceding discussion has focused mainly on the characteristics of $N$ Reactor that are important to the internal events analysis. The $N$ Reactor fire events core damage analysis [3-2] revealed some additional features of the $N$ Reactor construction that are important to the progression of fire-induced core damage events. First, fires in certain areas are capable of failing all control to the V-4 valves. These valves must actuate to depressurize the primary system in order for ECCS coolane to enter the core. Second, fires in other areas can damage control cables for ECCS, GSCS, HPI and the primary cooling system (PCS). While at least the ECCS control failures would be recoverable, it would be almost impossible to do so before the onset of core damage. Most other fireinduced core damage accidents were found to be single riser failure events.

The structures that are considered critical to the safety of $\mathrm{N}$ Reactor are designed to withstand a "safe shutdown earthquake" (SSE) with a peak ground acceleration of $0.25 \mathrm{~g}[3-1]$. Thus, it should not be surprising that virtually all of the seismically induced core damage accidents occur at peak ground accelerations that are higher than the N Reactor SSE [3-2]. This analysis considers three representative ground acceleration levels as shown in Table 3.1. Different types of failures are expected to occur at 
each of these ground acceleration levels. At the "low" ground acceleration level, the failure of the silo (this cuts off the supply of water to the ECCS and other systems) occurs most frequently, while the collapse of Building $182 \mathrm{~N}$ (this building houses the ECCS and other pumps) would occur more often at "intermediate" ground acceleration levels. At "high" ground acceleration levels, a multiple pressure tube rupture (MPTR) event can occur in which many pressure tubes and GSCS tubes are damaged by either core motion or the collapse of Building 105N. These features of the $\mathrm{N}$ Reactor construction are important to the progression of seismic accidents at $\mathbf{N}$ Reactor.

Table 3.1 N Reactor ground acceleration categories and mean annual frequency of exceedence [3-2].

\section{Ground Acceleration Class}

N Reactor Safe Shutdown Earthquake (SSE) [3-1]

low ground acceleration medium ground acceleration high ground acceleration
Representative Peak Ground Acceleration
$0.25 \mathrm{~g}$
$0.3 \mathrm{~g}$
$0.5 \mathrm{~g}$
$0.7 \mathrm{~g}$

Mean Annual Frequency of Exceedence

$5.0 \mathrm{E}-05 *$

$3.5 \mathrm{E}-05$

$5.6 \mathrm{E}-06$

8. 8E-07

$\star 5.0 \mathrm{E}-05=5.0 \times 10^{-5}$, and should be interpreted as follows: based on the mean seismic hazard curve [3-2], it is expected that a seismic event of magnitude $0.25 \mathrm{~g}$ or greater would be expected to occur with a probability of 5.0E-05 each year, or equivalently, approximately once each 20,000 years.

\subsubsection{Accident Progression Event Tree Structure}

The $\mathrm{N}$-Reactor APET is divided into seven sections based on various phases of accident progression. These are:

Initial Core and Primary System Status (events 1-15)

Initial Confinement System Status (events 16-23)

Initial Blowdown Accident Progression (events 24-32) 
Pre-Recovery Accident Progression (events 33-50)

Recovery Actions (events 51-54, 56-58)

Recovery Phase Accident Progression (events 55, 59-77)

Post Recovery Status and Source Term Issues (events 78-87)

A brief description of each of these sections is presented below.

The first section of the APET is the Initial Core and Primary System Status (events 1-15). This section is primarily an interface with the Level I PRA and the external events core damage analyses. The initial conditions for the reactor primary system and core are determined here. These include the status of the reactor protection system (RPS), electrical power, high pressure injection (HPI), main coolant pumps, the Emergency Core Cooling System (ECCS) and the Graphite and Shield Cooling System (GSCS). In addition, the Level I PRA accident sequence initiating event, the type of fire or seismic event to be analyzed, the break location (if applicable), and possible early pressure tube rupture are determined in this section. The section ends with the determination of the initial core damage state based on conditions similar to those used in the Level I PRA.

The second section of the APET is the Initial Confinement system Status (events 16-23). The demand for and operation of several major confinement systems are established in this section of the APET. The confinement pressure is determined based on MELCOR and HECTR confinement analyses, and is used to determine the demand for confinement isolation, fog sprays and vents. The possibility of dump tank, filter stack, confinement isolation system, forced ventilation system, fog spray and hydrogen mitigation system failures (given demand) are modeled here based on confinement system fault tree analyses and seismic considerations.

The third section of the APET is the Initial Blowdown Accident Progression (events 24-32). Much of this section is irrelevant for transient accident sequences as it models the response of the confinement to blowdown conditions. The operation and subsequent reisolation of the steam vents, and the establishment of the filtered release path are determined in this section of the APET. Additional events explore the possibility that the operator will, based on hydrogen mitigation procedures, isolate the steam vents based on hydrogen concentrations before they are demanded. Should confinement fail, the failure mode and continuing fog spray availability are determined here.

The fourth section of the APET is the Pre-Recovery Accident Progression (events 33-50). This could also be called "early" accident progression where "early" is defined to be the first two hours after the initiation of the accident. This is similar to the definition from the Level I PRA. This is because Hanford analyses show that scenarios in which GSCS never fails and ECCS is successful for two hours (but then fails) do 
not proceed to core damage. Events in this section of the APET deal with a variety of accident progression phenomena, including hydrogen generation and combustion, loss of the helium blanket, possible graphite combustion, vacuum breaker demand and operation, and filter path reisolation and survival. Confinement failure mode and continuing fog spray operation are determined here in the same manner as in the previous section of the APET.

The fifth section of the APET is the Recovery Actions (events 51-54, 56-58). Based on probabilistic and procedural guidance, the recovery of electrical power, GSCS, ECCS, HPI, fog sprays, filter release path, and the hydrogen mitigation system are considered in this section of the APET. The possible negative and positive aspects of system recovery are analyzed below.

The sixth section of the APET is the Recovery Phase Accident Progression (events 55, 59-77). This section of the APET could be considered "late" phase accident progression, and is intended to model the response of various systems to the recovery actions taken in the above section. The first several events in this section deal with the negative aspects of restarting ECCS or HPI cooling once the core heats up. These include the potential for generating a steam pressure spike, additional hydrogen generation and combustion due to fuel oxidation, pressure tube rupture, loss of helium blanket integrity, and possible graphite oxidation and fuel damage propagation. The response of the filter path to these phenomena is also considered in this section. At the end of this section, the final status of the core (total fuel damage) and confinement are determined. Confinement failure mode and continuing fog spray operation are determined here in the same manner as in earlier sections of the APET.

The final section of the APET is the Post Recovery Status and Source Term Issues (events 78-87). The final condition of the filter path and liquid disposal systems are summarized in this section of the APET. System events such as sump pump operation, valve operation, Liquid Effluent Retention Facility (LERF) status, 117N filter spray system operation, filter seal operation, and $D$ cell filter alignment summarize data from other sections of the APET into a form that is useful to the source term analysis as well as determine the status of systems that will affect the source term.

The above paragraphs present an overview of the APET that summarizes the phenomena and systems considered in the event tree analysis. An event by event description of the $\mathrm{N}$-Reactor APET is presented below. For each event, the possible "answers" (branches) are listed, followed by a brief discussion of some of the issues that are relevant to that event, and a listing of the logical structure that is used to determine the various branch probabilities.

\subsubsection{Notation}

In the interest of maintaining effective communication, a structure was developed for the description of the logical case structure that is 
used to determine the branch probabilities. The following notation will be used extensively in the APET description:

$3 / 6$ represents branch 6 of event 3 .

$3 / 6$ represents the logical "not" operation applied to branch 6 of event 3. This same operation is sometimes represented in other writings as $-3 / 6$.

$3 / 6,7$ represents $3 / 6$ or $3 / 7$, " or, logically, $3 / 6+3 / 7$.

$3 / 6 \& 4 / 2$ represents "both $3 / 6$ and $4 / 2, n$ or, logically, $3 / 6 \star 4 / 2$.

$3 / 6,7$ represents "not $(3 / 6$ or $3 / 7)$ ", which is the same as "not $3 / 6 \&$ not $3 / 7 "$, or $" 3 / 6 * 3 / 7 "$.

$3 / 6,7(0.99 / 0.01)$ means that the probability of $3 / 6$ should be 0.99 , and the probability of $3 / 7$ should be 0.01 .

$2 / 2 \Rightarrow 3 / 3$ means event $2 / 2$ always (with probability 1.0 ) leads to branch $3 / 3$, so the probability distribution is not explicitly written.

This shorthand notation can be confusing at first, but without it, the presentation of the case dependence would be very cumbersome and would not translate easily to the form used by the EVNTRE code package [3-3]. The reader should become familiar with this notation before attempting to read the detailed APET description that follows.

It is also extremely important that the reader understand how the EVNTRE code package analyzes the EVNTRE input. The code package does not require the logical input for the event tree dependencies to be exhaustive or logically exclusive. It does not require that one and only one of the logical conditions be satisfied. In fact, it requires that the last logical case be a catch-all "otherwise" case that is taken by all paths through the event tree that do not fit into any of the other logical conditions.

EVNTRE considers only the first logical pathway that is satisfied. Thus, it may be possible that, for a given path through the event tree, all logical eases could be satisfied by the particular events and branches that have come before. EVNTRE would consider only the results of the first logical case and ignore all of the other cases. This means that the earlier logical cases for an event can be used to "filter out" classes of events so that later cases can be made simpler. For example, if the first logical case for an event were to read "all small LOCAs," then all remaining cases implicitly contain the logic "not a small LOCA" along with whatever other logic is written. It is critical that the reader be aware of this fact when reading the logical structure presented in the following sections. 
The description of each event contains a table of possible outcomes, or branches, and their descriptions. The table contains the branch number for reference purposes when reading the logic for later events that are dependent on this one. It also contains an abbreviation for the branch. This abbreviation is used in the input to the EVNTRE code to give added readability to the input file and will be referred to occasionally in the description of later events to ensure clarity.

Probability values are also presented for each branch in each logical case developed in the event description. Each probability value is an average probability taken from the actual, realized branch splits in the EVNTRE frequency output report (see Appendix F). A branch probability for a given case represents the fraction of the frequency for which that branch was chosen given that the preceding events in that accident progression pathway caused that case to be chosen by the EVNTRE code. In other words, it represents the conditional probability that the specified branch was chosen given that the specified case was chosen. The probability represents an average over all Latin Hypercube sample members as computed by the EVNTRE code. The frequencies are affected by the truncation of paths based. on probability within EVNTRE and, as such, may vary slightly from the input probability values as documented in Appendix $E$ for all events that are not subject to modification by values from the Latin Hypercube sample.

\subsection{INITIAL CORE AND PRIMARY SYSTEM STATUS}

This part of the APET encompasses events 1 through 15 and is used primarily to interface the event tree to the Level I PRA and external events core damage analyses. The events in this section of the APET are:

1 Is the Reactor Protection System (RPS) Successful?

2 Is this accident sequence caused by fire, seismic, or internal events?

3 Is this a transient or a LOCA event?

4 If this is a seismic event, what type of a seismic event occurred?

5 If this is a LOCA event, where did the break occur?

6 Is AC power available?

7 Is damage caused by a riser failure, or early or late ECCS failure?

8 Why does the ECCS cooling failure involve more than a single riser?

9 Has a pressure tube rupture occurred?

10 Is the GSCS operating?

11 Is GSCS effectively cooling the core?

12 Is the HPI system operating?

13 Are the main coolant pumps supplying water to the core?

14 What is the overall status of flow to the core?

15 How much fuel damage occurs due to the initial core coolant flow?

As can be seen by the nature of the events (phrased as questions) above, this section of the APET sets up the "initial conditions" for the APET analysis. The probabilities for many of these events are calculated directly from the cut sets resulting from the Level I PRA analysis by the 
TEMAC code. TEMAC evaluates each of the cut sets based on the Level I frequency data and calculates fractions for use in EVNTRE based on the cut set frequencies. For example, the fraction for the first event is, physically, the fraction of all core damage events that are caused by ATWS (failure of the reactor protection system (RPS)). This is computed by TEMAC $[3-4,3-5]$ as the total frequency of all incidents where the RPS fails divided by the total core damage frequency. This information is calculated by TEMAC and fed directly into EVNTRE to be used as the probability for this event. Fractions are calculated in a similar manner from the Level I analysis for Events 3,6, 7, 8, 10, 12 and 13, and from the External Events analysis for Events 2, 3, 4, 6, 7, 8, 9, 10, and 12 . Additional information from the Level I PRA was distilled to obtain a probability distribution for Event 5.

Events 11,14 and 15 are summary events which simply combine the results of earlier events into a form that is more easily usable in the remainder of the APET. All probabilities in these events are either zero or unity because they do not actually represent new events, but rather summarize previous events. The fuel damage levels determined in Event 15 are nominal values based on the operation or failure of various systems. They are determined in a manner similar to the internal events Level I PRA [3-6], and are addressed in more detail under uncertainty analysis in Chapter 4 .

Event 1 - Is the Reactor Protection System (RPS) successful?

BRANCH ABBREV. DESCRIPTION

(1) Scram Reactor Protection System was successful

(2) nScram Anticipated Transient Without SCRAM (ATWS) event

This event is used to determine the fractional frequency of ATWS events among the accident sequences being analyzed. It is an independent event (that is, it does not depend on any other events in the APET). The TEMAC analysis analyzes the cut sets from the Level I PRA and external events core damage analyses to determine the conditional probability of an ATWS event. This probability split is fed into the EVNTRE code directly. The average probability of branch 2 (an ATWS event) is 0.0165 for the internal events analysis, and 0.000 for both the fire and seismic analyses based on the external events core damage analyses [3-2]. These zero probabilities were taken into account in the structure of the APET. Had they not been zero, the order of events 1 and 2 would have been reversed to account for the dependency.

The ATWS analysis in the APET assumes that all primary and ECCS systems continue to function normally throughout the accident progression. The reasons for this are several. First, no ECCS failures were modeled in conjunction with ATWS events in the Level I PRA. Second, ATWS events with ECCS failure would be extremely rare events (at least two orders of magnitude less likely than ATWS events with successful ECCS). Finally, since $N$ Reactor has a large negative void coefficient, ATWS events with ECCS failure would see neutronic shutdown conditions anyway and progress as 
if the RPS had functioned properly. The ATWS events with ECCS failure would be trivial in frequency and be very similar in accident progression characteristics to other, non-ATWS events that are already being studied. For this reason, ATWS events with ECCS failure are neglected in this APET analysis.

Event 2 - Is the accident sequence caused by fire, seismic or internal events?

BRANCH ABBREV, DESCRIPTION

(1) Int The core damage was caused by internal events

(2) Fire The core damage was caused by a fire

(3) Seismic The core damage was caused by a seismic event

This event determines the overall class of the accident sequence. For this analysis, the fire, seismic and internal events EVNTRE runs were all run separately, so the probability of each branch was either 1.00 or 0.00 , depending on the EVNTRE run. For example, for the internal events analysis, the probability distribution for this event would be

$$
2 / 1,2,3 \rightarrow(1.00 / 0.00 / 0.00) \text {. }
$$

The input probabilities for this event were manually constructed in this manner for each of the three EVNTRE analyses (internal events, fire events and seismic events). If all three types of accident sequences were to be analyzed simultaneously, split fractions would be taken from the results of a combined fire/seismic/internal events TEMAC analysis and would show nonzero values for each branch proportional to the relative frequencies of the three core damage frequencies. This is an independent event, except that all ATWS events $(1 / 2)$ are by definition internal events (see Event 1 ).

$1 / 2 \Rightarrow 2 / 1$

otherwise $\Rightarrow 2 / 1$ or $2 / 2$ or $2 / 3$ as discussed above

Event 3 - Is this a transient or a LOCA event?

$\begin{array}{lll}\text { BRANCH } & \text { ABBREV } & \text { DESCRIPTION } \\ \text { (1) } & \text { Trans } & \text { Transient initiator - no break in the primary system } \\ \text { (2) } & \text { LLOCA } & \text { Primary system is not intact - large LOCA } \\ \text { (3) } & \text { SLOCA } & \text { Primary system is not intact - small LOCA }\end{array}$

This event is used to determine the initial status of the primary cooling system (PCS). For N-Reactor, a small LOCA is defined to be any pipe break of four inches diameter or less because of the unusually large reserve capacity (more than $6000 \mathrm{gpm}$ ) of the High Pressure Injection (HPI) system to makeup reactor coolant system losses. The branch probabilities for ATWS events will be different from those events where RPS succeeds because the events come from different core damage sequences in the level I PRA. The same is true between internal. events and seismic accident sequences. The external events analysis found no LOCAs associated with 
fire events, so all fire-induced core damage accidents are classified as transients. The probability distributions are determined by the cut sets in the Leve1 I PRA [3-6] and external events core damage analyses [3-2] and calculated during the appropriate TEMAC analyses [3-7].

The four logical cases for this dependent event represent ATWS, internal events, fire and seismic core damage accident sequences, respectively. Note that the large LOCA ATWS core damage sequence was deleted from further analysis. This is discussed in detail in Chapter 4.

$$
\begin{aligned}
& 1 / 2 \Rightarrow 3 / 1,2,3(0.865 / 0.000 / 0.135) \\
& 2 / 1 \Rightarrow 3 / 1,2,3(0.692 / 0.051 / 0.257) \\
& 2 / 2 \Rightarrow 3 / 1 \\
& \text { otherwise (seismic) } \Rightarrow 3 / 1,2,3(0.671 / 0.187 / 0.142)
\end{aligned}
$$

\begin{tabular}{|c|c|c|}
\hline $\mathrm{RANCH}$ & ABBREV. & DESCRIPTION \\
\hline (1) & Norm & $\begin{array}{l}\text { The seismic event that occurred was not a member of } \\
\text { any of the special classes listed below (thus, } \\
\text { "normal") }\end{array}$ \\
\hline (2) & RVR & $\begin{array}{l}\text { A multiple pressure tube rupture (MPTR) seismic event } \\
\text { occurred (similar in effect to a reactor vessel } \\
\text { rupture (RVR) event in a commercial reactor) }\end{array}$ \\
\hline $\begin{array}{l}\text { (3) } \\
\text { (4) }\end{array}$ & $\begin{array}{l}\text { B-182 } \\
\text { LoSP } \\
\text { None }\end{array}$ & $\begin{array}{l}\text { A seismic event caused the collapse of Building } 182 \mathrm{~N} \\
\text { A loss of offsite power seismic event occurred } \\
\text { No seismic event occurred }\end{array}$ \\
\hline
\end{tabular}

Event 4 - What type of seismic event occurred?

This event is used to separate the seismic class of accident sequences into a number of sub-classes with different characteristics. An MPTR event is a special type of large LOCA in which many pressure tubes and GSCS tubes are damaged by either core motion or the collapse of Building $105 \mathrm{~N}$. Building $182 \mathrm{~N}$ collapse and loss of offsite power are both special classes from the transient primary system configuration. Building $182 \mathrm{~N}$ houses, among other things, the HPI pumps, the ECCS high lift diesel pumps, the fog epray diesel pumps (they supply GSCS water when GSCS is operating in its once-through mode) and the low pressure injection pumps (they supply makeup water to the GSCS in its recirculating mode). A loss of offsite power can affect the success probabilities for numerous other primary and confinement systems. There are no special classes of seismically induced small LOCAs. Branch 5 is used if the event is not seismically initiated. See Appendix C.13 for further discussion. The four logical cases for this event represent non-seismic events, seismic small LOCAs, seismic large LOCAs, and seismic transients, respectively. The probabilities used in this event are taken from the seismic events TEMAC analysis.

$$
\begin{aligned}
& \frac{2 / 3}{3 / 3} \rightarrow 4 / 5 \\
& 3 / 2 \rightarrow 4 / 1 \\
& \text { otherwise (seismic transients) } \rightarrow 4 / 1,3,4(0.000 / 0.729 / 0.271)
\end{aligned}
$$


Event 5 - Break Location

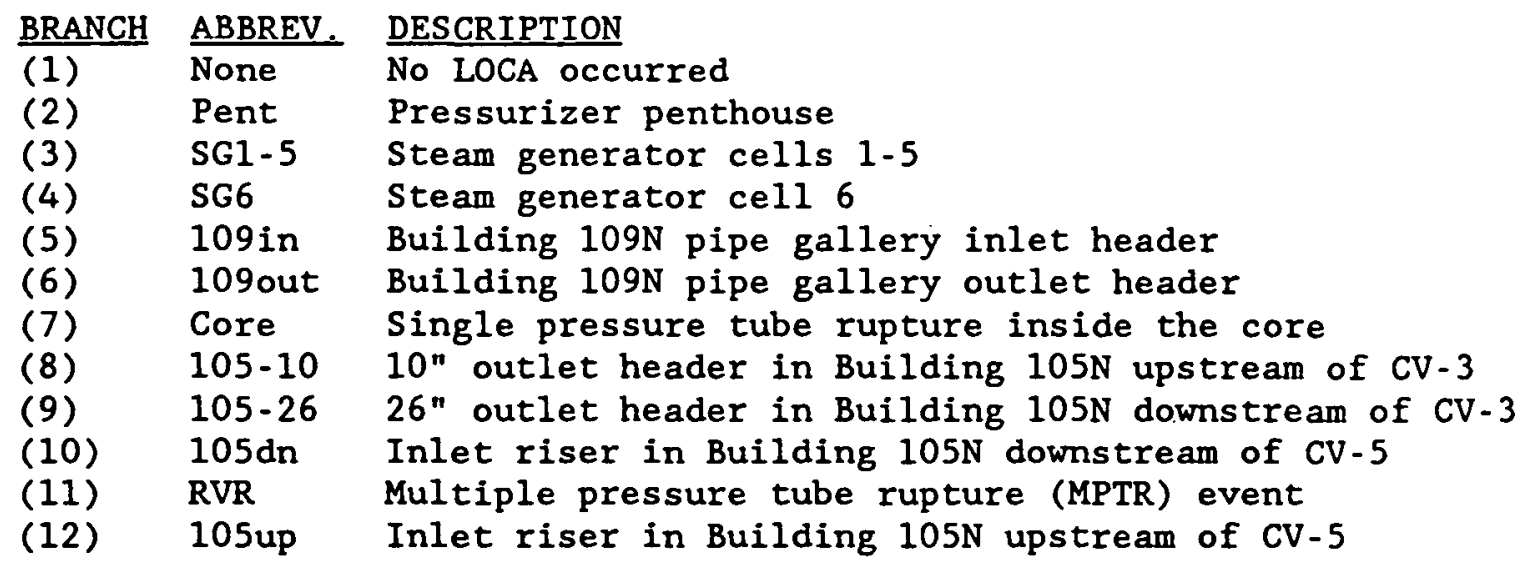

As stated previously, a small LOCA is defined to be any pipe break of four inches diameter or less because of the unusually large reserve capacity (more than $6000 \mathrm{gpm}$ ) of the High Pressure Injection (HPI) system to makeup reactor coolant system losses. Thus, a single pressure tube rupture is considered a small LOCA. A zero probability is assigned to any break location that is either not possible or not considered credible in the Level I analysis. For example, there is no LOCA in transient situations, so the probability of any branch other than branch 1 occurring is zero. Conversely, branch 1 must have a zero probability for all LOCA scenarios since by definition there is a loss of coolant.

While the event descriptors used above are indicative of the large pipes throughout the plant, there are often small pipes and valves in the same area that will draw coolant from the same location as the named large break. Thus, small breaks are assigned names that correspond to the large break locations they most closely resemble. For example, an RV-2 failure is assigned to branch 8 because it draws coolant from that location (even though it is not a break in the actual 10" line). In addition, the LOCA characteristics of the MPTR event are similar to an LWR reactor vessel rupture event, and is denoted by the abbreviation RVR in the computer models documented in Appendices $E$ and $F$.

The frequency of large and small pipe breaks in a given area will be different because of differing amounts of large and small pipe and differing numbers of large and small valves. The relative frequency between the different locations was assessed to be similar for both internal and seismic LOCAs (see Appendix C.13), so the same distributions were used for both cases.

The probabilities for the various branches are derived in Appendix $D$. While the absolute LOCA frequencies were sampled for the uncertainty analysis, the relative frequencies of the various locations were not. Thus, the probabilities listed below, unlike those listed for other events, are point estimate input values rather than mean values. The four dependent logical cases for this event represent seismic MPTR events, large LOCAs, small LOCAs and transient events, respectively. Note that since the same cases are used for both the seismic and internal events analyses, the 
truncation of accident progression pathways can cause the realized values to differ slightly between the two analyses.

$$
\begin{aligned}
& 4 / 2 \Rightarrow 5 / 11 \\
& 3 / 2 \Rightarrow 5 / 2-12(0.018 / 0.410 / 0.082 / 0.003 / 0.004 / 0.000 / 0.176 / \\
& 0.150 / 0.153 / 0.000 / 0.004) \\
& 3 / 3 \Rightarrow 5 / 2-12(0.000 / 0.602 / 0.120 / 0.000 / 0.000 / 4.4 E-5 / 0.246 / \\
& 0.000 / 0.032 / 0.000 / 0.000)
\end{aligned}
$$

Event 6 - Is AC Power Available?

\section{BRANCH ABBREV. DESCRIPTION}

(1) All-Pow All AC power is available

(2) $\quad n$-A-Bus Only $A C$ bus $A$ has failed

(3) $n$-B-Bus Only AC bus $B$ has failed

(4) $\quad n-A C$ Both $A C$ busses have failed

This event is used to determine the availability of AC power throughout the APET. The probabilities are different for each type of accident sequence to be analyzed. The probability distributions are determined by the cut sets in the Level I PRA [3-6] and external events core damage analyses [3-2] and calculated during the appropriate TEMAC analyses $[3-7]$.

Under normal operating conditions, the electrical A Bus is powered from offsite sources and $B$ Bus is powered from the $N$ Reactor turbogenerator. A large LOCA causes a sudden loss of steam to the turbogenerator as the primary side of the steam generator dries out. For this reason, all large LOCAs entail the loss of B Bus. In addition, most fire sequences would be expected to cause a loss of B Bus because the fires would cause the failure of the primary/secondary system function (i.e., demand ECCS actuation) before the oil-fired boilers could be brought on line to replace the steam from the steam generators.

The first four logical cases below are for seismic scenarios with large and small LOCAs, losses of offsite power, and other transients, respectively. The next case is for the fire analysis, followed by two ATWS cases (large LOCA and "other"). Finally, the internal events large LOCA, small LOCA and transient cases round out the structure. Note that AC power is assumed to succeed for ATWS events just as ECCS was assumed to succeed.

$$
\begin{array}{ll}
2 / 3 \& 3 / 2 & \rightarrow 6 / 2,4(0.016 / 0.984) \\
2 / 3 \& 3 / 3 & \Rightarrow 6 / 2,4(0.081 / 0.919) \\
2 / 3 \& 4 / 4 & \Rightarrow 6 / 1,2,3,4(0.155 / 0.145 / 0.068 / 0.632) \\
2 / 3(\text { transients) } & \rightarrow 6 / 1,2,3,4(0.000 / 0.361 / 0.000 / 0.639) \\
2 / 2 & \rightarrow 6 / 1,2,3,4(0.000 / 0.003 / 0.960 / 0.037) \\
1 / 2 \& 3 / 2 & \rightarrow 6 / 3 \\
1 / 2 & \rightarrow 6 / 1 \\
3 / 2 & \rightarrow 6 / 3,4(1.00 / 0.00) \\
3 / 3 \quad & \rightarrow 6 / 1,2,3(0.170 / 0.015 / 0.815) \\
\text { otherwise (transient) } \rightarrow 6 / 1,2,3(0.2999 / 0.0002 / 0.6999)
\end{array}
$$


Event 7 - What is the class of ECCS failure?

$\begin{array}{lll}\text { BRANCH } & \text { ABBREV } & \text { DESCRIPTION } \\ \text { (1) } & \text { fECCS } & \text { ECCS does not fail } \\ (2) & \text { ECCS-LF } & \text { ECCS functions initially, but fails after } 2 \text { hours } \\ (3) & 15-\text { LCCC } & \text { ECCS works, but a single riser failure occurs } \\ & & \text { initially, and ECCS fails after } 2 \text { hours } \\ (4) & 15-E C C S & \text { ECCS works, but a single riser failure occurs } \\ (5) & \text { nECCS } & \text { ECCS full-core success criteria have not been met }\end{array}$

This event determines the broad class of the ECCS failure. The probabilities are determined by the class of accident sequence being analyzed. The probability distributions are determined by the cut sets in the Level I PRA [3-6] and external events core damage analyses [3-2] and calculated during the appropriate TEMAC analyses [3-7]. The first branch is used during the analysis of ATWS events. Also, recall that all single riser failure core seismic accident sequences were neglected from the analysis because they are very low frequency events and exhibit low consequences. The seven logical cases for this event are for ATWS events, internally initiated transients, large LOCAs, and small LOCAs, fire events in areas that cause the loss of electrical bus B, all other fires, and all seismic events, respectively. Fires involving the loss of $B$ Bus are separated from other fires because the fire locations that were seen to cause single riser failure events are a subset of those where B Bus, rather than A Bus, would be expected to fail [3-2].

$$
\begin{aligned}
& 1 / 2 \rightarrow 7 / 1 \\
& 2 / 1 \& 3 / 1 \rightarrow 7 / 2,3,4,5(0.005 / 0.000 / 0.622 / 0.373) \\
& 2 / 1 \& 3 / 2 \rightarrow 7 / 2,3,4,5(0.000 / 0.000 / 0.751 / 0.249) \\
& 2 / 1 \& 3 / 3 \rightarrow 7 / 2,4,5(0.150 / 0.478 / 0.372) \\
& 2 / 2 \& 6 / 3 \rightarrow 7 / 4,5(0.079 / 0.921) \\
& 2 / 2 \quad \Rightarrow 7 / 4 \\
& \text { otherwise (seismic) } \rightarrow 7 / 5
\end{aligned}
$$

\begin{tabular}{|c|c|c|}
\hline 3RANCH & REV & ION \\
\hline (1) & $\overline{\text { ECCS - ok }}$ & $\begin{array}{l}\text { ECCS failure does not initially affect more than } \\
\text { single riser }\end{array}$ \\
\hline (2) & EC & The 3 H33 ECCS bypass switch is in the wrong position \\
\hline (3) & EC & The RWSV-620 main ECCS supply valve has failed \\
\hline (4) & EC. & No ECCS high lift diesel pumps started \\
\hline (5) & EC & Only one ECCS high lift diesel pump started \\
\hline (6) & $\mathbf{E}$ & $\begin{array}{l}\text { All v-3 valves on one side of the reactor failed to } \\
\text { open }\end{array}$ \\
\hline (7) & EC & $\begin{array}{l}\text { Three V-3 valves on the same side of the react } \\
\text { failed to open }\end{array}$ \\
\hline (8) & EC & $\begin{array}{l}\text { Two ECCS high lift diesel pumps started, but more } \\
\text { than one } V-3 \text { valve on one side of the reactor failed }\end{array}$ \\
\hline $\begin{array}{l}(9) \\
(10\end{array}$ & & $\begin{array}{l}\text { None of the } V-4 \text { valves opened } \\
\text { All V-4 valves on one side of the reactor failed }\end{array}$ \\
\hline
\end{tabular}

Event 8 - Why does the ECCS failure involve more than a single riser? 
This event determines the specific cause of an initial ECCS failure affecting more than a single riser. The probabilities are determined by the class of accident sequence being analyzed. The probability distributions are determined by the cut sets in the Level I PRA [3-6] and external events core damage analyses [3-2] and calculated during the appropriate TEMAC analyses [3-7]. The first branch is used in cases where ECCS fails initially in no more than a single riser. This includes scenarios where ECCS succeeds, where ECCS fails only after more than two hours and and single riser failure accidents.

The external events core damage analysis revealed that the possible ECCS failure mechanisms for these events involve the failure of far fewer components than internal events failure. The only failures caused by fires involve either all of the high lift diesel pumps or all of the $V-4$ valves. Also, all seismic events modeled in this analysis involve a total loss of ECCS flow to the core, whether from the collapse of Building $182 \mathrm{~N}$ or from the collapse of the silo. These are all modeled as if all ECCS high lift diesel pumps had failed because the net effect to the core is the same in either case (zero ECCS flow).

The first logical case for this event considers ATWS events and those ECCS failure events that fail cooling to no more than a single riser. The next three cases are for internally initiated transients, large LOCAs and small LOCAs, respectively, while the last two cases are for all fire and seismic core damage events, respectively.

$$
\begin{aligned}
& 7 / 5+1 / 2 \rightarrow 8 / 1 \\
& 2 / 1 \& 3 / 1 \rightarrow 8 / 2,3,5,6,8,10(0.185 / 0.003 / 0.219 / 0.116 / 0.092 / 0.385) \\
& 2 / 1 \& 3 / 2>8 / 2,3,5,6,8,10(0.022 / 0.004 / 0.330 / 0.132 / 0.120 / 0.392) \\
& 2 / 1 \& 3 / 3 \rightarrow 8 / 2,3,5,6,8,10(0.122 / 0.004 / 0.280 / 0.123 / 0.102 / 0.369) \\
& 2 / 2 \rightarrow 8 / 4,9(0.972 / 0.028) \\
& \text { otherwise (seismic) } \rightarrow 8 / 4
\end{aligned}
$$

Event 9 - Has a pressure tube rupture occurred?

\section{BRANCH ABBREV. DESCRIPTION}

(1) PT-OK All pressure tubes have remained intact

(2) PT-Rup One or more pressure tubes have ruptured - either as a small break LOCA initiating event or on ECCS refill

This event addresses the possibility that one or more pressure tubes will rupture prior to the initiation of delayed recovery actions. The pressure tubes were assessed during the expert judgement uncertainty elicitation to be more likely to fail in situations where ECCS is partially successful than in cases where ECCS has completely failed because water hammer effects are possible for partial ECCS cases. This phenomenon is caused by cold water coming into contact with heated fuel producing a pressure spike within the pressure tube. The pressure spike inside the pressure tube drives the water front back away from the heated fuel. The process may repeat many times causing pressure/temperature oscillations which could be of a sufficient magnitude to fail one or more pressure tubes 
by fatigue [3-8]. Note that this event considers pressure tube failure not just at the time of the initiating event, but at any time prior to the initiation of delayed recovery actions (two hours - see Section 3.7). The first dependent logical case for this event models situations where a pressure tube failure has already occurred as noted by the break location in Event 5. The second case is used when ECCS is supplying a significant amount of water to the core (although it is still failing to meet its success criteria), and the third case models all remaining situations.

The probabilities for this event are derived from expert judgement elicitations (see Appendix C.7) and were not sampled during the uncertainty analysis. The probabilities listed below, unlike those listed for other events, are point estimate input values rather than mean values. Note that since the same cases are used for the fire, seismic and internal events analyses, the truncation of accident progression pathways can cause the realized values to differ slightly between the analyses.

$$
\begin{array}{ll}
5 / 7+5 / 11 & >9 / 2 \\
8 / 5,6,7,8,10 & >9 / 1,2(0.995 / 0.005) \\
\text { otherwise } & \rightarrow 9 / 1,2(0.9999 / 0.0001)
\end{array}
$$

Event 10 - Is the GSCS operating?

$\frac{\text { BRANCH }}{\text { (1) GSBREV }} \frac{\text { DESCRIPTION }}{\text { The GSCS is operating to pump coolant through the }}$
(2) GSCS-f The GSCS has failed

This event considers whether the pumps, valves and pipes are successful in delivering GSCS coolant to the core. It does not consider the degree of success the GSCS has in cooling the core. That is examined in the next event. The branch probabilities will differ between the fire, seismic and internal events analyses as well as between the different primary system configurations. The probability distributions are determined by the cut sets in the Level I PRA [3-6] and external events core damage analyses [3-2] and calculated during the appropriate TEMAC analyses [3-7].

In many cases, the result of this event is pre-determined based on previous events. GSCS operates for ATWS scenarios (see Event 1) and single riser failures, and is irrelevant for cases where the ECCS functions fully. On the other hand, GSCS fails by definition in seismic MPTR events and seismic failures of Building $182 \mathrm{~N}$ as well as fires that damage the ECCS pumping capability [3-2]. It also fails by definition in delayed ECCS failure core damage events because, if it does not, the Level I PRA analysis states that core damage does not occur.

The eleven logical cases for this event are described as follows: the first case prevents GSCS failure for ATWS and single riser failure events. The second case ensures GSCS failure for MPTR and delayed ECCS failure events. The next three cases model internally initiated transients, large LOCAs and small LOCAs. The next two cases model fire events, while the 
remaining four model seismic large LOCAs, small LOCAs, failures of Building $182 \mathrm{~N}$, and other transients.

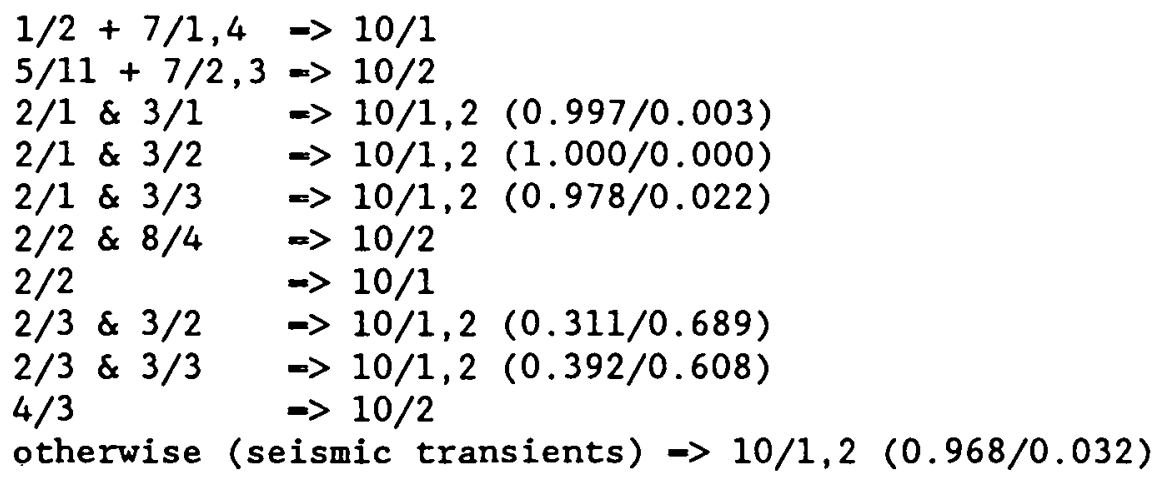

Event 11 - Is GSCS Effectively Cooling the Core?

BRANCH ABBREV. DESCRIPTION

(1) fGSCS GSCS is cooling the core at normal efficiency

(2) DegGSCS GSCS is operating at a degraded efficiency due to the

(3) nGSCS GSCS has failed

The operation and efficiency of the GSCS are determined in this summary event. Two conditions must occur for the GSCS to function at full efficiency. First, obviously, the system must pump coolant to and through the core. Second, the heat transfer from the graphite to the GSCS is enhanced by the presence of a helium blanket surrounding the graphite. If the helium is replaced by steam due to a pressure tube rupture, the thermal conductivity of the gap between the graphite and the GSCS tubes is reduced by a factor of approximately five. The heat transfer across this gap is the dominant thermal resistance for heat transfer to the GSCS, so loss of the helium blanket will degrade the heat removal by the GSCS [3-9]. The total efficiency of the GSCS is determined by the combination of these two factors. The three logical cases below implement these criteria.

$10 / 2 \quad \rightarrow 11 / 3$

$10 / 1 \& 9 / 1 \rightarrow 11 / 1$

otherwise (pressure tube rupture with GSCS operating) $\Rightarrow 11 / 2$

Event 12 - Is the HPI system operating?

BRANCH ABBREV. DESCRIPTION

(1) 4-HPI Full HPI flow is reaching the core

(2) 2-HPI 2 Pumps equivalent flow reaches core

(3) 1-HPI 1 Pump equivalent flow reaches core

(4) NoHPI No HPI flow reaches core

This is a summary event which takes into account all high pressure injection (HPI) flow dependencies. It has been noted that, in the $N$ Reactor Emergency Response Guide [3-10], ERG-B instructs the operators to 
unload and shutdown all HPI pumps in step 11. However, this ERG assumes that ECCS has succeeded, while this APET is only used for cases where ECCS has at least partially failed. Thus, it is assessed that in cases where ECCS has not met its success criteria, the operators will not shut down the HPI system.

The uncertainty assessment on core damage resulting from partial ECCS success (see Appendix C.3) found no basis for assessing that HPI flow would be able to prevent or even significantly reduce the amount of core damage that would occur given inadequate ECCS cooling. Thus, the primary function of HPI flow in the APET is to determine whether there is a steam source in the core to drive the released radionuclides out to the environment. For this reason, the particular amount of HPI flow is not as much of a concern as is the presence or absence of flow.

The success criteria for the HPI system (part of primary/secondary heat transfer success) from the Level I PRA [3-6] is that three of four pumps must operate during a small LOCA. Thus, by definition, a small LOCA core damage accident sequence must have no more than two HPI pumps operating. Also, since AC power bus $B$ is always lost during large LOCAs and since two HPI pumps are operated from each AC power bus, it is not possible to have more than two HPI pumps operating initially during a large LOCA accident sequence (the other pumps could, however, be recovered later). More than two pumps can operate, however, during transient accident sequences.

The branch probabilities for the transient case are determined by the cut sets in the Level I PRA and external events core damage analyses and calculated during the appropriate TEMAC analyses. The remaining branch probabilities are sampled based on uncertainty distributions presented in Appendix D.

The following statements summarize the logical cases used to model this event. Several plant states rule out any HPI flow. These are:

- failure of AC power (the HPI pumps are AC powered),

- full core damage seismic event (either the silo, which supplies water to the HPI pumps, or the building housing the pumps is destroyed),

- a fire where ECCS high lift diesel pumps are damaged (control cables for HPI pumps are co-located with those for the high lift pumps),

- initial ECCS success (operators are instructed to shut the system down), and

- a large LOCA outside of the check valves that surround the core (HPI is injected in the steam generator cells and will not reach the core for those break locations).

Other break locations that would be expected to affect HPI flow are:

- A large break in $4 / 10(105 \mathrm{dn})$ is in the inlet riser after the last checkvalve, so HPI is assessed to almost completely bypass the core. Small LOCAs in this area are broken connectors or spacers that are assessed to cause HPI to partially bypass the core. 
- Branch 4/8 (105-10) gives full HPI because the break is between the core outlet and CV-5, so water must go through the core to get to the break location.

$\begin{array}{ll}2 / 3+(2 / 2 \& 8 / 4)+6 / 4+8 / 1 & >12 / 4 \\ 3 / 2 \& 5 / 7,8 & \Rightarrow 12 / 4 \\ 3 / 3 \& 5 / 1,7,8 & \Rightarrow 12 / 3,4(0.980 / 0.020) \\ 5 / 7,8 & \Rightarrow 12 / 2,3,4(0.980 / 0.020 / 0.000) \\ \text { otherwise (transients) } & \Rightarrow 12 / 2,4(0.908 / 0.092)\end{array}$

Event 13 - Are the main coolant pumps supplying water to the core?

\section{BRANCH ABBREV. DESCRIPTION}

(1) fMCP Main coolant pumps are supplying water to the core

(2) nMCP No primary coolant flow

The primary coolant pumps can circulate water through the core provided that $A C$ power remains available and the primary system remains pressurized (the $V-4$ valves do not open as in events $8 / 2,9$ ). Thus, the main coolant pumps cannot maintain long term circulation if AC power fails or the V-4 valves open or a large LOCA occurs. Calculations [3-9] show that this condition can continue for a few hours and delay but not prevent core damage. Main coolant pump flow is, for this reason, treated as a partial core cooling failure. Failures that lead to main coolant pump failure are not considered because of the redundancy of the main coolant pumps, so this event is treated as a summary event and not sampled.

$$
\begin{array}{ll}
3 / 2+6 / 4+8 / 2,9 & \rightarrow 13 / 2 \\
\text { otherwise } & \rightarrow 13 / 1
\end{array}
$$

Event 14 - What is the overall status of flow to the core?

BRANCH ABBREV. DESCRIPTION

(1) fECCS Full ECCS flow (at most $1 / 16$ of the core is damaged initially)

(2) pECCS Partial flow (core cooling is adequate to reduce but not prevent core damage)

(3) nECCS No ECCS flow (core cooling is insufficient to significantly reduce core damage)

This event summarizes the initial cooling state of the core as a whole (discounting single riser failures). It takes into account ECCS and main coolant pump flow. HPI flow is neglected because it has been judged insufficient to reduce core damage. Branch 2 is admittedly a very broad category, but is actually more specific than might be thought. In the assessment of core damage due to partial ECCS flow, a large number of core flow situations were considered. These were classified according to an expected nominal amount of core damage, and any situation with an expected core damage that was approximately one-third (or more) lower than would be expected without any ECCS was classified as having partial ECCS flow. The remainder were classified as having no ECCS flow. An uncertainty 
distribution was then generated for the amount of fuel damage for the most conservative accident sequences in each class. It must be noted that these uncertainty distributions reflect the state of knowledge at the time they were constructed and, thus, may not include the results of analyses performed since that time. The uncertainty distributions are documented in detail in Appendix C.3.

The following statements summarize the logical case structure for this summary event:

- The following are the only ECCS failures that defeat all ECCS flow: $8 / 2,3,4$ (EC-3H33, EC-620, EC-nPmp). Also, 8/9 (EC-nV4) in the absence of a large LOCA completely defeats ECCS.

- If the ECCS failure is due to V-4 failure $8 / 9,10$ (EC-nV4, EC-4V4) and there is a large LOCA that is in 5/7,8,10 (not core, 105-10, $105 \mathrm{dn}$ ), then the lack of $\mathrm{V}-4$ valves opening will be made up for by the break area, resulting in full ECCS cooling to the core.

- ECCS succeeds globally in all analyzed ATWS events and single riser failures.

- All other ECCS failure and break location combinations are assessed to fall into the partial ECCS success category.

$\begin{array}{ll}\left.1 / 2+\frac{7 / 5}{(8 / 2,3,4+(8 / 9 \& 3 / 2)}\right) & \Rightarrow 14 / 1 \\ 13 / 2 \& 14 / 3 \\ 3 / 2 \& 8 / 9,10 \& 5 / 7,8,10 & \rightarrow 14 / 1 \\ \text { otherwise } & \Rightarrow 14 / 2\end{array}$

Event 15 - How much initial fuel damage occurs?

\begin{tabular}{|c|c|c|}
\hline BRANCH & ABBREV, & DESCRIPTION \\
\hline (1) & O\&IFD & $\begin{array}{l}\text { Initial fuel damage either does not occur or is below } \\
\text { the level that would be of concern for offsite } \\
\text { releases }\end{array}$ \\
\hline $\begin{array}{l}(2) \\
(3)\end{array}$ & $\begin{array}{l}28 \text { IFD } \\
138 \text { IFD }\end{array}$ & $\begin{array}{l}28 \text { of fuel (nominal) fails initially } \\
138 \text { of fuel (nominal) fails initially }\end{array}$ \\
\hline (4) & 258 IFD & 258 of fuel (nominal) fails initially \\
\hline (5) & 508 IFD & 508 of fuel (nominal) fails initially \\
\hline (6) & 67\&IFD & 678 of fuel (nominal) fails initially \\
\hline (7) & 100\&IFD & 1008 of fuel (nominal) fails initially \\
\hline 8 & TWS - FD & uel damage class for ATWS events \\
\hline
\end{tabular}

This event determines the nominal amount of fuel damage sustained as a result of the initiating events. It summarizes the operation of the RPS, ECCS and GSCS, as determined in the preceding events, to make this determination. The values displayed are nominal fractions of the fuel mass that experience clad failure. These nominal values are derived from the uncertainty analysis as described in Appendix C. 3 and are based on MELCOR [3-11] and other calculations as discussed there. The actual fuel damage amounts are sampled in the uncertainty analysis and used in the source term analysis. The nominal fuel damage values are representative of the distributions and are used in the APET analysis to refer to the core cooling states they represent. 
The nominal fuel damage categories correspond to the following system configurations:

28 - A single riser failure occurs in one of the inlet risers to fail ECCS cooling to $1 / 16$ of the core.

138 - ECCS is partially successful, and GSCS is operating at full efficiency.

258 - ECCS has completely failed, but GSCS is operating at full efficiency.

508 - ECCS is partially successful, but GSCS is either operating at a degraded efficiency or is not operating at all.

678 - ECCS has completely failed, but GSCS is operating at a degraded efficiency because the helium blanket around the core has failed.

1008 - ECCS and GSCS have both completely failed.

ATWS - The nominal value for fuel damage during an ATWS events is 50 and is based on liquid levels calculated during Westinghouse Hanford's ATWS analysis [3-12], although a broad uncertainty range on this value was used for the analysis.

The eight logical cases below represent, in reverse order, these eight system configurations and assign them to the appropriate fuel damage branches.

$\begin{array}{ll}1 / 2 & \rightarrow>15 / 8 \\ 11 / 3 \& 14 / 3 & \rightarrow 15 / 7 \\ 11 / 2 \& 14 / 3 & \rightarrow 15 / 6 \\ 11 / 2,3 \& 14 / 2 & \rightarrow 15 / 5 \\ 11 / 1 \& 14 / 3 & \rightarrow 15 / 4 \\ 11 / 1 \& 14 / 2 & \rightarrow 15 / 3 \\ 7 / 3,4 & \rightarrow 15 / 2 \\ \text { otherwise } & \rightarrow 15 / 1\end{array}$

\subsection{INITIAL CONFINEMENT SYSTEM STATUS}

This section of the APET serves to interface the APET analysis with the confinement system fault tree analysis [3-13] and the external events core damage analysis [3-2]. Events 16 through 23 consider the demand for and initial status of many of the confinement systems which will be used to mitigate the consequences of any fuel damage event. These systems include confinement isolation, fog sprays, steam vents, the EDT, the filter release stack, and the hydrogen mitigation system. The events in this section of the APET are:

16 What is the confinement pressure due to the initiating event?

17 Does the EDT function?

18 Does the filter release stack remain intact?

19 Does the confinement isolation system successfully operate?

20 If the confinement is compromised, what is the compromise location? 
The confinement pressure is determined in Event 16 based on MELCOR and HECTR confinement analyses for various initiating events [3-9], and is used to determine the demand for confinement isolation, fog sprays and steam vents. Given that a system is demanded, its failure rate is determined based on the confinement system fault tree analysis results. The cut sets from that analysis are analyzed by the TEMAC code and split fractions are calculated for use by EVNTRE in order to incorporate confinement system uncertainties into the APET analysis (see Reference 3-7 for details).

Events 17 and 18 were included in the APET analysis for seismic accident sequences. While the EDT could fail during a non-seismic event, such a failure would be rare by comparison to failure given that a significant seismic event had occurred, and filter stack failure would not be expected to occur without a seismic event. Probabilities for these events vary with the estimated peak ground acceleration and are based on an estimated seismic response (see Appendix C.13).

Event 16 - What is the confinement pressure due to the initiating event?

\section{BRANCH ABBREV, DESCRIPTION}

(1) Lo-pres Pressure is below confinement trip point (2" water)

(2) Conf-tp Pressure is between the confinement trip point and

(4) Vent-tp the spray trip point (10" water) Pressure is between the spray trip point and the steam vent opening pressure ( 1.5 psig)

Vent-tp Pressure is greater than the steam vent opening pressure

The initial confinement pressure is determined in Event 16 based on MELCOR [3-14] confinement calculations for the different initiating events [3-9]. This information is used to determine the demand for confinement isolation, fog sprays and steam vents.

Calculations indicate that the blowdown for any large LOCA will pressurize the confinement enough to demand that the steam vents open. Any transient will not develop a high enough confinement pressure to demand any confinement systems because the primary system will depressurize through the V-4 or RV-2 valves into the EDT. A small LOCA caused by stuck open RV2 valves (5/8) will act as a transient for confinement purposes because coolant is released to the EDT. All other small LOCAs will demand sprays and are likely to demand that the steam vents open.

The probabilities for this event are point estimates and were derived from the confinement MELCOR calculations. The probabilities related to the small LOCA case are unimportant to the overall results because the banana wall can successfully vent small LOCA steam sources without challenging the confinement. Thus, it does not matter if the steam vents are "demanded" because even if the venting function fails, the confinement is not 
compromised. This is discussed in more detail in Event 26. The three logical cases for this event model transients (and those small LOCAs that act as transients), large LOCAs and small LOCAs, respectively.

$$
\begin{aligned}
& 3 / 1+(3 / 3 \& 5 / 8) \rightarrow 16 / 1 \\
& 3 / 2 \Rightarrow 16 / 4 \\
& \text { otherwise (other small LOCAs) } \Rightarrow 16 / 3,4(0.100 / 0.900)
\end{aligned}
$$

\begin{tabular}{|c|c|c|}
\hline BRANCH & ABBREV. & DESCRIPTION \\
\hline (1) & DTok & No damage to piping or EDT \\
\hline (2) & DT - Atm & $\begin{array}{l}\text { Releases through dump line scrubbed by a pool, the } \\
\text { released to the environment (atmospheric release) }\end{array}$ \\
\hline (3) & DT-LiqR & $\begin{array}{l}\text { Releases through the dump line are made directly } \\
\text { the environment without retention (liquid release) }\end{array}$ \\
\hline 4) & $\mathrm{T}-\mathrm{NA}$ & No EDT activity because the $\mathrm{V}^{\prime}$ 's never opened \\
\hline
\end{tabular}

Event 17 - Does the emergency dump tank function?

This event determines not only whether the EDT remains initially intact, but also whether any effluents would flow to the EDT during the course of the accident. If the $V-4$ valves do not open $(8 / 2,9)$ or a seismic MPTR event occurs ( $4 / 2)$, then the EDT is not applicable because liquids can not get from the core to the EDT. The failure of the EDT on demand for non-seismic core damage accident sequences is modeled based on the TEMAC analysis of the confinement system fault tree results [3-7].

Seismic events other than MPTR can also damage the EDT. Seismic events were placed into three categories based on the peak ground accelerations that are most likely to cause the event. Transients are generally caused by low ground accelerations (up to $-0.3 \mathrm{~g}$ - slightly more severe than the $0.25 \mathrm{~g} \mathrm{~N}$ Reactor SSE), while small LOCAs and Building $182 \mathrm{~N}$ failures are usually caused by intermediate ground accelerations $(-0.5 \mathrm{~g})$, and large LOCAs and MPTR events are usually caused only by high ground accelerations ( $-0.7 \mathrm{~g}$ and above). An estimated seismic response for the EDT was used for each of the ground acceleration levels. The seismic failure branch probabilities are developed in Appendix C.13 and used as point estimates in the APET analysis. Uncertainty (aside from the uncertainty of the seismic hazard curves as expressed by the variations in the core damage frequency and the relative frequencies of the seismic core damage accident sequences) is not propagated for these quantities.

The five logical cases below are arranged logically as follows: the first case considers those accident progression pathways in which the EDT is not demanded because either the $V-4$ valves did not open or a MPTR event occurred. The second case considers high ground acceleration large LOCA events, while the third case considers intermediate ground acceleration small LOCA and Building $182 \mathrm{~N}$ failure events, and the fourth case considers other low ground acceleration transient accident sequences. The final case is used for non-seismic accident sequences that demand the EDT. 


$$
\begin{array}{ll}
8 / 2,9+4 / 2 & \Rightarrow 17 / 4 \\
2 / 3 \& 3 / 2 & \Rightarrow 17 / 1,2,3(0.010 / 0.495 / 0.495) \\
2 / 3 \&(3 / 3+4 / 3) & >17 / 1,2,3(0.500 / 0.250 / 0.250) \\
2 / 3 & \Rightarrow 17 / 1,2,3(0.990 / 0.005 / 0.005) \\
\text { otherwise } & \Rightarrow 17 / 1,2,3(0.999 / 0.0001 / 0.0009)
\end{array}
$$

Event 18 - Does the filter release stack remain intact?

$$
\begin{array}{lll}
\text { BRANCH } & \text { ABBREV } & \text { DESCRIPTION } \\
\text { (1) } & \text { StkOK } & \text { No damage to filter path and stack } \\
\text { (2) } & \text { StkP1g } & \text { The stack is plugged } \\
\text { (3) } & \text { StkTpl } & \text { The stack has toppled }
\end{array}
$$

The filter stack remains intact in internal events $(2 / 1)$ and fire accident sequences $(2 / 2)$. However, in seismic events, there is a possibility that the stack can be damaged. When stacks of this type are damaged, they typically topple at a point approximately 608 of the way up from the bottom [3-2], although there is a remote possibility that the stack could become plugged as the materials from the damaged portion of the stack fall down.

An estimated seismic response for the EDT was used for each of the three ground acceleration levels described in Event 17. These are each considered separately in the logic structure below. The seismic failure branch probabilities are developed in Appendix C.13 and used as point estimates in the APET analysis. Uncertainty (aside from the uncertainty of the seismic hazard curves as expressed by the variations in the core damage frequency and the relative frequencies of the seismic core damage accident sequences) is not propagated for these quantities.

The four logical cases below are arranged logically as follows: the first case considers high ground acceleration large LOCA events, while the second case considers intermediate ground acceleration small LOCA and Building $182 \mathrm{~N}$ failure events, and the third case considers other low ground acceleration transient accident sequences. The final case is used for nonseismic accident sequences.

$$
\begin{array}{ll}
2 / 3 \& 3 / 2 & \rightarrow 18 / 1,2,3(0.050 / 0.010 / 0.940) \\
2 / 3 \&(3 / 3+4 / 3) & \Rightarrow 18 / 1,2,3(0.500 / 0.005 / 0.495) \\
2 / 3 & \rightarrow 18 / 1,2,3(0.950 / 0.000 / 0.050) \\
\text { otherwise (internal events or fire) } \rightarrow 18 / 1
\end{array}
$$


Event 19 - Does the confinement isolation system successfully operate?

\begin{tabular}{|c|c|c|}
\hline $\mathrm{RAl}$ & BBREV & DESCRIPTION \\
\hline $\begin{array}{l}(1) \\
(2)\end{array}$ & $\begin{array}{l}\text { Iso } \\
\text { nIs }\end{array}$ & $\begin{array}{l}\text { Confinement has successfully isolated } \\
\text { Confinement does not isolate because a bypass switch } \\
\text { is in the wrong position ( } 3 \mathrm{H} 33 \text { or } 1 \mathrm{H} 45 \text { ) }\end{array}$ \\
\hline 3) & $\mathrm{nIs}-\mathrm{V} 18$ & $\begin{array}{l}\text { Confinement fails to isolate because the filter path } \\
\text { remains open }\end{array}$ \\
\hline b) & ok & $\begin{array}{l}\text { Confinement other than the filter path fails to } \\
\text { isolate }\end{array}$ \\
\hline 5) & & $\begin{array}{l}\text { Confinement is initially significantly } \mathrm{cc} \\
\text { General confinement isolation failure }\end{array}$ \\
\hline
\end{tabular}

During normal operation, the $\mathrm{N}$ Reactor confinement building operates in a vented mode where outside air is circulated through the building and vented back to the environment through filters [3-15]. All of these paths to the outside environment are designed to close using isolation valves during accident conditions. If one of these paths remains open, it becomes a direct path to the environment for any released fission products. This event considers whether the confinement isolation system is able to successfully isolate the confinement building from the outside environment.

This event incorporates all mechanisms for isolation failure as examined in the confinement systems fault tree analysis. Failure to isolate the filter path is separated from failure to isolate the rest of the confinement because filter path isolation failure can imply filter element failure if a LOCA occurs. Filter element failure is unrecoverable, while most other failures may be recoverable. The "initial confinement compromise" event represents cases where doors or other significant openings may have been left open inadvertently. In this case, even if the isolation system works perfectly, the confinement will not be isolated because another pathway out of the confinement exists. The probability for this branch was calculated from historical plant data as described in Reference 3-16. This probability is used as a point estimate.

The confinement isolation system is actuated by either the ECCS actuation signal or a measured confinement building pressure of greater than two Inches water. If the $3 \mathrm{H} 33$ ECCS bypass switch is mispositioned, then the ECCS actuation signal is not generated. Thus, if both ECCS and pressure fail to trip confinement isolation $(8 / 2 \& 16 / 1)$, then the confinement fails to isolate because no isolation signal is generated. This is modeled in the fifth logical case below.

Fire and seismic events can also cause confinement isolation failure. Fires in Room 6, Room 8 and Corridor 1 all cause confinement isolation failure by damaging the control cables for the confinement isolation valves. Also, large LOCA and MPTR seismic events can be associated with confinement isolation failures because collapse of Buildings $109 \mathrm{~N}$ and $105 \mathrm{~N}$ can cause large LOCAs and MPTR events, respectively [3-17].

The first two logical cases below account for fire events in locations that can damage the control cables required to successfully isolate the confinement. Fires in other locations are subject to the "random" 
isolation failures modeled in the final "otherwise" case. The third and fourth cases allow for seismic damage to the confinement buildings in MPTR and large loca accidents, respectively. The fifth case models the failure of the confinement isolation function in transient accidents where the $3 \mathrm{H} 33$ ECCS bypass switch is improperly positioned. The final case models the confinement isolation function when there is no accident-related condition to prevent the isolation function from occurring. It considers equipment failures and initial confinement compromise as discussed above. The nonzero probability for branch 2 in this case accounts for the failure to properly position the $1 \mathrm{H} 45$ bypass switch.

The number of possible accident progression pathways in the external events analyses can be much larger than for the internal events analysis. Thus, it is desirable to minimize the number of possible accident progression pathways while still maintaining the possibility for "random" failures of the confinement isolation system in addition to the other, accident-induced confinement failures. This was done by constructing a composite random confinement failure fraction from point estimate values of the "otherwise" case and using it in each of the first four logical cases. Branch 4 (confinement failure not affecting the path to the filters) was used to represent this composite confinement failure rate because it conservatively releases effluents directly to the environment and represents the largest single source of random confinement failures.

The probability of confinement isolation failure for internal events is modeled based on the TEMAC analysis of the confinement system fault tree results. The failure probabilities for external events are determined from the TEMAC analyses of the external events core damage results [3-7].

$$
\begin{aligned}
& 2 / 2 \& 8 / 4 \rightarrow 19 / 1,4,6(0.923 / 0.018 / 0.059) \\
& 2 / 2 \& 8 / 9 \rightarrow 19 / 1,4,6(0.472 / 0.018 / 0.510) \\
& 2 / 3 \& 4 / 2 \rightarrow 19 / 1,4,6(0.766 / 0.018 / 0.216) \\
& 2 / 3 \& 3 / 2 \rightarrow 19 / 1,4,6(0.914 / 0.016 / 0.070) \\
& 8 / 2 \& 16 / 1 \rightarrow 19 / 2 \\
& \text { otherwise } \rightarrow 19 / 1.6(0.982 / 0.001 / 0.003 / 0.009 / 0.005 / 0.0001)
\end{aligned}
$$

Event 20 - What is the confinement compromise location?

$\begin{array}{lll}\text { BRANCH } & \text { ABBREV } & \text { DESCRIPTION } \\ \text { (1) } & \text { ConfOK } & \text { Confinement is not compromised } \\ \text { (2) } & \text { Envir } & \text { Initial compromise is directly to the environment } \\ (3) & \text { Zone } & \text { Initial compromise is to another zone }\end{array}$

If the confinement isolation function fails for any reason including seismic building failure (as modeled in Event 19) a release path to the environment is established. This path can be either direct, as in the case of the failure of an isolation valve or building failure $(19 / 2,3,4,6)$, or through another radiation protection zone of the plant [3-18]. This latter mode is used for cases where a door or other opening is inadvertently left open $(19 / 5)$. Branch 1 for this event is used when the isolation function was successful. This event is a summary event, so no uncertainty assessment was performed on it. 


$$
\begin{array}{ll}
19 / 2,3,4,6 & \rightarrow 20 / 2 \\
19 / 5 & \Rightarrow 20 / 3 \\
\text { otherwise } & >20 / 1
\end{array}
$$

Event 21 - Does the forced ventilation system successfully shut down?

\section{BRANCH ABBREV, DESCRIPTION}

(1). FV The forced ventilation system remained on

(2) nFV The forced ventilation system successfully shut down

As part of the confinement isolation function, a number of large ventilation fans for both the $105 \mathrm{~N}$ and $109 \mathrm{~N}$ buildings are designed to shut down [3-15]. If $A C$ power remains available to operate the fans $(6 / 4)$ and no confinement isolation signal is generated $(19 / 2)$, the forced ventilation system will continue to operate and force released radionuclides out to the environment. Although failure of the relays that control the fans could be examined, they are neglected for the purposes of this analysis due to their low failure rate relative to the other confinement failure components. considered in the APET analysis. This causes this event to be a summary event with all probabilities either zero or one. No uncertainty analysis is performed on summary events.

$19 / 2 \& \underline{6 / 4} \Rightarrow 21 / 1$

otherwise $\Rightarrow 21 / 2$

Event 22 - Does the fog spray system operate?

BRANCH ABBREV, DESCRIPTION

(1) Spr All fog sprays actuate initially

(2) nSpr All fog sprays failed - whether demanded or not

(3) Spr-ND Fog sprays were not demanded, but will operate if demanded later in the accident progression

The operating procedures for the $\mathrm{N}$ Reactor fog spray system indicate that it is intended for use only as a confinement building pressure reduction device and not specifically for radionuclide decontamination of the confinement building atmosphere. Thus, the APET implementation of the fog spray function does not allow for spray actuation without a confinement pressure demand as established in Event 16 [3-19].

An examination of the fault tree analysis for the $105 \mathrm{~N}$ and $109 \mathrm{~N}$ pipe gallery fog sprays indicates that almost all of the failure frequency for both systems is related to the failure of the common diesel pumps that feed water to the fog spray system [3-13]. For this reason, fog spray function is considered as an "all or nothing" proposition in the APET analysis.

A number of other systems have the option of taking water from the fog spray diesel pumps. The failure rates for these systems are increased if the fog spray diesel pumps are not available. The fog spray diesel pumps do run under accident conditions even if the fog sprays themselves are not 
demanded. For this reason, the fog spray function in this event considers whether the fog sprays would operate if demanded (whether they were demanded). This allows other systems to determine whether the fog spray diesels are functioning without requiring that the fog sprays themselves are operating.

The fog spray diesel pumps supply the water to the GSCS in oncethrough mode, so if the GSCS is unavailable 10/4 (nGSCS), then fog spray diesel pump failure is a possible cause. The Level I PRA results [3-6] are examined using TEMAC to determine a representative probability that fog spray diesel pump failure is a contributing factor to the loss of GSCS function (see Reference 3-7 for the TEMAC analysis discussion).

Fire events can also cause fog spray failure. Fires in Room 6 , Room 8 and Corridor 19 all cause some sort of fog spray failure by damaging the control cables for the spray valves [3-17]. In each of these cases, however, the diesel pumps would start. The conservatism introduced by placing each of these fires in the fog spray failure category is small, however, because many of these same fires also cause confinement failure and make the function of the other systems that can use fog spray water irrelevant. It should be noted that, since no fires induce LOCA events, the fog sprays never actuate initially during fire accident sequences.

This model always associates seismic events with fog spray failure. The fog spray diesel pumps, like most other plant systems, draw water from the silo. Silo failure is by far the dominant cause of ECCS failure and cuts across most water-using plant systems.

When examining the eight logical cases for this event, it must be remembered that the true failure state for this event is branch 2 because the fog spray pumps operate in branch 3 (even though no spray water is injected into the confinement). Thus, the first logical case precludes any fog spray operation for seismic events as described above. The next three cases implement the results of the fire analysis. Cases 5 and 6 are used when the confinement pressures are not high enough to demand fog spray operation (case 5 correlates fog spray failure to failure to establish the GSCS once-through mode of operation). Cases 7 and 8 are the same as cases 5 and 6 except that, since confinement pressures are high enough to demand fog spray operation, the success state is branch 1 rather than branch 3 as before.

$\begin{array}{ll}2 / 3 & \rightarrow 22 / 2 \\ 2 / 2 \& 8 / 4 \& 19 / 1 & \rightarrow 22 / 2,3(0.653 / 0.347) \\ 2 / 2 \& 8 / 4 & \rightarrow 22 / 2,3(0.714 / 0.286) \\ 2 / 2 \& 8 / 9 \& 19 / 1 & \Rightarrow 22 / 2 \\ 11 / 3 \& 16 / 1,2 & \rightarrow 22 / 2,3(0.137 / 0.863) \\ 16 / 1,2 & \rightarrow 22 / 2,3(0.004 / 0.996) \\ 11 / 3 & \rightarrow 22 / 1,2(0.857 / 0.143) \\ \text { otherwise }(16 / 3,4) & \rightarrow 22 / 1,2(0.996 / 0.004)\end{array}$


Event 23 - In what mode does the hydrogen mitigation system operate?

BRANCH ABBREV. DESCRIPTION

(1) HM-OK Hydrogen mitigation system is operating in the "normal" mode ( $3 \mathrm{k}$ cfm nitrogen, 1 mixing fan)

(2) HM-3k No mixing fans started - only $3 k$ nitrogen purge

(3) HM-40k 40k cfm nitrogen inert and a mixing fan are running

(4) HM-Mix Only the mixing system is running (no nitrogen)

(5) HM-20k 20k cfm nitrogen inert and a mixing fan are running

(6) nHM The entire hydrogen mitigation system is not running

The APET analysis was performed under the assumption and with the assurance from Westinghouse Hanford that the hydrogen mitigation system would be fully installed as designed before $N$ Reactor would be re-started. This is the basis for Event 23. Failure data for this event was obtained by manual extraction of data from a fault tree analysis of the hydrogen mitigation system $[3-20]$. The fault trees from that report were not entered into the TEMAC analysis, so point estimate failure probabilities were used and uncertainty in the failure rate of the system was not considered (uncertainty in the effectiveness of the system was considered in the phenomenology section).

The fault tree analysis provided probabilities for system failure due to internal events. However, failures due to fire and seismic events were not considered in that analysis and are difficult to accurately assess because major portions of this system are not yet installed. This analysis assumed that the most recently available plans for the installation of the system would be followed. These plans call for the control cables for the nitrogen injection subsystem to be run such that they avoid all of the core damage fire areas identified in the external events PRA analysis and for the nitrogen tanks and piping to be seismically qualified to the $\mathrm{N}$ Reactor SSE level of $0.25 \mathrm{~g}$. This means that increased nitrogen system failure is not expected in fire-induced core damage events, but should occur in seismic events because almost all seismic core damage events occur at ground acceleration levels higher than the $N$ Reactor SSE. The results of this study could change significantly if either of these two assessments were to change. The bases for the probability values that arise from the seismic assumptions are discussed in Appendix C.13.

The planned procedures for hydrogen mitigation system operation [3-20] call for using the $40 \mathrm{k}$ inert system only in cases where neither ECCS nor GSCS are operating (unmitigated accidents), with the $20 \mathrm{k}$ inert system as a backup mode representing the failure of one of the two $20 \mathrm{k}$ nitrogen trains. The "normal" mode is to be used for all other core damage events where at least 28 hydrogen is measured. The nitrogen system can only be started manually, while the mixing fan is designed to start automatically on low primary system pressure regardless of hydrogen concentration [3-20].

The first six cases represent seismic events with varying ground acceleration and GSCS function because different ground accelerations fail the nitrogen tanks differently, and different GSCS states determine the mode of hydrogen mitigation system operation. The ground acceleration logic is similar to that of Events 17 and 18 . The next four cases 
represent the different possibilities of GSCS success (measured in terms of fuel damage states) and $A C$ power failure because $A C$ power failure causes a slightly increased probability of mixing fan failure over cases where AC power is available. The next case is used in the event of a single riser failure in which the V-4 valves operate successfully. Single riser failure events do not produce enough hydrogen to demand the nitrogen injection system. The otherwise case is taken only when the $V-4$ valves have failed to open and the reactor remains at high pressure. This event is distinguished from the others because the mixing fans are tied to reactor primary system pressure. For all fires, the hydrogen mitigation system fails with the same probabilities used in the internal events analysis.

$\begin{array}{ll}2 / 3 \& 3 / 2 \& 11 / 1 & \Rightarrow 23 / 3,4,5,6(0.043 / 0.900 / 0.007 / 0.050) \\ 2 / 3 \& 3 / 2 & \Rightarrow 23 / 1,4,6(0.047 / 0.906 / 0.047) \\ 2 / 3 \&(3 / 3+4 / 3) \& 11 / 1 & \Rightarrow 23 / 3,4,5,6(0.430 / 0.490 / 0.070 / 0.010) \\ 2 / 3 \&(3 / 3+4 / 3) & \Rightarrow 23 / 1,4,6(0.500 / 0.490 / 0.010) \\ 2 / 3 \& 11 / 1 & \Rightarrow 23 / 3,4,5,6(0.851 / 0.010 / 0.129 / 0.010) \\ 2 / 3 & \Rightarrow 23 / 1,4,6(0.980 / 0.010 / 0.010) \\ 6 / 4 \& 15 / 5,6,7,8 & \rightarrow 23 / 3,4,5,6(0.849 / 0.005 / 0.136 / 0.010) \\ 6 / 4 \& 15 / 1,2 & \Rightarrow 23 / 1,2,4,6(0.985 / 0.001 / 0.004 / 0.010) \\ 15 / 5,6,7,8 & \Rightarrow 23 / 3,4,5,6(0.859 / 0.005 / 0.136 / 0.0001) \\ \frac{15 / 1,2}{8 / 2,9} & \Rightarrow 23 / 1,2,4,6(0.995 / 0.001 / 0.004 / 0.0001) \\ \text { otherwise } & \Rightarrow 23 / 4,6(0.998 / 0.002)\end{array}$

\subsection{INITIAL BLOWDOWN ACCIDENT PROGRESSION}

The N-Reactor confinement system, unlike a conventional containment structure, was designed to vent the steam blowdown from a pipe break to the environment, then re-isolate to confine any radionuclides that would evolve thereafter. Events 24 through 32 model the venting and re-isolation of the confinement, as well as any confinement building failures that would occur as the result of a failure to successfully vent the confinement. Phenomenological models and confinement system response information are combined in this section of the APET. Most of this section of the APET is irrelevant during transients as it models the response of the confinement to blowdown conditions that do not exist during transients.

The events in this section of the APET are:

24 Does hydrogen concentration reach 38 before steam vents are demanded?

25 Does the operator isolate the vents based on 38 hydrogen?

26 If demanded, do the steam vents open?

27 Did all steam vent isolations close after the blowdown?

28 Does confinement fail during the blowdown phase?

29 What is the size of the confinement failure?

30 What is the location of the confinement failure?

31 Do the operating fog sprays survive the confinement failure?

32 Is the filter release path established after the blowdown? 
Events 28 through 31 are used several times in different forms throughout the APET. They define whether the confinement building fails and, if so, the size and location of the failure. In addition, it is possible that a particularly violent confinement failure could disrupt the function of the fog spray system either by severing pipes, by redirecting spray heads to cause gaps in coverage, or by significantly bypassing spray coverage area due to the confinement failure. The data sources for these events are described in Appendix $C$.

Event 24 - Does the $\mathrm{H}_{2}$ concentration reach 38 before the vents are demanded?

BRANCH ABBREV, DESCRIPTION

(1) 38-H2 38 Hydrogen level reached before vents are demanded

(2) $\mathrm{n} 38-\mathrm{H} 2 \quad 38$ Hydrogen level not reached before vents are demanded

This event determines whether the 38 hydrogen level is physically reached before venting is demanded. This is important because, under current procedures, the operator is instructed to isolate the steam vents when the hydrogen concentration reaches 38 . These procedures are almost certain to be changed when the full hydrogen mitigation system is installed because the proposed procedures for the nitrogen system call for it to be started when the 28 hydrogen level is reached. However, the existing procedures are modeled in this case because of possible negative effects should the operators isolate the steam vents before the blowdown has progressed to the point where it can no longer challenge the confinement integrity.

Large and smail LOCAs demand venting quickly when they occur to the confinement, so the 38 level will not be reached before venting is demanded. Also, the supporting calculations [3-9] predict that the 38 hydrogen level will never be reached during single riser failure events. If there is a break in the pressurizer penthouse and the mixing system is not runnimg, then the 38 leve 1 will be reached in that area (see Appendix C.1). All other situations are transients that will not demand the sleail vents prior to the initiation of recovery actions. Point estimate probabilities are used for this event because this is a summary event with only zero and one probabilities.

$$
\begin{aligned}
3 / 2+(3 / 3 \& 16 / 4)+15 / 1,2 & \rightarrow 24 / 2 \\
5 / 2 \& 23 / 1,3 & \rightarrow 24 / 1 \\
\text { otherwise } & \rightarrow 24 / 2
\end{aligned}
$$

Event 25 - Does the operator isolate the vents based on 38 hydrogen?

BRANCH ABBREV. DESCRIPTION

(1) H2-Iso Operator isolates vents before they are demanded

(2) H2-nIso nperator does not isolate vents before they are demanded 
This event determines whether the operator actually isolates the vents before they are demanded. A point estimate error of omission probability is used to model the operator failing to isolate the vents when procedures tell him to do so. No error is modeled for the case when the 38 hydrogen level is not reached because, in almost all of these cases, the steam vents are either demanded very quickly (within the first few minutes following the accident initiator) or are not demanded at all (transients).

$$
\begin{array}{lll}
24 / 1 & \Rightarrow 25 / 1,2 \quad(0.998 / 0.002) \\
\text { otherwise } & \Rightarrow 25 / 2
\end{array}
$$

Event 26 - Do the steam vents open if demanded?

\section{BRANCH ABBREV. DESCRIPTION}

(1) Vent-Op Venting is successful

(2) Vnt-nop Venting is not successful

(3) Vent-NA Venting is not applicable because it is not demanded

This event determines whether the steam vents open on demand. The failure to open probability is the probability of failure for two steam vents [3-13] and is sampled in the TEMAC analysis of the confinement system fault trees [3-7]. It has been postulated that a loss of AC power could cause the accumulators for hydraulic steam vent isolation valves to become depleted and the isolation valves to block the steam vents. This possibility was discounted for this analysis because, in all cases where steam vents are demanded, confinement pressures are expected to cause the demand to occur within the first few minutes following accident initiation. In addition, the longer it takes for the steam vents to be demanded, the smaller the blowdown source into the confinement, so fewer steam vents have to succeed in order for the venting function to protect the confinement. Finally, supporting calculations [3-9] indicate that small LOCA sources can be successfully vented through the banana wall without the operation of steam vents and without challenging the confinement. For these reasons, steam vent isolation valve accumulator depletion was discounted as a method for failure of the steam venting function.

The first logical case for this event prevents the steam vents from opening if they are not demanded. The second case considers whether the operators isolated the steam vents before they could open. The final case models failure of the steam venting function given that the vents are demanded and not previously isolated.

$$
\begin{array}{ll}
\frac{16 / 4}{25 / 1} & \Rightarrow 26 / 3 \\
\text { otherwise } & \Rightarrow 26 / 2 \\
& >26 / 1,2(0.999 / 0.001)
\end{array}
$$


Event 27 - Did all steam vent isolations close after the blowdown?

BRANCH ABBREV. DESCRIPTION

(1) Vent-Cl All vents reclosed

(2) Vnt-nCl Not all vents reclosed

(3) Vent-NA Vents reclosing is not applicable because venting was not applicable

This event considers whether the steam vents are successfully reisolated before significant radionuclide release to the confinement occurs. The probability of failure to reisolate all steam vent openings is based on the failure of both of the isolation valves inside each vent opening to close as computed in the TEMAC analysis of the confinement system fault tree analysis results [3-7]. The hydraulic isolation valves "fail" in the closed position, so $A C$ power to the hydraulic power packs is not required to close the valves [3-13]. The only power that is required to close these valves is DC control power to actuate the mechanisms that drain hydraulic pressure from the valve cylinders.

The first logical case for this event states that the valves do not need to be isolated if the steam vents were never demanded in the first place. The second case considers the placement of the bypass switches that can prevent confinement isolation from occurring. The conditional probability of vent isolation failure is higher if the confinement has failed to successfully isolate initially because of common sensors and actuation components. This is handled in the third case, and the fourth case examines vent isolation failure for all other cases.

$$
\begin{array}{ll}
26 / 3 & \Rightarrow 27 / 3 \\
19 / 2 & \Rightarrow 27 / 2 \\
19 / 1.5 & \Rightarrow 27 / 1,2(0.987 / 0.013) \\
\text { otherwise } & >27 / 1,2(0.999 / 0.001)
\end{array}
$$

Event 28 - Do the confinement buildings fail during the blowdown phase?

BRANCH ABRRFIL DESCRIPTION
$\mathrm{C}-\mathrm{OK}$
Confinement is intact
(2) CF-Init
Confinement was compromised during the initial
CF-BD isolation

This event is used to determine if the confinement building failed during either the initial confinement isolation of blowdown accident progression. The confinement can only be intact at this point if the Inltial confinement isolation was successful, the vents did not fail to open, and the vents did not fail to close. The cases where the confinement will fail during the blowdown phase are clearly defined and the uncertainty is very small. Therefore, point estimate probabilities of zero and one are used for this event.

Supporting calculations [3-9] indicate that small LOCA steam sources into the confinement can be successfully vented through the banana wall 
without the operation of steam vents and without challenging the confinement. This is true for all pressure sources that take longer than a few minutes for the pressure to rise to the confinement failure pressure. Thus, even if the steam vents fail to open and fog sprays fail to come on in a small LOCA or transient scenario, the confinement should not fail due to overpressure.

The first logical case for this event picks out the cases where the confinement has remained intact until this time (isolation succeeds, the vents do not fail to open in a large LOCA, and all vents reclose). The second case models confinement failure during the blowdown, and the "otherwise" case picks up those cases where the confinement failed to isolate initially in Event 19.

$$
\begin{array}{ll}
19 / 1 \&(\underline{26 / 2}+\underline{3 / 2}) \& \underline{27 / 2} \rightarrow 28 / 1 \\
27 / 2+26 / 2 & \rightarrow 28 / 3 \\
\text { otherwise } & \Rightarrow 28 / 2
\end{array}
$$

Event 29 - What is the size of the confinement building failure?

\section{BRANCH ABBREV, DESCRIPTION}

(1) C-OK Confinement has not failed

(2) C-Leak Confinement failure is a leak

(3) C-Rupt Confinement failure is a rupture

The APET analysis defined a confinement building rupture to be a confinement building failure such that the filter path has no decontaminating effect on the gaseous releases from the plant. A leak is defined such that, if the filter release path were to be in operation, competing flow paths would be set up between the filter path and the confinement failure. This distinction is, however, superficial because the binning input in Section 3.9 is set up using the conservatism that the "competition" between these paths is minimal and that the outflow from the confinement is dominated by the flow out the confinement building failure.

All failures of the confinement to initially isolate are classified as ruptures because of the large areas that would remain open to the environment. Steam vents that fail to isolate also fall into this category based on their size. Overpressurization confinement building fallures are also ruptures because of the large, sudden pressure increases caused by large LOCA events (small LOCAs vent through the banana wall before confinement failure can occur, see Appendix C). For all other cases, the confinement remains intact as modeled in Event 28.

$$
28 / 2+(3 / 2 \& 28 / 3) \rightarrow 29 / 3
$$

otherwise $\quad \rightarrow 29 / 1$


Event 30 - What is the location of the confinement building failure?

$\begin{array}{lll}\text { BRANCH } & \text { ABBREV. } & \text { DESCRIPTION } \\ \text { (1) } & \text { Conf-OK } & \text { No confinement compromise } \\ \text { (2) } & \text { Envir } & \text { Confinement compromise is directly to the environment } \\ \text { (3) } & \text { Zone } & \text { Confinement compromise is to another zone }\end{array}$

It makes a significant difference to the source term analysis whether a confinement building failure occurs directly to the environment or to another radiation protection zone of the $\mathrm{N}$ Reactor plant because, if the failure is to another zone, there is additional time for the released radionuclides to condense or settle out of the gas stream before being released to the environment. The only confinement building failure that can be confidently said to occur to another zone of the plant in these sections of the APET is the failure to initially isolate the confinement due to an initial compromise such as a Zone I door being left ajar. This was considered in Event 20 and is followed here in the otherwise case as a summary.

The first logical case for this event ensures that accident progression pathways without confinement failure in Event 29 do not show confinement failure in this event. The second case ensures that accident progression pathways with previous environmental releases (modeled in Event 20) continue in that state. The third case forces any confinement rupture events that occur during the blowdown accident progression to cause releases to the environment (confinement leak events do not occur in this section of the APET). The final case allows releases to other plant zones from Zone I doors being left ajar to continue in that state.

$$
\begin{array}{ll}
29 / 1 & \Rightarrow 30 / 1 \\
20 / 2 & \Rightarrow 30 / 2 \\
28 / 3 \& 29 / 3 & \Rightarrow 30 / 2 \\
\text { otherwise } & >30 / 3
\end{array}
$$

Event 31 - Do the operating fog sprays survive?

\section{BRANCH ABBREV, DESCRIPTION}

(1) Spr-nCh Spray configuration is unchanged

(2) nSpr-E The spray system is rendered inoperable or ineffective by a confinement failure

It is possible that a particularly violent confinement failure could disrupt the function of the fog spray system either by severing pipes, by redirecting spray heads to cause gaps in coverage, or by causing the confinement atmosphere to significantly bypass the spray coverage area due to the confinement failure. The only event in these sections of the APET that is violent enough to cause fog spray failure is confinement rupture due to an unvented large LOCA. The data sources for these events are described in Appendix $C$.

$$
\begin{aligned}
& 28 / 3 \& 29 / 3 \rightarrow 31 / 1,2(0.978 / 0.022) \\
& \text { otherwise } \rightarrow 31 / 1
\end{aligned}
$$


Event 32 - Is the filter release path established after the blowdown?

\section{BRANCH ABBREV, DESCRIPTION}

(1) Fpath Filter path successfully established

(2) nFpath Filter path not established, but recoverable

(3) Fpath-NA Filter path does not matter since confinement rupture has occurred, or the path does not open and is unrecoverable.

The blowdown phase concludes with the establishment of the filter release path. The path is the same as that used during normal reactor operations except that a different filter bank is placed into service and no fans are used to force air through the filters (which filter bank is in service is determined in Event 85 ). Instead, natural convection, the volumetric displacement from the fog spray water or the nitrogen addition from the hydrogen mitigation system are the only mechanisms available to push the effluent gases towards the filters. This allows a much longer residence time for decontamination of the effluent gases in the confinement building before they pass to the filters. It also, however, means that failures of the confinement building (even the isolation system) would be expected to cause significant, unfiltered releases to the environment because of the short distances required to reach the environment compared to the long filter release path and the fact that there are no fans pulling the effluent gases towards the filters.

Failures of the confinement isolation system are often recoverable. If these are the only confinement failures, the filter path could become operable later if the confinement isolation were achieved. For this reason, isolation failures cause the filter path to fail such that it could be recovered later. Other confinement failures, however, are unrecoverable, so effective filter path operation is also unrecoverable.

The exception to filter path and confinement isolation recoverability for isolation failures is the filter path isolation valve itself. If this valve fails to function properly, the filter path is unrecoverable. This is a conservative assessment because, if the valve fails to close initially, a small pressure increase would actually be required to damage the filters or the filter path. Such a pressure increase does occur during LOCA events, but may not occur during transients. If the valve closes but fails to reopen, the filter path is unrecoverable because the valve is located inside a radiologically contaminated area.

The first logical case for this event assigns as unrecoverable the cases where the confinement building has failed, and the second case assigns as recoverable those cases where confinement isolation has failed. The next two cases are for the conditional probability that the filter path will fail to be established given that the steam vents failed to close (the two systems have common actuation components and sensors) and that AC power has failed (a filter path support system, mainly for the confinemett isolation valve leading to the filter path), respectively. The final case is the independent failure probability for the filter path to be 
established. The probabilities for the last three cases are all taken from the TEMAC analysis of the confinement system fault tree analysis results (see Reference 3-13 for the confinement systems fault tree analysis and Reference 3-7 for the TEMAC analysis of $i t)$. This event determines only whether the filter release path exists and is useful. Whether there are any filters in the path is asked near the end of the tree.

$$
\begin{aligned}
& (\underline{28 / 1} \& 29 / 3)+18 / 2 \quad \rightarrow 32 / 3 \\
& 19 / 2,4,5,6+(27 / 2 \& 19 / 1) \Rightarrow 32 / 2 \\
& 27 / 2 \quad \rightarrow 32 / 1,2(0.713 / 0.287) \\
& 6 / 4 \quad \rightarrow 32 / 1,2(0.999 / 0.001) \\
& \text { otherwise } \quad \Rightarrow 32 / 1,2(0.999 / 0.001)
\end{aligned}
$$

\subsection{PRE-RECOVERY ACCIDENT PROGRESSION}

Accident progression phenomenology for $N$ Reactor is modeled in two phases in the APET analysis: pre-recovery ("early") and post-recovery ("late"). The distinction between these phases is made because recovery actions at $\mathrm{N}$ Reactor can cause accident progression phenomena to occur that otherwise would not. For example, it is possible that "recovery" of ECCS could cause a steam pressure spike large enough to fail the confinement building. For this reason, both pre- and post-recovery accident progression are modeled. Recovery is defined to occur at two hours after the occurrence of the initiating event. This will be discussed in further detail in Section 3.6.

Events in this section of the APET (Events 33-50) model phenomena including hydrogen generation and combustion, loss of the helium blanket, possible graphite oxidation, vacuum breaker demand and operation, and filter pach reisulation and survival. The events in this section of the APET are:

33 Is hydrogen flammable in the confinement building?

34 Is hydrogen flammable in the pipe gallery or emergency dump tank?

35 Is the hydrogen detonable?

36 Dnes the hydrogen in the confinement building ignite?

37 Does the hydrogen in the pipe gallery or emergency dump tank 1gnite?

38 Does the hydrogen in the confinement building detonate?

39 Does a detonation occur in the emergency dump tank?

40 Does the helium blanket surrounding the graphite stack remain intact?

41 Has oxidation begun on the graphite stack?

42 Are the vacuum breakers demanded?

43 Do the vacuum breakers open (if demanded)?

44 Do the vacuum breakers reclose?

45 Does the filter exhaust path reisolate to prevent filter damage?

46 Does confinement fail during the pre-recovery phase?

47 What is the size of the confinement failure?

48 What is the location of the confinement failure?

49 Do the operating fog sprays survive the confinement fallure?

50 Is the filter exhaust path open? 
The hydrogen modeling in this section of the APET is worthy of special note. The seven events described above allow for hydrogen flammability in up to three locations simultaneously, with detonability, ignition and detonation considered separately for each location. The three locations considered are the EDT, the pipe gallery, and the LOCA location (if applicable). The EDT can become flammable as hydrogen follows the coolant out of the core, through the V-4 valves and to the EDT. The pipe gallery can become flammable because the return line from the EDT comes into the confinement near the top of the pipe gallery. Finally, the LOCA location can become flammable as hydrogen follows the coolant out through the break into the confinement. This situation is made worse if all of the V-4 valves fail to open because all of the coolant and hydrogen flow out through the break rather than being split between the break and the EDT as would occur if some of the $V-4$ valves were to open. The action of the hydrogen mitigation system is considered as a mitigative factor in the hydrogen analysis. The underlying analysis for the hydrogen analysis is found in Appendix $C$.

Event 33 - Is hydrogen flammable in the confinement building?

$\begin{array}{lll}\text { BRANCH } & \text { ABBREV } & \text { DESCRIPTION } \\ \text { (1) } & \text { H-Mit } & \begin{array}{l}\text { Hydrogen has been mitigated or is otherwise rendered } \\ \text { non-flammable }\end{array} \\ \text { (2) } & \text { H-Pent } & \text { Hydrogen is flammable in the pressurizer penthouse } \\ \text { (3) } & \text { H-SG } & \text { Hydrogen is flammable in the steam generator cells } \\ \text { (4) } & \text { H-Barr } & \text { Hydrogen is flammable in the pipe barrier space } \\ \text { (5) } & \text { H-105 } & \begin{array}{l}\text { Hydrogen is flammable in large areas of the } 105 N \\ \text { building }\end{array}\end{array}$

This event and the following event summarizes a large amount of information to determine whether hydrogen is flammable in the confinement. As described above, hydrogen can be flammable in three locations simultaneously at $N$ Reactor: the vicinity of a pipe break, the EDT and the pipe gallery. The latter two locations are dealt with in the next event. This event summarizes hydrogen flammability in the vicinity of a primary system break. Several factors go into the flammability analysis. These include:

- the amount of hydrogen produced as determined within the APET by the amount of fuel damage,

- the ability of the hydrogen produced inside the core to travel to the break location as influenced by the position of the V-4 valves,

- the ability of the hydrogen released in an area to become flammable in the absence of mitigation as determined from the geometry of the area into which the hydrogen is being released, and

- the effects of various mitigating factors such as fog spray mixing, fan-induced mixing, nitrogen and forced ventilation sweep-out, and nitrogen and steam inerting of the atmosphere.

The first logical case for this event filters out several nonflammable cases. These are single riser failure events (insufficient 
hydrogen production), failure of forced ventilation to shut down (large air flow sweeps out hydrogen), and steam generator cell 6 when the nitrogen addition system is functioning (nitrogen is injected into the confinement through cell 6). Following that are cases related to specific break locations. Specifically, the second case states that the penthouse becomes flammable if the hydrogen mixing fans do not operate or the break is a small LOCA (the only small LOCA pipes in the penthouse are high in the penthouse, and the hydrogen elicitees expressed doubt as to the effectiveness of the mixing fans at that location).

The steam generator cells can become flammable when a break occurs there and the nitrogen system does not operate, and are more likely to become so if the $V-4$ valves do not open (this forces all hydrogen out through the break instead of dividing it with the EDT). The third case prevents flammability in the steam generator cells if the nitrogen injection system operates fully. The fourth case ensures flammability if the V-4 valves fail to open, and the fifth case models all other large LOCAs in the steam generator cells (small LOCAs are modeled in the "otherwise" case). The pipe barrier space becomes flammable whenever a pipe in that area breaks and ECCS does not succeed. This is modeled in case six.

Flammability in Building $105 \mathrm{~N}$ can be reduced by the mixing action of the fog sprays as well as the nitrogen addition system. Case seven models this, while case eight ensures flammability if the V-4 valves fail to open (the entire hydrogen mass will collect in Building 105N), and case nine models large LOCAs in this location.

The logic structure for the first nine cases handles those LOCA situations with non-trivial frequencies where the confinement building (other than the pipe gallery) would be expected to become flammable. Thus, the "otherwise" case models all other cases as not being flammable. The detailed explanation of hydrogen phenomenology as well as the justification behind the probabilities is beyond the scope of this report section, but is shown in Appendix C. (The logic structure is based on several MELCOR [3-14] and HECTR [3-21,3-22] calculations which are documented in References $3-9$ and 3-23.)

$\left(5 / 4 \& \frac{23 / 4,6}{2}\right)+15 / 1,2+21 / 1$

$\Rightarrow 33 / 1$

$5 / 2 \&(3 / 3+23 / 1,4)$

$\Rightarrow 33 / 2$

$5 / 3,4 \& 23 / 3,5$

$\Rightarrow 33 / 1$

$5 / 3,4 \& 8 / 2,9$

$\rightarrow 33 / 3$

$5 / 3,4 \& 3 / 2$

$5 / 7,11$

$\Rightarrow 33 / 1,3(0.889 / 0.111)$

$5 / 8,9,10,12$

$\rightarrow 33 / 4$

$5 / 8,9,10,12 \& 8 / 2,9$

$5 / 8,9,10,12 \& 3 / 2$

$\Rightarrow 33 / 1$

$\rightarrow 33 / 5$

$\Rightarrow 33 / 1.5(0.952 / 0.048)$

otherwise

$->33 / 1$ 
Event 34 - Is hydrogen flammable in the pipe gallery or emergency dump tank?

\author{
BRANCH ABBREV. DESCRIPTION \\ (1) $n-m-H 2$ Hydrogen is not flammable in either location \\ (2) H-PGal Hydrogen is flammable in the pipe gallery \\ (3) H-Dump Hydrogen is flammable in the EDT \\ (4) H-PG-DT Hydrogen is flammable in both the pipe gallery and \\ the EDT
}

The discussion of mechanisms that aid or mitigate hydrogen flammability from Event 33 is also applicable here because this event examines the same phenomena in different locations. The EDT can become flammable because hydrogen flows with the coolant out of the core, through the V-4 valves and to the EDT. It can collect there and, since the atmosphere inside the EDT is not inerted or purged, become flammable. The pipe gallery can become flammable because the return line from the EDT comes into the confinement near the top of the pipe gallery. It can also become flammable because of hydrogen released by a LOCA in the pipe gallery. Pipe gallery flammability should not be a major problem, however, as the operation of either the fog spray system or the hydrogen mitigation nitrogen system is predicted to mitigate the effect. However, the buoyancy of the hydrogen and the very high location of the EDT return line combine to reduce the possibility of mitigation through natural mixing phenomena.

The first logical case for this event filters out the non-flammable single riser failure events (insufficient hydrogen production). After that, flammability in the EDT is essentially controlled by the position of the V-4 valves. If the V-4 valves fail closed, as in events $8 / 2$ or $8 / 9$, the EDT can not become flammable, but if some $V-4$ valves open, the EDT can become flammable. Pipe gallery flammability is more involved to determine. The pipe gallery can become flammable if there is either a pipe break in that location or the EDT is on line because of the exit point of that pipe. Forced ventilation, fog sprays and the hydrogen mitigation nitrogen system all work to reduce the possibility of flammability. Failures in these systems are modeled in the fourth case. This event superimposes the above criteria to obtain flammability information for both the EDT and the pipe gallery. The detailed explanation of hydrogen phenomenology as well as the justification behind the probabilities is beyond the scope of this report section, but is shown in Appendix $C$.

\begin{tabular}{|c|c|}
\hline $\begin{array}{l}15 / 1,2 \\
21 / 1 \& \frac{8 / 2,9}{8 / 2,9} \\
5 / 5,6 \& 8 / 2,9 \& 23 / 1,3,4 \\
\frac{8 / 2,9}{8 / 2,9} \\
\frac{\text { otherwise }}{22 / 1} \& 23\end{array}$ & $\begin{array}{l}\Rightarrow 34 / 1 \\
\Rightarrow 34 / 3 \\
\Rightarrow 34 / 1,2(0.963 / 0.037) \\
\Rightarrow 34 / 3,4(0.941 / 0.059) \\
\Rightarrow 34 / 3 \\
\Rightarrow 34 / 1\end{array}$ \\
\hline
\end{tabular}


Event 35 - Is the hydrogen detonable?
BRANCH
(1)
ABBREV,
DESCRIPTION
(2)
$\mathrm{H}-\mathrm{Det}$
Hydrogen near the break location is not detonable
Hydrogen near the break location is detonable

The initial evaluation of hydrogen phenomenology revealed four areas where flammable hydrogen could become detonable: the pipe barrier space, the pressurizer penthouse, the area in the pipe gallery near the EDT return line, and the EDT itself. All other areas were assessed to become deflagrable but not detonable. The EDT and pressurizer penthouse were assessed to be detonable whenever flammable. The probability of detonability is less than one, however, for the pipe barrier space and the pipe gallery. Detonability is established for the pressurizer penthouse and pipe barrier spaces as applicable in this event. Each can become detonable only if a LOCA pipe break occurs in that location. The analysis behind the detonability findings and probabilities are presented in Appendix $C$.

There are no APET events to determine EDT and pipe gallery. detonability. Rather, the remaining hydrogen events in the APET are based on the assessment that the EDT is detonable whenever it is flammable. The situation for the pipe gallery is more complex. The large volume of the pipe gallery combined with the turbulence and mixing that would occur during a LOCA precludes detonability during pipe gallery LOCA events. The high release point associated with the EDT return line, however, combined with a possible lack of mixing phenomena in non-LOCA events, could cause a detonable mixture to occur in the area near the EDT return line. The small amount of hydrogen that would be involved in such a mixture would not, however, be expected to challenge the integrity of the confinement building unless it were to detonate (then the shock wave could damage the confinement). For this reason, the probability of pipe gallery "flammability" for non-LOCA events was adjusted to include detonability and deflagration-to-detonation transition, and a pipe gallery detonability event was not used in the APET. In other words, since deflagration events in this area would not challenge the confinement, deflagrations were neglected for non-LOCA events. The probability of flammability stated in Event 34 for non-LOCA events actually represents the probability that a detonation will occur if the hydrogen is ignited (deflagrations during LOCA events could, however, challenge the confinement and are not neglected). The logic in the remainder of the APET determines whether pipe gallery flammability is based on a LOCA or the EDT return line and adapts accordingly. The logic related to the EDT return line is discussed in detail in Appendix $C$.

$$
\begin{array}{ll}
33 / 2 & \Rightarrow 35 / 2 \\
33 / 4 & \Rightarrow 35 / 1,2(0.475 / 0.525) \\
\text { otherwise } & >35 / 1
\end{array}
$$


Event 36 - Does hydrogen in the confinement building ignite?

\section{BRANCH ABBREV. DESCRIPTION}

(1) H-nIgn Hydrogen near the break location does not ignite

(2) H-Ign Hydrogen near the break location does ignite

This event determines whether flammable hydrogen near a LOCA break location will ignite during the first two hours. The probability of ignition is effected by a number of factors. These include:

- flammability (non-flammable mixtures cannot be ignited),

- detonability (detonable mixtures are more sensitive and, thus, more easily ignited),

- steam concentration (steam makes ignition more difficult),

- electrical power (electrical sparks can cause ignition), and

- sources of mechanical sparks.

These phenomena would be expected to vary between accident progression pathways and even between areas of the plant in the same accident progression pathway. For this reason, the probability of ignition varies. The detailed derivation of the logic structure as well as the justification behind the probabilities is beyond the scope of this report section, but is shown in Appendix C. A summary of the logical structure used in the APET analysis, however, is presented below.

The first logical case disallows ignition if the confinement building atmosphere is not flammable. The next three cases are for different conditions in the pressurizer penthouse and steam generator cells (these were assessed to have similar ignition probabilities given similar confinement conditions). The conditions are: with electrical power available, and detonable and non-detonable without electrical power. The next case describes the pipe barrier space (which is always detonable when flammable and is very close to the hot reactor core and effluent gas stream). The last two cases model Building $105 \mathrm{~N}$ with electrical power failed and available, respectively.

$\begin{array}{ll}33 / 1 & \rightarrow 36 / 1 \\ 33 / 2,3 \& 6 / 4 & \rightarrow 36 / 1,2(0.508 / 0.492) \\ 33 / 2 \& 35 / 2 \& 6 / 4 & \rightarrow 36 / 1,2(0.979 / 0.021) \\ 33 / 2,3 & \rightarrow 36 / 1,2(0.974 / 0.026) \\ 33 / 4 & \Rightarrow 36 / 1,2(0.107 / 0.893) \\ 33 / 5 \& 6 / 4 & \rightarrow 36 / 1,2(0.074 / 0.926) \\ \text { otherwise } & \Rightarrow 36 / 2\end{array}$

Event 37 - Does hydrogen in the emergency dump tank or pipe gallery ignite?

BRANCH ABBREV. DESCRIPTION

(1) H-nIgn Ignition does not occur in either location

(2) PG-Ign Hydrogen in the pipe gallery ignites

(3) DT-Ign Hydrogen in the EDT ignites

(4) PG-DT-I Hydrogen ignites in both the EDT and the pipe gallery 
The discussion of ignition phenomenology in Event 36 is also applicable here because this event examines the same phenomena in different locations. This event considers ignition given that the EDT, the pipe gallery, or both are flammable. Flammability in these areas are independent from each other due to the separation between them. The EDT contains no electrical equipment, but does contain vacuum breakers and other mechanical equipment that could cause sparks to ignite a flamnable hydrogen mixture. The pipe gallery does contain electrical equipment, so the availability of electrical power will effect the ignition probability in this area. The ignition probability for the pipe gallery is independent of whether the hydrogen is released from a LOCA or from the EDT return line. The ignition probabilities are discussed in detail in Appendix $C$.

The first logical case below prohibits ignition when neither the pipe gallery nor the EDT are flammable. The next two cases examine ignition when only the pipe gallery is flammable. The fourth case determines ignition when only the EDT is flammable, and the last two cases are used when both areas are flammable.

$$
\begin{array}{ll}
34 / 1 & \rightarrow 37 / 1 \\
34 / 2 \& 6 / 4 & \rightarrow 37 / 1,2(0.500 / 0.500) \\
34 / 2 & \Rightarrow 37 / 1,2(0.950 / 0.050) \\
34 / 3 & \Rightarrow 37 / 1,3(0.974 / 0.026) \\
34 / 4 \& \frac{6 / 4}{} \rightarrow 37 / 1,2,3,4(0.495 / 0.495 / 0.005 / 0.005) \\
\text { otherwise } \rightarrow 37 / 1,2,3,4(0.941 / 0.049 / 0.009 / 0.001)
\end{array}
$$

Event 38 - Does the hydrogen near the break location detonate?

$\begin{array}{lll}\text { BRANCH } & \text { ABBREV. } & \text { DESCRIPTION } \\ \text { (1) } & \begin{array}{l}\text { H-nBurn } \\ \text { Hydrogen near the break location does not burn } \\ \text { Hydrogen near the break location burns in a } \\ \text { (3) }\end{array} \text { Heflagration } \\ \text { H-Det } & \begin{array}{l}\text { Hydrogen near the break location burns in a } \\ \text { detonation }\end{array}\end{array}$

Even if hydrogen is detonable and ignites, it may not detonate. This event models whether hydrogen that becomes detonable in the vicinity of a LOCA break location and ignites actually detonates. The process of deflagration to detonation transition (DDT) depends mainly on the strength of the hydrogen-air mixture, the geometry of the flammable volume, and whether there is significant mixing of the gases in the volume. The detailed description of the justification behind the probabilities used in this event is beyond the scope of this report section, but is shown in Appendix $C$.

The first two logical cases preclude ignition and detonation in cases where the mixture does not ignite or is not detonable, respectively. The next two cases consider penthouse detonations in the presence and absence of mixing phenomena. The final case is used to determine whether the hydrogen in the plpe barxier space detonates. 


$\begin{array}{ll}36 / 1 & \Rightarrow 38 / 1 \\ 35 / 1 & \Rightarrow 38 / 2 \\ 33 / 2 \& 23 / 2.6 & \Rightarrow 38 / 2,3(0.842 / 0.158) \\ 33 / 2 & \Rightarrow 38 / 2,3(0.301 / 0.699) \\ \text { otherwise } & \Rightarrow 38 / 2,3(0.977 / 0.023)\end{array}$

Event 39 - Does a detonation occur in the EDT?
BRANCH ABBREV.
DESCRIPTION
(1)
(2)
DT-nBrn
Hydrogen in the EDT does not burn
(3) DT-Det
Hydrogen in the EDT burns in a deflagration
Hydrogen in the EDT burns in. a detonation

The phenomenological discussion in Event 38 is also appropriate here because this event considers the same phenomena in different locations. This analysis models the EDT as being detonable whenever it is flammable. However, even if it ignites, DDT must occur within the EDT in order for a detonation to occur. The justification behind the probabilities used in this event is beyond the scope of this report section, but is shown in Appendix $C$.

The first logical case determines whether the EDT atmosphere detonates given that it has ignited. The other case precludes detonation because ignition did not occur.

$$
\begin{array}{ll}
37 / 3,4 & \Rightarrow 39 / 2,3 \quad(0.761 / 0.239) \\
\text { otherwise } & >39 / 1
\end{array}
$$

Event 40 - Does the helium blanket surrounding the core remain intact?

$$
\begin{array}{lll}
\text { BRANCH } & \text { ABBREV, } & \text { DESCRIPTION } \\
\text { (1) } & \text { He-OK } & \text { Helium blanket remains intact } \\
\text { (2) } & \text { He-Lost } \text { Helium blanket fails }
\end{array}
$$

The interior of the core graphite stack is kept inert by a helium blanket during normal operations to prevent oxidation of the graphite and to enhance the heat transfer between the graphite and the GSCS tubes. If the helium blanket is lost, the efficiency with which the GSCS cools the graphite stack is significantly reduced [3-9]. The helium blanket can be lost by a pressure tube rupture event. It can also be lost by a hydrogen detonation inside the pipe barrier space because the rupture disk for the helium system is located in that area. Hydrogen deflagrations, however, are not expected to cause the rupture disk to fail because the disk is rated to withstand 10 psig overpressure from the barrier space without failing, and the blowout panels in the barrier space are expected to relieve the pressure from a deflagration without challenging the rupture disk. The disk is not, however, expected to withstand the shock wave from a detonation. 
$9 / 2+11 / 2+(33 / 4 \& 38 / 3) \Rightarrow 40 / 2$

otherwise $\quad \Rightarrow 40 / 1$

Event 41 - Has oxidation of the graphite stack begun?

BRANCH ABBREV. DESCRIPTION

(1) Gr-nOx No graphite oxidation

(2) Gr-sOx Slow, self-sustaining graphite oxidation has started

(3) Gr-Fire Rapid, self-sustaining graphite oxidation (graphite fire) has started

The prevention of self-sustaining graphite oxidation has been an issue for all graphite-moderated nuclear reactors including $N$ Reactor. Three different means are used to help prevent graphite oxidation at $N$ reactor. First, the graphite temperatures are kept relatively low by the action of a second, independent graphite cooling system (the GSCS). Second, the atmosphere surrounding the graphite stack is kept inerted by a helium cover gas system. Third, even in the event of failure of the first two systems, the paths for air to progress into the core and oxidation products to escape are severely limited by the steel thermal shield and concrete biological shield walls that surround the reactor. In order for significant air to reach the graphite stack, pressure tubes would have to break both inside and outside the shield walls to provide a path for gas exchange.

Graphite oxidation events are not expected to occur within the first two hours of any accident sequence at $N$ Reactor because it takes more than two hours to heat the outer regions of the graphite stack to the temperatures where self-sustaining graphite oxidation would occur. In addition, in all accident sequences except the seismic MPTR event, a very slow rate of air ingress into the core is expected to further reduce the possibility of self-sustaining oxidation. These findings are expanded in a more detailed discussion of graphite oxidation in Appendix $C$.

The logic fur this event states that graphite oxidation can start within the first two hours only when all coolant flow to the core has failed. In addition, oxidation will not occur if the confinement has remained intact and the hydrogen mitigation system is injecting at least $20 \mathrm{k}$ cfm into the confinement because this sweeps oxygen out of the confinement building to inert the atmosphere. Hydrogen detonations can not cause graphite oxidation because the geometry of the pipe barrier space and the massive iron and concrete shielding around the reactor direct the force of explosions away from the reactor core [3-24]. Graphite oxidation is considered very unlikely within the first two hours because an adiabatic core heatup calculation that neglected the reflector regions generated a core average graphite temperature of less than $850^{\circ} \mathrm{C}$ at two hours $[3-25$, 3-1]. Point estimate probabilities were used in this event because the graphite oxidation event during the recovery phase will dominate both in frequency and uncertainty. 
$11 / 3 \& 14 / 3 \& 12 / 4 \&(\underline{29 / 1}+\underline{23 / 3,5}) \Rightarrow 41 / 1,2,3(0.999 / 0.001 / 0.0001)$

otherwise

$\Rightarrow 41 / 1$

Event 42 - Are the vacuum breakers demanded?

BRANCH ABBREV. DESCRIPTION

(1) vBr-Dem Vacuum breakers were demanded

(2). $\quad \mathrm{Br}-\mathrm{nDem}$ Vacuum breakers were not demanded

This event determines whether the confinement vacuum breakers are demanded prior to the initiation of recovery actions. The operation of the fog spray system both cools the confinement atmosphere and condenses out atmospheric steam from a LOCA blowdown. If fog sprays do not operate, then the vacuum breakers will not be demanded. However, the vacuum breakers can be demanded if sprays operate in an isolated confinement building. This is true regardless of whether a steam environment exists in the confinement building because simple ideal gas calculations show that cooling a sealed confinement building full of an ideal gas from $310 \mathrm{~K}$ to $299 \mathrm{~K}\left(99^{\circ} \mathrm{F}\right.$ to $\left.79^{\circ} \mathrm{F}\right)$. produces the rated vacuum for the filter path downstream of the isolation valves $(-.5 \mathrm{psig})$, while cooling it from $333 \mathrm{~K}$ to $288 \mathrm{~K}\left(140^{\circ} \mathrm{F}\right.$ to $\left.60^{\circ} \mathrm{F}\right)$ produces the rated building vacuum $(-2.0 \mathrm{psig})$. The spray system pumps water that is drawn, ultimately, from the river into the confinement building, so long term operation of the sprays could cool the building atmosphere to those temperatures, and condensation of steam from the atmosphere would only add to the vacuum drawn on the building.

If the confinement building did not successfully isolate or has failed, then demand for the vacuum breakers is unlikely because the pressure can be equalized through the already open air paths because most of these paths are larger than a fully open vacuum breaker. The probabilities used in this event were assessed to be significantly conservative and were not refined further because previous analyses of the $N$ Reactor APET have shown that the extremely high reliability of the vacuum breakers (see Event 43 ) renders the APET results very insensitive to their actual values. Point estimate probabilities were used during the uncertainty analysis for this same reason.

The first $\log 1 \mathrm{cal}$ case for this event filters out situations where the fog sprays do not operate. The second case is used when the confinement building has remained successfully isolated, and the last case is used when it has not.

$$
\begin{aligned}
& \frac{22 / 1}{19 / 1} \& 29 / 1 \rightarrow 42 / 2 \\
& \text { otherwise } \rightarrow 42 / 1 \\
& \text { > }
\end{aligned}
$$


Event 43 - Do the vacuum breakers open if demanded?

BRANCH ABBREV. DESCRIPTION

(1) $\mathrm{vBr}-\mathrm{Op}$ Vacuum breakers were demanded and opened

(2) $\quad \mathrm{BB}-\mathrm{nOp}$ Vacuum breakers were demanded but did not open

(3) $\quad \mathrm{BBr}-\mathrm{NA}$ Vacuum breakers were not demanded - not applicable

The demand for the vacuum breakers to open is established in Event 42. This event determines whether they actually open given that they are demanded. Should they fail to open, there is a chance that the confinement building will fail due to vacuum. The confinement system fault tree analysis results showed that the highly redundant vacuum breaker system would be expected to fail on demand with a probability of less than $1.0 \times 10^{-7}[3-13]$. An extremely conservative probability (compared to the value from the fault tree analysis) was used for the APET analysis as a point estimate. Uncertainty was not calculated for this event because it was found to have no significant effect on the overall outcome of the APET analysis.

The first logical case for this event filters out all situations where the vacuum breakers are not demanded, while the other case considers the possibility of vacuum breaker failure given that they are demanded.

$42 / 2 \quad \Rightarrow 43 / 3$

otherwise $\Rightarrow 43 / 1,2(0.9999 / 0.0001)$

Event 44 - Do the vacuum breakers reclose?

BRANCH ABBREV. DESCRIPTION

(1) $\mathrm{vBr}-\mathrm{Cl}$ Vacuum breakers opened and reclosed

(2) $\mathrm{vBr}-\mathrm{nCl}$ Vacuum breakers opened but failed to reclose

(3) $\mathrm{vBr}-\mathrm{NA}$ Vacuum breakers did not open, so closing is not applicable

If any of the vacuum breakers open, there is a possibility that one or more might fail to reclose. This event models that possibility.

If the vacuum breakers are not demanded, then there is no possibility that they might fail to reclose. However, it is always possible that the vacuum breakers could be demanded and fail to open enough to cause success of the vacuum reduction function, yet fail to reclose. This possibility is modeled in this event. The failure of any vacuum breaker to reclose constitutes a rupture-type confinement failure to the environment based on the size of the vacuum breakers.

The first logical case filters out those cases where the vacuum breakers are not demanded, while the other case models the probability of the breakers to reclose given that they are demanded. The data for this case is calculated during the TEMAC analysis of the results of the confinement systems fault tree analysis and fed directly into the APET analysis $[3-7]$. 
$43 / 3 \quad \rightarrow 44 / 3$

otherwise $\rightarrow 44 / 1,2(0.9999 / 0.0001)$

Event 45 - Does the filter path reisolate in time to prevent filter damage?

BRANCH ABBREV. DESCRIPTION

(1) C-ReIso Filter path reisolates in time to prevent filter

(2) C-nRIso Filter path reisolation is called for, but reisolation does not occur in time to prevent filter damage

(3) C-nCh Filter path reisolation is not called for

The confinement system is designed to reisolate the filter path any time the confinement pressure rises above 15" water [3-15]. This event determines whether the isolation function occurs quickly enough to protect the filters and the low pressure section of the filter path from being damaged by the increased pressure. Conditions during the accident progression which could demand the reisolation function are hydrogen and graphite oxidation events. The other events in this section of the APET either would not demand isolation (vacuum-related), or are secondary events caused by hydrogen events (helium system failure). The effectiveness of the isolation mechanism during these demands was analyzed and is discussed in more detail in Appendix $C$, but a glimpse of those results is presented below.

Hydrogen detonations produce shock waves that travel so quickly that there is no possibility for the filter path isolation valve to close in time to prevent filter or filter path damage. Hydrogen deflagrations can occur more slowly and do not produce a shock wave, so, depending on the amount of hydrogen present, they may occur slowly enough to allow the filter path to isolate in time to prevent filter damage. Graphite oxidation events occur more slowly still, so the speed of the isolation mechanism is of limited importance. The logical cases for this event model hydrogen detonations first, hydrogen deflagrations second, graphite oxidation third and cases where isolation is not demanded are last. The probabilities were sampled from the uncertainty distributions presented in Appendix C.

$\begin{array}{lll}38 / 3 & \rightarrow 45 / 2 \\ 38 / 2+37 / 2,4 & \rightarrow 45 / 1,2(0.647 / 0.353) \\ 41 / 3 & \rightarrow 45 / 1,2(0.867 / 0.133) \\ \text { otherwise } & \Rightarrow 45 / 3\end{array}$


Event 46 - Does confinement fail during pre-recovery accident progression?
BRANCH
ABBREV.
DESCRIPTION
(1)
$\mathrm{C}-\mathrm{nCh}$
Confinement condition did not change
(2) $\quad \mathrm{CF}-\mathrm{H} 2$
Confinement failed based on a hydrogen event
(3) $\quad$ CF-Vac
(4)
CF-GrOx
Confinement failed based on a vacuum event
Confinement failed based on graphite oxidation

This event determines whether any of the previous accident progression events (hydrogen, graphite fire or vacuum) caused the confinement buildings to fail in their ability to maintain isolation. This event and those that follow are identical to events 28-32 in the previous section of the APET but for different phenomena. This event does not consider whether the confinement is intact, but only whether any phenomena have occurred since the end of the blowdown phase that would fail the confinement buildings. The next event determines whether the building is still intact. Note also that while this event considers confinement isolation, it considers only the confinement buildings and not the EDT or the filters. While they are required to function in order for confinement of the radionuclides to be accomplished, they are considered in other APET events. Thus, the term "confinement" should be interpreted throughout the remainder of the APET to refer to the ability of the confinement buildings to maintain isolation and not to the overall confinement of radionuclides.

Hydrogen events can fail the confinement in two different ways. First, the shock waves from a detonation will cause failure of the confinement building. Second, if a deflagration occurs, the overpressurization of the building or even of a confined local area can cause building failure. Deflagrations from large hydrogen volumes are more likely to cause building failure than are those from small volumes because of the increased energy released from burning large volumes of hydrogen. For this reason, the probability of building failure is higher for deflagrations in the pipe gallery than for other areas of the plant.

Confinement failure always occurs when a vacuum breaker fails to close because that failure leaves a direct path open to the environment. Also, the high reliability of the vacuum breakers in opening allows for a conservative treatment of confinement building failure when the breakers fail to open. Rapid graphite oxidation always fails confinement because of the large amounts of heat generated, and slow graphite oxidation can cause confinement failure for this same reason. This conservative treatment is possible because the frequency of late graphite oxidation far dominates that of early oxidation.

The logical cases must be arranged for the EVNTRE input in order of decreasing failure probability for EVNTRE to use the probability for the event most likely to cause a failure. The probability distributions for confinement failure for various events are described in more detail in Appendix $C$. Those distributions were sampled for this event during the uncertainty analysis. 
The first and eighth logical cases for this event are used to analyze graphite oxidation events. The second, third, sixth and seventh cases consider hydrogen phenomena. Note that the probability of confinement failure given a hydrogen event in the pipe gallery differs depending on whether a LOCA event is responsible for the hydrogen release. The fourth and fifth cases are used by vacuum events, and the last case is for accident progression pathways where no events occur to challenge the confinement during the pre-recovery phase.

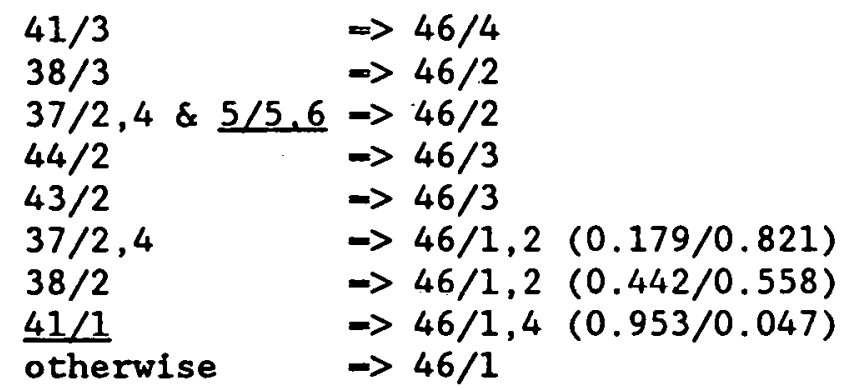

Event 47 - What is the size of the confinement failure?
BRANCH ABBREV. DESCRIPTION
(1). Conf-OK Confinement has not failed
(2) C-Leak Confinement failure is a leak
(3) C-Rupt Confinement failure is a rupture

This evene is similar to Event 29, and considers what (if any) size failure has occurred to the confinement building up to this point. Thus, it summarizes failures both in and prior to the pre-recovery accident progression analysis.

The APET analysis defines a confinement building rupture to be a confinement building failure such that the filter path has no decontaminating effect on the gaseous releases from the plant. A leak is defined such that, if the filter release path were to be in operation, competing flow paths would be set up between the filter path and the confinement failure. This distinction is, however, superficial because the binning input in Section 3.9 is set up using the conservatism that the "competition" between these paths is minimal and that the outflow from the confinement is dominated by the flow out the confinement building failure.

This event summarizes the entire APET to this point, so previous confinement building ruptures (from Event 29) must be factored in. Leaks must also be factored in as long as they have not been turned into ruptures by later events. The only way the confinement can continue to be intact is if there is no previous confinement leak or rupture and confinement failure is not cited in Event 46 above.

Whether an event causes a confinement leak or rupture depends on how energetic the event is. In general, those events that are more likely to cause confinement failures in Event 46 are more likely to cause confinement 
ruptures. For this analysis, hydrogen detonations and graphite oxidation are always assessed to cause confinement building ruptures. Vacuum breakers failing to reclose are also classified as ruptures due to the size of the opening that would be left to the environment. Other confinement failure mechanisms could also cause leaks. The probability distributions for confinement failure for various events are described in more detail in Appendix $C$. Those distributions were sampled for this event during the uncertainty analysis.

The first and fifth logical cases for this event are used to analyze graphite oxidation events. The first, second, and third cases consider hydrogen phenomena, and the first and fourth cases are used by vacuum events. The first case also includes previous confinement ruptures, while the sixth case includes previous confinement leaks. The last case is for accident progression pathways where the confinement building remains intact at the initiation of recovery actions.

$\begin{array}{ll}29 / 3+38 / 3+41 / 3+44 / 2 & \Rightarrow 47 / 3 \\ 37 / 2,4 \& 46 / 1 & \Rightarrow 47 / 2,3(0.434 / 0.566) \\ 38 / 2 \& 46 / 1 & \Rightarrow 47 / 2,3(0.792 / 0.208) \\ 43 / 2 \& 46 / 1 & \Rightarrow 47 / 3 \\ 41 / 1 \& 46 / 1 & \Rightarrow 47 / 3 \\ 29 / 2 & \Rightarrow 47 / 2 \\ \text { otherwise } & \Rightarrow 47 / 1\end{array}$

Event 48 - What is the location of the confinement building failure?

BRANCH ABBREV. DESCRIPTION

(1) Conf-OK No confinement compromise

(2) Envir Confinement compromise is directly to the environment

(3) Zone Confinement compromise is to another zone

This event is similar to Event 30 , and considers whether a confinement building failure leads directly to the environment or through another radiation protection zone to the environment. It summarizes failures prior to the initiation of recovery actions. This event considers only the confinement buildings and not the EDT or other non-building isolation failure release pathways.

This event summarizes the entire APET to this point, so previous confinement building failures (from Event 30) must be factored in. The only way the confinement can continue to be intact is if there is no previous confinement failure and confinement failure is not cited in Event 46 above. If a previous failure has occurred to the environment, it must be factored into this event. If a previous failure has occurred to cause releases to be directed to another radiation protection zone, it will continue to exist as long as nothing has happened during this section of the APET to cause a release pathway directly to the environment. This event is therefore not sampled in the uncertainty analysis. 


$\begin{aligned} 47 / 1 & \\ 30 / 2+(46 / 1 \&(47 / 3+44 / 2)) & \Rightarrow 48 / 1 \\ 46 / 1 \& 47 / 2 & \Rightarrow 48 / 2,3(0.900 / 0.100) \\ \text { otherwise } & \Rightarrow 48 / 3\end{aligned}$

Event 49 - Does the fog sprays system remain functional?

BRANCH ABBREV, DESCRIPTION

(1) Spr-nCh Spray configuration is unchanged.

(2) nSpr No spray on

It is possible that a particularly violent confinement failure could disrupt the function of the fog spray system either by severing pipes, by redirecting spray heads to cause gaps in coverage, or by causing the confinement atmosphere to significantly bypass the spray coverage area due to the confinement failure. The uncertainty analysis as detailed in Appendix $C$ considered three different types of confinement failure: "static" conditions (including local deflagrations), rapid pressurizations (such as global deflagrations at high hydrogen concentrations), and shock. events (detonations). Each confinement failure mechanism was placed into one of these categories for the purposes of this event.

The first logical case for this event filters out situations where the confinement does not fail during this section of the APET. The second case considers static confinement failure situations caused by vacuum or graphite oxidation, while the third handles shock events. The fourth case also handles "static" conditions, although this time the conditions are caused by hydrogen deflagrations. The probability distributions for fog spray failure are described in more detail in Appendix $C$. Those distributions were sampled for this event during the uncertainty analysis.

$$
\begin{array}{ll}
47 / 1 & \rightarrow 49 / 1 \\
46 / 3,4 \& 47 / 3 & \rightarrow 49 / 1,2(0.984 / 0.016) \\
38 / 3 \& 46 / 2 \& 47 / 3 \rightarrow 49 / 1,2(0.004 / 0.996) \\
\text { otherwise (deflagrations) } \Rightarrow 49 / 1,2(0.970 / 0.030)
\end{array}
$$

Event 50 - Is the filter exhaust path open?

\section{BRANCH ABBREV, DESCRIPTION}

(1) FP-nCh Filter path condition has not changed.

(2) CV18-Cl Filter path isolation valves have drifted closed

(3) FP-Fail Regardless of previous condition, the filter path is no longer available and is not recoverable.

This event determines whether the filter release path was affected by the events of the pre-recovery accident progression. This event asks only whether the pathway is available. Whether there are any filters in the path is handled near the end of the tree. 
Two things could have happened to change the status or efficacy of the path. First, a confinement rupture could have occurred to cause the gas effluent stream to bypass the filter path and render it useless. This failure cannot be recovered without recovery from the confinement failure. This portion of this event is a summary event.

A second mechanism for failure of the filter path is a loss of AC power. Tests of the filter path isolation valves indicated that there is a small probability that the hydraulic accumulators on those valves will become depleted over a period of several hours if $A C$ power is not available to power the hydraulic power packs and prevent accumulator depletion. These valves fail closed on loss of hydraulic pressure. Thus, the filter path can drift closed on a loss of $A C$ power [3-13]. This failure is recoverable if $A C$ power is restored. The second case for this event models this process, but only prior to the initiation of recovery actions (approximately two hours). Point estimate probability values are used for this event. Failures later in the accident progression are modeled in Event 57 based on the TEMAC analysis of the confinement system fault tree analysis results. Uncertainties are sampled in that event.

The first logical case for this event filters out confinement rupture failures. The second case considers the valve drift phenomenon, and the third maintains the status quo, because this event does not summarize previous filter path failures, but only considers new failures during this section of the APET.

$$
\begin{array}{ll}
47 / 3 & \Rightarrow 50 / 3 \\
6 / 4 & \Rightarrow 50 / 1,2(0.999 / 0.001) \\
\text { otherwise } & >50 / 1
\end{array}
$$

\subsection{RECOVERY ACTIONS}

In the APET analysis, the dividing time between the "early" and "late" phases of the accident progression is the time of recovery. Recovery is assumed to occur approximately two hours after the initial onset of core damage. This time is significant for several reasons. The Level I PRA [3-6] used this definition of "early" and "late" because two hours marks the time after which the GSCS can prevent core damage without assistance from the ECCS. This makes for a natural delineation point. In addition, support calculations [3-9] indicate that the differences between full core accident sequences with and without GSCS are relatively minor for the first two hours because most of this time is spent heating the large thermal mass of the graphite stack. Core temperatures and hydrogen generation rates are reasonably similar for hoth cases during this time. Later, however, the accident sequences progress by very different courses. Thus, the two hour point forms a natural dividing point between "early" and "late" from an accident progression point of view.

While putting recovery actions at the two hour point may seem arbitrary, it is in fact a reasonable and conservative approach. A later recovery time (but still well before the 24 hour modeling time) would not 
significantly affect the "late" accident progression because all of the modeled phenomena that can occur during a later recovery action can also occur if recovery is performed at the two hour mark because, by that time, the core temperatures are already high enough to support the phenomena. On the other hand, earlier recovery actions may not see these high core temperatures and could fail to produce some of the accident progression phenomenology. Thus, a two hour recovery time is conservative from this perspective. In addition, most recovery actions that would take longer than two hours to accomplish involve significant amounts of labor and would probably not be completed in time to be of significant use during the time scale of interest for the APET analysis.

Based on probabilistic and procedural guidance, the recovery of electrical power, GSCS, ECCS, HPI, fog sprays, filter release path, and the hydrogen mitigation system are allowed for in this section of the APET (Events 51-58). The events in this section of the APET are:

51 Is AC power available for recovery and post-recovery actions?

52 Does GSCS operate during the post-recovery phase?

53 Does ECCS operate during the post-recovery phase?

54 Does high pressure injection operate during the post-recovery phase?

55 Does recovery cause a confinement steam pressure spike to occur?

56 Do fog sprays operate during the post-recovery phase?

57 Does the filter release path operate during the post-recovery phase?

58 Does hydrogen mitigation operate during the post-recovery phase?

In many studies, the term "recovery" is used to denote the positive steps an operator can take to recover (or "make things better") during an accident. "Negative recovery" actions are not normally an issue. At $N$ Reactor, however, it is possible for the operators to take actions believing that they are assisting recovery efforts when in fact they are making the situation worse. This is because the pressure tubes must remain intact in order for the GSCS to effectively limit core damage as predicted by deterministic analyses. If one or more pressure tubes were to fail, the helium blanket that normally surrounds the core would be replaced by steam which has a significantly lower thermal conductivity than helium. If ECCS were to be "recovered", there is a high probability that at least one of the pressure tubes would fail and, if the GSCS were operating, its efficiency would be significantly decreased. This would cause additional core damage. These negative aspects of ECCS recovery are considered along with the usual positive aspects in the following section of the APET.

Event 55 is technically not a recovery event, but it establishes whether an ECCS recovery event or other pressure tube rupture event causes the confinement pressure to rise to the point where the fog spray system would be demanded. A pressure tube failure opens a path for the generated steam to be released to the confinement atmosphere. Calculations [3-9] show that the pressure rise could be high enough to demand the fog spray system or possibly even challenge the confinement. For this reason, a post-recovery phenomenology event is inserted into the recovery section of the APET to allow primary system recovery actions to cause a recoveryrelated demand on the confinement systems. 
Event 51 - Is electrical power available for recovery actions?

\section{BRANCH ABBREV, DESCRIPTION}

(1) All-Pow All electric power is operating

(2) $n$-A-Bus Only AC Bus $A$ has failed

(3) n-B-Bus Only AC Bus B has failed

(4) $\mathrm{n}-\mathrm{AC}$ Both $\mathrm{AC}$ busses have failed

The delayed recovery of a failed AC power supply can have a major effect on the progression of a commercial reactor accident because it allows electrically operated equipment to be restarted to mitigate accident consequences. At $N$ Reactor, however, the effect is smaller because of the large number of diesel pumps and air and hydraulic accumulators in the plant. This event considers the recovery of AC power from losses initiated either outside or within the plant itself. The loss of an electrical bus during the accident progression is not considered because no mechanisms have been found for electrical bus failure that are not directly tied to the initiating event.

For internally initiated accident sequences, the delayed recovery of a lost electrical bus is highly probable if the other bus is still functional. Facilities and procedures exist to tie both AC power busses to the same onsite or offsite power source, and two separate offsite power sources exist (the $\mathrm{N}$-tap and the $\mathrm{K}-\mathrm{N}$ tie). These procedures are exercised frequently. The recovery procedures take approximately 15 minutes to accomplish, so are of no value to the Level I PRA analysis, but can be of significant value during the APET analysis.

Given a loss of both AC busses, the probability of electrical power recovery is dominated by the recovery of offsite power. The bus ties mentioned above mean that recovery of both electrical busses is little more difficult than the recovery of a single bus, so it was assessed that any offsite $A C$ power recovery would recover both busses. The data and logical structure for this event are described in Appendix C (as obtained from data in NUREG-1032 [3-26]), and sampled during the uncertainty analysis.

For fire and seismic accident sequences, however, recovery of lost AC power busses is almost impossible. Seismic events damage not only the offsite electrical grid, but also the power bus mountings within the plant itself [3-2]. Recovery of major incidents like this would be almost impossible within the 24 hour period of analysis for the APET. Likewise, most fire events that cause electrical losses occur in switching equipment, and rerouting power around that equipment would be difficult. It may be slightly conservative, but no electrical recovery is allowed for seismic and fire events.

The first logical case for this event retains complete electrical function when there is no electrical failure. The second and third cases consider recovery from the loss of Bus A for internally and externally initiated accident sequences, respectively. The fourth, fifth, sixth and last cases consider the same recoveries, but for losses of Bus $B$ and all AC power, respectively. 

$6 / 1 \quad \Rightarrow 51 / 1$
$2 / 1 \& 6 / 2 \Rightarrow 51 / 1,2(0.941 / 0.059)$
$6 / 2 \quad \rightarrow 51 / 2$
$2 / 1 \& 6 / 3 \rightarrow 51 / 1,3(0.934 / 0.066)$
$6 / 3 \quad \Rightarrow 51 / 3$
$2 / 1 \& 6 / 4 \rightarrow 51 / 1,4(0.650 / 0.350)$
otherwise $\Rightarrow 51 / 4$

Event 52 - Does GSCS operate during the recovery phase?

$\begin{array}{lll}\frac{B R A N C H}{(1)} & \frac{A B B R E V}{\text { fGSCS }} & \begin{array}{l}\text { DESCRIPTION } \\ \text { GSCS operates at full efficiency during recovery } \\ \text { phase }\end{array} \\ \text { (2) DegGSCS } & \begin{array}{l}\text { GSCS is operating but degraded (loss of helium } \\ \text { blanket) }\end{array} \\ \text { (3) nGSCS } & \text { GSCS is not operating }\end{array}$

There is significant uncertainty among analysts who have studied $N$ Reactor as to the length of time it would take to recover the GSCS cooling function once the system was physically restarted (given that it had been without coolant for at least several minutes), but it is generally agreed that cooling will not approach the normal efficiency for at least a few hours thereafter. This is because steam binding inside the GSCS tubes prevents GSCS coolant from entering the tubes until they can be cooled enough to prevent the coolant from flashing to steam. In addition, if electrical power failures did not contribute to the GSCS failure, then the most dominant causes of GSCS failure are due to valve failures and orifice plugging. Recovery of these failures would be very difficult and is not considered credible for this analysis. GSCS recovery is discussed in more detail in Appendix $C$.

This event summarizes the operation of the GSCS following the initiation of recovery actions and, thus, must summarize previous GSCS operation even if the conditions remain unchanged. The first logical case for this event precludes GSCS recovery for externally initiated accident sequences. It also ensures GSCS failure for accident sequences in which delayed ECCS failure occurs because GSCS must fail for core damage to occur based on the definition of core damage bin 21 from the Level I PRA [3-6]. The second case ensures that cases where GSCS is operating but the core helium cover gas system has failed are labeled for degraded GSCS performance. The third case ensures the continued operation of GSCS at full efficiency given that it was originally operating in that mode. The fourth and fifth cases allow a very small probability for GSCS recovery given that electrical power originally failed but was at least partially recovered in Event 51, and the last case flags all other GSCS failure cases as unrecoverable. All probabilities used in this event are point estimates because the APET analysis results have been found to be insensitive to the actual GSCS recovery probability as long as it remains small. 


$\begin{array}{ll}(\underline{2 / 1} \& 11 / 3)+7 / 2,3 & \rightarrow 52 / 3 \\ 11 / 2+(11 / 3 \& 40 / 2) & \Rightarrow 52 / 2 \\ 11 / 1 & \\ 6 / 4 \& 51 / 4 \& 40 / 2 & \Rightarrow 52 / 1 \\ 6 / 4 \& \underline{51 / 4} & \Rightarrow 52 / 2,3(0.01 / 0.99) \\ \text { otherwise } & \Rightarrow 52 / 3\end{array}$

Event 53 - Does ECCS operate during the recovery phase?
BRANCH ABBREV
(1) $\quad$ EECCS
(2) pECCS
DESCRIPTION
(3) nECCS
ECCS operates during the recovery phase
Partial ECCS flow exists during the recovery phase
No ECCS flow exists during the recovery phase

This event determines the flow status of the ECCS following the initiation of recovery actions and, hence, must consider the possible initiation or recovery of ECCS after core damage has already occurred. The operators are instructed in the Emergency Response Guides not to initiate ECCS if the core has been without cooling flow for seven or more minutes [3-10]. However, many ECCS failures provide partial ECCS flow to the core while not meeting the ECCS success criteria. In other situations, the configuration of the plant controls would prevent the operator from knowing when seven minutes had elapsed. The above situations show why delayed ECCS recovery is important to the APET analysis.

A more complete discussion of ECCS recovery can be found in Appendix C. Several underlying assessments for this discussion are:

- Operators will try to recover full ECCS function (rather than maintain or stop ECCS flow) if ECCS is in a partial flow configuration,

- Recovery of components inside Zone I of the confinement building is not possible,

- Recovery of fire-damaged or seismically damaged equipment is not possible,

- Recovery of diesel pumps within the time frame modeled in the APET analysis is almost impossible, and

- Operators will sometimes violate ECCS initiation rules if faced with the choice between starting ECCS and an unmitigated core heatup (both ECCS and GSCS fail).

The above assessments allow only three ECCS failures to be recovered: the 3H33 ECCS bypass switch (located in the main control room), V-3 valve failures (located outside Zone $I$ ), and ECCS diesel pump failures caused by fires in Corridor 19 (the pumps can be started locally in Building 182N). Seismic events are specifically not recoverable because almost all involve failure of the silo structure. This fails suction to the ECCS high lift diesel pumps. Other events are assigned a small recovery probability as a conservatism to allow for consideration of phenomenology related to delayed ECCS recovery. 
Since this event summarizes the condition of the ECCS after two hours, it must also consider delayed ECCS failure events. In this accident sequence, GSCS fails and ECCS succeeds for at least two hours before failing. Failure was taken in the Level I analysis to mean failure of ECCS to meet its initial success criteria and was always caused by failure of two of the three diesel pumps to run. However, later research indicates that a single diesel pump can supply more than enough water to keep the core cool after two hours, so failure of all three pumps would be required for core damage to occur. For this reason, a portion of the frequency for accident sequences that are predicted by the Level I PRA to result in fuel damage are predicted by this analysis to result in successful core cooling. The probability of ECCS "failure" for this case is the probability that the third ECCS diesel pump will fail to run because the core damage frequency for this accident sequence from the Level I PRA analysis [3-6] already considers the failure of the first two pumps. This situation is described in more detail in Appendix $C$.

The first logical case for this event allows for ECCS recovery when fire has damaged the control cables to the diesel pumps (the main fire location referred to here is Corridor 19, which also fails GSCS cooling as in the three core damage levels specified for this case). The recovery is accomplished through local actuation in Building $182 \mathrm{~N}$. The second case describes delayed ECCS failures as discussed above. The third case precludes ECCS recovery for seismic accident sequences. The fourth, ninth and tenth cases maintain the previous status of ECCS with a slight possibility of recovery for the accident progression reasons stated above. The fifth, sixth and seventh cases allow for ECCS recovery when the failure was caused by the $3 \mathrm{H} 33$ bypass switch, and the eighth case allows for recovery of the V-3 valves. Cases six and eight are worthy of special note because these cases implement the assessment (from the uncertainty elicitation) that the operators are much more likely to attempt delayed ECCS recovery if they are faced with an unmitigated core heatup due to the failure of the GSCS even though this is in violation of the written procedures. The probabilities for this event are sampled from uncertainty analyses presented in Appendix C.

$\begin{array}{ll}2 / 2 \& 15 / 5,6,7 & \Rightarrow 53 / 1,3(0.562 / 0.438) \\ 7 / 2,3 & \Rightarrow 53 / 1,3(0.803 / 0.197) \\ 14 / 3 \&(5 / 10+2 / 3) & \rightarrow 53 / 3 \\ 14 / 1 & \rightarrow 53 / 1 \\ 8 / 2 \& 3 / 2 & \Rightarrow 53 / 1,3(0.709 / 0.291) \\ 8 / 2 \& 52 / 1 & \rightarrow 53 / 1,3(0.326 / 0.674) \\ 8 / 2 & \Rightarrow 53 / 1,3(0.014 / 0.986) \\ 52 / 1 \& 8 / 6,7,8 & \Rightarrow 53 / 1,2(0.316 / 0.684) \\ 14 / 2 & \rightarrow 53 / 1,2(0.005 / 0.995) \\ \text { otherwise } & \rightarrow 53 / 1,2,3(0.001 / 0.004 / 0.995)\end{array}$


Event 54 - Does HPI operate during the recovery phase?

\section{BRANCH ABBREV, DESCRIPTION}

(1) 4/4-HPI HPI operates fully during the recovery phase

(2) 2/4-HPI Flow from 2 HPI pumps reaches the core

(3) 1/4-HPI Flow from 1 HPI pump reaches the core

(4) nHPI No HPI flow reaches the core during the recovery phase

The uncertainty assessment on core damage resulting from partial ECCS success found no basis for assessing that HPI flow would be able to prevent or even significantly reduce the amount of core damage that would occur given inadequate ECCS cooling. Thus, the primary function of HPI flow in the APET is to determine whether there is a steam source in the core to drive the released radionuclides out to the environment. For this reason, the particular amount of HPI flow is not as much of a concern as is the presence or absence of flow.

The operators are not instructed to recover HPI, and procedures are not available for doing so. For this reason, HPI recovery is treated in a manner similar to GSCS recovery with a possibility of recovery upon the restoration of electrical power and a very small probability of recovery (for phenomenological reasons similar to ECCS recovery above) for all other cases. All probabilities used in this event are point estimates because the APET analysis results have been found to be insensitive to the actual HPI recovery probability as long as it remains small (see Appendix C).

The first and second logical cases rule out HPI function when electrical power is unavailable (the pumps are electric), when ECCS is operating fully (the operators are instructed to shut HPI down), for fire and seismic scenarios where either the pumps, the control cables or the water sources are damaged, and for large LOCAs where the coolant would not be able to reach the core. The remaining cases consider HPI recovery from various degraded states with electrical power available in differing configurations. The last case is used only when HPI was fully functioning during the pre-recovery accident progression.

\begin{tabular}{|c|c|}
\hline $\begin{array}{l}(2 / 2 \& 8 / 4)+2 / 3+8 / 1+51, \\
3 / 2 \& 5 / 1,7,8 \\
12 / 4 \& 6 / 4 \& 51 / 1 \\
12 / 4 \& 6 / 4 \& 51 / 4 \\
12 / 4 \& 12 / 3 \\
3 / 3 \& \underline{51 / 1} \\
12 / 3 \& 12 \% 31 / 1 \\
12 / 3 \& \underline{51} \\
12 / 2 \& \text { otherwise }(12 / 1)\end{array}$ & $\begin{array}{l}\Rightarrow 54 / 4 \\
\Rightarrow 54 / 1,4(0.010 / 0.990) \\
\Rightarrow 54 / 2,4(0.010 / 0.990) \\
\Rightarrow 54 / 1,4(0.010 / 0.990) \\
\Rightarrow 54 / 3 \\
\Rightarrow 54 / 2,3(0.006 / 0.994) \\
\Rightarrow 54 / 1,3(0.009 / 0.991) \\
\Rightarrow 54 / 2 \\
\Rightarrow 54 / 1,2(0.010 / 0.990) \\
\Rightarrow 54 / 1\end{array}$ \\
\hline
\end{tabular}


Event 55 - Does recovery cause a steam pressure spike in the confinement?

BRANCH ABBREV, DESCRIPTION

(1) StmP-NA No steam spike occurred in confinement

(2) StmP>5 A steam spike occurred, max pressure more than 5 psig

(3) StmP<5 A steam spike occurred, max pressure less than 5 psig

Recovery of ECCS at $\mathrm{N}$ Reactor can be a violent process as cold water is introduced into pressure tubes that can be at temperatures near $1500 \mathrm{~K}$ [3-27]. The water would have a difficult time getting into and through the core due to steam binding similar to that described for the GSCS. Water hammer phenomena can also be caused when steam binding occurs if the steam pressure is enough to force the coolant front backwards out of the core [3-28]. When the coolant comes back into the pressure tube, the process repeats with oscillatory pressure spikes. Both of these phenomena would be expected to cause pressures high enough to fail pressure tubes (see Appendix C).

When pressure tubes fail, the ECCS coolant can then flow over the hot graphite and vaporize. The steam will flow out of the graphite stack and into the confinement building that is already isolated and, for this reason, cannot release the steam pressure. An added effect is that the filter release path (the only remaining pathway to the environment) is designed to isolate any time the confinement pressure reaches 15 inches water [3-15]. This completes the sealing of the confinement building. The increased pressure will be sufficient to demand fog spray operation and could be enough to challenge the integrity of the confinement building. The logic and data for this event are presented in Appendix $C$, and the data was sampled during the uncertainty analysis.

The first logical case for this event considers recovery when ECCS has completely failed initially, while the second considers recovery when ECCS has partially failed initially. The last case is used when delayed ECCS recovery does not occur.

$$
\begin{aligned}
& 14 / 3 \& \frac{53 / 3}{53 / 1} \rightarrow 55 / 2,3(0.672 / 0.328) \\
& 14 / 2 \& 55 / 3 \\
& \text { otherwise } \rightarrow 55 / 1
\end{aligned}
$$

Event 56 - Do fog sprays operate during the recovery phase?

BRANCH ABBREV. DESCRIPTION

(1) Spr Full fog spray function occurs during recovery phase

(2) nSpr Fog sprays do not operate during the recovery phase

This event summarizes whether the fog spray system operates after the initiation of recovery actions. Event 22 determines whether the system will operate if demanded, and events $16,38,41$ and 55 will all demand fog spray operation based on pressure increases. Procedures call for the fog spray system to be actuated only in response to high confinement building pressures. 
The first logical case for this event filters out several accident progression pathways in which the fog sprays would be unavailable during, the recovery phase due to previous system failures. These are initial spray failure (Event 22) and spray failures caused by confinement building failures (Events 31 and 49). The second case keeps the sprays on if they were demanded initially and allows new pressure demands to cause the actuation of spray function. Note that a new demand for spray actuation can only cause successful spray function if the initial spray event is in the state where sprays are not operating but would operate if demanded $(22 / 3)$. The last case is used only when fog spray operation is not demanded by any of these mechanisms. This is a summary event, so all probabilities are either zero or one and are not sampled.

$$
\begin{array}{ll}
22 / 2+31 / 2+49 / 2 & \Rightarrow 56 / 2 \\
22 / 1+22 / 3 \&(\underline{38 / 1}+41 / 1+55 / 1) & \Rightarrow 56 / 1 \\
\text { otherwise (sprays not demanded) } & \Rightarrow 56 / 2
\end{array}
$$

Event 57 - Does the filter release path operate during recovery?

$$
\begin{array}{lll}
\text { BRANCH } & \text { ABBREV } & \text { DESCRIPTION } \\
\text { (1) } & \text { FPath } & \text { Filter path operates } \\
(2) & \text { nFPath } & \text { Filter path does not operate } \\
(3) & \text { Path-NA } & \text { Filter path is not applicable }
\end{array}
$$

This event summarizes the status of the filter release path after recovery actions have been performed. This event only asks whether the path can be established. Whether the filters in the path are damaged is determined at the end of the APET.

The filter release path can fail in three ways, and two of these are recoverable. Confinement building failures can cause the exhaust gas stream to bypass the filter path and unrecoverably fail its function. Failures of the confinement to isolate initially also causes bypass of the filter path, but many of these can be recovered. Finally, if electrical power is lost, the confinement isolation valves that lead to the filter path can drift closed due to loss of hydraulic pressure from the accumulators (see Event 50). Accumulator depletion events from Event 50 are automatically recovered here if electrical power has been restored in Event 51. Otherwise, the probability that accumulator depletion will occur during the remainder of the accident progression must be applied here. That probability is derived in the TEMAC analysis of the confinement system fault tree analysis results and is passed directly into the EVNTRE analysis with uncertainties sampled.

The first logical case for this event keeps track of the unrecoverable filter path failures. The second case precludes filter path failure when the path is already established and electrical power is available. The third and fourth cases allow for recovery of various initial confinement isolation failures, and the last case continues filter path failure when the above conditions have nut been met. 


$\begin{array}{ll}32 / 3+50 / 3+(50 / 2 \& 51 / 4) & \Rightarrow 57 / 3 \\ 32 / 1 & \Rightarrow 57 / 1 \\ 19 / 2 \& 51 / 4 & \Rightarrow 57 / 1,2(0.993 / 0.007) \\ 19 / 4 \& \underline{51 / 4} & \\ \text { otherwise } & \Rightarrow 57 / 1,2(0.005 / 0.995) \\ & \Rightarrow 57 / 2\end{array}$

Event 58 - Does the hydrogen mitigation system operate during recovery?

BRANCH ABBREV, DESCRIPTION

(1) HM-OK Hydrogen mitigation system is operating in the

(2) HM-3k No mixing fans started - only $3 k$ nitrogen purge

(3) HM-40k 40k inert system and mixing fans are running

(4) HM-Mix Only mixing system is running - no nitrogen

(5) HM-20k 20k inert system and mixing fans are running

(6) nHM The entire system is not running

This event determines the status of the hydrogen mitigation system after recovery actions have been performed. As such, it must summarize the current state of the mitigation system and allow for recovery actions as appropriate. For this study, recovery of the hydrogen mitigation system is limited to delayed actuation of the nitrogen injection system. Other actions may be possible, but uncertainties about the configuration of the final, installed system make their analysis difficult, and neglecting them is a conservative measure.

The logical structure for this event is designed to maintain the hydrogen mitigation system in its current mode of operation unless conditions have changed so that a different mode of operation would be appropriate $[3-20]$. Thus, the first two logical cases maintain the mitigation system in the $40 \mathrm{k} \mathrm{cfm}$ and $20 \mathrm{k} \mathrm{cfm}$ nitrogen injection modes, and the sixth, seventh and eighth cases maintain other, less stringent modes of operation. The third and fourth cases preserve the current operating modes of the system because the system has already been demanded and has failed to meet that demand. The fifth case allows for the nitrogen inert function to be started should either GSCS or ECCS function be affected by either the accident progression or recovery events. Here, as in Event 23 , the probabilities for mitigation system failure are taken from the fault tree analysis of the hydrogen mitigation system and are used as point estimates without uncertainty.

\begin{tabular}{|c|c|}
\hline $\begin{array}{l}23 / 3 \\
23 / 5 \\
23 / 4 \&(15 / 1,2+2 / 1) \\
23 / 6 \&\left(\frac{8 / 2,9}{(53 / 3} \& \underline{2 / 1}\right) \\
(\underline{52 / 1})) \& \underline{23 / 2} \\
23 / 1 \\
23 / 2 \\
\text { otherwise }\end{array}$ & $\begin{array}{l}\Rightarrow 58 / 3 \\
\Rightarrow 58 / 5 \\
\Rightarrow 58 / 4 \\
\Rightarrow 58 / 6 \\
\Rightarrow 58 / 3,4,5,6(0.859 / 0.005 / 0.136 / 0.0001) \\
\Rightarrow 58 / 1 \\
\Rightarrow 58 / 2 \\
\Rightarrow 58 / 6\end{array}$ \\
\hline
\end{tabular}




\subsection{RECOVERY PHASE ACCIDENT PROGRESSION}

This section of the APET (Events 55,59-77) could be considered "late" phase accident progression, and is intended to model the response of various systems to the recovery actions as well as those phenomena that would occur late in the accident progression whether recovery actions are taken. Event 55 is described in the preceding section because it effects the operation of certain confinement systems during the recovery phase. The events in this section of the APET are:

55 Does recovery cause a steam pressure spike to occur in the confinement?

59 Is pressure tube integrity maintained for all pressure tubes?

60 What is the total hydrogen production?

61 Is hydrogen flammable in the confinement building near a pipe break?

62 Is hydrogen flammable in the pipe gallery?

63 Is hydrogen flammable in the emergency dump tank?

64 Is a detonable hydrogen mixture present?

65 Does the hydrogen near the break ignite?

66 Does the pipe gallery or emergency dump tank ignite?

67 Does the hydrogen near the break detonate?

68 Does a detonation occur in the emergency dump tank?

69 Is the the helium blanket around the core lost?

70 Has oxidation of the graphite stack begun?

71 Does fuel damage propagate to supposedly unaffected core regions?

72 What is the total amount of fuel that fails during the accident progression?

73 Does the filter exhaust path reisolate to prevent filter damage?

74 Does confinement fail during the pre-recovery phase?

75 What is the size of the confinement failure?

76 What is the location of the confinement failure?

77 Do the operating fog sprays survive the confinement failure?

All of these except Event 55 are described below.

Events 55 and 59 model two of the negative aspects of restarting the ECCS and HPI systems: steam spikes and pressure tube failures. Hydrogen can be produced by the additional water from the recovery events as well as linger from the events that occurred prior to recovery. Events 60-68 model post-recovery hydrogen phenomenology in much the same way as events 33-39 did prior to recovery. After considering other accident progression phenomena, this section of the analysis ends with the determination of the final amount of core damage and the final state of the confinement buildings.

Event 59 - Does pressure tube failure occur due to water injection?

BRANCH ABBREV, DESCRIPTION

(1) Tube-nFr No pressure tubes failed due to recovery actions

(2) Tube-Fr One or more pressure tubes failed as a result of renovery actions 
This event determines whether the pressure tubes remained intact during the recovery actions. It does not, however, summarize the current condition of the core pressure tubes, but considers only recovery-induced pressure tube failures. Pressure tubes can fail in one of two ways. First, the steam spike described in Event 55 contains the implicit assumption that one or more pressure tubes have failed in order to allow the steam to be released to the confinement and cause the steam spike. The other mechanism for pressure tube failure is dissolution of the pressure tube by molten uranium $[3-29,3-30]$. While this process takes a few hours at high temperatures, it would occur when both ECCS and GSCS continue in a failed condition for a long period of time. These mechanisms are described and probability distributions are presented in Appendix $C$.

The first two logical cases for this event model the two pressure tube failure mechanisms described in the paragraph above, respectively. The third case is used when neither pressure tube failure mechanism is appropriate.

$$
\begin{array}{ll}
55 / 2,3 & \rightarrow 59 / 2 \\
52 / 3 \& 14 / 3 \& 53 / 3 & \rightarrow 59 / 1,2(0.0001 / 0.9999) \\
\text { otherwise } & \rightarrow 59 / 1
\end{array}
$$

Event 60 - What is the total hydrogen production?

$\begin{array}{lll}\frac{\text { BRANCH }}{(1)} & \frac{\text { ABBREV }}{<230 \mathrm{H} 2} & \begin{array}{l}\text { DESCRIPTION } \\ \text { GSCS is operating at full efficiency, so total } \\ \text { hydrogen production is less than } 230 \mathrm{~kg}\end{array} \\ \text { (2) } & <1200 \mathrm{H} 2 & \begin{array}{l}\text { GSCS is operating in a degraded mode, so total } \\ \text { hydrogen production could approach } 1200 \mathrm{~kg}\end{array} \\ \text { (3) } & <2000 \mathrm{H} 2 & \begin{array}{l}\text { GSCS has failed, but ECCS has been recovered to cool } \\ \text { the core, so hydrogen total production could approach }\end{array} \\ (4) & >2000 \mathrm{H} 2 \begin{array}{l}2000 \mathrm{~kg} \\ \text { unmitigated accident, so total hydrogen production } \\ \text { could exceed } 2000 \mathrm{~kg}\end{array}\end{array}$

Unlike the pre-recovery accident progression, different core conditions can produce significantly different amounts of hydrogen in the post-recovery accident progression [3-31]. The amount of hydrogen generated in each of these cases was determined in the supporting calculations [3-9] and is described briefly in Appendix C.

The first branch above $(<230 \mathrm{~kg}$ ) represents the "nominal" N Reactor design basis accident where GSCS is operating but ECCS has failed. The other three branches represent conditions worse than that design basis accident. The distinctions between them are not, however, critical because the hydrogen uncertainty analysis determined that any hydrogen generation that was greater than the first branch was large enough to cause global hydrogen burns within the confinement building, and that the phenomenological differences between them would not justify further investigation. This event is a summary event, and the four logical cases below implement the logic from the branch descriptions above in reverse 
order. Single riser failure events end up in the final case, although their hydrogen production is much less than $230 \mathrm{~kg}$ and is, according to Appendix C, below the level of concern for hydrogen phenomenology. These events are called out separately in the hydrogen phenomenology events below.

$\begin{array}{ll}14 / 3 \& 52 / 3 \& 53 / 3 & \rightarrow 60 / 4 \\ 14 / 3 \& 52 / 3 \& 53 / 3 & \Rightarrow 60 / 3 \\ 52 / 2,3 \& 14 / 2,3 & \rightarrow 60 / 2 \\ \text { otherwise } & \rightarrow 60 / 1\end{array}$

Event 61 - Is hydrogen flammable in the confinement building?

\section{BRANCH ABBREV, DESCRIPTION}

(1) H-Mit Hydrogen has been mitigated or is otherwise rendered non-flammable

(2) H-Pent Hydrogen is flammable in the pressurizer penthouse

(3) H-SG Hydrogen is flammable in the steam generator cells

(4) H-Barr Hydrogen is flammable in the pipe barrier space

(5) H-105 Hydrogen is flammable in large areas of the $105 \mathrm{~N}$ building

This event serves the same purpose and considers the same phenomenology as Event 33 did prior to the initiation of recovery activities. It summarizes a large amount of information to determine whether hydrogen is flammable in the confinement. Hydrogen can be flammable in three locations simultaneously at $N$ Reactor: the vicinity of a pipe break, the EDT and the pipe gallery. The latter two locations are dealt with in the next event. This event summarizes hydrogen flammability in the vicinity of a primary system break. Hydrogen can become flammable in that area either by being released there during the post-recovery accident progression, or as lingering unburned hydrogen from the prerecovery progression. Accident progression pathways where more than $230 \mathrm{~kg}$ of hydrogen are produced are dealt with separately in events 64 and 67 below.

The hydrogen generation rate decreases over time and, while this event merely states that the atmosphere either was or was not flammable, the real situation would be that hydrogen could be flammable early, but mixing and leakage from the confinement could render it non-flammable at a later time. This event, therefore, is an attempt to model a dynamic quantity with a static parameter. The hydrogen uncertainty analysis assessed that the probability of an area being flammable decreased with time during the postrecovery phase, so areas that were not previously flammable would not be expected to become flammable later in the accident progression provided that the GSCS remains functional. However, areas that were flammable during the pre-recovery phase would remain flammable for at least some portion of the post-recovery accident progression provided that the hydrogen had not been previously consumed by ignition. Other factors, such as the nitrogen injection system and the amount of fuel damage, could preclude flammability, but no mechanisms were found to be present for the $N$ 
Reactor design basis accident to render non-flammable areas flammable after recovery.

The above logic is implemented as follows: the first logical case for this event filters out several non-flammable configurations. These are single riser failure events (insufficient hydrogen production), nitrogen injection in the $20 \mathrm{k}$ or $40 \mathrm{k} \mathrm{cfm}$ mode (inert atmosphere), previous hydrogen ignition (consumes the available hydrogen and, for the design basis accident, hydrogen is produced very slowly and is not likely to build up to flammable levels again as documented in Appendix $C$ ), previous hydrogen mitigation, and delayed core damage (extremely slow acting, so very low hydrogen production rate). If the confinement has not been rendered non-flammable, then any location that was previously flammable remains so. This is implemented by the next four cases. The detailed derivation of the logic structure as well as the justification behind the probabilities is beyond the scope of this report section, but is shown in Appendix $C$.

$\begin{array}{ll}15 / 1,2+58 / 3,5+33 / 1+36 / 2+7 / 2,3 & \Rightarrow 61 / 1 \\ 33 / 2 & \Rightarrow 61 / 2 \\ 33 / 3 & \\ 33 / 4 & >61 / 3 \\ \text { otherwise (33/5) } & \Rightarrow 61 / 4 \\ & \Rightarrow 61 / 5\end{array}$

Event 62 - Is hydrogen flammable in the pipe gallery?

BRANCH ABBREV, DESCRIPTION

(1) $\mathrm{nH}-\mathrm{PGal}$ Hydrogen is not flammable in the pipe gallery

(2) H-PGal Hydrogen is flammable in the pipe gallery

This event serves the same purpose and considers the same phenomenology as Event 34 did prior to the initiation of recovery activities. The flammability models it uses are the same as those described in Event 61 above because it examines the same phenomena in a different location. In summary, if the pipe gallery was previously flammable and did not ignite, it will remain flammable provided that the other mechanisms for non-flammability described above do not occur.

The first logical case for this event filters out several nonflammable configurations. These are single riser failure events (insufficient hydrogen production), nitrogen injection in the $20 \mathrm{k}$ or $40 \mathrm{k}$ cfm mode (inert atmosphere), previous hydrogen ignition (consumes the available hydrogen), a non-flammable pipe gallery during the pre-recovery phase, and delayed core damage (extremely slow acting, so very low hydrogen production rate). The second case determines that the pipe gallery is flammable if none of the non-flammability conditions are met. The detailed derivation of the logic structure as well as the justification behind the probabilities is beyond the scope of this report. section, but is shown in Appendix C.

$$
\begin{aligned}
& 15 / 1,2+34 / 1,3+37 / 2,4+58 / 3,5+7 / 2,3 \rightarrow 62 / 1 \\
& \text { otherwise } \quad \rightarrow 62 / 2
\end{aligned}
$$


Event 63 - Is hydrogen flammable in the emergency dump tank?
BRANCH
(1)
ABBREV
nH-DT
H-DT
DESCRIPTION
Hydrogen is not flammable in the EDT
Hydrogen is flammable in the EDT

This event serves the same purpose and considers the same phenomenology as Event 34 did prior to the initiation of recovery activities. The flammability models it uses are the same as those described in Events 61 and 62 above because it examines the same phenomena in a different location. In summary, if the EDT was previously flammable and did not ignite, it will remain flammable provided that the other mechanisms for non-flammability described above do not occur. The EDT atmosphere is likely to become inerted by steam at some time late in the accident progression.

The first logical case for this event filters out several nonflammable configurations. These are single riser failure events, previous hydrogen ignition, a non-flammable EDT during the pre-recovery phase, and delayed core damage. The hydrogen mitigation nitrogen system does not affect the EDT. The second case determines that the EDT is flammable if none of the non-flammability conditions are met. The logic structure is defined and the probabilities are justified in Appendix $C$.

$$
\begin{array}{ll}
15 / 1,2+34 / 1,2+37 / 3,4+7 / 2,3 & \Rightarrow 63 / 1 \\
\text { otherwise } & \Rightarrow 63 / 2
\end{array}
$$

Event 64 - Is a detonable hydrogen mixture present?

BRANCH ABBREV, DESCRIPTION

(1) H-nDet Hydrogen near the break location is not detonable

(2) H-Det Hydrogen near the break location is detonable

The post-recovery hydrogen detonability event is very similar in function to Event 35 , and very similar in logic to the preceding three events. The hydrngen generation rate decreases over time and, like the preceding events, this event is an attempt to model a dynamic quantity with a static parameter. The analysis assessed that the probability of a detonable mixture occurring decreases over time, so that only those areas that were detonable during the pre-recovery phase can be detonable later. The nitrogen injection system becomes fully effective by the time of recovery and is able to preclude detonability if it is operating.

Unlike previous events, large hydrogen source terms are analyzed in this event. The hydrogen uncertainty analysis assessed that large areas of the confinement would become detonable if upwards of $1000 \mathrm{~kg}$ of hydrogen were to be injected into the confinement building, but that the atmosphere into which it is released would be unable to support oxidation (inert) if the nitrogen injection system were operating in either the $20 \mathrm{k}$ or $40 \mathrm{k} \mathrm{cfm}$ modes as designed. In the absence of this nitrogen, though, the confinement building atmosphere would become widely detonable. 
The assessment in Event 35 determined that the EDT and pressurizer penthouse would be detonable whenever flammable. The pipe barrier space and pipe gallery near the EDT return line could also become detonable. The remaining hydrogen events in the APET are based on the assessment that the EDT is detonable whenever it is flammable and, as discussed in Event 35, the probability of pipe gallery flammability near the EDT return line implicitly contains the probability of detonability and DDT. Detonability is established for the pressurizer penthouse, pipe barrier spaces, and global areas as applicable in this event. The analysis behind the detonability findings and probabilities are presented in Appendix $C$.

The first logical case for this event precludes detonability in cases where the nitrogen system is operating in either the $20 \mathrm{k}$ or $40 \mathrm{k} \mathrm{cfm}$ mode. The second case holds as detonable any cases where the penthouse is flammable, or where the barrier space was previously detonable and continues to be flammable. It also holds as detonable any case where the hydrogen source term is significantly greater than $230 \mathrm{~kg}$. The final case considers all other situations not detonable.

$$
\begin{aligned}
& 58 / 3,5 \quad \Rightarrow 64 / 1 \\
& 61 / 2+\underline{60 / 1}+(61 / 4 \& 35 / 2) \Rightarrow 64 / 2 \\
& \text { otherwise } \quad \Rightarrow 64 / 1
\end{aligned}
$$

Event 65 - Does hydrogen in the confinement building ignite?

$$
\begin{array}{lll}
\text { BRANCH } & \text { ABBREV } & \text { DESCRIPTION } \\
\text { (1) } & \text { H-nIgn } & \text { Hydrogen near the break location does not ignite } \\
\text { (2) } & \text { H-Ign } & \text { Hydrogen near the break location does ignite }
\end{array}
$$

This event is similar in function to Event 36 , but is considerably simpler in logic. The hydrogen uncertainty analysis in Appendix $C$ held that the very long exposure time ( 22 hours) made ignition almost certain in any instance where flammable hydrogen was present in the confinement building. Thus, the first logical case states that ignition does not occur if there is not a flammable hydrogen mixture within the confinement, while the second case states that all flammable mixtures inside the confinement building ignite at some time during the post-recovery accident progression.

$$
\begin{array}{ll}
61 / 1 & \Rightarrow 65 / 1 \\
\text { otherwise } & \Rightarrow 65 / 2
\end{array}
$$

Event 66 - Does hydrogen in the emergency dump tank or pipe gallery ignite?

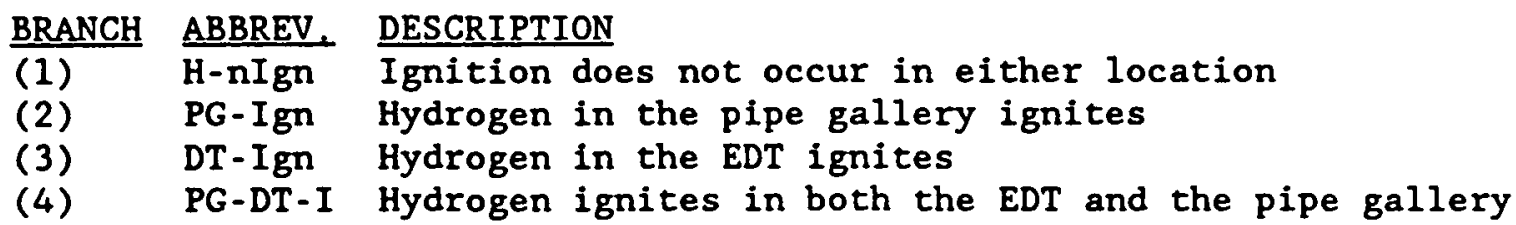


This event is identical in function to Event 37 in the pre-recovery accident progression analysis. It determines whether flammable hydrogen in the EDT and pipe gallery ignite. As stated above, the hydrogen uncertainty analysis in Appendix $C$ held that the very long exposure time (22 hours) made ignition almost certain in any instance where flammable hydrogen was present in the confinement building, although the fewer ignition mechanisms and the possibility of steam inerting made ignition within the EDT less than a sure thing. The EDT contains no electrical equipment, but does contain vacuum breakers and other mechanical equipment that could cause sparks to ignite a flammable hydrogen mixture. The probability of EDT ignition is taken from the hydrogen uncertainty analysis in Appendix $C$ and is sampled as part of the uncertainty analysis.

There are four possible combinations of EDT and pipe gallery flammability, and each has a logical case in the APET input. The first case is when both are flammable, the second is when only the pipe gallery is flammable, the third case is when only the EDT is flammable, and the last case is when neither is flammable. The sampling is simplified by the fact that the probability of pipe gallery ignition was assessed to be one. whenever it is flammable.

$$
\begin{array}{ll}
62 / 2 \& 63 / 2 & >66 / 2,4 \quad(0.829 / 0.171) \\
62 / 2 & \Rightarrow 66 / 2 \\
63 / 2 & \Rightarrow 66 / 1,3 \quad(0.829 / 0.171) \\
\text { otherwise } & \Rightarrow 66 / 1
\end{array}
$$

Event 67 - Does the hydrogen near the break location detonate?

\section{BRANCH ABBREV. DESCRIPTION}

(1) H-nBurn Hydrogen near the break location does not burn

(2) H-Def Hydrogen near the break location burns in a

(3) H-Det Hydrogen near the break location burns in a detonation

This event models deflagration to detonation transition (DDT) just as Event 38 did prior to the initiation of recovery activities. The purpose and logic of this event are identical to that in Event 38 with the lone exception that logic was added to consider globally detonable mixtures (from the very high hydrogen source terms). The detailed description of the justification behind the probabilities used in this event is shown in Appendix C.

The first logical case causes globally detonable hydrogen mixtures (in which at least $1000 \mathrm{~kg}$ of hydrogen are produced and the confinement atmosphere is not inerted to preclude hydrogen combustion) to detonate with a probability of one. The next two logical cases preclude ignition and detonation in cases where the mixture does not ignite or is not detonable, respectively. The next two cases consider penthouse detonations in the presence and absence of mixing phenomena. The final case is used to determine whether the hydrogen in the pipe barrier space detonates. 


$\begin{array}{ll}\frac{60 / 1}{65 / 1} \& 64 / 2 & \rightarrow 67 / 3 \\ 64 / 1 & \Rightarrow 67 / 1 \\ 61 / 2 \& 23 / 2,6 & \rightarrow 67 / 2 \\ 61 / 2 & \Rightarrow 67 / 2,3(0.750 / 0.250) \\ \text { otherwise (barrier space) } \rightarrow 67 / 2,3(0.930 / 0.070)\end{array}$

Event 68 - Does a detonation occur in the emergency dump tank?

BRANCH ABBREV. DESCRIPTION

(1) DT-nBrn Hydrogen in the EDT does not burn

(2) DT-Def Hydrogen in the EDT burns in a deflagration

(3) DT-Det Hydrogen in the EDT burns in a detonation

This event models deflagration to detonation transition (DDT) in the EDT. The purpose and logic of this event are identical to that in Event 39. The detailed description of the justification behind the probabilities used in this event is shown in Appendix $C$.

The first logical case determines whether the EDT atmosphere detonates given that it has ignited. The other case precludes both detonation and deflagration because ignition did not occur.

$66 / 3,4 \quad \Rightarrow 68 / 2,3(0.930 / 0.070)$

otherwise $\rightarrow 68 / 1$

Event 69 - Is the helium blanket around the core lost?

BRANCH ABBREV, DESCRIPTION

(1) He-OK Helium blanket remains intact

(2) He-Lost Helium blanket fails

This event is similar in function and logic to Event 40 . It determines whether the helium cover gas system has remained functional throughout the accident progression. The helium cover gas system enhances the heat transfer between the graphite and the GSCS tubes. If the helium blanket is lost, the efficiency with which the GSCS cools the graphite stack is significantly reduced [3-9]. The helium blanket can be lost by a pressure tube rupture event or by pressure tube failure resulting from late ECCS actuation or recovery. It can also be lost by a hydrogen detonation inside the pipe barrier space because the rupture disk for the helium system is located in that area.

The first logical case for this event considers all possible cases where one or more pressure tubes have failed or where the rupture disk on the helium cover gas system has failed. It is much like Event 40 . The second case is used when the conditions for helium system failure do not occur. This is a summary event, so uncertainty is not considered for this event and ail probabilities are either zero or one. 
$9 / 2+11 / 2+40 / 2+52 / 2+59 / 2+(61 / 4 \& 67 / 3) \rightarrow 69 / 2$

otherwise $\quad \Rightarrow 69 / 1$

Event 70 - Has oxidation of the graphite stack begun?

BRANCH ABBREV, DESCRIPTION

(1) LGr-nOx No graphite oxidation

(2) LGr-Fir Rapid, self-sustaining graphite oxidation (graphite fire) has started

(3) LGr-sOx Slow, self-sustaining graphite oxidation has started

This event is similar in function to Event 41 , and determines whether the graphite moderator stack begins to oxidize during the first 24 hours following the initiation of an accident. Self-sustaining graphite oxidation would aggravate an already bad situation by adding a chemical energy source to the decay heat energy that is usually responsible for core damage accidents.

Three different mechanisms are used to help prevent graphite oxidation at $\mathrm{N}$ Reactor. The graphite temperatures are kept relatively low by the action of the GSCS. The atmosphere around the graphite stack is normally kept inert by the action of the helium cover gas system. Finally, at $N$ Reactor, even if the other two systems fail, the paths available for air ingress into the core region and for oxidation products to escape are almost always severely limited by the steel and concrete shield walls that surround the reactor.

Two possible graphite oxidation scenarios were found during the graphite oxidation uncertainty analysis as documented in Appendix $C$. These are unmitigated accident sequences and multiple pressure tube rupture (MPTR) events. MPTR events are really a special case of the unmitigated accident sequence where, in many cases, a large number of pressure tubes and GSCS tubes are sheared off by relative motion between the core and the shield walls. This could open up large air paths between the confinement bullding atmosphere and the graphite stack itself, and would fail both the GSCS and the cover gas systems. In addition, since this event is predicted to occur mainly at high ground acceleration levels, it is very unlikely that both the hydrogen mitigation nitrogen system and the confinement buildings would both remain intact. They could provide an inert atmosphere and preclude oxidation if they were to remain functional (this is modeled in the first logical case below). Because of the the large areas that could be available for air ingress, the uncertainty analysts could not justify a conclusion that graphite oxidation would not occur in the absence of an inert atmosphere.

Unmitigated accident sequences can also cause graphite oxidation to begin because of the intense temperatures that can occur when all forced cooling to the reactor fails. The only heat transfer mechanism from the core to the confinement atmosphere is heat conduction from the core and through the slifeld walls. This is an inefficient process that requires high core temperatures to drive the conduction. Pressure tubes would be 
expected to fail inside the core at the high temperatures, but a second failure outside the core would be required to allow gases to circulate into and out of the core. Mechanisms for large air flows to occur into the core seemed rather far fetched to the graphite oxidation uncertainty analysts. For this reason, the probability distributions in Appendix $C$ show a considerably lower probability of oxidation for unmitigated accident sequences than for MPTR events.

Unmitigated events occur only when there is no coolant flow to the core from any source including ECCS and HPI. The reasons for HPI by itself to be assessed to preclude graphite oxidation are that the latent heat from vaporization of several thousand gallons of water per minute would help hold down the graphite temperatures, but more importantly, because the steam produced by the flashing coolant would drive air away from the graphite. The steam-carbon reaction is endothermic, so self-sustaining oxidation is not possible from this reaction.

The first logical case for this event precludes graphite oxidation if the confinement building has remained intact and the hydrogen mitigation system nitrogen subsystem operates in either the $20 \mathrm{k}$ or $40 \mathrm{k} \mathrm{cfm}$ modes, because of an inert confinement atmosphere. The second case models MPTR events, while the third models unmitigated accident sequences. The final case is used by all accident progression pathways in which the conditions for graphite oxidation do not occur.

$$
\begin{array}{ll}
58 / 3,5 \& 47 / 1 \& \underline{55 / 2} & \rightarrow 70 / 1 \\
4 / 2 & \rightarrow 70 / 2,3(0.490 / 0.510) \\
11 / 3 \& 14 / 3 \& 52 / 3 \& 53 / 3 \& 54 / 4 & \rightarrow 70 / 1,2,3(0.895 / 0.005 / 0.100) \\
\text { otherwise } & \rightarrow 70 / 1
\end{array}
$$

Event 71 - Does core damage propagate to the "unaffected" core region?

\section{BRANCH ABBREV, DESCRIPTION}

(1) nFDprop No fuel damage propagation occurs

(2) 1008FDP Fuel damage propagates to the entire core

(3) 2xFDP Fuel damage is approximately double what would be expected

Several mechanisms have been proposed by which regions of the core that apparently have adequate cooling would progress to fuel damage. These mechanisms include propagating pressure tube failures, induced GSCS failures, coolant flow diversion, and various molten fuel-related issues. These events are considered at length in Appendix $C$ and were found to have minimal credibility. However, this event serves as a place where some small probability of occurrence can be assigned to them as a group so that they can be included in the APET analysis.

Two fuel damage propagation possibilities are allowed for this event: "small scale" fuel damage propagation, which states that the actual amount of core damage is roughly double what is predicted by the cooling system models used in this analysis (minimum of 258 fuel damage), and "large scalen fuel damage propagation, which states that, regardless of the 
cooling configuration, the core proceeds to 1008 fuel damage. The probabilities for these propagation conditions are sampled for the uncertainty analysis from distributions that are described in Appendix $C$.

The first logical case for this event uses "fuel damage propagation" to ensure that all graphite oxidation events proceed to 1008 core damage. The second case states that, as a minimum, pressure tube ruptures caused by the late actuation of ECCS double the amount of core damage. This is a minimum increase in fuel damage. The increase in fuel damage can be greater if the configuration of core cooling would demand the greater amount of fuel damage. The final case is used to model fuel damage propagation by the mechanisms listed above regardless of the core cooling configuration.

$$
\begin{array}{ll}
41 / 2,3+70 / 2,3 & >71 / 2 \\
59 / 2 & \rightarrow 71 / 3 \\
\text { otherwise } & >71 / 1,2,3(0.998 / 0.0001 / 0.002)
\end{array}
$$

Event 72 - What is the total amount of fuel that fails during the accident progression?

$\begin{array}{lll}\text { BRANCH } & \text { ABBREV } & \text { DESCRIPTION } \\ \text { (1) } & \text { 08IFD } & \begin{array}{l}\text { Fuel damage either does not occur or is below the } \\ \text { level that would be of concern for offsite releases }\end{array} \\ \text { (2) } & 28 \text { IFD } & 28 \text { of fuel (nominal) fails } \\ (3) & 138 \text { IFD } & 138 \text { of fuel (nominal) fails } \\ (4) & 258 I F D & 258 \text { of fuel (nominal) fails } \\ (5) & 508 I F D & 508 \text { of fuel (nominal) fails } \\ (6) & 678 \text { IFD } & 678 \text { of fuel (nominal) fails } \\ (7) & 1008 I F D & 1008 \text { of fuel (nominal) fails } \\ (8) & \text { ATWS-FD } & \text { A special fuel damage class for ATWS accident } \\ \text { sequences }\end{array}$

This event is determines the nominal amount of fuel damage sustained during the entire accident progression. It is similar to Event 15, and it summarizes the operation of the RPS, ECCS, GSCS, fuel damage propagation, and all other events that can affect the state of the core. The nominal values are derived from the uncertainty analysis as described in Appendix C. The actual fuel damage amounts are sampled in the uncertainty analysis and used in the source term analysis. The nominal fuel damage values are representative of the distributions and are used in the APET analysis to refer to the core cooling states they represent.

The nominal fuel damage categories correspond to the same cooling system configuracions as described in Event 15. Those are:

28 - A single riser failure occurs in one of the inlet risers to fail ECCS cooling to $1 / 16$ of the core.

138 - ECCS is partially successful, and GSCS is operating at full efficiency.

258 - ECCS has completely failed, but GSCS is operating at full efficiency. 
508 - ECCS is partially successful, but GSCS is either operating at a degraded efficiency or is not operating at all.

67\% - ECCS has completely failed, but GSCS is operating at a degraded efficiency because the helium blanket around the core has failed.

1008 - ECCS and GSCS have both completely failed.

ATWS - The nominal value for fuel damage during an ATWS events is 508 and is based on liquid levels calculated during Westinghouse Hanford's ATWS analysis [3-12], although a broad uncertainty range on this value was used for the analysis.

Note that small scale fuel damage propagation doubles the initial amount of fuel damage subject to a minimum of 258 total fuel damage, and that large scale fuel damage propagation proceeds to 1008 fuel damage. Also, it is impossible for the core to proceed from a higher to a lower fuel damage fraction because this event represents the total amount of fuel damage sustained during the accident progression rather than an incremental amount of fuel damage sustained since Event 15. The logical cases for this event merely implement the logic described in the above list of cooling system conditions in the reverse order.

$\begin{array}{ll}15 / 8 & \\ 15 / 7+(52 / 3 \& 53 / 3)+71 / 2+(15 / 5,6 \& 71 / 3) & \rightarrow 72 / 8 \\ 15 / 6+(52 / 2 \& 53 / 3)+(52 / 1 \& 69 / 2 \& 53 / 3) & ->72 / 6 \\ 15 / 5+(15 / 4 \& 71 / 3)+(52 / 2,3 \& 53 / 2) & \\ 15 / 4+71 / 3+53 / 3 & \Rightarrow 72 / 5 \\ 15 / 3+53 / 2 & \rightarrow 72 / 4 \\ 15 / 2 & \rightarrow 72 / 3 \\ \text { otherwise }(15 / 1) & ->72 / 2 \\ \end{array}$

Event 73 - Does the filter path reisolate in time to prevent filter damage?

BRANCH ABBREV. DESCRIPTION

(1) C-ReIso Filter path reisolates in time to prevent filter damage

(2) C-nRIso Filter path reisolation is called for, but reisolation does not occur in time to prevent filter damage

(3) C-nCh Filter path reisolation is not called for

The confinement system is designed to reisolate the filter path any time the confinement pressure rises above 15" water. This event determines whether the isolation function occurs quickly enough to protect the filters and the low pressure section of the filter path from being damaged by the increased pressure. Conditions during the post-recovery accident progression that could demand the reisolation function are hydrogen combustion, graphite oxidation and steam pressure spikes. The other events in this section of the APET, such as pressure tube failure and helium cover gas system failure, do not necessarily cause pressure increases, although pressure increases could initiate or occur in combination with them. The effectiveness of the isolation system during these demands is discussed in much more detail in Appendix $C$. 
Hydrogen detonations produce shock waves that travel so quickly that there is no possibility for the filter path isolation valve to close in time to prevent filter or filter path damage. Hydrogen deflagrations occur more slowly and do not produce shock waves, so they can occur slowly enough to allow the filter path to isolate in time to prevent filter damage. The pressure at which fog sprays are demanded is very close to the pressure at which filter path reisolation is demanded, so all steam pressure spikes are assessed to demand reisolation. Steam pressure spikes are unlikely to occur faster than effective reisolation because the path has to be able to isolate fast enough to prevent a large LOCA event from damaging the filter path [3-18]. Graphite oxidation events occur more slowly still, so the speed of the isolation mechanism is of limited importance.

The logical cases for this event are hydrogen detonations first, all hydrogen deflagrations second, graphite oxidation third, steam pressure spikes fourth, and cases where isolation is not demanded are last. The probabilities were sampled from the uncertainty distributions presented in Appendix $C$.

$$
\begin{array}{lll}
67 / 3 & \rightarrow 73 / 2 \\
67 / 2+66 / 2,4 & \rightarrow 73 / 1,2(0.640 / 0.360) \\
70 / 2 & \Rightarrow 73 / 1,2(0.855 / 0.145) \\
55 / 2,3 & \Rightarrow 73 / 1,2(0.980 / 0.020) \\
\text { otherwise } & \Rightarrow 73 / 3
\end{array}
$$

Event 74 - Does confinement fail during the recovery phase?
BRANCH
(1)
ABBREV, DESCRIPTION
(2)
C-nCh Confinement condition did not change
CF-Rec
Confinement was stressed and failed

This event determines whether any of the pre-recovery accident progression events (hydrogen, graphite fire or steam spike) caused the confinement buildings to fail. This event and those that follow are identical to events $28-32$ and 46-49 in previous sections of the APET. This event does not determine whether the confinement is intact, but only whether any phenomena have occurred during the post-recovery accident progression that would fail the confinement buildings. The next event determines whether the building is still intact. Note also that while this event considers confinement isolation, it considers only the confinement buildings and not the EDT or the filters. While they are required to function in order for confinement of the radionuclides to be accomplished, they are considered in other APET events. Thus, the term "confinement" should be interpreted throughout the remainder of the APET to refer to the ability of the confinement buildings to maintain isolation and not to the overall confinement of radionuclides.

Graphite oxidation and hydrogen combustion are discussed as mechanisms for confinement building failure in Event 46 and Appendix C. Pressure tube rupture and large scale fuel damage propagation by themselves do not cause confinement failure, but the things that cause them contribuce to 
confinement failure. Steam pressure spikes can cause confinement building failure because the increased pressures from late ECCS initiation occur in an isolated confinement (assuming the building has not already failed) in which filter path isolation is also demanded. Two mechanisms remain to relieve the pressure: the banana wall and building failure. The banana wall was assessed earlier to have sufficient venting capacity to relieve the pressure increase from a small LOCA, but the steam source from dumping full ECCS flow over a hot core and into the confinement would be considerably above the capacity of the banana wall to vent. Supporting calculations [3-9] indicate that pressures considerably above the failure point of the confinement are achievable during ECCS recovery, so the uncertainty analysis in Appendix $C$ held that confinement building failure is to be expected for these accident progression pathways.

The first logical case for this event considers all accident progression events that are expected to fail the confinement with certainty. These are rapid graphite oxidation and hydrogen detonations (including those in the pipe gallery near the return line from the EDT under non-LOCA conditions). The second and fourth cases model hydrogen deflagrations. The third case models steam pressure spikes associated with delayed ECCS recovery. The fifth case considers slow graphite oxidation, and the last case is for accident progression pathways in which no events occur during the post-recovery accident progression to challenge the confinement. The probability distributions for confinement failure are described in Appendix $C$ and sampled for this event during the uncertainty analysis. The order of the cases was selected to ensure that the EVNTRE code used evaluated the cases in order of increasing probability of confinement survival (that is, when more than one event capable of causing confinement failure occurs, the confinement failure probability used will be associated with the event most likely to fail the confinement).

$\begin{array}{ll}67 / 3+70 / 2+(66 / 2,4 \& 5 / 5,6) & >74 / 2 \\ 66 / 2,4 & \Rightarrow 74 / 1,2(0.166 / 0.834) \\ 55 / 2 & \Rightarrow 74 / 1,2(0.211 / 0.789) \\ 67 / 2 & \\ 70 / 1 & \Rightarrow 74 / 1,2(0.472 / 0.529) \\ \text { otherwise } & >74 / 1,2(0.954 / 0.046) \\ & >74 / 1\end{array}$

Event 75 - What is the size of the confinement failure?

BRANCH ABBREV. DESCRIPTION

(1) C-OK Confinement has not failed

(2) C-Leak Confinement failure is a leak

(3) C-Rupt Confinement failure is a rupture

This event is similar in structure and function to events 29 and 47 in earlier sections of the APET. It is used to determine what (if any) size failure has occurred to the confinement building during the entire accident progression. It must, then, summarize confinement failures both here and in all previous sections of the APET and factor in previous confinement bullding leaks and ruptures from Event 47 as well as recent confinement failure events from Event 74. 
The APET analysis defines a rupture to mean confinement building failure such that the filter path has no decontaminating effect on the gaseous releases from the plant, while a leak is defined such that competing flow paths would be set up between the filter path and the failure. The distinction is superficial, though, because the binning input in section 3.9 uses the conservatism that the the outflow from the confinement is dominated by the flow out of the confinement building failure even for confinement leaks.

Whether an event causes a confinement leak or rupture depends on how energetic the event is. For this analysis, hydrogen detonations, graphite oxidation and steam spikes are assessed to cause confinement building ruptures. Hydrogen deflagrations could cause leaks or ruptures. The probability distributions for confinement failure for various events are described in more detail in Appendix $C$. Those distributions were sampled for this event during the uncertainty analysis.

The first logical case for this event is used to analyze hydrogen detonations and steam pressure spikes. The first, second, and third cases consider hydrogen phenomena, and the fourth case is used to analyze graphite oxidation events. The first case also includes previous confinement ruptures, while the fifth case includes previous confinement leaks. The last case is for accident progression pathways where the confinement building remains intact at the end of the entire' 24 hour accident progression.

\begin{tabular}{ll}
$47 / 3+67 / 3+(55 / 2 \& 74 / 2)$ & $>75 / 3$ \\
$66 / 2,4 \& 74 / 2$ & \\
$67 / 2 \& 74 / 2$ & $\Rightarrow 75 / 2,3(0.434 / 0.566)$ \\
$70 / 1 \& 74 / 2$ & $\Rightarrow 75 / 2,3(0.788 / 0.212)$ \\
\hline $47 / 2$ & $\Rightarrow 75 / 3$ \\
otherwise & $\rightarrow 75 / 2$ \\
& $\Rightarrow 75 / 1$
\end{tabular}

Event 76 - What is the location of the confinement failure?

BRANCH ABBREV, DESCRIPTION

(1) ConfoK No confinement compromise

(2) Envir Confinement compromise is directly to the environment

(3) Zone Confinement compromise is to another zone

This event is similar to Events 30 and 48 , and considers whether a confinement building failure leads directly to the environment or through another radiation protection zone to the environment. It summarizes confinement building failures during the entire accident progression analysis, so it must factor in previous confinement building failures from Event 46 as well as recent confinement failure events from Event 74.

If a previous failure has occurred to cause releases to be directed to another radiation protection zone, it will continue to exist as long as nothing has happened during this section of the APET to cause a release 
pathway directly to the environment. This event is not sampled in the uncertainty analysis.

$$
\begin{array}{ll}
48 / 2+(74 / 2 \& 75 / 3) & >76 / 2 \\
74 / 2 \& 75 / 2 & \Rightarrow 76 / 2,3(0.901 / 0.099) \\
48 / 3 & \Rightarrow 76 / 3 \\
\text { otherwise } & \Rightarrow 76 / 1
\end{array}
$$

Event 77 - Do the operating fog sprays survive the confinement failure?

\section{BRANCH ABBREV}

(1) Spr-nCh Spray configuration is unchanged

(2) nSpr-L Sprays all fail late

This event is similar in function and logic to events 31 and 49 in previous sections of the APET. It determines whether the function of the fog spray system is disrupted when the confinement fails. The fog spray function failure could be caused either by severing pipes, by redirecting spray heads to cause gaps in coverage, or by causing the confinement atmosphere to bypass the spray coverage area. Failure of the fog spray function is discussed and probabilities are developed in more detail in Appendix $C$. The probability distributions described in that appendix were sampled during the uncertainty analysis.

The first logical case for this event considers hydrogen detonations, while the second case considers deflagrations that fail confinement. The third case is used to model all other modes of post-recovery confinement failure including graphite oxidation and steam pressure spikes. The last case is used in situations where the confinement does not fail during this section of the APET.

$$
\begin{aligned}
& 74 / 2 \& 75 / 3 \& 67 / 3 \quad \Rightarrow 77 / 1,2(0.009 / 0.991) \\
& 74 / 2 \& 75 / 3 \&(66 / 2,4+67 / 2) \rightarrow 77 / 1 ; 2(0.828 / 0.172) \\
& 74 / 2 \& 75 / 3 \quad \rightarrow 77 / 1,2(0.989 / 0.011) \\
& \text { otherwise } \quad \Rightarrow 77 / 1
\end{aligned}
$$

\subsection{POST-RECOVERY STATUS AND SOURCE TERM ISSUES}

The post-recovery status and source term issues section of the APET summarizes the condition of systems that are important to the source term model but do not impact the remainder of the accident progression analysis. The systems analyzed here are the filter system and the liquid disposal systems. These systems are summarized in APET Events 78-87. The events in this section of the APET are:

78 Is the liquid effluent system aligned to $1312 \mathrm{~N}$ or $1325 \mathrm{~N}$ ?

79 Does the Zone I lift station operate?

80 Does liquid effluent flow to $1325 \mathrm{~N}$ ?

81 Does the Liquid Effluent Retention Facility (LERF) 1312N function?

82 Are the filters bypassed?

83 How heavily loaded are the filters? 
Events 78 through 81 summarize the operation of the liquid effluent system. This system includes the $1325 \mathrm{~N}$ Liquid Waste Disposal Facility (LWDF), the 1312N Liquid Effluent Retention Facility (LERF), the lift station diesel driven sump pumps, and their associated equipment. Events 82 through 87 model the filter system. The system model considers filter bypass, filter loading, filter media integrity, filter cell alignment, operation of the filter sprays, and filter burning. The filter system model implemented in the APET analysis is documented in Appendix $C$.

Event 78 - Is the liquid effluent system aligned to $1312 \mathrm{~N}$ or $1325 \mathrm{~N}$ ?

BRANCH ABBREV, DESCRIPTION

(1) $1312 \mathrm{~N}$ The diversion from $1325 \mathrm{~N}$ to $1312 \mathrm{~N}$ is successful

(2) $1325 \mathrm{~N}$ The diversion from $1325 \mathrm{~N}$ to $1312 \mathrm{~N}$ is not successful

During normal operations, the mildly contaminated liquid waste is sent directly to the $1325 \mathrm{~N}$ liquid waste disposal facility (LWDF). This facility is not designed to handle heavily contaminated liquids. The direct path to $1325 \mathrm{~N}$ is normally isolated on ECCS actuation. If isolation functions properly, the liquid effluent is directed to the Liquid Effluent Retention Facility (LERF) [3-32]. The LERF is a large, plastic lined and covered basin that is designed to minimize the release of radionuclides by preventing evaporation and ground seepage. If the path to $1325 \mathrm{~N}$ fails to isolate, heavily contaminated water from the EDT and/or sumps will be sent to the LWDF. Effluent water is also passively sent to the LWDF through a weir arrangement if flow to the LERF is blocked. The probabilities for this event are taken from the TEMAC analysis [3-7] of the confinement system fault tree results [3-13]. The first logical case below models the failure to generate an ECCS actuation signal due to the mispositioning of the 3 H33 ECCS bypass switch, while the second case models all other cases where the ECCS actuation signal is successfully generated.

$$
\begin{array}{ll}
8 / 2 & \rightarrow 78 / 2 \\
\text { otherwise } & \rightarrow 78 / 1,2 \quad(0.993 / 0.007)
\end{array}
$$

Evont 79 - Does the Zone I lift station operate?

\section{BRANCH ABBREV, DESCRIPTION}

(1) SUMP-OP Sump pumps are demanded and operate properly

(2) SmpFail Sump pumps are demanded but fail to operate properly

(3) SUMP-NA Sump pumps are not demanded

The diesel driven lift station is used to pump liquids from the Zone I sump areas to the liquid waste handling facilities. If the lift station falls lu operato on demand, the water in the sump areas can, after a significant period of time, overflow into the banana wall and into the fuel 
handling facilities. There are no high water level alarms in these areas, and water level indications for the sump areas in the control room do not provide readings at the levels that would be expected to occur under accident conditions even if the lift station were operating correctly. If water continues to be added to the sumps by the fog sprays, the fuel handling area would overflow. The released liquids would flow out of the building and down the embankment behind. It is possible that these liquids could cause a release of radionuclides to the river. It should be noted that a significant amount of this liquid would flow through gravity drains to the liquid waste handling facilities rather than being released from the building. The calculation of the amount of liquid released (versus the amount that flows to the liquid waste handling facilities) as well as its radionuclide contamination are computed in the source term model and are documented in Appendix $\mathrm{C}$ of the $\mathrm{N}$ Reactor Level II/III Probabilistic Risk Assessment final report [3-7]. This event determines only that the lift station pump has failed and, hence, that liquid releases are possible.

Volumetric calculations revealed that the lift station will be demanded if the fog sprays operate for more than a couple of hours. They also indicated that the flow from a large LOCA would not demand the lift station unless it were combined with either fog spray operation or with ECCS flowing out of the primary system within the confinement building. There are currently no procedural guidelines for turning off the fog spray system.

The lift station consists primarily of a single diesel driven pump. Electric pumps are available and could be valved into service to remove the liquid effluents, but they are located at an elovation ouch that they would be likely to be flooded out by the liquid levels expected during accident conditions. In addition, if that pump area were to become flooded, the overflowing liquids could place equipment in other radiation protection zones at risk of flooding. For this reason, the electric pumps are not considered as backup pumps to the lift station diesel pump. Thus, fog spray operation in conjunction with a single component failure (the diesel driven lift station pump) can cause a release to the river. The probability of lift station pump failure is taken from the TEMAC analysis [3-7] of the confinement system fault tree results [3-13].

$22 / 1+56 / 1 \rightarrow 79 / 1.2(0.957 / 0.043)$

otherwise $\rightarrow 79 / 3$

Event 80 - Does liquid effluent flow to $1325 \mathrm{~N}$ ?

BRANCH ABBREV, DESCRIPTION

(1) 1312Flo Liquid effluents flow to the 1312N LERF

(2) 1325F10 Liquid effluents flow to the $1325 \mathrm{~N}$ LWDF

(3) No-Flo No liquid effluents are produced by the plant

Liquids are released from the $\mathrm{N}$ Reactor plant by three methods: flow draining excess water from the EDT, flow through a gravity drain from the Zone I sumps, and liquids pumped from the lift station. All of these flows come together and are routed to the 1312N LERF if the path to the 1325N 
LWDF isolates as designed and as modeled in Event 78 . Otherwise, all liquid effluents are sent to the 1325N LWDF. This event determines whether the liquid effluents are sent to the LWDF or the LERF.

Liquids can reach the EDT whenever the V-4 valves are open $(\underline{8 / 2,9})$, and can reach the gravity drain whenever the lift station is demanded $(79 / 3)$ or when a LOCA occurs $(3 / 2,3)$. The first two logical cases for this event model flow to the LWDF and LERF, respectively. The third case is used when there is no liquid waste flow. This event is a summary event, so all probabilities are either zero or one and are not sampled.

$$
\begin{aligned}
78 / 2 \&(79 / 3 & +8 / 2,9 \\
\frac{79 / 3}{3}+\frac{8 / 2,9}{3 / 2,3} & \rightarrow 80 / 2 \\
\text { otherwise } & \rightarrow 80 / 1 \\
& \rightarrow 80 / 3
\end{aligned}
$$

Event 81 - Does the 1312N LERF function properly?
BRANCH
ABBREV
LERF - OK
LERF - Lk
DESCRIPTION
(2)
LERF functions as designed
LERF-NA
the LERF cover

This event determines whether the LERF cover remains intact given that liquid effluents are routed there. The modes of LERF cover failure and the probability thereof are discussed in Appendix C. That probability distribution is sampled in the uncertainty analysis. Event 80 determines whether flow is actually reaching the LERF.

$$
\begin{array}{ll}
80 / 1 & \rightarrow 81 / 1,2(0.9999 / 0.0001) \\
\text { otherwise } \rightarrow 81 / 3
\end{array}
$$

Event 82 - Are the filters bypassed?

\section{BRANCH ABBREV, DESCRIPTION}

(1) nByp Filter bypass does not occur

(2) F-pByp The filters are partially bypassed by a confinement leak at some time during the accident progression

F-Byp-L The filters are not bypassed prior to recovery, but are completely bypassed after a confinement failure during the recovery or post-recovery accident progression

(4) F-Byp The filters are completely bypassed prior to recovery

This event is used to determine whether the filter elements see the radioactive releases that they are supposed to be filtering. If the confinement buildings have ruptured, then gas flow is assessed to completely bypass the filters while leaks diminish the flow to the filters. This difference is superficial because the binning input combines leak and rupture effects into a single category by setting the flow to the filters to zero for confinement leaks. This event is a summary of confinement 
failure throughout the accident progression and, thus, is not sampled in the uncertainty analysis.

$\begin{array}{ll}\frac{45 / 2}{75 / 2} \& 73 / 2 \& 75 / 1 & \Rightarrow 82 / 1 \\ 45 / 2+47 / 3 & \Rightarrow 82 / 2 \\ \text { otherwise (late confinement rupture) } \Rightarrow 82 / 3\end{array}$

Event 83 - How heavily loaded are the filters?

BRANCH ABBREV, DESCRIPTION

(1) FL-Low Filter loading is below the level where filter sprays would be demanded

(2) FL-SP-E Filter loading will demand filter sprays prior to the initiation of recovery actions

(3). FL-SP-L Filter loading will demand filter sprays during the recovery or post-recovery phase accident progression

(4) FLHL-FE Filter loading is adequate to cause aerosol loading failure of the HEPA filters prior to the initiation of recovery actions

(5) FLHL-FL Filter loading is adequate to cause aerosol loading failure of the HEPA filters, but only after the beginning of the recovery phase accident progression

The filtration uncertainty analysis determined that there were three main mechanisms by which filter media failure could occur: installation errors, filter overload, and filter spray operation. The filtor oprays 1mpinge directly on the filter media as well as the walls and ceilings of the filter cell. Spraying water on the HEPA filters causes them to become very weak so that even normal gas flow rates through them can cause failure. This event determines whether the filter elements become loaded so heavily that they either fail outright or would demand the actuation of the filter spray system.

The load experienced by the filters is affected by two main factors: the amount of fission products and other aerosols released during the accident progression, and the amount of decontamination that occurs before the gas effluent stream reaches the filters. The release of fission products from the core is most directly related to the amount of fuel damage that occurs. The WHC filter effectiveness evaluation considered only a design basis accident in which ECCS completely failed but GSCS succeeded. This accident is similar to the 258 nominal fuel damage accident sequence in the APET. The results from this analysis were scaled downward to consider the effects of single riser failure accident sequences on the filters, and the result was that single riser failure events should not load the filters even to the point where the filter sprays should be demanded. The results were also scaled up (with extra conservatism added to account for the higher core temperatures) to consider filter loading for cases where the GSCS and ECCS both failed. This assessment was carried out only for the pre-recovery phase accident progression, and the filters were assessed to always fail during the post-recovery accident progression because no deterministic analysis was available to show filter performance 
in unmitigated accident sequences and extrapolation from the lesser core damage analysis for long times would be unreliable due to the large differences in accident progression expected between these scenarios. The scaling analyses are presented in more detail in the descriptions of the uncertainty issues in Chapter 4 and Appendix $C$.

N Reactor filter effectiveness was examined [3-33] and estimated loadings were determined for several different confinement atmosphere and filter flow conditions. The uncertainty analysis calculated an approximate decontamination factor (DF) from these results for the gas stream between its release into the confinement and its reaching the filters, and used this information as the basis for an uncertainty distribution for the DF required to prevent the filter sprays from being demanded. This DF could be achieved by either the EDT or the fog sprays (building settling DF was neglected). The uncertainty distributions for the fog spray and EDT DFs were overlaid onto the distribution for the required $D F$ to obtain a distribution for the probability that filter sprays would be demanded given that these systems either were or were not operating. This was done for each core cooling configuration as described above. The process and the resulting uncertainty distributions are discussed in more detail in Chapter. 4 and Appendix $C$.

The results of the above analysis are implemented as logical cases in the structure below. The first case keeps single riser and non-core damage events from loading the filters. The second and third cases cause automatic filter failure due to aerosol loading for accident progression pathways involving graphite oxidation. The fourth, fifth, sixth and seventh cases model accident progression pathways where the GSCS operates successfully or where a delayed ECCS failure occurs (these were assessed to be similar in accident progression because the late ECCS failure accident sequence has a very low decay heat level and progresses very slowly). The cases represent filter loading where both the EDT and the fog sprays operate, only the fog sprays operate, only the EDT operates, and neither the fog sprays nor the EDT operate, respectively. The remaining four cases represent the same fog spray and EDT configurations for GSCS failure and ATWS accident progression pathways.

$$
\begin{aligned}
& 72 / 1,2 \quad \Rightarrow 83 / 1 \\
& 70 / 1 \quad \Rightarrow 83 / 5 \\
& \underline{41 / 1} \quad \rightarrow 83 / 4
\end{aligned}
$$

$(72 / 3,4+7 / 2,3) \&(3 / 1+(3 / 3 \& 8 / 2,9))$

$\& 22 / 1 \& 31 / 1 \& 49 / 1 \& 56 / 1 \& 77 / 1 \Rightarrow 83 / 1,2,3$

$(72 / 3,4+7 / 2,3) \& 3 / 2,3 \& 22 / 1 \& 31 / 1$ $\& 49 / 1 \& 56 / 1 \& 77 / 1 \quad \Rightarrow 83 / 1,2,3$

$(72 / 3,4+7 / 2,3) \&(3 / 1+(3 / 3 \& 8 / 2,9)) \rightarrow 83 / 1,2,3$ $(0.632 / 0.288 / 0.080)$

$72 / 3,4+7 / 2,3 \quad \rightarrow 83 / 2,5(0.701 / 0.299)$ 


$$
\begin{aligned}
\begin{array}{l}
72 / 5,6,7,8 \&\left(3 / 1+\left(3 / 3 \& \frac{8 / 2,9}{2}\right)\right) \\
\& 22 / 1 \& 31 / 1 \& 49 / 1 \& 56 / 1 \& 77 / 1
\end{array} & \rightarrow 83 / 2,5(0.297 / 0.703) \\
72 / 5,6,7,8 \& 3 / 2,3 \& 22 / 1 \& 31 / 1 \& 49 / 1 & \\
\& 56 / 1 \& 77 / 1 & \Rightarrow 83 / 2,5(0.744 / 0.256) \\
72 / 5,6,7,8 \&(3 / 1+(3 / 3 \& 8 / 2,9)) & \rightarrow 83 / 2,5(0.363 / 0.637) \\
\text { otherwise }(72 / 5,6,7,8) & \rightarrow 83 / 4
\end{aligned}
$$

Event 84 - Do the filter sprays operate?
BRANCH
(1)
(2)
ABBREV, DESCRIPTION
FSpr Filter sprays are demanded and operate
(3)
nFSpr
Filter sprays are demanded but do not operate
FSpr-NA
Filter sprays are not demanded

Event 83 determines whether the filter sprays are demanded. Given that they are demanded, this event determines whether they actually operate. The probabilities for this event are determined by the TEMAC analysis of the confinement system fault tree analysis results and are included in the uncertainty analysis. This event does not consider whether the filters have survived to this point $[3-18]$.

The first logical case for this event filters out those accident progression pathways where the filter sprays are not demanded. The second and third cases model the increased probability of filter spray failure given that support systems consisting of one or more electrical busses and the fog sprays have failed. The last case considers other random failures of the filter spray system. The modeling for these cases is taken directly from a TEMAC analysis of the confinement system fault tree cut sets, and the probabilities are sampled during the integrated uncertainty analysis.

$$
\begin{array}{ll}
83 / 1 & \rightarrow 84 / 3 \\
(6 / 2,4+51 / 2,4) \& 22 / 2 & \rightarrow 84 / 2 \\
(6 / 3+51 / 3) \& 22 / 2 & \Rightarrow 84 / 1,2(0.736 / 0.264) \\
\text { otherwise } & \Rightarrow 84 / 1,2(0.998 / 0.002)
\end{array}
$$

Event 85 - D Cell Filter Operating

$$
\begin{array}{lll}
\text { BRANCH } & \text { ABBREV } & \frac{\text { DESCRIPTION }}{\text { (1) }} \\
\text { Fil-D } & \text { D-cell filter is operating } \\
\text { (2) } & \text { Fil-AB } & \text { D-Cell filter is not operating }
\end{array}
$$

Part of the automatic sequence that is initiated by confinement isolation is designed to realign the filter pathway from its normal operating alignment ( $f l o w$ is sent through cells $A$ and $B$ ) to its accident condition alignment (flow is sent through cell $D$ ). The reason for the realignment is that the $D$ filter cell charcoal beds are impregnated with potassium iodide to increase the absorption of iodine from the effluent gas 
stream while the beds in cells $A$ and $B$ are not. This event determines whether the alignment takes place as intended. The probabilities for this event are determined by the-TEMAC analysis of the confinement system fault tree analysis results and are included in the uncertainty analysis. The doors on $D$ cell are counterweighted to remain open given a loss of electrical power.

Common sensors and actuation components between the confinement isolation system, the steam vent closure system and the filter cell alignment system cause increased probabilities for the failure to align to cell $D$ when one or both of these systems have failed. This is modeled in the first three logical cases below. The fourth case considers other random failures of the filter cell alignment system. The case structure and probabilities for this event are taken from the TEMAC analysis of the confinement system fault tree analysis results and are sampled in the uncertainty analysis.

$$
\begin{array}{ll}
\frac{19 / 1.5}{27 / 2+32 / 2}(27 / 2+32 / 2) & \Rightarrow 85 / 2 \\
19 / 1.5 & \Rightarrow 85 / 1,2(0.909 / 0.091) \\
\text { otherwise } & \Rightarrow 85 / 1,2(0.982 / 0.018) \\
& \Rightarrow 85 / 1,2(0.993 / 0.007)
\end{array}
$$

Event 86 - Do the filters remain intact?

\section{BRANCH ABBREV, DESCRIPTION \\ (1) Fil-OK Filter media failure does not occur \\ (2) Fil-F Filter media failure occurs}

This event models failures of the filter media that are not related to filter loading or spray actuation. It considers such factors as defective filter media, filter installation errors, and failure of the sealing mechanisms that hold the filter elements in place. The probabilities for this event are determined by the TEMAC analysis of the confinement system fault tree analysis results [3-13] and are included in the uncertainty analysis [3-7]. The first logical case below bypasses this event if the filters are bypassed by the effluent gas flow during the entire accident progression because the filtration function is failed regardless of the condition of the filter cells. The other case considers the filter media failures described above.

$$
\begin{array}{ll}
82 / 4 & \Rightarrow 86 / 2 \\
\text { otherwise } & \rightarrow 86 / 1,2(0.998 / 0.002)
\end{array}
$$


Event 87 - How effective are the filters?

BRANCH ABBREV, DESCRIPTION

(1) F-1008 Filters operate at or very near the design effectiveness for the entire accident progression

(2) F-Early Filters operate at design efficiency until the

beginning of the recovery phase accident progression

(3) $\quad F<1008$ Filters are partially bypassed by a confinement leak

(4) F-08 Filters are bypassed by a confinement building

(5) F-Burn Filters burned during the post-recovery accident progression

This event summarizes all of the filtration events (events 82 through 86) to determine the overall filter performance for the accident progression pathway. The first logical case for this event determines that the filters burn if the filter sprays were demanded but did not actuate based on an assessment in Appendix $C$. The second case considers all accident progression pathways where the filtration function fails prior to the initiation of recovery actions. These include accident progression pathways where the filter media fails, the filters are bypassed or the filter sprays operate prior to the initiation of recovery actions. The third case considers all accident progression pathways where the filters survive and are active during the pre-recovery phase but fail during the post-recovery accident progression. These include accident progression pathways where the filters are bypassed or the filter sprays actuate after recovery. The fourth case models cases where the filters are bypassed by a confinement building failure during the entire accident progression. The last case considers cases where the filters are partially bypassed by a confinement leak. The difference between this and the filters being fully bypassed during the entire accident progression is superficial for the actual analysis because the binning input combines leak and rupture effects into a single category by setting the flow to the filters to zero for confinement leaks. This event is a summary event, so no sampling of probabilities occurs.

$\begin{array}{ll}84 / 2 & \rightarrow 87 / 5 \\ 82 / 4+83 / 2,4+86 / 2+18 / 2 & ->87 / 4 \\ 82 / 3+83 / 3,5 & \Rightarrow 87 / 2 \\ 82 / 2 & ->87 / 3 \\ \text { otherwise } & ->87 / 1\end{array}$

This completes the description of the $N$ Reactor APET. A vast amount of data has been distilled to arrive at this description of $\mathrm{N}$ Reactor accident progression, and a large amount of information is generated on each pass through the APET during solution (each pass produces one accident progression pathway). This information must be distilled to a manageable level for the source term analysis. This distillation process combines similar accident progression pathways into accident progression bins. The process by which this is done is discussed in the next section. 


\subsection{BINNING OF ACCIDENT PROGRESSION EVENT TREE OUTPUT}

Each possible path through the APET represents a comprehensive description of a unique accident progression pathway because a unique sequence of accident progression events occurred to cause it. However, two unique accident progression pathways in the APET may differ only in ways that are not distinguishable by the source term analysis. For example, it makes very little difference whether a vacuum breaker fails to reclose if, in the same accident progression pathway, the confinement initially failed to isolate. More than five million accident progression pathways were analyzed in the APET analysis during the uncertainty analysis (these were developed from approximately 30 internal events, 15 fire events and 32 seismic events Level I accident sequences). The large number of accident progression pathways needs to be reduced to a more manageable size if for no other reason than that the computer must consider all of these simultaneously during the final risk and regression analysis calculations. Clearly, accident progression pathways that the source term analysis considers to be indistinguishable should be combined to avoid unnecessary computational duplication.

The reduction in the number of accident progression pathways is accomplished through a technique known as "binning." Under this technique, combinations of APET events are identified that are important to the source term analysis. The EVNTRE code package determines a value for each of these parameters and saves only that information. Continuous values are not allowed for these parameters, but only discrete values. Thus, all APET accident progression pathways that have the same discrete values for the binning parameters are lumped together as a single accident progression bin for the source term analysis.

The parameters mentioned above are called "dimensions" by the EVNTRE code. Examples of dimensions are, "How much fuel damage occurred?" and "How did the filter system perform?" The discrete "answers" to these questions are called "attributes." Examples of attributes for the first dimension above would be discrete levels of core damage such as 08,28 , $138,268,508$, and 1008. Each accident progression pathway must fit into one attribute for each dimension. If it does not, the EVNTRE code aborts. The dimensions and attributes and their associated conditional probabilities are the only information that is retained after the completion of the EVNTRE code. This information is passed from the APET analysis to the source term calculation.

The structure of the binning system was determined during extensive consultations between the accident progression analyst and the source term analyst. The purpose of these discussions was to ensure that all major phenomena affecting the source term were effectively communicated during the analysis. The result was a binning system with 14 dimensions. These are listed and discussed below. Each attribute is assigned a letter designator. These letter designators are used in the EVNTRE output that is presented in the appendix. They are also used in the input and output for the source term and risk calculations presented in the full PRA report $[3-7]$. 
The logical structure for the binning input is described along with the dimensions and attributes below. The notation is the same as that used in the APET description above. However, the binning input, unlike the EVNTRE event input, must be logically exhaustive because "otherwise" cases are not allowed. An example of how the individual attribute descriptors are combined to form an accident progression description can be found following the description of the descriptors and attributes.

\section{Dimension 1 - Primary Coolant System Integrity}

The first dimension determines whether the primary system has remained intact throughout the accident progression. It is used to tell the source term model how radionuclides are released to the confinement. It is important to the source term analysis because the EDT scrubs many radionuclides from the liquid effluents that pass through it. This scrubbing does not occur if the coolant flows directly to the confinement building. The different attributes are used to define how the coolant flow is divided between the EDT and the direct path to the confinement building.

The letters before the attribute names are used throughout the source term and risk analyses in the first position to determine the value of this dimension. This dimension has four attributes:

$\begin{array}{cll}\frac{\text { ATTRIBUTE }}{\text { A }} & \begin{array}{l}\text { ABBREV } \\ \text { LLOCA }\end{array} & \begin{array}{l}\text { DESCRIPTION } \\ \text { large LOCA (including multiple pressure tube rupture } \\ \text { seismic initiating events), } \\ \text { small LOCA (including single pressure tube rupture } \\ \text { initiating events), } \\ \text { pressure tube rupture (other than as an initiating } \\ \text { event), and }\end{array} \\ \text { C } & \text { PLOCA } & \begin{array}{l}\text { "transient," which indicates that the primary system } \\ \text { remained intact. }\end{array} \\ \text { D } & \text { Trans }\end{array}$

These attributes are determined based on the events in the APET according to the following logical cases: large and small LOCAs can be determined directly by the initiating event, while pressure tube failures must be determined by examining the two pressure tube failure events. The primary system remains intact in any accident progression pathway that does not involve either a LOCA or a pressure tube rupture.

$$
\begin{array}{ll}
3 / 2 & \Rightarrow \mathrm{A} \\
3 / 3 & \Rightarrow \mathrm{B} \\
9 / 2+59 / 2 & \Rightarrow \mathrm{C} \\
3 / 1 & \Rightarrow \mathrm{D}
\end{array}
$$

Dimension 2 - Initial Fuel Damage

This dimension communicates the amount of "initial" fuel damage to the source term model. Fuel damage is determined as the ratio of the fuel mass in elements in which the clad has failed to the total fuel mass initially 
in the core. Fuel damage information impacts the source term analysis because radionuclides are not released from intact fuel. Thus, the total radioactive release from the core is directly correlated to this information.

Dimension 2 considers only the fuel damage as a result of the core cooling configuration at the onset of core damage. Thus, even though the APET considers delayed ECCS failure as having zero initial fuel damage, the binning analysis sees the delayed core damage as initial fuel damage because there is no core damage during the delay time.

The letters before the attribute names are used throughout the source term and risk analyses in the second position to determine the value of this dimension. The dimension has eight attributes that represent "nominal" core damage states of:

\begin{tabular}{|c|c|c|}
\hline ATTRIBUTE & ABBREV, & DESCRIPTION \\
\hline A & O\&IFD & 08 initial fuel damage, \\
\hline B & 28 IFD & 28 initial fuel damage, \\
\hline C & 138 IFD & 138 initial fuel damage, \\
\hline D & 258 IFD & 258 initial fuel damage, \\
\hline $\mathbf{E}$ & 508 IFD & 50 initial fuel damage, \\
\hline $\bar{F}$ & 1008 IFD & 1008 initial fuel damage, \\
\hline G & ATWS - FD & ATWS fuel damage, and \\
\hline H & $67 \&$ IFD & 678 initial \\
\hline
\end{tabular}

These nominal fuel damage states are based on the status of the various core cooling systems. The nominal value for fuel damage during an ATWS accident sequence is 508 and is based on liquid levels calculated during Westinghouse Hanford's ATWS analysis [3-12], although a broad uncertainty range on this value was used for the analysis. The nominal fuel damage amounts described above are varied as uncertain parameters during the uncertainty analysis.

The above attributes are determined based on the events in the APET according to the following logical cases: the first case is used for 1008 initial fuel damage as well as late ECCS failures that proceed to 1008 fuel damage. The remaining seven cases directly copy the results of Event 15 into the appropriate attributes for this dimension.

$\begin{array}{ll}15 / 7+(72 / 7 \& 7 / 2,3) & \Rightarrow \mathrm{F} \\ 15 / 1 & \Rightarrow \mathrm{A} \\ 15 / 2 & \Rightarrow \mathrm{B} \\ 15 / 3 & \Rightarrow \mathrm{C} \\ 15 / 4 & \Rightarrow \mathrm{D} \\ 15 / 5 & \Rightarrow \mathrm{E} \\ 15 / 8 & \Rightarrow \mathrm{G} \\ 15 / 6 & \Rightarrow \mathrm{H}\end{array}$


Dimension 3 - Emergency Core Cooling System Status

This dimension summarizes the status of the emergency coolant flow through the core during the accident progression. The information is used to determine whether a water or steam flow is present to sweep the radionuclides out of the core. If no flow is present, many radionuclides are decontaminated from the effluent stream by condensation and settling. Steam or water flow decreases the time the effluent gases spend inside the primary system and, hence, the time available for effluent decontamination. This information is important to the source term analysis.

The letters before the attribute names are used throughout the source term and risk analyses in the third position to determine the value of this dimension. The dimension has four attributes:

\begin{tabular}{|c|c|c|}
\hline ATTRIBUTE & ABBREV & DESCRIPTION \\
\hline$A$ & $\overline{1-R i s e F}$ & single riser failure, \\
\hline B & pECCS & $\begin{array}{l}\text { ECCS and/or other sources supply water to the core, but } \\
\text { the supplies are insufficient to prevent core damage, }\end{array}$ \\
\hline C & ECCS - FL & $\begin{array}{l}\text { ECCS succeeds for at least two hours before failing, } \\
\text { and }\end{array}$ \\
\hline D & nEccs & $\begin{array}{l}\text { all sources of water to the core either fail or are } \\
\text { shut off. }\end{array}$ \\
\hline
\end{tabular}

After the completion of the original APET-source term model interface, it was seen to be desirable to communicate certain special fire cases to the source term model. These cases represent fires that damage the control cables to the ECCS high lift diesel pumps. The reason these fires form a special case is that delayed ECCS recovery would be expected to occur significantly sooner in this situation than in other types of accidents because the pumps could be started locally in Building 182N. The source term analyst determined that this could be factored into the source term analysis if the information were to be passed from the APET analysis. This interface was accomplished by passing an apparently contradictory accident progression bin to the source term analysis in which the amount of initial fuel damage is set to 1008 (dimension 2, attribute $F$ ) combined with an ECCS status of single riser failure (dimension 3, attribute A). A detailed search was performed on a sample APET uncertainty analysis to ensure that no other legitimate accident progression bins would contain such a combination of bins before this method was finalized for transferring this piece of special information. The special logic for this transfer is implemented in the third logical case below.

The above attributes are determined based on the events in the APET according to the following logical cases: the first two cases describe single riser failures and delayed ECCS failures, respectively. The third case is used to communicate the special fire core damage information described above to the source term model. The fourth case determines whether any water enters the core during the accident progression from either ECCS or HPI because, although HPI does not lessen core damage, it would push radionuclides out of the core. The last case is used for accident progression pathways where no coolant whatsoever flows into the core. 


$\begin{array}{ll}7 / 3,4 & \Rightarrow \mathrm{A} \\ 7 / 2 & \\ 2 / 2 \& 15 / 7 \& 14 / 3 \& \underline{52 / 2} \& 53 / 3 & \Rightarrow \mathrm{A} \\ 14 / 1,2+12 / 4+54 / 4 & \Rightarrow \mathrm{B} \\ 14 / 3 & \Rightarrow \mathrm{D}\end{array}$

Dimension 4 - Graphite and Shield Cooling System Status

This dimension summarizes the status of the GSCS coolant flow through the core. GSCS information is important to the source term model because the GSCS status determines an approximate failed fuel temperature profile. The radioactive releases from the fuel are based on this temperature as correlated to the GSCS status. Since this affects the release of radionuclides from the fuel, it has a first-order effect on the source term analysis.

The letters before the attribute names are used throughout the source term and risk analyses in the fourth position to determine the value of this dimension. The dimension has three attributes:
ATTRIBUTE ABBREV, DESCRIPTION
A fGSCS GSCS operates at normal efficiency throughout the accident progression,
B degGSCS the helium blanket is lost at some time, so GSCS operates at degraded efficiency once the helium blanket is lost, and
C nGSCS GSCS did not operate for at least some portion of the accident progression.

The above attributes are determined based on the events in the APET according to the following logical cases: the first case filters out events where GSCS works both during the pre- and post-recovery accident progression phases. The second and third cases are used when GSCS was degraded or failed during at least some portion of the accident progression, respectively.

$$
\begin{aligned}
& 11 / 1 \& 52 / 1 \rightarrow \mathrm{A} \\
& 11 / 2+52 / 2 \rightarrow \mathrm{B} \\
& 11 / 3+52 / 3 \rightarrow \mathrm{C}
\end{aligned}
$$

Dimension 5: Emergency Dump Tank Status

The fifth dimension monitors the status of the EDT. The EDT scrubs many radionuclides from the liquid effluents that pass through it. This will cause radionuclides to accumulate in the liquid in the EDT. If the EDT fails, radioactive liquids or gases can be released to the environment. This dimension allows the source term model to consider EDT failure as a mechanism for releasing fission products to the environment from the liquid effluenl stream. 
The letters before the attribute names are used throughout the source term and risk analyses in the fifth position to determine the value of this dimension. The dimension has six attributes:

\begin{tabular}{|c|c|c|}
\hline ATTRIBUTE & ABBREV. & DESCRIPTION \\
\hline $\mathrm{A}$ & $\mathrm{nV} 4 \mathrm{~s}$ & $\begin{array}{l}\text { the EDT is not applicable because the flow to it is } \\
\text { blocked, }\end{array}$ \\
\hline B & DTf-Cok & $\begin{array}{l}\text { the EDT fails and releases liquid directly to the } \\
\text { environment, but the remainder of the confinement has } \\
\text { not failed, }\end{array}$ \\
\hline C & DTf-Cf & $\begin{array}{l}\text { same as } B \text {, but with a failure of the confinement } \\
\text { building, }\end{array}$ \\
\hline D & Vtf-Cok & $\begin{array}{l}\text { the EDT venting system fails and allows fission product } \\
\text { vapors to be released to the environment, but the } \\
\text { remainder of the confinement has not failed, }\end{array}$ \\
\hline $\mathbf{E}$ & Vtf-Cf & $\begin{array}{l}\text { same as } D \text {, but with a failure of the confinement } \\
\text { building, and }\end{array}$ \\
\hline $\mathbf{F}$ & DT-V4ok & EDT functions as intended. \\
\hline
\end{tabular}

The above attributes are determined based on the events in the APET according to the following logical cases: the first case is used when no liquid effluents can reach the EDT. The second and fourth cases are used when the EDT fails and the confinement remains intact, and the third and fifth cases are used when the EDT fails in the same accident progression pathway as a confinement failure. The last case is used when liquid is flowing to the EDT and it remains intact. Hydrogen combustion events within the EDT are assessed to always cause EDT failure with detonations faliling the tank itself and deflagrations allowing vapors to be released to the environment.

$$
\begin{array}{ll}
8 / 2,9+17 / 4 & \rightarrow \mathrm{A} \\
(17 / 3+39 / 3+68 / 3) \& 75 / 1 & \rightarrow \mathrm{B} \\
17 / 3+39 / 3+68 / 3 & \rightarrow \mathrm{C} \\
(17 / 2+39 / 2+68 / 2) \& 75 / 1 & \rightarrow \mathrm{D} \\
17 / 2+39 / 2+68 / 2 & \rightarrow \mathrm{E} \\
17 / 1 \& 39 / 1 \& 68 / 1 \& \underline{8 / 2.9} & \rightarrow \mathrm{F}
\end{array}
$$

\section{Dimension 6 - Fog Spray Status}

This dimension describes the operation of the fog spray system. The fog spray system provides pressure reduction through steam condensation inside the confinement building. It also provides some decontamination to the confinement building atmosphere. It is the latter effect that is important to the source term analysis. It should be noted that current procedures only call for fog spray system actuation in response to a rise in the confinement building pressure. Thus, fog spray actuation and the associated confinement decontamination are not expected in cases where the primary system remains intact. 
The letters before the attribute names are used throughout the source term and risk analyses in the sixth position to determine the value of this dimension. The dimension has four attributes:

ATTRIBUTE ABBREV, DESCRIPTION

A Sp-Erly sprays operate early but fail during the recovery phase,

B Sp-late sprays are not demanded early but operate during the recovery phase,

C Spr-Op sprays operate during the entire accident progression, and

D no-Spr sprays do not operate at all.

The above attributes are determined based on the events in the APET according to the following logical cases:

$\begin{array}{ll}22 / 1 \& 31 / 1 \& 49 / 1 \&(56 / 1+77 / 1) & \rightarrow \mathrm{A} \\ (\underline{22 / 1}+\underline{31 / 1}+\underline{49 / 1}) \& 56 / 1 \& 77 / 1 & \Rightarrow \mathrm{B} \\ 22 / 1 \& 31 / 1 \& 49 / 1 \& 56 / 1 \& 77 / 1 & \Rightarrow \mathrm{C} \\ (\underline{22 / 1}+\underline{31 / 1}+\underline{49 / 1}) \&(\underline{56 / 1}+\underline{77 / 1}) & \rightarrow \mathrm{D}\end{array}$

The cases summarize the results of the three pre-recovery fog spray operation events $(21,31$ and 49 ) and the two post-recovery events (56 and 71) to determine whether the fog sprays operated early (first case), late (second case), both (third case) or neither (last case).

Dimension 7 - Nitrogen System Status

This dimension summarizes the status of the nitrogen purge function of hydrogen mitigation system. As discussed above for the primary system, condensation and settling provide decontamination of radionuclides in atmospheres. These effects are highly dependent on the time the effluent gases spend in confined areas. Since $N$ Reactor has a filtered, vented confinement rather than a containment building, there is always expected to be gas flow through the building. The velnrity of the gas flow determines the residence time of the gases in the various confinement areas. The nitrogen system adds gases to the confinement atmosphere. This forces the confinement atmosphere out through either the filter system or an existing building failure location and decreases the time the atmosphere spends inside the building for decontamination. Thus, nitrogen system operation is important to the source term analysis.

The letters before the attribute names are used throughout the source term and risk analyses in the seventh position to determine the value of this dimension. The dimension has two attributes:

$\begin{array}{lll}\frac{\text { ATTRIBUTE }}{\text { A }} & \frac{\text { ABBREV }}{\text { Nit }} & \frac{\text { DESCRIPTION }}{\text { the nitrogen system operates in some mode during the }} \text { entire accident progression, and } \\ \text { the nitrogen system does not operate. }\end{array}$


The operation of the nitrogen system is not further subdivided because the planned nitrogen system operating procedures call for the system to be activated very shortly after the onset of core damage (whenever that occurs), and recovery of failures in the system is modeled in the APET as being very unlikely. Thus, if the nitrogen system operates at all, it will be actuated shortly after the onset of core damage and operate throughout the accident.

The above attributes are determined based on the events in the APET according to the following logical cases: any accident progression pathway with any hydrogen mitigation system nitrogen operating in either hydrogen mitigation event ( 23 or 58 ) uses the first case, and all others use the second case.

$$
\begin{array}{ll}
23 / 1,2,3,5+58 / 1,2,3,5 & \Rightarrow \mathrm{A} \\
23 / 4,6+58 / 4,6 & \Rightarrow \mathrm{B}
\end{array}
$$

\section{Dimension 8 - Filter System Status}

This dimension monitors the operation of the filter system. The filter system is the last line of defense between the contaminated confinement atmosphere and the environment, so failure of the filter system would radically increase the magnitude of the radionuclide release during a core damage accident sequence.

The letters before the attribute names are used throughout the source term and risk analyses in the eighth position to determine the value of

\begin{tabular}{|c|c|c|}
\hline ATTRIBUTE & ABBREV. & DESCRIPTION \\
\hline A & Fil-Op & $\begin{array}{l}\text { the filters operate at the expected efficiency durin } \\
\text { the entire accident progression, }\end{array}$ \\
\hline B & F-Burn & $\begin{array}{l}\text { the filters burn during the recovery phase due to th } \\
\text { failure of the filter spray system, }\end{array}$ \\
\hline C & Fil-FL & $\begin{array}{l}\text { the filters operate as above early in the accider } \\
\text { progression but fail due to either loading or filt } \\
\text { spray impingement during the recovery phase, and }\end{array}$ \\
\hline D & Fil-Oz & $\begin{array}{l}\text { the filters are ineffective during the entire accider } \\
\text { progression due either to filter media failure or } \\
\text { failure of the confinement that causes the effluer } \\
\text { gases to bypass filter path. }\end{array}$ \\
\hline
\end{tabular}
this dimension. 'the dimension has four attributes:

The above attributes are determined based on the events in the APET by a direct mapping of the results of Event 87 into the attributes of this dimension. It should be noted that all partial as well as total filter bypass accident progression pathways are routed to the Fil-08 attribute. This effectively prevents competition for the gas stream between the filter path and the confinement failure and routes all gases out the confinement failure. This may be a significant conservatism in the overall analysis. It was adopted because the elicitees in the uncertainty analysis felt that a reasonable assessment of the competition between the filter pathway and 
any confinement failure would require a much more exact knowledge of the location and size of the failure than the information in the APET would be able to provide. In addition, the results of previous numerical calculations attempting to predict such flows varied between programs and even, occasionally, between nodalizations for the same code. The elicitees felt that, while the current treatment might be significantly conservative, it would be almost impossible to justify any less conservative approach without embarking on a significant (even multi-year) research effort. This forms the basis for binning all confinement leaks into the confinement rupture category as is done in the fourth logical case below.

$$
\begin{aligned}
& 87 / 1 \quad \rightarrow A \\
& 87 / 5 \rightarrow B \\
& 87 / 2 \rightarrow C \\
& 87 / 3,4 \rightarrow \text { D }
\end{aligned}
$$

Dimension 9 - Confinement Building Status

This dimension is used to communicate the cause of confinement building fallure. The cause of confinement building failure is important because it affects the energy of the radionuclide release to the environment. The release energy affects the consequence calculations because it affects the buoyancy and dispersal of the release plume as it is tracked by the consequence model. The behavior and location of the release plume determines which segment of the population is at risk as a result of any radioactive release and what radiation they would be exposed to.

\begin{tabular}{|c|c|c|}
\hline TTRIBUTE & BBREV, & DESCRIPTION \\
\hline A & Conf-Ok & the confinement does not fail, \\
\hline B & $=0 x$ & $\begin{array}{l}\text { the confinement fails due to a global hydrogen } \\
\text { detonation while graphite oxidation occurs, }\end{array}$ \\
\hline C & GDetnox & $\begin{array}{l}\text { a global detonation fails confinement without graphite } \\
\text { oxidation, }\end{array}$ \\
\hline D & EOx & $\begin{array}{l}\text { the confinement fails due to a local hydrogen } \\
\text { detonation or a global deflagration while graphite } \\
\text { oxidation occurs. }\end{array}$ \\
\hline $\mathbf{E}$ & GDefnOx & $\begin{array}{l}\text { a local detonation or global deflagration fail } \\
\text { confinement without graphite oxidation, }\end{array}$ \\
\hline $\mathbf{F}$ & LDef-Ox & $\begin{array}{l}\text { the confinement fails due to a local deflagration while } \\
\text { graphite oxidation occurs, or any other graphite } \\
\text { oxidation event. }\end{array}$ \\
\hline G & LDefnox & $\begin{array}{l}\text { a local deflagration fails the confinement without } \\
\text { graphite oxidation, and }\end{array}$ \\
\hline $\mathbf{H}$ & oth & $\begin{array}{l}\text { the confinement fails due to a non-hydrogen ev } \\
\text { without graphite oxidation. }\end{array}$ \\
\hline
\end{tabular}

The letters before the attribute names are used throughout the source term and risk analyses in the ninth position to determine the value of this dimension. The dimension has eight attributes:

The above attributes are determined based on the events in the APET according to the following logical structure: events 41 and 70 determine 
graphite oxidation, and are necessary components of attributes $B, D$ and $F$, and cannot occur in attributes $A, C, E, G$ and $H$. Attributes $B$ and $C$ are identical except for the graphite oxidation, as are $D$ and $E$, and $F$ and $G$. Events 33 and 61 determine the location of a hydrogen event, and 38 and 67 determine whether the event was a detonation or a deflagration. Events 37 and 66 determine whether the pipe gallery has experienced a hydrogen event, and 39 and 68 determine whether the EDT experienced a detonation or a deflagration. Events 46 and 74 determine whether the confinement failed as a result of the hydrogen events (some can occur without failing the confinement). Event 75 determines whether the confinement buildings were damaged at any point in the accident progression pathway, and Event 17 determines the initial state of the EDT. This information provides a guide to the logical structure below. Attributes $B, D$ and $F$ all have the form of (some hydrogen event) and (confinement building failure) and (graphite oxidation). Attributes $C, E$ and $G$ all have the same form without the graphite oxidation. Attribute $H$ is used whenever there is a confinement failure that does not occur in conjunction with a hydrogen or graphite oxidation event.

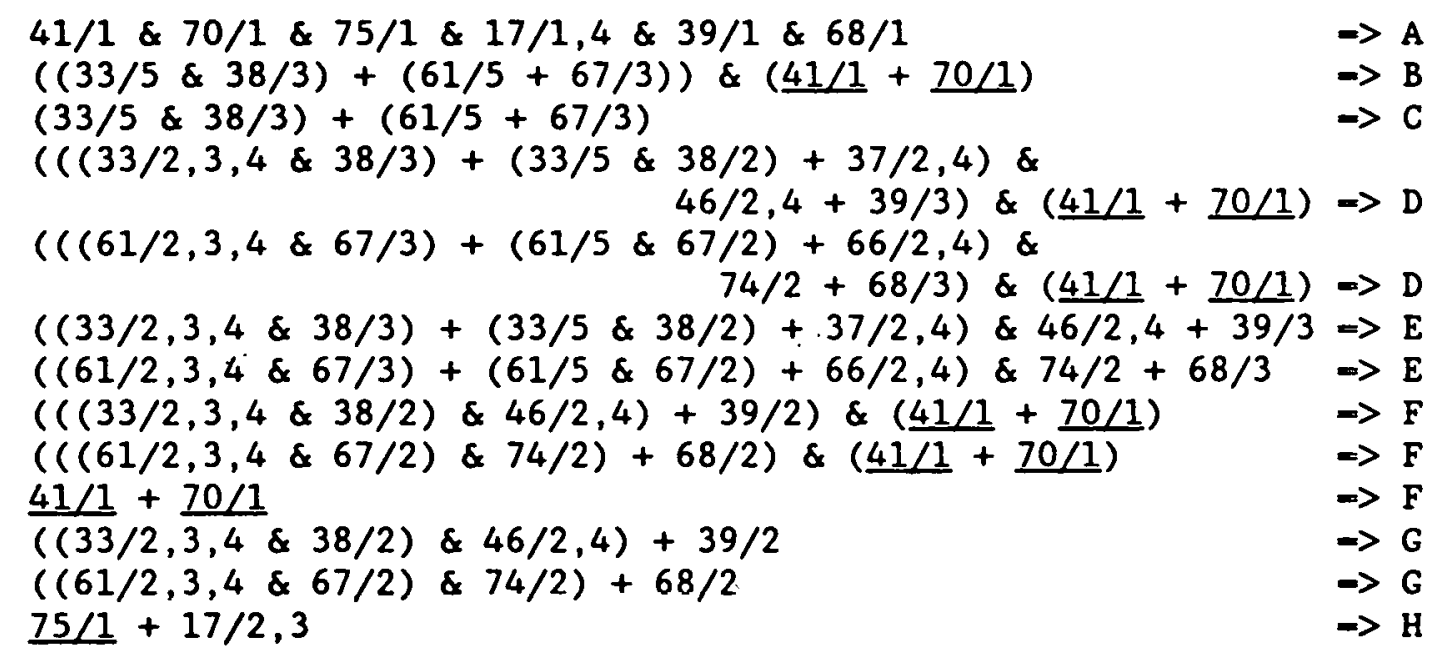

Dimension 10 - Time of Failure of the Confinement Function

This dimension is used to communicate the time of failure of the confinement function. The confinement function is different from what has been discussed previously in this chapter under the name "confinement". The failure of the confinement function, as used in this dimension, is defined to mean the failure of any structure (except the filters themselves) that is designed to prevent gaseous effluents from reaching the environment. This definition, then, includes both the confinement building and the EDT because failure of the EDT will allow effluents to pass directly from the core to the environment if any of the $V-4$ valves open as designed. The time of failure is important to the consequence model because it affects the amount of time available for warning and evacuating the public. A longer time between fuel damage and confinement failure allows more time for emergency response, so lower consequences would be expected. 
The letters before the attribute names are used throughout the source term and risk analyses in the tenth position to determine the value of this dimension. The dimension has four attributes:

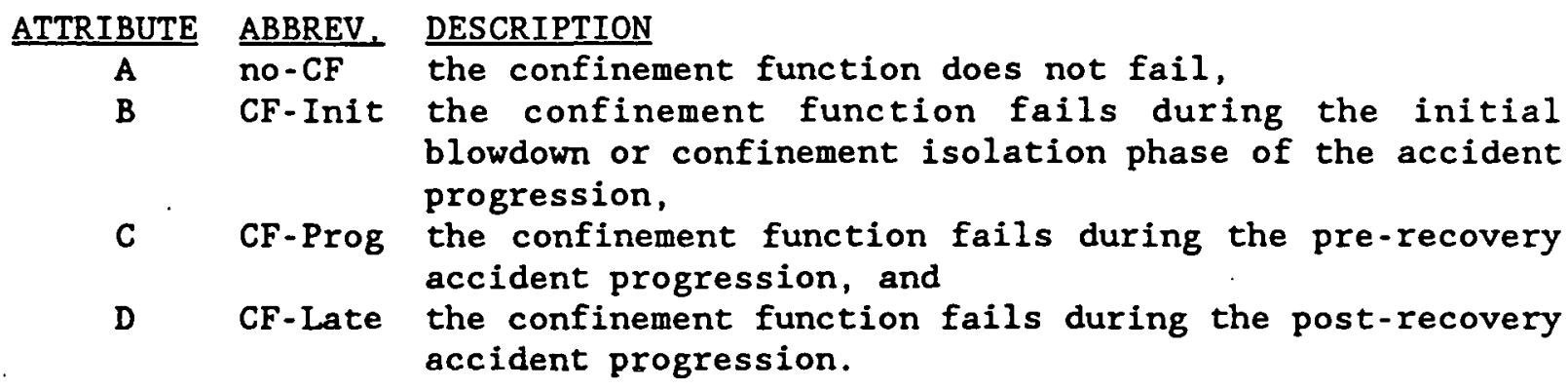

D CF-Late the confinement function fails during the post-recovery accident progression.

The pre- and post-recovery accident progression phases are discussed earlier in this chapter.

The above attributes are determined based on the events in the APET according to the following logical cases: the first case considers the initial EDT condition, the initial confinement compromise location, and any blowdown confinement failure. The second case considers pre-recovery confinement failure and EDT hydrogen events. The third case considers post-recovery confinement failure and EDT hydrogen events. The last case considers only accident progression pathways where the EDT and confinement remain intact throughout the accident progression.

$$
\begin{aligned}
\frac{20 / 1}{46 / 1}+\frac{28 / 1}{39 / 1}+17 / 2,3 & \rightarrow B \\
\frac{75 / 1}{75 / 1}+68 / 1 & \rightarrow C \\
& \rightarrow D \\
& \rightarrow \text { A }
\end{aligned}
$$

Dimension 11 - Release Location

Dimension 11 describes the location of the radioactive release from Lone I of the cunfinement building. The elevation and location of the release are important to the consequence calculations. A release from the top of the filter release stack lifts and disperses the release, while a release through other radiation protection zones of the plant allows additional time for decontamination by radionuclide condensation and settling.

The letters before the attribute names are used throughout the source term and risk analyses in the eleventh position to determine the value of this dimension. The dimension has three atributec: 


\section{ATTRIBUTE ABBREV, DESCRIPTION}

$A$ Stk-Rel any release occurs at the top of the filter release stack because no confinement failure occurred,

B L-Env a confinement failure occurred, and the release was directly to the environment at the elevation of the confinement building, and

C L-Zone a confinement failure occurred, but the release from Zone I goes through other zones of the confinement building before reaching the environment.

The above attributes are determined based on the events in the APET by directly mapping events 75 and 76 into the attributes of Dimension 11 .

$$
\begin{array}{ll}
75 / 1 & \rightarrow A \\
75 / 1 \& 76 / 2 & \rightarrow B \\
75 / 1 \& 76 / 3 & \rightarrow C
\end{array}
$$

\section{Dimension 12 - Liquid Effluent Status}

This dimension monitors the performance of the liquid effluent system. This system and its importance to the source term analysis are discussed in detail in Section 3.8 .

The letters before the attribute names are used throughout the source term and risk analyses in the twelfth position to determine the value of this dimension. The dimension has four attributes:

\section{ATTRIBUTE ABBREV, DESCRIPTION}

A LERF-Op the lift station and LERF all function as designed,

B L-River the lift station fails and causes the Zone I sumps to overflow through the fuel handling area and possibly to the river,

C L-1325 the automatic system failed to align the liquid system to the LERF, so radioactive water is being sent to the $1325 \mathrm{~N} L W D F$, and

D L-Air all portions of the liquid system succeeded, but the plastic LERF cover liner failed to remain intact.

The above attributes are determined based on the events in the APET according to the following logical cases: the first case is used for all accident progression pathways where the EDT has failed in some manner. If the EDT fails during the accident progression, then LERF failure is considered irrelevant because both have similar effects on the source term. The second case considers lift station failure, while the third considers failure to align the liquid effluent system from the LWDF to the LERF. The fourth case is used when failure of the LERF is the only liquid effluent system failure. The last case is used to model cases where the liquid effluent system operates as designed. 


$\begin{array}{ll}\frac{17 / 1}{79 / 2}+39 / 1+68 / 1 & \rightarrow \mathrm{A} \\ 80 / 2 & \rightarrow \mathrm{B} \\ 81 / 2 & \rightarrow \mathrm{C} \\ 80 / 1,3 & \rightarrow \mathrm{D} \\ & \rightarrow \mathrm{A}\end{array}$

Dimension 13 - Filter Stack Height

This dimension considers the condition of the filter release stack after a seismic event. Seismic activity can cause the filter release stack to be toppled. This typically occurs at a point approximately 608 up from the bottom. A toppling of the release stack would change the height at which effluents are released from the plant in cases where the confinement building remains intact, and this would have an impact on the consequence calculations by changing the dispersal and altitude of the release.

The letters before the attribute names are used throughout the source term and risk analyses in the thirteenth position to determine the value of this dimension. The dimension has two attributes:

\section{ATTRIBUTE ABBREV, DESCRIPTION \\ $A$ Stk-OK the filter release stack remains intact, and \\ B Sk-Fail the filter release stack is toppled.}

The above attributes are determined based on the events in the APET by directly mapping Event 18 into the proper attributes of Dimension 13.

$$
\begin{aligned}
& 18 / 3 \rightarrow A \\
& 18 / 3 \rightarrow B
\end{aligned}
$$

Dimension 14 - Total Fuel Damage

This dimension communicates the amount of "total" fuel damage to the source term model. Fuel damage is determined as the ratio of the fuel mass in elements in which the clad has falled to the total fuel mass initially in the core. Dimension 14 considers all fuel damage mechanisms that occur at any time during the accident progression. The impact of fuel damage information on the source term analysis is discussed under Dimension 2 above.

The letters before the attribute names are used throughout the source term and risk analyses in the last position to determine the value of this dimension. The dimension has eight attributes that represent "nominal" core damage states of: 


\begin{tabular}{cll} 
ATTRIBUTE & ABBREV & DESCRIPTION \\
\cline { 1 - 2 } A & O\&IFD & 08 total fuel damage, \\
B & $28 I F D$ & 28 total fuel damage, \\
C & 138 IFD & 138 total fuel damage, \\
D & 258 IFD & 258 total fuel damage, \\
E & $508 I F D$ & 508 total fuel damage, \\
F & $1008 I F D$ & 1008 total fuel damage, \\
G & ATWS-FD & ATWS fuel damage, and \\
H & $678 I F D$ & 678 total fuel damage.
\end{tabular}

These nominal fuel damage states are based on the status of the various core cooling systems. The nominal value for fuel damage during an ATWS accident sequence is 508 and is based on liquid levels calculated during Westinghouse Hanford's ATWS analysis [3-12], although a broad uncertainty range on this value was used for the analysis. The nominal fuel damage amounts described above are varied as uncertain parameters during the integrated uncertainty analysis. The source term analysis obtains the fuel damage uncertainty results by first reading the attributes for Dimensions 2 and 14 and then selecting from the Latin Hypercube sample vector the appropriate variables representing the actual amount of fuel damage for those attributes. in that sample member. In this way, the uncertainties associated with the amounts of initial and total fuel damage are propagated through to the consequence and risk analysis phases of the Level II/III PRA analysis.

The above attributes are determined based on the events in the APET by directly mapping Event 72 into the proper attributes of Dimension 14.

$$
\begin{aligned}
& 72 / 1 \rightarrow A \\
& 72 / 2 \rightarrow B \\
& 72 / 3 \rightarrow C \\
& 72 / 4 \rightarrow D \\
& 72 / 5 \rightarrow E \\
& 72 / 7 \rightarrow F \\
& 72 / 8 \rightarrow G \\
& 72 / 6 \rightarrow H
\end{aligned}
$$

The letters that represent the attributes for the 14 dimensions are combined in a 14 letter symbol for an accident progression bin. This symbol describes the progression of one unique (in the eyes of the $N$ Reactor source term model) N Reactor accident progression. Each character, in order, represents the value of one dimension. For example, ADBAFCAAAAABAD would be an accident progression with: 


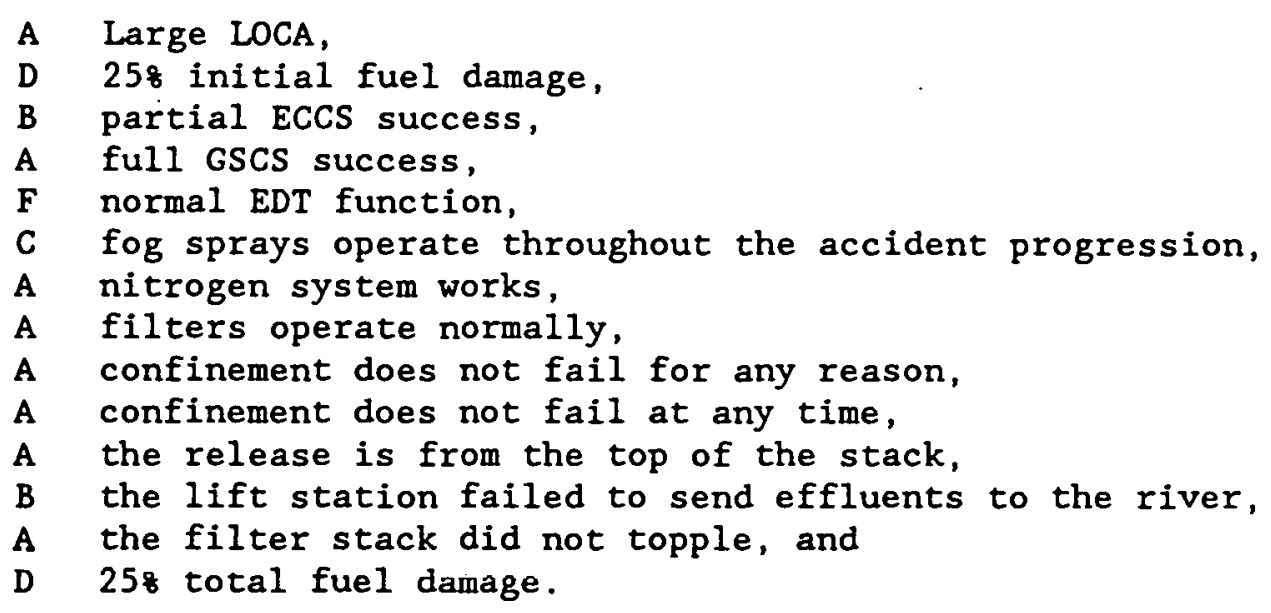

These 14 character descriptions appear throughout the tabular APET results in the appendices and are interpreted in all cases as above.

The above discussions summarize the dimensions and attributes used in the $N$ Reactor accident progression analysis and present some insight into why each is important either to the source term or to the consequence calculations, but it does not discuss the reasons for the overall structure of the binning system. The binning system must cover all aspects of the accident progression that are relevant to the source term and consequence calculations because it is the only information that is passed on from the APET to these analyses. It is useful, then to see how this binning structure follows the course of the accident progression to encompass all aspects of the accident progression.

An accident sequence begins with a core damage event that causes a release of radionuclides to occur. The radionuclides must first escape from the primary system, then traverse the confinement system and face various decontaminating influences before they can be released from the plant. An ordering for these events is presented in Table 3.2. An examination of the table shows the relationship between the binning structure and the important snurr.e term phenomena. This reveals much about the reasoning behind the selection of the particular binning structure and about how it encompasses the important source term and consequence issues.

In summary, the binning structure is important to the overall PRA analysis because it represents the only accident progression information that is maintained and passed on to the source term and consequence analyses. The binning structure for $\mathrm{N}$ Reactor was described and justified above, and found to adequately encompass the phenomena important to the source rerm and consequence calculations. The APFT, as described in Section 4.3, and the binning structure as described here, form the basis for the accident progression analysis for the N Reactor PRA. 
Table 3.2 Summary of the $N$ Reactor binning structure by comparison to important source term phenomena.

How many radionuclides are released from the fuel?

Determined by:

- Fraction of the core in which the fuel cladding fails (Dimensions 2, 14)

- Fuel temperature profile as determined principally by the status of the GSCS (Dimension 4)

How are the radionuclides released from the primary system to the confinement?

Determined by:

- Presence or absence of a LOCA or pressure tube rupture (Dimension 1)

- Availability of an ECCS or HPI water or steam source to drive the radionuclides out of the primary system (Dimension 3 )

How are the radionuclides scrubbed outside of the primary system before they are released to the environment?

Determined by:

- Availability of the EDT (Dimension 5)

- Action of the fog spray system (Dimension 6)

- Residence time in the confinement as determined mainly by the nitrogen purge system (Dimension 7)

- Residence time in other radiation protection zones (Dimension 11)

- Action of the filter system (Dimension 8)

How, where, when and with what energy does the release of radionuclides to the environment occur?

Determined by:

- Cause of confinement building failure (Dimension 9)

- Time of confinement building failure (Dimension 10)

- Location of release (Dimension 11)

- Height of the filter release stack (Dimension 13)

- Release pathway for liquids to the environment from the EDT (Dimension 5) or the LERF (Dimension 12) 


\subsection{SUMMARY}

The preceding descriptions of the events in the $\mathrm{N}$ Reactor APET make it clear that the APET is truly a distillation of a vast amount of data into a usable, discrete logical structure. The data comes from the Level I PRA, the external events core damage analysis, the confinement systems fault tree analysis, the hydrogen mitigation fault tree analysis, and uncertainty analyses as presented in Appendix $C$. The APET determines the complete progression of any $N$ Reactor accident starting with a description of the condition of the primary and confinement systems and progressing through blowdown, early phenomenology, recovery actions, late phenomenology, and the primary and confinement system responses to the phenomenology and recovery actions. The binning process takes the detailed accident progression and provides an overview or summary in the form of an accident progression bin for use by the source term analysis.

It is also easy to see how the number of possible paths through the APET can number in the multiple thousands, and even the millions when uncertainty is calculated. There is always a desire to model systems and phenomena in more detail to glean a finer picture of the accident progression, but this must be balanced against the effort that would be involved in evaluating an even more detailed event tree, because adding even a simple, two branch event can double the number of paths through the APET (and, hence, the computational effort required to solve it). The APET is not designed to present a detailed analysis of every possible accident progression pathway, but rather to show the relative frequencies of many different of accident progression pathways while maintaining as much detail as is necessary to preserve the realism of the analysis.

The goal, then, is to provide a basic analysis for all types of accident sequences that the reactor could experience and to use that information both to calculate risk and to make informed decisions about which hardware and procedural modifications would provide the greatest safety improvement given the resources available. The APET described in this chapter provides the framework for that analysis. 


\subsection{REFERENCES}

3-1. UNC Nuclear Industries, Inc., N Reactor Updated Safety Analysis Report (NUSAR), WHC-SP-0297, February 1978 .

3-2. J. A. Lambright, et al., Analysis of Core Damage Frequency due to External Events at the DOE $N$ Reactor, SAND89-1147, Sandia National Laboratories, 1990.

3-3. J. M. Griesmeyer and L. N. Smith, A Reference Manual for the Event Progression and Analysis Code (EVNTRE), NUREG/CR-5174, SAND88-1607, Sandia National Laboratories, September 1989.

3-4. R. L. Iman and M. J. Shortencarier, A User's Guide for the Top Event Matrix Analysis Code (TEMAC), NUREG/CR-4598, SAND86-0960, Sandia National Laboratories, August 1986.

3-5. R. L. Iman, Memorandum to File, "Modifications to TEMAC," Sandia National Laboratories, January 17, 1989.

3-6. M. D. Zentner, et al., N Reactor Level 1 Probabilistic Risk Assessment: Final Report, WHC-EP-0322, Westinghouse Hanford, August 1988 .

3-7. D. M. Kuñsman, et al., Level III Probabilistic Risk Assessment for $\mathrm{N}$ Reactor, WHC-MR-0045, SAND89-2102, Sandia National Laboratories, April 1990.

3-8. C. J. Everline, C. F. Dahms, and M. B. Richards, Prohahilistir Failure Assessment of the N Reactor Pressure Tubes - Phase IV Report, SD-NR-ER-043, Westinghouse Hanford Co., March 1989.

3-9. L. A. Miller, et al, N Reactor Probabilistic Risk Assessment Supporting Calculations, SAND89-2101, Sandia National Laboratories, 1990.

3-10. United Nuclear Industries, Inc., "ECS Actuation During Normal Operation," Emergency Response Guides, WHC-CM-4-1, Vol. 2, Guide No. B, Issued May 12, 1987, as updated through June 1989.

3-11. G. D. Wyss, "N-Reactor Core (CRN) Package Users' Guide," Draft Version 1.8XA, Sandia National Laboratories, March 17, 1989.

3-12. K. Sathyanarayana and D. M. Ogden, Analysis of $N$ Reactor Anticipated Transients Without SCRAM, WHC-SP-0457, Westinghouse Hanford, March 1989 .

3-13. G. A. Coles, N Reactor Confinement Systems Sequence Analysis Including Recovery, WHC-SD-NR-RA-001, Westinghouse Hanford, March 1990 . 
3-14. J. E. Kelly, "Advances in MELCOR Modeling and Analysis," Transactions of the 16th Water Reactor Safety Information Meeting, NUREG/CP-0096, U.S. Nuclear Regulatory Commission, Gaithersburg, Maryland, October $24-27,1988$.

3-15. Westinghouse Hanford Co., $N$ Reactor Process Standards, WHC-CM-6-12, February 1982, as updated through June 1989.

3-16. J. Clark, "Hanford Action Item Status after Trip," memo to A. Kolaczkowski, Science Applications International Corp., March 17, 1988 .

3-17. G. A. Coles, "Effect of Fire Sequences on Confinement System for $N$ Reactor Level 2 Probabilistic Risk Assessment," Letter to D. M. Kunsman and G. D. Wyss, WHC Correspondence \#8953739, Westinghouse Hanford Co., June 21, 1989.

3-18. UNC Nuclear Industries, NReactor Plant Manual, UNI-M-94, March 1979, as updated through June 1989.

3-19. United Nuclear Industries, Inc., "Zone I Confinement Trip," Emergency Response Guides, WHC-CM-4-1, Vol. 2, Guide No. L, Issued May 12, 1987, as updated through June 1989.

3-20. S. E. Lindberg, Hydrogen Mitigation System Risk Analysis, WHC-SD-NR-RA-002, Westinghouse Hanford, October 1988.

3-21. A. L. Camp and S. E. Dingman, HECTR Version 1,5N-A Modification of HECTR Version 1.5 for Application to N Reactor, SAND87-0027, Sandia National Laboratories, May 1987.

3-22. L. A. Miller, Sandia National Laboratories, letter to Sam Wood, Westinghouse Hanford Co., subject: "HECTR Version 1.51N," February 17,1988 .

3-23. A. C. Payne, Jr. and A. L. Camp, Parametric HECTR Calculations of Hydrogen Transport and Combustion at $N$ Reactor, SAND86-2630, Sandia National Laboratories, June 1987.

3-24. J. T. Long, "Review of $N$ Reactor Level 2/3 Probabilistic Risk Assessment Initial Point Estimate of Risk," memo to E. J. Krejci, Westinghouse Hanford Co., March 22, 1989.

3-25. M. B. Richards and A. W. Barsell, Catalysis Effects by Impurities on the Steam-Graphite Reaction under $\mathrm{N}$ Reactor Accident Conditions, GA-C19161, Rev. 1, General Atomics Corp., April 1988.

3-26. P. W. Baranowshy, Evaluation of Station Blackout Accidents at Nuclear Power Plants, NUREG-1032, U. S. Nuclear Regulatory Commission, June 1988 . 
3-27. F. J. Heard, et al., $N$ Reactor Safety Enhancement Final Report: Hydrogen Generation and Thermal Analysis of the Hydrogen Mitigation Design Basis Accident, WHC-SP-0096, Westinghouse Hanford, December 1987.

3-28. M. J. Thurgood, An Evaluation of Condensation Induced Water Hammer During ECCS Injection in N-Reactor, NAI-8708-1, Numerical Applications, Inc., November 1987.

3-29. K. R: Birney, "Pressure Tube Considerations Relating to Molten Uranium," memo to D. M. Ogden dated January 7, 1987, Westinghouse Hanford, formerly UNC Nuclear Industries.

3-30. E. H. Randlev, et al., Molten Uranium/Zircaloy Interaction Studies, WHC-SP-0338, Westinghouse Hanford Co., August 1988.

3-31. Safety Issues at the Defense Production Reactors: A Report to the Department of Energy, National Academy of Sciences, National Academy Press, Washington, D.C., 1987.

3-32. R. A. Paasch, et al., Project $H-797 \ldots N$ Reactor 1312-N Liquid Effluent Retention Facility, WHC-SP-0221, Westinghouse Hanford, January 1988.

3-33. L. D. Muhlestein, D. W. Jeppson, J. D. McCormack, N Reactor Filter System Fission-Product Retention Assessment, WHC-SP-0233, Westinghouse Hanford Co., June 1988. 


\section{INCORPORATION OF UNCERTAINTY}

This chapter describes how uncertainties were incorporated into the analysis of the accident progression event tree (APET). The overall uncertainty analysis method used throughout the $\mathrm{N}$ Reactor Level II/III PRA uses a stratified Monte Carlo sampling technique known as Latin Hypercube Sampling (LHS) that is discussed in Reference 4-1. The uncertainty is represented by the sample members of the various distributions and propagated through the entire PRA: Obviously to perform an uncertainty analysis, it is necessary to define probability distributions to be sampled.

The APET analysis uses four sources of uncertainty information: internally initiated primary system response data from the Internal Events Level I PRA [4-2], fire and seismically initiated primary system response data from the External Events Level I PRA [4-3], confinement system response from the Confinement Systems Fault Tree Analysis [4-4, 4-5], and phenomenological uncertainty. The first three sources of data and their incorporation into the analysis are discussed in the first two sections. The third section describes the phenomenological uncertainty analysis for the APET portion of the Level II/III PRA analysis.

\subsection{INTERFACE WITH SYSTEMS ANALYSIS}

The modeling of core damage in the Level I portion of the PRA forms an important part of the analysis of risk. It is at this stage that the various accidents which could lead to core damage and to release of radionuclides are defined and quantified, and it is these accident sequences for which the APET must be exercised to identify the potential accident progression pathways they have for eventual source term analysis. This section describes the process by which the quantification of the Level I uncertainty analysis was linked to the APET uncertainty analysis.

\subsubsection{Introduction}

As mentioned above, two Level I PRA analyses of core damage were performed for $N$ reactor, internal initiators [4-2], and external initiators [4-3]. Those sequences with dominant core damage frequencies or high consequence potentials from each of these analyses formed initial conditions for the Level III risk analysis [4-1]. The APET and source term analyses were conducted within the context of these sequences (e.g., status of confinement cooling and filter systems, timing of core damage, status of the core and primary cooling systems). The frequencies of these sequences and their probabilistic uncertainties were incorporated into the APET. 


\subsubsection{Level I - Internal Event Systems Analysis}

Table 4.1 contains a list of the accident sequences identified in the N Reactor Level I PRA for internal events [4-2] which were included in the APET analysis. All sequences with point estimate frequencies from the Level I PRA of greater than $1.0 \times 10^{-7} / \mathrm{yr}$ (Table 9-2 of Reference 4-2) and those sequences with lesser frequencies but high consequence potential were selected for inclusion in the analysis. The high consequence, low frequency criterion was any sequence in plant damage state bin $Z$ ( 1008 core damage; see Reference 4-2 for an explanation of the damage state bins and sequence notation). The exceptions to this criterion were sequences $L 7$ and L8, which were excluded because of their extremely low frequencies (less than $1.0 \times 10^{-10} / \mathrm{yr}$. ).

\subsubsection{Level I - External Event Analysis}

Table 4.2 contains a list of the fire sequences included in the APET analysis. All of the sequences from the $\mathrm{N}$ Reactor fire Level I study were included [4-3]. The seismic analysis had to be slightly modified for the APET analysis [4-6]. The dominant sequences in the seismic Level I analysis were not defined in sufficiently specific terms with respect to the various system failures considered in the APET analysis. For this reason, the seismic sequences were redefined so that the status of specific systems for each sequence were known. The result was an expansion of 10 sequences into 32 . The results of this are shown in Tables 4.3 and 4.4.

The size of the fire and seismic sequences (in terms of number of cut sets, number of basic events, and distributions) was very small compared to the internal event. PRA sequences. Thus, there was no need (based on the number of cut sets) to screen any of the sequences based on low frequency or risk potential. However, the computational complexity of the seismic APET analysis had to be reduced because of the extremely large number of accident progression pathways associated with not only the 32 accident sequences, but also the consideration of seismic failure of the emergency dump tank, the hydrogen mitigation system nitrogen tanks and the filter release stack. For this reason, all $1 / 16$ core accidents were dropped from the seismic analysis before the 32 seismic accident sequences were defined. This has an extremely small impact on the overall results because a large fraction of the overall seismic core damage frequency is related to GSCS failure events while the $1 / 16$ core accidents make up less than 28 of the overall core damage frequency and have been shown through the internal events analysis to have an extremely low risk potential.

None of the 32 full core seismic accident sequences were dropped from consideration in the LHS sampling analysis even though several of them show extremely low frequencies. However, when split fractions were generated in the TEMAC analysis, some of the lowest frequency sequences were combined with similar, higher frequéncy sequences to simplify the analysis. 
Table 4.1. N Reactor Level I Dominant Sequences - Internal Event*

Sequences

$\begin{array}{ll}\text { L6 } & 1.03 \mathrm{E}-08 \mathrm{t} \\ \mathrm{L} 2 & 2.36 \mathrm{E}-08\end{array}$

Frequency $\left(Y_{Y^{-1}}\right)$

Mean 95 th

\section{$6.15 E-06$}

1. $90 \mathrm{E}-06$

1. $19 \mathrm{E}-07$

$7.16 \mathrm{E}-06$
$7.42 \mathrm{E}-06$
$2.28 \mathrm{E}-07$

2.94E-05

1. 35E-05

$6.24 \mathrm{E}-06$

8. 22E-07

$6.74 \mathrm{E}-07$

4. 36E- 07

1.72E-07

9. $20 \mathrm{E}-05$

5.10E-05

3. $09 \mathrm{E}-06$

1. 78E-06

1. $21 \mathrm{E}-06$

2. $54 \mathrm{E}-07$

9. $62 \mathrm{E}-08$

$7.17 \mathrm{E}-08$

2. $12 \mathrm{E}-09$

$3.47 \mathrm{E}-09$

1. 15E-06

2. $02 \mathrm{E}-07$
$5.48 \mathrm{E}-05$

5.65E-05

1. $91 \mathrm{E}-05$

2.17E-06

1.81E-06

1.15E-06

8.58E-07

1. $25 \mathrm{E}-04$

1. $77 \mathrm{E}-04$

$1.08 \mathrm{E}-05$

6.67E-06

$3.57 \mathrm{E}-06$

4. $57 \mathrm{E}-07$

2. $65 \mathrm{E}-07$

1. $93 \mathrm{E}-07$

$1.94 \mathrm{E}-06$

4. $23 \mathrm{E}-08$
Original Level I

8.00E-07

9.10E-07

$1.63 \mathrm{E}-08$

6.60E-06

$7.60 \mathrm{E}-06$

$1.40 \mathrm{E}-06$

$1.70 \mathrm{E}-07$

$1.30 \mathrm{E}-07$

1. $10 \mathrm{E}-07$

8.00E-08

2.00E- 05

$2.50 \mathrm{E}-05$

$7.67 \mathrm{E}-07$

4. $00 \mathrm{E}-07$

$3.70 \mathrm{E}-07$

4. $27 \mathrm{E}-08$

$2.45 \mathrm{E}-08$

3. $21 \mathrm{E}-08$

$2.90 \mathrm{E}-7$

4. $23 \mathrm{E}-8$

\footnotetext{
* The frequencies reported in the original internal events Level I report [3-1] are point estimates.
} $t 1.03 E-08=1.03 \times 10^{-0 B}$ 
Table 4.2 N Reactor Dominant Fire Area

Core Damage Sequences and Uncertainties

Sequence

Corridor 19 and B1dg. 107 Basement

Main Control Room

Bldg. 105, Corridor 1

Bldg. 184, Inst. Air

Compressor Cable Runs

Bldg. 151, Switchgear

Bldg. 105, Room 6

Bldg. 105, Room 8

Bldg. 105, Room 9

Bldg. 153, Main Floor

"B" Switchgear

Bldg. 153, Main Floor

"A" Switchgear

Bldg. 184, Inst. Air

Compressor Area

Control Room Bldg. 182-N

Cell Access Corridor

Bldg. 184, Control Room

Outlet Piping Area

$\overline{1.8 \mathrm{E}-06}=1.8 \times 10^{-6}$
Frequency $\left(\mathrm{Yr}^{-1}\right)$

$\begin{array}{clll}5 \text { th } & 50 \text { th } & 95 \text { th } & \text { Mean } \\ 1.8 E-06 * & 6.9 E-06 & 2.7 E-05 & 9.7 E-06\end{array}$

$5.6 \mathrm{E}-10$

2.8E-06

2. 5E-05

$6.5 E-06$

1. 4E-08

8. 7E-08

$6.7 E-07$

$1.8 \mathrm{E}-07$

2. $3 E-08$

3.4E-07

5. 3E-06

1. 3E-06

$3.4 E-10$

1. $7 \mathrm{E}-07$

2. $9 \mathrm{E}-06$

8.0E-07

2. OE-08

2. OE-07

2. 4E-06

$6.5 E-07$

1. $2 E-10$

4. 7E- 08

6.1E-07

$1.6 \mathrm{E}-07$

2. OE-11

3. $6 E-08$

$6.9 \mathrm{E}-07$

$1.6 \mathrm{E}-07$

8. $2 E-11$

3. 1E- 08

6. OE- 07

1. 5E-07

3. $8 \mathrm{E}-11$

$1.4 \mathrm{E}-08$

2. 5E-07

6. $0 \mathrm{E}-08$

4. OE- 10

7. OE-09

1. $2 \mathrm{E}-07$

3. $2 E-08$

$5.0 E-13$

3. 2E-09

1. OE- 07

2. 8E-08

3. $7 \mathrm{E}-10$

5. 2E-09

8. 7 E-08

2. 2E-08

$3.4 E-13$

2.1E-09

8. $6 \mathrm{E}-08$

2. 1E-08

$6.4 \mathrm{E}-11$

3. 5E-09

$6.1 E-08$

$1.6 E-08$ 
Table 4.3 N Reactor Dominant Seismic Core Damage Sequences

Level I Sequences Redefined Sequences

Multiple Pressure

-ube Rupture (MPTR-

See Section 4.6.3)

Large LOCA L6**

$\stackrel{\ddagger}{\vdots}$

Large LOCA L7

Small LOCA S9

ECCS Gystem Status

$\begin{array}{lll}\text { Fail } & \text { Fail } & \text { None } \\ \text { Fail } & \text { Fail } & \text { A Only } \\ \text { Fail } & \text { Fail } & \text { None }\end{array}$

Fail Fail A Only

Fail OK

Fail OK

Fail OK

Fail OK

Fail

Fail

Fail

Fail

Fail

Fail

Fail

Fail

Fai1

Fail

Fail

Fail

\section{None}

A Only

None

A Only

Fa11

Fail

Fail

Fail

OK

OK

OK

OK

Fail

Fail

Fail

Fail
None

A Only

None

A Only

None

A Only

B Only

OK

\section{None}

A Only

B Only

OK

Fail
Fail
OK
OK

OK

Fat1

Fail

OK

OK

Fa11

Fal1

OK

$\mathrm{OK}$

\section{OK}

$\mathrm{OK}$

OK

OK

OK

OK

OK

OK
Mean Frequency $\left(\mathrm{Yr}^{-1}\right)$
$7.87 \mathrm{E}-07 *$
3. $94 \mathrm{E}-11$
7. $06 \mathrm{E}-06$
$4.50 \mathrm{E}-08$
3. $13 \mathrm{E}-10$
3. $65 \mathrm{E}-13$
2. $84 \mathrm{E}-08$
$3.65 \mathrm{E}-13$
$5.46 \mathrm{E}-10$
3. $63 \mathrm{E}-13$
5. $21 \mathrm{E}-09$
3. $66 \mathrm{E}-13$
1. $50 \mathrm{E}-06$
1. $93 \mathrm{E}-08$
2. $36 \mathrm{E}-10$
4. $34 \mathrm{E}-10$
2. $02 \mathrm{E}-06$
2. $28 \mathrm{E}-08$
$2.08 \mathrm{E}-09$

1. $80 \mathrm{E}-08$

\footnotetext{
$* 7.87$ E-07 $=7.87 \times 10^{-7}$

** Sequences L6, L7, S8, S9, T8, and T9 are defined in Reference 3-2 and are identical to their definitions in the Level I PRA [3-1].
} 
Table 4.3 N Reactor Dominant Seismic Core Damage Sequences (Cont.)

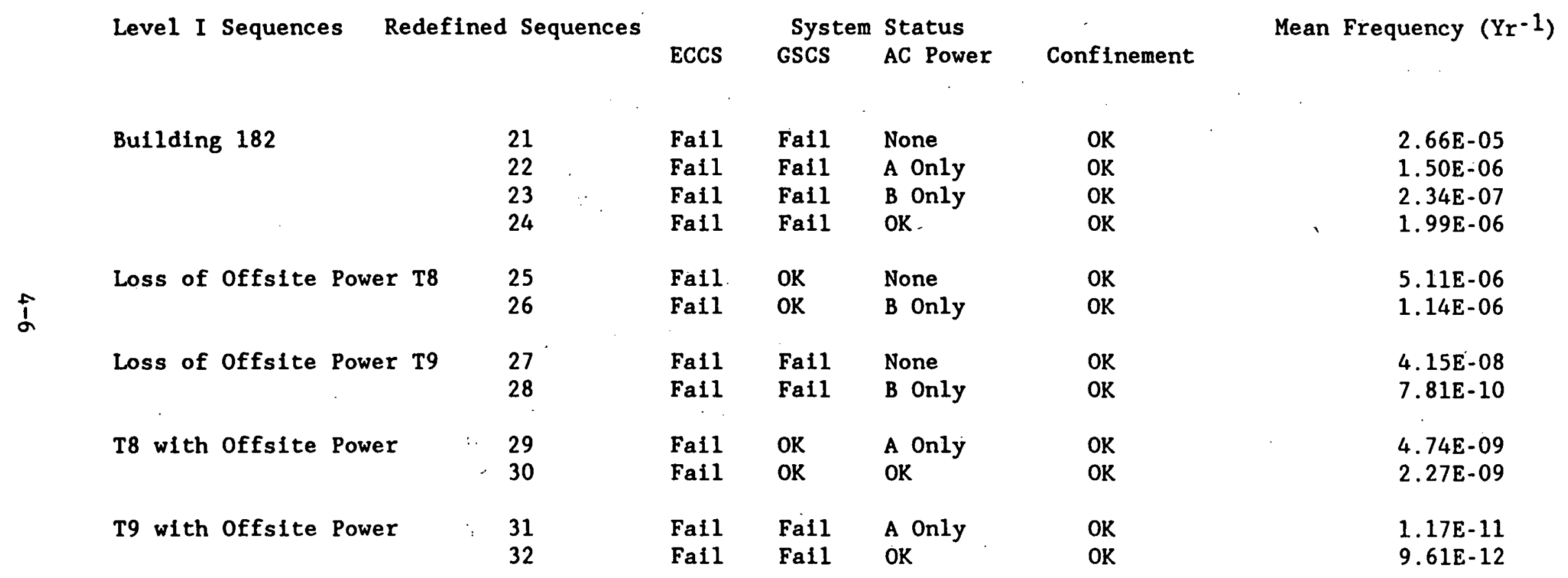


Tatle 4.4 N Reactor Dominant Seismic Core Damage Frequencies and Uncertainties

\section{Sequence}

Level I Sequences

Multiple Pressure

Tube Rupture (MPTR -

See Section 4.6.3)

Large LOCA L6**

Large LOCA L7

I

Sna11 LOCA S8

Sma11 LOCA S9
Redefined Sequences

5 th

$$
\begin{aligned}
& 0 \\
& 3.19 \mathrm{E}-15 \\
& 0 \\
& 3.06 \mathrm{E}-14
\end{aligned}
$$

0

$2.79 E-15$

0

2.79E-15

0

$2.79 \mathrm{E}-15$

0

$2.79 \mathrm{E}-15$

$2.77 \mathrm{E}-11$

9. $86 \mathrm{E}-13$

$7.45 \mathrm{E}-15$

8. $23 \mathrm{E}-15$

$1.71 \mathrm{E}-10$

$4.36 \mathrm{E}-13$

$4.49 \mathrm{E}-14$

$2.01 E-13$

Frequency $\left(\mathrm{Yr}^{-1}\right)$

50 th

8.07E-09

$1.01 \mathrm{E}-13$

$2.61 \mathrm{E}-07$

$4.66 \mathrm{E}-11$

1. $83 \mathrm{E}-13$

$6.21 \mathrm{E}-14$

$6.75 \mathrm{E}-13$

$6.21 \mathrm{E}-14$

2. $13 \mathrm{E}-13$

$6.21 \mathrm{E}-14$

9. $42 \mathrm{E}-13$

$6.21 \mathrm{E}-14$

4. $78 \mathrm{E}-08$

2. $13 \mathrm{E}-10$

4. $55 \mathrm{E}-13$

8. $51 \mathrm{E}-13$

1. 08E-07

$2.95 \mathrm{E}-10$

1. $78 \mathrm{E}-11$

$1.57 \mathrm{E}-10$ 95th

$1.93 \mathrm{E}-06$

1. $32 \mathrm{E}-11$

2. $92 \mathrm{E}-05$

5. $78 \mathrm{E}-08$

2. $23 E-10$

1. $44 \mathrm{E}-12$

2. $10 \mathrm{E}-09$

1. $44 \mathrm{E}-12$

3. $38 \mathrm{E}-10$

$1.44 \mathrm{E}-12$

2. $68 \mathrm{E}-09$

$1.44 \mathrm{E}-12$

2. $98 \mathrm{E}-06$

2. $40 \mathrm{E}-08$

$1.07 \mathrm{E}-10$

4. $08 \mathrm{E}-10$

5. 82E-06

$5.74 \mathrm{E}-08$

4. 81E-09

$4.27 \mathrm{E}-08$
Mean

7. $87 \mathrm{E}-07 *$

3. $94 \mathrm{E}-11$

$7.06 \mathrm{E}-06$

4. $50 \mathrm{E}-08$

3. $13 \mathrm{E}-10$

3. $65 \mathrm{E}-13$

2. $84 \mathrm{E}-08$

3. $65 \mathrm{E}-13$

$5.46 \mathrm{E}-10$

3. $63 \mathrm{E}-13$

5. 21E-09

3. $66 \mathrm{E}-13$

1. 50E-06

1. $93 \mathrm{E}-08$

2. $36 \mathrm{E}-10$

4. $31 \mathrm{E}-10$

2.02E-06

2. $22 \mathrm{E}-08$

2.08E-09

1. $80 \mathrm{E}-08$

\footnotetext{
$\star 7.87 \mathrm{E}-07=7.87 \times 10^{-7}$
}

** Sequences L6, L7, S8, S9, T8, and T9 are defined in Reference 3-2 and are identical to their definitions in the Level I PRA [3-1]. 
Table 4.4 N Reactor Dominant Seismic Core Damage Frequencies and Uncertainties (Cont.)

\section{Sequence}

Level I Sequences

Building 182

Loss of offsite

$\hat{j}$
Redefined Sequences

21

22

23

24

25

26

Loss of Offisite Power T9

T8 with offsite Power

29

30

T9 with offsite Power

31

\section{Frequency $\left(\mathrm{Yr}^{-1}\right)$}

50 th

95th

Mean

$\begin{array}{lll}2.14 \mathrm{E}-06 & 9.59 \mathrm{E}-05 & 2.66 \mathrm{E}-05 \\ 4.44 \mathrm{E}-08 & 5.66 \mathrm{E}-06 & 1.50 \mathrm{E}-06 \\ 4.60 \mathrm{E}-09 & 9.68 \mathrm{E}-07 & 2.34 \mathrm{E}-07 \\ 4.86 \mathrm{E}-08 & 7.20 \mathrm{E}-06 & 1.99 \mathrm{E}-06\end{array}$

$7.67 \mathrm{E}-09$

3. $83 \mathrm{E}-09$

2.93E-07

1. 10E- 07

1.32E-05

3. $68 \mathrm{E}-06$

5.11E-06

1.14E-06

\section{$1.51 E-12$}

4.58E-14

\section{$7.54 \mathrm{E}-10$}

6. $91 \mathrm{E}-12$

6.70E-08

4. 15E-08

$7.81 \mathrm{E}-10$

$1.49 \mathrm{E}-13$

2. $16 \mathrm{E}-11$

1. $38 \mathrm{E}-11$

4.13E-09

1. $96 \mathrm{E}-09$

4.74E-09

2. 27E-09

3. $35 \mathrm{E}-15$

3. $29 \mathrm{E}-15$

1. $22 \mathrm{E}-13$

8. $26 \mathrm{E}-12$

3. $77 \mathrm{E}-12$

1. $17 \mathrm{E}-11$

9. $61 \mathrm{E}-12$ 


\subsubsection{Level I - Confinement Systems}

A systems analysis was performed on the $\mathrm{N}$ Reactor confinement systems to quantify various combinations of confinement system failures. The analysis [4-4] developed "confinement sequence" equations which were conditional on the occurrence of the core damage sequences. These sequence equations contained only demand and time related failures. No initiating event frequencies or core damage events were included. These sequences were quantified separately from the Level I accident sequences. This analysis of the confinement systems, performed by WHC personnel, assessed the failure probability for the various confinement systems given that a demand for their operation had occurred. The confinement system fault trees were solved first for individual systems, then re-solved for multiple combinations of systems to account for any common support or actuation components between systems. The work was revised and expanded for the external events analyses $[4-5,4-6]$.

\subsection{UNCERTAINTY ANALYSIS INTERFACE}

The Level III PRA incorporates an integrated uncertainty analysis in the estimate of risk. As discussed in the Level III PRA report [4-1], an integrated uncertainty analysis ensures that the uncertainty from the various segments of the analysis - core damage, accident progression, and source term - are propagated through the modeling process so that the uncertainty of the estimate of risk reflects the uncertainty of all aspects of the analysis.

A stratified Monte Carlo sampling method, Latin Hypercube Sampling (LHS) [4-7], was the method used to sample the probability distributions for all of the model parameters for which uncertainty existed. The parameter distributions from the various segments of the analysis were sampled in a single LHS run. This LHS matrix of parameter observations was used as an input to several stages of the analysis. It is fundamental to the LHS method that all parameter samples are drawn in a single run to ensure that only desired conrrelations exist in the matrix of observations. Thus, separate LHS sampling for the Level I basic events, the APET parameters, the source term variables, etc. was not done. The result was that a single LHS matrix with 262 positions (variables) from the Level I analyses (from both the Internal and External analyses) and the Level II/III analyses was generated.

\subsubsection{Internal Event Uncertainty Analysis}

An uncertainty quantification was done for each Level I accident sequence equation. The results of this quantification contained a probability distribution of the frequency of each sequence. The probability distributions characterize the uncertainty of the frequency estimates which is derived from the individual probability models of the basic events. The mean, 5 th, and 95 th quantiles for each sequence frequency distribution are shown on Table 4.1 . 
The uncertainty quantification is developed by defining a probability distribution for each basic event and initiating event in the sequence models. Many basic events can be modeled by the exact same random variable. For example, there may be several events such as "MOV XX fails to open." If the data used to develop the distribution for this type of fault comes from a body of data which includes experience from all such components, then all "MOV fails to open" events are modeled with the same distribution. The sampling of each of these events in the LHS run would be correlated so that all such events would have the exact same sample. The correlation of basic event uncertainty sampling to probability distributions was the same as was done in the original Level: I PRA.

Once distributions and correlations had been defined, the LHS code [4-7] was used to draw observations from each distribution. The resulting LHS matrix of random variable observations was used along with the accident sequence equations as input to TEMAC $[4-8,4-9]$. The Top Event Matrix Analysis Code (TEMAC) is used in conjunction with the LHS code to quantify the accident sequence models and to generate various calculations needed in the Level II/III analyses. TEMAC produces a sample of the frequency distributions for the following:

- Total core damage frequency,

- Individual sequence frequencies,

- Split fraction frequencies.

The uncertainty analysis for the confinement sequences was performed in the same manner as the uncertainty analysis for the Level I sequence equations.

\subsubsection{Propagation of Sequence Frequencies and Split Fractions}

The accident sequence models produced by a Level $I$ analysis quite literally form the starting point for the accident progression analysis. The sequence models have estimated frequencies of occurrence which provide a probabilistic measure upon which Level II/III quantification is started. The Level I sequence models also define much of the very structure of the Level II/III models. The sequence equations represent combinations of events which establish the status of the accident mitigation systems at $N$ Reactor and the conditions which must be responded to. Much of the structure of the APET analysis (and source term analysis as well) is shaped specifically to address the Level I events.

The integrated risk analysis approach links the entire PRA process together, from the Level I accident analysis to the Level III consequence and risk analysis. As discussed above, the results of the Level $I$ analysis define much of the structure of the Level II/III analyses. Thus, the characteristics of the Level I results are incorporated into those analyses when the APET and source term models are developed. However, the quantitative uncertainty of the Level I analysis must be incorporated into the integrated analysis of risk as well. This is done by sampling the probability distributions of the sequence frequencies and other Level I quantities which are relevant to the Level II/III analyses. These samples 
are passed as input to the models used in the Level II/III analyses. In the case of the APET analysis, the split fractions from the Level I PRA as well as the confinement systems fault tree analysis are passed directly as input to the EVNTRE code $[4-10]$.

In the integrated risk uncertainty method employed in this PRA, as described in Reference 4-1, the total core damage and individual sequence frequency samples are passed on to the risk portions of the Level II/III analyses, and the split fraction frequencies are used as input by the APET. A split fraction is a ratio of the frequencies of two groups of cut sets. The ratio is usually, but not exclusively, defined in such a way that the cut sets which form the numerator are a subset of the cut sets of the denominator. An example of a split fraction which is needed in the APET is the ratio of the ATWS sequence frequencies to the total core damage frequency. An example of a split fraction for a confinement component where the numerator cut sets are not a subset of those that form the denominator is the ratio of all cut sets in which a particular electrical power bus has failed to the probability that the particular electrical bus will fail. This represents the probability that the confinement component will fail given that the particular electrical bus has failed.

The split fractions of interest are defined from the questions asked in the APET. The value of a split fraction represents the probability that an accident progresses down a specific branch of the APET. TEMAC generates a sample of $\mathrm{N}$ observations for each split fraction and sequence (500 observations were generated for this analysis). In this fashion, the uncertainty of the Level $I$ analysis is passed on to the accident progression and other portions of the Level II/III analysis.

TEMAC creates an output file which contains the statistical samples for all of the accident sequences and related split fractions needed in the APET analysis. As stated above, this output file is used as an input file by EVNTRE. The cut sets of the denominators of the split fractions usually are grouped into accident sequence blocks (e.g., all LOSP sequences, all transients, and the entire core damage model). Examples of split fractions defined with respect to total core damage frequency in the APET analysis are :

- Failure to SCRAM

- Specific failure modes of ECCS

- Specific failure modes of GSCS

- Specific type of fire or seismic event

- Failure of confinement isolation

- Status of electrical power

- Initiator (Large LOCA, Small LOCA transient)

- Liquid Effluent System alignment

- Steam Vent failure

The systems and events represented in the definitions of the split fractions is indicative of the significant impact of the Level I analysis on the APET analysis. The total core damage frequency, which forms the denominator in these split fractions, is the total frequency of all core 
damage events analyzed in a particular event tree analysis, and not the total over all such analyses.

The sequence and split fraction quantification was done in two parts. A separate quantification was done on the internal events analysis, while the fire and seismic sequences and split fractions were combined to form one external event quantification. The reader is referred to chapter 3 of Reference 4-1 for a more detailed description of this process.

\subsection{INCORPORATION OF PHENOMENOLOGICAL UNCERTAINTY}

As mentioned in the introduction to this chapter, phenomenological uncertainties are also included in the uncertainty analysis of the accident progression. The description of the phenomenological issues for which uncertainty distributions were generated and the reasons for these selections are described in this section. This section is a summary of Appendix C.

A number of issues were considered for use in the uncertainty analysis. The original list of possibilities was obtained by examination of previous studies, by consideration of the comments of an external review group to earlier draft versions of this analysis, and by literature review. This list of phenomena was used to determine the phenomenological events to be included in the actual APET analysis as well as those to be analyzed further during the uncertainty analysis.

Twelve major issues were identified for uncertainty analysis in the accident progression analysis. These are:

1) Hydrogen generation, transport, mitigation, and ignition,

2) Confinement building failure and fog spray system survival,

3) Fuel damage for partial ECCS and partial core accidents,

4) Fuel damage due to late ECCS failure,

5) Fuel damage propagation between uncooled and cooled core regions,

6) Core response to late ECCS actuation,

7) Pressure tube failure,

8) Graphite oxidation,

9) Recovery of failed systems,

10) Filter path reisolation,

11) Filter performance, and

12) LERF plastic liner failure.

These were selected as uncertainty issues because each represents a possibility for either a significant additional release of radionuclides from the fuel, a release of radionuclides to the environment, or a major change in the progression of the accident. The method used for selection of these issues from all APET issues is not glamorous or profound, but rather practical. Many candidate issues were determined based on an examination of all phenomena modeled in the APET. An examination of the candidate issues was done to find the issues that had both the potential to significantly change the accident progression and significant uncertainties associated with them. This was done by examining the results of a point 
estimate APET analysis and selecting issues that were both uncertain and significant to the results of the point estimate analysis. The above list of issues, together with hardware failure uncertainty issues from the Level $I$ and confinement fault tree analyses, represents those uncertain phenomena with significant potential to change the accident progression. They were included in the uncertainty analysis on that basis.

\subsubsection{Hydrogen Phenomenology}

The $\mathrm{N}$ Reactor confinement building is constructed with a design rating of 5 psig overpressure. Hydrogen detonations and deflagrations, should they occur, would be expected to present a serious challenge to the integrity of such a building. This has been noted in previous studies [4-11]. The issue of hydrogen phenomenology consists of several subissues. These include analysis of the amount and rate of hydrogen production that would be expected for various accident progression pathways, analysis of hydrogen transport and mitigation phenomena to determine hydrogen flammability, analysis of hydrogen detonability given that a flammable mixture occurs, determination of the probability of ignition for various configurations, and, given that ignition occurs, the probability that the mixture detonates. The probabilities for this uncertainty issue were generated through an expert elicitation process that is documented in Appendix C. Due to the length and complexity of the issue resolution, only the issues and phenomena considered are discussed here.

The broad issue of hydrogen phenomenology was broken down into five sub-issues. These are:

a) amount of hydrogen generated,

b) hydrogen transport and flammability,

c) hydrogen detonability,

d) hydrogen ignition, and

e) deflagration-to-detonation transition

The hydrogen transport sub-issue was by far the most complex because it considered not only hydrogen transport, but also the activity of the hydrogen mitigation system.

The first sub-issue was determined mainly through the use of MELCOR calculations. These were based on the Hydrogen Mitigation Design Basis Accident (HMDBA) [4-12] as extended for additional, non-design basis core cooling conditions. The MELCOR calculations are documented in the supporting calculations report [4-13]. They showed that single riser failures would be expected to produce less than $30 \mathrm{~kg}$ of hydrogen during a 24 hour accident, and, hased on the low hydrogen concentrations calculated from this hydrogen generation rate, hydrogen combustion events for single riser failures were excluded from further consideration. The expected hydrogen production during full core damage accident sequences would be between 80 and $130 \mathrm{~kg}$ during the first two hours, and between 230 and 2500 $\mathrm{kg}$ during a 24 hour accident, depending on the status of the GSCS. This information was used in the hydrogen transport and flammability assessment. 
The hydrogen transport and flammability assessment determined probability distributions for hydrogen flammability in various areas of the plant by considering supporting calculations from the HECTR [4-13, 4-14], MELCOR [4-13], and COBRA-NC [4-15] code packages. The assessment allowed for hydrogen flammability in three areas simultaneously: the emergency dump tank, the pipe gallery near the return line from the dump tank, and near the location of a pipe break. The analysis considered the action of the nitrogen purge and inert systems as well as the mixing fan function of the hydrogen mitigation system as well as the mixing effects of the fog spray system. The results of this analysis caused significant modifications to the APET structure because previously hydrogen flammability was considered only for locations near pipe breaks.

The hydrogen mitigation system is designed to reduce the probability that a hydrogen combustion event will cause confinement failure by preventing the hydrogen concentrations inside the confinement building from reaching flammable levels or, alternatively, by reducing the oxygen content of the atmosphere such that combustion cannot occur. This is done by ventilating the pressurizer penthouse area and injecting a nitrogen source into the confinement building to purge it of hydrogen as the hydrogen is released into the confinement from the core. This system is of significant benefit in many scenarios and has the added effect of helping to keep the filters cool by providing a steady gas flow over the filter elements.

Hydrogen detonability is closely related to hydrogen flammability because the same factors effect both issues: hydrogen concentration, mixing, and inerting. For most accident progression pathways, hydrogen was assessed to be detonable only under very limited circumstances because hydrogen concentrations were assessed to remain below the detonable range.

Hydrogen ignition is a very significant issue because the confinement can not be damaged by a hydrogen event if the hydrogen does not ignite. Ignition can occur by a number of mechanisms including sparks from electrical equipment, static discharge, sparks from mechanical objects moving about, and hot surfaces and airborne particles. The assessment notes that the energy required to ignite flammable hydrogen is extremely small (millijoules), and that any of these mechanisms is capable of supplying more than enough energy for ignition. The static discharge and mechanical contributions were generally small when compared to the contribution from electrical equipment for situations when power was available, but formed the basis for ignition in the absence of power. It is noteworthy that most large areas, when flammable, were assessed to be likely to ignite when power was available because of the availability of electrically powered equipment in those areas.

The final portion of the assessment considered deflagration-todetonation transition (DDT) because, even if a detonable mixture ignites, it will not always detonate (cause a shock wave to be produced), but will sometimes deflagrate (cause a subsonic pressure wave without a shock wave). This analysis considered the probable richness of the hydrogen-air mixture as well as the geometry of the flammable areas to determine probability distributions for the occurrence of DDT. A more complete discussion of the 
justification for and the results of the entire hydrogen assessment can be found in Appendix $C$. The response of the confinement building and filter path to hydrogen events is considered separately in other uncertainty issues.

\subsubsection{Confinement Bullding Failure}

Various energetic events, such as steam spikes, hydrogen events and graphite oxidation, are expected to challenge the integrity of the confinement building. Particularly violent building failures could have the additional effect of rendering the fog spray system inoperable or ineffective either by destroying the spray system piping, by twisting the structure such that the fog spray heads are no longer aimed to provide building coverage, or by violating confinement integrity so severely as to cause the spray-covered areas to be bypassed by the contaminated gas stream. Each of these cases are considered under this uncertainty issue.

The confinement building failure analysis considered the following events as possibly causing confinement building failure: hydrogen detonations, hydrogen deflagrations, graphite oxidation, steam spikes and vacuum breaker failure. Data in support of the uncertainty analysis were gathered from supporting calculations [4-13] as well as previously published studies [4-14]. In general, hydrogen events and steam spikes were assessed to be most likely to cause confinement building failure while graphite oxidation events were less likely to do so. Vacuum breaker failure was conservatively assumed to always fail confinement as it was shown by the confinement systems fault tree analysis to be an extremely rare event [4-4], and no additional analysis was justified. A table of the assessed probabilities and comments on their. justification is presented in Appendix $C$.

Fog spray system failure was considered in this assessment only as a result of the confinement building failure. Other failures were considered in the confinement systems fault tree analysis. It was assessed that only very rapid events such as hydrogen events would be violent enough to cause fog spray failure, and that the system would be very likely to survive most deflagrations because of the relatively low pressures and the lack of a shock wave. However, detonations would be very likely to cause fog spray function failure as described above because of the added destructive force associated with the shock wave. A table of the assessed probabilities and comments on their justification is presented in Appendix C.

\subsubsection{Fuel Damage for Partial Eccs Accidents}

Most internally initiated $\mathrm{N}$ Reactor severe accident sequences do not involve complete failure of the ECCS, but rather show ECCS operating in some degraded state. MELCOR and RELAP calculations have demonstrated that steam cooling provides significant heat removal in many of these situations [4-13], while geometric constraints limit core damage in other situations. The amount of core damage has a first order impact on the source term for radionuclides because it directly impacts the amount of fuel available for 
radioactive release. This issue deals with the uncertainties associated with fuel damage amounts for the partially cooled N Reactor core.

The $N$ Reactor is different from most commercial nuclear reactors because most fuel damage accidents result in damage to only portions of the core. The reason for this is that about half of the core damage frequency involves failure of ECCS flow to $1 / 16$ of the core as caused by a failed ECCS check valve, and more than 908 of the remaining core damage frequency involves success of the GSCS. Furthermore, most ECCS failures involve the failure of enough valves to open or pumps to start, but some valves open and some pumps start such that cooling to the core does not meet the ECCS success criteria. This combination of factors means that the vast majority of N Reactor accident sequences result in only limited core damage. However; the large uncertainties associated with the geometry of the failed fuel prevent the use of meaningful deterministic calculations to obtain the exact amount of fuel damage, so the amount of core damage is determined under the uncertainty assessment.

At least eight different partial ECCS accident sequences were considered during the uncertainty analysis. These are:

a) GSCS succeeds throughout the accident, but ECCS fails completely,

b) GSCS is degraded due to a pressure tube failure, and ECCS has failed completely,

c) ECCS cooling fails in a single inlet riser ( $1 / 16$ of the core),

d) all V-4 valves fail on one side of the reactor,

e) all V-3 valves fail on one side of the reactor,

f) two ECCS diesel pumps fail to start or run,

g) one ECCS diesel pump fails to start or run, and two V-3 valves fail to open on the same side of the core, and

h) only high pressure injection (HPI), and not ECCS, is available to cool the core.

Most of the above accident sequences were considered both with GSCS success and GSCS failure. In addition, ATWS with successful ECCS function was examined for core damage. Calculations [4-13] based on conservative liquid and gas heat transfer assumptions [4-12] provided a basis for the uncertainty assessments for cases with little or no ECCS flow, while pressure drop calculations provided guidance for the amount of the core that would be liquid-filled in other cases where ECCS flow was significant. The details of the assessments and probability distributions are given in tables in Appendix $C$.

\subsubsection{Fuel Damage for Late ECCS Fallure}

A unique feature of the $N$ Reactor is that the ECCS function can fail after two hours and, if the GSCS is functional, core damage is still prevented. However, if GSCS also fails, core damage can occur. This situation is called core damage bin $\mathrm{Zl}$, or delayed core damage in the $\mathrm{N}$ Reactor Level I PRA report, and is described there as proceeding to "up to 1008 core damage" [4-2]. Since these accident sequences have non-trivial frequencies, a conservative assessment of 1008 core damage for all of them 
would have a significant and artificial effect on the results of this study.

It was noted during the assessment that most of the delayed ECCS failures are caused by the failure of a diesel ECCS pump to run for the full 24 hours, but that this situation still leaves one pump operating. It was further noted that the Emergency Response Guides [4-16] allow the operators to reduce ECCS flow to $5000 \mathrm{gpm}$ at two hours. Analyses show that this flow is sufficient to prevent fuel damage if ECCS has succeeded for the first two hours. A single ECCS diesel pump can supply more than 14000 gpm. It was assessed that, as long as a single ECCS diesel pump remains operational during the entire 24 hour accident duration, core damage would be kept to levels that are below the level at which any out-of-plant consequences would be expected. The engineering judgment concerning this issue is presented in more detail in Appendix $C$.

If the remaining diesel pump were also to fail, the operator would have at his disposal a number of means by which the required amount of coolant could be injected to cool the core. However, the operator is not provided procedural guidance on how to bring these systems on line in the Emergency Response Guides. It is conservative to assume that the operator would not bring these systems on line and that the core would boil dry leading to 1008 core damage. This conservatism, however, should not artificially bias the results of the study as severely as the original assumptions would have because the cut sets that have all ECCS pumps failing after two hours would form only a very small fraction of the frequency of the original "delayed ECCS failure" accident sequences. Procedural modifications in the Emergency Response Guides could reduce the probability of this accident sequence to essentially zero if guidance were given for the startup and alignment of the additional water sources.

\subsubsection{Fuel Damage Propagation}

A number of phenomena have been proposed to cause the propagation of fuel damage from an uncooled pressure tube to one that is apparently adequately cooled. The possibility of each of these mechanisms is remote at best, but they deserve some consideration in the analysis. This issue provides an opportunity to consider these mechanisms and to assign them as a group some probability of occurrence.

The following issues were considered as possible mechanisms of fuel damage propagation:

a) "cascading" pressure tube failures,

b) coolant diversion by pressure tube failure,

c) water hammer ("chugging") induced multiple pressure tube failures,

d) molten fuel attack on pressure tubes and graphite blocks,

e) molten fuel relocation from one pressure tube to another,

f) molten fuel relocation within the graphite stack, and

g) pressure tube fallure inducing full GSCS failure. 
A lengthy discussion of why each of these mechanisms are considered less than credible is presented in Appendix $C$ along with an assessed probability distribution for any one of the mechanisms to occur. Briefly, however, mechanisms a), b) and c) are all considered unlikely based on the results of pressure tube integrity studies (see section 4.3.7) that indicate that the phenomena would occur only at pressure tube temperatures that were high enough to fail the fuel even before the phenomena would occur. Mechanisms d), e), f) and $g$ ) are discounted based on the reaction rates and the particular geometry of the graphite stack (mechanism $g$ does cause increased fuel damage because the efficiency of heat transfer from the graphite to the GSCS is decreased, but this is different from full GSCS failure, which proceeds to 1008 core damage).

\subsubsection{Confinement Response to Late ECCS Actuation}

Starting the ECCS after core damage has occurred can cause a number of undesirable effects for both the core and the confinement building. The effects in the core can include both pressure tube failure and the possibility of additional core damage. These are discussed in the Sections 4.3.5 and 4.3.7. The effects in the confinement building can include additional hydrogen production (see Section 4.3.1) and a steam-induced pressure spike inside the building. The steam spike issue is considered in this section.

Three questions are relevant for this issue:

a) Does the pressure exceed the fog spray demand setpoint (10" water)?

b) Does the pressure exceed the filter reisolation setpoint (15" water)?

c) Does the pressure exceed the building rupture pressure (at least 5 psig)?

The third question is especially relevant because, since both the building and the primary system would likely be depressurized before the ECCS was started, the steam vents would be isolated and unavailable to relieve a large steam pressure event. The banana wall is described in Section 1.2 of Reference $4-1$ and could relieve the pressure from a small event (the magnitude of a small LOCA), but not a major event [4-13]. Thus, if a large steam spike were to occur, the possibility of confinement failure could be very real.

Research on the issue was done using MELCOR to obtain a rough idea of the maximum pressure that could possibly be achieved under these conditions if the confinement were to remain intact. The calculation [4-13] indicated that a pressure of 16 psig could be achieved under certain circumstances, but smaller pressures would be expected in most cases. Based on this information, it was assessed that events a) and $b$ ) above would always occur, and that event c) above would occur on the average about 808 of the time. The reader is referred to Appendix $C$ for a more thorough discussion of this issue. 


\subsubsection{Pressure Tube Fallure}

Questions were raised by the National Academy of Sciences [4-11] and others about the fundamental assumption of many previous $\mathrm{N}$ Reactor core analysis studies: that the pressure tubes would remain intact for the entire duration of the accident. Pressure tube failure would mean degradation of the GSCS function due to loss of the helium blanket. This would cause additional fuel damage. Thus, the issue of pressure tube integrity is very important to the overall results of the risk assessment.

Four mechanisms were evaluated for their potential to cause pressure tube failure. These are:

a) dissolution of the pressure tube by molten uranium,

b) molten fuel-coolant interactions (MFCI) inside a pressure tube,

c) tube yield due to a pressure spike or water hammer event, and

d) failure due to a pre-existing defect.

Failure mode a) above was determined to be applicable only late in the accident for very high temperature core damage events. This conclusion was based on the results of experimental studies [4-17] that indicated that the rate of attack from the uranium is not significant except at pressure tube temperatures above $1600 \mathrm{~K}$. In all analyzed scenarios (except GSCS failure scenarios) where pressure tube failure was assessed to be likely, other failure modes were assessed to be more significant contributors to the pressure tube failure probability than the dissolution mode.

Failure mode b) was assessed to be likely to occur only in cases where the fuel had become molten in the inlet regions of the core and water was later introduced to the pressure tube. If the fuel is molten in the middle of the core but not the inlet region, it would be almost impossible for significant amounts of liquid water to reach the molten fuel and cause an MFCI event. Even if the inlet fuel is molten, the geometry of the pressure tube would not allow for the "classical" MFCI steam explosion to occur bccause of the limited amount of water that can come in contact with the molten fuel at any given time. For this reason, only "mini" MFCI events are considered credible, and these only with a small probability. This does not, however, rule out pressure tube failure due to steam pressure spikes, as will be discussed below.

The dominant mode of pressure tube failure for most delayed ECCS restart events was assecsed to be failure mode c). Pressure tube strength studies indicate that the yield strength decreases rapidly at temperatures greater than $-1200 \mathrm{~K}$. In addition, water hamner studies indicate that pressures above the yield point are achievable by both steam spikes and water hammer events. Mechanical cycling was also assessed to be a possible yield failure mechanism due to the possibility of repeated water hammer events. Thus, if ECCS is restarted, pressure tube failure due to yield in failure mode c) was assessed to be almost certain to occur. 
Failure mode d) was assessed to be the dominant mode of failure for low temperature accidents. A non-destructive eddy current examination of all pressure tubes was recently completed to demonstrate the integrity of the $\mathrm{N}$ Reactor pressure tubes. However, these techniques typically fail to detect up to 58 of the existing flaws when they are applied. For this reason, this study assumed that at least one pressure tube flaw with a depth of at least $20 \%$ of the pressure tube thickness would be present in the core at the time of the accident. Such a defect might propagate to pressure tube failure under thermal shock or pressure spike events. Pressure spike events were noted to be a very unlikely cause of defect propagation because the pressure tubes had already been exposed to higher pressures during normal operation than would be expected during a steam spike. Thermal shock was assessed to be more likely to cause defect propagation, although even in this case the probability of pressure tube failure due to defect propagation was always assessed to be less than 0.10 .

The pressure tube failure assessment considered more than 15 different accident sequences. The discussion of these sequences would be far more lengthy than would be appropriate in this context. The reader is referred to Appendix $C$ for a more complete discussion.

\subsubsection{Graphite Oxidation}

The condition of the graphite stack can have a profound impact on the amount of radionuclides released from the reactor. If the helium cover gas for the stack is lost, the heat removal efficiency of the GSCS suffers. This results in more fuel damage and a higher source term. More important from a source term perspective, however, is the possibility that a sequence of events could cause a self-sustaining oxidation reaction to begin within the graphite stack. The heat from such a reaction could cause a significant additional release of radionuclides from the reactor. The uncertainty in the graphite oxidation phenomena was considered in the APET analysis for this reason.

Two graphite oxidation reactions were considered: steam-graphite and air-graphite. The steam-graphite reaction was ruled out as a possible mechanism for a self-sustaining oxidation reaction because, although the reaction produces flammable gases, it is endothermic in nature and, hence, can not be self-sustaining. The graphite-air reaction, however, is exothermic and could become self-sustaining if not limited by air availability.

The assessment determined that there were two factors that would determine whether or not a self-sustaining graphite oxidation reaction could occur: graphite temperature and oxygen availability. Expected temperatures under accident conditions do become high enough so that the possibility of oxidation cannot be ruled out, but in all but the total loss of cooling case (where ECCS and GSCS both completely fail), the high temperatures are limited to a very small section of the graphite stack. In addition, the stack is inert due to the presence of the helium blanket in most of these cases and, when it is not, the area available for air ingress 
is very small. This limits the oxidation rate to a minimal level. The little air that would get into the graphite stack under such conditions would be consumed by the graphite in the reflector before it could reach the core region. Thus, any oxidation energy would be deposited away from the fuel, and the long characteristic time for heat conduction in the graphite stack would, for all practical purposes, render the core unaffected. For these reasons, graphite oxidation is not considered credible in situations where there is a significant degree of cooling available to the core from either the GSCS or the ECCS.

In cases where the ECCS and GSCS both fail, temperature arguments can not be used to eliminate the possibility of graphite oxidation because the graphite stack is subjected to an unmitigated heat-up. However, in cases where a seismic event has not occurred, it is difficult to obtain a pathway by which air inflow and oxidation product outflow are favorable. The large concrete and steel biological and thermal shields that surround the reactor make the gas flow extremely difficult in the absence of multiple pressure tube failures. Even in the latter case, the pressure tubes would have to fail both outside and inside the core to allow air first into the pressure tubes from the outside and then from the pressure tubes into the graphite stack. For these reasons, the probability of graphite oxidation under these conditions was assessed to be less than 0.20 with a median of 0.10 .

The most likely scenario for self-sustaining graphite oxidation is a seismic pressure tube rupture event. This event would only be expected to occur in the most severe seismic events with a mean frequency of less than once per hundred thousand years [4-3]. In such a scenario, many pressure tubes are sheared off by the translational motion between the graphite stack and shield walls. This could provide a large total opening on each side of the core for gas transfer and set up a flow path that would be favorable to oxidation. The assessment found that it could not defend a conclusion that the graphite would not oxidize under these conditions, although the oxidation would not be likely to occur for several hours. The APET analysis used the conservative assumption that all such events would result in self-sustaining graphite oxidation after several hours. It should be noted that it may be possible to take mitigation actions to prevent or reduce the possibility of graphite oxidation if such a cataclysmic seismic event were to occur, but those actions were not considered in the assessment. For more discussion about this issue, the reader is referred to Appendix $C$.

\subsubsection{Recovery Actions}

As discussed in Chapter 3, recovery actions are assumed to take place approximately two hours after the onset of core damage and can involve several systems. It is important to note that these are not recovery actions to prevent fuel damage--those were considered in the internal events and external events Level I PRAs [4-2, 4-3]--but rather recovery actions either to minimize the amount of fuel damaged or to recover confinement systems which could mitigate the accident. The systems considered for such actions are electrical power, GSCS, ECCS, high pressure injection, fog sprays, the filter release path and the hydrogen mitigation 
system. Also, the "recovery" of some systems can cause negative as well as positive effects. The recovery uncertainty issue, therefore, must consider not only whether or not the system can be recovered, but also whether or not the operators will actually attempt to accomplish the recovery. In some cases, such as the fog spray system, the filter path and the hydrogen mitigation system, the "recovery" actions can be caused by a new demand on the system. For example, a pressure tube rupture can cause a demand for fog spray actuation if the system had not been previously demanded. These actions are accounted for in the APET itself and not in the uncertainty analysis.

Systems were identified that would be essentially unrecoverable under any situation either due to the failure mode (i.e. diesel pump failure), equipment location (i.e. components most likely to fail being located inside the radiologically contaminated confinement building), physical phenomena (i.e. the inability of GSCS water to penetrate the core due to steam binding), or a lack of procedures (i.e. the operators are not given instructions to recover high pressure injection). These systems were assigned a recovery probability less than or equal to 0.01 for accident progression pathways where recovery would be all but impossible. Conditions were also identified under which recovery would be essentially automatic. For example, if the confinement isolation valve to the filter path failed due to a loss of $A C$ power and hydraulic accumulator depletion, AC power recovery would automatically cause recovery of the filter path as we11.

The recovery of other failures was assessed based on the probable failure location and type. For example, V- 3 valve failure causes partial ECCS failure. The valves are accessible and almost all failures are related to the motive means and control circuits rather than the inner workings of the valve, so recovery is usually possible. Also, the operators would be likely to continue recovery efforts even after the onset of core damage because the core is already receiving partial ECCS flow. After accounting for the recovery factor from the Level I PRA and the above facts, a recovery probability of approximately 0.25 was assigned for $V-3$ valve failure. This recovery probability is in addition to that given in the Level I PRA analysis because that study only considers recovery actions during the first seven minutes of the accident sequence (the time before fuel damage would occur in a large LOCA accident sequence with complete ECCS fallure), while many recovery actions would take longer than that to complete. Similar analyses were carried out for other components.

Human factors were also considered in the recovery assessment. This was especially true for recovery of the $3 \mathrm{H} 33$ ECCS bypass switch. In this case, the recovery event was decomposed into two sub-cases:

a) Does the operator know that fuel damage has occurred?

b) Does the operator go against procedures and start ECCS?

The operator was assessed to be very likely to start the ECCS if he did not know that fuel damage had occurred, but very. unlikely to do so if he knew that the core had been damaged because his procedures told him not to. The issue becomes even more clouded when the GSCS fails, because the operator 
is faced with the possibility of an unmitigated core heatup if he does not violate procedures by starting the ECCS. For this case, the assessed probability distribution was bimodal (i.e., with two peaks) because certain operators would almost always start ECCS, and others would almost never start ECCS. The uncertainty, then, is related to which operators are in the control room at the time of the accident. It should be noted that this uncertainty issue is the only major exception to the general assumption that the operators will not perform actions in direct violation of the written procedures.

The recovery assessment considered many different accidents. The discussion of these accidents would be far more lengthy than would be appropriate in this context. The reader is referred to Appendix $C$ for a more complete discussion.

\subsubsection{Filter Path Reisolation}

The confinement filter path is designed to reisolate any time the confinement pressure rises above 15 inches of water. The reisolation is designed to prevent an overpressure event from damaging the filters. However, events such as hydrogen detonations occur so quickly that the path would not be able to close quickly enough to prevent damage from occurring to the filter path downstream of the isolation valves. The path is designed to isolate quickly enough that a large LOCA event will not damage the filters. This was used as the basis of comparison for the events analyzed in this assessment.

The following events were considered as having the potential to demand filter path reisolation:

a) hydrogen detonations,

b) hydrogen deflagrations,

c) graphite oxidation events, and

d) pressure tube failure events.

Estimatcd preceure wave transit times were examined for many of the events and compared to an estimated large LOCA pressure wave transit time from the LOCA location to the isolation valves. Based on these calculations, it was determined that graphite oxidation events and pressure tube failure events would not create pressure spikes that would challenge the filter path reisolation function, while hydrogen combustion events would be likely to occur too quickly for the isolation function to be successful. The reader is referred to Appendix $C$ for a more complete discussion of this uncertainty assessment.

\subsubsection{Filter Performance}

The performance of the filter system has a major impact on the overall release of radioactivity from the plant during a severe accident because it forme the last line of defense between the contaminated confinement building atmosphere and the outside environment. The filters are designed 
to be the only path by which radioactive gases can leave the building and, as such, they are called upon to remove as much of the radioactive contamination as possible from the gas stream.

The filtration function is accomplished by a three stage filter system [4-18]. The first stage is a demister that removes any entrained moisture from the gas stream to protect the HEPA and charcoal stages. Since many fission products are likely to be trapped in the entrained moisture and since many larger aerosols will be trapped in this stage, the demister performs an important scrubbing function. The second stage of the filter system is a high efficiency particle absorber (HEPA) stage. This stage removes the extremely fine aerosol particles passed by the demister stage but leaves vapors unattenuated. The third stage charcoal filter removes fission product vapors, especially halogens, from the gas train. This leaves only noble gases unattenuated and, since these are not chemically reactive, they pose a far smaller threat to the health of the surrounding population.

The first stage in the uncertainty assessment for the filter system was to determine the possible modes of filtration function failure. These were assessed to be:

a) bypass of the filters by the exhaust gas stream,

b) pre-existing filter defect or installation error,

c) filter element failure due to hot and humid operating conditions,

d) filter plugging or element failure due to excessive aerosol loading,

e) filter overheating and/or burning, and

f) filter element failure due to the action of the filter spray system.

Mechanisms a) and b) were analyzed in the confinement systems fault tree analysis [4-4], and are independently implemented in the APET analysis from that source. Mechanism a) also considers the results of other confinement failure events in the APET such as hydrogen events and steam spikes. Mechanism c) was assessed to be unimportant because temperature and humidity conditions much more severe than those expected during $N$ Reactor accidents [4-13] would be required to significantly degrade filter performance during the time period analyzed for the accident progression.

Filter failure mechanisms d), e) and $f$ ) are all closely tied to the loading of the filters with fission products over the course of the accident progression. A filter loading assessment was performed previously by Westinghouse Hanford [4-18] to analyze these issues. This analysis considered the filter loading for a design basis accident in which a large LOCA had occurred and the GSCS, fog spray and the hydrogen mitigation system nitrogen injection systems were operating as designed. This analysis concluded that the filters would remain adequately cooled without the aid of the filter spray system as long as a gas flow of at least -1000 cfm is maintained. The analysis did not, however, consider "beyond design basis". accidents where one or more of the above systems did not function. While these accidents are relatively rare, they can be very significant in the overall risk result because they can be more severe than the design basis accidents. 
If the decay heat from the radionuclides trapped on the filters causes them to overheat, a spray system automatically actuates to spray the filter cells (including the filter media itself) with a fine water mist to cool the filters. HEPA filters are significantly weaker when wet than when dry. While numerous tests have been conducted on $N$ Reactor filters, filtration efficiency and flow resistance data for wet filters has not been reported. It is possible that the filters would survive under such conditions, but even a very small failure of the filter media would drastically reduce the filtration efficiency because the effluent gases would flow preferentially through the failure. For this reason and because of the lack of alternative data, it was assessed that filter spray actuation would cause filter media failure, and that failure of the filter sprays would lead to the destruction of the filters by burning because of the insufficient removal of decay heat. These assessments are admittedly quite conservative, but most other models for these phenomena would run the risk of being non-conservative, and frequently by amounts that would be difficult to predict.

The uncertainty assessment was begun by computing factors to represent the approximate differences between the radionuclide release from the core in Reference 4-18 and that of the core state in question. Also, an approximation of the decontamination factor (DF) was calculated for the fog spray system as modeled in that reference. Based on this information, probability distributions were developed to represent the DF that would be required between the point where the radionuclides are released from the primary system and when they reach the filters. This DF would have to be provided by either the emergency dump tank or the fog spray system. If the actual $D F$ is less than the required DF, the filter decay heat loading will cause the filter sprays to be demanded. The required DFs were generated for several core damage states based on the expected differences in radionuclide release from the core.

While the required DFs to prevent filter spray actuation are uncertain, the DFs that are provided by the fog sprays and the emergency dump tank are also uncertain in and of themselves, and are discussed as source term issues in Chapter 5 of the $N$ Reactor Level II/III Probabilistic Risk Assessment final report [4-1]. The uncertainty distributions for the provided DFs (from Chapter 5 of Reference 4-1) were overlaid on the distributions for the required DFs to obtain an uncertainty distribution for the probability of filter spray actuation in various situations. This process is described in more detail in Appendix C. The Latin Hypercube sample members from these distributions were generated to induce a strong, intentional correlation between the probability that filter sprays will not be demanded, the fog spray system DF and dump tank DF uncertainty distributions. The physical reason for this correlation is that a high dump tank or spray DF would mean that the filters are exposed to a lower fission product load and, hence, have a higher probability of not demanding the filter sprays. The converse is also true. It should be noted that other, more minor decontamination mechanisms, such as the building settling $D F$, were neglected during the analysis, and the fog spray and dump tank DFs were only considered in the APET if the respective systems were indeed operating. 
Some recovery actions are possible but are not considered in this analysis due to a lack of procedures. The possible recovery actions include switching filter banks on filter failure or burning, and isolation of cells in which filters are burning to prevent the released radionuclides from reaching the environment. This latter action could also help extinguish the filter fire. A determination of possible recovery actions and procedures could significantly reduce radioactive source terms for accident progression pathways in which the filters fail. Since filter failure occurs more often in high filter load, high core damage accident sequences (especially those where GSCS fails), this could have an impact on the overall risk result.

\subsubsection{LERF Plastic LIner Fallure}

The plastic liner of the LERF would be expected to fail with some small probability in any accident where effluent water is generated. The $N$ Reactor Liquid Effluent Retention Facility is described in Reference 4-19. It consists of a very large excavated basin which is lined with a thick (60 mils), flexible, stretchable plastic liner. A second layer of this liner material forms a flexible top for the facility. The LERF is designed to hold at least 908 of the radioactive liquids generated by the plant during any postulated plant accident. The 908 figure is based on the ECCS flow prescription from ERG-B as found in the $\mathrm{N}$ Reactor Emergency Response Guides [4-16]. A ventilation system allows gases generated by the liquids in the LERF to be released to the environment through charcoal filters. Liquids enter the facility through a weir and standpipe system that passively diverts the liquids to other facilities or, as a last resort, to the ground once the LERF is full. Thus, failure of the LERF cover system by overpressurization is not considered credible. Liner failure due to other causes is the subject of this uncertainty issue.

The mechanisms considered for LERF liner failure are:

a) installation defects,

b) weather and age-related defects, and

c) material quality defects.

Each of these was examined in some detail in Appendix $C$, and the reader is referred to that appendix for a more complete discussion. The failure probability was assessed to be less than or equal to a standard aboveground tank because it is extremely well-supported and flexible.

\subsection{SUMMARY}

The uncertainties incorporated in and propagated through the APET analysis encompass a large number of different areas. Uncertainties from both the internal and external events Level I PRA analyses are propagated through the APET analysis. This is accomplished through a TEMAC analysis on the cut sets and accident sequences from the Level I PRA analyses. The split fractions generated by TEMAC form the basis for the initial 
conditions for the APET analysis. A TEMAC analysis is also performed on the cut sets generated by the confinement systems fault tree analysis. The split fractions from that analysis form the basis for many of the systemic uncertainties considered in the APET analysis. The underlying uncertainty distributions for the basic events in all of these cut sets are stratified Latin Hypercube Monte Carlo samples that are generated by the LHS program based on distributions supplied by the respective fault tree analyses.

In addition to the systemic uncertainties described above, the APET analysis considers phenomenological uncertainties for a number of issues related to the progression of postulated severe accidents at $\mathbb{N}$ Reactor. These issues include hydrogen and graphite combustion phenomenology, filter performance, confinement building performance, core fuel damage and pressure tube failure phenomena, and the behavior of the liquid effluent handling facilities. These issues were selected because they represented those issues that had both a significant potential to increase the radioactive releases from the plant and significant uncertainties associated with them.

Both types of uncertainty were included in the APET analysis through the use of Latin Hypercube sampling. The effects of the uncertainty as well as a list of those uncertainties that dominate the overall uncertainty results are discussed in Chapter 5. 


\subsection{REFERENCES}

4-1. D. M. Kunsman, et al., Level III Probabilistic Risk Assessment for $N$ Reactor, WHC-MR-0045, SAND89-2102, Prepared by Sandia National Laboratories for Westinghouse Hanford, April 1990.

4-2. M. D. Zenter, et al., N Reactor Level I Probabilistic Risk Assessment: Final Report, Westinghouse Hanford Company, Hanford, Washington, WHC-EP-0322, August 1988.

4-3. J. A. Lambright, et al., Analysis of Core Damage Frequency Due to External Events at the DOE $N$ Reactor, Sandia National Laboratories, Albuquerque, New Mexico, SAND89-1147, 1990.

4-4. G. A. Coles, N Reactor Confinement Systems Sequence Analysis Including Recovery, WHC-SD-NR-RA-001, Westinghouse Hanford, March 1990 .

4-5. G. A. Coles, "Effect of Fire Sequences on Confinement Systems for $N$ Reactor Level 2 Probabilistic Risk Assessment," Letter to D. $M$. Kunsman and G. D. Wyss, WHC Correspondence \#8953739, Westinghouse Hanford Co., June 21, 1989.

4-6. G. D. Wyss, "Research on APET Integration of Seismic PRA Results," Memo to T. A. Wheeler, Sandia National Laboratories, September 8, 1989.

4-7. R. L. Iman and M. J. Shortencarier, A FORTRAN 77 Program and User's Guide for the Generation of Latin Hypercube and Random Samples for Use with Computer Models, NUREG/CR-3624, SAND83-2365, Sandia National Laboratories, June 1984.

4-8. R. L. Iman and M. S. Shortencarier, A User's Guide for the Top Event Matrix Analysis Code (TEMAC), Sandia National Laboratories, Albuquerque, New Mexico, NUREG/CR-4598, August 1986.

4-9. R. I. Iman, "Modifications to TEMAC," Memo to file, Sandia National Laboratories, January 17, 1989.

4-10. J. M. Griesmeyer and L. N. Smith, A Reference Manual for the Event Progression Analysis Code (EVNTRE), NUREG/CR-5174, SAND88-1607, Sandia National Laboratories, September 1989.

4-11. Safety Issues at the Defense Production Reactors: A Report to the Department of Energy, National Academy of Sciences, National Academy Press, Washington, D.C., 1987.

4-12. F. J. Heard, et al., N Reactor Safety Enhancement Final Report: Hydrogen Generation and Thermal Analysis of the Hydrogen Mitigation Design Basis Accident, WHC-SP-0096, Westinghouse Hanford, December 1987. 
4-13. L. A. Miller, et al., N Reactor Probabilistic Risk Assessment Supporting Calculations, SAND89-2101, Sandia National Laboratories, 1990.

4-14. A. C. Payne and A. L. Camp, Parametric HECTR calculations of Hydrogen Transport and Combustion at $\mathrm{N}$ Reactor, SAND86-2630, Sandia National Laboratories, June 1987.

4-15. T. L. George, et al., An Assessment of the $N$ Reactor Hydrogen Mitigation System Effectiveness, NAI-8708-2, Numerical Applications, Inc., January 1988.

4-16. United Nuclear Industries, Inc., "ECS Actuation During Normal Operation," Emergency Response Guides, WHC-CM-4-1, Vo1. 2, Guide No. B, Issued May 12, 1987, as updated through June 1989.

4-17. K. R. Birney, "Pressure Tube Considerations Relating to Molten Uranium," memo to D. M. Ogden dated January 7, 1987, Westinghouse Hanford, formerly UNC Nuclear Industries.

4-18. L. D. Muhlestein, D. W. Jeppson, J. D. McCormack, N Reactor Filter System Fission-Product Retention Assessment, WHC-SP-0233, Westinghouse Hanford, June 1988.

4-19. R. A. Paasch, et al., Project H-797 $\ldots \mathrm{N}$ Reactor 1312-N Liquid Effluent Retention Facility, WHC-SP-0221, Westinghouse Hanford, January 1988 . 


\section{RESULTS OF THE ACCIDENT PROGRESSION EVENT TREE ANALYSIS}

As discussed in detail in the $\mathrm{N}$ Reactor Level III Probabilistic Risk Assessment report [5-1], the $\mathrm{N}$ Reactor APET was solved in the uncertainty mode for internally initiated accidents as well as those initiated by fire and seismic external events. In each case the distributions for the uncertain events were represented using a Monte Carlo sample consisting of 500 sample members. These were processed by the TEMAC code package [5-2, 5-3] to obtain results for each sample member for the Level I PRA and confinement system fault tree analyses as discussed in Chapters 3 and 4, and processed by the EVNTRE code package [5-4] to determine the various accident progression possibilities at $\mathrm{N}$ Reactor. The results of the three EVNTRE analyses are presented below.

It should be noted that the $\mathrm{N}$ Reactor external events core damage analysis [5-5] considered more causes of core damage than just fire and seismic events. Events such as flooding of the Columbia River, the collapse of dams upstream of $\mathrm{N}$ Reactor on the Columbia River, and storm damage were all analyzed for their potential to cause core damage in that report. They were found to have insignificant core damage frequencies and were not, for that reason, carried through the Level II/III PRA analysis.

The results presented in these three sections are averages over all Monte Carlo sample members, but are separated by accident classification (internal events, fires and seismic events are described separately). They are intended to provide insights into which events and systems are driving the accident progression. The fractions presented are mean fractions of the conditional accident probability unless otherwise stated for a given table. They are not fractions from a list of accidents without regard for the probability of occurrence. Thus, when it says that 268 of all internally initiated accidents are initiated by small LOCAs, the statement should be interpreted to mean that if there were many thousands of reactors identical to $\mathrm{N}$ Reactor in the world, and 100 of them were to have internally initiated core damaging accidents, we would expect that about 26 of them would have been initiated by small LOCAs. The results are intended to show approximately how often the cause of core damage is a small LOCA given that the core has been damaged in some way.

\subsection{ACCIDENTS INITIATED BY INTERNAL EVENTS}

In the final integrated risk uncertainty calculations, all accidents due to internal initiators were examined in a single execution of the EVNTRE code. Thus, this analysis considered large and small LOCAs as well as transients, and ATWS events as well as accidents where the reactor protection system succeeds. This method allows an accurate comparison between the different types of accidents because data from the same sample member is used to assess all accidents together. 
The initiator frequencies are determined by the analysis discussed in Chapter 4. The mean fractions of the conditional core damage frequency attributable to each type of accident initiator (large LOCA, small LOCA or Transient) are presented in Table 5.1. This table also presents the probability that the reactor protection system (RPS) succeeds in driving in either the control rods or balls to render the reactor subcritical for each type of initiator. It should be noted that the Large LOCA ATWS accident was deleted from the analysis because of its very low frequency. This accounts for the probability of RPS success being 1.00 for large LOCA accidents. The probability calculated in the Level I PRA analysis [5-6] for large LOCA RPS success was close to the small LOCA value.

Table 5.1 Accident initiator and reactor protection system (RPS) success fractions for the internal events analysis.

\begin{tabular}{|c|c|c|}
\hline Initiator & $\begin{array}{l}\text { Fraction of Conditional } \\
\text { Core Damage Frequency } \\
\text { For Each Initiator } \\
\end{array}$ & $\begin{array}{l}\text { Fraction with } \\
\text { RPS Success }\end{array}$ \\
\hline $\begin{array}{l}\text { Large LOCA } \\
\text { Small LOCA } \\
\text { Transient }\end{array}$ & $\begin{array}{l}0.050 \\
0.255 \\
0.695\end{array}$ & $\begin{array}{l}1.000 \\
0.991 \\
0.979\end{array}$ \\
\hline
\end{tabular}

A significant finding of this analysis is that almost no internally initiated core damage accidents at $N$ Reactor would damage all of the fuel in the core. In fact, more than 508 of the accidents would involve less than 78 of the reactor because they involve either late ECCS failure (as described in Appendix C.4), the failure of cooling to a single riser $(1 / 16$ of the core), or because the LOCA break is of a size and in a location such that the V-4 valve failure that caused ECCS failure is compensated for by the break. Table 5.2 describes fuel damage. In that table, the first column represents accidents where fuel damage amounts are below the level at which offsite consequences would be expected, the second column represents the same with single riser failures added, and the third column represents adds accidents where GSCS succeeds. The results in this table are particularly significant in that they represent a significant difference from most commercial reactors. In commercial reactor risk analysis, there is no justifiable basis for saying that a fuel damage event would result in damage to only a small portion of the core. This basis does, however, exist with $N$ Reactor. It is significant to the overall risk assessment because fission products are not released in significant quantities from fuel that has not failed. This has a direct relationship to the radioactive release, consequences and risk. The amount of fuel damage for each accident initiator is shown in further detail in Figure 5.1 . 
Table 5.2 Average fuel damage fractions and quantiles for the accident initiators for the internal events analysis.

Initiator

Large LOCA

Sma11 LOCA

Transient

\section{Quantile}

0.05

0.25

0.50 (median)

0.75

0.95

mean

Quantile

0.05

0.25

0.50 (median)

0.75

0.95

mean

\begin{tabular}{ccc}
\multicolumn{3}{c}{ mean values } \\
\hline 18 Damage & $\leq 78$ Damage & $\leq 408$ Damage \\
0.065 & 0.818 & 0.999 \\
0.120 & 0.595 & 0.958 \\
0.004 & 0.611 & 0.977
\end{tabular}

Fraction of accidents with $<78$ fuel damage Large LOCA

Sma11 LOCA

Transient
0.416
0.093
0.112
0.760
0.451
0.399
0.867
0.648
0.661
0.913
0.817
0.826
0.954
0.958
0.945
0.818
0.595
0.611

Fraction of accidents with $7-408$ fuel damage

Large LOCA
0.046
0.085
0.132
0.236
0.580

0.181
Small LOCA

0.039

0.150

0.298

0.512

0.875

0.363
Transient

$$
0.051
$$

0.166

0.322

0.550

0.821

0.366 
Figure 5.1

TOTAL FUEL DAMAGE FOR INTERNAL INITIATORS

Mean Core Damage Frequency $=1.418^{\circ} 10^{-4}$

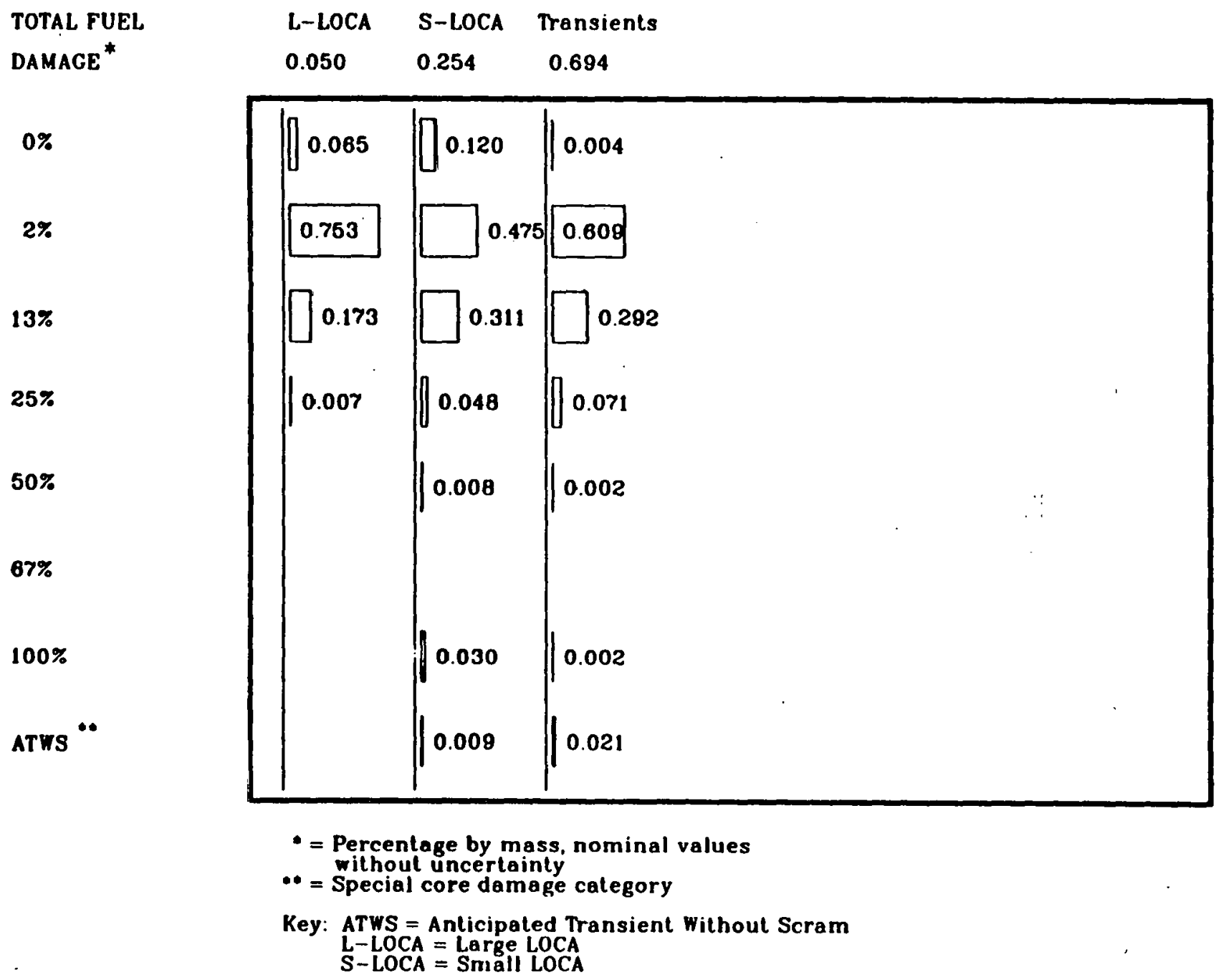


The format of Figure 5.1 is typical of those found throughout this chapter and deserves some additional explanation. The mean core damage frequency is the average core damage frequency calculated for all initiating events in the internal events analysis and is in units of a number of core damage events per year. The fraction below each initiator heading (L-LOCA, S-LOCA or Transients) the average fraction of the total internal events core damage frequency that is caused by that initiator. The fraction for each fuel damage category under each initiator represents the average fraction of events for that initiator that result in that total fuel damage category. Thus, for example, an average of 6.68 of the large LOCA conditional core damage frequency would be expected to result in $0 \%$ total fuel damage. The size of each box is proportional to the accompanying fraction value.

The fuel damage categories on the left side of the figure represent nominal amounts of core damage for the various states of core cooling and are in units of the percentage of the entire core, by mass, that experiences fuel clad failure. Of course, the actual amount of core damage for each of these classifications is an uncertainty issue and varies for each sample member in the Latin Hypercube analysis. The 0o total fuel damage category represents the following two cases: 1) a large LOCA occurs but the LOCA location compensates for the failure of one or more V-4 valves to open, and 2) a delayed ECCS failure has occurred in which one of the high lift diesel pumps has remained running to prevent core damage. The 28 fuel damage class is representative of single riser failure accidents. The 138 and 258 fuel damage classes represent different ECCS failure modes when GSCS remains operational. The 508 and 1008 fuel damage classes represent the same ECCS failure modes as the 138 and 258 fuel damage classes, respectively, but with the GSCS failed. The $67 \%$ fuel damage class represents the failure of ECCS cooling combined with GSCS degradation on account of a failure of the helium cover gas system. Finally, the ATWS fuel damage class is a special fuel damage category for events in which the RPS fails (it has its own, special uncertainty distribution and is unrelated to the condition of the ECCS and GSCS).

The uncertainty section of Table 5.2 should be read as a cumulative probability distribution. The quantile in the left column represents the probability that the true fraction of core damage in that category is less than or equal to the fraction in the adjoining column. For example, the 0.75 quantile for Large LOCAs resulting in less than 78 total fuel damage is 0.91 . This means that the analysis showed that, for the internal events analysis, the fraction of all large LOCA core damage accidents resulting in less than 78 total fuel damage was less than or equal to 0.91 in 758 of the sample members analyzed, or, conversely, that for 258 of the samples, more than 0.91 of these large LOCA core damage accidents resulted in less than 78 total fuel damage. Note that it is not correct to add the fractions on a quantile between the two core damage categories to obtain an uncertainty distribution for the fraction of the accident frequency that would have less than 408 core damage. This should be obvious because, in some cases, this would give a fraction greater than one. This is because a negative correlation exists between the distributions. Physically, this occurs because sample members that contain a large fraction of accidents in which 
less than 78 of the core is damaged must necessarily contain a smaller fraction of the other types of accidents because the results are displayed in terms of fractions and the fractions must sum to unity. All uncertainty distributions in the remainder of this chapter will be in this form.

An examination of the uncertainty results in Table 5.2 reveals a very wide uncertainty range for the two fuel damage categories printed. This wide uncertainty range is a result of the large error factors used in the Level I PRA analysis (see Reference 5-6) because these results mainly summarize the core damage results found in the Level I PRA. Most of the basic events in the Level I PRA analysis were modeled using lognormal distributions with error factors of 10, rather than error factors of 3 as is typical in commercial reactor PRA studies where there is a larger base of data to draw information from.

Another important core damage result is that the vast majority of ECCS failures actually involve only a partial failure of the ECCS. In these situations, there is a failure to get enough cooling into the core to prevent core damage because of individual valve or pump failures. These are shown as the 138 and 508 core damage categories in Figure 5.1. The positive effect of this is to reduce the amount of core damage expected in many accidents because one side of the reactor often has ECCS "success" while the other side fails. The negative effect of this is that the limited ECCS flow is more than enough to sweep many of the radionuclides out of the primary system piping and lessen the decontamination effect of the primary system on the effluent stream. ECCS fails completely in less than 108 of internally initiated core damage accidents, but when it does, the failure is almost always (more than 998 of the conditional frequency) due to the human error failure to position the 3 H33 ECCS bypass switch correctly. This can be reduced very easily with procedural or minor hardware modifications.

Graphite oxidation was found in the internal events analysis to be insignificant. In fact, based on the criteria set forth in Appendix $C$, not a single graphite oxidation event was found to have a conditional probability greater than 0.00001 . That is, given that an internally initiated core damaging accident has occurred, the probability that it will not involve significant graphite oxidation is expected to be at least 0.99999. This is not, however, true for fire and seismic accidents as will be seen in the following sections.

To this point, the discussion of results has focused on the core, rather than the confinement, but the confinement also has a major role to play in the reduction of any radioactive release. The first thing that must happen in order for the confinement to be effective in this role is for the normal confinement ventilation paths to isolate successfully. However, as depicted in Table 5.3, the failure of this function is responsible for a large fraction of the failures of the confinement to successfully perform its design function. Isolation failures in the LOCA cases are almost exclusively due to failures in the isolation mechanisms themselves, while transient isolation failures are dominated by the $3 \mathrm{H} 33$ ECCS bypass switch. In the LOCA cases, the pressure increase inside the confinement building causes confinement isolation to be demanded regardless 
of the position of the $3 \mathrm{H} 33$ switch. However, there is no such rise in transient situations, so the $3 \mathrm{H} 33$ switch effectively prevents not only ECCS actuation but also confinement isolation. This dependent failure between ECCS and confinement is a very significant accident both in terms of probability and possible consequences, but the contribution from the failure of the isolation mechanisms themselves is also notable. The dominant causes of confinement failure after successful initial confinement isolation are hydrogen events (especially in the emergency dump tank) and pressure spikes associated with a delayed recovery of ECCS. These will be discussed in more detail later in this chapter.

Uncertainty ranges are also presented in Table 5.3 for overall confinement failure and confinement isolation failure. The range covers about an order of magnitude in all cases. Isolation failure, while a significant contributor in all cases, is not dominant except in the transient initiator as described above. The width of the uncertainty can be attributed to large lognormal error factors for the initial confinement isolation failure, along with uncertainties in hydrogen and steam spike phenomena as presented in Appendix $C$.

The confinement failure results from Table 5.3 are shown in more detail in Figures 5.2 and 5.3. It is interesting to note in Figure 5.3 that the confinement initially fails to isolate in almost all of the 258 and $67 \%$ core damage accidents. These low frequency accidents are caused mainly by the $3 \mathrm{H} 33$ ECCS bypass switch and together account for no more than 78 of the total core damage frequency.

Hydrogen events are responsible for about half of those confinement failures that are not caused by confinement isolation failure, with most of the remainder being caused by steam spikes when ECCS is actuated at the time of delayed recovery. A notable fraction of the hydrogen events occur when the hydrogen generated in the core collects in the emergency dump tank (EDT) and ignites to cause EDT failure. This is shown in Table 5.4. Although it is possible for the dump tank to fail by non-hydrogen mechanisms, these mechanisms occur at least an order of magnitude less frequently than the dump tank failures seen here. Hydrogen events in the dump tank are among the more uncertain events in the APET analysis, and the treatment afforded in the APET analysis is believed to be realistic if slightly on the conservative side given the uncertainties in the underlying events. Dump tank hydrogen events are discussed in further detail in Appendix $C$.

The hydrogen mitigation system appears to have a significant effect in reducing the likelihood of hydrogen events in the confinement building itself, although it does not completely preclude their occurrence. It also does much to help a seemingly unrelated system: the filter system. The injection of nitrogen into the confinement forces a gas flow past the filter that can help to keep the filters cooled. This reduces the probability of filter failure because the filter sprays are not demanded as often as they would be if the gas flow from this system was not present. 
Table 5.3 Confinement failure* and isolation failure fractions and quantiles for the accident initiators for the internal events analysis.

\begin{tabular}{cccc} 
& \multicolumn{3}{c}{$\begin{array}{c}\text { Fraction of Accidents Involving Failure of } \\
\text { the Confinement to Achieve Initial Isolation }\end{array}$} \\
\cline { 2 - 3 } & $\frac{\text { Large LOCA }}{\text { Quall LOCA }}$ & $\frac{\text { Transient }}{0.014}$ \\
\hline 0.05 & 0.000 & $\frac{\text { Smal10 }}{0.010}$ & 0.025 \\
0.25 & 0.008 & 0.014 & 0.047 \\
0.50 (median) & 0.012 & 0.020 & 0.104 \\
0.75 & 0.018 & 0.033 & 0.294 \\
0.95 & 0.039 & 0.073 & \\
mean & 0.019 & 0.028 & 0.085
\end{tabular}

\begin{tabular}{cccc} 
& \multicolumn{3}{c}{ Fraction of Accidents Involving Confinement Failure } \\
\cline { 2 - 3 } Quantile & Large LOCA & Sma11 LOCA & Transient \\
\hline 0.05 & 0.005 & 0.018 & 0.028 \\
0.25 & 0.020 & 0.037 & 0.066 \\
0.50 (median) & 0.036 & 0.073 & 0.128 \\
0.75 & 0.066 & 0.136 & 0.208 \\
0.95 & 0.163 & 0.291 & 0.428 \\
mean & 0.055 & 0.102 & 0.159
\end{tabular}

\begin{tabular}{|c|c|c|}
\hline \multicolumn{3}{|c|}{$\begin{array}{l}\text { Percent of Confinement Failure Events Caused } \\
\text { by Initial Confinement Isolation Failure*t }\end{array}$} \\
\hline Large LOCA & Sma11 LOCA & Transient \\
\hline 358 & 278 & 538 \\
\hline
\end{tabular}

\footnotetext{
* The confinement function is defined in this table and throughout the report to include the integrity of the emergency dump tank.

* Computed as a ratio of mean values.
} 
Figure 5.2

CONFINEMENT FAILURE FOR INTERNAL INITIATORS

Mean Core Damage Frequency $=1.418^{\circ} 10^{-6}$

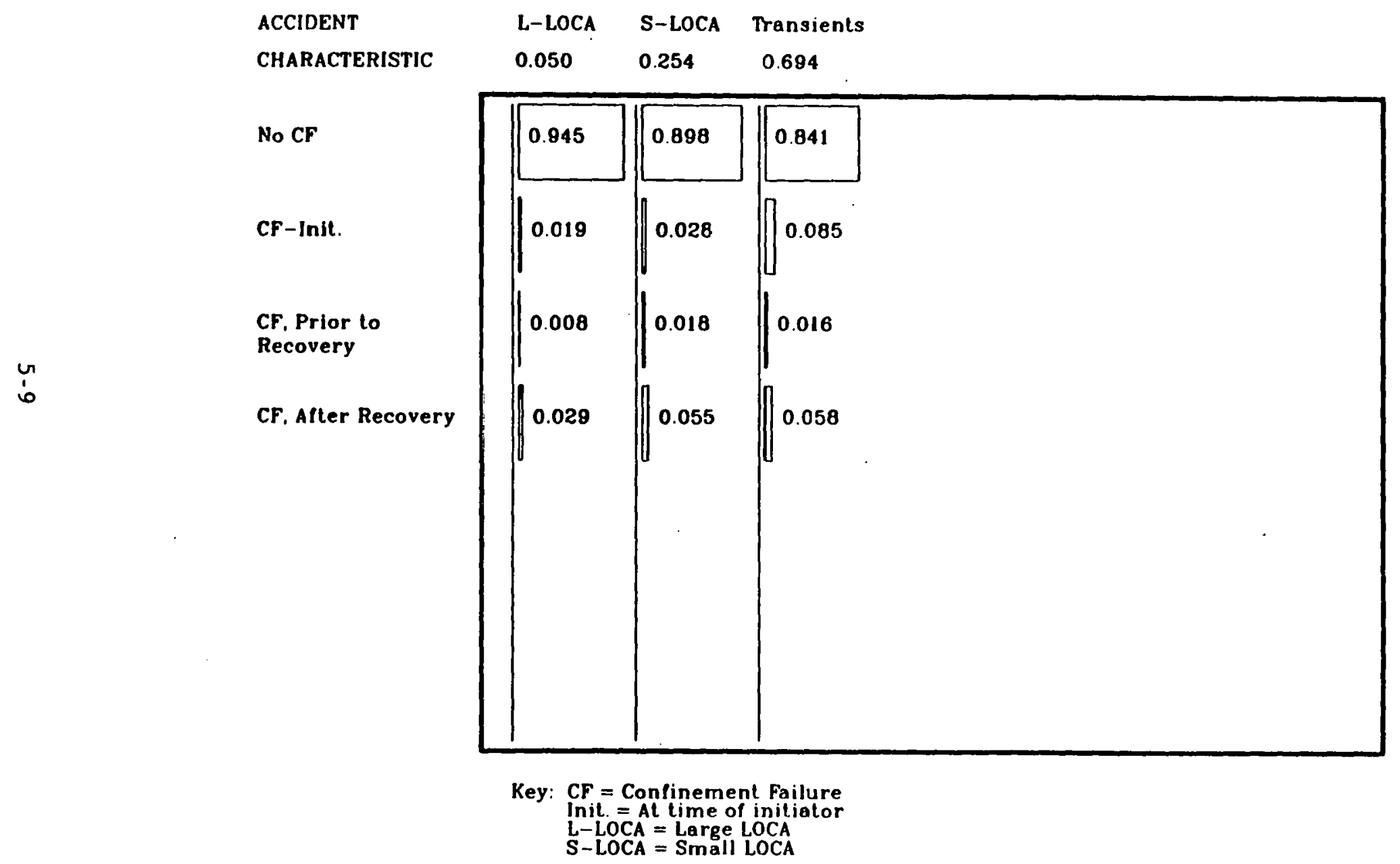


Figure 5.3

CONFINEMENT FAILURE FOR INTERNAL INITIATORS

Mean Core Damage Frequency $=1.418 \cdot 10^{-4}$

TOTAL FUEL DAMAGE

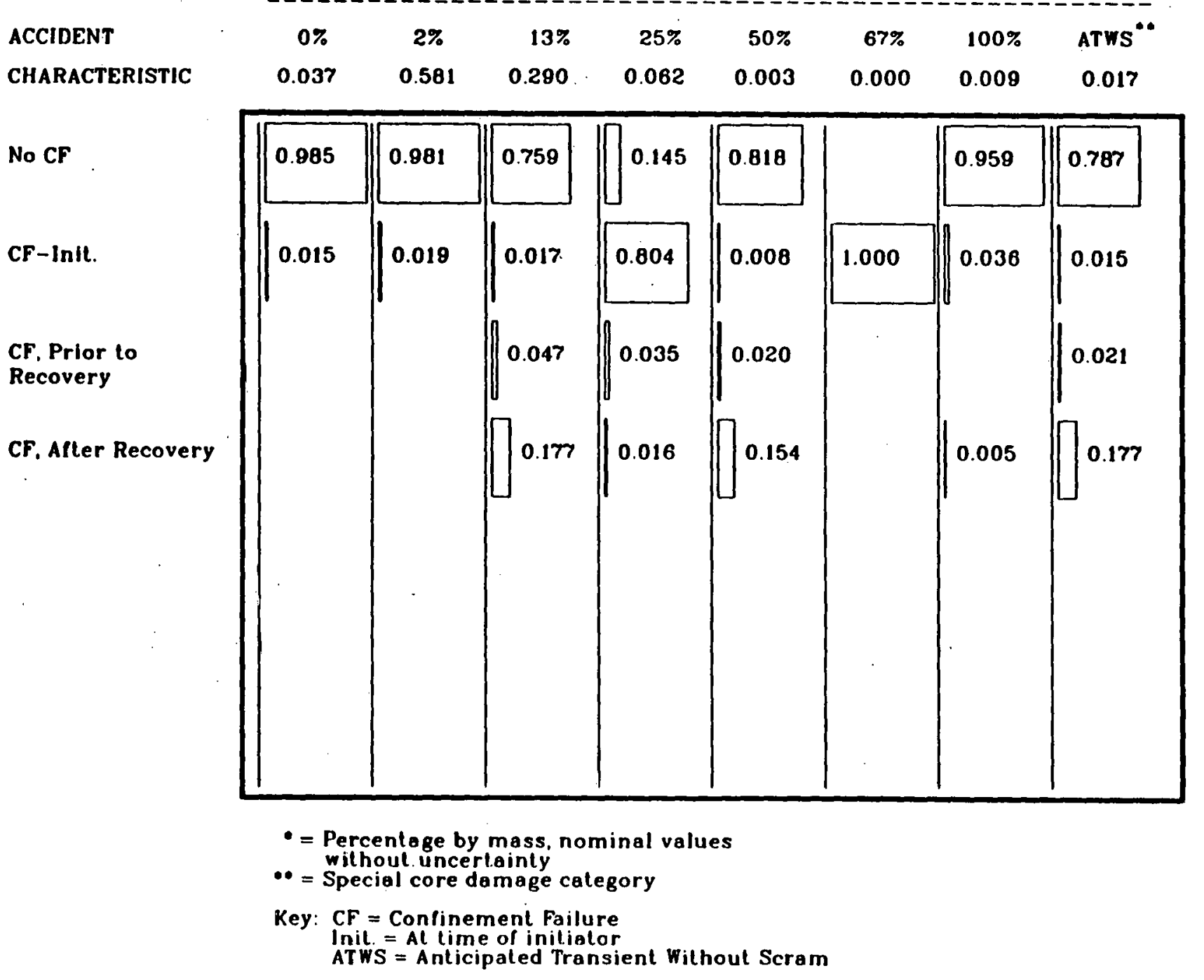


Table 5.4 Confinement and dump tank mean failure fractions for accident initiators for the internal events analysis.

\begin{tabular}{lccccc} 
Initiator & $\begin{array}{c}\text { Confinement } \\
\text { Failure }\end{array}$ & $\begin{array}{c}\text { \& Dump } \\
\text { Tank Only }\end{array}$ & $\begin{array}{c}\text { \& Building } \\
\text { Failure Only }\end{array}$ & $\begin{array}{c}\text { \& Both Tank, } \\
\text { Building Fail }\end{array}$ \\
\cline { 2 - 2 } Large LOCA & 0.055 & & 628 & 368 & 28 \\
Small LOCA & 0.102 & & 608 & 388 & 28 \\
Transient & 0.159 & & 398 & 588 & 38
\end{tabular}

* Building failure includes initial confinement isolation failure and all subsequent confinement building failures.

The status of the dump tank as a function of core damage is presented in Figure 5.4. The 258 and 678 core damage cases are again notable for the fact that the EDT is not demanded in almost all of these accidents. This is again because of the 3H33 ECCS bypass switch. In these cases, the V-4 valves never open, so there is no flow from the core to the EDT in these accidents.

Another significant finding of the APET analysis relates to the release of contaminated liquids from the plant. The mechanism for this release is as follows: the fog spray system takes water from outside the confinement building and pumps it into the building through spray heads. The operators are not instructed by their procedures to ever turn off the fog spray system, and the control room indication of water level in the confinement building sumps does not cover the range of levels expected during accident conditions. If the single diesel-driven lift station (sump pump) fails, the water will overflow the sumps in a few hours and flow out through the banana wall, and into the fuel handling area. This area has very little room for additional water and would soon overflow itself. Any overflow from this area would flow out of the building and down the embankments behind the building. It would be possible for these liquids to reach the river, although this is by no means certain because much of the water could be absorbed by the soil or held back by the embankments in that area. This scenario would occur in 1.28 of the internally initiated accidents, and in 4.48 of the accidents when the fog spray system operates. It is also very easy to take corrective action to reduce this conditional 
Figure 5.4

EMERGENCY DUMP TANK STATUS FOR INTERNAL INITIATORS

Mean Core Damage Prequency $=1.410^{*} 10^{-1}$

TOTAL FUEL DAMAGE*

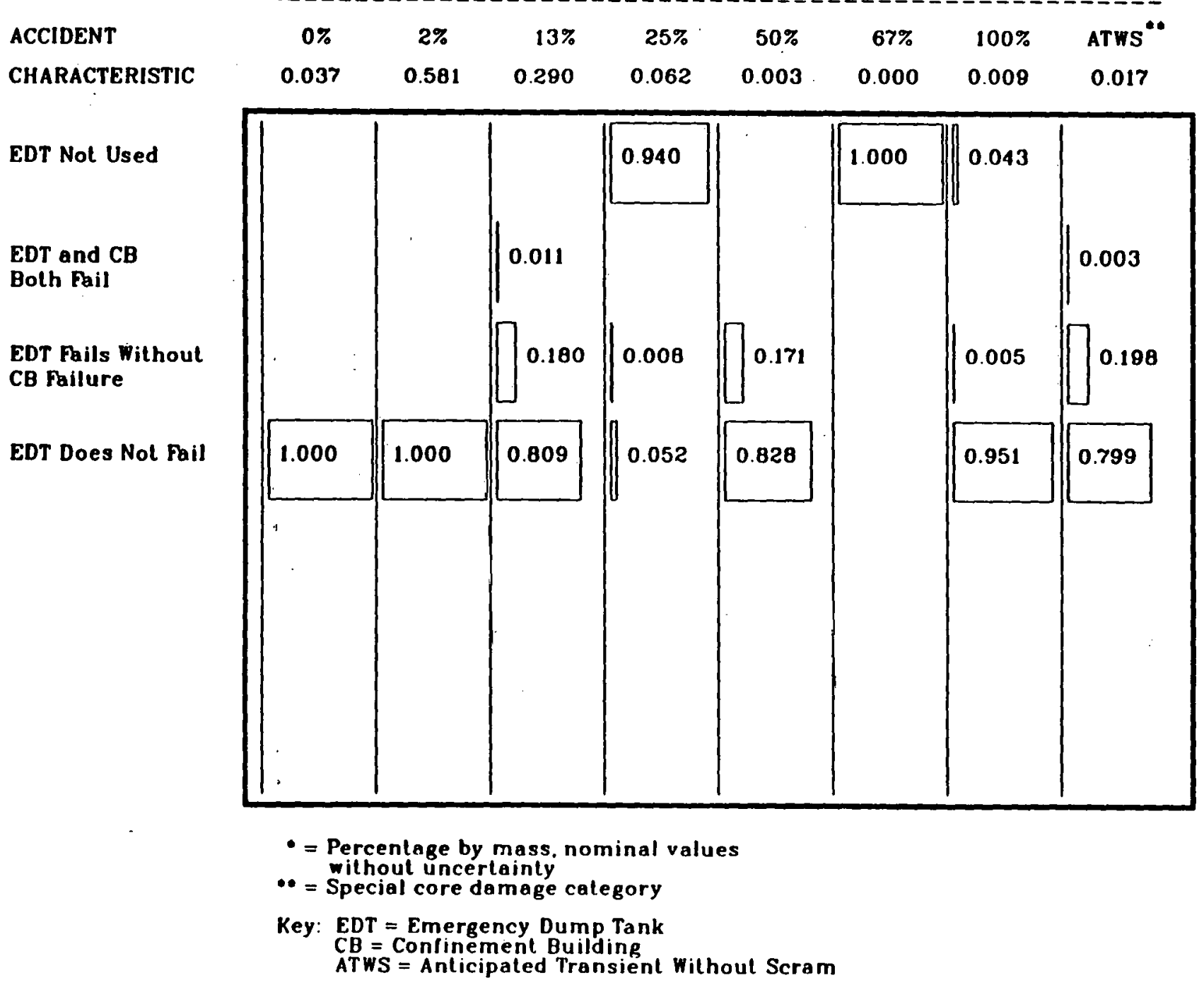


probability either by installing better monitoring equipment in the sump areas, by installing an additional sump pump for the lift station, or both. It should be noted that the assessments used in this analysis are slightly conservative in that the effects of some small drain lines are neglected. The radiological effects of such a release, as assessed for the $N$ Reactor Level II/III PRA analysis, are discussed in Chapter 5 of Reference 5-1. Further analysis of the actual liquid flow locations would have to be done to determine more exactly the extent and contamination (including dilution and plateout of the contaminants) of the liquid releases if corrective actions were to be taken in this area.

The filter system is the last barrier between the contaminated confinement and the environment for gaseous effluents. The statistics on filter failure were originally generated for use by the source term analysis and, for this reason, a confinement building failure that would bypass the filters is considered a failure of the filtration function. In reality, 658 of the "filtration failures" are confinement failures that cause effluents to be released without reaching the filters themselves. This causes the filter failure numbers to be higher than would be otherwise expected. Most of the remaining filter failures are due to the filter loading model and the action of the filter sprays as discussed in Chapter 4 and Appendix C.11.

The filtration function summarizes several separate effects: confinement building integrity, filter element loading and possible failure, filter spray operation, and filter soaking. Even after considering all of these failure mechanisms, the APET analysis revealed that the filtration function is successful in 798 of the expected internally initiated accidents. Of the remaining 218,658 actually involve confinement building failure as discussed above. The rest are filter media failures, the vast majority of which are caused by the actuation of the fllter spray system. The remaining failures are filter element failures caused either by installation errors or by random filter media failure (possibly a pre-existing filter media defect). This distribution of causes of filtration failure is expected.

A correlaliui was noted between the amount of core damage and the success of the filtration function. In Table 5.5, filter function is almost independent of accident initiator, but is strongly dependent on the amount of core damage. This correlation is due to the large decay heat loads delivered to the filters by the radionuclides released in accident progression pathways with large amounts of failed fuel. The filtration failures in the less than 78 fuel damage category are due almost exclusively to the failure of the confinement building to isolate at the beginning of the accident. This bypasses the filters. 
Table 5.5 Filtration failure mean fractions and quantiles for accident initiators and core damage amounts for the internal events analysis.

\begin{tabular}{|c|c|c|c|}
\hline \multirow[b]{2}{*}{ Initiator } & \multicolumn{2}{|c|}{$\begin{array}{l}\text { Mean Fraction of accidents } \\
\text { where filtration succeeds }\end{array}$} & $\begin{array}{c}\text { Fraction of } \\
\text { Internal Events }\end{array}$ \\
\hline & $\begin{array}{l}\text { Until } \\
\text { Recovery }\end{array}$ & $\begin{array}{l}\text { Entire } \\
\text { Accident }\end{array}$ & $\begin{array}{l}\text { Accidents Represented } \\
\text { by These Statistics }\end{array}$ \\
\hline Large LOCA & 0.868 & 0.831 & 0.050 \\
\hline Small LOCA & 0.874 & 0.825 & 0.255 \\
\hline Transient & 0.812 & 0.772 & 0.695 \\
\hline Core Damage & & $\therefore$ & \\
\hline$<78$ Core Damage & 0.980 & 0.980 & 0.617 \\
\hline 7 - 408 Core Damag & 0.581 & 0.501 & 0.352 \\
\hline$>408$ Core Damage & 0.323 & $0.000 *$ & 0.029 \\
\hline
\end{tabular}

\begin{tabular}{cccc} 
& \multicolumn{3}{c}{ Fraction of Accidents with } \\
Quantile & \multicolumn{2}{c}{ Filtration } & Succeeding until Recovery \\
\cline { 2 - 4 } & $\frac{\text { Large LOCA }}{0.05}$ & $\frac{\text { Small LOCA }}{0.557}$ & $\frac{\text { Transient }}{0.389}$ \\
0.25 & 0.594 & 0.842 & 0.745 \\
0.50 (median) & 0.939 & 0.936 & 0.880 \\
0.75 & 0.954 & 0.968 & 0.947 \\
0.95 & 0.983 & 0.984 & 0.977 \\
mean & 0.868 & 0.874 & 0.812
\end{tabular}

\begin{tabular}{cccc} 
& \multicolumn{3}{c}{ Fraction of Accidents with } \\
Quantile & Filtration Succeeding During Entire Accident \\
\cline { 2 - 4 } 0.05 & $\frac{\text { Large LOCA }}{0.491}$ & $\frac{\text { Small LOCA }}{0.414}$ & $\frac{\text { Transient }}{0.272}$ \\
0.25 & 0.792 & 0.749 & 0.693 \\
0.50 (median) & 0.875 & 0.896 & 0.851 \\
0.75 & 0.918 & 0.952 & 0.932 \\
0.95 & 0.958 & 0.978 & 0.970 \\
mean & 0.831 & 0.825 & 0.772
\end{tabular}

\footnotetext{
- The uncertainty analysis assessed that the filter sprays would always be demanded late in accidents where GSCS failed because of an excessive fission product and decay heat loading. This table does not consider the delayed core damage accident.
} 
The uncertainty in filter system performance is driven principally by the uncertainty in the spray and dump tank DFs required to insure that filter cooling is sufficient. These uncertainties are compounded by the fact that the spray and dump tank DFs themselves are uncertain and can vary by orders of magnitude. The issue of filter cooling is discussed in Appendix C.11. Additional uncertainty is due to the fact that there is a very strong correlation between filter failure and the amount of fuel damage because of filter loading issues. The filters are expected to operate normally in single riser accidents unless they are bypassed, yet, the fraction of single riser accidents in the analysis was shown in Table 5.2 to vary from 0.11 to 0.90 for the transient accident initiator. This wide variation is due to the large error factors used in the Level I PRA analysis. This strongly influences the uncertainty in filter performance because it determines whether or not filter loading is such that cooling can become a problem.

The filtration results are shown in more detail in Figure 5.5. The 1008 core damage column is especially worthy of note because the filters operate throughout the accident 638 of the time. This represents the delayed core damage accidents as discussed in Appendix C.4. These accidents develop very slowly, and would take many times longer to release radionuclides from the core than would the immediate 1008 fuel damage accidents. This translates to a longer period of time to load the filters and a reduced decay heat load on the filters as they are loaded. This, in turn, reduces the possibility that the filter sprays will actuate and cause failure of the filtration function. For this reason, it was assessed that the delayed core damage accident is not likely to fail the filters -especially within the 24 hour time frame of the APET analysis.

In summary, the internal events APET analysis revealed several important items about potential radioactive releases from $N$ Reactor. First, the 3 H33 ECCS bypass switch is a significant contributor to both core damage and confinement failure because it defeats both the ECCS actuation and confinement isolation functions in transient accident sequences. Second, a significant number of accidents do not proceed to assessable core damage, and more than 968 of the internally initiated core damage accidents damage less than half of the core. Third, about half of all confinement failures are caused by failure of the confinement to initially isolate and not by accident progression events. Fourth, the filter system operates successfully about 808 of the time, and, for many accidents where it fails, it fails only because the confinement building does not remain intact to prevent the filters from being bypassed by the effluent gas stream. Fifth, the uncertainty in many sections of the APET analysis, especially total fuel damage, is dominated by the uncertainty in the internal event Level I PRA basic events. Finally, 4.48 of the internally initiated core damage accidents in which the fog spray system functions result in liquid releases from the plant that could enter the Columbia River. This problem, however, is easily fixed by adding either a backup to the existing diesel lift station sump pump, improved sump water level monitoring capabilities, or both. 
Figure 5.5

FILTER PERFORMANCE FOR INTERNAL INITIATORS

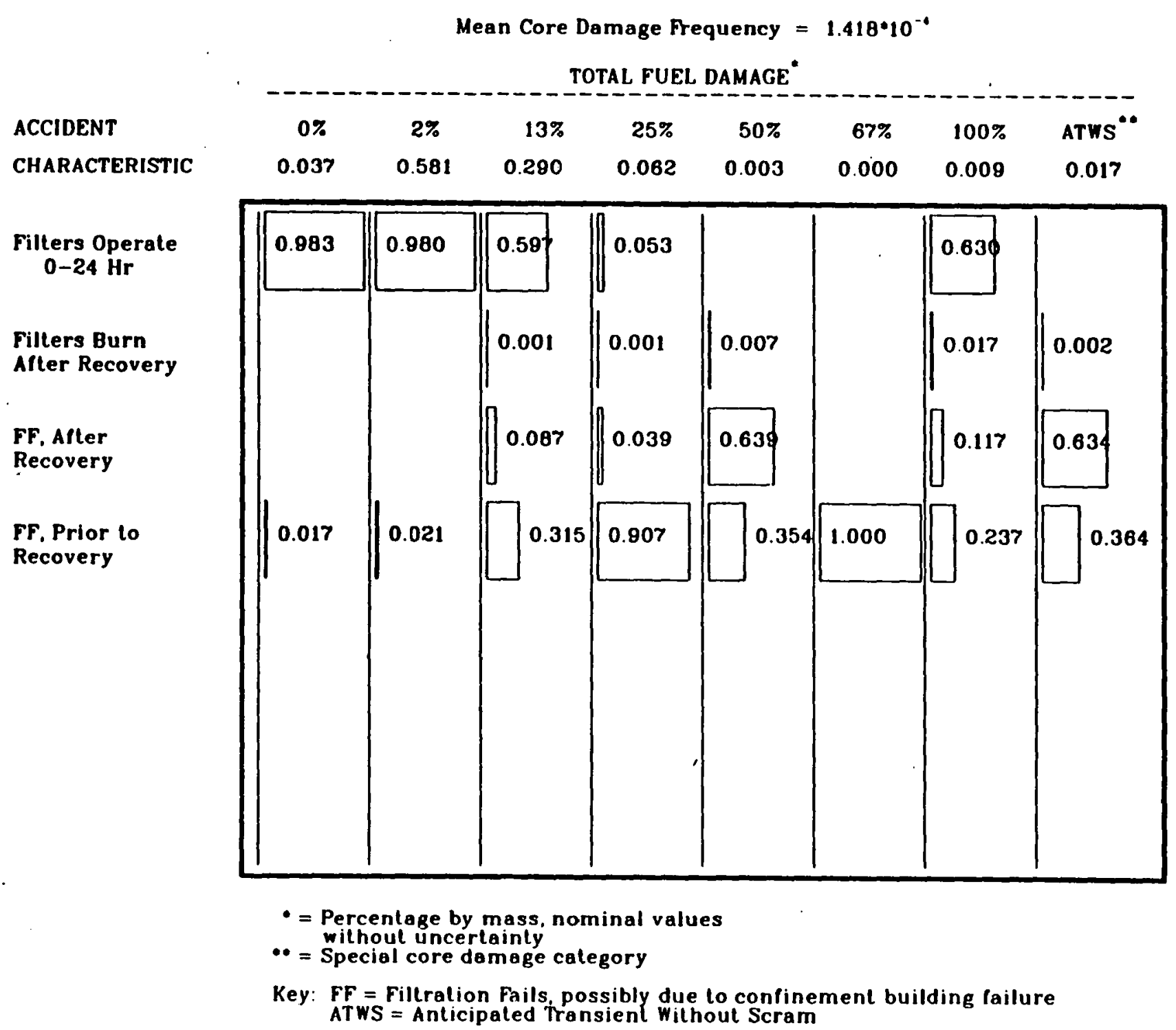




\subsection{ACCIDENTS INITIATED BY FIRES}

The $N$ Reactor fire analysis differs in many major ways from the internal events analysis. While 500 Latin Hypercube sample members were considered in each analysis, all accidents initiated by fires were of the "transient" type as assessed in Reference 5-5. Also, since the most frequent core damaging fires (Corridor 19, the 109 Building basement, the Main Control Room, and Building 105 Corridor 1) disrupt both ECCS and GSCS, more than half of the fire-initiated core damage sequences proceed to 1008 core damage. In addition, fires in locations such as Building 105 Corridor 1 damage primary coolant system, ECCS, GSCS and confinement isolation cabling. This means that a single fire can both cause core damage and prevent the confinement from isolating to contain the radioactive releases. This is similar to the $3 \mathrm{H} 33$ ECCS bypass switch in the internal events analysis, although that did not disable GSCS. The frequencies and descriptions for the different locations where fires can cause core damage are described in Chapter 4.

The analysis of core damage induced by fire events [5-5] was performed in accordance with standard fire analysis methods. One particular aspect of this analysis methodology that should be noted here is that it is impossible to determine ahead of time whether a fire will cause control cables to fail as "open" circuits or as hot short circuits. In fact, it is possible that there will be cross-connections between the control circuits for various systems whose cables run through the same cable trays. Since these effects are impossible to predict, it is typically assumed that all systems that are controlled by cables that are affected by fire are rendered inoperable by the fire. This is a conservative approach that can result in seemingly strange combinations of systems being declared inoperable, but it would not be possible to prove that any less conservative approach had actually considered the worst possible combination of system failures that could arise from such a fire.

Westinghouse Hanford has committed to the installation of fire protection improvements in a number of areas of the plant including Corridor 19 and the main control room. This analysis was performed as if those improvements had already been installed. The effects of those improvements were to significantly reduce the overall fire core damage frequency and to reduce the fraction of core damaging fires that would be expected to lead to 1008 core damage. The results of this portion of the analysis would not be valid if $\mathrm{N}$ Reactor were to operate without these fire protection improvements first being completed.

The $\mathrm{N}$ Reactor fire analysis contains a higher frequency of 1008 core damage events than does the internal events analysis. In the fire analysis, approximately 868 of the fire-induced core damage events result in 1008 core damage, while, for the internal events analysis, the figure was less than 28. This is because a few of the major fire areas contain cables that, if damaged, would prevent both the ECCS and GSCS from functioning. Most of the remaining frequency is in $1 / 16$ core accidents in which the fire fails the primary system and, coincident with the fire, one 
CV-2R fails to open on the ECCS actuation. ATWS events, on the other hand, accounted for approximately 18 of all internal events core damage frequency while no ATWS accidents were found in the fire analysis (aside from random RPS failure which, when combined with a fire initiator, would be more than 10,000 times less likely than the other fire core damage accidents).

The uncertainty in the amount of fuel damage produced during fire accidents is shown in Table 5.6. In some sense, the uncertainty is as wide as in the internal events analysis, although the uncertainty is between very small numbers and is, thus, far less significant. The major uncertainty in the fire analysis is in the fire initiation frequency for the different plant areas. Since fires in several plant areas would proceed to 1008 core damage, the uncertainty in the fraction of accidents that proceed to that core damage state is relatively small.

Table 5.6 Core damage uncertainty for fire-initiated accidents.

\begin{tabular}{ccc} 
& \multicolumn{2}{c}{$\begin{array}{c}\text { Fraction of Fire Accidents } \\
\text { and Core Damage Classes }\end{array}$} \\
\cline { 2 - 3 } Quantile & $\leq 78$ Damage & $\geq 408$ Damage \\
\hline 0.05 & 0.004 & 0.516 \\
0.25 & 0.018 & 0.808 \\
0.50 (median) & 0.057 & 0.922 \\
0.75 & 0.148 & 0.969 \\
0.95 & 0.422 & 0.992 \\
mean & 0.116 & 0.860
\end{tabular}

Another difference from the internal events analysis is that almost all fire-related ECCS failures are total ECCS failures, while, for the internal events analysis', almost all failures were partial failures. The reason for this is that control cables for similar equipment tend to run together and are susceptible to common fire damage while the internal events analysis ECCS failures are almost always failures of individual components. An example of this is Corridor 19. The control cables for all ECCS diesel pumps and the high pressure injection pumps, among other things, run through that corridor leaving all to be effected by a single fire event.

The vast majority of fire-induced core damage events would occur such that delayed ECCS recovery would be possible. It is possible, for example, to start the ECCS diesel pumps manually in Building $182 \mathrm{~N}$ even if the control cables in Corridor 19 are lost. Since almost all fire-induced accidents involve a complete loss of cooling to the core, the operators would certainly be tempted to start the pumps locally to avoid an unmitigated core heatup and the possibility of graphite oxidation. If 
delayed recovery of ECCS were to be attempted, it would be done soon after the onset of core damage. The results of the delayed recovery as predicted by the APET analysis are notable, and are discussed below.

The internal events analysis showed that the confinement survived the entire accident nearly 868 of the time. The confinement survives in 598 of fire-induced core damage accidents, but only 248 of the confinement failures occur prior to the recovery phase accident progression. The reason for the large number of late confinement failures is that delayed ECCS recovery occurs often and ECCS water is dumped onto an overheated core. This damages pressure tubes and creates a steam spike that can overpressurize the confinement to cause failure. This analysis was conservative because the delayed ECCS recovery would be likely to occur earlier and with the core at lower temperatures than those assumed for the internal events analysis. This is because many of the fires effect the function of the diesel pumps, and these failures can be recovered in the $182 \mathrm{~N}$ Building.

Confinement status as a function of core damage is shown in Figure 5.6. The uncertainty in confinement survival for fire-initiated accidents is shown in Table 5.7. Uncertainty about hydrogen events and steam spikes from ECCS recovery contribute to the uncertainty in confinement survival. The ECCS recovery issue plays a strong role in the uncertainty about late confinement failures because the uncertainty distribution for the recovery event itself is bimodal (that is, it has two peaks). This causes a spread in the tails of the uncertainty distribution of those events that are dependent on ECCS recovery. The recovery uncertainty distribution is discussed further in Appendix $C$.

Table 5.7 Confinement survival uncertainty for fire-initiated accidents.

\begin{tabular}{ccc} 
Quantile & $\begin{array}{c}\text { Fraction of Fire-Induced Core } \\
\text { Damage Accidents in which } \\
\text { Confinement Function is Successful }\end{array}$ \\
\cline { 2 - 3 } 0.05 & $\frac{\text { Until Recovery }}{0.319}$ & $\frac{\text { Ful1 Accident }}{0.226}$ \\
0.25 & 0.530 & 0.422 \\
0.50 (median) & 0.710 & 0.598 \\
0.75 & 0.873 & 0.758 \\
0.95 & 0.976 & 0.902 \\
mean & 0.896 & 0.587
\end{tabular}


Figure 5.6

CONFINEMENT FAILURE FOR FIRE INITIATORS

Mean Core Damage Frequency $=1.939{ }^{\circ} 10^{-0}$

TOTAL FUEL DAMAGE

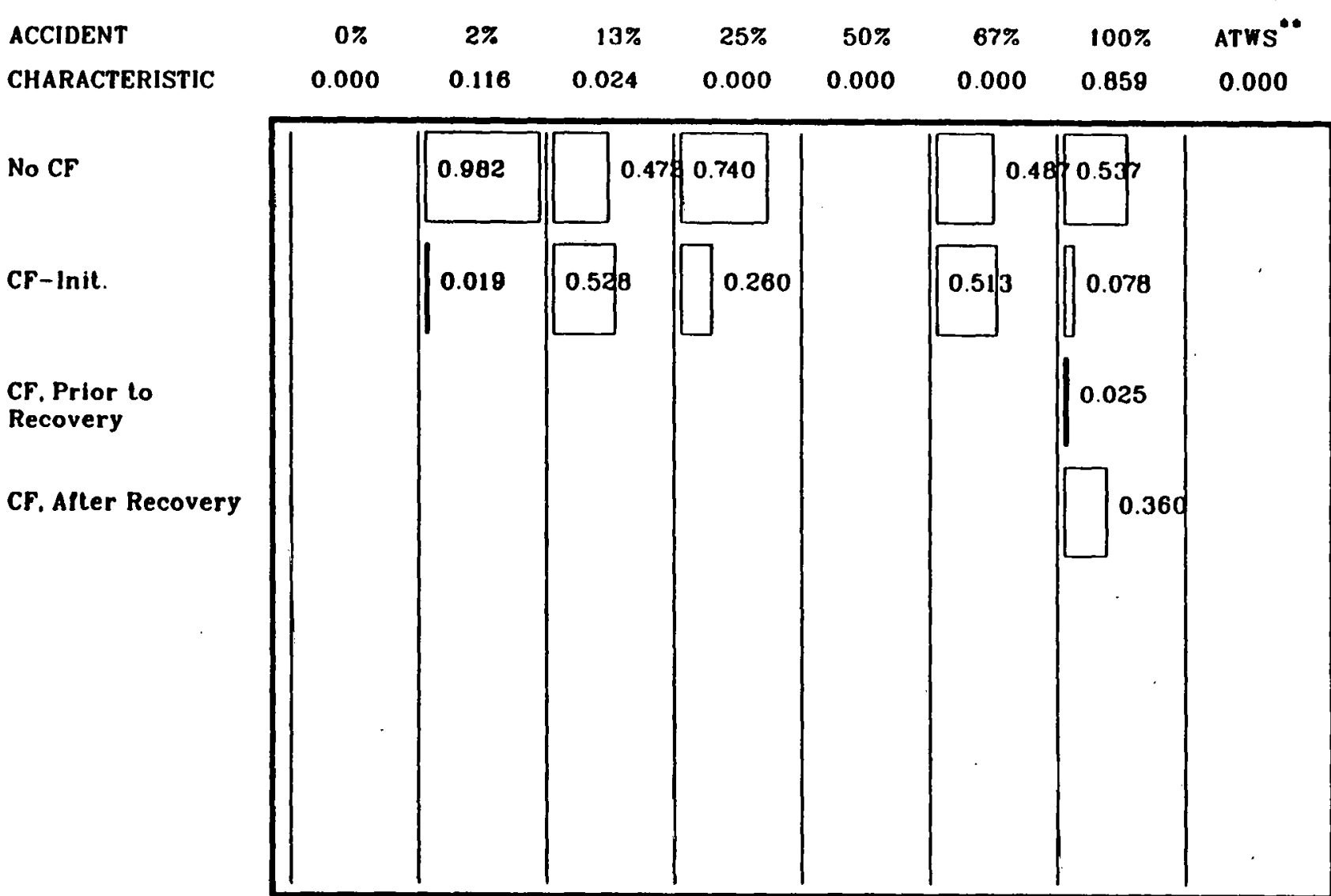

- = Percentage bj mass, nominal values

** = Special core damage category

Key: $\mathrm{CF}=$ Confinement Failure

ATWS = Anticipated Transient Without Scram 
Another significant effect of the total cooling failures that are predicted to dominate the fire analysis is that graphite oxidation is predicted to occur on the average in 0.38 of all fire-initiated core damage accidents. This is based on the assessment in the uncertainty analysis that graphite oxidation would occur in approximately 108 of the accidents in which both GSCS and ECCS remain completely non-functional during the entire accident. Delayed ECCS recovery was assessed to essentially preclude self-sustaining graphite oxidation by cooling the core and inerting the graphite stack of oxygen even if it could produce a steam spike to fail the confinement building. The uncertainty distribution assigned to delayed ECCS recovery under this situation represents the conscious decision between these two possibilities by the operators on duty at any given time.

It should be noted that successful nitrogen inerting of the confinement atmosphere (nitrogen inert system operation along with confinement isolation) by the hydrogen mitigation system was assessed to preclude graphite oxidation because of the lack of oxygen in the confinement atmosphere to support the graphite oxidation process. The success of the hydrogen mitigation nitrogen injection system reduced the frequency of graphite oxidation events by up to an order of magnitude when compared to an analysis of the plant without nitrogen injection. Clearly this is a significant change that reduces the overall plant risk.

The emergency dump tank also fails more often in the fire analysis than in the internal events analysis. The dump tank fails in an average of 178 of the fire accidents. Most dump tank failures here are associated with hydrogen combustion events as they were in the internal events analysis. The failure frequency is higher here because the average amount of fuel damage (and, hence, hydrogen production) is much greater here than in the internal events analysis. This frequency could be reduced if the dump tank were inerted with a nitrogen flow as is currently done for the remainder of the confinement.

The dump tank failure results for fire-induced core damage are shown graphically in Figure 5.7. The high fraction of accidents in the "EDT is not used" category for the 138, 258 and 508 core damage categories is due to a low frequency category of fires that damage the control cables for the $V-4$ valves. These fires prevent the V-4 valves from opening and, as a result, prevent liquid from going to the dump tank.

Liquid releases due to lift station diesel pump failure occur in 0.78 of fire accidents, as opposed to 1.28 of the internal events accidents. This is because all fire-induced accidents are transients, and transients do not achieve the confinement pressures needed to demand fog spray operation unless a pressure tube rupture occurs. In addition, damage occurs to fog spray control cables in some fire accidents to prevent fog spray actuation even if pressures would normally demand them. It should be noted that the lift station diesel pumps still failed in 4.48 of the accident pathways for which they were demanded. 
Figure 5.7

EMERGENCY DUMP TANK STATUS FOR FIRE INITIATORS

Mean Core Damage Frequency $=1.939 \cdot 10^{-6}$

TOTAL FUEL DAMAGE

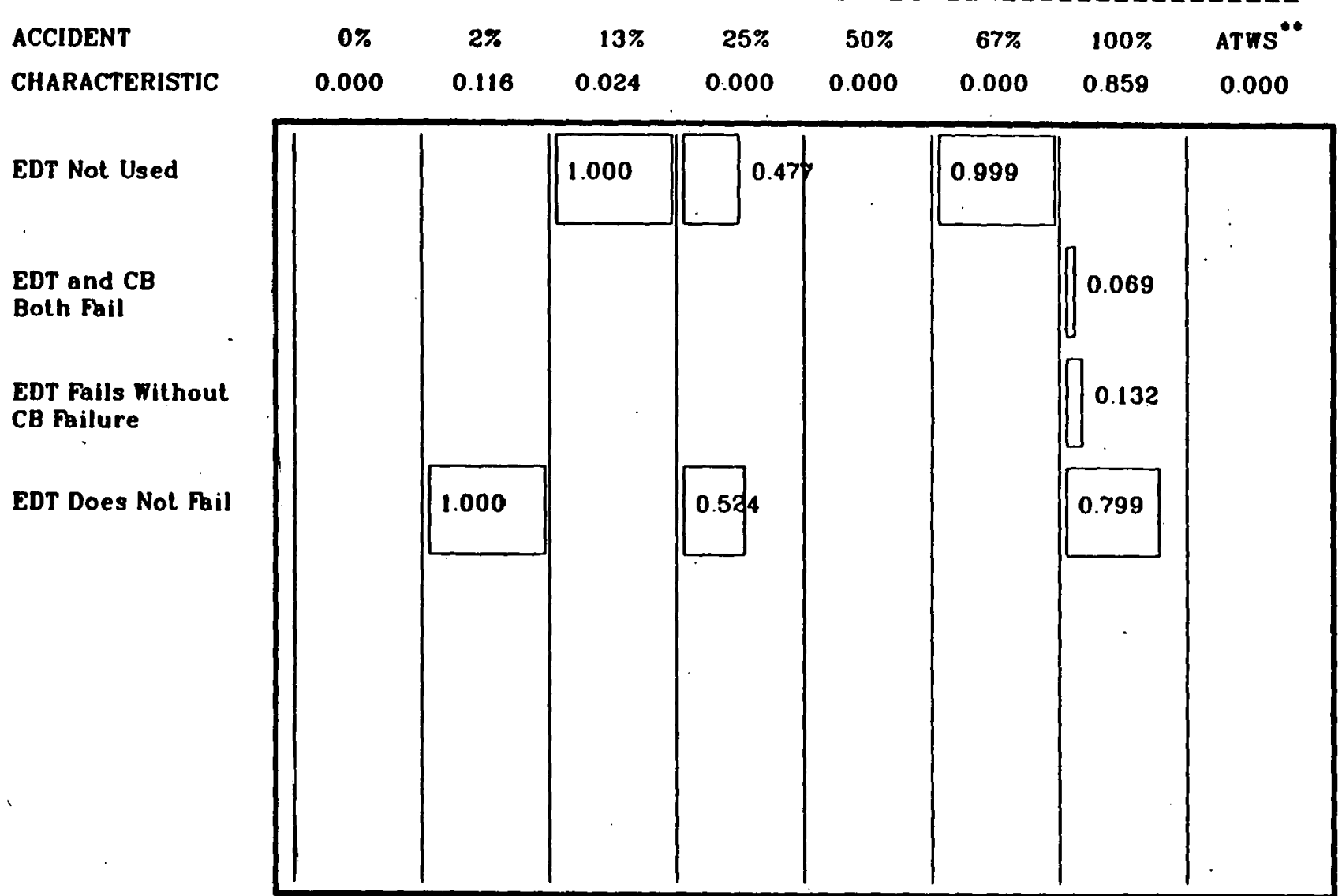

- = Percentage by mass, nominal values without uncertainl.y

- = Special core damage category

Key: EDT = Emergency Dump Tank

CB = Confinement Building
ATWS = Anticipaled Transient Without Scram 
The large fraction of fire events with 1008 fuel damage resulted in increased filter failure over the internal events analysis. The APET analysis predicts that the filters will survive the entire accident in 128 of fire induced accidents (almost all of these accidents affect only a single riser and, thus, do not severely load the filters). The filtration function succeeds until recovery in 698 of the fire events, while the filtration function fails prior to recovery in the remaining 318 . This is because 868 of fire-induced core damage accidents are expected to proceed to 1008 core damage. The higher filtration failure rate is expected because the uncertainty analysis assessed that the filter sprays would always be demanded late in all 1008 core damage accidents because of an excessive fission product and decay heat loading (see Appendix C.11).

The uncertainty in filter performance for the fire analysis is presented in Table 5.8. Filter survival throughout the accident is strongly correlated to the amount of core damage because of the high loading demands placed on the filters during 1008 core damage accidents. Filter survival to the time of recovery is almost totally correlated to the uncertainty in the spray and dump tank DFs required to insure that filter cooling is sufficient. The spray and dump tank DFs themselves are uncertain and can vary by orders of magnitude to add to the uncertainty in early filter performance.

Table 5.8 Filtration success uncertainty for fire-initiated accidents.

\begin{tabular}{ccc} 
& \multicolumn{2}{c}{$\begin{array}{c}\text { Fraction of Fire Accidents } \\
\text { and Filtration Success }\end{array}$} \\
\cline { 2 - 3 } Quantile & To Recovery & Full Accident \\
\hline 0.05 & 0.121 & 0.005 \\
0.25 & 0.460 & 0.021 \\
0.50 (median) & 0.818 & 0.063 \\
0.75 & 0.949 & 0.162 \\
0.95 & 0.978 & 0.420 \\
mean & 0.691 & 0.121
\end{tabular}

The APET analysis for $\mathrm{N}$ Reactor accidents induced by fires shows much more core damage, confinement failure and filter failure than did the internal events analysis. The most significant fire locations from a frequency perspective are Corridor 19 and the main control room. These areas together account for more than $80 \%$ of the total mean core damage frequency. Fires in either of these locations damage the primary cooling system, ECCS, and GSCS (Corridor 19 also damages the fog sprays) and result in $100 \%$ coite damage. While this analysis was performed using core damage frequencies that are based on fire protection improvements in Corridor 19 
that have been committed to by WHC, this corridor is still expected to be the most likely place for a core-damaging fire to occur (about half of the total fire frequency). It is important to note, however, that confinement isolation functions would not be affected by fires in either of these locations. This significantly mitigates any release that might occur from such an event.

\subsection{ACCIDENTS INITIATED BY SEISMIC EVENTS}

The seismic APET analysis was more like the internal events analysis in that a wide variety of accidents were analyzed for both LOCA and transient accident initiators. In reality, five different accident initiators were considered: transients (including loss of offsite power and others), seismic failure of Building 182N, small LOCA, large LOCA and multiple pressure tube rupture (MPTR) events. The MPTR event is somewhat similar to the reactor vessel rupture event in commercial light water reactors. It is caused by relative motion between the core graphite stack and the shield walls that surround the reactor that can shear off large numbers of pressure tubes and GSCS tubes at the shield wall to produce a total cooling failure or, alternatively, by the gross collapse of Building $105 \mathrm{~N}$ to fail all cooling systems as they enter the core.

The five accident initiators were grouped into three general ground acceleration categories:

low ground acceleration: medium ground acceleration: high ground acceleration:

Transients, Building $182 \mathrm{~N}$ and small LOCAs, and large LOCAs and MPTR events,

and into three APET primary system configuration categories:

Transient: small LOCAs: large LOCAs:
Transients and Building $182 \mathrm{~N}$ events, small LOCAs, and large LOCAs and MPTR events.

The primary system configuration categories were chosen based on the existence and size of the breach in the primary system. Thus, for example, the transient primary system configuration represents those seismically induced core damage accidents in which no LOCA or primary system breach occurs.

The ground acceleration categories were chosen to allow for different failure probabilities for certain confinement systems based on the strength of the seismic event. Each initiator was placed into the category where it most frequently occurred. The seismic ground acceleration categories are defined based on representative ground accelerations. These ground accelerations and the mean value of the frequency with which a seismic event of that magnitude or greater would be expected are shown in Table 5.9 [5-5]. As can be seen from the table, the seismic events that would be expected to cause core damage are exceedingly rare and very severe. 
Table 5.9 N Reactor ground acceleration categories and mean annual frequency of exceedence [5-5].

\section{Ground Acceleration Class}

N Reactor Safe Shutdown Earthquake (SSE) [5-7]

low ground acceleration medium ground acceleration high ground acceleration
Representative

Peak Ground

Acceleration
Mean Annual Frequency
of Exceedence

* 5.0E-05 - $5.0 \times 10^{-5}$, and should be interpreted as follows: based on the mean seismic hazard curve [5-5], it is expected that a seismic event of magnitude $0.25 \mathrm{~g}$ or greater would be expected to occur with a probability of 5.0E-05 each year, or equivalently, approximately once each 20,000 years.

The average initiator fractions for the seismic analysis are presented in Table 5.10. That table shows a strong correlation between ground acceleration and the amount of fuel damage that occurs. It should be noted that a few single riser accidents were presented in the external events core damage report [5-5], but these were neglected from the APET seismic analysis because of their very small frequencies and the low consequences associated with single riser accidents. Reference 5-5 found no seismically induced core damage accidents in which the rearton protection system fails, so ATWS events are not analyzed in the seismic APET analysis. The amount of fuel damage for each primary system configuration is shown graphically in Figure 5.8 .

The dominant cause of uncertainty in this table is the underlying uncertainty in the seismic hazard curve. These curves represent the frequency with which earthquakes of a given magnitude occur. The effects of all other uncertainties on the fuel damage, such as random component failures, are dominated by the hazard curvo. 
Table 5.10 Accident initiator and fuel damage fraction means and quantiles for the seismic APET analysis.

\begin{tabular}{lc}
$\begin{array}{l}\text { Primary System } \\
\text { Configuration }\end{array}$ & $\begin{array}{l}\text { Fraction of Core } \\
\text { Damage Frequency }\end{array}$ \\
\cline { 1 - 1 } Large LOCA* & 0.190 \\
Small LOCA & 0.136 \\
Transient & 0.674
\end{tabular}

\section{Quantile}

\subsection{5}

0.25

0.50 (median)

0.75

0.95

mean

\section{Fraction of accidents with $<408$ fuel damage}

Large LOCA*

Sma11 LOCA

0.000

0.000

0.460

1.000

1.000

0.387
Transient

0.001

0.025

0.100

0.477

0.982

0.263

* In this and the following tables in this section, transient includes Building 182N collapse, and large LOCA includes MPTR events. 
Figure 5.8

TOTAL FUEL DAMAGE FOR SEISMIC INITIATORS

Mean Core Damage Frequency $=1.671^{\circ} 10^{-4}$

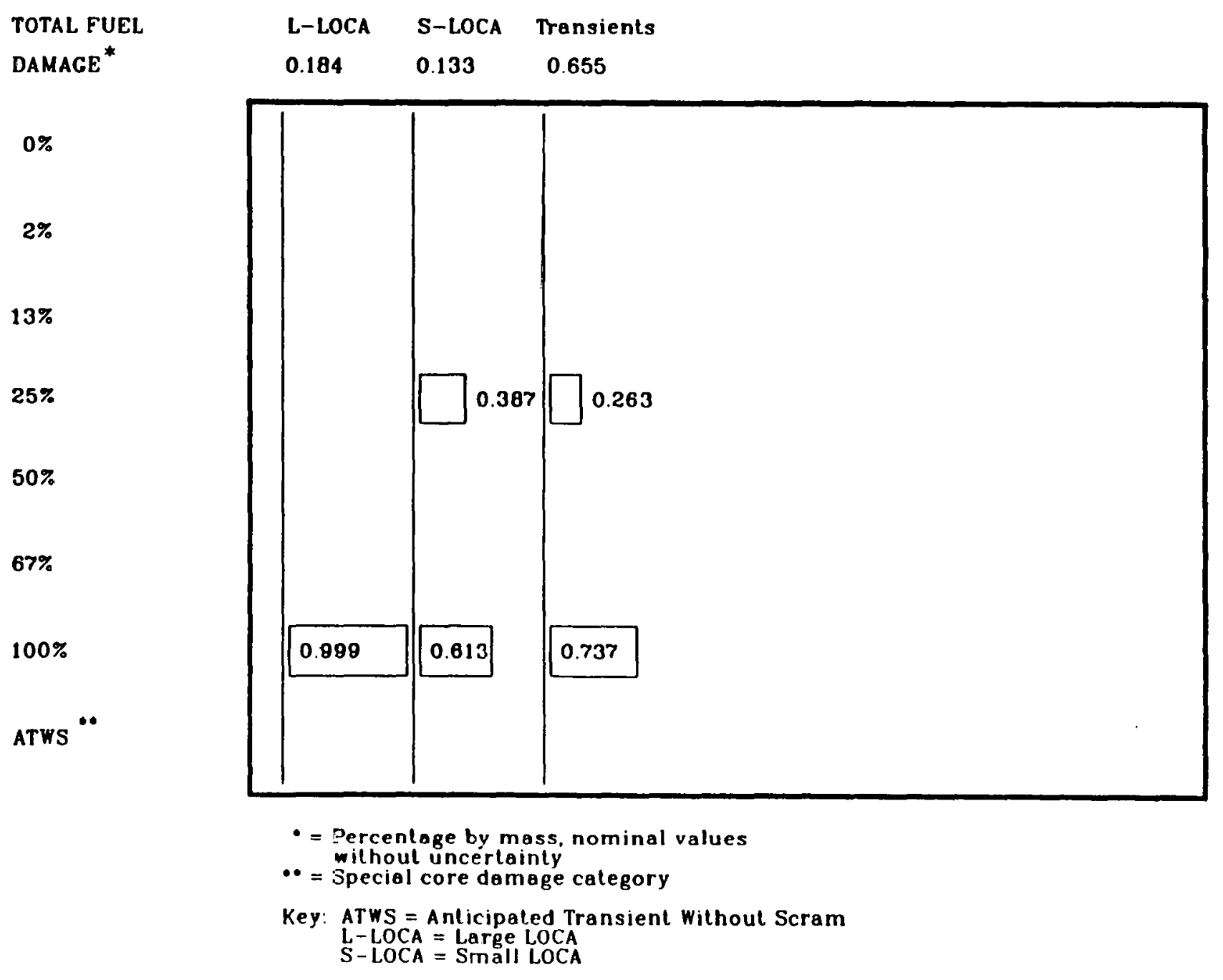


Table 5.11 examines the effects of various tank failures on the overall reactor accident. While the silo is a tank that fails in most seismic core damage accidents, it is noticeably absent from this table because its failure is analyzed in the external events core damage analysis [5-5] as a contributor to the overall seismic core damage frequency. Silo failure destroys the source of water for all once-through cooling systems for the duration of the accident. In cases where GSCS succeeds, its success is typically in the recirculation mode. The other major cause of ECCS failure is collapse of Building 182N. This building contains the fog spray diesel pumps, the ECCS diesel high lift pumps and the high pressure injection pumps. Clearly neither of these failures are recoverable because either the pump house or the common single source of coolant is damaged.

Table 5.11 Tank failure fractions by primary system configuration for the seismic APET analysis.

$\begin{array}{lccc}\begin{array}{l}\text { Primary System } \\ \text { Configuration }\end{array} & \begin{array}{c}\text { Fraction with } \\ \text { EDT Failure }\end{array} & \begin{array}{c}\text { Fraction with } \\ \text { Nitrogen Tank Failure }\end{array} \\ \text { Large LOCA } & 0.99 * & 0.95 \\ \text { Small LOCA } & 0.59 & 0.49 \\ \text { Transient } & 0.49 & 0.02\end{array}$

This number includes only cases where water flows to the EDT. In 998 of large LOCA accidents, water does not flow to the EDT because upstream piping inside confinement ruptures, diverting flow away from the EDT. This reduces the effect of EDT failure on the confinement failure results in Table 5.12.

The EDT and the hydrogen mitigation system nitrogen tanks would both be expected to survive the $\mathrm{N}$ Reactor SSE $(0.25 \mathrm{~g})$. A preliminary assessment of these tanks indicated that they could be classed as $0.5 \mathrm{~g}$ structures. This was translated into the ground acceleration categories considered in the APET analysis (see Appendix C.13) and the probabilities of failure for both tanks were assessed to be similar with an average failure probability of 0.01 for low, 0.50 for intermediate, and 0.99 for high ground accelerations. The correlation between tank failure and ground acceleration can be seen for both the nitrogen tanks and the EDT. The EDT failure probabilities listed in Table 5.11 are often higher than the "random" failure values because of failures caused by hydrogen combustion events. A fundamental assumption of this analysis is that failure of a nitrogen tank does not cause additional damage. It is possible that the 
failure of the tank at high pressures would be violent enough to damage other structures, but since the tanks were not installed at the time of the analysis, the likelihood of this occurring could not be assessed, although it is our understanding that the tanks are not to be located near the confinement or $182 \mathrm{~N}$ buildings. The validity of this assumption would need to be checked after installation of the system is complete.

The function of the EDT is shown for each primary system configuration in Figure 5.9. It is worth noting that the EDT is not used in many of the large LOCA primary system configuration accidents. In the previous analyses, this has always been related to failure of the $V-4$ valves to open. In this case, it is related to MPTR accidents. In these accidents, the piping is sheared off around or inside the reactor itself. This prevents water from getting out of the reactor and to the EDT.

The fraction of the accident frequency for which the confinement fails is also well correlated to the ground acceleration as shown in Table 5.12 and Figure 5.10 below. It should be noted that confinement success is defined to include the proper functioning of the EDT, so these figures do not reflect the actual fraction of events with building failure, but overall, the confinement building survived intact about $27 \%$ of the time. Most late confinement failures are due to hydrogen events because of the failure of the nitrogen tanks in the hydrogen mitigation system. It must be remembered that the term "initial confinement failure" represents all of the following: failure of the building to isolate, initial failure of the EDT (when it is used in the accident), or gross failure of the confinement building as a result of the seismic event.

There is one apparent inconsistency in the confinement failure data: the mean values for initial confinement failure. The data in Table 5.12 show that the confinement function is apparently less likely to fail initially in large LOCA accidents (most likely at high ground acceleration levels) than either small LOCA or transient accidents (most likely at low and intermediate ground acceleration levels). One would expect that initial confinement failure fractions would increase with ground acceleration - especially since the gross failure of either Building $109 \mathrm{~N}$ or Building $105 \mathrm{~N}$ proceeds dircotly to a large LOCA or MPTR event, respectively, without other contributing failures. This apparent discrepancy is due to the fact that the EDT is expected to fail with a significant frequency at all beyond design basis (SSE) ground acceleration levels and that EDT failure implies failure of the confinement function if effluents are allowed to flow to the EDT. In most transient and small LOCA events, effluents can get to the EDT, so this EDT failure implies failure of the confinement function. However, most large LOCA events are MPTR events that shear off primary system piping either inside the reactor shield walls or inside Bullding 105N. This prevents the effluents from reaching the EDT, so EDT failure does not imply failure of the confinement function. Failure of the confinement buildings is, however, more probable for large LOCA events. 
Figure 5.9

EMERGENCY DUMP TANK STATUS FOR SEISMIC INITIATORS

Mean Core Damage Frequency $=1.671^{*} 10^{-4}$

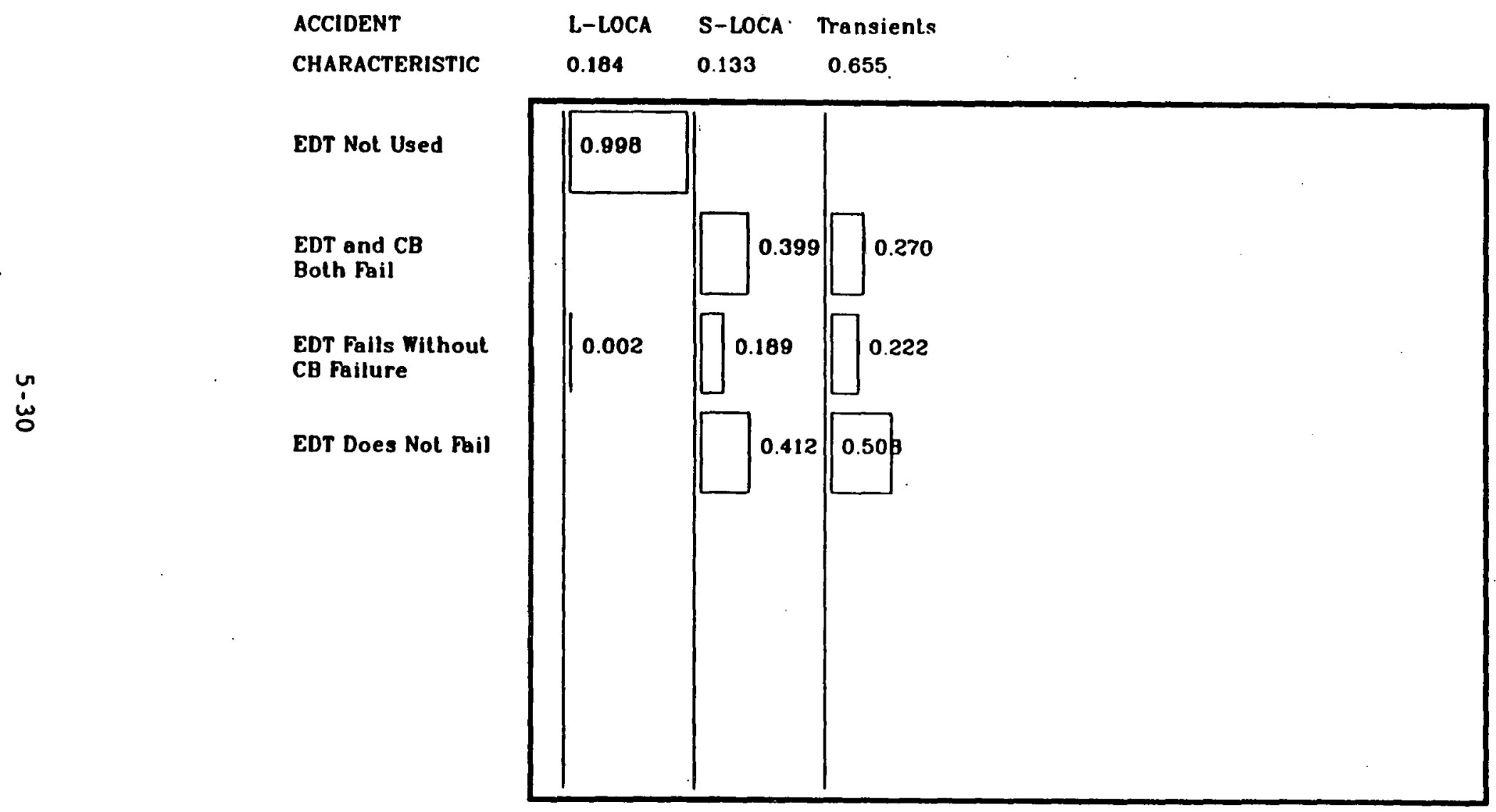

Key: $E D T=$ Emergency Dump Tank $\mathrm{CB}=$ Confinement Building

$S-L O C A=$ Small LOCA 
Table 5.12 Confinement failure fractions by primary system configuration for the seismic APET analysis.

\begin{tabular}{|c|c|c|c|}
\hline $\begin{array}{l}\text { Primary System } \\
\text { Configuration }\end{array}$ & $\begin{array}{c}\text { Initial Conf. } \\
\text { Failure* }\end{array}$ & $\begin{array}{c}\text { Early Conf. } \\
\text { Failure* }\end{array}$ & $\begin{array}{c}\text { Total Conf. } \\
\text { Failure* }\end{array}$ \\
\hline Large LOCA & 0.235 & 0.568 & 0.983 \\
\hline Small LOCA & 0.501 & 0.510 & 0.721 \\
\hline Transient & 0.374 & 0.395 & 0.655 \\
\hline
\end{tabular}

\begin{tabular}{|c|c|c|c|}
\hline \multirow[b]{2}{*}{ Quantile } & \multicolumn{3}{|c|}{ Fraction of Accidents with Initial Failure } \\
\hline & Large LOCA & Sma11 LOCA & Transient \\
\hline 0.05 & 0.000 & 0.000 & 0.025 \\
\hline 0.25 & 0.011 & 0.000 & 0.254 \\
\hline 0.50 (median) & 0.020 & 0.495 & 0.449 \\
\hline 0.75 & 0.446 & 0.503 & 0.494 \\
\hline 0.95 & 1.000 & 0.511 & 0.507 \\
\hline mean & 0.235 & 0.501 & 0.374 \\
\hline
\end{tabular}

\begin{tabular}{|c|c|c|c|}
\hline \multirow{2}{*}{\multicolumn{4}{|c|}{ action of $A C$}} \\
\hline & Large LOCA & Small LOCA & Iransient \\
\hline 0.05 & 0.964 & 0.435 & 0.229 \\
\hline 0.25 & 0.979 & 0.600 & 0.529 \\
\hline 0.50 (median) & 0.998 & 0.744 & 0.721 \\
\hline 0.75 & 1.000 & 0.826 & 0.774 \\
\hline 0.95 & 1.000 & 1.000 & 0.866 \\
\hline mean & 0.983 & 0.721 & 0.655 \\
\hline
\end{tabular}

\footnotetext{
* Confinement failure includes dump tank failure. Initial denotes at the time of accident initiation, while early denotes the first two hours of the accident. Cases where no flow reaches the EDT are not considered EDT or confinement failure unless oller events ocr.ur to fail the confinement buildings.
} 
Figure 5.10

CONFINEMENT FAILURE FOR SEISMIC INITIATORS

Mean Core Damage Frequency $=1.671 * 10^{-\bullet}$

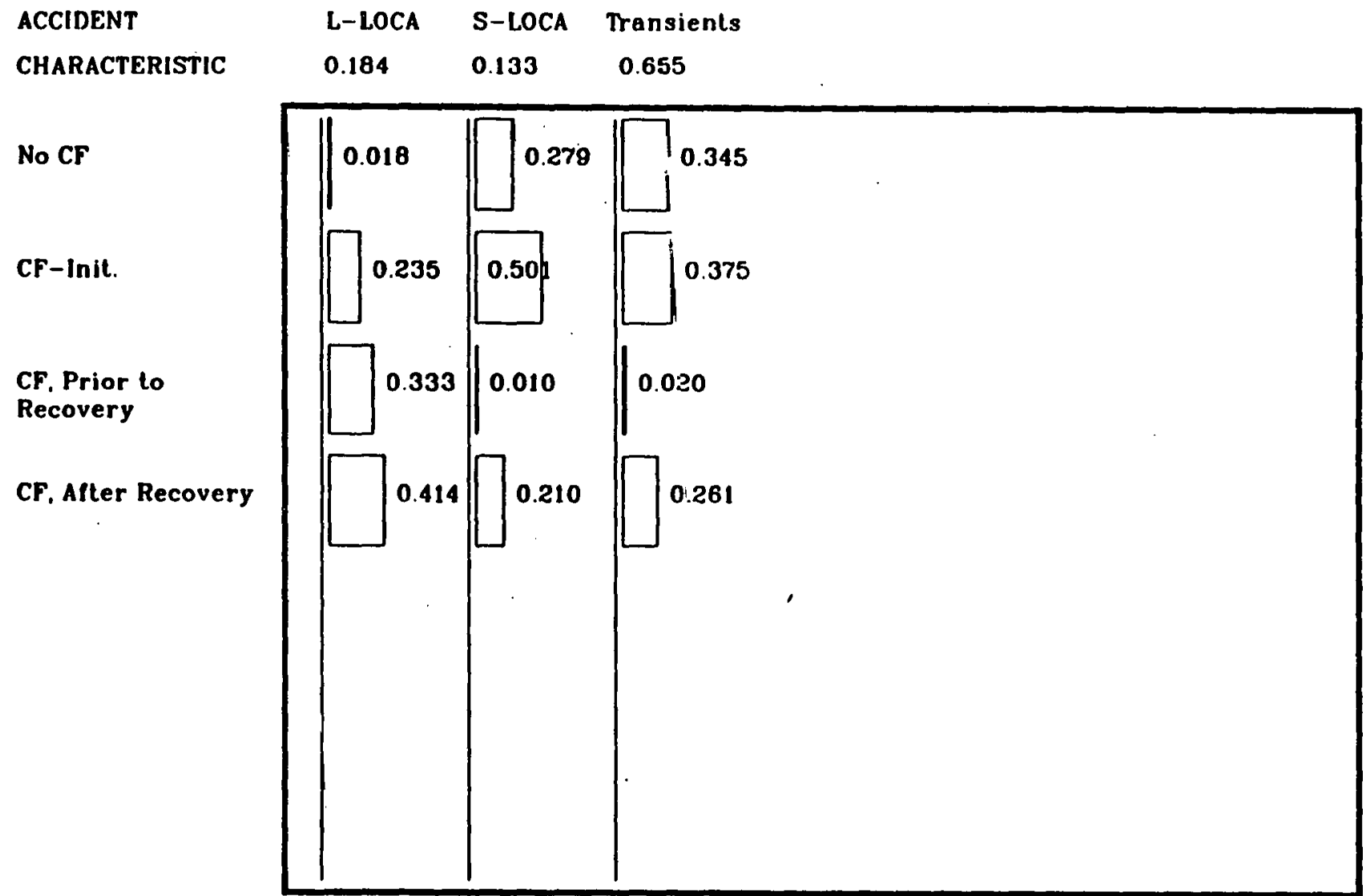

Key: $\mathrm{CF}=$ Confinement Failure Init. = At time of initiator S-LOCA = Small LOCA 
The uncertainty in the survivability of the confinement is also shown in Table 5.12. There is very wide uncertainty about whether or not the confinement fails initially, but, over the course of the accident, the uncertainty lessens because either the confinement or the EDT is expected to fail in most cases where either medium or high ground acceleration is experienced. The wide uncertainty in the "transient" column is due to the fact that the primary system configuration is the same for both Building 182N failures and other general transients, but the ground acceleration is low for the latter and medium for the former. Thus, uncertainty in the hazard curve accounts for most of the uncertainty in the transient primary system configuration. The uncertainty in the small LOCA column is actually wider, but most of the spread is in the tails of the distribution while, for transients, the spread is throughout the distribution. The long tails on the small LOCA distribution are related to the long tails on the seismic hazard curves.

Graphite oxidation was assessed to be much more likely to occur in seismic MPTR accidents than in other types of accidents (see Appendix C). The large number of broken pressure tubes and the possibility for breaks to occur both inside and outside the core allows a much greater chance for adequate alr ingress area and oxidation product exhaust area to for a selfsustaining graphite oxidation reaction to occur. Thus, the graphite oxidation results presented in Table 5.13 are not surprising. The MPTR event forms much of the frequency for the large LOCA primary system configuration and is far more conducive to graphite oxidation than any other accident analyzed.

Table 5.13 Graphite oxidation fractions by primary system configuration for the seismic APET analysis.

Primary System Configuration

Large LOCA

Small LOCA

Transient
Fraction of frequency with Graphite Oxidation

0.977

0.024

0.037 
The filtration function success fractions and uncertainties are presented in Table 5.14. There are two dominant modes of failure for the filtration function for the seismic analysis. The first is a confinement failure that allows the effluent gas stream to bypass the filters. The second is a failure of the filter spray function that leads to filter burning. This is far more prevalent in the seismic case because the water supply for the filter spray system is effected by damage to the silo. The other filter failure modes were almost never seen in the seismic analysis.

The uncertainty in filtration success for the seismic analysis is primarily attributed to uncertainty in the survivability of the confinement building. The filter spray system does contribute to filter failure for the lower core damage accidents where the confinement remains intact, but the confinement failure bypassing the filters causes most of the filtration function failures and most of the uncertainty in the filtration issue for the seismic analysis.

In summary, the different ground accelerations considered during the APET seismic analysis were seen to have significant effects on the types of core damage accidents that would occur. at $\mathbf{N}$ Reactor. Smaller seismic events (near the $N$ Reactor SSE) produce accidents that are similar to the internal events analysis because seismically qualified components normally survive such an event. Thus, core damage is rare for smaller seismic events, and when it occurs, there are major contributions from random failures similar to those seen in the internal events analysis. Above this level, however, the accidents are significantly different because most accidents involve the failure of the silo. This acts as a common failure to cut across many systems and affect the primary, ECCS, confinement systems, and in many cases contributes to GSCS failure. In addition, the EDT and hydrogen mitigation nitrogen tanks would be prone to failure at higher ground accelerations. This enhances the path to the environment for the fission products and adds the possibility that serious hydrogen combustion events might damage the confinement. The seriousness of most confinement-related events was, as expected, seen to increase as ground acceleration increased. As with all seismic analyses, there is much uncertainty in the underlying seismic hazard curves. These curves represent the frequency with which earthquakes of a given magnitude occur. Thus, the absolute frequency of a given seismic event or ground acceleration is relatively uncertain, but, given that a specific seismic event has occurred (i.e. large LOCA or MPTR), the assessment of its progression from the APET analysis is accurate. 
Table 5.14 Filtration success fractions by primary system configuration and core damage amount for the seismic APET analysis.

Primary System Configuration

Large LOCA

Small LOCA

Transient
Fraction with

Filter Success

$<0.01 *$

0.204

0.154

0.57
Fraction of

Seismic Accidents

0.190

0.136

0.674

0.231

0.769
$<408$ Core Damage

$>408$ Core Damage

$$
<0.01 *
$$

\begin{tabular}{ccc} 
& \multicolumn{2}{c}{$\begin{array}{c}\text { Fraction of Accidents with Filtration } \\
\text { Succeeding During Entire Accident }\end{array}$} \\
\cline { 2 - 3 } Quantile & $\frac{\text { Sma11 LOCA }}{0.000}$ & $\frac{\text { Transient }}{0.000}$ \\
0.05 & 0.000 & 0.004 \\
0.25 & 0.000 & 0.035 \\
0.50 (median) & 0.238 & 0.206 \\
0.75 & 1.000 & 0.296 \\
0.95 & 0.204 & 0.154 \\
mean & &
\end{tabular}

\footnotetext{
\# The uncertainty analysis assessed that, in accidents where GSCS failed, fission product and decay heat loading would almost always lead to failure of the filtration function as discussed in Appendix C.11.
} 


\subsection{SUMMARY}

The $\mathrm{N}$ Reactor accident progression analysis was performed in three separate phases: internal events, fire, and seismic. The results of these analyses were passed on in a machine readable form to the source term analysis. Examination of the results outside the context of the risk analysis process yielded several significant insights. Among these insights were:

- The 3 H33 ECCS bypass switch is a significant contributor to both core damage and confinement failure because, for transients, it defeats both the ECCS actuation and confinement isolation functions.

- A significant number of accidents do not proceed to assessable core damage, and about 968 of all internally initiated core damage accidents damage less than half of the core.

- About half of all confinement failures during internally initiated accidents are caused by failure of the confinement to initially isolate and not by accident progression events.

- The filter system operates successfully for 798 of the internally initiated accidents, and, for many accidents where it fails, it fails only because the confinement building does not remain intact to prevent the filters from being bypassed by the effluent gas stream.

- Approximately 4.48 of all core damage accidents (regardless of initiator) in which the fog spray system functions result in liquid releases from the plant that could enter the Columbia River. This problem, however, is easily fixed by either adding a backup to the existing diesel lift station sump pump, improving the sump water level monitoring capabilities, or both.

- The APET analysis for $N$ Reactor accidents induced by fires shows much more core damage, confinement failure and filter failure than did the internal events analysis.

- The most significant two fire locations from a frequency perspective are Corridor 19 and the main control room. Fires in these areas damage the primary cooling system, ECCS, and GSCS, (Corridor 19 also affects the fog spray system), but do not affect confinement isolation functions.

- The external events core damage analysis showed that extremely rare high ground acceleration $(\geq 0.5 \mathrm{~g})$ typically produce more severe core damage levels than do lower ground acceleration levels. The APET analysis showed that the confinement also fails more often during these accidents high ground acceleration accidents. 
- The seismic accidents are significantly different from the internally initiated accidents because most accidents involve the failure of the silo. This acts as a common failure to cut across many systems and effect the primary, ECCS, GSCS and confinement systems.

- The EDT and hydrogen mitigation nitrogen tanks would be prone to failure for ground accelerations significantly higher than the SSE in seismic accidents.

- The seriousness of most confinement-related events was, as expected, seen to increase as ground acceleration increased. 


\subsection{REFERENCES}

5-1. D. M. Kunsman, et al., Level III Probabilistic Risk Assessment for $N$ Reactor, WHC-MR-0045, SAND89-2102, Prepared by Sandia National Laboratories for Westinghouse Hanford, April 1990.

5-2. R. L. Iman and M. J. Shortencarier, A User's Guide for the Top Event Matrix Analysis Code (TEMAC), NUREG/CR-4598, SAND86-0960, Sandia National Laboratories, August 1986.

5-3. R. L. Iman, Memorandum to File, "Modifications to TEMAC," Sandia National Laboratories, January 17, 1989.

5-4. J. M. Griesmeyer and L. N. Smith, A Reference Manual for the Event Progression Analysis Code (EVNTRE), NUREG/CR-5174, SAND88-1607, Sandia National Laboratories, September 1989.

5-5. J. A. Lambright, et al., Analysis of Core Damage Frequency due to External Events at the DOE $N$ Reactor, SAND89-1147, Sandia National Laboratories, 1990.

5-6. M. D. Zentner, et al., N Reactor Level 1 Probabilistic Risk Assessment: Final Report, WHC-EP-0322, Westinghouse Hanford, August 1988 .

5-7. UNC Nuclear Industries, Inc., N Reactor Updated Safety Analysis Report (NUSAR), WHC-SP-0297, February 1978. 


\section{SUMMARY AND CONCLUSIONS}

The accident progression analysis for the $N$ Reactor Level III PRA represents the current state of the art. The method used is complex and incorporates detailed information about the plant design, operating characteristics, phenomenological calculations, experiments, severe accident research, and expert judgment. The use of this method and the supporting information yield results and uncertainties that are technically defensible.

More important than the actual numbers, though, the insights we have gleaned from our study of possible accident progression at $N$ Reactor have been presented throughout this report. The insights explore the character of the phenomenological processes and plant features which could cause potential problems in the accident progression and ultimately result in risk to the general public and Hanford Reservation workers. At the same time, however, insights have also been given into the phenomenological processes and plant features which mitigate such problems. Perhaps these latter kind have not been discussed enough because by its nature a PRA presents the risk, and the drivers to that risk, of the plant as it is designed, and does not speculate on how much larger the risk might be if mitigative design features were not present.

\subsection{ACCIDENT PROGRESSION INSIGHTS}

The accident progression at $N$ Reactor can be very complex, with many possible outcomes to be considered. As discussed in detail in the $N$ Reactor Level III PRA [6-1], the overall risk from $N$ Reactor tends to be very low. The nature of the core design, the confinement design, and the expected accident progression all contribute to these findings. As a result of these low risk estimates, only a small effort has been devoted to identifying significant risk reduction alternatives that involve changes to the core cooling and confinement systems in order to improve the accident progression outcome. These are discussed in the next section.

The results of a PRA analysis are not measured only in terms of the final quantitative results, but must also include the additional insights gained into the operation of the plant under both normal and accident conditions. Several significant technical insights were gained in the course of the $N$ Reactor accident progression analysis. These include:

- A large fraction (in excess of 958) of the internally initiated accident sequences involve damage to less than the full core, thus reducing the source term potential. This result ensues from three factors: much of the accident sequence frequency contains damage to a single riser only; the GSCS system usually operates; and, even in situations where ECCS has failed, the failure is generally one of failing to meet the entire success criterion and some water is still being injected into the core. This is not true, however, for many 
fire and seismically initiated core damage events because many fires affect both the ECCS and GSCS control cables, and many seismic events damage components and buildings that are required by both the ECCS and GSCS.

- The confinement maintains its integrity for a large majority of the internally initiated core damage accident sequences (more than 858 of the frequency). Furthermore, should the confinement boundary be compromised, it is likely (up to 508) that the failure will occur initially with the failure of the confinement isolation system. The systemic problem--the $3 \mathrm{H} 33$ bypass switch--was identified and discussed in this report. Its being left in the wrong position defeats automatic actuation of ECCS, which would actuate the confinement isolation function for transient accidents.

- The analysis has been conducted with the entire hydrogen mitigation system in place. This system was found to significantly reduce the frequency of hydrogen combustion events in most areas of the confinement that might otherwise lead to confinement failure.

- A large fraction of the phenomenologically induced confinement failures (approximately 508 for the internal events analysis) are caused by hydrogen combustion events inside the emergency dump tank. The hydrogen is swept out of the core to the dump tank with the coolant after the V-4 valves open and collects there to become flammable. This assessment is also true for the external events analyses, although to a somewhat lesser extent.

- Fallures in the hydrogen mitigation system during severe, high ground acceleration ( $\geq 0.5 \mathrm{~g}$ - at least twice the $\mathrm{N}$ Reactor SSE) seismic events contribute significantly to an increased confinement failure rate for those events. The failures are typically associated with the nitrogen storage tanks for the confinement purge and inert systems.

- N Reactor ATWS sequences do not involve high power excursions, so the character of the $\mathrm{N}$ Reactor ATWS accident progression is simllar to that of other, non-ATWS, sequences.

- The multiple pressure tube rupture event plays a very important role in the seismic analysis because it not only fails all cooling to the core, but it can also open up significant air ingress pathways into the graphite stack and allow graphite oxidation to occur. This is only seen in the highest ground acceleration (as much as three times the $\mathbf{N}$ Reactor SSE) seismic scenarios. These high acceleration sequences are the most rare and have the most uncertain frequencies.

- Graphite oxidation is not important for internal events, but is seen in a few fire and several seismic accident progression pathways because of the large fraction of events that involve ECCS and GSCS failure. This unmitigated heatup event can cause graphite oxidation to occur, although it is unlikely. 
- Induced pressure tube rupture events caused by the actuation of the ECCS after significant core heatup has occurred is important to the accident progression for the internal events and fire scenarios. This could cause a steam pressure spike within the confinement of sufficient magnitude to cause failure of the confinement building.

- The filter system is expected to operate successfully about 808 of the internally initiated accidents, and, for many accidents where it fails, it fails only because the confinement does not remain intact to prevent the filters from being bypassed by the effluent gas stream.

- Approximately 4.48 of all core damage accidents (regardless of initiator) in which the fog spray system functions could result in liquid releases from the plant that could enter the Columbia River.

- The most significant two fire locations from a frequency perspective are Corridor 19 and the main control room. Fires in these areas damage the primary cooling system, ECCS, and GSCS, (Corridor 19 also affects the fog spray system), but do not affect confinement isolation functions.

\subsection{POSSIBLE PLANT IMPROVEMENTS}

Current operating procedures and features that tend to prevent or mitigate severe accidents are already included in the analysis. This includes the hydrogen mitigation system, as currently designed. The hydrogen mitigation system had a significant impact on the risk, eliminating confinement failures in many instances. Its exact impact is unknown, since a calculation was not performed without the system in place; however, it is believed to be very important.

It is important to note that these improvements could reduce plant risk, but the risk numbers are sufficiently low that no specific recommendations are being made for plant improvements. Future changes at the plant to improve accident progression outcomes include:

- The hydrogen mitigation system effectiveness could be enhanced by inerting the gas plenum in the emergency dump tank and increasing the seismic qualification of the nitrogen tanks against higher $g$ earthquake threats. As the system is not completely installed, we made assumptions concerning its installation with respect to the latter point.

- Fire events in Corridor 19 and the main control room are dominant from a frequency standpoint and causes failure of the ECCS, and GSCS. While this analysis was performed with currently planned fire protection improvements installed, additional fire suppression equipment in these areas would further reduce the risk from fire initiated core damage accidents. 
- The $3 \mathrm{H} 33$ ECCS bypass switch is a significant contributor to ECCS failure and confinement failure. Minor hardware or procedural changes could be made to greatly reduce the frequency of this scenario.

- Additional seismic qualification of the emergency dump tank would reduce the impact of certain seismic accidents.

- The expected frequency of liquid releases from the plant could be all but eliminated by the addition of a backup to the existing lift station diesel sump pump, improving the sump water level monitoring capabilities, or both.

\subsection{CONCLUSIONS}

The accident progression analysis for the $N$ Reactor PRA has been a major effort using state-of-the-art PRA technology. While there are limitations in all such analyses, we have taken particular care to clearly identify the assumptions and limitations specific to this study. Every effort was made to be comprehensive in the consideration of systems and phenomena, resulting in extremely complex models of accident progression. Whenever possible, the results are based on definitive experiments, data, and calculations; expert judgments are clearly identified when they are used. We believe that this work has resulted in realistic, as opposed to unduly conservative or nonconservative results. The uncertainty ranges depict the region within which the true answer should be expected to lie for the accidents considered in this study. With the above discussion in mina, we believe that the general findings of this study are robust and, while the numbers may change, small changes in the models will not alter the basic conclusions.

\subsection{REFERENCES}

6-1. D. M. Kunsman, et al., Leve1 III Probabilistic Risk Assessment for $N$ Reactor, WHC-MR-0045, SAND89-2102, Sandia National Laboratories, April 1990. 


\section{APPENDIX A:}

\section{INTERFACE WITH INTERNAL EVENTS UNCERTAINTY ANALYSIS}

\section{A.1 IATIN HYPERCUBE SAMPLE PROCESSING}

The EVNTRE code package [A-1], when run in sampling mode, must interface with the Latin Hypercube analysis by reading in the sample file that is output by the IHS program and applying a pointer file to direct the values into the proper events, cases and branches for EVNTRE. However, the LHS sample file contains much information that is not needed by the APET analysis. Th1s information is related to the Level I PRA results and/or the source term analysis. In order to conserve disk space and to reduce the complexity of the pointer file, many of the unused values are stripped out of the LHS output file before it is picked up by EVNTRE. This is done by removing certain vector locations ("column") from each observation of the LHS sample matrix. For the APET analysis, columns 1-26, 28-52, 120-175, and 183 forward were removed before the APET analysis. Thus, the pointer file positions for the LHS sample file correspond to only columns 27, 53-119 and 176-182 of the original LHS sample file. The pointer file is listed in Appendix $\mathrm{E}$. The original LHS sample file is presented in the appendix of Reference A-2.

A simple FORTRAN program was used to perform this operation. It is listed in the file LHS_CONV.FOR on the following pages.

\section{A. 2 PROCESSING OF TEMAC RESULTS}

The $N$ Reactor TEMAC analysis for the internal events analysis was done in two parts. The first part examined the Level I PRA cut sets and accident sequences to produce split fractions for many of the first 15 events in the APET. This was done in one TEMAC run. A second TEMAC run was performed to analyze the confinement system cut sets and provide split fractions for the confinement events of the APET. These two runs produced two separate output files that are presented separately in the appendix of Reference A-2.

The EVNTRE code package, however, can only accept input from two uncertainty files. One of these is the LHS sample file. Thus, the two TEMAC output files must be merged into a single file so that both can be used in the APET analysis. This is accomplished by a small FORTRAN program that is listed in the file MERGEl-INT.FOR on the following pages. This program writes the Level I TEMAC output variables as the first 74 variables in the resulting file and the confinement TEMAC output as the remaining 32 variables. These are then accessed by EVNTRE based on the pointer file in Appendix E. 
This program also checks the TEMAC split fraction data as it is processed to insure that none of the split fractions have "probabilities" greater than one, and warns the user should this occur. Split fraction values slightly greater than one occurred occasionally when split fractions were constructed for the confinement system analysis due to the effect of recovery actions. For example, to determine a split fraction that represents the conditional failure probability of system $A$ given that system $B$ had failed, a split fraction is constructed by dividing the probability that both have failed by the probability that $B$ has failed. This number is by definition less than one. However, when recovery actions are applied to the cut sets, they are typically applied only to cut sets with mean probabilities greater than a "cutoff" value (often $10^{-8}$ per occurrence). Since the numerator represents the failure of both $A$ and $B$, it must contain all of the cut sets of the denominator, but each cut set will have additional basic events to represent the failure of system $A$. These corresponding cut sets have lower probabilities than those found in the denominator due to the presence of the additional basic events. Consequently, it is possible to have recovery applied to a cut set in the denominator and not to the corresponding cut set in the numerator. This creates a problem when the recovery/non-recovery disparity occurs in conjunction with an LHS sample member that causes all other basic events in the numerator cut set to be large and that cut set dominates the numerator. Since all cut sets are combined using the "or" function, the split fraction value will be greater than one. This program sets the value of such a split fraction to one and warns the user. The user must then make sure that any such occurrences are legitimate. Two split fractions showed this behavior in the $N$ Reactor confinement analysis in a total of six LHS sample members. In no case was the resulting split fraction value greater than 1.1, and all were found to be legitimate through cut set analysis.

\section{A. 3 REFERENCES}

A-1. J. M. Griesmeyer and L. N. Smith, A Reference Manual for the Event Progression Analysis Code (EVNTRE), SAND88-1607, NUREG/CR-5174, U. S. Nuclear Regulatory Commission, September 1989.

A-2. D. M. Kunsman, et. al., Level III Probabilistic Risk Assessment for $N$ Reactor, SAND89-2102, WHC-MR-0045, Westinghouse Hanford, April 1990. 
The following text is a listing of the FORTRAN program TEMAC_CONV as found in the file LHS_CONV.FOR and described earlier in this appendix.

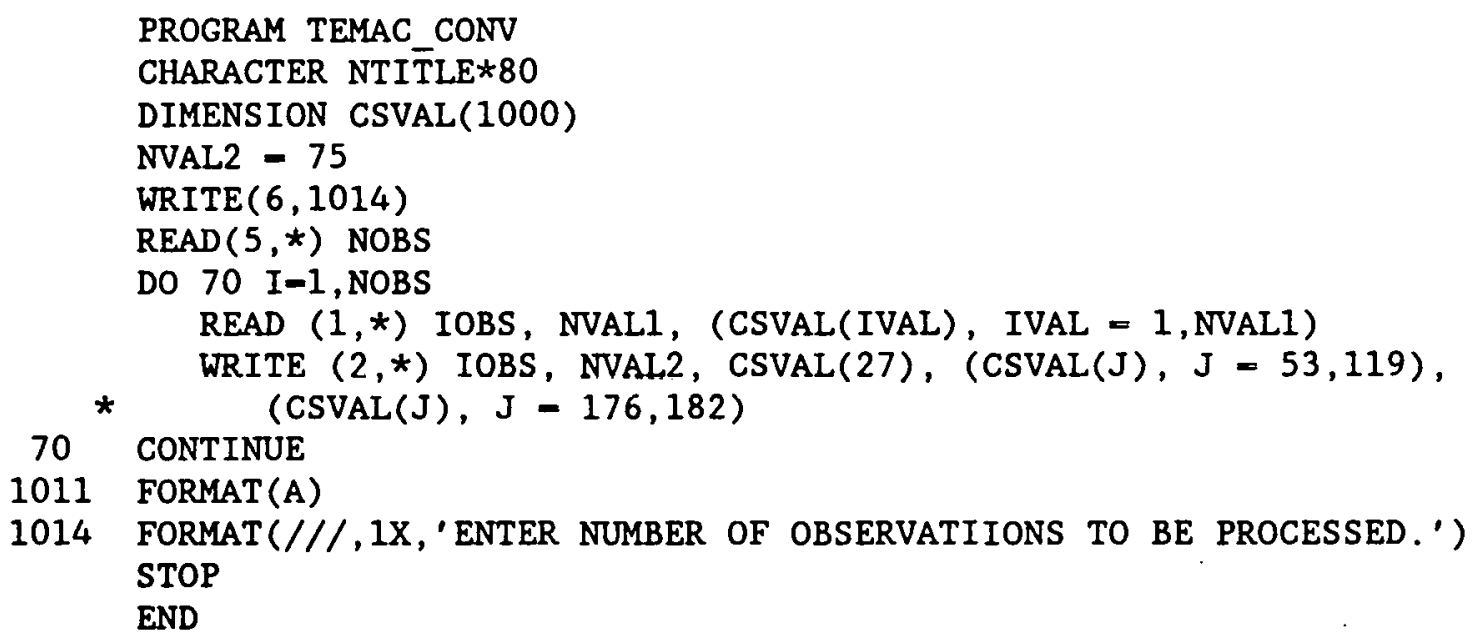


The following text is a listing of the FORTRAN program MERGE as found in the file MERGE1-INT.FOR and described earlier in this appendix.

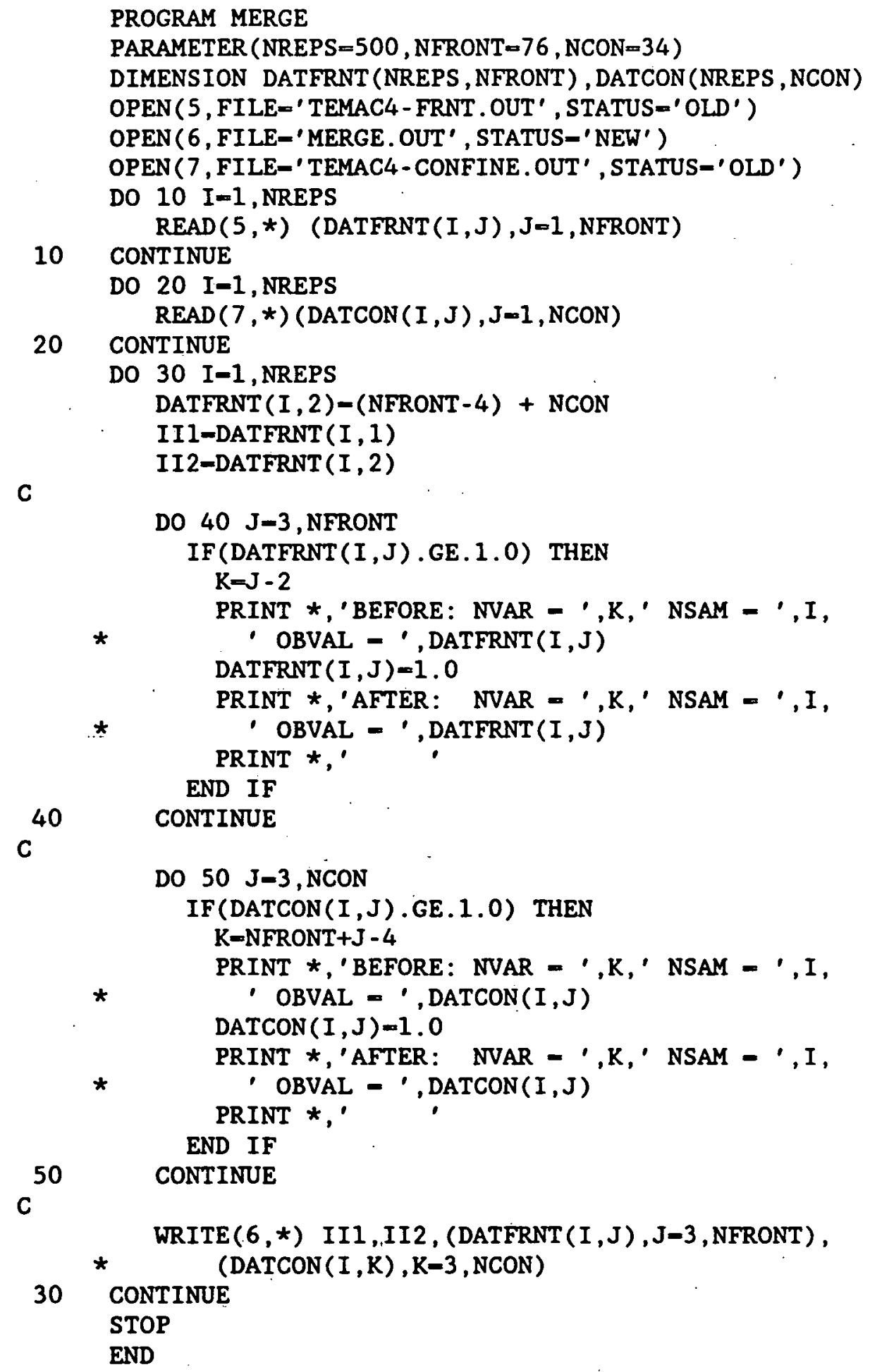

C

DO $40 \mathrm{~J}-3$, NFRONT

IF (DATFRNT (I,J) .GE.1.0) THEN 


\section{APPENDIX B :}

\section{INTERFACE WITH EXTERNAL EVENTS UNCERTAINTY ANALYSIS}

\section{B.1 LATIN HYPERCUBE SAMPLE PROCESSING}

The EVNTRE code package [B-1], when run in sampling mode, must interface with the Latin Hypercube analysis by reading in the sample file that is output by the LHS program and applying a pointer file to direct the values into the proper events, cases and branches for EVNTRE. However, the LHS sample file contains much information that is not needed by the APET analysis. This information is related to the Level I PRA results and/or the source term analysis. In order to conserve disk space and to reduce the complexity of the pointer file, many of the unused values are stripped out of the LHS output file before it is picked up by EVNTRE. This is done by removing certain vector locations ("columns") from each observation of the LHS sample matrix. For the external events APET analysis, columns $1-26,28-52,120-175$, and 183 forward were removed before the APET analysis. Thus, the pointer file positions for the LHS sample file correspond to only columns 27,53-119 and 176-182 of the original LHS sample file. The pointer file is listed in Appendix E. The original LHS sample file is presented in the appendix of Reference B-2.

A simple FORTRAN program was used to perform this operation. It is listed in the file LHS_CONV.FOR on the following pages.

\section{B.2 PROCESSING OF TEMAC RESULTS}

The $\mathrm{N}$ Reactor TEMAC analysis for the external events analysis was done in two parts. The first part examined the external events core damage analysis cut sets and accident sequences (both fire and seismic accidents together) to produce split fractions for many of the first 15 events in the APET. This was done in one TEMAC run. The EVNTRE APET input is designed such that both the fire and seismic data can be read into the analysis simultaneously and the APET logic compensates and uses the proper data for the analysis.

A second TEMAC run was performed to analyze the confinement system cut sets and provide split fractions for the confinement events of the APET. These two runs produced two separate output files that are presented separately in the appendix of Reference B-2.

The EVNTRE code package, however, can only accept input from two uncertainty files. One of these is the LHS sample file. Thus, the two TEMAC output files must be merged into a single file so that both can be used in the APET analysis. This is accomplished by a small FORTRAN program that is listed in the file MERGE-EXT.FOR on the following pages. This program writes the external events core damage andysis TEMAC output 
variables as the first 43 variables in the resulting file and the confinement TEMAC output as the remaining 32 variables. These are then accessed by EVNTRE based on the pointer file in Appendix E.

This program also checks the TEMAC split fraction data as it is processed to insure that none of the split fractions have "probabilities" greater than one, and warns the user should this occur. Split fraction values slightly greater than one occurred occasionally when split fractions were constructed for the confinement system analysis due to the effect of recovery-actions. For example, to determine a split fraction that represents the conditional failure probability of system A given that system $B$ had falled, a split fraction is constructed by dividing the probability that both have failed by the probability that $B$ has failed. This number is by definition less than one. However, when recovery actions are applied to the cut sets, they are typically applied only to cut sets with mean probabilities greater than a "cutoff" value (often 10-8 per occurrence). Since the numerator represents the failure of both $A$ and $B$, it must contain all of the cut sets of the denominator, but each cut set will have additional basic events to represent the failure of system $A$. These corresponding cut sets have lower probabilities than those found in the denominator due to the presence of the additional basic events. Consequently, it is possible to have recovery applied to a cut set in the denominator and not to the corresponding cut set in the numerator. This creates a problem when the recovery/non-recovery disparity occurs in conjunction with an LHS sample member that causes all other basic events in the numerator cut set to be large and that cut set dominates the numerator. Since all cut sets are combined using the "or" function, the split fraction value will be greater than one. This program sets the value of such a split traction to one and warns the user. The user must then make sure that any such occurrences are legitimate. Two split fractions showed this behavior in the $N$ Reactor confinement analysis in a total of six LHS sample members. In no case was the resulting split fraction value greater than 1.1 , and all were found to be legitimate through cut set analysis.

\section{B. 3 REFERENCES}

B-1. J. M. Griesmeyer and L. N. Smith, A Reference Manual for the Event Progression Analysis Code (EVNTRE), SAND88-1607, NUREG/CR-5174, U. S. Nuclear Regulatory Commission, September 1989.

B-2. D. M. Kunsman, et. al., Leve1 III Probabilistic Risk Assessment for $N$ Reactor, SAND89-2102, WHC-MR-0045, Westinghouse Hanford, April 1990. 
The following text is a listing of the FORTRAN program TEMAC_CONV as found in the file LHS_CONV.FOR and described earlier in this appendix.

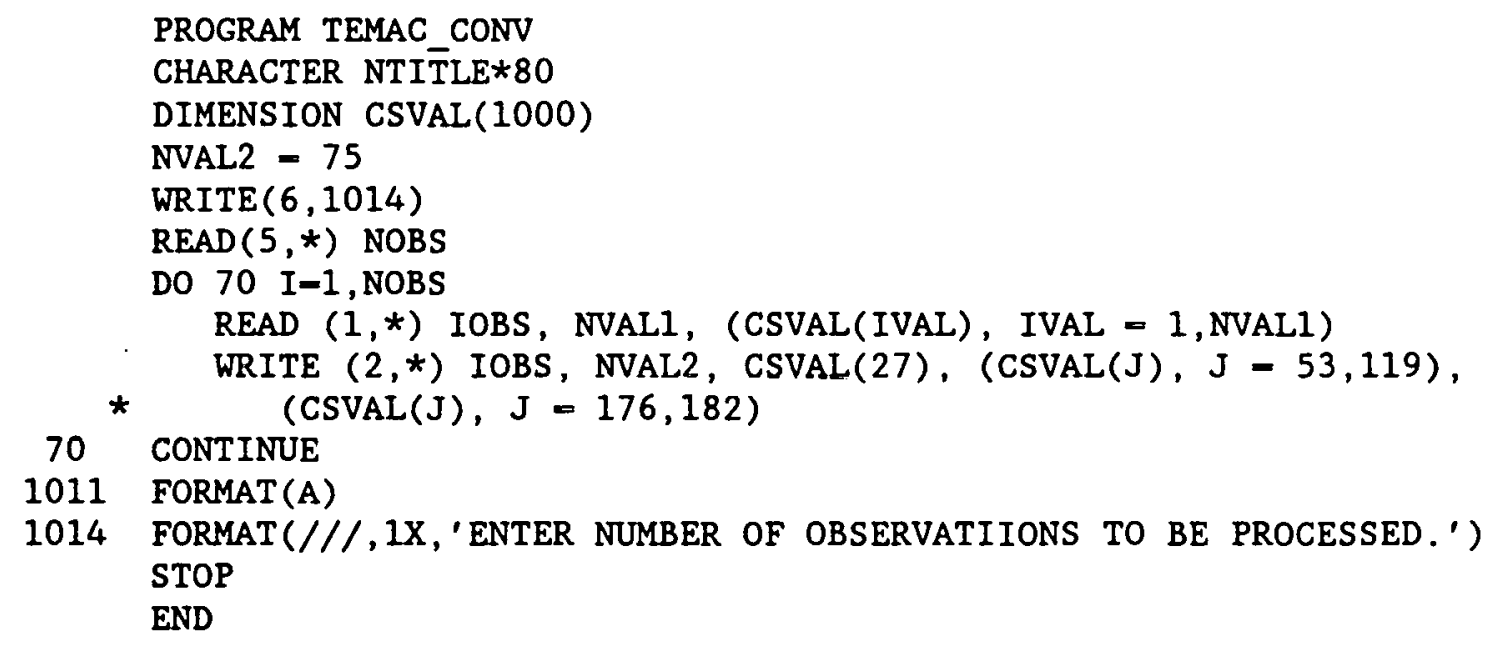


The following text is a listing of the FORTRAN program MERGE as found in the file MERGE1-EXT.FOR and described earlier in this appendix.

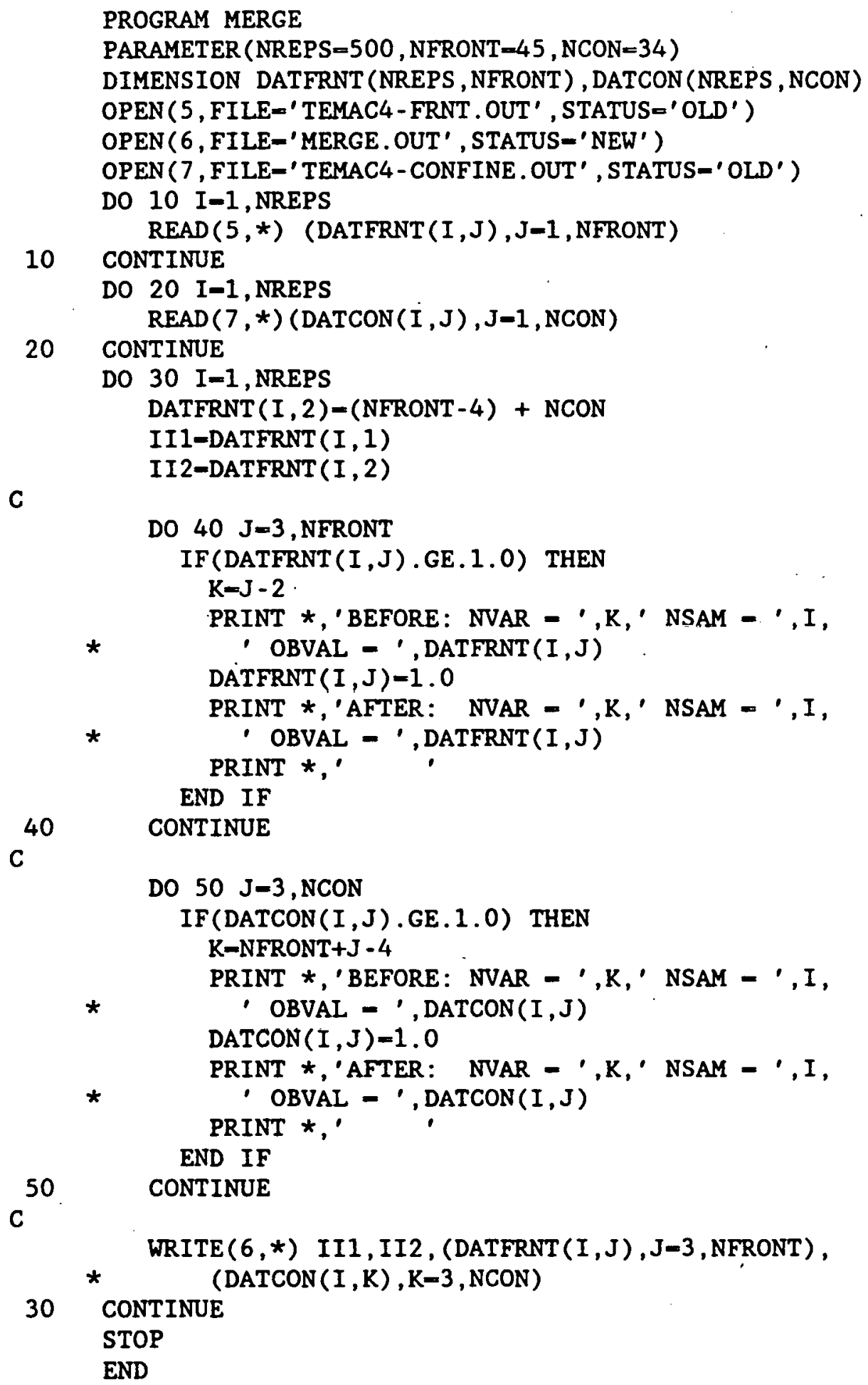




\author{
APPENDIX C \\ SUPPORTING INFORMATION FOR THE N REACTOR APET
}

This appendix describes the issues addressed for the uncertainty analysis for the $\mathrm{N}$ Reactor Accident Progression Event Tree analysis. The thirteen issues that were considered are summarized in the paragraphs below. The following sections address each of the issues and the results in detail.

\title{
Issue 1: Hydrogen
}

Hydrogen detonations and deflagrations, should they occur, would be expected to present a serious challenge to the integrity of the confinement building. Distributions were developed to describe the relative probabilities of the various types of hydrogen events for different system configurations. The hydrogen issue depends on the amount of fuel damaged, the availability of AC power, the location of the primary system breach, and the status of the hydrogen mitigation system and the fog spray system.

\section{Issue 2: Confinement Failure and Fog Spray Survival}

Various energetic events, such as steam spikes, hydrogen events and graphite combustion, are expected to challenge the integrity of the confinement building. Certain types of building failures could have the additional effect of rendering the fog spray system inoperable or ineffective. Probability distributions were developed to model the various modes of confinement building and fog spray system failure for different types of energetic events.

\section{Issue 3: Fuel Damage for Partial ECCS and Partial Core Accidents}

Most $\mathbf{N}$-Reactor severe accident scenarios do not involve complete failure of the ECCS, but rather show ECCS operating in some degraded state. MELCOR and RELAP calculations have demonstrated that steam cooling provides significant heat removal in many of these situations. Probability distributions were developed lo describe the total amount of core damage given that ECCS is operating in one of its degraded modes (including the failure of a CV-2R valve to upen).

Issue 4: Fuel Damage due to Late ECCS Failure

Previous analyses assessed that the failure of the ECCS function which occurs at least two hourg after reactor trip, when combined with a GSCS failure, proceeds to up to 1008 core damage. It has been noted, huwever, 
that most of these failures involve only a degradation of the ECCS function, and that, since the decay heat load is relatively low by this time, coolant demands will also be reduced. Probability distributions were developed to determine the extent of core damage for these scenarios.

Issue 5: Fuel Damage Propagation

The APET allows for the possibility that fuel damage will propagate from areas of the core which are not receiving adequate cooling to those which apparently are receiving adequate cooling based on the occurrence of phenomena that are considered less than credible. A probability distribution was developed to attempt to account for these phenomena.

\section{Issue 6: Core Responses to Late ECCS Initiation}

Injecting water into a portion of the core that has been heated to the point of fuel damage is expected to contribute to a number of undesirable effects. These include pressure tube failure, the production of a steam spike in confinement, hydrogen production, and possibly additional core damage. Probability distributions were developed to model these phenomena for various initial core damage and ECCS flow situations.

\section{Issue 7: Pressure Tube Failure}

Water hammer and other phenomena have been postulated for some partial ECCS flow scenarios which could lead to pressure tube failure due to thermal and mechanical cycling. Probability distributions were developed to model this possibility for the major partial ECCS success modes.

\section{Issue 8: Graphite Oxidation}

Unmitigated accidents present the possibility that the graphite moderator stack could begin to oxidize or even burn under certain circumstances. Probability distributions were developed to predict these possibilities.

\section{Issue 9: Systems Recovery}

The APET considers the possibility that several primary and confinement systems could be recovered during the accident. Probability distributions were developed to show the probability that each system will be recovered within the first two hours after reactor trip. Consideration was given to both the recoverability of the systems and the probability that the operator would actually try to recover the system. 
Issue 10: Filter Path Reisolation

The confinement filter path is designed to reisolate any time the confinement pressure rises above 15 inches of water. However, certain events (such as hydrogen detonations) occur so quickly that the path would not be able to close quickly enough to prevent damage from occurring to the filter path downstream of the isolation valves. Probability distributions were developed to model the effectiveness of the reisolation function for the phenomena which would be expected to demand it.

\section{Issue 11: Filter Performance}

The performance of the filter system has a major impact on the overall release of radioactivity from the plant during a severe accident. Probabllity distributions were developed to model the expected filter system performance under various accident conditions.

\section{Issue 12: LERF Plastic Liner Failure}

The plastic liner of the LERF would be expected to fail with some small probability in any accident sequence that produced liquid effluents. An estimate of the probability of this plastic liner failing was developed.

\section{Issue 13: Seismic Assessment of Confinement Structures}

The external events core damage analysis did not consider the failure of structures that were not directly involved in producing core damage. However, a number of these structures are important in the retention of radionuclides within the confinement system. Probabilities for these structures to fail were developed for this issue. 


\section{C.1 HYDROGEN PHENOMENOLOGY}

\section{C.1.1 Issue Definition}

Hydrogen detonations and deflagrations, should they occur, would be expected to present a serious challenge to the integrity of the confinement building. The presence of the hydrogen mitigation system is expected to keep hydrogen from having a major impact on the overall plant risk, but a detailed hydrogen analysis is required to lend credibility to the overall results of the APET analysis.

Distributions are needed to describe, the relative probabilities of hydrogen events for different system configurations. This issue should consider all relevant aspects of hydrogen generation, transport, mitigation and ignition. Hydrogen phenomenology needs to be addressed in both the pre-recovery (approximately the first two hours) and post-recovery (up to 24 hours) phases of the accident. The following should be specifically considered:

1) Hydrogen generation

(a) core thermal/hydraulic conditions

(b) amount of fuel damaged

(c) uncertainties in the surface area of the uranium fuel extruded on clad failure

2) Hydrogen transport

(a) location of primary system breach

(b) mixing by sprays and the mitigation system mixing fans

3) Hydrogen mitigation

(a) nitrogen system effectiveness

(b) detonability versus deflagrability

4) Hydrogen ignition

(a) availability of ignition sources

(b) differences between situations where AC power is and is not available

(c) effects of radionuclides (especially aerosols) on the probability of ignition

\section{C.1.2 Issue Resolution Process}

A. I. Camp, S. E. Dingman, and L. A. Miller of Sandia Labs were selected to be elicited on the issue of hydrogen phenomenology because of their broad experience and knowledge in analyzing hydrogen phenomena. Camp and Dingman are the authors of HECTR, a major hydrogen phenomenology computer code sponsored by the U. S. Nuclear Regulatory Commission, and did extensive research into hydrogen phenomenology in support of that project. Miller has done extensive work modeling material transport in the $\mathrm{N}$ Reactor confinement using the MELCOR and HECTR code packages. 
The elicitees were given complete freedom to define the issue and the structure of the final results to be included in the APET analysis within the framework of the previous section. They developed a set of events that were, with minor modifications, included directly into the APET. They provided probability distributions for some of the huge number of possible conditions within the confinement and guidance for combining the remaining cases into a logical structure that could be used in the APET analysis.

\section{C.1.3 Issue Analysis and Resolution}

The elicitees set up a framework for analysis of the hydrogen phenomenology issue as a series of questions to be analyzed. The questions they determined would adequately represent the phenomena in question are:

1) Is Hydrogen Flammable in the First Two Hours?

2) Is Flammable Hydrogen Detonable in First Two Hours?

3) Does Hydrogen Ignite in the First Two Hours?

4) Does Ignited Hydrogen Deflagrate or Detonate in First Two Hours?

5) Does Hydrogen Fail Confinement Late?

6) Is Hydrogen Flammable Late in the Accident?

7) Is Flammable Hydrogen Detonable in First 24 Hours?

8) Does Hydrogen Ignite in the First 24 Hours?

9) Does Ignited Hydrogen Deflagrate or Detonate in the First 24 Hours?

Each of these questions are analyzed in detail in the following subsections.

\section{C.1.3.1 Is Hydrogen Flammable in the First Two Hours?}

The contents of this section are taken from an issue writeup for this. question by A. L. Camp, S. E. Dingman and L. A. Miller dated September 26, 1989. This question addresses the probability of a flammable mixture in several locations during the first two hours. The flammability limits used in this analysis were 48 hydrogen for deflagrability, and 118 hydrogen for detonability. The nine possible branches for this question are:

Branch Abbrev.

(1) H-Mit

(2) H-Pent

(3) H-Pgal

(4) H-SG

(5) H-Bar

(6) H-105

(7) H-Dump

(8) H-109

(9) H-Mix

\section{Description}

Hydrogen is not flammable anywhere.

Hydrogen is flammable in the penthouse

Hydrogen is flammable in the Pipe Gallery

Hydrogen is flammable in a steam generator cell or the aux cell

Hydrogen is flammable in the barrier space

Hydrogen is flammable in the 105 building

Hydrogen is flammable in the Dump Tank

Hydrogen is flammable throughout the 109 building

Hydrogen is flammable throughout the confinement 
Relevant Factors and Background Information

The answers to this question depend on a number of previous questions dealing with hydrogen generation, release location, and purging and mixing mechanisms. For example, questions $5,8,9,14,17,21,22,23,24,31$, 32 , and the amount of hydrogen generated based on the state of the ECCS and GSCS could all impact the flammability question.

Several reports were consulted in determining the probabilities and probability distributions including:

1) SAND86-2630 Parametric HECTR Calculations of Hydrogen Transport and Combustion at $\mathbf{N}$ Reactor,

2) WHC-SP-0078 Interim Evaluation of the Proposed Hydrogen Mitigation System for $N$ Reactor,

3) NAI-8708-2 An Assessment of the $\mathrm{N}$ Reactor Hydrogen Mitigation System Effectiveness,

4) WHC-SP-0238 HECTR Analys is of the N Reactor Hydrogen-Mitigation System, and

5) SAND89-2101 N Reactor Probabilistic Risk Assessment Supporting Calculations.

From the information obtained, there are a number of key points that apply to many different cases. These will be discussed here and not. repeat.en for each case.

Based on Reference 5, the total amount of hydrogen generated in the first two hours will not exceed $150 \mathrm{Kg}$. This restriction limits the size of the flammable volumes and precludes global flammability. Later in the analysis, this will also limit detonability in medium and large sized volumes.

In many of the sequences, the V4 valves are either one-half or completely open. In these cases most of the hydrogen ( $>758$ for large LOCAs and $>908$ for small LOCAs) will go to the dump tank. Therefore only LOCAs in small compartments can be flammable at the break location. On the other hand, dump tank flammability and pipe gallery flammability at the dump tank return line must be considered. For the remainder of the hydrogen flammability discussion, the term "V4s, Open" will refer to either one-half or all of the $\mathrm{V} 4$ valves open.

Large LOCAs, and to a lesser extent small LOCAs, will tend to purge the confinement of oxygen during the blowdown process. If the nitrogen system functions with sufficient flow to prevent air inflow during subsequent condensation immediately after the blowdown, then most of the 
confinement will be inert during the entire sequence. Generally, we believe that either of two modes will accomplish this:

1) $20000 \mathrm{cfm}$ nitrogen for two hours followed by $1000 \mathrm{cfm}$ for the rest of the accident, or

2) $40000 \mathrm{cfm}$ nitrogen for one hour followed by $1000 \mathrm{cfm}$ for the rest of the accident.

In the remaining discussions, nitrogen mitigation will refer to either of the above modes. Conservatively, we did not give credit for the $3000 \mathrm{cfm}$ mode (this is included in "no nitrogen system" in the discussions below). The time to purge the confinement in this mode is many hours. In a small fraction of the cases, the confinement ventilation system remains operable. This system will be very effective in removing hydrogen from the confinement.

In the discussions below, point estimates (to be used as mean values) are provided for most cases. We believe that the key uncertainties will be in the ignition probability treated in a later question. We also believe that for most of the questions, the differences between zero and small numbers, such as $10^{-2}$, are not of significance in this analysis. The same holds true for numbers such as 1.0 and 0.9 ; we have assigned 1.0 with no uncertainty to such cases. If the risk analysis indicates that these assumptions are not correct, then full uncertainty distributions will be provided at that time.

\section{Penthouse}

Hydrogen may be introduced into the penthouse in three ways: 1) the pipe break may be located in the penthouse, 2) hydrogen in the 105 building may be transported into the penthouse through the mitigation system fan, and 3 ) hydrogen in the pipe gallery may rise into the penthouse. In order to simplify the problem, the latter two ways of introducing hydrogen into the penthouse are not addressed here. There are no mechanisms in the penthouse to increase the flammability of mixtures entering that space, e.g., a substantial condensation mechanism. As will be shown later, flammable mixtures in the 105 building are more likely to ignite at that location than after transport to the penthouse. Also, since flammable mixtures in the pipe gallery are not likely to be significantly more concentrated upon entering the penthouse, and burns in the pipe gallery are more serious due to the larger volume involved, hydrogen in the pipe gallery will be assessed for flammability at that location and not with respect to the penthouse.

For the case in which the break is located in the penthouse, there are a number of key factors that affect the flammability. First, due to the small volume, sufficient hydrogen to achieve flammability can be present even if the V4 valves are open. Second, large LOCAs can only occur low in the penthouse (below the mitigation fan exit), while small LOCAs can occur high in the penthouse (above the mitigation fan exit). If the fan 15 on, 
releases low in the penthouse will be swept out, while COBRA-NC calculations in Reference 3 indicate that high releases can result in flammability even with the fan operating.

All of the cases with releases in the penthouse have been assigned values of either zero or one for the probability of a flammable mixture. These two groups of cases are presented below.

\section{Inflammable Cases}

Case 1: Large LOCA, V-4's operate, mixing fan

With the V4 valves open, most of the hydrogen will go to the dump tank. The hydrogen that is released through the break will be released below the level of the mixing fan (which will displace the volume of the penthouse in 100 seconds). With a relatively small amount of hydrogen in confinement, the air coming in from the 105 building to the penthouse will be an air-steam-nitrogen mixture (depending on the nitrogen system operation), but will contain very little hydrogen. Thus, the effect will be to rapidly purge the penthouse of hydrogen. Therefore, we have assigned a probability of 0.0 for flammability in this case.

\section{Case 2: Small LOCA, V-4's operate, mixing fan}

COBRA-NC calculations indicate that high releases can result in flammable amounts of hydrogen $(-248)$ in the penthouse even with the mixing fans operating. However, with the V4 valves open, less than $10 \%$ of this amount of hydrogen is released to the penthouse. Generally, the concentration in confinement calculations for $\mathrm{N}$ Reactor is proportional to the release. A simple proportioning results in 2.48 hydrogen for this case. In fact the amount of hydrogen is probably much less than 108 of the total generated in this case. Therefore-we assign 0.0 as the probability of a flammable mixture.

Case 3: Large LOCA, V-4's do not open, mixing fan

A HECTR calculation with the upper limit of early hydrogen release in the penthouse and the mixing fan operating showed that the hydrogen concentrations were kept below 48 in the penthouse. This calculation is subject to the limitations of control volume codes. The fact that the inlet of the mixing duct is located at the $65 \mathrm{ft}$ level in the penthouse and is tilted up at a 45 degree angle support the well mixed assumption as does the high velocity of the air from the fan. Also, this fan is capable of turning over the volume of the penthouse in $100 \mathrm{~s}$. The mixture entering from the 105 building will contain little or no hydrogen, since there is insufficient hydrogen to fill the pipe gallery and the 105 building. As a result, we assess the probability of a flammable mixture in this case to be 0.0 . 
Case 4: Large LOCA, V-4's operate, no mixing fan, no nitrogen system

Without the mixing fans, sufficient hydrogen will be released into the penthouse to achieve flammability, even with the $\mathrm{V} 4$ valves open. Without the nitrogen system operating, the presence of oxygen is also likely. Therefore, we have assigned a probability of 1.0 for flammability in this case.

Case 5: Small LOCA, V-4's operate, no mixing fan, no nitrogen system

The arguments for case 4 hold here also, even though less hydrogen will be released to the penthouse.

Case 6: Small LOCA, V-4's do not operate, mixing fan

In this case all of the hydrogen is released through the break. The COBRA-NC calculations noted earlier indicate that high hydrogen concentrations can occur near the top of the penthouse for high releases, such as could occur for a small LOCA. These concentrations occur even with the mitigation fan operating. Therefore, a probability of 1.0 for a flammable mixture was assigned for this case.

Case 7: V-4's do not open, no mixing fan, no nitrogen system

In this case all of the hydrogen is release through the break. Since the mixing fan is not operating, there are fewer differences between the large and small LOCAs. Without the nitrogen system, oxygen will be present in confinement, and can eventually mix with the hydrogen in the penthouse.

A HECTR calculation with a hydrogen release of $90 \mathrm{~kg}$ in 2 hours and fan operation that begins at $2500 \mathrm{~s}$ predicts a hydrogen concentration of over 248 in the pressurizer penthouse before the fans begin operating. Based on these calculations, the probability of a flammable mixture in the penthouse under these conditions is 1.0 for both production cases.

\section{Pipe Gallery}

Hydrogen may enter the pipe gallery through four pathways: 1) a pipe break in the pipe gallery, 2) transport from a steam generator cell in which a break is located, 3) transport from the Emergency Dump Tank (EDT), or 4) transport from the penthouse if a break occurs there. Cases 2 and 4 will be considered in the branch addressing flammability in the entire 109 building. For this pipe gallery branch, the direct introduction of hydrogen into the pipe gallery by a break located there (case 1) will be considered first. Next, the transport of hydrogen from the dump tank into the pipe gallery will be considered.

The key factors affecting hydrogen flammability in the pipe gallery for a pipe break located in the pipe gallery are the mixing mechanisms, 
potential for inerting, and amount of hydrogen released to the region. Flammable regions would be more likely if the hydrogen remained more concentrated near the release location than if the hydrogen mixed with surrounding gases. The possible locations for pipe breaks in the pipe gallery are all fairly low in the region. This favors mixing of the released hydrogen, rather than forming a stratified layer. Spray operation will further mix the region, practically guaranteeing a well-mixed region. Operation of the mitigation system at either 20,000 or 40,000 cfm or operation of the building ventilation system at $77,000 \mathrm{cfm}$ will also provide a well-mixed region. Operating the nitrogen purge system at either 20,000 or $40,000 \mathrm{cfm}$ appears to be sufficient to deplete the oxygen below the flammability level before flammable mixtures of hydrogen would accumulate, whereas purge at $3000 \mathrm{cfm}$ would not be adequate. In addition, the pipe gallery would remain inert throughout the transient for a LOCA in the pipe gallery with the nitrogen purge operating at 20,000 or $40,000 \mathrm{cfm}$. For these cases, the pipe gallery would be voided of oxygen during the blowdown and replaced by steam. Subsequent operation of the nitrogen mitigation system at a sufficiently high flow rate will prevent the reentry of oxygen, leaving an inert region.

The amount of hydrogen released through a pipe break in the pipe gallery will be strongly affected by the state of the V-4's. If either all or half of the $V-4^{\prime} \mathrm{s}$ are open, the majority of the hydrogen release is predicted to enter the dump tank. For cases in which the V-4's open, the majority of the hydrogen generated during the accident would be released to the dump tank, rather than directly into confinement. The hydrogen would then enter the pipe gallery at a high elevation. This release location has much less potential for mixing without active mechanisms such as the sprays or nitrogen purge. Thus, there is a higher likelihood of achieving a flammable mixture.

The cases below are presented in four groups. The first two groups deal with flammability as a result of hydrogen emanating directly from a pipe break in the pipe gallery, and the second two groups deal with hydrogen entering the pipe gallery from the dump tank return line. For each of the two types of groups the inflammable and potentially flammable cases are identified.

\section{Inflammable Cases for Pipe Gallery LOCAs}

\section{Case 1: Forced Ventilation On}

The hydrogen concentration in the pipe gallery from the injection of $150 \mathrm{~kg}$ of hydrogen (the upper limit of production case 2) would be 98. However, the forced ventilation system $(77000 \mathrm{cfm})$ will turn over the volume of the entire 109 building in approximately $30 \mathrm{~min}$. Because the building is so well mixed the hydrogen is very likely to be well mixed within the 109 building; further, the hydrogen is being swept out by the ventilation system. For these reasons the probability of a flammable mixture has been set to 0.0 during the first two hours. 
Case 2: V-4's open

With the V-4's open, the majority of the hydrogen will be released to the dump tank. The small fraction of hydrogen released to the pipe gallery will tend to be well-mixed as a result of the low release location and the convection forces present. Thus, a probability of 0.0 for flammability has been assigned for this case. Note that this case only addresses flammability from hydrogen originating at the break and does not account for return of hydrogen from the dump tank. That condition is addressed in a later case.

Case 3: V-4's fail closed, Nitrogen System on

For these conditions, the pipe gallery is expected to be inert because the nitrogen purge prevents the re-entry of oxygen into the confinement following the LOCA. Thus, the probability of flammability was assigned a value of 0.0 for this case.

\section{Flammable Cases for Pipe Gallery LOCAs}

Case 4: V-4's fail closed, sprays on, no nitrogen system

For this case, the LOCA will initially purge the oxygen from confinement, but it will be drawn back in as the steam condenses. The sprays will lead to a well mixed 109 building, but the majority of the hydrogen will be released directly into the pipe gallery. A HECTR run with a source injection rate of twice the NUSAR rate (approximately $180 \mathrm{~kg}$ in $13000 \mathrm{~s})$ [Ref. 1, p. 5-1] predicts that the maximum hydrogen concentration in the confinement will be 3.68 at $3.6 \mathrm{hr}$. This source is of greater magnitude than the production case 2 but it is stretched out over a longer time period. However, no hydrogen had escaped from the confinement during the first $13000 \mathrm{~s}$. Based on this calculation the following probability distribution was obtained for production case 2.

\begin{tabular}{|c|c|}
\hline Quantile & Total Probability \\
\hline 0.0 & 1.E-5 \\
\hline .25 & 1.E-3 \\
\hline .50 & 5.E-2 \\
\hline .75 & 7.E-2 \\
\hline 1.0 & 1.E-1 \\
\hline
\end{tabular}

Case 5: V-4's fail closed, sprays off, no nitrogen system

For these conditions, there is little potential for mixing: the released hydrogen and the nitrogen purge does not function to maintain an inert region. Note that this case is differentiated from the $V-4^{\prime} \mathrm{s}$ open case because more hydrogen is released directly to the pipe gallery with the $V-4$ 's closed than with them open.

A HECTR calculation similar to the one used to quantify case 4 predicts that the maximum hydrogen concentration will be 1.98 at $1.7 \mathrm{hr}$. 
The differences between this calculation and the case 4 calculation are that the sprays are not operating and the hydrogen injection rate was the NUSAR rate [Ref 1, p 5-9] Extending this to twice the NUSAR source yields 3.88. The following probability distribution was developed for production case 2 .

\begin{tabular}{cc} 
Quantile & Total Probability \\
\hline 0.0 & $1 . E-4$ \\
.25 & $5 . E-3$ \\
.5 & $5 . E-2$ \\
.75 & $1 . E-1$ \\
1.0 & $2 . E-1$
\end{tabular}

\section{Inflammable Cases - Dump Tank Return Line}

Case 6: V-4's open, transient or break anywhere in confinement, sprays on

For this case, most of the hydrogen will initially be released to the dump tank, but the hydrogen will subsequently flow into the pipe gallery. The hydrogen will be released high in the pipe gallery, but the sprays will result in a well-mixed region. Since a fraction of the hydrogen will be held up in the dump tank, and the remaining hydrogen will be well-mixed in the 109 building, a flammable mixture is not expected to be formed. Thus, the probability of forming a flammable mixture in the pipe gallery for these conditions was assigned a value of 0.0 .

Case 7: V-4's open, transient or break anywhere in confinement, forced ventilation or nitrogen system operating

For this case, most of the hydrogen will initially be released to the dump tank, but the hydrogen will subsequently flow into the pipe gallery. The hydrogen will be released high in the pipe gallery, but the forced ventilation or nitrogen purge will result in a well-mixed region. Since a fraction of the hydrogen will be held up in the dump tank, and the remaining hydrogen will be well-mixed in the 109 building, a flammable mixture will probably not be formed. In addition, for the $\mathrm{N}_{2}$ purge cases, the confinement will most likely be inert. Thus, the probability of forming a flammable mixture in the pipe gallery for these conditions was assigned a value of 0.0 .

\section{Flammable Cases - Dump Tank Return Line}

Case 8: V-4's open, transient or break anywhere in confinement, forced ventilation off, sprays off, no nitrogen system

For this case, most of the hydrogen will initially be released to the dump tank, but the hydrogen will subsequently flow into the pipe gallery. The hydrogen will be released high in the pipe gallery, and for this case there will be little potential for mixing the release. Thus, the geometry is conducive to forming a flammable or even detonable mixture. Analyses are not available to aid in the quantification of this case, so a wide 
uncertainty is reflected in the quantification. The following distribution should be used.

\begin{tabular}{|c|c|}
\hline Quantile & Total Probability \\
\hline 0.0 & 0.0 \\
\hline .25 & .25 \\
\hline .5 & .5 \\
\hline .75 & .75 \\
\hline 1.0 & 1.0 \\
\hline
\end{tabular}

\section{Steam Generator Cell or Aux Cell}

For this analysis, breaks in the different steam generator cells are treated as if they occurred in a typical cell (i.e. treated as cell 1 - 5). This is a conservative treatment since steam generator cell 6 (which contains the inlet of the nitrogen system) could be rapidly mixed and inerted.

Hydrogen may be introduced into the steam generator cell due to a pipe break in the pipe gallery or in a steam generator cell. Since the hydrogen concentration will be higher in the pipe gallery if the break is located there, this possibility will be considered in the branch for hydrogen flammability throughout the 109 building.

Flammability in the steam generator cell depends on: 1) the production of hydrogen, 2) the state of the V-4 valves, and 3) mixing mechanisms. The only mixing mechanisms available are the nitrogen system, forced ventilation, and natural convection since there are no automatic sprays or fans in the steam generator cells. The primary coolant pumps are assumed to be operating in this analysis since this will maximize the amount of hydrogen released through the break.

HECTR was used to analyze a scenario with a hydrogen source based on a MELCOR calculation that maximized oxidation (production case 1 ) and included the $3000 \mathrm{cfm}$ nitrogen source in the same steam generator cell. During the first two hours a small amount of the nitrogen reached other steam generator cells; however, to be conservative, the other steam generator cells will be assumed to be unmixed if the 3,000 $\mathrm{cm}$ source is on.

The cases have been grouped below into inflammable and potentially flammable groups.

\section{Inflammable Cases}

Case 1: V-4 valves open, forced ventilation on

For this case, most of the hydrogen will be released to the dump tank. Further, the ventilation system will rapidly mix and purge the hydrogen. 
Therefore, the probability of a flammable mixture has been assessed to be 0.0 .

Case 2: V-4 valves open, nitrogen system on

In this case, the steam generator cells will be well mixed and nitrogen inerting will prevent the reintroduction of oxygen following the blowdown. Further, with the V-4's open, most of the hydrogen will be released to the dump tank, so the probability of attaining a flammable mixture was assigned a value of 0.0 .

Case 3: SLOCA, V-4 valves open, no nitrogen system

With the V4 valves open, most of the hydrogen is released directly to the dump tank. Although the steam generator cells will not necessarily be well mixed, only a small fraction of the hydrogen enters the steam generator cells. Thus, the region will most likely not accumulate sufficient hydrogen for combustion, and the probability of being flammable was assigned a value of 0.0 for this case.

Case 4: V-4 valves fail, nitrogen system on

For this case, the LOCA will initially purge the region of all oxygen, and the nitrogen purge will subsequently prevent re-entry of oxygen. Thus, despite the fact that additional hydrogen will be introduced into the steam generator cell, a probability of forming a flammable mixture was assigned a value of 0.0 for this case.

\section{Flammable Cases}

Case 5: LLOCA, V-4 valves open, no nitrogen system

For these conditions, the steam generator cells will not necessarily be well mixed. Thus, although the $V-4^{\prime}$ 's being open will direct most of the hydrogen to the dump tank, the fraction released directly into the relatively small steam generator cell could be flammable, particularly if mixing is not complete. Thus, the following probability distribution is assigned for this case.

\begin{tabular}{cc} 
Quantile & Probability \\
\hline 0.0 & 0.0 \\
0.25 & 0.05 \\
0.5 & 0.1 \\
0.75 & 0.15 \\
1.0 & 0.3
\end{tabular}

Case 6: V-4 valves fail, forced ventilation on

In this case, the steam generator cells will be well mixed but complete purging or inerting may not occur. The results of a HECTR calculation with the NUSAR source in steam generator cell 6 and confinement 
sprays on was used to guide the quantification of this branch. It should be noted that the calculation used a very detailed nodalization of the steam generator cell and the duct between it and the pipe gallery to ensure that the mixing between the two volumes was modeled accurately. The results show that the maximum hydrogen concentration in cell 6 would be 28 [Ref. 1, p. 5-9]. Extrapolating to twice the NUSAR source yields a concentration of 48 (the lower flammability limit). Doubling the NUSAR source results in $140 \mathrm{~kg}$ of hydrogen at $2 \mathrm{hr}$ whereas the integral production case 2 hydrogen is $150 \mathrm{~kg}$ at $2 \mathrm{hr}$. For this branch, forced ventilation will also be operating. With the forced ventilation on, the hydrogen is not only mixed and transported through the confinement, it is removed from the building through the exhaust fans (the forced ventilation system turns over the volume of the 109 building in 30 minutes). The probability distribution for this case is shown below.

$\begin{array}{cc}\text { Quantile } & \text { Total Probability } \\ & 0 . \\ .25 & .05 \\ .5 & .1 \\ .75 & .2 \\ 1.0 & .3\end{array}$

Case 7: V-4 valves fail, forced ventilation off, no nitrogen system

For these conditions, the LOCA will initially purge the oxygen from the confinement, but without forced ventilation the subsequent steam condensation in confinement will reduce the pressure and draw air back in from the environment. In addition, without forced ventilation, the steam generator cells will not necessarily be well mixed. Thus, the probability of forming a flammable mixture was assigned a value of 1.0 for this case.

\section{Pipe Barrier Space}

Hydrogen may enter the pipe barrier space if the pipe break is located in the core, in the 105 inlet header downstream of $\mathrm{CV}-5$, or if the break is somewhere else in the 105 building. Only the first two break locations will be considered in the analysis for the pipe barrier space since concentrations in the 105 building resulting in flammable concentrations in the pipe barrier space will be considered in the case of hydrogen flammability throughout the 105 building.

Once again the flammability of the mixture depends on: 1) mixing mechanisms, 2) hydrogen production, and 3) the state of the V-4 valves. Since the pipe barrier space is basically an enclosed space, there are no mixing mechanisms such as forced ventilation, sprays, or nitrogen mixing due to the mitigation system; thus, the case structure for a given production case is fairly simple.

Each of the cases is self explanatory but it must be kept in mind that a small LOCA may be a break in the core or a break in the 105 inlet riser 
downstream of the CV-5's. The large LOCA can only occur in the 105 inlet riser downstream of the CV-5's.

A HECTR calculation was performed with the NUSAR hydrogen source injected into the pipe barrier space. The maximum hydrogen concentration was 6.5\%. Extending this to twice the NUSAR hydrogen source (approximately the production case 2 source), the hydrogen concentration would reach a maximum of 13\%. Because of the small volume and the relative isolation of the pipe barrier space from the mixing and mitigating mechanisms, the probability of a flammable mixture in the pipe barrier space is assessed to be 1.0 for breaks that occur there.

\section{Building}

Hydrogen may enter the 105 building from any break in the 105 building; however, the ones considered here are those breaks that are in the 105 building but were not analyzed in the pipe barrier space branch. The breaks in the pipe barrier space were treated separately because the enclosure is much smaller, increasing the probability of a flammable mixture.

The breaks considered are a large LOCA in the $26^{n}$ outlet header outside of CV-3's, a large LOCA in the 105 inlet riser upstream of the CV$5^{\prime} \mathrm{s}$, and a small or large LOCA in the 10" outlet header inside CV-3's. These breaks may be grouped into two types of breaks: 1) those in which flow is though the break and V-4 valves (assuming they npen) and 2) thoso in which filow is primarily through the break. Group 1 includes the large LOCAs in the 26" outlet header outside of the CV-3's and in the 105 inlet riser upstream of the CV-5's and the small LOCA in the 10" outlet header inside the $\mathrm{CV}-3^{\prime} \mathrm{s}$. Group 2 includes the large LOCA in the 10" outlet header inside the CV-3.

Flammability in the 105 building depends on the combination of break group and V-4 valve position, the presence of mixing mechanisms (i.e. forced ventilation, sprays, the hydrogen mitigation system), and the amount of hydrogen released. The cases below are presented in two groups, those that are inflammable and those that are flammable with some probability.

\section{Inflammable Cases}

\section{Case 1: forced ventilation on}

The concentration of hydrogen in a volume the size of the 105 building if $150 \mathrm{~kg}$ of hydrogen (production case 2) were introduced would be 108 , implying that a flammable mixture may occur. The forced ventilation system in the 105 building has a volumetric flow rate of $100,000 \mathrm{cfm}$; this will turn over the 105 building volume in 7.6 minutes. Based on this knowledge the probability of a flammable mixture in these cases is assessed to be 0.0 . 
Case 2: Break Group 1, Large LOCA, V-4 valves open, sprays on

In this case the confinement will be well mixed by the sprays. Further, most of the hydrogen in this case will go to the dump tank. Therefore, the probability of a flammable mixture is assessed to be 0.0

Case 3: Break Group 1, Small LOCA, V-4 valves open

In this case nearly all of the hydrogen will go to the dump tank. Given the large volume of the 105 building and the convection forces present (hot surfaces and condensation), the probability of a flammable mixture is assessed to be 0.0 .

Case 4: nitrogen system on

In this case the confinement will be well mixed by the hydrogen mitigation system (the $40000 \mathrm{cfm}$ and $20000 \mathrm{cfm}$ nitrogen purges will turn over the volume of the confinement in 77 minutes and 155 minutes, respectively) Unlike case 1, nitrogen inerting is possible since the forced ventilation system is not on. Following blowdown, the nitrogen system will prevent oxygen from reentering the 105 building. Therefore, the probability of a flammable mixture is assessed to be 0.0

\section{Flammable Cases}

Case 5: Break Group 1, Large LOCA, V-4 valves open, fans on, no nitrogen system

Even with the V4 valves open, up to 258 of the hydrogen may enter the 105 building in a large LOCA. Without the nitrogen system, only the fans will provide mixing of hydrogen with the rest of the confinement. Sufficient hydrogen is available to produce a flammable mixture in the 105 building, although not if mixed within the 109 and 105 buildings by the fan. Because of timing considerations, some probability of flammability is considered.

\begin{tabular}{lc} 
Quantile & Probability \\
\hline 0.0 & 0.0 \\
0.25 & 0.025 \\
0.5 & 0.05 \\
0.75 & 0.075 \\
1.0 & 0.15
\end{tabular}

Case 6: Break Group 1, Large LOCA, V-4 valves open, fans off, no nitrogen system

This case is similar to case 5, except that the fans are not working. The only mixing is provided by convection from hot surfaces and 
condensation. Therefore, the probability of flammability is higher than for case 5 .

\begin{tabular}{ll} 
Quantile & Probability \\
\hline 0.0 & 0.0 \\
0.25 & 0.05 \\
0.5 & 0.1 \\
0.75 & 0.15 \\
1.0 & 0.3
\end{tabular}

Case 7: Break group 1, V-4 valves fail closed, no fans, no nitrogen system or Break group 2, no fans, no nitrogen system

This case is similar to case 2 with the exception of the state of the V-4 valves and the addition of the break 2 cases which are equivalent to the break 1 cases with V-4 valve failure. The small and large break LOCA's are not differentiated because all flow will be out the break. A HECTR calculation was performed with the NUSAR hydrogen source injected into the pipe barrier space [Ref 1, p. 5-3]. The maximum hydrogen concentration with the exception of the pipe barrier space was 2.88. Extrapolating this to twice the NUSAR hydrogen source yields a maximum hydrogen concentration of 5.68. Even with mixing by the sprays, if available, there is sufficient hydrogen for flammability. Since the integral hydrogen source can be greater than the NUSAR source at $2 \mathrm{hr}$, a value of 1.0 was assigned to the probability of flammability for this case.

Case 8: Break group 1, V-4 valves fail closed, fans on, no nitrogen system or Break group 2, fans on, nn nitrogen oyotem

With the fans on, there will be additional mixing with the 109 building. Given that there is not a large excess of hydrogen according to the calculation discussed for case 7 , there is some probability that a flammable mixture will not occur. Therefore, the probability distribution below was developed.

\begin{tabular}{|c|c|}
\hline Quantile & Probability \\
\hline 0.0 & 0.2 \\
\hline 0.25 & 0.4 \\
\hline 0.5 & 0.6 \\
\hline 0.75 & 0.8 \\
\hline 1.0 & 1.0 \\
\hline
\end{tabular}

\section{Dump Tank}

Hydrogen may enter the dump tank through the dump line or through the vent from the confinement; however, only the pathway through the dump line will be considered since the probability of getting a flammable mixture in the dump tank via hydrogen transport through the confinement vent is much smaller than the probability due to transport through the dump line. 
The flammability of the mixture depends on the status of the dump line path, the status of the V-4 valves, and the size of the break (given that there is one). The dump line must be intact and the $V-4$ valves must have opened for hydrogen to accumulate in the dump tank. There are no mixing mechanisms in the dump tank.

A MELCOR model of the dump tank and the pipe gallery was used to determine the hydrogen concentrations in both buildings. for the upper limit of production case 1 ( $90 \mathrm{~kg}$ in $2 \mathrm{hr}$ ). The dump tank was initially filled to the LERF overflow level and then hydrogen was injected into the control volume. The mole fraction of hydrogen in the dump tank increased rapidly and a two hours it was .55. The mole fraction of oxygen decreased and was approximately .08 at 2 hours. Some hydrogen was transported to the pipe gallery and at 2 hours the mole fraction was .005. Based on this calculation the probability of a flammable mixture in the dump tank is assessed to be 1.0 for all cases with the V4 valves open and the dump tank path intact, and 0.0 for cases with the V4 valves closed.

\section{Building}

Although the case structure for this branch has been developed, a simple calculation of the hydrogen concentration in the 109 building if 150 $\mathrm{kg}$ are introduced shows that the concentration would be $2.8 \%$. For this reason a probability of 0.0 has been assigned in all cases for flammability during the first two hours.

\section{5 and luy Buildings}

The mass of hydrogen necessary for a 48 concentration in the entire confinement is $286 \mathrm{~kg}$. Since Reference 5 indicated that the maximum amount of hydrogen produced in the first two hours without ECCS and GSCS was 150 $\mathrm{kg}$ (the upper limit of production case 2), this branch will have a zero probability for flammability in the first two hours for all cases based on the fact that this amount of hydrogen only produces a 2.88 hydrogen concentration in building 109 alone - without the inclusion of building 105.

\section{C.1.3.2 Flammable Hydrogen Detonable in First Two Hours?}

The contents of this section are taken from an issue writeup for this question by L. A. Miller, A. L. Camp and S. F. Dingman dated September 25, 1989. This question addresses the probability of a detonable mixture in the first two hours provided that nothing (such as a prior deflagration) precludes it. The two possible branches for this question are:

Branch Abbrev, Description
(1) Henter
Hydrogen Not Detonable
(2) $\mathrm{H} \cdot \operatorname{det}$
Hydrogen De Conable Sumewhere 


\section{Relevant Factors and Background Information}

The answers to this question depend primarily on the answers to Hydrogen Question 1. The probabilities are given for each appropriate case in Question 1. The references identified in Hydrogen Question 1 were also used here. In addition, SAND82-1137, NUREG/CR-2726 Light Water Reactor Hydrogen Manual was used.

Only a single location is considered to be potentially detonable at a given time because the amount of hydrogen released during the first two hours is not believed to be sufficient to cause flammable conditions in two locations. Further, with an upper limit of $150 \mathrm{~kg}$ of hydrogen, only relatively small volumes can achieve a detonable mixture (>118). For example, an 118 hydrogen mixture in air (the lower detonability limit used) containing $150 \mathrm{~kg}$ of hydrogen at 1 atmosphere pressure and a temperature of $320 \mathrm{~K}$ occupies a volume of $17903 \mathrm{~m}^{3}$. Thus, detonable mixtures throughout either the pipe gallery or the 105 building are not possible, since their volumes are larger than this.

For smaller volumes, numerous code calculations indicate the propensity for hydrogen to mix in many cases. Fans and sprays will both induce significant mixing. Further, natural convection will be significant, due to condensation on the walls and also the presence of hot surfaces, particularly in the 105 building. Most of the separate compartments are connected by large openings that allows for substantial intercompartment mixing. Finally, the purging of oxygen to levels below about 98 or the presence of steam above about 308 will also render a mixture nondetonable.

With the above considerations in mind, each potentially flammable location is considered below. The probabilities below apply to all cases in hydrogen question 1 in which a flammable mixture is considered possible (obviously, nonflammable mixtures are also nondetonable).

\section{Penthouse}

Because of the small volume of the Penthouse, and the results of code calculations using HECTR and COBRA-NC (with hydrogen concentrations above 248), it appears that high hydrogen concentrations are possible for virtually all cases where flammability is possible. In some cases it is difficult to ascertain the availability of oxygen. We will conservatively assume that oxygen will be present and available to mix with the hydrogen in the penthouse. Therefore, a probability of 1.0 that the mixture will be detonable is assigned. 


\section{Pipe Gallery}

\section{Case 1: V4 valves closed}

The maximum concentration of hydrogen in the pipe gallery would be 98 if $150 \mathrm{~kg}$ of hydrogen was well mixed within the pipe gallery, as a result of pipe breaks in the pipe gallery or in adjacent compartments. This hydrogen will also mix with other locations, and major stratification is not anticipated. For these reasons, we have assigned a probability of 0.0 for the formation of detonable mixtures in the pipe gallery in cases with the V4 valves closed.

Case 2: V4 valves open, no sprays or mitigation.

In this case most of the hydrogen will go to the dump tank. From there, some will be transported back to the pipe gallery near the top. Without sprays or mitigation, mixing will be limited and some stratification near the top is possible. No specific calculations are available to address this case. Therefore, the probability distribution below is assigned to the case.

\begin{tabular}{cc} 
Quantile & Probability \\
\hline 0.0 & 0.0 \\
0.25 & 0.25 \\
0.5 & 0.5 \\
0.75 & 0.75 \\
1.0 & 1.0
\end{tabular}

\section{$\underline{\text { Steam Generator Cel1 }}$}

The probability of hydrogen not being detonable is based on a HECTR calculation using the NUSAR source term. This calculation included a detailed nodalization to examine mixing between the steam generator cells and the pipe gallery. A substantial amount of mixing with the pipe gallery is predicted to occur. The maximum hydrogen concentration was approximately 28 in the calculation. Extrapolating to the maximum hydrogen production case yields a value of approximately 48 hydrogen. Therefore, the probability of a detonable mixture is assigned a value of 0.0 for all mixtures in the steam generator cells during the first two hours.

\section{Pipe Barrier Space}

A HECTR calculation was performed releasing the NUSAR hydrogen source into the pipe barrier space. A maximum hydrogen concentration of 6.58 resulted. Extrapolating for the maximum hydrogen production case yields a hydrogen concentration of 138. This is in the weakly detonable range of 11 - 188, so the possibility of detonation can not be discounted. However, there will also be high concentrations of steam present, and the oxygen may 
be partially depleted. Therefore, the probability distribution shown below is assigned to this case.

\begin{tabular}{|c|c|}
\hline Quantile & Probabilit \\
\hline 0.0 & 0.0 \\
\hline 0.25 & 0.3 \\
\hline 0.5 & 0.6 \\
\hline 0.75 & 0.7 \\
\hline 1.0 & 1.0 \\
\hline
\end{tabular}

\section{Building}

A maximum hydrogen concentration in the 105 building would be approximately 108 if $150 \mathrm{~kg}$ of hydrogen was well mixed within the building. Because of transport out of the building and into other parts of confinement, less than $150 \mathrm{~kg}$ of hydrogen will be present at any one time. Further, the hot surfaces around the reactor will promote convection in the event that the sprays or fan are not operating. Therefore the probability of a detonable mixture was assessed to be 0.0

\section{Emergency Dump Tank}

A MELCOR calculation was performed that released approximately $90 \mathrm{~kg}$ of hydrogen into the dump tank over a two hour period. Even accounting for flow back to the pipe gallery, hydrogen concentrations as high as 558 were calculated. Eventually, the oxygen may be purged from the dump tank. However, during the first two hours we assess the probability of a detonable mixture to be 1.0 .

\section{Building}

The maximum concentration of hydrogen in the 109 building with $150 \mathrm{~kg}$ of hydrogen is below the flammability limit. Therefore, the probability of a mixture that is detonable throughout the 109 bullding is 0.0

\section{5 and 109 Building}

The same argument as for the 109 building detonability branch holds for this branch. The value assigned to the probability of a detonable mixture is 0.0 


\section{C.1.3.2 Does Hydrogen Ignite in the First Two Hours?}

The contents of this section are taken from an issue writeup for this question by A. L. Camp dated September 17, 1989. This question addresses the ignition probability for early hydrogen releases. The two possible branches for this question are:

Branch Abbrev, Description

(1) H-noig Ignition does not occur

(2) H-ig Ignition does occur

\section{Relevant Factors}

The answers to this question are a function of several previous questions. If there are no flammable mixtures present, then branch 1 is always chosen. Given that a flammable mixture exists, there are three types of ignition sources to consider: 1) electrical sources (function of $\mathrm{AC} / \mathrm{DC}$ power and colocated components, or even static discharges), 2) mechanical sources (items that can move around and create sparks, and 3 ) hot gas and debris from the break. These ignitions sources are considered separately for each potential location of hydrogen flammability.

The actual probability of ignition, given flammability and some ignition source, is also a function of the mixture sensitivity (hydrogen concentration). Mixtures closer to stoichiometric are easier to ignite. Thus, detonable mixtures are considered easier to ignite than those that are merely flammable.

\section{Background Information}

In developing the probability distributions for this issue, a number of information sources were reviewed. NUREG/CR-2726, Light Water Reactor Hydrogen Manual, indicates that small amounts of spark energy (millijoules) are required to ignite hydrogen and that hydrogen will spontaneously ignite in temperacures above $500 \mathrm{C}$ (higher temperatures for lean, steam-rich mixtures).

Another source of information is the soon to be published NUREG/CR-4551, which is a supporting document to NUREG-1150. NUREG/CR-4551 contains expert judgments concerning hydrogen ignition during station blackout events at the Sequoyah and Grand Gulf nuclear power plants (igniter systems failed). Three experts provided estimates of ignition probabilities for cases where there were no clearly identifiable ignition sources. Generally, 1gnillun was alwayo oonsidered a possibility, with probabilities distributions covering the $0-208$ range for some conditions and $0-758$ for others. Significant variability was due to 1) different hydrogen concentrations, and 2) identifiable mechanical mechanisms, such as ice-condenser doors. While these cases are not precisely the same as the cases to be considered for $\mathrm{N}$ Reactor, they do indicate that hydrogen ignition 15 possible under a variety of conditions and without deliberate ignition systems. 
Consultation with the MELCOR analysts for the $N$ Reactor PRA program indicate that, early in the accident, hydrogen exit temperatures could be in the range of 290 - $320 \mathrm{C}$. In most cases, the calculations show that the hydrogen will be in contact with liquid water before it exits the break, thus keeping the exit temperature low. On the other hand, for releases near the reactor core, e.g., in the pipe barrier space, the ignition probability is assessed to be very high.

A review of the plant design indicated four possible mechanical components that could cause sparks:

1. Vacuum breakers in the 105 and 109 building,

2. Cross vents between the 105 and 109 buildings,

3. Valve in the filter vent system, and

4. Vacuum breaker between dump tank and confinement.

A detailed review of electrical components was not performed; however, it was assumed that electrical sources are present in most locations. These sources include instrumentation and valves. For this analysis, AC sources are the only ones considered likely to cause sparks. Active DC components that cycle on and off are assumed to all be located outside confinement. No information is available regarding static discharges. At least one accidental ignition has occurred at the FLAME facility at Sandia as a result of static discharge, and the NUREG-1150 experts considered that as a possibility, especially since even high gas velocities from a pipe break can lead to static discharge.

\section{Assessments for Each Case}

The cases are defined by the answers to three previous questions: 1 ) any $A C$ power, 2) location of flammable mixture, and 3) whether the mixture is detonable. In addition, for the Pressurizer Penthouse in cases without AC power, the hydrogen mitigation fans will be considered as a source of AC power (of low probability since the fan motors will be sealed). If other $A C$ is available, the mitigation fans will represent a negligible contribution.

Initially 30 different cases were identified, based on the questions above. Those cases were collapsed down to 13 cases by combining all cases with similar probability distributions.

For some cases, the assessment is decomposed into the probabilities of ignition from each individual cause. In those cases recomposition by sampling the individual distributions will be required - simply adding the probabilities at each quantile is not correct. 
Case 1: Penthouse, No AC Power, Not Detonable, No Mitigation Fans or SG Ce11, No AC Power, Not Detonable

For the penthouse, no specific mechanical means of causing sparks has been identified. Without any source of AC power, we are left with hot effluent from the break, static discharge, and the possibility that some mechanical source may have been overlooked. In the first two hours, MELCOR results indicate that the temperature of the effluent will be well below $500 \mathrm{C}$; thus, that mechanism has been discounted. A very low probability of ignition during the first two hours is thus expected.

No specific mechanical sources have been identified in the SG Cells or Aux Cell. The static probability should be similar to the pressurizer penthouse. High temperature effluents are not expected during the first two hours. Ignoring the effects of sprays, these cells should behave the same as the penthouse.

\begin{tabular}{ccc} 
Quantile & Static Discharge & Mechanical \\
\hline 0.0 & $1 . E-6$ & $1 . E-5$ \\
.25 & $1 . E-5$ & $1 . E-3$ \\
.5 & $5 . E-4$ & 0.01 \\
.75 & 0.01 & 0.05 \\
1.0 & 0.05 & 0.1
\end{tabular}

These probabilities are on a log scale, and interpolations should be done in a log-linear manner.

Case 2: Penthouse, No AC Power, Not Detonable, Mitigation Fans Available or Penthouse, No AC Power, Detonable, No Mitigation Fans

With the mitigation fans available, the probabilities in case 1 are increased in two ways. First, electrical discharges are increased due to the fan motors, and increased gas velocities which increase the static discharge probabilities. Second, the increased gas velocities increase the probability of mechanical sparks due to things moving around. Overall, the probabilities remilin fairly low.

With a detonable mixture, a much smaller spark is needed to light off the mixture. This sensitivity was assessed to increase the ignition probability from Case 1 by the same amount as having the mitigation system on; therefore, the two cases were combined.

$\begin{array}{ccc}\text { Quantile } & \text { Static }+ \text { Elect. } & \frac{\text { Mechanical }}{0.0} \\ .25 & 1 . E-5 & 5 . E-5 \\ .5 & 1 . E-4 & 1 . \text { E-3 } \\ .75 & 1 . E-3 & 0.02 \\ 1.0 & 0.05 & 0.07 \\ & 0.15 & 0.15\end{array}$

These probabilities are on a log scale, and interpolations should be done in a log-linear manner. 
Case 3: Penthouse, No AC Power, Detonable, Mitigation Fans Available

For this case, the probabilities are slightly higher than for Cases 1 and 2 , because the mixture is extremely sensitive and the mitigation fans are available to provide possible spark sources and enhance the mechanical motions of loose items.

\begin{tabular}{ccc} 
Quantile & Static +Elect. & Mechanical \\
\hline 0.0 & $1 . E-4$ & $1 . E-4$ \\
.25 & $1 . E-3$ & $2 . E-3$ \\
.5 & 0.01 & 0.05 \\
.75 & 0.07 & 0.1 \\
1.0 & 0.15 & 0.2
\end{tabular}

These probabilities are on a $\log$ scale, and interpolations should be done in a log-linear manner.

Case 4: Penthouse, AC Available or Pipe Gallery, AC Available or SG Cell, AC Available

With AC power available and a substantial amount of electrical equipment in the penthouse, the probability of ignition from AC sources is assessed to be much larger than the probability from static or mechanical sources. Also, since the spark from any electrical equipment will almost certainly be energetic enough to light the mixture, no differentiation between detonable and nondetonable mixtures was made. Similar arguments hold for the SG Cells. For the pipe gallery, the AC contribution may be lower, but that is offset by a higher mechanical contribution.

\begin{tabular}{cc} 
Quantile & Total Probability \\
\hline 0.0 & 0.0 \\
.25 & 0.3 \\
.5 & 0.5 \\
.75 & 0.7 \\
1.0 & 1.0
\end{tabular}

These probabilities are on a linear scale, and interpolations should be done in a linear manner. 
Case 5: Pipe Gallery, No AC Power, Detonable

Detonable cases in the pipe gallery will involve the top portions, which is fairly open. Most of the mechanical sources are fairly high in the pipe gallery and will be considered as ignition sources in this case.

\begin{tabular}{|c|c|c|}
\hline Quantile & Static Discharge & Mechanical \\
\hline 0.0 & 1.E-5 & 1.E-3 \\
\hline .25 & 1.E-4 & 0.01 \\
\hline .5 & 1.E-3 & 0.05 \\
\hline .75 & 0.05 & 0.15 \\
\hline 1.0 & 0.15 & 0.4 \\
\hline
\end{tabular}

These probabilities are on a log scale, and interpolations should be done in a log-linear manner.

Case 6: Barrier Space or 105 Building, No AC Power

The close proximity of the pipe barrier space to the reactor core means that any fuel damage scenario is likely to cause very hot material to be in this region. Thus, ignition is considered very likely, independent of the detonability of the mixture and independent of the presence or absence of $A C$ or $D C$ power.

The case of the entire 105 building includes the pipe barrier space along with the rest of the 105 building. Therefore, the total probability will be larger than for just the barrier space. However, the likelihood of hot effluents is the same as for the barrier space, and the additional mechanical sources are small in comparison. Therefore, the same probability is used.

$\begin{array}{cc}\text { Quantile } & \text { Total Probability } \\ & 0.5 \\ .25 & 0.8 \\ .5 & 0.9 \\ .75 & 0.95 \\ 1.0 & 1.0\end{array}$

These probabilities are on a linear scale, and interpolations should be done in a linear manner.

Case 7: 105 Building, AC Power

With both $A C$ power and hot effluents and materials present in the 105 building, the probability of ignition is assessed to be 1.0 .

Case 8: Dump Tank, Not Detonable

For the dump tank, no AC or DC sources of significance were identified. Also, the clean, open configuration and low flow velocities of 
hydrogen within the dump tank make static sources unlikely. The most likely source would appear to be mechanical sparks coming from the vacuum breakers into confinement. Therefore, the only differentiation in case structure here is for detonable versus nondetonable mixtures.

\begin{tabular}{cc} 
Quantile & Total Probability \\
\hline 0.0 & 1. E -6 \\
.25 & 5. E -4 \\
.5 & 5. E -3 \\
.75 & 0.02 \\
1.0 & 0.1
\end{tabular}

These probabilities are on a $\log$ scale, and interpolations should be done in a log-linear manner.

Case 9: Dump Tank, Detonable

For the detonable case, the bottom and middle of the distribution is increased, due to the enhanced mixture sensitivity.

\begin{tabular}{cc} 
Quantile & Total Probability \\
\hline 0.0 & $1 . E-5$ \\
.25 & $1 . E-3$ \\
.5 & 0.01 \\
.75 & 0.05 \\
1.0 & 0.1
\end{tabular}

Thésè probabilities are on a log scale, and interpolations should be done in a log-linear manner.

Case 10: Mixed in 109 Building, No AC Power

For this case, the mixture is assumed to exist in the pipe gallery, the penthouse, and six cells. Therefore, the probability is determined by combining the probabilities from Cases 2, 4, and 1, with the latter included six times. The appropriate equation is:

$$
P_{18}=1-\left(1-P_{p h}\right)\left(1-P_{p g}\right)\left(1-P_{s g}\right)^{6}
$$

where

$$
\begin{aligned}
& P_{18}=\text { Case } 10 \text { ignition probability } \\
& P_{p h} \text { - Case } 2 \text { ignition probability for the penthouse } \\
& P_{p 8} \text { - Case } 4 \text { ignition probability for the pipe gallery } \\
& P_{s 8} \text { - Case } 1 \text { ignition probability for the SG cell }
\end{aligned}
$$

The new distribution for Case 10 should be formed assuming independence among the distributions, except for the six cells which should be fully correlated. 
Case 11: Mixed in 109 Building, AC Available

The approach for case 11 is the same as for case 10, except that different cases are combined. The variables in the Case 10 equation should be replaced with:

$$
\begin{aligned}
& P_{1 \mathrm{~g}}=\text { Case } 11 \text { ignition probability } \\
& \mathrm{P}_{\mathrm{ph}}=\text { Case } 4 \text { ignition probability for the penthouse } \\
& \mathrm{P}_{\mathrm{pg}}=\text { Case } 4 \text { ignition probability for the pipe gallery } \\
& \mathrm{P}_{\mathrm{sg}}=\text { Case } 4 \text { ignition probability for the } S G \text { cell }
\end{aligned}
$$

In this case all of the variables using the Case 4 distribution should be fully correlated.

\section{Case 12: Mixed in Entire Confinement}

Because the probabilities from Cases 6 and 7 (Barrier Space and 105 Building) are so high, when combined with the 109 building probabilities, the probability for this case is assessed to be 1.0 .

\section{C.1.3.4 Does Ignited Hydrogen Deflagrate or Detonate in First Two Hours?}

The contents of this section are taken from an issue writeup for this question by A. L. Camp and L. A. Miller dated September 17, 1989. This

\begin{tabular}{|c|c|c|}
\hline Branch & Abbrev. & Description \\
\hline $\begin{array}{l}(1) \\
(2)\end{array}$ & $\begin{array}{l}\text { H-def } \\
\text { H-det }\end{array}$ & $\begin{array}{l}\text { Deflagration occu } \\
\text { Detonation occurs }\end{array}$ \\
\hline
\end{tabular}
question addresses the likelihood of a detonation in potentially detonable mixtures, given that ignition has occurred. The two possible branches for this question are:

\section{Relevant Factors}

The answers to this question are dependent on the answers to several previous questions. First, if a detonable mixture is not present anywhere or ignition does not occur, then this question should be skipped. Given that a detonable mixture is ignited, then the previous questions that matter are:

1. Location - This determines the size of the volume involved and the obstacles and confinement that may influence deflagration to detonation transition (DDT).

2. Sprays - This influences the turbulence level.

3. Mitigation System - The fans and the gas injection will supply turbulence. 
Along with these factors, it is important to note whether the mixture is weakly detonable (about 11 - 188 hydrogen or with significant fractions of dilutents) or strongly detonable. Further, multiple events must be considered. That is, an initial deflagration does not preclude a detonation at a later time unless all of the hydrogen is consumed. On the other hand, one detonation will be considered adequate to fail confinement, so that additional events do not need to be treated. In all cases it is assumed that DDT is necessary for a detonation to occur, i.e., there are no ignition sources strong enough to lead to direct initiation.

\section{Background Information}

There is a tremendous amount of background information on DDT and hydrogen detonations. The references consulted include:

1. NUREG/CR-4803, The Possibility of Local Detonations During Degraded-Core Accidents in the Bellefonte Nuclear Power Plant.

2. NUREG/CR-2726, Light Water Reactor Hydrogen Manual.

3. Nuclear Science and Engineering: 93, 321-347, A Critical Review of Recent Large-Scale Experiments on Hydrogen-Air Detonations.

4. NUREG/CR-4551, Evaluation of Severe Accident Risks: Quantification of Major Input Parameters, Volume 2, to be published.

The latter reference is a supporting document to NUREG-1150, and rantains input from several experts and reference to still other supporting documents not cited here. Specifically, detonation probabilities were determined for various accident sequences at the Grand Gulf and Sequoyah nuclear power plants. The results in Reference 4 are different for each specific case, based on most of the same factors identified above. However, it is important to note that in many cases high detonation probabilities ( $>508$ ) were considered possible.

Specifically for $N$ Reactor, each of the possible locations where detonable mixtures are present contain obstacles conducive to detonations. These obstacles include piping, grating, and components. The only exception to this is the dump tank.

The mixing fan provides turbulence to the penthouse, while the sprays could provide turbulence to all of the regions except the penthouse and the dump tank. Note, however, that the sprays are automatic in some regions and manual in others.

On the positive side, most of the regions considered, except the dump tank, include a large amount of venting that tends to reduce the likelihood of detonations. However, it is important to note that venting that draws flow past obstacles can also be detrimental.

Generally, sensitive mixtures will be considered likely to detonate in all of these regions except the dump tank, which is a very clean, open 
volume. The probability of DDT in weakly detonable mixtures will be considered below on a case by case basis.

The assessments themselves are based on the approach in Reference 1 , with some differences in the classification breakdown.

\section{Case 1: Penthouse, Mixing Fan Available}

The penthouse is open on the bottom, providing a significant amount of venting. The pressurizer, associated piping, and gratings are present in the area as obstacles. Also, the mixing fan blows turbulent air directly into the penthouse from the 105 building. While most of the applicable sequences could result in high hydrogen concentrations, oxygen will be limiting in some cases, and prior burns could preclude highly detonable mixtures in other cases.

Overall, the geometry is favorable for detonations - Class 2 or 3 in the scheme of Reference 1. Obstacles are present; however, the venting somewhat counteracts this. The mixture can vary, but will likely be in the range of class 2 to class 4. This produces an overall Results class of 3 or 4 , probably closer to 4 .

The detonation probabilities below for weak and strong mixtures are conditional on the presence of the mixture. The fraction of mixtures assessed to be strongly detonable, SF, is 0.25 . The total probability is determined by the following equation:

$$
P_{d}=S F * P_{s}+(1-S F) * P_{w}
$$

where

$$
\begin{aligned}
& P_{d}=\text { Total Detonation Probability } \\
& S F=\text { Fraction of detonable mixtures that are strongly detonable } \\
& P_{s}=\text { Detonation Probability for strong mixtures } \\
& P_{w}=\text { Detonation Probability for weak mixtures }
\end{aligned}
$$

\begin{tabular}{|c|c|c|c|}
\hline Quantile & Weak Mixtures & Strong Mixtures & Total Probability \\
\hline 0.0 & 0.0 & 0.1 & 0.025 \\
\hline .25 & 0.05 & 0.25 & 0.1 \\
\hline .5 & 0.1 & 0.5 & 0.2 \\
\hline .75 & 0.15 & 0.75 & 0.3 \\
\hline 1.0 & 0.25 & 1.0 & 0.4375 \\
\hline
\end{tabular}

The distributions for weak and strong mixtures are assumed to be fully correlated, since the factors that affect them, such as geometry, ignition location, and turbulence, are the same. Therefore, the above equation can be used at each quantile.

This distribution should be linearly interpolated in between the given quantiles. 


\section{Case 2: Penthouse, No Mitigation}

Without the mitigation system, strongly detonable mixtures are more likely. In order to simplify the analysis, a simple split fraction is used to identify the fraction of mixtures that are strongly detonable.

A split fraction of .9 and .1 was assigned to the probability of a strongly detonable and a weakly detonable mixture, respectively. A small value was assigned to the weakly detonable mixture to allow for break locations near the bottom of the penthouse which would allow more mixing with the pipe gallery atmosphere. A value of .9 was assigned to a strongly detonable mixture because HECTR calculations show a hydrogen concentration of 248 early in the release if the mitigation fans are not operating. Since the expected concentration is in the strongly detonable region, multiple deflagrations may take place if the mixture deflagrates first.

Given a weak mixture, the detonation probability is less than for Case 1 due to the lack of turbulence from the mitigation system. The results class is approximately 4. On the other hand, with a mixture class of 1 or 2 and a larger fraction of the penthouse involved, the results class for strong mixtures becomes 1 or 2 .

Using the method discussed for Case 1 and with a value for SF of 0.9 , we obtain the results shown below.

\begin{tabular}{ccc} 
Quantile & Weak Mixture \\
\cline { 1 - 1 } 0.0 & & 0.0 \\
.25 & & 0.1 \\
.5 & & 0.2 \\
.75 & & 0.3 \\
1.0 & & 0.4
\end{tabular}

\begin{tabular}{cc} 
Strong Mixture & Total Probability \\
\cline { 1 - 2 } 0.25 & 0.23 \\
0.5 & 0.46 \\
0.75 & 0.70 \\
0.875 & 0.82 \\
1.0 & 0.94
\end{tabular}

These probabilities are on a linear scale, and all interpolations should be done in a linear manner.

\section{Case 3: Dump Tank}

The dump tank is a clean, open geometry, with no identifiable mechanisms for generating turbulence, except for the turbulence generated in the combustion process. Therefore, the detonation probability for weak mixtures is very small.

The split fraction for strong versus weak mixtures is .95 versus .05 . The reason this split fraction was chosen was that the mixture is strongly detonable during the majority of the time after transport into the tank begins. However, in the early portion of the accident the mixture is weakly detonable. Multiple deflagrations could preclude strongly detonable mixtures in some cases. 
The geometric class here is felt to be 3 or 4 , yielding a Results class of 4 or 5 for weak mixtures and about 3 for strong mixtures. The equation presented for Case 1 is used to determine the total probability.

\begin{tabular}{|c|c|c|c|}
\hline Quantile & Weak Mixture & Strong Mixture & Total Probability \\
\hline 0.0 & 0.0 & 0.0 & 0.0 \\
\hline .25 & 0.005 & 0.125 & 0.12 \\
\hline .5 & 0.01 & 0.25 & 0.24 \\
\hline .75 & 0.03 & 0.375 & 0.36 \\
\hline 1.0 & 0.05 & 0.5 & 0.48 \\
\hline
\end{tabular}

These probabilities are on a linear scale, and all interpolations should be done in a linear manner.

Case 4: Pipe Gallery, No Sprays or Mitigation or Pipe Gallery, No Sprays, 3k Nitrogen Purge with no Fans

Detonable mixtures in the pipe gallery during the first two hours can only form at the top as a result of return from the dump tank. Without the sprays or mitigation, mixing will be limited to natural convection and some detonable mixtures are considered possible. The mixtures are likely to be weakly detonable, due to the large mixing volume that could participate and the holdup of hydrogen in the dump tank.

For mixtures in the upper region, the geometric class is about 3 . The results classes would then be about 3 or 4 .

In this case the fraction of strong mixtures, SF, is assessed to be 0.1 , and the probabilities are shown below.

$\begin{array}{ccccc}\text { Quantile } & \text { Weak Mixture } & & \text { Strong Mixture } & \text { Total Probability } \\ 0.0 & 0.0 & 0.0 & 0.0 \\ .25 & 0.05 & 0.125 & 0.0575 \\ .5 & 0.1 & 0.25 & 0.115 \\ .75 & 0.15 & 0.375 & 0.1725 \\ 1.0 & 0.2 & 0.5 & 0.23\end{array}$

These probabilities are on a linear scale, and all interpolations should be done in a linear manner.

\section{Case 5: Barrier Space}

The pipe barrier space itself is a reasonably clean, open volume. However, the paths out of the space involve the gas travelling out through a number of different holes. The travel of gas through these holes will generate turbulence and create vortices that could represent detonation sources.

Based on the HECTR calculation described in hydrogen question 1 for the pipe barrier space, the maximum hydrogen concentration in the first two hours would be 138 which is weakly detonable. However, hydrogen released 
into the pipe barrier space would be extremely hot (above the ignition temperature) and the mixture would probably deflagrate before detonating. The possibility of a continuously burning situation exists in this location. Therefore, the probability of a detonation is considered to be small.

$\begin{array}{cc}\text { Quantile } & \text { Total Probability } \\ & 0.0 \\ .25 & 0.01 \\ .5 & 0.05 \\ .75 & 0.10 \\ 1.0 & 0.15\end{array}$

\section{C.1.3.5 Does Hydrogen Fall Confinement Late?}

The contents of this section are taken from an issue writeup for this question by A. L. Camp and S. E. Dingman dated September 25, 1989. This is the first in a series of questions that ascertain the importance of hydrogen in confinement up to 24 hours into the accident. The three possible branches for this question are:

Branch Abbrev, Description

(1) H-nfail No confinement failure late due to hydrogen combustion

(2) H-fail Confinement fails due to late hydrogen combustion

(3) H-unk Subsequent questions will be addressed related to hydrogen

\section{Relevant Factors and Background Information}

For long term accidents, large quantities of hydrogen can be generated. On the other hand, ample time exists for the hydrogen mitigation system to purge the confinement of oxygen. We have grouped the accident sequences into a small number of groups with straightforward responses to the above question.

Case 1: Nitrogen System Operating, V-4 valves closed

Over a long time period, the nitrogen system can be expected to function to purge the confinement of oxygen, so that the mixture will be inert regardless of the quantity of hydrogen present. With the V-4's closed, hydrogen will not be entering the dump tank, so it will not be flammable. Therefore, branch 1 of this question will be selected with probability 1.0 . 
Case 2: Nitrogen System Operating, V-4 valves open

All of these cases will be included in Branch 3 above with a probability of 1.0. This case is unresolved here because of the possibility of flammable mixtures in the dump tank, even with the nitrogen system operating. Subsequent questions will resolve this issue.

Case 3: Nitrogen System Not Operating, less than $230 \mathrm{~kg}$ of Hydrogen

All of these cases will be included in Branch 3 above with a probability of 1.0. Subsequent questions will resolve this issue.

Case 4: Nitrogen System Not Operating, More than $230 \mathrm{~kg}$ of Hydrogen

Quantities of hydrogen up to $2000 \mathrm{~kg}$ have been postulated for long term accidents. Without the nitrogen system operating, sufficient oxygen to support combustion will probably be present. In these cases large flammable volumes may be present. Over a 24 hour period, it is likely that ignition will eventually occur from one of the sources identified in earlier questions. The presence of hot particulates becomes more likely as time passes. With large quantities of hydrogen involved in a burn, the difference between a deflagration and a detonation becomes irrelevant. With a 5 psig design pressure for the confinement, failure is likely. Multiple or continuous burning could alleviate this situation; however, we will conservatively neglect those effects. Thus, for this case, Branch 2 is selected with probability 1.0 .

\section{C.1.3.6 Is Hydrogen Flammable Late in the Accident?}

The contents of this section are taken from an issue writeup for this question by A. L. Camp and S. E. Dingman dated September 25, 1989. This question addresses the probability of a flammable mixture late in the accident (up to 24 hours). The nine possible branches for this question are:

Branch Abbrev.

(1) H-mit

(2) H-pent

(3) H-pgal

(4) H-sg

(5) H-bar

(6) $\mathrm{H}-1.05$

(7) H-dump

(8) H-109

(9) H-mix
Description

Hydrogen is not flammable anywhere

Hydrogen is flammable in the penthouse

Hydrogen is flammable in the pipe gallery

Hydrogen is flammable in a steam generator cell or the aux cell

Hydrogen is flammable in the pipe barrier space

Hydrogen is rlammablc in the 105 building

Hydrogen is flammable in the dump tank

Hydrogen is flammable throughout the 109 building

Hydrogen is flammable throughout the confinement 


\section{Relevant Factors and Background Information}

Two groups of cases are possible for this question. The first group of cases considers a maximum of $230 \mathrm{~kg}$ of hydrogen over a 24 hour period. Further, the nitrogen system has not functioned at either the 20000 or $40000 \mathrm{cfm}$ flow rates. With these restrictions in mind, much of the information discussed for Hydrogen Question 1 is relevant here also and will not be repeated. One key difference is that prior burns during the early phase can prevent late flammability. Otherwise, cases that were flammable early are likely to be flammable late also, since most of these cases involve slowly changing conditions after the first two hours. In most cases the difference between $150 \mathrm{~kg}$ of hydrogen early and $230 \mathrm{~kg}$ of hydrogen over a 24 hour period is minor.

The second group of cases considers flammability in the dump tank with hydrogen releases that could exceed $230 \mathrm{~kg}$. $230 \mathrm{~kg}$ is sufficient to form a flammable mixture in the dump tank, and the cases with additional hydrogen will also be flammable, but at a higher concentration. If a previous burn has occurred in the dump tank, however, the oxygen will be depleted, leaving a non-flammable mixture.

\section{Group 1 Cases: No Nitrogen System, Less than $230 \mathrm{~kg}$ of Hydrogen}

Penthouse, Pipe Gallery, SG Cell, 105 Building, 109 Building, Entire Confinement

\section{Case 1: Not Flammable Early}

If conditions did not favor flammability early, the incremental amount of hydrogen (from a maximum hydrogen production of $150 \mathrm{~kg}$ in the first two hours to $230 \mathrm{~kg}$ over 24 hours) will not change that, based on examination of the potentially flammable cases in hydrogen question 1 . Therefore the probability of late flammability for this case is 0.0 .

\section{Case 2: Flammable Early, No Burn}

This case will be flammable with a probability of 1.0 .

Case 3: Flammable Early, Burn(s)

Early burns can consume both the hydrogen and the oxygen present in the penthouse. Because the additional late release of hydrogen is very slow in this case, the probability of late flammability is assessed to be 0.0 . 


\section{Pipe Barrier Space}

The pipe barrier space is flammable early in all cases where the break is located in the barrier space. Therefore, the first case above for the penthouse is not applicable here.

Case 1: No Burn Early

The probability of late flammability is 1.0

Case 2: Early Burn(s) occur

The probability of late flammability is 0.0

Dump Tank

The dump tank is not affected by the nitrogen system, so that the case structure is slightly different.

Case 1: Not Flammable Early

The probability of late flammability is 0.0

Case 2: Flammable Early, No Burns

The probability of late flammability is 1.0

Case 3: Flammable Early, Burn(s)

Even if the hydrogen is not fully consumed, the oxygen will soon be gone from the dump tank. Thercfore, the probability of a flammable mixture late is 0.0 .

\section{Group 2 Cases: Nitrogen System. V-4 Valves Open}

Dump Tank

Case 1: No Early Burns

Due to the large quantity of hydrogen present, a flammable mixture is almost guaranteed, although eventually the oxygen will be purged out, leaving a hydrogen and steam rich mixture. However, the probability of a flammable mixture is asscsood here to be 1.0 


\section{Case 2: Early Burns occur}

If early burns occur in the dump tank, the oxygen will be consumed and late flammability will not be possible. Thus, the probability of a flammable mixture is 0.0 .

\section{C.1.3.7 Is Flammable Hydrogen Detonable In First 24 Hours?}

The contents of this section are taken from an issue writeup for this question by S. E. Dingman and A. L. Camp dated September 25, 1989. This question addresses the probability of forming a detonable mixture in the first 24 hours. Although it is possible for large hydrogen quantities to be released during long term accidents, most of these cases would either be inflammable because of nitrogen inerting or the cases would have previously led to confinement failure in hydrogen question 5. Basically, this question addresses the likelihood of forming a detonable mixture in the dump tank late in the accident (even if nitrogen inerting is available for the confinement) or in the confinement for lower releases of hydrogen (230 $\mathrm{kg}$ ) that are not guaranteed to fail confinement. The two possible branches for this question are:

\section{Branch Abbrev, Description}
(1)
H-ndet
Hydrogen Not Detonable
(2) H-det
Hydrogen Detonable Somewhere

\section{Relevant Factors and Background Information}

This question considers a maximum of $230 \mathrm{~kg}$ of hydrogen for detonability in all regions except for the dump tank which may have a larger release of hydrogen. The incremental release of hydrogen between 2 and 24 hours is therefore small, and would not be expected to greatly affect the likelihood of forming a detonable mixture. Thus, for all regions except the dump tank, it would be expected that only mixtures that were detonable early and that did not burn would be detonable late. For the dump tank, even if the region were not detonable early, with the large late hydrogen release it would become detonable. As currently configured, the dump tank contains a detonable mixture early for all cases; thus, it collapses into the same logic applicable to the other regions.

Case 1: Detonable early and No burn

A probability of 1.0 is assigned to branch 2 .

Case 2: Not detonable early or Early burn

A probability of 1.0 is assigned to branch 1 . 


\section{C.1.3.8 Does Hydrogen Ignite in the First 24 Hours?}

The contents of this section are taken from an issue writeup for this question by A. L. Camp and S. E. Dingman dated September 25, 1989. This question addresses the ignition probability for late hydrogen releases. The two possible branches for this question are:

Branch Abbrev. Description

(1) H-noig Ignition does not occur

(2) H-ig Ignition does occur

\section{Relevant Factors}

Except for the dump tank, this question deals only with cases where the nitrogen inerting system has not functioned. For most of confinement, there is sufficient potential for ignition that ignition will most likely occur during a 24 hour period. For the dump tank, there is much less potential for ignition, so it is considered as a separate case.

\section{A11 Regions Except Dump Tank}

A probability of 1.0 is assigned to branch 2 .

\section{Dump Tank}

If the dump tank did not ignite early, conditions favoring ignition probably do not exist. This is countered by long exposure time. Therefore the probability of ignition in the dump tank during 24 hours is:

\begin{tabular}{cc} 
Quantile & Probability \\
\hline 0.0 & 0.001 \\
.25 & 0.01 \\
.5 & 0.1 \\
.75 & 0.3 \\
1.0 & 0.6
\end{tabular}

\section{C.1.3.9 Does Ignited Hydrogen Deflagrate or Detonate in First 24 Hours?}

The contents of this section are taken from an issue writeup for this question by A. L. Camp and S. E. Dingman dated September 25, 1989. This question addresses the likelihood of a detonation occurring in potentially detonable mixtures, given that ignition has occurred. The two possible branches for this question are:

Branch Abbrev. Description

(1) H-def Deflagration occurs

(2) H-det Detonation occurs 


\section{Relevant Factors and Background Information}

Except for the dump tank, this question only applies to cases with less than $230 \mathrm{~kg}$ of hydrogen and with the nitrogen system inoperable. Because the incremental amount of hydrogen compared to the first two hours is not large and conditions late are changing slowly, the cases are not substantially different from those considered in hydrogen question 4.

The dump tank, however, will be somewhat different as a result of changing mixtures. Over time, hydrogen and steam will tend to purge the dump tank of oxygen, yielding weaker mixtures. Therefore, some modification to the hydrogen question 4 results is necessary.

\section{Case 1: Al1 Locations Except Dump Tank}

Use the hydrogen question 4 quantification results.

Case 2: Dump Tank

In hydrogen question 4, the split fraction for strong versus weak mixtures was 0.95 . For this case, with the oxygen concentration declining with time and the steam concentration increasing, that split fraction is modified to be 0.25 . The resulting distribution, using the method for hydrogen question 4 , then becomes:

\begin{tabular}{|c|c|c|c|}
\hline Quant1le & Weak Mixture & Strong Mixture & Total Probability \\
\hline 0.0 & U. U & 0.0 & 0.0 \\
\hline .25 & 0.005 & 0.125 & 0.035 \\
\hline .5 & 0.01 & 0.25 & 0.07 \\
\hline .75 & 0.03 & 0.375 & 0.11625 \\
\hline 1.0 & 0.05 & 0.5 & 0.1625 \\
\hline
\end{tabular}

\section{C.1.4 Application of Results}

A few of the probability distributions in the above analyses were not implemented in the Latin Hypercube (LHS) analysis for hydrogen because they were only applicable to low probability events such as the continued operation of the forced ventilation system. With one exception (discussed below), all others are implemented in the analysis. The implementation details for LHS can be found in the appendices to SAND89-2102, the final $N$ Reactor Risk Analysis Report.

The issue that is not implemented directly from the above distributions is that of hydrogen flammability in the pipe gallery at the end of the dump tank return line. The elicitees agreed that a deflagration under those conditions would involve only a very thin layer of hydrogen near the roof of that area, and that there would be more than enough volume in that building to absorb any pressure increase that would occur as a result of a deflagration in that area. Therefore, they assigned a probability of zero for confinement building failure as a result of a 
hydrogen deflagration due to hydrogen from the dump tank return line. However, the shock wave produced by a hydrogen detonation in that region would almost surely cause a failure of the confinement function. That is, a detonation would almost surely cause a hole in the building of sufficient size to cause a significant release of radionuclides to the environment. For this reason, the probability distributions in the above section were synthesized into one "aggregate" probability distribution based on the small event tree below.

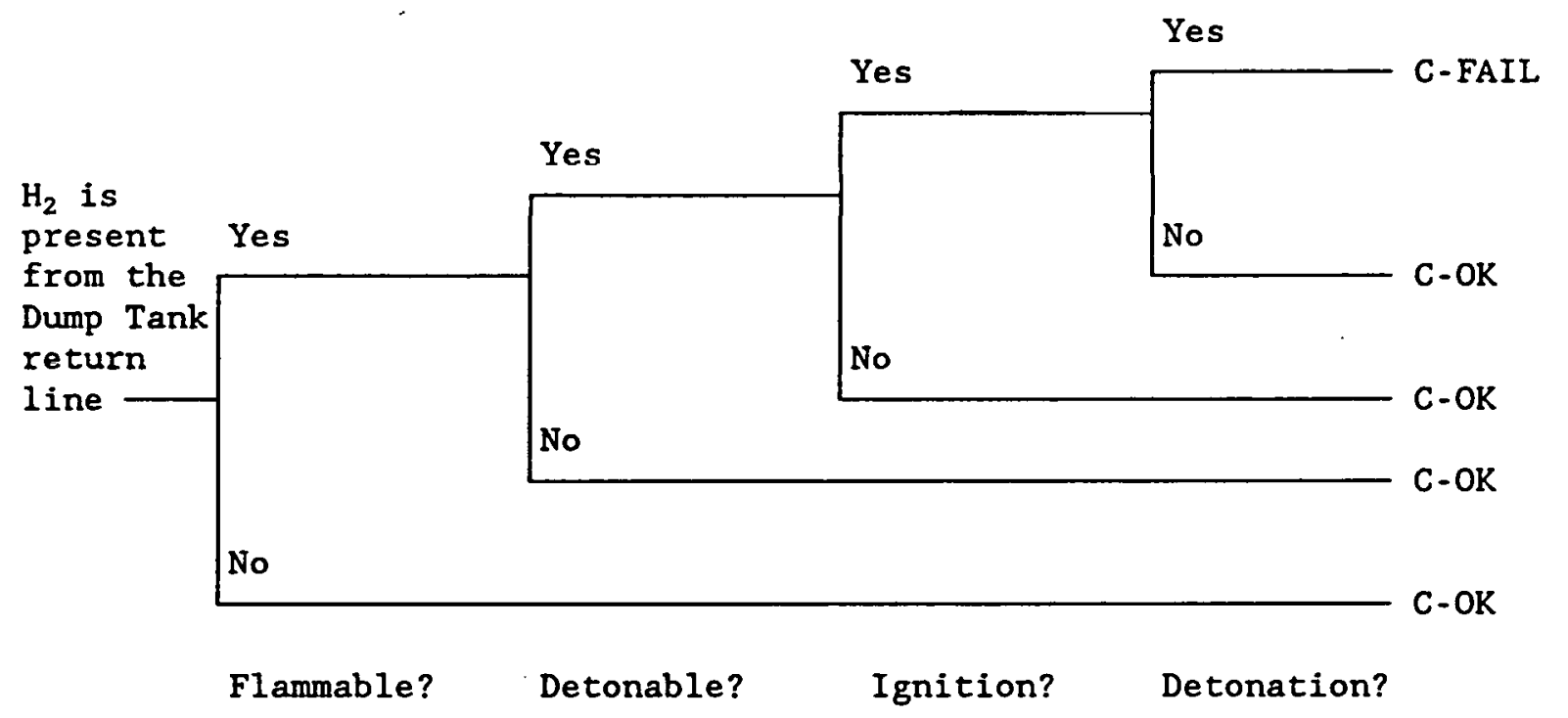

By the event tree above, the only combination of events that can lead to a failure of the confinement function for hydrogen events caused by the hydrogen released from the dump tank return line is that the hydrogen is flammable and detonable, it ignites and undergoes deflagration-todetonation transition (DDT). It was the consensus of the elicitees that all of the hydrogen issues and prohahility distributions are very tightly correlated because the principal uncertainty is the amount of hydrogen that is available for combustion. This uncertainty affects all areas of the hydrogen phenomenology including the probability of ignition. Thus, since al1 of the hydrogen issues can be thought of as being based on one underlying random variable (the amount of hydrogen), the same quantile would be chosen from each of the distributions, and it would for this reason be appropriate to simply "and" together the similar quantiles of the component distributions to obtain the aggregate distribution. This process 1s showr in the table below. Note that ignition is modeled in a separate APET event and, for this reason, is not included in the aggregate probability distribution below. The detonation probability, then, should 
be thought of as the conditional probability that detonation will occur if the mixture ignites.

\begin{tabular}{|c|c|c|c|c|}
\hline Quantile & Flammable & Detonable & Detonation & Aggregate \\
\hline 0.00 & 0.00 & 0.00 & 0.00 & 0.00 \\
\hline .25 & 0.25 & 0.25 & 0.0575 & 0.0144 \\
\hline .50 & 0.50 & 0.50 & 0.115 & 0.0575 \\
\hline .75 & 0.75 & 0.75 & 0.1725 & 0.097 \\
\hline 1.00 & 1.00 & 1.00 & 0.23 & 0.23 \\
\hline
\end{tabular}

By aggregating the distributions in this way, it was possible to make the evient tree hydrogen logic much simpler. The question of hydrogen combustion in the pipe gallery could then be interpreted as, "Does a hydrogen combustion event that could possibly threaten the integrity of the confinement occur in the pipe gallery?" and the additional logic of determining the detonability and, should ignition occur, whether or not DDT occurs were removed from the tree. It should be noted that deflagrations that involve hydrogen from a pipe break in the pipe gallery could cause confinement failure as these would be expected to involve a much larger fraction of the confinement volume. These are treated using the explicit distributions presented in the preceding sections. The elicitees stated, however, that hydrogen detonations were not possible in these pipe break situations because the amount of hydrogen that is produced early in the accident is not sufficient to make the volume globally detonable. 


\section{C.2 CONFINEMENT FAILURE AND FOG SPRAY SURVIVAL}

\section{C.2.1 Issue Definition}

Both systemic and energetic events can lead to the failure of the confinement to mitigate radioactive releases to the environment. Systemic events include the failure of various vents and valves to function as designed. Systemic confinement failure effects are generally straight forward and do not usually propagate to other systems such as the fog spray system. Energetic events such as steam spikes, hydrogen events and graphite combustion are also expected to challenge the integrity of the confinement building, and certain types of building failures could have the additional effect of rendering the fog spray system inoperable or ineffective. This would occur if the fog spray piping were sheared or if the confinement building failure occurred such that a number of spray heads were to end up pointing towards the roof walls. It must be remembered that the banana wall will relieve slow pressure rises, so not all pressure excursions will lead to confinement failure.

Probability distributions are needed to model confinement building and fog spray failure for different types of energetic events. The following should be specifically considered:

a) The probability that the confinement building has failed

b) The probability that the failure is a leak (which causes a competition between the filtered release path and the confinement failure) versus a rupture (in which case the filter path is of minimal effectiveness)

c) The probability that the building failure is directly to the environment versus another radiation protection zone of the plant

d) The probability that the fog spray system will survive

The following energetic events need to be addressed:

a) Hydrogen detonations and deflagrations,

b) Graphite oxidation (can the Intense heat cause the building to fail?), and

c) Steam spikes (are these too fast for the banana wall to relieve?).

\section{C.2.2 Issue Resolution Process}

L. A. Miller and D. M. Kunsman of Sandia National Laboratories were selected to be elicited on these issues. Miller has done extensive work in modeling the $\mathbf{N}$ Reactor confinement response to various accident conditions and was selected to address the issue of confinement building failure. Kunsman has significant previous experience in assessing demolition effects and survivability and was selected to address the issue of fog spray system failure. 
The elicitees were given freedom to define the issue and the structure of the final results to be included in the APET analysis within the framework of the previous section. They determined the appropriate causal events and provided probability distributions based on these events. Their causal relationships are used as the bases for the logical structure of the APET case structure for these events.

\section{C.2.3 Issue Analysis and Resolution}

The elicitees conducted their research and presented their results separately. The causes and probabilities for confinement failure are presented in C.2.3.1, while the fog spray failure results are presented in C.2.3.2 below.

\section{C.2.3.1 Confinement Failure}

The contents of this section are taken from an issue writeup on the causes and probabilities of confinement failure by $L$. A. Miller dated September 22, 1989.

Energetic events such as steam spikes, hydrogen events, and graphite combustion are expected to challenge the integrity of the confinement building. If the building does fail, it may fail as either a leak or a rupture, where a leak results in competition between the filtered release path and the confinement failure and a rupture eliminates the effect of the filter path.

\section{Background Information}

1. A. C. Payne, A. L. Camp, Parametric HECTR Calculations of Hydrogen Transport and Combustion at N Reactor, SAND86-2630, Sandia National Laboratories, June 1987.

2. L. A. Miller, $N$ Reactor Probabilistic Risk Analysis Supporting Calculations, SAND89-2101, Sandia National Laboratories, 1990.

\section{Leaks and Ruptures}

The differentiation between leaks and ruptures based on the criteria above is not well defined. In the world of commercial reactors, where high pressure containment buildings are evaluated, the difference between a leak and a rupture is that a rupture depressurizes the building while a leak merely halts the pressure increase. HECTR calculations for $\mathrm{N}$ Reactor showed that, given the same confinement configuration, a confinement building failure could be subject to inflow or outflow based on different weather conditions (ambient temperature). It is recommended that the distinction between confinement leaks and ruptures be done away with unless a more specific definition can be used. If this is not done, then the following probability distribution can be used to represent the large 
uncertainty in the fraction of the effluent flow that goes out the failure for a leak. This defines a rupture to be any confinement failure where more than 508 of the flow goes out the confinement failure.

$\begin{array}{cc}\text { Quantile } & \text { Probability } \\ 0.0 & 0.1 \\ 1.0 & 0.5\end{array}$

\section{Hydrogen Detonations}

The probability of a confinement failure given that a detonation occurred anywhere in the confinement was assigned a value of 1.0 because of the high pressure rises and shock waves that are generated by detonations. Given that the building failed a value of 1.0 was assigned to the probability that the failure was a rupture.

\section{Hydrogen Deflagrations}

The probability of a confinement failure given that a deflagration occurred depends on the location of the deflagration. For the purposes of this issue two "representative" areas were considered. One area is the 109 building and represents the consequences to the building if the pipe gallery, 109 building, or entire confinement is involved in a deflagration. The other area is the 105 building and represents the consequences to the building if the 105 building, steam generator cells, or penthouse is involved in a deflagration.

The probability distribution developed for a deflagration in the 109 building is based on HECTR results for cases with an initial concentration in the 109 building. The results show that a pressure increase of more than 5 psig will occur if the initial concentration is greater than 58 . The banana wall is not believed to relieve enough of the pressure quickly enough to prevent confinement failure. The probability of building failure is shown below for a deflagration in the 109 building (and for the other areas this area represents). The uncertainty is due to uncertainty in the hydrogen concentration that would be generated during an accident and the actual failure pressure of the building ( 5 psig was used as representative).

\begin{tabular}{cc} 
Quantile & Probability \\
\hline 0.0 & 0.0 \\
.1 & 0.3 \\
.25 & 0.6 \\
.5 & 0.8 \\
1.0 & 1.0
\end{tabular}


The probability distribution for a rupture given that the building has failed is given below (the probability of a leak is 1 - the probability of a rupture).

\begin{tabular}{ccc} 
Quantile & Probability \\
\hline 0.0 & & 0.0 \\
.25 & 0.3 \\
.5 & 0.7 \\
.75 & 0.8 \\
1.0 & 1.0
\end{tabular}

The second area is the 105 building and is also representative of the penthouse and the steam generator cells. The distribution developed for this area is based on HECTR calculations with an initial hydrogen concentration in the 109 building. These calculations showed that a hydrogen concentration of more than 108 was necessary to result in a pressure increase of more than 5 psig following a deflagration. The failure probability distribution is shown below, and is based principally on the uncertainty in the amount of hydrogen that would be available for combustion.

\begin{tabular}{cc} 
Quantile & Probability \\
\hline 0.0 & 0.0 \\
.25 & 0.3 \\
.5 & 0.5 \\
.75 & 0.6 \\
.9 & 0.9 \\
1.0 & 1.0
\end{tabular}

The probability distribution for a confinement rupture given that the confinement has failed is shown below.

\begin{tabular}{cc} 
Quantile & Probability \\
\hline 0.0 & 0.0 \\
.5 & 0.2 \\
.75 & 0.3 \\
1.0 & 0.5
\end{tabular}

\section{Graphite Oxidation}

Graphite oxidation is not expected to lead to confinement failure based on a pressure increase, however, a distribution was assigned to the probability that it would cause some sort of a confinement failure.

$\begin{array}{cc}\text { Quantile } & \text { Probability } \\ 0.0 & 0.0 \\ 1.0 & 0.1\end{array}$




\section{$\underline{\text { Steam Spike }}$}

The probability that a steam spike will cause a pressure increase greater than 5 psig is developed in Appendix C.6. The banana wall is not expected to relieve quickly enough to make a significant difference. Assuming that a pressure of 5 psig results in a confinement failure the following distribution was developed for the probability that the failure is a rupture.

\begin{tabular}{|c|c|}
\hline Quantile & Probability \\
\hline 0.0 & 0.2 \\
\hline .25 & 0.6 \\
\hline .5 & 0.8 \\
\hline 1.0 & 1.0 \\
\hline
\end{tabular}

\section{C.2.3.2 Fog Spray Failure}

\section{Background Information}

1. A. C. Payne, A. L. Camp, Parametric HECTR Calculations of Hydrogen Transport and Combustion at N Reactor, SAND86-2630, Sandia National Laboratories, June 1987.

2. NUREG/CR-2726, Light Water Reactor Hydrogen Manual.

\section{Discussion}

The contents of this section are taken from written and oral comments received from D. M. Kunsman the week of September 8, 1989 on the subject of fog spray survivability given confinement building failure.

The $\mathrm{N}$ Reactor confinement buildings (105N and $109 \mathrm{~N}$ together) were built with a total volume of approximately $3.2 \times 10^{6} \mathrm{ft}^{3}$ and a design pressure of 5 psig. The fog epray system is designed as a building pressure suppression system and is mounted to withstand the "normal" operating conditions that would be expected during an accident. It is possible, however, that abnormal accident conditions related to failure of the confinement buildings could cause the fog spray system to fail. In this analysis, failure is meant in a more general sense than simply the failure of water to come out of the spray heads. Failure includes any situation that would render the sprays ineffective for either their pressure suppression function or for the removal of radionuclides from the confinement building atmosphere. Such could include the breakage of spray system pipes during a confinement failure, but it could also include failures that cause major gaps in the volumetric coverage of the confinement building atmosphere by the sprays or redirection of the spray heads away from the building atmosphere altogether. Such failures are the subject of this uncertainty analysis. 
The APET analysis does not consider partial states of fog spray success because most of the component failures that cause fog spray failure affect all parts of the fog spray system. This would not be necessarily true for fog spray failures caused by confinement building failure. However, this analysis will not attempt to determine a degree of fog spray success because such further subdivision of the results would not be justified based on the knowledge available. Also, subdivision of the analysis beyond the three cases presented below will not be attempted because such would be too scenario specific.

Two different major types of loadings are possible to cause confinement building failure (a third case that is between these will be described later). These are "quasi-static" loadings and dynamic loadings. Quasi-static loads are those generated by relatively benign pressure phenomena that cause pressure increases that can not be vented through the banana wall. Examples of this are graphite oxidation, steam pressure spikes and localized hydrogen deflagrations. While the latter two may not seem like quasi-static loadings, the pressure rises do not involve shock waves and are not expected to produce pressures that are many times the building failure pressure. Thus, they are expected to cause less violent building failures.

Dynamic building loadings are caused by impulse loads where shock waves can cause very large openings in the building. Shock waves can be caused by hydrogen detonations and classical steam explosion events. However, classical steam explosions have been ruled out as not credible for $N$ Reactor because the geometry does not exist for a large mass of molten material to be dropped into a pool of water. The only credible mechanism for the generation of shock waves inside the $\mathrm{N}$ Reactor confinement is hydrogen detonation.

With some extrapolation from the hydrogen analysis in Appendix C.1, it can be determined that the total hydrogen production for an accident where GSCS is successful $(230 \mathrm{~kg})$, the confinement building can reach a maximum global hydrogen concentration of -3.58 . Even if this were to ignite, it would still produce a confinement load that could be characterized as quasi-static. This is supported by HECTR calculations [Ref. 1, p. 6-6] that show that $300 \mathrm{~kg}$ of hydrogen would have to be burned globally within the confinement to produce a pressure of 10 psig. While this is true for global burns, it is not true if the same $230 \mathrm{~kg}$ is placed in a localized area.

Global hydrogen burns of significantly more than $230 \mathrm{~kg}$ (such as the $1200 \mathrm{~kg}$ produced when GSCS is degraded) can, according to the hydrogen manual [Ref. 2, Fig. 2-10, p. 2-29], produce pressures of 50-90 psig. A deflagration that produced this high a pressure would be expected to cause damage similar to a detonation. It will be classified as a dynamic load. 
A probability distribution for fog spray survival during quasi-static loading confinement failures is given below.

\begin{tabular}{|c|c|}
\hline Quantile & Probability \\
\hline 0.0 & 0.90 \\
\hline 0.5 & 0.99 \\
\hline 1.0 & 1.00 \\
\hline
\end{tabular}

The quantiles represent a cumulative probability distribution. This distribution should be interpreted as a loguniform distribution between 0.90 and 0.99 , and another loguniform distribution between 0.99 and 1.00 . It represents the assessment that the fog sprays are at worst likely to survive such a static loading.

A probability distribution for fog spray survival during confinement failures caused by dynamic loadings is given below.

\begin{tabular}{cc} 
Quantile & Probability \\
\hline 0.0 & 0.0 \\
.25 & 0.001 \\
.50 & 0.01 \\
.75 & 0.03 \\
1.0 & 0.1
\end{tabular}

This distribution represents the assessment that fog spray survival is at best unlikely and, more realistically, only remotely possible given that a detonation or severe overpressure event occurs to fail the confinement building. It may be possible to argue that a detonation in the penthouse would not fail the fog spray function, but such an assertion would be difficult to prove because of the amount and velocity of the debris that could possibly be generated during such an event.

A third case can occur that is in some ways between the other two. This is the case of a local deflagration where a large amount of hydrogen is built up in a small area. Such an event could produce the types of pressures seen in the large global deflagration discussed above in a small area. Case 6B in Ref. 1 analyzed a deflagration in steam generator cell 6 (with a volume of $-230,000 \mathrm{ft}^{3}$ ) with $-90 \mathrm{~kg}$ of hydrogen. The burn was slow $(-12 \mathrm{sec})$ due to the high concentration of steam in the volume and reached a pressure that was 918 of the 10 psig design limit for the steam generator cells. Putting $230 \mathrm{~kg}$ in that same volume would produce a maximum pressure of 20-25 psig. This is a significant overpressure that would be more likely to fail the fog spray system than would a quasi-static load. A 
probability distribution for fog spray function survival during a large, localized deflagration is presented below.

\begin{tabular}{cc} 
Quantile & Probabilit \\
\hline 0.0 & 0.5 \\
.25 & 0.7 \\
.50 & 0.9 \\
.75 & 0.95 \\
1.0 & 0.99
\end{tabular}

This distribution represents the assessment that the fog spray system is on the whole likely to survive such a local deflagration event.

The major uncertainties in fog spray survival are related to the response of the fog spray system itself, so the three distributions above should be considered correlated when sampled.

\section{C.2.4 Application of Results}

The above probability distributions in both sections were implemented in the Latin Hypercube uncertainty analysis and sampled for use directly in the APET analysis EVNTRE runs. 


\section{C.3 FUEL DAMAGE FOR PARTIAL ECCS AND PARTIAL CORE ACCIDENTS}

\section{C.3.1 Issue Definition}

Most $\mathrm{N}$-Reactor severe accident scenarios do not involve complete failure of the ECCS, but rather show ECCS operating in some degraded state. MELCOR and RELAP calculations have demonstrated that steam cooling provides significant heat removal in many of these situations. Probability distributions are needed to describe the total amount of core damage given that ECCS is operating in one of its degraded modes.

Cases where GSCS succeeds as well as cases where GSCS fails must be considered for all of the following degraded ECCS modes:

1) ECcs succeeds, but cooling is lost to $1 / 16$ of the core due to the failure of a $C V-2 R$ valve to open. Since these accidents always involve less than 78 of the core, is it necessary to consider the effects of the presence or absence of HPI flow, or is the conservative approach of ignoring HPI flow adequate?

2) A common mode failure occurs where all V-3 or all V-4 valves on one side of the core fail to open. Normal ECCS flow occurs to the other side of the core.

3) ECCS operates normally except that only one high lift pump starts. This pump delivers approximately $11000 \mathrm{gpm}$ to the core.

4) The ECCS supply consists of two high lift pumps, but two $V-3$ valves on the same side of the core fail to open. Flow rates are unknown, but should be approximately half of that provided by two pumps with all V-3 valves open on the affected side of the reactor.

5) Scenario 2) is the dominant partial ECCS scenario for full core accidents, and the analysis involved in 2) seems to be more straight-forward and defensible during review than that involved in 3) and 4). As an alternative to 3) and 4), can it be said that the results from 2) represent a conservative upper bound on the total amount of fuel damage in 3) and 4)? Scenarios 2), 3) and 4) account for 65-708, 8-128, and 9-128, respectively, of all core accidents which lead to core damage.

6) ECCS fails completely, but two HPI pumps operate and deliver 3600 gpm of cooling to the core through the top of the inlet riser. How does this relate to 2), 3) and 4) above? This scenario accounts for 3-208 of all full core accidents which lead to core damage.

\section{C.3.2 Issue Resolution Process}

S. E. Dingman, and L. A. Miller of Sandia National Laboratories, and C. J. Shaffer of Science and Engineering Associates (under contract to Sandia National Laboratories) were selected to be elicited on these issues. 
All were heavily involved in modeling the $\mathrm{N}$ Reactor primary system for the N Reactor MELCOR benchmark effort and were selected for their extensive familiarity with the MELCOR, RELAP and TRUMP analyses that had been conducted for $\mathrm{N}$ Reactor. The issue was presented to them as described above and they were asked to provide input as to either the resolution or restatement of the issue. They were content to consider the issue as presented above.

\section{C.3.3 Issue Analysis and Resolution}

\section{Background Information}

1. L. A. Miller, et. al., N Reactor Probabilistic Risk Assessment Supporting Calculations, SAND89-2101, Sandia National Laboratories, 1990.

2. M. A. Bolander, Simulation of a Cold Leg Manifold Break Sequence in the $N$ Reactor with Failure of an ECCS CV-2R Valve, EGG-TFM-7988, Idaho National Engineering Laboratory, February 1988.

3. M. A. Bolander and C. D. Fletcher, Simulation of Cold Leg Manifold Break and Station Blackout Sequences in the $N$ Reactor, EGG-TFM7891, Idaho National Engineering Laboratory, February 1988.

4. M. A. Bolander, et. al., Simulation of Cold Leg Manifold Break and Station Blackout Revised Sequences for Reduced ECCS in the $N$ Reactor, EGG-TFM-7962, Idaho National Engineering Laboratory, February 1988.

5. K. Sathyanarayana and D. M. Ogden, Analysis of $N$ Reactor Anticipated Transients Without SCRAM, WHC-SP:0457, Westinghouse Hanford, March 1989.

\section{Discussion}

A number of the degraded ECCS modes above involve core-wide degradation of the coolant flow rather than full flow to certain areas of the core and no flow to others. The latter case is significantly easier to analyze than the former because it places a theoretical cap on the amount of fuel damage that can occur during the accident, while the former does not. In order to allow a more meaningful discussion of these core-wide degraded flow cases, S. E. Dingman performed a number of single pressure tube calculations using MELCOR to determine the steam flow rates required to keep fuel temperatures below given levels. Calculations were also done to determine the pressure drop across the core for given ECCS coolant injection rates. These calculations and the resulting method for estimating core damage based on coolant flow rates are described in Section 4.1.1 of the supporting calculations document [1]: These methods were used as one input to the issue elicitation for fuel damage discussed below. 
The following discussion and the included probability distributions are taken from an issue writeup prepared by $C$. J. Shaffer and amended to reflect the comments received during oral discussions of the fuel damage issue that occurred during the week of September 8, 1989.

Degraded Mode 1: ECCS succeeds, but one CV-2R valves fails to open.

What is the probability distribution of fuel damage considering success or failure of the GSCS and/or HPI?

Since ECC is successful to 15 risers then fuel failure is limited to $1 / 16$ of the core (6.258). Cooling to the riser with the failed CV-2R valve is available from primary system depressurization, ECC entering by way of the hot leg riser flush line, graphite lateral heat conduction, and GSCS/HPI when available.

Judgements on this mode could be made by relying heavily on MELCOR NReactor experience, especially the T4 calculation which assumes that the GSCS continues to operate but the HPI fails. In the T4 calculation, the flush lines allowed $2-3 \mathrm{~kg} / \mathrm{sec}$ ECC to flow to the blocked riser which in addition to the depressurization flows, prevented fuel damage with a significant margin for error.

The blocked riser atmosphere was not trapped and prevented from flowing through the hot leg piping to the dump manifold upstream of the V-4 valves. The hot leg piping associated with the blocked riser voided early in the calculation and remained voided down to the horizontal section of the six inch pipe which allowed gases to flow into the dump manifold where they were either condensed or entrained. The hot leg piping associated with the unblocked risers was nearly voided, as well, meaning that there was not a significant hydrostatic head to refill the blocked hot leg piping. The unblocked outlet riser level was maintained near the top by the ECC which provided a significant hydrostatic pressure head to force water though the flush line to the blocked riser. Water in the blocked outlet riser flowed into the bottom of the blocked core where it was boiled to steam. A large portion of this steam then flowed out of the core by means of the blocked inlet riser and back through the voided upper core regions providing cooling to these regions.

The flush line flow was under predicted since the flush line was modeled with an unrealistically high flow resistance for numerical stability. But even at this flow rate the water level in the outlet riser remained above the top of the core and the inlet riser was reflooding.

The cooling by the depressurization flows is very important in determining the extent of fuel damage. One sensitivity to the $T 4$ calculation assumed the total failure of the ECCS such that the blocked riser was cooled only by the depressurization flows and conduction heat transfer. Fuel failure was still not predicted.

The MELCOR T4 transient calculation did not predict any ECC entering the blocked inlet riser by means of the CV-5 valves. The CV-3 valves 
closed shortly after the V-4 valves opened and remained closed throughout the remainder of the calculation. The pressure in the hot leg manifold was the highest pressure in the primary system.

It has been noted that the phenomenon of reverse flow through the $\mathrm{V}-4$ valves is a significant departure from the behavior predicted in RELAP analyses. However, the RELAP analyses [2] all end at approximately 600 seconds into the accident and this reverse flow does not begin in the MELCOR calculations until -900 seconds into the accident. Also, the RELAP calculations examined were all large LOCA calculations where the depressurization flow through the core did not occur. The depressurization flows are critical to maintaining cooling to the affected riser until either the reverse flow occurs or the ECCS refills the primary system and forces water into the affected riser through the CV-5 valves.

The success of the HPI to provide a continuous flow of $3600 \mathrm{gpm}$ to the primary system means that about $227 \mathrm{~kg} / \mathrm{sec}$ of water will flow through the CV-5 valves into the inlet risers after the primary system from the CV-3 to the CV-5 has reflooded but water should flow before reflooding is complete. If this water was divided evenly over the 16 risers, then about $14.2 \mathrm{~kg} / \mathrm{sec}$ would flow into the blocked riser inlet. Then there is a question of timing. The T4 calculation showed that about 258 of the original water in this section of the primary system remained after system depressurization. Given the $3600 \mathrm{gpm}$ flow rate, it would take about 26 minutes to completely reflood the primary system from the CV-3 to CV-5 valves. Therefore, it should take something less than 26 minutes to provide a steady HPI flow into the inlet risers.

The GSCS core cooling and lateral heat conduction have their most important effects on long term heat transfer. During the short term, the effects would be much less since both of these mechanisms depend upon heat conduction through the graphite. Their effect on short term fuel damage would be of a more minor nature than the cooling due to the flush line ECC and depressurization flows. In the long term, when conduction heat transfer has had time to significantly effect temperatures, the blocked riser should be reflooded or nearly so by the flush line ECC flow and if available the HPI flows. In other words, the highest probability of fuel damage given that the GSCS fails is still at a relatively early part of the accident. So the probabilities for GSCS failure and success will not look greatly different.

This mode was broken down into the following sub-modes for analysis.

$\begin{array}{lll} & \text { GSCS } & \text { HPI } \\ \text { A. } & \text { YES } & \text { NO } \\ \text { B. } & \text { YES } & \text { YES } \\ \text { C. } & \text { NO } & \text { NO } \\ \text { D. } & \text { NO } & \text { YES }\end{array}$

Further, it helps to divide the blocked riser fuel into relative zones of probability. The highest probability of fuel damage occurs in fuel tubes with high power factors near the top of the core. The corresponding tubes 
at the bottom of the core will receive much more cooling and so have a much lower probability of failure. There is also an axial dependence. Zone I consists of six out ten axial nodes for six tubes in the one-sixteenth of the core. Zone II consists of the same six axial nodes but in the rest of the tubes in the upper half of the blocked riser. Zone III consists of the last four axial nodes in all of the tubes of the upper half and Zone IV is the lower half of the blocked riser. The resulting core volume fractions are $0.0035,0.0152,0.0126$, and 0.0313 . The following are preliminary fuel failure probabilities. These are intended to be used as a basis for discussion by the other elicitees.

\begin{tabular}{|c|c|c|c|c|c|c|c|}
\hline \multirow{3}{*}{ Zones } & Percent & Fuel & & Perce & \multicolumn{2}{|c|}{ Cumulative } & Probabilit \\
\hline & Damage & & A & B & C & D & Ave \\
\hline & 0 & & 80 & 83 & 77 & 80 & 80 \\
\hline I & .35 & - & 95 & 97 & 93 & 95 & 95 \\
\hline I, II & 1.87 & & 99 & 99.5 & 98.5 & 99 & 99 \\
\hline$I, I I, I I I$ & 3.13 & & 99.8 & 99.9 & 99.7 & 99.8 & 99.8 \\
\hline$I$ - IV & 6.25 & & 100 & 100 & 100 & 100 & 100 \\
\hline & $>6.25$ & & 0 & 0 & 0 & 0 & 0 \\
\hline
\end{tabular}

The probabilities are given for each one of the sub-modes $A$ to $D$. However, since the differences between sub-modes is much smaller than the perceived uncertainty, an average is listed for degraded mode 1 . In general, there is a high probability that fuel damage would be zero and that if some damage did occur, it would probably be limited to the highest powered fuel cells.

During discussions with the other elicitees, the above distributions were revised to account for some of the discrepancies between the MELCOR, RELAP and TRUMP analyses. The RELAP and TRUMP analyses both predicted higher core damage levels than did MELCOR, although TRUMP does not account for convective (steam) cooling and RELAP [3,4] does not account for lateral heat conduction and the action of the GSCS. These modeling limitations explain some of the differences between MELCOR, but the above probability distributions were made more conservative by accounting considering these results.

\begin{tabular}{|c|c|c|c|}
\hline \multirow[b]{2}{*}{ Quantile } & \multicolumn{3}{|c|}{8 Core Damage by Mass } \\
\hline & Miller & Shaffer & Dingman \\
\hline 0.0 & 0.0 & 0.0 & 0.0 \\
\hline .25 & 1.5 & 0.0 & 1.5 \\
\hline .50 & 2.0 & 0.0 & 2.0 \\
\hline .60 & & 0.0 & \\
\hline $\begin{array}{l}.75 \\
.80\end{array}$ & 2.5 & & 2.5 \\
\hline .95 & & 0.35 & \\
\hline .99 & & 1.9 & \\
\hline .998 & & 3.1 & \\
\hline 1.0 & 6.25 & 6.25 & 6.25 \\
\hline
\end{tabular}


Degraded Mode 2: A common mode failure occurs where all V-3 valves on one side of the core fail to open.

Calculations were not done for this degraded mode but it can be related to mode 1 . The failure mechanism for mode 2 is the same as the mode where all eight $C V-2 R$ valves fail on one side and the probabilities should be similar to those for mode 1 except that the they are roughly eight times larger.

In this failure mode, ECC is successful to the eight risers on one side which physically limits core damage to 508. I feel that the arguments presented for mode 1 are applicable for mode 2. This does requires the flush line flow to be eight times larger than for mode 1, but this should not be a problem. All of the fuel associated with the outer most riser has lower power factors will be considered not to contain any Zone 1 or 2 fuel which reduces the probabilities slightly. Also mode 1 considered the blocked riser to be the highest powered riser.

It is possible to consider this accident from a different perspective: since the GSCS is working and ECCS has completely failed on one side of the reactor, this mode is similar to the hydrogen mitigation design basis accident for one half of the core. That accident showed a point estimate of 278 fuel damage. An uncertainty analysis based on this observation and the comparison between the MELCOR and TRUMP analyses of this accident would produce a slightly more conservative (higher fuel damage) probability distribution.

\begin{tabular}{|c|c|c|c|}
\hline \multirow[b]{2}{*}{ Uuantile } & \multicolumn{3}{|c|}{ Gore Damago by Mass } \\
\hline & Miller & Shaffer & Dingman \\
\hline 0.0 & 0.0 & 0.0 & 0.0 \\
\hline .25 & 10 & 0.0 & 10 \\
\hline .50 & 16 & 0.0 & 16 \\
\hline .60 & & 0.0 & \\
\hline .75 & 20 & & 20 \\
\hline .95 & & 2.5 & \\
\hline . 998 & & 15 & \\
\hline 1.0 & 50 & 50 & 50 \\
\hline
\end{tabular}

Degraded Mode 3: A common mode failure occurs where all V-4 valves on one side of the core fail to open.

Since a calculation is not available for this mode of core damage, it is necessary to estimate how this type of transient sequence will progress. A transient sequence is initiated but when the $V-4$ valves are actuated, all of the V-4 valves on one side (failed side) of the core fail to open. The primary system associated with unfailed side of the reactor will depressurize normally through its $V-4$ valves but the failed side will have to depressurize to the unfailed side through either the outlet riser flush line or the steam generators. The steam generator portion of the primary system will depressurize through the unfailed side of the core because of the higher pressure in the failed side. 
The ECC to the failed side will flow from the failed side through the flush line or through the steam generators to the unfailed side. The flush line is a ten inch manifold on each side of the reactor with a six-inch connecting crossover pipe. The ten inch outlet riser flush line has $78 \%$ of the flow area of the eight combined four-inch pipes which carry ECC into the bottom of the inlet risers but the six-inch crossover pipe has only 298 of the flow area. The ECC flowing to the failed side could therefore be considerably degraded by the higher flow resistance of the flush line. The failed side reactor pressure will remain higher than the unfailed side which will further reduce the ECC flow and the failed side of the reactor will not benefit nearly as much from the depressurization flows because the steam generators will depressurize through the unfailed side. Since the flow restriction for the fail side is downstream of the reactor, the inlet riser should completely fill with water which would force some water through all of the tubes and provide some convective cooling to the entire reactor.

Using the algorithm developed by S. E. Dingman for the maximum fuel damage for partial ECC accidents (conservative assumptions throughout), an ECC flow rate of $10660 \mathrm{gpm}$ (to entire core) is sufficient to limit the maximum fuel temperature to $1150 \mathrm{~K}$. The fuel is normally considered to fail at $1300 \mathrm{~K}$. The upper temperature limit for the algorithm is $1150 \mathrm{~K}$ so it is not possible to specify the flow needed to prevent fuel from exceeding $1300 \mathrm{~K}$ but the T4 transient calculations indicate that a small flow can have a significant effect at the higher temperatures. The lower bound for fuel damage must be zero.

The upper bound for fuel damage is much more uncertain. Assuming that the failed side ECC flow rate is equal to 298 of the undegraded ECC flow (based on the pipe area ratio) then about $4000 \mathrm{gpm}$ of ECC will flow through the failed side of the reactor. This is equivalent to $8000 \mathrm{gpm}$ for the entire reactor and according to the algorithm will limit fuel damage to about 258 of one side. The specification of an upper bound of 508 for one side or 258 for the entire reactor should be more than conservative.

In addition it should be noted that the ECCS success criteria for the V-4 valves is that une valve must open on each side of the reactor. Thus, the flush line alone presents approximately half of the success criteria flow area for water flow out of the reactor. This would place additional conservatism in the partial ECCS flow calculation cited above. Based on these assessments, the elicitees agreed that it would be conservative to use the results for Degraded Mode 2 above to describe fuel damage in this situation.

Degraded Mode 4: ECCS operates normally except that only one high lift pump start.

The analytical basic for estimating the fuel damage probabilities for mode 4 comes from the maximum fuel damage study for partial ECC by $S$. E. Dingman. In this study, an algorithm was developed which estimates the maximum fuel damage (conse'rvative assumptions used throughout) as a function of the fuel failure temperature and the total ECC flow injected. 
The algorithm was developed for maximum temperatures from $850 \mathrm{~K}$ to $1150 \mathrm{~K}$. A linear extrapolation to higher temperatures was not possible since the pressure differential goes to zero at about $1190 \mathrm{~K}$, i.e., the algorithm does not hold for the estimated $1300 \mathrm{~K}$ fuel temperature.

This mode assumes that $11000 \mathrm{gPm}$ (conservative lower bound) of ECC is supplied to the core. The actual ECC flow would be approximately $15000 \mathrm{gpm}$ based on WHC pump tests. The $11000 \mathrm{gpm}$ flow rate, according to the algorithm would limit the maximum temperature to $1137 \mathrm{~K}$ (163 K below the failure temperature). The best estimate and lower bound for fuel damage would then be zero. However, if the fuel damage temperature is specified at $1100 \mathrm{~K}$, then the estimated maximum core damaged is 208 . In other words, the fraction of core damage is very sensitivity to the damage temperature which indicates the need to consider the uncertainty in these estimates.

An upper bound core damage of 258 of the core would be conservative. Based on these assessments, the elicitees agreed that it would be conservative to use the results for Degraded Mode 2 above to describe fuel damage in this situation.

Degraded Mode 5: The ECCS supply consists of two high lift pumps, but two $\mathrm{V}-3$ valves on the same side of the core fail to open.

The success of two high lift pumps implies that 22000 gpm of ECC flow would be supplied to the reactor if all V-3 valves are working and would be sufficient to prevent fuel damage. The two failed $V-3$ valves on one side will reduce the flow to that side by a factor with an uppor bound of two. This means that at least $5500 \mathrm{gpm}$ of flow goes the side with the failed valves which is equivalent to $11000 \mathrm{gpm}$ for the entire core. This means that core damage probabilities can be estimated as one half of those for mode 4 since only one half of the core is at risk. Based on these assessments, the elicitees agreed that it would be conservative to use the results for Degraded Mode 2 above to describe fuel damage in this situation.

Degraded Mode 6: ECCS fails completely but two HPI pumps operate and deliver $3600 \mathrm{gpm}$ to the inlet risers.

In this mode, the ECC will partially fill the inlet riser and force water through the lower core. Some tubes at the bottom will be completely cooled, some tubes partially cooled, but most of the core will suffer fuel damage. The effect of a small amount of cooling delivered to the inlet riser would be different than a small amount delivered to the outlet riser by the flush line as in the T4 transient calculation. When the water is delivered to the outlet riser, the steam generated in the lower core is forced to flow into the inlet riser where it must flow back through the upper core providing steam cooling to the upper core. But steam generated by water delivered to the inlet riser, flows directly to the outlet riser and bypasses the upper core and so provides minimal steam cooling to the upper core. 
The uncertainty of this mode is such that the core damage bounds should be set to the limits of zero and 1008 but with small probabilities for the extremes. The algorithm developed by S. E. Dingman for partial ECC cases indicates that $2 / 3$ of the core would be damaged given that fue 1 damage occurs at $1150 \mathrm{~K}$. The GSCS success will effect this distribution because some of the lower power fuel may not fail at all if the GSCS operates. Based on these assessments, the elicitees agreed that it would be conservative to ignore the contribution of HPI as a mitigating factor and lump this mode into a mode with ECCS completely failed to describe fuel damage in this situation.

\section{Additional Case 1: ECCS fails completely but GSCS succeeds.}

This cooling failure mode has been analyzed using both TRUMP and MELCOR with similar results. The point estimate of fuel damage from such an accident would be between 25 and 308 . An examination of those calculations and the sensitivity calculations that were done in support of those efforts to determine a probability distribution for fuel damage under this situation. The dominant uncertainty is the efficiency of the GSCS in removing heat from the core. Tied up in this uncertainty is uncertainty in the thermal conductivity of the graphite as it has been affected by radiation, and the performance of the gap between the graphite and the GSCS tubes. The probability distributions below were elicited.

\begin{tabular}{|c|c|c|c|}
\hline \multirow[b]{2}{*}{ Quantile } & \multicolumn{3}{|c|}{8 Core Damage by Mass } \\
\hline & Miller & Shaffer & Dingman \\
\hline 0.0 & 0.0 & 0.0 & 0.0 \\
\hline .05 & & 0.0 & \\
\hline .25 & & 12 & 15 \\
\hline .50 & 25 & 20 & 25 \\
\hline $\begin{array}{r}.75 \\
.90\end{array}$ & 30 & $\begin{array}{l}30 \\
40\end{array}$ & 35 \\
\hline .99 & 50 & 50 & 50 \\
\hline 1.0 & 100 & 100 & 100 \\
\hline
\end{tabular}

Additional Case 2: ECCS fails completely and GSCS operates in a degraded mode because of a loss of the helium cover gas system.

The helium cover gas that surrounds the graphite stack enhances the thermal conductivity of the gap between the graphite and the GSCS tubes. This gap is the dominant thermal resistance for GSCS performance. If the helium cover gas is replaced by steam (as in the case of a pressure tube rupture event), the thermal conductivity of that gap would decrease by a factor of 4 to 8. A similar effect is obeerved for air. A MELCOR analysis of this scenario was performed and is documented in the supporting calculations document [Miller et al, SAND89-2101]. These calculations showed that a point estimate of the amount of core damage that would be sustained in such an accident would be 658 . Broad uncertainties should be placed on this because of the nodalizations required by the MELCOR analysis are considerably coarser than those that would be used in a TRUMP analysis, and a TRUMP analysis of this scenario is not available. 
The following probability distribution was constructed for this scenario and agreed upon by consensus of the elicitees.

\begin{tabular}{|c|c|}
\hline Quantile & 8 Core Damage \\
\hline 0.0 & 25 \\
\hline .25 & 50 \\
\hline .50 & 65 \\
\hline .75 & 75 \\
\hline $\begin{array}{l}.95 \\
1.0\end{array}$ & $\begin{array}{r}90 \\
100\end{array}$ \\
\hline
\end{tabular}

\section{Additional Case 3: ATWS}

As discussed in Chapter 3, the APET analysis considers only ATWS accidents where ECCS is fully functional because ECCS failure, when combined with SCRAM failure, is a very rare event and, since the reactor has a negative void coefficient of reactivity, an ATWS ECCS failure accident would behave just like a normal ECCS failure event. For this reason, a probability distribution is needed to represent fuel damage for ATWS accidents where ECCS is successful.

Westinghouse Hanford analyzed several ATWS events using the RELAP computer code [5] and determined that the ECCS would be likely to be able to fill approximately half of the reactor, but pressure caused by the steaming of the ECCS coolant could cause the ECCS to fail to refill the remainder of the reactor. This would imply that approximately half of the fuel would be damaged. The RELAP model, however, used a point neutron kinetics representation of the neutronic power of the reactor. This representation would assign far too much power to the uncooled portion of the core because, in reality, there is not enough moderator in that area to support a critical nuclear reaction. Thus, it is believed that the -50 z fuel damage figure may be conservative. It is also highly uncertain. Thus, a broad uncertainty range on this value was used for the analysis. The following probability distribution was constructed for this scenario and agreed upon by consensus of the elicitees.

\begin{tabular}{ccc}
$\frac{\text { Quantile }}{0.0}$ & Core Damage \\
\cline { 1 - 2 } .25 & 0 \\
.50 & 35 \\
.75 & 50 \\
1.0 & 70 \\
& 100
\end{tabular}

Additional Case 4: Degraded ECCS with failure of GSCS - Degraded modes 1-6 above

Six degraded ECCS modes were considered for fuel damage analysis above when GSCS was functional. These six modes can also exist when GSCS has failed. The elicitees considered these modes and determined that the following statements could be made: 
1) There is no reason to believe that an accident where both ECCS and GSCS flows to the core have completely failed will proceed to anything less than 1008 core damage. The small frequency of GSCS failure events indicates that taking all as 1008 core damage will not introduce excessive conservatism into the analysis.

2) The first degraded mode (single riser failure) is essentially unaffected by GSCS failure because of the lateral heat conduction mechanism, the depressurization flows (if applicable), and the possibility of reverse coolant flow through the $V-4$ valves or forward reflooding through the $\mathrm{CV}-5$ valves.

3) There is no reason to believe that the second degraded mode (common mode V-3 failure on one side of the reactor) will not proceed to its maximum core damage amount ( 50 z since only half of the reactor is uncooled), although this could be conservative because of the lateral conduction to the unaffected half of the core. The small frequency of GSCS failure events indicates that taking these as 508 core damage will not introduce excessive conservatism into the analysis.

4) The previous assessments that stated that degraded modes 3,4 and 5 are less severe than degraded mode 2 are still valid for the GSCS failure case. Steam cooling is believed to be effective in keeping core damage to below 50 f for the cases where the whole core is effected, and the half-core cases can not proceed to more than 508 core damage. The small frequency of GSCS failure events indicates that taking all as 508 core damage will not introduce excessive conservatism into the analysis.

5) In degraded mode six, HPI will by itself not limit core damage, and should be ignored just as it was in the GSCS operating cases.

Therefore, the following summary is possible for GSCS failed accidents: In this analysis, it is conservative (but not excessively so) to consider all accidents where ECCS fails completely as 100 \% fuel damage regardless of the state of HPI, and to consider all partial ECCS success cases as 508 fuel damage.

\section{Correlation}

The uncertainties in these probability distributions are all generally related to the efficiency of steam cooling and the efficacy of the GSCS, so there should be a strong correlation between these probability distributions when they are sampled.

\section{C.3.4 Application of Results}

In all cases whcrc a single distribution is given, it is used to determine the amount of fuel damage that will be passed from the APET 
analysis for use in the source term analysis. Where there are multiple distributions, all were usually in relatively close agreement, so the most conservative distribution was used rather than integrating all of the distributions into a single distribution. The conservatism introduced by this is small because the distributions are all relatively close to each other. 


\section{4 FUEL DAMAGE DUE TO IATE ECCS FAILURE \\ C.4.1 Issue Definition}

In a scenario where GSCS has failed and ECCS succeeds for two hours but fails sometime thereafter, the Level I PRA analysis states that the result is "up to" $100 \%$ core damage. This describes a scenario which makes up core damage bin 21 (delayed core damage) from the Level I PRA. The 100\% core damage assumption is conservative and is based on language in the Level I PRA which states that "1008 of the fuel may be affected" (emphasis added) in accident scenarios which are placed into bin $\mathrm{Zl}$. The purpose of this issue is to determine a probability distribution for the expected amount of core damage given a bin $\mathrm{Zl}$ accident.

It has been noted that the failures which the Level I PRA categorized as bin $\mathrm{Zl}$ accident sequences generally involve only a degradation of the ECCS function. Specifically, more than 998 of the frequency in Level I PRA core damage bin $\mathrm{zl}$ involve the failure of two of the three high lift diesel pumps to run for 24 hours, but one pump continues to run. However, since the point estimate probability of the third pump failing during these 22 hours is $0.076,92.48$ of the frequency of bin $\mathrm{Zl}$ would involve only a degraded ECCS state with at least $12000 \mathrm{gpm}$ available for cooling the core. The decay heat load is relatively low after two hours, so the ECCS function may still succeed even with the reduced coolant flow. In particular, Emergency Response Guide ERG-B states that ECCS flow may be reduced to 5000 gpm after two hours if certain bulk outlet coolant temperature conditions can be satisfied.

The above scenario describes 92.48 of all late ECCS failures, but a number of cases exist in which all three ECCS pumps fall to run for the full 24 hour period considered in the Level I PRA. In some of these cases, however, additional sources of coolant may be available to the operators after two hours which were not available at the time of accident initiation. For example, if $A C$ power is available, three HPI pumps can supply more than $5000 \mathrm{gpm}$ to the core as long as the cold leg remains intact. Other coolant syslems could bo configured to inject water into the ECCS, and a coolant tie to the B-Reactor site could be activated. Many of these additional sources of coolant have been added since the beginning of the N-Reactor PRA project. The reduced decay heat load at this time means that the operators would have significant time to take action before core damage would be expected.

The following is an incomplete list of uncertain issues which are believed to be of importance to late ECCS failure scenarios:

- core flow rate required to prevent core damage

- availability of systems which might be used to supply water should ECCS fail

- the time available for the operators to take the required actions

- the abllity and likelihood of the operators to take the required actions during the time avallable 
A Probability distribution is needed to model the extent of core damage for scenarios which exhibit late loss of ECCS function as defined by the Level I PRA. The accident sequence begins with the core full of water and at low pressure since ECCS has succeeded for two hours. The HPI system and main coolant pumps have been shut down as per the instructions in Emergency Response Guide ERG-B. The GSCS failed very early in the accident due to unspecified causes. AC power is available on both busses because none of the bin 21 accident sequences involve loss of offsite power, and the operators would have time to recover B-Bus from offsite power given a loss of thermal flywheel.

If the location of a pipe break could change the resulting core damage state, the following distribution of break locations should be used:

Transients: no breaks

Small LOCAs: 978 are between the core outlet and main coolant pump inlets

38 are in the $\mathrm{V}-11$ valves

Large LOCAS 678 are between the core outlet and main coolant pump inlets

168 are in the steam generator cells on the outlet side of the main coolant pumps

158 are in the inlet risers near the $C V-2 R$ valves

28 are in the pressurizer penthouse

\section{C.4.2 Issue Resolution Process}

This issue as described above was elicited at Westinghouse Hanford on Thursday, July 20, 1989. The elicitation on this issue occurred after much of the elicitation on Issue 7 (Pressure Tube Failure) was completed, and some of the success criteria discussion from that elicitation were used. The elicitation was attended by John Long (PNL, formerly of WHC). Don Ogden (WHC) and Gary Smith (WHC). While the statements below are attributed to specific people, it should be noted that all conclusions reached in this discussion were supported by a unanimous consensus of those in attendance and should be considered as such.

\section{C.4.3 Issue Analysis and Resolution}

\section{Background Information}

1. Roblyer, S. P., Decay Heating Powers of $\mathrm{N}$ Reactor Mark IV and Mark IA Fuels for Updated Safety Analysis Studies, WHC-SP-0037, Westinghouse Hanford, March 1988.

2. Westinghouse Hanford, Emergency Response Guides, WHC-CM-4-1, Volume 2, June 1981, as updated through June 1989 
3. Zentner, M. D., et. al., N Reactor Level I Probabilistic Risk Assessment: Final Report, WHC-EP-0322, Westinghouse Hanford, August 1988

Discussion

The issue was decomposed into four sub-problems for analysis. These are: (1) Time Available for Action, (2) Flow Required to Prevent Core Damage, (3) Support System Availability, and (4) Available Coolant Sources. Each of these is discussed in detail below.

\section{Time Available for Action}

The following consensus opinion is based on a discussion of the subproblem by Gary Smith.

Numerous analyses form the basis of what is called the "seven minute criterion." This instructs the operators not to initiate ECCS flow if it can not be established within seven minutes after it is demanded. The analyses predict that the earliest credible time for fuel damage to occur is 500 seconds after ECCS demand based on an assumption that no coolant flow exists in the core between 100 and 400 seconds after ECCS demand (the first 100 seconds are the blowdown period). A safety factor was applied to arrive at the seven minute ( 420 second) criterion.

The above criterion is calculated by setting the time of ECCS demand to the same time as the reactor trip. In the delayed ECCS failure scenario, GSCS has failed, but ECCS operates successfully for at least two hours before failing, so the fuel, pressure tubes, and graphite stack have all cooled significantly from their operating temperatures. It is, then, very conservative to assume that the total amount of energy required to heat the core to the point of fuel failure at the two hour point is the same as it was at the time of the reactor trip because this neglects the removal of sensible heat from the reactor during the first two hours.

The decay power level of the reactor at the time of the reactor trip is slightly more than 68 of the pre-trip operating power, while the decay power level two hours after the reactor trip is less than 1.58 [1]. Thus, at the two hour point it takes at least 4 times as long to deliver the same amount of energy to the core as it does just after the reactor trip. Hence, the reactor should be able to survive without coolant for 4 times as long, or $4 \star(400 \mathrm{sec}-100 \mathrm{sec})$, or 1200 seconds (20 minutes). This calculation does not consider the reduced sensible heat load (discussed above) or the coolant boiloff time. Since the core is initially full of water when ECCS fails, this adds conservatism to the calculation. Thus, it is assessed that the operators will have more than 20 minutes to take action upon the loss of all ECCS function before core damage can occur given that ECCS fallure occurs al two hourc after the reactor trip. The time to act will be longer if the ECCS failure occurs more than two hours 
after the reactor trip because the decay heat and sensible heat loads are reduced even further.

\section{Flow Required to Prevent Core Damage}

The following consensus opinion is based on a discussion of the subproblem by Don Ogden.

Many deterministic safety analyses were performed before the adoption of the core flow requirements as presented in Emergency Response Guide ERG-B [2], and the coolant flows required under ERG-B should be viewed as the distillation of that research with a margin of safety already applied. These flow requirements have been supported by other, more recent analyses which were used in the discussion and elicitation of APET Expert Judgement Issue 7 earlier in the day (July 20) and are referenced in the documentation of that elicitation.

Emergency Response Guide ERG-B states that ECCS flow may be reduced to 4,300 gallons per minute to the core at two hours after SCRAM, and to 4000 gallons per minute after an additional fifteen minutes have passed. It must be noted that this flow rate assumes that all inlet risers remain intact and that bulk coolant outlet temperature conditions can be satisfied. However, the analyses and safety margins used in the generation of the flow rate table are assessed to be sufficient that core damage will not occur if the tabulated coolant flow rates are followed for all accident sequences which do not involve inlet riser breakage.

\section{Support System Availability}

The following consensus opinion is based on a discussion of the subproblem by John Long.

AC Power: Based on historical data for the Hanford site, no offsite power interruptions have been of such a duration that any AC-related system would still be affected after two hours. In all historical cases, all ACrelated systems were recovered within fifteen minutes of the original loss of offsite power. Procedures are in place to restore operation of all AC powered systems within ten minutes of the restoration of offsite power. Thus, it is highly likely that full AC power will be available for core cooling system recovery long before the two hour point in the accident.

Circulating Raw Water (CRW) System: While the ECCS High Lift Diesel Pumps (HLDP's) normally draw water from the ECCS Low lift Diesel Pumps (LLDP's), they are also able to draw water from the CRW system upon the opening of a single manual valve in building 182. The CRW system uses electric pumps to draw water from the river and would be likely to be available as long as $A C$ power is available. The 20 minute minimum available action time would make this type of a recovery action highly probable. 
Demineralized Water: The other major support system for some of the additional sources of coolant which might be used to supplement a degraded or failed ECCS are the filtered and demineralized water systems. These systems use electric pumps and would likely be available as long as AC power is available. The Demineralized water system would be required for High Pressure Injection (HPI) to be used as an additional source of coolant.

\section{Available Coolant Sources}

- The following consensus opinion is based on a discussion of the subproblem by John Long.

There are many systems available to the operators which could be used to provide additional coolant to the core to supplement a degraded or failed ECCS. These systems, along with the operating requirements, are described below.

Single ECCS HLDP: The Level I PRA [3] used the same ECCS success criteria at two hours after SCRAM as was used immediately after SCRAM. This is extremely conservative because the ECCS must supply at least 22,000 gpm initially, while, as discussed above, about $4000 \mathrm{gpm}$ are sufficient after two hours. The issue presentation states that 92.48 of the delayed ECCS failure sequence frequency involves cases where a single ECCS HLDP would continue to run for the duration of the accident. Pump tests have shown that a single HLDP can provide at least $15,000 \mathrm{gpm}$ to the core. Thus, no core damage will occur as long as a single HLDP remains operating for the duration of the accident given that two pumps operate for the first two hours.

High Pressure Injection (HPI) System: The operators are instructed in Emergency Response Guide ERG-B to shut down the HPI system once adequate ECCS cooling is established. While there are no procedures to restart the HPI system upon the degradation or loss of ECCS function, the system could be restarted based on other procedures in the control room to provide additional core cooling as long as the cold $\log$ piping has remained intact. No actions outside the control room are required. The HPI system consists of four electric pumps which are capable of providing $1800 \mathrm{gpm}$ each. Thus, the operation of three pumps would provide adequate core cooling at the two hour point, and two pumps would be likely to provide adequate core cooling after a few more hours had passed. The HPI system requires the support of $A C$ power and the Demineralized Water System.

Filtered Water (FW) System: Connections are available between the ECCS manifold and the Filtered Water system. The FW pumps are electrically driven, and when a single manual valve in building $182 \mathrm{~N}$ is opened, filtered water flows into the outlet manifold from the HLDP's and follows the normal ECCS path through the core. The 20 minute minimum available action time would make this type of a recovery action highly probable.

Export System: A recently installed connection is between the ECCS manifold and the water export system. The export system connects the $N$ 
Reactor site with water systems at Hanford areas $B$ and $D$. The system contains two settling basins with capacities of 25,000,000 gallons each as well as electric pumps at both $B$ and $D$ areas and diesel pumps at $B$ area. $A$ single manual valve must be opened from a below-grade valve station near building $109 \mathrm{~N}$ to allow coolant to enter the ECCS manifold from the export system. The coolant would then follow the normal ECCS path through the core. The export system pumps are designed to start automatically based on pressure indications. In addition, $B$ area is attended during most shifts, so manual actuation is also possible. Each set of pumps is capable of providing $3500-4000 \mathrm{gpm}$ to the core, so the system can provide adequate coolant flow to prevent delayed core damage.

\section{Consensus Issue Resolution}

It is the consensus of the panel that core damage will not occur when one ECCS HLDP continues to operate. This situation constitutes 92.48 of the delayed ECCS failure sequence frequency as discussed in the issue description. It was also noted that, for the remaining 7.68 of the delayed ECCS failure sequence frequency, both HLDP's would not be expected to fail at the same time. Rather, one would be expected to continue to operate for some time after the failure of the other two pumps, because simultaneous random failures to run are extremely rare after two hours of successful operation, and common mode failures are very unlikely to allow the pumps to run for two hours, then fail simultaneously at some later time. This could provide significantly more time for the operators to take action to protect against effects of the possible failure of the third HLDP (core damage) than the 20 minute minimum available action time described above, which begins on complete loss of ECCS.

While all of the other systems described above are capable of providing coolant to the core in the case of a degradation or failure of the ECCS function, the actuation of each of them would require actions by the operators which are outside of procedures. However, additional engineering and management personnel would undoubtedly be on hand by the two hour point for any event which had demanded ECCS, and it is believed that these people would consider the use of the described systems to prevent core damage. Based on the discussion in the previous paragraph, they would almost certainly have the time to implement these plans. Thus, the panel believes that core damage based on delayed ECCS failure is extremely unlikely to occur.

It would, however, be non-conservative to take credit in the PRA for the use of systems for which procedures have not yet been established. For this reason, it is the assessment of the panel that the Level II PRA should take the conservative approach that core damage will result when all three ECCS HLDP's fail and GSCS is not operating. The panel further recommends that control room procedures be established for the actuation of these additional cooling systems as an additional protection against these possible delayed core damage scenarios. 


\section{C.4.4 Application of Results}

The above issue resolution was implemented in the APET analysis by using the unconditional failure probability for a single ECCS HLDP as the probability that core damage occurs given that an accident has occurred that was assessed by the Level I PRA to result in a bin $Z 1$ core damage state. Those that do not progress to core damage (i.e., one ECCS HLDP continues to run), will end the APET analysis with zero core damage while those that do progress to core damage will conservatively progress to 1008 core damage. The ECCS HLDP failure rate is sampled in the Latin Hypercube analysis for the Level I PRA and is picked up directly by the APET for use in this analysis in the "status of ECCS during the recovery phase" APET event. 


\section{5 FUel Damage PROPagation}

\section{C.5.1 Issue Definition}

$N$ Reactor differs from commercial reactors in that it is possible to credibly model many core damage accidents in which less than 1008 core damage occurs. The extent of core damage for various cooling conditions is an uncertainty issue unto itself. A fundamental assessment of that analysis appears to be the fact that fuel damage will not propagate from pressure tubes that are undercooled to those that are apparently adequately cooled. A number of mechanisms have been proposed to cause this propagation, but assessments of these phenomena have generally agreed that they are less than completely credible. However, each of these mechanisms deserves individual discussion to justify why it is not considered a credible fuel damage mechanism. Finally, a probability distribution needs to be developed to encompass the possibility that one of these mechanisms may indeed occur. It should be emphasized that no actual scenarios are known to cause this type of propagation because all fuel damage scenarios which are considered credible are included in the APET in other ways.

A few of the scenarios that should be considered in a fuel damage propagation analysis should include the following: A severe pressure tube rupture event has been postulated which might locally disrupt the graphite stack and rupture additional pressure tubes. This could possibly end up diverting a significant portion of the degraded ECCS flow away from the core causing additional fuel damage. A second postulated scenario includes GSCS tube rupture due to thermal stresses. The GSCS tube temperatures required to produce such stresses could occur as the result of a pressure tube break destroying the helium blanket or through GSCS restoration after a period of failure. A third postulated scenario includes GSCS tubes being sheared off due to the thermal expansion of the graphite stack.

Fuel damage propagation is examined in the APET using two different possibilities. Large scale fuel damage propagation is assumed to always result in 1008 core damage. Small scale fuel damage propagation is assumed to result in a doubling of the expected amount of fuel damage with a minimum of 258 core damage. A probability distribution needs to be developed to attempt to account for the possibility of small scale and large scale fuel damage propagation.

\section{C.5.2 Issue Resolution Process}

S. E. Dingman, L. A. Miller, and G. D. Wyss, all of Sandia National Laboratories, and C. J. Shaffer of Science and Engineering Associates (under contract to Sandia National Laboratories) were selected to be elicited on these issues. All were heavily involved in modeling the $N$ Reactor primary system for the $N$ Reactor MELCOR benchmark effort and were selected for their extensive familiarity with the MELCOR, RELAP and TRUMP analyses that had been conducted for $\mathrm{N}$ Reactor. The issue was presented to them and, after some discussion, they reformulated it to be as described 
above. They were then asked to provide input as to the resolution of the issue.

The issue was presented to the elicitees on August 28, 1989. At that time other issues were also presented, and the elicitees panel felt that it would be most profitable for each panel member to write up background information about one issue for review at a final elicitation meeting. The following represents the compilation of background information for the fuel damage propagation issue as compiled by Gregory Wyss, with additions based on oral and written comments received during the final elicitation meeting.

\section{C.5.3 Issue Analysis and Resolution}

\section{Background Information}

1. UNC Nuclear Industries, Inc., $\mathbf{N}$ Reactor Updated Safety Analysis Report (NUSAR), WHC-SP-0297, February 1978.

2. K. R. Birney, "Pressure Tube Considerations Relating to Molten Uranium," memo to D. M. Ogden dated January 7, 1987, Westinghouse Hanford, formerly UNC Nuclear Industries.

3. F. J. Heard, et al., N Reactor Safety Enhancement Final Report: Hydrogen Generation and Thermal Analysis of the Hydrogen Mitigation Design Basis Accident, WHC-SP-0096, Westinghouse Hanford, December 1987 .

4. E. H. Randklev, et. al., Molten Uranium/Zircaloy Interaction Studies, WHC-SP-0338, Westinghouse Hanford, August 1988.

5. K. R. Birney, "Molten Uranium - Graphite Interactions," memo to W. J. Quapp dated February 17, 1987, Westinghouse Hanford, formerly UNC Nuclear Industries.

\section{Discussion}

A number of mechanisms have been proposed to cause the propagation of fuel damage from an uncooled pressure tube to one that is apparently adequately cooled. The possibility of each of these mechanisms is remote at best, however, they deserve individual discussion to justify why they are not considered credible fuel damage mechanisms. Finally, a probability distribution will be developed to encompass the remote possibility that one of these mechanlsus may indecd occur. 
Pressure Tube Failure

The unique pressure tube design of $\mathrm{N}$ Reactor has led to considerable speculation about what types of events might cause the failure of a few pressure tubes to propagate to the entire core. A detailed analysis of this possibility was performed in the NUSAR [1], and pressure tube damage propagation was found to be essentially impossible because each pressure tube is surrounded by its own thick graphite sheath. Thus, for one damaged pressure tube to damage another, it must first destroy the graphite sheath that surrounds the other pressure tube. The limited open spaces available inside the graphite lattice limit the credibility of this phenomenon.

An additional postulated pressure tube-related fuel damage propagation scenario involves a pressure tube rupture event causing the failure of the GSCS. In such a scenario, the pressure tube failure destroys a number of GSCS tubes, which is assumed to destroy GSCS function. Analyses similar to that described above show that a pressure tube failure event can in fact damage the two GSCS tubes directly above and below the pressure tube failure point, but nothing further away than that. Multiple pressure tube failures are expected only in seismic scenarios or when the primary system has been depressurized for ECCS action. Pressure tube failures at ECCS system pressures are expected to be far less violent and, thus, not to damage the GSCS tubes. The GSCS consists of hundreds of $0.75^{\prime \prime}$ diameter tubes running through the graphite stack, and the GSCS system capacity is such that the failure of a few tubes is not expected to significantly degrade GSCS function for the rest of the core. For this reason, pressure tube failure propagating to GSCS system failure is considered impossible.

The possibility has also been raised that coolant could be diverted from cooled sections of the core and flow out through broken pressure tubes. This could caused "unaffected" regions of the core to lose cooling and fail fuel. This possibility was considered in the expert judgement elicitation on pressure tube failure, at which time it was decided that it would take several pressure tube failures in a single riser to divert enough cooling from the rest of the riser to affect the core damage state. Furthermore, these pressure tube failures would have to be below the liquid level in the riser. Since, as discussed above, multiple pressure tube failures are only expected to occur in areas where fuel has already failed, and these areas are by definition above the liquid level in the riser, and since the ECCS has enough reserve capacity to make up for the flow diverted from several broken pressure tubes in each riser, the possibility that this effect could cause fuel damage propagation is seen as very remote.

A water hammer ("chugging") phenomenon has been postulated by which multiple pressure tube failure events might occur. In this scenario, coolant attempts to enter a hot, voided region of the core. Flashing occurs at the coolant front to cause a pressure spike large enough to force the coolant backward out of the core. Such a scenario would be cyclical in nature and has been postulated to cause multiple pressure tube failures by the fatigue mechanism. The water hammer phenomenon was discussed at great 
length during the expert judgement elicitation for the pressure tube failure issue, and the reader is referred to that section for a more complete discussion. The result of that elicitation, however, was that the pressure tubes would only fail under high temperature conditions where the fuel inside the pressure tube had already failed. This is because the material properties of the pressure tube begin to degrade at temperatures above the fuel failure temperature. Water hammer is not considered a credible mechanism for causing additional fuel failure because the high pressure tube temperatures required to allow water hammer-induced pressure tube failures do not exist in areas where fuel damage has not already occurred.

\section{Molten Fuel-Related Issues}

Accident scenarios that involve molten fuel have been the source of much discussion in regard to fuel damage propagation issues. The horizontal pressure tube geometry and the fact that each pressure tube is surrounded by a thick layer of graphite has led many to the analogy that each fuel element is essentially surrounded by its own "core catcher" which prevents the fuel from migrating out of the core and interacting with other structures. Others, however, have postulated molten fuel scenarios which they contend could cause fuel damage propagation. These scenarios are discussed below.

The most obvious molten fuel issue relates to the possibility that the molten fuel will interact with the Zircaloy pressure tubes and the graphite stack. Studies of these phenomena $[2,4,5]$ indicate that they only occur at very high temperatures $(\rightarrow 1550 \mathrm{~K})$. If molten fuel is available at these temperatures, pressure tube failure would be expected to occur within a few hours due to dissolution of the zircaloy by the molten fuel, but the graphite should remain intact for a long time ( $>50$ hours). These interactions are not violent in nature, so they should be confined to the pressure tube with the molten fuel. Therefore, fuel damage propagation due to fuel/structure interactions is not credible.

Other moltcn fuel scenarins involve liquid fuel relocating into other locations to cause damage propagation. One such scenario would involve molten fuel running out of a pressure tube, down the riser and plugging either the riser or other pressure tubes to cause fuel damage propagation. In order for this to occur, however, the molten fuel would have to flow an average of 50 feet just to reach the riser. This fuel must flow through the reflector regions of the core which are considerably colder than the normally fueled region of the core. Calculations [3] indicate that these regions are cold enough that any molten fuel reaching the area would be refrozen long before it could get out of the core. This precludes fuel damage propagation.

An issue of potentially more concern is that molten fuel could flow down inside the graphite stack to aggravate an accident scenario. If there no event has occurred to breach the graphite surrounding a pressure tube, it is almost impossible for the molten fuel to flow down inside the core. Rather, it will remain inslde the graphite pressure tube sleeve becausc 
these graphite blocks are compression fitted together with trunions to leave only fine cracks in compression between the blocks. The graphite blocks are typically colder than the fuel, so the fuel would be expected to initially freeze in these cracks. However, even if the fuel remelts, the compression fitting technique means that molten fuel will not escape the pressure tube channel in significant quantities as long as the graphite channel remains intact.

In cases where the pressure tube graphite block does not remain intact (such as a pressure tube rupture small LOCA event), the fuel could be expected to flow out of the tube and down into the graphite stack. In order for this to cause fuel damage propagation, however, this fuel must work its way through the pressure tube graphite for another pressure tube and attack the pressure tube itself. The compression fitting technique described above prevents this from occurring. The GSCS tubes also flow through their own graphite blocks which, while possibly not compression fitted, do pose a barrier to the molten fuel attack. When the capacity of the GSCS to make up for several failed GSCS tubes is considered, it becomes clear that, while such an event would cause a degradation in the GSCS performance due to the loss of the helium cover gas system, it should not cause either a total loss of GSCS or additional fuel damage propagation (beyond the level normally expected for accidents where GSCS is degraded).

\section{Probability Distributions for Fuel Damage Propagation}

The above discussion leads to the conclusion that fuel damage propagation, if it can occur at all, will be an extremely rare event. The Accident Progression Event Tree (APET) allows for two fuel damage propagation alternatives: small scale and large scale fuel damage propagation. Small scale propagation is defined to be the case where the actual amount of fuel damage is approximately twice what would be expected based on the core cooling state. Large scale propagation is defined to be a situation where, regardless of the core damage conditions expected based on the core cooling state, the core actually proceeds to 1008 core damage.

The purpose of the fuel damage propagation elicitation is to assign a probability that a mechanism exists to significantly increase core damage above the amount that would be expected based on the core cooling state. The scenarios presented above were all considered in this analysis and found to be either not credible or a very remote possibility. The analysts were unable to think of any other mechanisms by which propagation might occur. The crux of the elicitation, therefore, comes down to an assessment of the probability that one of these events would actually occur. It is the assessment of this analyst that a reasonable point estimate of this would be no greater that 0.001 , with lower and upper bounds of 0.000 and 0.005 . This probability should be divided between small and large scale propagation with approximately 108 of the probability allotted to large scale propagation. The probability distribution is assessed to be: 


\begin{tabular}{ccr} 
Quantile & Small Scale & Large Scale \\
\hline 0.00 & 0.0000 & 0.00000 \\
0.25 & 0.0004 & 0.00004 \\
0.50 & 0.0010 & 0.00010 \\
0.75 & 0.0020 & 0.00020 \\
1.00 & 0.0050 & 0.00050
\end{tabular}

These probability distributions are not independent, but should be very highly correlated.

\section{C.5.4 Application of Results}

The above results appear directly in the APET as uncertainty input for the event "Does fuel damage propagate to the 'unaffected' region of the core?" (APET event 72). 


\section{C.6 CORE RESPONSES TO LATE ECCS INITIATION}

\section{C.6.1 Issue Definftion}

Dumping water onto a portion of the core that has been heated to the point of fuel damage is expected to contribute to a number of undesirable effects. These include pressure tube failure, the production of a steam spike in confinement, hydrogen production, and possibly additional core damage. Probability distributions need to be developed to model these phenomena for the various initial core damage and ECCS flow situations.

The following questions are asked in the APET related to the possibility of late ECCS actuation:

1) Does the injection of water cause a steam spike in the confinement building with a peak pressure greater than 10 inches water? Is the peak confinement pressure from the spike greater than 5 psig?

2) Does a pressure tube fail due to water injection?

3) How much additional fuel fails due to water injection?

\section{C.6.2 Issue Resolution Process}

L. A. Miller and G. D. Wyss of Sandia National Laboratories and C. J. Shaffer of Science and Engineering Assnciates (under contiact to Sändia National Laboratories) were selected to be elicited on these issues. All were heavily involved in modeling the $\mathrm{N}$ Reactor primary and confinement systems for the $N$ Reactor MELCOR benchmark effort and were selected for their extensive familiarity with the MELCOR, HECTR and other analyses that had been conducted for $N$ Reactor. The issue was presented to them as described above and they were asked to provide input as to either the resolution or restatement of the issue. They were content to consider the issue as presented above.

\section{C.6.3 Issue Analysis and Resolution}

\section{Background}

1. L. A. Miller, et. al., N Reactor Probabilistic Risk Assessment Supporting Calculations, SAND89-2101, Sandia National Laboratories, 1990.

\section{Discussion}

The following is based on an issue writeup by L. A. Miller dated August 31, 1989 as amended based on oral and written comments. 
The primary question to be considered in this issue is whether the injection of water into the core causes a steam spike in the confinement building which results in a peak pressure greater than the spray actuation pressure criteria $\left(10^{\prime \prime} \mathrm{H}_{2} \mathrm{O}\right.$ ) or the building failure pressure criteria ( 5 psig).

A simple MELCOR calculation was performed to investigate the rate of steam production if the ECCS was initiated after the fuel had begun to fail. The model consisted of a 30 node core, as in the Hydrogen Mitigation Design Basis Accident, and control volumes for the core, the inlet risers, the Graphite and Shield Cooling System (GSCS), and an environment for the steam from the GSCS. The outlet of the core was directly connected to a 15 volume confinement model to determine the pressure rise in the confinement caused by steam generation in the core. All core temperatures (fuel and graphite) were initialized to $1300 \mathrm{~K}$ and the core was dry. This calculation is documented in the $N$ Reactor PRA supporting calculations document [1].

A number of factors can be considered that make this calculation the upper bound for a steam generation rate. First, steam binding in the core was unrealistically low, so water was able to penetrate the core more quickly than would be expected. Second, an unrestricted flow path was available for the steam to escape from the core and move into the confinement building atmosphere. The real path for the steam would be more difficult and restricted - either through the primary system to a break location, or through the graphite stack internals and out to the building atmosphere. Third, the fuel and graphite temperature was uniformly set to $1300 \mathrm{~K}$, which is hotter than many sections of the core are expected to become in most accidents. Finally, blockage of the pressure tube and fuel channels by extruded fuel, which would be an additional restriction for the steam, was not modeled. However, a realistic water source was used and the graphite reflectors, thermal and biological shields were not modeled in this calculation, and these could contribute an unmodeled addition to the steaming rate. Also, by the time recovery actions would be taken to restore ECCS flow to a damaged core, the confinement building would be sealed. Any significant pressure increase would also demand reisolation of the confinement, so any reasunably rapid pressure increase will stand a chance of failing the confinement.

This calculation predicted a pressure rise of 16 psig in the reactor building in the first few seconds after ECCS actuation. Since the pressure rise occurs quickly, and the banana wall takes more than a minute to relieve if confinement integrity is to be maintained, the banana wall is not considered to be capable of mitigaling it. While the calculation was very simplistic, the possibility of a steam spike causing a pressure rise of more than 5 psig can not be discounted. MELCOR transfers all of the heat directly to the liquid water (heat is not transferred to the atmosphere), steaming it, therefore, this is a conservative calculation with respect to steam production.

Based on the calculation and the uncertainty in it the following distrlbution was accepted by thc elicitees for the probability of a steam spike causing a pressure increase of more than 5 psig. The probability of 
a steam spike causing a pressure increase of more than 10 inches of water (where fog sprays would be demanded) was assigned a value of 1.0 .

$\begin{array}{cc}\text { Quantile } & \text { Total Probability } \\ & 0.0 \\ .25 & 0.5 \\ .5 & 0.8 \\ .75 & 0.85 \\ 1.0 & 1.0\end{array}$

These probabilities are on a linear scale, and interpolation should be done in a linear manner. They are based on the perception that the steam pressure rise is likely to cause confinement failure, but that there is considerable uncertainty in that result based on the conservatism of the calculation used in the analysis. It was also noted, however, that it may not be possible to perform a significantly less conservative calculation because of vast uncertainties associated with the available flow areas in regions of the core where fuel has failed and been extruded into the coolant channels.

\section{C.6.4 Application of Results}

The above results were applied directly in the event "Does recovery cause a steam pressure spike in the confinement?" (APET event 55) for the case where ECCS is recovered from a completely failed state.

The elicitees did not want to commit to a core damage result other than to note that the amount of fuel damage would be expected to increase because of the loss of the helium cover gas and its effect on the operation of the GSCS. It would be reasonable to state that the amount of fuel damage could double as a result of the late initiation of ECCS, or that ECCS would be only partially effective (due to failed fuel partially blocking pressure tubes) and that GSCS degradation would cause a corresponding increase in fuel damage. 


\section{C.7 PRESSURE TUBE FAILURE}

\section{C.7.1 Issue Definition}

Everline et. al. assessed the probability of failure for the N-Reactor Zircaloy pressure tubes under normal operating conditions and seismic loadings (SD-NR-ER-043). However, that analysis did not consider the additional pressure tube loadings that are possible under accident conditions. For example, when ECCS is actuated under station blackout conditions, there can be a substantial period during which the top few rows of pressure tubes are steam filled. Certain outside reviewers have postulated water hammer ("chugging") phenomena during the time ECCS is trying to refill these pressure tubes which could lead to thermal and mechanical cycling and, possibly, to pressure tube failure. In this scenario, water comes into contact with hot fuel, and the rapid boiling at the liquid front causes a pressure increase which drives the liquid front back out of the reactor. This could occur repeatedly, and has been hypothesized as a mechanism for thermal/mechanical failure of the pressure tubes. These conditions would be significantly worse in cases where ECCS flow to the core does not meet the success criteria because the period during which the top rows of pressure tubes are uncovered could be much longer, allowing more time for the pressure tubes to heat up.

It has been stated by Westinghouse Hanford personnel during meetings (June 13, 1989) that additional pressure tube analyses have been conducted since the Everline study which incorporate additional information about the stresses anticipated due to thermal, mechanical and water hammer phenomena. These phenomena need to be considered in order to present a complete picture of core behavior during partial ECCS scenarios and late ECCS restart events.

The following is an incomplete list of uncertain physical phenomena which are believed to contribute to pressure tube failure phenomena:

- thermal and mechanical stresses in the pressure tube

- quenching of hot pressure tubes and/or fuel

- flow oscillations ("chugging")

- pressure spikes away from the coolant front which are caused by quenching or chugging near the coolant front

- radiation damage and embrittlement of the pressure tubes

- pre-existing defects in the pressure tubes

- stresses induced in the tubes by graphite movement (caused by radiation damage to or thermal expansion of the graphite stack)

For the following discussion, pressure tube failure is defined to be the state where at least one pressure tube has failed anywhere in the reactor piping system at any time during the accident. If GSCS function is assessed to make a significant difference in any of the probability distributions, separate distributions should be made for GSCS operating and GSCS failed scenarios. The following should also be considered: 
1) Determine probability distributions for pressure tube failure under the following accident conditions:

a) ECCS has failed initially such that the total coolant flow into the core is clearly insufficient to significantly reduce core temperatures $(1000-4000 \mathrm{gpm})$. The reactor continues in this state indefinitely.

b) ECCS has failed initially such that the total coolant flow into the core is substantial, but does not meet ECCS success criteria $(-10000 \mathrm{gpm})$. The reactor continues in this state indefinitely.

c) ECCS has succeeded initially except that a CV-2R valve has failed to open. Coolant is flowing into the blocked riser only by backflow through the V-4 header. The reactor continues in this state indefinitely.

d) Identical to case (c) above, except that high pressure injection is also supplying coolant to the affected riser.

e) Identical to case (c) above, except that the failed (closed) CV-2R valve opens after an undetermined period to flood the previously blocked riser.

f) ECCS is not established initially (totally failed), but is started after a significant amount of fuel has failed.

A suggested decomposition of the problem is to consider first the probability that the postulated chugging phenomenon (or another thermal/mechanical cycling process) occurs, and then to consider the probability of pressure tube failure given that chugging does or does not occur.

2) Discuss the expected effects of pressure tube rupture at low ( -300 psig) primary system pressures for the following scenarios:

a) partial ECCS flow has been established and a pressure tube fails before substantial core damage has occurred

b) ECCS is actuated after significant core damage has occurred

\section{C.7.2 Issue Resolution Process}

The above described issue was elicited at Westinghouse Hanford on Thursday and Friday, July 20 and 21, 1989. The normative and substantive experts for this elicitation were T. A. Wheeler and G. D. Wyss, respectively, both of Sandia National Laboratories. The expert panel for the elicitation consisted of Keith Scott (WHC), John Long (PNL, formerly of WHC), Don Ogden (WHC), Gary Smith (WHC), and Marv Thurgood (WHC). The elicitation was preceded by approximately one hour of normative training and twenty minutes of issue description as presented by the normative and 
substantive experts. The results of that elicitation are described in the following section.

\section{C.7.3 Issue Analysis and Resolution}

\section{Background Information}

1. F. J. Heard, et al., N Reactor Safety Enhancement Final Report: Hydrogen Generation and Thermal Analysis of the Hydrogen Mitigation Design Basis Accident, WHC-SP-0096, Westinghouse Hanford, December 1987.

2. J. C. Chapman and R. A. Callow, Emergency Response Guide B ECCS Guideline Evaluation Analys is for N Reactor, EGG-EAST-8385, Idaho National Engineering Laboratory, March 1989.

3. M. A. Bolander, et. al., Simulation of Cold Leg Manifold Break and Station Blackout Revised Sequences for Reduced ECCS in the $N$ Reactor, EGG-TFM-7962, Idaho National Engineering Laboratory, February 1988.

4. I. Harris, et. al., Pressure Tube Rupture Evaluations: Supplement 1, WHC-SD-NR-ER-034, Prepared by Impell for Westinghouse Hanford, December 1987.

5. K. Scott, et. a1., Probabilistic Estimate of Pressure Tube Rupture Propagation, WHC-SD-NR-ER-011, Westinghouse Hanford, January 1988.

6. M. B. Richards and A. W. Barse11, Catalysis Effects by Impurities on the Steam-Graphite Reaction under N Reactor Accident Conditions, GA-C19161, General Atomics, April 1988.

7. M. B. Richards, Issues Related to Hydrogen Generated from MetalSteam Reactions under $N$ Reactor Accident Conditions, GA-C19273, General Ntomics, May 1988 .

8. M. J. Thurgood, Final Report: Re-Evaluation of Condensation Induced Water Hammer During ECCS Injection in $N$ Reactor, NAI-8804-5, Numerical Applications, Inc., February 1989.

9. C. J. Everline and M. B. Richards, Probabilistic Failure Assessment of the $N$ Reactor Pressure Tubes - Phase IV Report, SD-NR-ER-043, Westinghouse Hanford, March 1989.

10. K. R. Birney, "Pressure Tube Considerations Relating to Molten Uranium," memo to D. M. Ogden dated January 7, 1987, Westinghouse Hanford, formerly UNC Nuclear Industries.

11. E. H. Randklev, et. al., Molten Uranium/Zircaloy Interaction Studies, WHC-SP-0338, Westinghouse Hanford, August 1988. 
General Discussion - Pressure Tube Failure Mechanisms

The following discussion represents the consensus opinion of the panel as to the possible pressure tube failure mechanisms.

An area of concern for pressure tube failure amongst outside groups has been the possibility of propagation of a pre-existing pressure tube defect to the point of failure. This concern is being addressed through an eddy current testing program which is currently being performed on all pressure tubes at $\mathrm{N}$ Reactor and will be completed before restart. An assessment of the techniques being used indicates that pressure tube flaws up to 50 mils deep for axial flaws or 100 mils deep for circumfrential flaws on the outside surface of a pressure tube are the limits of detection for the system. Sensitivity is considerably better on the inner tube surface. The pressure tube itself is 270 mils thick, and any tube which shows a detected flaw will be replaced. It is assessed that up to 58 of the existing pressure tube flaws above these limits will not be detected by the examination process. This is comparable to the results expected from other applications of the eddy current testing technique. The possibility of a pre-existing defect propagating into a fracture is quantified further in the elicitations.

A more dominant failure mechanism is that of mechanical cycling in the pressure tube connectors. Studies have shown that cyclical water hammer (or, as some have called it, "chugging") can in some accident conditions fail the pressure tube connectors in as few as 100 cycles. These conditions, however, always involve elevated temperatures because, at lower pressure tube temperatures (around $1000 \mathrm{~F}$, where the elastic limit is -30 ksi), all analyzed phenomena (including thermal shock, mechanical stresses, pressure spikes and water hammer simultaneously) produce pressure tube stresses which are below the elastic limit for the Zircaloy pressure tube material. As the pressure tube temperature becomes significantly increased, however, the tube properties degrade. The degraded mechanical properties combined with the higher pressure spikes expected when liquid coolant interacts with very high temperature reactor components makes pressure tube failure increasingly likely as the component temperatures increase. These effects will be quantified further in the remainder of the elicitation.

Additional failure mechanisms have been identified by other analyses. These mechanisms are pressure spikes in which a pressure tube fails due to yield as in the water hammer case above, dissolution of the pressure tube by molten uranium, which occurs at temperatures greater than $2300 \mathrm{~F}$ over a period of several hours, and molten fuel-coolant interactions (MFCI), in which a pressure tube again fails due to yield, but the pressure spike is based on a different mechanism. MFCI at $N$ Reactor is considerably different from the "classical" MFCI scenario in which molten fuel drops into a coolant pool to create a "steam explosion." N Reactor's horizontal geometry precludes such an event. Instead, a small MFCI event is possible in which liquid coolant is able to traverse enough of the pressure tube without being vaporized to come into contact with molten fuel, mix, and create a large, localized pressure spike. Such a scenario could be very 
difficult to achieve because, in many cases where molten fuel is not present over the entire length of a pressure tube, coolant must flow past hot, solid fuel which has been extruded into the coolant channels on failure of the clad. This extruded fuel tends to block the penetration of coolant into the pressure tube and would help to prevent MFCI events. This prevention effect is less pronounced for low exposure fuel since it tends to extrude less uranium into the coolant channels on failure. Another MFCI mitigation mechanism would be the formation of an oxide film over the surface of the molten fuel. This would help to prevent mixing between the coolant and the fuel, but can not be relied upon because the oxide film could be blown off during pressure spikes which might precede an MFCI event.

Based on the above discussion, it was decided by the panel that the following decomposition of the pressure tube failure question was appropriate:

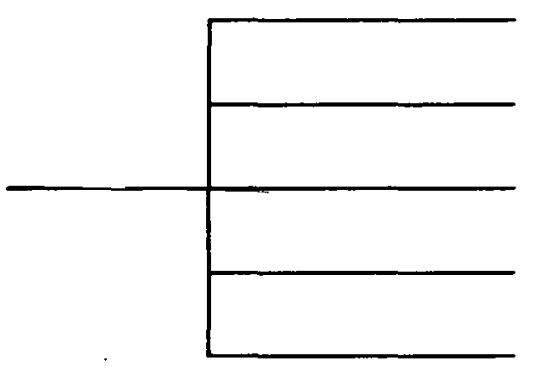

U/Zr Dissolution

MFCI

Yield

Pre-Existing Defect

No Tube Failure

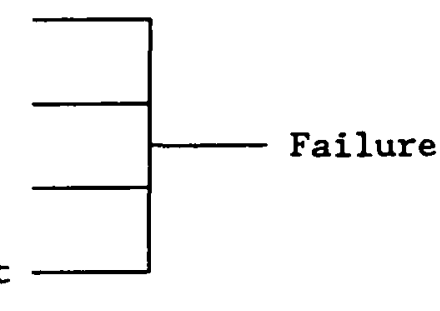

Success

\section{Elicitation: Issue 7. Part 1f}

Issue: Determine the probability that at least one pressure tube will fail somewhere in the core when ECCS is not established initially (totally failed) but is started after a significant amount of fuel has failed.

Discussion: The panel decided that it was necessary to break this issue down into three time regimes: early (where pressure tube temperatures are still below $-1400 \mathrm{~F}$ ), intermediate (molten fuel may exist, but not in the inlet region of the core), and late.

Early Reflood With GSCS: The characteristics of the "early" time regime for reflooding the core are that fuel failure has occurred, but little molten fuel is present and, as a result, the pressure tube temperature is following the graphite temperature rather than the fuel temperature. Thus, the pressure tube temperature is expected to remain relatively low $(1200-1400 \mathrm{~F})$. I was the consensus of the panel that, under these conditions, the uranium dissolution mode of pressure tube failure is not credible because of low temperatures. In addition, the MFCI and yield modes of failure are very unlikely. Water hammer pressures of 300-1000 psig are achievable based on research by Marv Thurgood.

A full description of the case to be elicited here is as follows: ECCS fails initially, then starts when there is at least 108 core damage. 
The maximum fuel temperature is $-1900 \mathrm{~F}$, and calculations show that this situation occurs at about 1000 seconds into the accident and that the peak pressure tube temperatures at this time are $-1500 \mathrm{~F}$. GSCS is operating, and the primary system has blown down, so the current system pressure is -40 psig. For this elicitation, any probability less than 0.01 is effectively zero.

In discussing the issue of pressure tube failure due to MFCI, John Long assessed that MFCI is very unlikely (almost zero probability) in this situation because very little if any molten fuel is available for interaction (most would be refrozen to the pressure tube walls), so the probability that MFCI would cause pressure tube failure was assessed to be <0.01. Gary Smith, however, assessed that the fuel from the failed inner elements would not resolidify because it is insulated from the pressure tube, so the probability that MFCI could cause pressure tube failure was assessed to be 0.05 . Marv Thurgood believed that the uranium extruded from failed fuel would prevent water from reaching the molten fuel, and that steam cooling would prevent MFCI from occurring, so the probability that MFCI could cause pressure tube failure was assessed to be 0.01 . Don Ogden agreed with the reasoning of and the probability assigned by Gary Smith, while Keith Scott did not wish to comment on this issue.

Pressure tube failure due to yield is based on a phenomena where pressure spikes generated by means other than MFCI stress the tube to the point where it yields causing failure. This is especially important in the water hammer scenarios, and depends heavily on the temperature of the pressure tube as this controls the degradation of the material yield strength. Keith Scott stated that his research indicated that the yield strength curves for Zircaloy begin to drop rapidly at $-1300 \mathrm{~F}$, and by $1500 \mathrm{~F}$, the pressure tubes should be able to withstand -2000 psig. Since the assessment of water hammer does not indicate that pressures above 1000 psig are achievable, the probability of yield failure should be quite low. However, if the tubes get up to $-1650 \mathrm{~F}$, then the yield strength is closer to 1000 psig. The yield strength curve is very steep in this range, so he assessed that there was a high degree of uncertainty in this issue (based on uncertainty in the pressure tube temperature) and assigned a probability range of $0.05-0.20$ to this issue with a median of 0.10 . It was the assessment of John Long that nearby pressure tubes containing fuel with only the inner element failed would provide a large enough flow area to relieve any pressure rises that might occur and that steam cooling would decrease the pressure tube temperature to below the yield point. In addition, the failure of both the inner and outer fuel elements would plug both flow channels with nearly frozen uranium that may transmit pressure, but would not allow water to have rapid access to the liquid uranium. This would also limit the peak pressure, so the probability that yielding could cause pressure tube failure was assessed to be 0.01 for this case. Gary Smith assessed that, for fuel with both the inner and outer elements failed, the coolant channels would likely be plugged and would prevent pressure relief and steam cooling. This would expose the pressure tube (with a somewhat reduced yield strength) to any water hammer that might occur, so the probability that yielding could cause pressure tube failure was assessed to be 0.05 . Don Ogden felt that high pressures were possible due to water hammer and also felt that steam cooling was unlikely, but that 
the temperature profile was well characterized by the computer models, so the probability that yielding could cause pressure tube failure was assessed to be 0.05 . Marv Thurgood believed that significant pressures are possible from water hammer (up to $1000 \mathrm{psig}$ ), so the probability that yielding could cause pressure tube failure was assessed to be 0.05 .

Failure due to pre-existing defects in the pressure tube is based on the assumption that at least one significant defect escaped detection during the eddy current testing of the pressure tubes. These defects can propagate into tube failures when stresses are added to the tubes due to thermal shock or mechanical (pressure-induced) effects. It is the assessment of Gary Smith that as the pressure tube temperature is increased to more that $1000 \mathrm{~F}$, the defects in the pressure tubes would be annealed away to decrease the possibility of failure, but, since the end regions of the tubes would not have reached that temperature, failure is still posstble, so the probability that a pre-existing defect could cause pressure tube failure was assessed to be 0.01 . John Long believed that liquid coolant would hit and quench the parts of the pressure tubes that had not yet been annealed and cause thermal shock in the pressure tube, so the probability that a pre-existing defect could cause pressure tube failure was assessed to be 0.03 . Don Ogden agreed with the thermal shock assessment and added that water hammer could cause high pressures in combination with the thermal stresses, so the probability that a preexisting defect could cause pressure tube failure was assessed to be 0.10 . Marv Thurgood stated that, from his water hammer and thermal shock analyses, the high pressures and temperatures expected during these scenarios caused stresses slightly in excess of the ASME code, but the conservatisms built into the code gave him confidence that the pressure tubes would be likely to survive, so the probability that a pre-existing defect could cause pressure tube failure was assessed to be 0.05 . Keith Scott agreed with the assessment of Marv Thurgood, so the probability that a pre-existing defect could cause pressure tube failure was assessed to be 0.05 .

None of the panel members wished to change their assessments, so the elicited probability distributions were aggregated. In any case where a panel member declined to give a probahility, the average of the other panel members' probabilities was used. The aggregation process is summarized in the following table. 


\begin{tabular}{|c|c|c|c|c|c|}
\hline & J. Long & G. Smith & D. Ogden & M. Thurgood & K. Scott \\
\hline Dissolution & 0.00 & 0.00 & 0.00 & 0.00 & 0.00 \\
\hline MFCI & 0.01 & 0.05 & 0.05 & 0.01 & $(0.03) *$ \\
\hline Yield & 0.01 & 0.05 & 0.05 & 0.05 & 0.10 \\
\hline Defect & 0.03 & 0.01 & 0.10 & 0.05 & 0.05 \\
\hline & $\ldots$ & $\ldots \ldots$ & $\ldots$ & $\ldots \ldots$ & $\cdots$ \\
\hline Failure & 0.05 & 0.11 & 0.20 & 0.11 & 0.18 \\
\hline
\end{tabular}

Average failure probability: 0.13
Success
0.95
0.89
0.80
0.89
0.82

Average success probability: 0.87

Declined comment; used average of other panel members' probabilities

Following the elicitation of point estimates of failure probabilities, the panel members were asked to place uncertainties on their answers. When asked for a lower bound, John Long stated that he felt that it was possible that the true answer was zero (that is, the pressure tubes would never fail in such scenarios). This result is certainly the limiting case, and no other panel members felt confident assigning a tighter lower bound, so all panel members used zero as the lower bound.

There was a much wider spread in the upper bounds. John Long felt that the upper bound could not be greater than a probability of 0.07 based on his ideas about pressure relief and steam cooling. Don Ogden and Keith Scott, however, both felt that there was a considerable amount of uncertainty in the final results based on uncertainties in the true temperatures and the strength of the pressure tubes. All other panel members agreed that the uncertainties are large. Because of the large uncertainties involved, several of the panel members felt that the use of a uniform ("flat") distribution between the bounds of the uncertainty distribution was appropriate. They were informed that this would, in certain instances, produce probability distributions with medians that are different from the point estimates described above. They replied that they were uncertain enough about the overall outcome that changing the point estimate by a small amount would not bother them. 
The uncertainty elicitation produced the following verbal descriptors:

Don Ogden: Uniform distribution between 0.00 and 0.30 .

Gary Smith: Uniform distribution with a mean to match his point estimate $(0.13)$.

Keith Scott: Uniform distribution between 0.00 and 0.30 .

Marv Thurgood: Normal distribution around 0.15 with bounds at 0.00 and 0.30 .

John Long: Bounds of 0.00 and 0.07 , with first, second and third quartiles at $0.04,0.05$ and 0.06 , respectively.

Late Reflood Without GSCS: Analyses show that the late regime begins at about two hours into the accident when GSCS has failed. The late time regime is characterized by molten fuel in the inlet regions of the core. In these situations, computer analyses indicate that pressure tube temperatures will track the fuel temperature due to the intimate contact between the molten fuel and the pressure tube and, thus, be $-2000 \mathrm{~F}$. Research by Keith Scott indicated that the material properties of the pressure tubes degrade rapidly at high temperatures, and that at $2000 \mathrm{~F}$, the pressure tubes could fail due to yield at pressures as low as 300 psig. In addition, Marv Thurgood indicated that pressures of 1000 psig are achievable by water hammer phenomena. Thus, it was the consensus opinion of the panel that at least one pressure tube will almost certainly fail under these conditions.

Intermediate Reflood Without GSCS: The intermediate time regime is characterized by the absence of molten fuel from the inlet region of the pressure tubes. During the intermediate time regime, molten fuel will, however, almost certainly exist in other parts of the core. TRUMP analyses show that after the early phase the pressure tube temperature begins to follow the fuel temperature more closely than the graphite temperature. The melting point of uranium is more than $2000 \mathrm{~F}$, and computer analyses predict pressure tube temperatures of more than $1700 \mathrm{~F}$ during this time. Keith Scott indicated that the material properties of the pressure tubes degrade rapidly at temperatures abovo $-1300 \mathrm{~F}$, and that by $-1650 \mathrm{~F}$ the pressure tube would likely withstand only 1000 psig before failing due to yield. Thus, at the temperatures of interest during this time regime, the pressure tube would be expected to fail during any water hammer or major pressure spike event. It was the consensus opinion of the panel that at least one pressure tube will almost certainly fail under these conditions.

Late Reflood with Operating GSCS: The case to be elicited here can be described as follows: ECCS has completely failed for the first two hours of the accident, then starts when there is approximately 258 core damage. GSCS has functioned throughout the accident. Calculations [1] show that the maximum fuel temperatures are in the inlet region and are slightly higher than the melting point of uranium $(-2075 \mathrm{~F})$, and the maximum pressure tube temperatures are $-1850 \mathrm{~F}$. For this elicitation, any probabilicy less than 0.01 is effectively zero. 
The elevated temperatures associated with this scenario raised the possibility in the minds of some panel members that the uranium/zircaloy dissolution mode of pressure tube failure might be possible. No panel member placed the probability of failure by this mode at greater than 18 , and Gary Smith believed that the temperatures were still too low for this to be an effective pressure tube failure mechanism. As will be seen in the following paragraphs, this mode of failure will still not have a significant impact on the overall probability of pressure tube failure.

The probability of pressure tube failure due to a pre-existing defect was assessed by all panel members to be similar to or less than that elicited in the "early" reflood scenario above. The temperatures would be higher in this case than the former, and this would help to anneal away any defects which might cause failure, and other effects were assessed to dominate the failure probability. No panel member placed the probability of failure by this mode at greater than 18. This mode of failure will also not have a significant impact on the overall probability of pressure tube failure based on the discussion below.

Don Ogden assessed the the remaining fallure mechanisms as follows: Pressure tube failure due to MFCI was assessed to be more likely for this case than for the early reflood case elicited previously. This is due to the possible presence of of molten fuel in the region of the pressure tubes near the inlet which is without GSCS cooling. Two studies had been performed by outside organizations to determine whether or not MFCI was possible, and that the studies disagreed about the result. He stated that the conditions in this scenario are very good for MFCI if the phenomena were possible, so the probability that MFCI could cause pressure tube failure was assessed to be 0.50 . In addition, non-MFCI pressure spikes with a duration of several seconds are likely to occur and, since the high temperature will have weakened the pressure tubes by at least a factor of six ( $1900 \mathrm{~F}$ v.s. $1600 \mathrm{~F})$, failure is likely to occur [2]. He assessed the probability of failure due to yield to be 0.80 . His assessment of the probability of uranium/Zircaloy dissolution or a pre-existing defect causing failure was -0.01 based on the discussion in the preceding paragraphs.

The synthesis of the above probabilities into an overall failure probability is complicated by the fact that a simple addition of the probabilities gives a result that is greater than one. If, however, the various failure mechanisms are viewed as independent events of which none may occur to achieve the success, the synthesis can be viewed as

$$
P_{\text {Success }}=\left(1-P_{U / Z r}\right) \star\left(1-P_{\text {MFCI }}\right) \star\left(1-P_{Y_{1 \theta 1 d}}\right) *\left(1-P_{\text {Defect }}\right)
$$

where $P_{\text {Success }}$ is the probability that all pressure tubes will survive intact, and $P_{U / Z I}, P_{M F C I}, P_{Y 1 e l d}$, and $P_{\text {Defect }}$ are the probabilities that at least one pressure tube will fail due to uranium/Zircaloy dissolution, MFCI, yield and pre-existing defects, respectively. The synthesis of the above probabilities by this method gives $P_{\text {Success }}=0.098$. When this result was presented to Don Ogden, he stated that he believed that the actual Psuccess would be 0.01 . The ensuing discussion revealed that, in his 
judgement, there was a strong correlation between the MFCI and yield modes of pressure tube failure which would account for the very small $\mathrm{P}_{\text {Success. }}$.

John Long agreed with many of the sentiments voiced by Don Ogden, and added that a pressure pulse may originate from an MFCI or water hammer in one pressure tube and fail a different, higher temperature pressure tube by the yield mechanism. He preferred to assign a probability that ali pressure tubes would remain intact rather than a pressure tube failure probability, and this he assessed to be 0.01 . This position and probability assessment was shared by Marv Thurgood based on similar reasoning.

Gary Smith initially disagreed with Don Ogden's assessment of the failure probability. His disagreement was based on a belief that a small amount of molten fuel, tight geometries, and an oxldizing environment would minimize the possibility of MFCI by reducing the amount of mixing between fuel and coolant. In particular, he did not believe that a coolable geometry would exist due to the massive amounts of failed fuel which would be present. Don Ogden, however, contended that at least one outer channel of one fuel tube (there are 1003 fuel tubes) is likely to remain unblocked, and that this would provide a relatively easy path for water to traverse to get to molten fuel, and, based on John Long's arguments, pressure tube failure due to MFCI or water hammer-induced yield was very likely. After some discussion, Gary Smith became convinced that the other arguments were indeed valid and decided to join in the consensus opinion that $P_{\text {Success }}$ would not be greater than 0.01 .

After the elicitation, the panel looked at the overall results of all of the elicitations and noted that in all cases examined, pressure tube failure seems to be dominated by the pressure tube temperature. In particular, when the graphite temperature increases to the point where the pressure tube is not cooled sufficiently to continue resolidifying molten fuel, pressure tube failure during core reflood becomes significantly more probable. This typically occurs approximately one hour into an accident in which ECCS is lost. The process is relatively insensitive to most other factors because the graphite and pressure tube temperatures increase (with a corresponding degradation of the matorial properties) on a time scale which can not be effectively mitigated by the activity of the GSCS and other factors.

\section{Elicitation of Issue 7. Part 1b}

Issue: Determine the probability that at least one pressure tube will fail somewhere in the core when ECCS has failed initially such that a single ECCS High Lift Diesel Pump (HLDP) is operating. The reactor continues in this state indefinitely.

Discussion: It was the consensus of the panel that the presence or absence of GSCS in this scenario will not make a significant difference in the probability of pressure tube failure. 
Don Ogden summarized the available deterministic calculations as follows: For cases where ECCS flow is not sufficient to fully flood the core, a liquid level is established in the inlet riser which is sufficient to support the two-phase fluid in the outlet riser. The core refills slowly over the course of a few hours as the decay heat load decreases. Steam cooling is generally ineffective. There are significant differences between cases where the cold leg is and is not intact. Coolant can be forced through the remainder of the primary system and into the core through the CV-5 valves to quench the top of the core when the cold leg is intact, but the core is isolated from the rest of the primary when the cold leg is broken. The panel therefore decided to break the issue down into two cases: intact cold leg and broken cold leg.

Broken Cold Leg: A full description of the case to be elicited here is as follows: The primary system depressurizes through the $V-4$ valves and a break in the cold leg manifold. ECCS actuation is demanded, but two of the three ECCS HLDP's fail to run. The operating HLDP operates normally for the duration of the accident. Gary Smith indicated that system tests have shown that a single HLDP can deliver $-16000 \mathrm{gpm}$ to the core for emergency cooling, and that under these conditions, the expected core pressure would be < 50 psig. The cold leg break prevents flow to the core through the CV-5 valves. For this elicitation, probabilities must be less than 0.001 to be effectively zero.

It was the consensus of the panel that pressure tube failure due to uranium/Zircaloy dissolution is not possible for this scenario because the high temperatures ( $>2200 \mathrm{~F}$ ) and relatively long time frames (a few hours) required for this mechanism to occur will not be present in this seenario.

Gary Smith discussed the possibility of MFCI and stated that as the liquid level rises in the core, there will be a transition region between the cooled and uncooled regions of the core in which any molten fuel would freeze. The energy would be transferred from this region (which would be only a few rows of tubes high) to the cooled region by conduction cooling through the graphite stack and steam cooling as the bits of liquid begin to penetrate the outer ends of the tubes. For this reason he believed that MFCI is not credible in this situation. He also believed that the pressure tubes in a region which is just starting to be cooled (and, hence, is receiving the greatest pressure pulses) would be cool and, therefore, in their region of good material strength.

Marv Thurgood stated that he had done some research into pressure tube integrity issues related to water hammer and mechanical stresses. His analyses showed that water hammer would be most likely to occur in cases where the ECCS flow to the core was degraded, including cases such as this one. His analyses were conducted for non-LOCA station blackout conditions, which cause higher primary system pressures ( -250 psig), and the analyses showed that the ASME code could be met for all computed conditions. The greatest risk of failure came from the pressure tube connectors, which could fail after -100 water hammer cycles due to fatigue. However, he believed that the lower system pressure for the current case will allow the connector stresses to remain within the elastic limit of the connector material. This would make yield failure of the pressure tubes very 
unlikely. He agreed with Gary Smith's assessment that MFCI will not occur, and assessed the probability that any pressure tube would fail due to the yield mechanism to be 0.002 based primarily on the uncertainties involved in the analysis. He believed that a pressure tube could fail due a preexisting defect with about the same probability due to pressure spikes, and assessed the probability that any pressure tube could fail by this mechanism to be 0.002 . Thus, he assessed that the overall probability that a pressure tube would fail under these conditions to be 0.004 .

John Long assessed that steam cooling would help keep the pressure tube temperatures below the point where declining material strength became important. He believed that thermal and mechanical cycling on pre-existing defects would be the dominant cause of pressure tube failure, and that uranium/Zircaloy dissolution, MFCI and yield would not contribute to the failure frequency. He assessed that the probability that all pressure tubes would survive an accident of this type to be greater than 0.99 , and declined to be more specific. This number is chiefly due to uncertainty about the effects of thermal cycling. Don Ogden and Gary Smith were involved in the discussion leading up to John Long's assessment and concurred with the reasons and probabilities developed in that assessment.

When the panel was asked to arrive at an uncertainty distribution, it was the consensus of the panel that the failure probability of 0.01 was a very conservative estimate and should be used as an upper bound. The conservatism in the estimate is based on the fact that conservative assumptions were used in the deterministic code calculations for developing the station blackout core conditions, and even with these conservatisms, the ASME codes (which have much conservatism built in) were met. A uniform distribution between 0.00 and 0.01 was selected as best describing the current state of knowledge because it provides the maximum amount of uncertainty.

Intact Cold Leg: A full description of the case to be elicited here is as follows: The primary system depressurizes through the $V-4$ valves due to a demand for ECCS actuation. Two of the three ECCS HLDP's fail to run. The operaling HLDP opcrates normally for the duration of the accident. For this elicitation, probabilities must be less than 0.001 to be effectively zero.

Don Ogden summarized the deterministic calculations which have been modeled situations similar to this one. A concern was that if some water were to enter the core region through the CV-5 valves, it would enter at the top of the core and could encounter hot fuel. This could raise the probability of MFCI and yield-based failures significantly. The only way for this to occur is if the $V-4$ valves are not able to accommodate a flow of 16,000 $\mathrm{gpm}$ between the core and the dump tank. This would cause some of the ECCS coolant to pass out of the core through the CV-3 valves, reflood the primary loop and force coolant back into the core through the CV-5 valves.

Calculations [3] show that the V-4 valves are able to accommodate a flow of $16,000 \mathrm{gpm}$. If some of the ECCS coolant should escape through the 
CV-3 valves, Gary Smith indicated that it is doubtful that the reduced ECCS head of a single HLDP is sufficient to push the water through the primary system loop with only one ECCS pump operating. In addition, the degraded coolant flow would cause a very long fill time for the remainder of the primary system. By the time any such refill would occur, it would almost certainly be after the core itself has been refilled (this should occur in less than one hour). Therefore, the MFCI issue is no more important in this case than the previous.

One final matter of concern was the primary system pressure. Since this case does not involve a LOCA, the core pressure should be somewhat higher than that expected in the cold leg break case. The expected system pressure in this case is $<70$ psig (versus $<50$ psig above). This pressure increase is not sufficient to place the pressure tube material in danger of exceeding the elastic limit. Thus, the yield mode of failure should be similar in probability for this case to the case above.

Based on these discussions, it was the consensus of the panel that the probability of pressure tube failure for this case is not significantly different from the cold leg break case described above, and the probability distribution generated above describes this case as well.

\section{Elicitation of Issue 7. Part 1c}

Issue: Determine the probability that at least one pressure tube will fail somewhere in the core when ECCS has succeeded initially except that one CV-2R has failed to open. Coolant flow is available to the blocked riser only by backflow. The reactor continues in this state indefinitely.

Discussion: Don Ogden discussed the available deterministic calculations as follows: TRAC and RELAP calculations both predict that reverse flow through the flush line can not penetrate the core because the liquid level supported in the outlet riser of the affected region of the core is below the level of the bottom pressure tube. The CV-5 and the blocked CV-2R valves cause the affected region of the core to act as a pressurizer to prevent inflow. The hotter the affected region gets, the tougher it becomes to achieve reverse flow. For cold leg break sequences, there is no other way to get coolant to the affected region of the core, but when the cold leg is intact, ECCS and HPI pumping will cause the primary system to refill and push water onto the core through the $C V-5$ valves.

It was the consensus of the panel that the presence or absence of GSCS in this scenario would not make a significant difference in the probability of pressure tube failure. The panel decided to break the issue down into two cases: intact cold leg and broken cold leg.

Broken Cold Leg: Since no water can get to the affected region and pressure tube temperatures are not expected to climb to the point where the uranium/Zircaloy dissolution mechanism would cause pressure tube failure, the panel assessed by consensus that the pressure tubes will remain intact based on the lack of a failure mechanism. 
Intact Cold Leg: Don Ogden stated that there is a higher degree of uncertainty for this scenario because it is not known if or when water will come into the core through the CV-5 valves. Under the worst circumstances, he believed that conditions in the core would not be as conducive to pressure tube failure as the intermediate subcase of Issue 7 Case $1 f$, but could be worse than the early subcase. The issue comes down to when water gets into the failed riser.

A full description of the case to be elicited here is as follows: ECCS has succeeded initially ( 3 HLDP's are running) except that one CV-2R has failed to open. AC power is available and there has not been a large LOCA.

Don Ogden stated that for these conditions, it takes about 600 seconds to blow down the primary system, and about 800 seconds after that to heat the fuel in the affected riser to the point of failure. With 3 HLDP's running, it is possible that the primary system will be refilled with ECCS water and begin to open the CV-5 valves and quench the affected riser before any fuel damage occurs. In any case, the worst expected pressure tube temperatures would occur if fuel were to fail, and these would be $-1500 \mathrm{~F}$.

In the general discussion that followed, John Long asserted that the low temperatures precluded uranium/Zircaloy dissolution and MFCI as pressure tube failure mechanisms. He also believed that the low temperatures for this scenario were far more certain than those of the early subcase of Case $1 f$ and that the expected flooding rate is significantly lower than that case. Thus, the expected pressure spikes would be lower. For this reason, he expected pressure tube failure due to pre-existing defects to be the dominant mode of failure, but that yield failure was also possible. He assessed the probability of pressure tube failure due to pre-existing defects to be bounded by 0.01 , and yield to also be bounded by 0.01 . Additional discussion lead to the consensus assessment of the panel that the total probability of pressure tube failure for this scenario would be at most 0.02 , and that a uniform probability distribution between 0.00 and 0.02 would be representative of the state of kuvwledge becausc it contains the maximum uncertainty. It was once again noted that uranium/Zircaloy dissolution and MFCI are not considered credible in this scenario.

\section{Elicitation of Issue 7, Case 1d}

Issue: Determine the probability that at least one pressure tube will fail somewhere in the core when ECCS has succeeded initially except that one $\mathrm{CV}-2 \mathrm{R}$ has failed to open. High Pressure Injection (HPI) is supplying coolant to the affected riser. The reactor continues in this state indefinitely.

Discussion: General discussion of this issue by the panel rapidly reached the consensus that treating this issue as identical to Case $1 \mathrm{c}$ above is conservative but not unreasonable. The reasoning behind this statement is that the HPI flow will refill the primary system while the ECCS refills the 
core. This would refill the inlet risers approximately half the time required by three HLDP's alone. This would mean that core temperatures would be lower when coolant reached the fuel in the affected riser than in Case $1 c$, so treating this case as Case $1 c$ is conservative. At the same time, there would be some time for core heatup and Case lc was assessed to have a low probability of pressure tube failure which was dominated by preexisting defects. Thus, the probability of pressure tube failure could not be significantly reduced, and the conservatism of treating Case $1 \mathrm{~d}$ as Case $1 c$ is not unreasonable.

\section{Elicitation of Issue 7. Case le}

Issue: Determine the probability that at least one pressure tube will fail somewhere in the core when ECCS has succeeded initially except that one $C V-2 R$ has failed to open. After an undetermined amount of time, the CV-2R valve reopens to flood the affected riser with water.

Discussion: Cases $1 \mathrm{c}$ and $1 \mathrm{~d}$ discussed the possibility that the core would be reflooded by flow through the CV-5 valves in cases where there has been no large LOCA. Hence, the panel decided to break this case down into two subcases: intact primary system and large LOCA.

Large LOCA: If a large LOCA has occurred (especially in the cold leg), there is no possibility of reflooding the loop and establishing flow through the CV-5 valves. The immediate consensus of the panel was that reopening the $\mathrm{CV}-2 \mathrm{R}$ valve under these circumstances would have a probability of pressure tube failure which is similar to Case $1 f$. The panel noted that the consequences of this event, however, would be much different because this case involves only 64 tubes versus 1003 in Case if.

Intact Primary System: It was proposed to the panel that the possibility of flow through the CV-5 valves would make this case similar to Case lc above. The panel discussed this option as follows: The operation of the HLDP's refloods the primary system and quenches the affected region of the core in a relatively short time. This makes Case le similar to Case lc. However, the opening of a CV-2R valve would cause a much higher flow into the affected riser. This would cause higher pressure spikes and increase the probability of pressure tube failure. The "worst case" would occur if a CV-2R were to open just before flow came in through the CV-5 valves because the core would be at its highest possible temperature, then hit with a large amount of water. The temperatures would be similar to Case 1c, so uranium/Zircaloy dissolution and MFCI are not assessed to be credible pressure tube failure mechanisms for this scenario.

It was the consensus assessment of the panel that pressure tube failure due to yield and pre-existing defects would each be more likely here than in Case $1 c$ by a factor of approximately two. Additional discussion lead to the consensus assessment of the panel that the total probability of pressure tube failure for this scenario would be at most 0.04 , and that a uniform probability distribution between 0.00 and 0.04 would be representative of the state of knowledge because it contains the maximum uncertainty. 


\section{Elicitation of Issue 7 . Case 1a}

Issue: Determine the probability that at least one pressure tube will fail somewhere in the core when ECCS has failed (totally) initially and the only coolant being supplied to the core is from two HPI pumps (3000-4000 gpm). The reactor continues in this state indefinitely.

Discussion: It was noted by the panel that HPI water is injected into the primary coolant system just upstream of the main coolant pumps and would be unlikely to be able to enter the core if a large LOCA were to occur anywhere between the $\mathrm{CV}-3$ valves on the outlet side of the core and the CV-5 valves on the inlet side. Since this comprises almost all of the large break frequency, it was assessed by the panel that the case described here could not occur in a large LOCA situation.

Another possible scenario which could produce a situation of this type would be a high pressure boiloff scenario. In this scenario the reactor remains at high pressure either because of a failure of all V-4 valves to open or because the ECCS trip was bypassed and ECCS was not started manually. If this situation were to continue and primary/secondary heat transfer were not possible, eventually the primary coolant would boil off and expose the core for heatup and eventual core damage. This would take a long time (hours) if the primary coolant pumps were left running because the entire primary system would have to be brought to saturation before the core can dry out. When the primary coolant pumps are shut off, however, the core begins to act as a pressurizer to keep coolant out of the core as in Case 1c above (natural coolant circulation is not possible in $N$ Reactor). The decay heat would boil the water out of the core and dryout would occur more quickly. High Pressure Injection will delay but not prevent these effects. In either case, it was assessed by the panel that as long as the primary system remains at high pressure (> $1500 \mathrm{psig}$ ), the time to between the uncovery of the top row of pressure tubes to pressure tube failure will be short compared to the time required to boll off the required amount of coolant. The high pressures involved are likely to cause failure of the pressure tuhes by the yield mechanism based on the reduced strength of the zircaloy pressure tube material at very high temperatures. In addition, actuation of ECCS at this time would be similar to Case 1f, which was discussed earlier. Finally, if this condition contlnues indefinitely, uranium/Zircaloy dissolution will come into play, although this takes much time at very high temperatures. The consensus of the panel was that the pressure tubes would almost certainly fail in such a situation, and that early yield failure would be the dominant mechanism because of the high pressures and temperatures involved.

Another situation in which a flow of this magnitude might occur would be a low pressure scenario in which either the V-4 valves had opened or a pipe break had occurred in a location which would depressurize the system but not affect HPI flow to the core. In this situation, it would take about four hours for the HPI flow to reflood the core because the cold leg of the primary system has to be refilled and much sensible heat has to be removed from the core before the liquid can effectively penetrate the core 
(if it can penetrate at all due to failed fuel). MFCI was assessed to be unlikely to occur due to the low flow rates involved, but yield failure was assessed to be almost certain after approximately three hours regardless of whether or not GSCS functioned. The yield failures would occur due to water hammer in the inlet regions of the core which are not cooled by the GSCS. If the pressure tubes do not fail due to yield, uranium/Zircaloy dissolution will fail the tubes in the case where GSCS has failed, but not when GSCS functions.

\section{Elicitation of Issue 7. Case 1, Other Miscellaneous Topics}

Issue: Determine the probability that at least one pressure tube will fail somewhere in the core when the primary system has depressurized and ECCS fails and remains failed indefinitely. No other coolant is available to the primary system.

Discussion: It was the immediate consensus assessment of the panel that if GSCS has failed, then pressure tube failure would almost certainly occur in the 2-3 hour time frame based on the uranium/Zircaloy dissolution mechanism. Yield fallures are not assessed to be important since the primary system is at low pressure and no coolant is available to cause a water hammer or pressure spike. This is the "adiabatic heatup" scenario.

If GSCS succeeds in the above case, then pressure tube failure would take many hours to occur and, if it were to occur, it would be based on the uranium/Zircaloy dissolution mechanism in the end regions of the core that are not reached by the GSCS. Yield failures are- not assessed to be important since the primary system is at low pressure and no coolant is available to cause a water hammer or pressure spike. The temperatures which are required for the uranium/Zircaloy dissolution mechanism to occur are only achievable in the inlet region of the core which is not reached by the GSCS, although computations have shown that this type of failure is very unlikely (this is the classic "design basis accident" case).

Issue: Determine the probability that at least one pressure tube will fail somewhere in the core when the ECCS barely fails to meet its success criteria with the failure of two V-3 valves on the same side of the core to open and the failure of one ECCS HLDP to start or run.

Discussion: It was proposed to the panel that this situation be considered using the probability distribution of Case 1b. The panel believed that this treatment would be vastly conservative since cooling is provided to both sides of the core and the ECCS barely missed its success criteria. It was the consensus assessment of the panel that there was almost no possibility of pressure tube failure under these conditions based on the analyses done by Marv Thurgood. 
Issue: Determine the probability that at least one pressure tube will fail somewhere in the core when the ECCS barely fails to meet its success criteria with the failure of all four V-4 valves on the same side of the core to open.

Discussion: The ECCS success criteria for the V-4 valves is that one V-4 valve must open on each side of the reactor for success. In the event of a common mode or other failure of all four $\mathrm{V}-4$ valves on the same side of the core to open, the initial depressurization of the primary system is affected very little because the affected side of the core can depressurize through the rest of the primary system. In addition, there is a flush line which connects the bottom of all 16 risers together through which depressurization and ECCS coolant flow could be accomplished. The minimum section of the flush line is 6" diameter, while the throat diameter for a

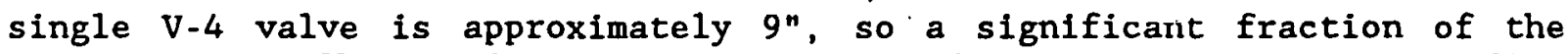
required ECCS flow could exit the core in that manner. Additional flow would occur from the affected side of the core through, the primary system, and exit the system by flowing through the unaffected side of the core. This is a significant amount of cooling which, while possibly not enough to prevent core damage, will keep core temperatures down in the affected region. For this reason, it was the consensus assessment of the panel that there is almost zero probability of pressure tube failure in such a scenario.

\section{Elicitation of Issue 7, Case 2}

Issue: Discuss the expected effects of pressure tube rupture at low $(<300$ psig) primary system pressures when ECCS flow has been established and a pressure tube fails before major core damage has occurred, and contrast this to the case where pressure tubes fail because ECCS is actuated after major core damage has occurred.

Discussion: The following discussion contains the thoughts of Gary Smith, Don Ogden, Marv Thurgood and John Long. It represents a consensus of the panel as no objections were raised to the points as they were made.

The research that has been conducted into the effects of pressure tube failure have concentrated on situations where a pressure tube would rupture under normal operating conditions with the reactor at power. These analyses have shown that a postulated double-ended guillotine break from operating conditions would release $-1500 \mathrm{gpm}$ into the graphite stack as the system depressurized. The breaking of a few GSCS tubes can not be discounted, but disruption of the action of enough control rods to cause an ATWS event can not occur. Also, the forces involved in a rupture event are not great enough to move a control rod out of position $[4,5]$. Another postulated event with possible reactivity implications involves filling the areas between the graphite blocks with water to make up for the moderation lost by draining the core. This is not possible because the channels through the graphite carry the water away through the rupture disk in the helium blanket gas system. This will remove the water from the graphite stark to the confinement and preclude a buildup of water on the outside of the pressure tubes. 
The amount of coolant that would be released from the primary system once it has depressurized to the normal ECCS operating pressure is reduced from the $-1500 \mathrm{gpm}$ discussed above to about $350 \mathrm{gpm}$. Since the normal ECCS flow through a riser is $-1500 \mathrm{gpm}$, it is possible that about 208 of the ECCS coolant for a given riser would be diverted through the break. This is not expected to threaten the core with fuel damage.

The reaction between steam and graphite (carbon) is an endothermic reaction which produces flammable gases by the reaction

$$
\mathrm{H}_{2} \mathrm{O}+\mathrm{C} \rightarrow \mathrm{H}_{2}+\mathrm{CO} \text {. }
$$

At high graphite temperatures this reaction can produce significant amounts of flammable gases and release them to the confinement $[6,7]$. Previous studies, however, have found the Zircaloy and uranium metal-water reactions to dominate the carbon reaction for accidents at $\mathrm{N}$ Reactor.

The possibility of one broken pressure tube propagating to involve a larger section of the core has been raised by a number of parties. This has been postulated to occur because the stresses from one pressure tube rupture event could augment the residual stresses in another pressure tube and cause that tube to fail as well. Studies by Keith Scott indicated that residual stresses were not found to contribute significantly to the postulated pressure tube failure scenarios. The effects of residual stresses were factored into the generation of the above elicitations.

It was assessed that it would take a very energetic event to cause the failure of multiple pressure tubes. The only type of event that would fit such a description is the MFCI event. The large amounts of open space inside the graphite stack and the wide spacing between pressure tubes makes such propagation possible but unlikely.

The most significant difference between early pressure tube failures and those which occur due to a delay in the actuation of the ECCS is that early pressure tube fallures are expected to result in only a single or at most a few pressure tubes failing, while delayed ECCS actuation is likely to fail many pressure tubes. Early pressure tube failures are expected to fail only a few tubes because the events which cause early fallures occur at lower temperatures and are less energetic in nature. The effects of such events would be dissipated in the open spaces within the graphite stack. In addition, the lower temperatures would mean that other pressure tubes are not yet weakened due to thermal effects and would be able to withstand the pressure spikes generated within the primary system itself without failing. This is a significant result because the ECCS can make up for the coolant diversion when a few pressure tubes are involve and continue to cool the remainder of the core. 
Issue: Discuss the types of phenomena which could cause GSCS tubes to fail during an accident sequence.

Discussion: While the comments below are attributed to specific people, the represent a consensus opinion of the panel as no objections were raised to the points as they were made.

Gary Smith noted that some have questioned the displacement of the GSCS tubes that would occur as the temperature of the graphite stack was raised from normal operating temperatures to an accident temperature such as $-1500 \mathrm{~F}$. The calculated maximum GSCS tube displacement for such a sequence, however, is only 1.5 inches. This should not challenge the tubes because they are quite flexible and heating enhances the flexibility. In addition, the GSCS pipe supports are seismically qualified, so the GSCS tubes are well-supported where they enter and exit the core. IrradiationInduced growth of the graphite stack is also assessed to pose no challenge to the tubes for reasons similar to the above and also due to the monitoring of that growth and its effects.

John Long assessed that there is a significant possibility that under certain circumstances GSCS tubes could crack or fracture inside the core. In cases where bulk boiling would occur in the GSCS or where the GSCS tubes are allowed to heat up significantly before being hit with cold water, failure may occur. The bulk boiling scenario could occur in a station blackout scenario because it takes 20 seconds to achieve a dump of the GSCS, and, without coolant circulation, bulk boiling would be expected to occur by that time. Previous GSCS tube failures have all been leaks and have not failed the rupture disk in the helium cover gas system, but in a GSCS tube rupture event, the blowdown from the GSCS would be expected to break the rupture disk (based on flashing of coolant), but flow through the core should continue in the remaining intact tubes, and some cooling would be expected as the GSCS coolant is released into the graphite stack. The latter cooling would come chiefly from the vaporization of that coolant. It should be noted that the GSCS is a low pressure system, so GSCS tube failure will not cause significant damage to the graphite stack structure. It should be further noted that GSCS tubes run through specially bored channels in llie graphite (much like thnse for the pressure tubes), and this graphite "shel1" should restrict the amount of coolant which would egress from a failed tube into the graphite stack.

Don Ogden added that an accident in which GSCS is not operating heats up the graphite rapidly enough that within one hour the restoration of the GSCS function will be impossible. This occurs because the when GSCS water hits hot GSCS tubes, 1t would flash and create a large backpressure which would prevent the GSCS water from penetrating the core. Only as the GSCS tube is cooled can the quench front move down the tube and into the core. Even if the GSCS tube remains intact, it could take several hours for the coolant to completely penetrate the core and establish a liquid flow. 


\section{C.7.4 Application of Results}

A few of the probability distributions in the above analyses were not implemented in the Latin Hypercube (LHS) analysis for pressure tube failure because they were not applicable to the time frame of interest in the APET analysis. All others are implemented in the analysis. The implementation details for LHS can be found in the appendices to SAND89-2102, the final $\mathrm{N}$ Reactor Risk Analysis Report. 


\section{8 GRAPHITE OXIDATION}

\section{C.8.1 Issue Definition}

Unmitigated accidents present the possibility that the graphite moderator stack could begin to oxidize or even burn under certain circumstances. This issue has taken on an added political importance due to the Soviet reactor accident at Chernobyl.

A number of scenarios for the onset of graphite oxidation have been dismissed based on physical considerations. A hydrogen detonation in the core is dismissed based on the very small volumes available for the burn. A hydrogen detonation in the pipe barrier space has been dismissed based on the presence of panels which would act as blowout panels to direct the detonation energy away from the well-protected core. No other credible internal events scenarios have been found which would lead to graphite oxidation based on a serious core disruption.

Probability distributions are needed to predict these possibilities for both the early and late phases of the accident for each primary system condition considered. The early phase of the accident is defined to be the first two hours following the reactor trip. The late phase begins at that time and continues until 24 hours after the reactor trip.

Primary system conditions to be considered:

1) ECCS and GSCS have both completely failed.

2) ECCS has completely failed and a pressure tube has failed. Thus, although fluid is running through the GSCS tubes, the heat transfer coefficient from the graphite to the GSCS is degraded by a factor of approximately 5 .

3) A seismic multiple pressure tube rupture (MPTR) event has occurred.

4) Any other situations which might cause graphite oxidation to begin.

\section{C.8.2 Issue Resolution Process}

The above stated issue was presented to S. E. Dingman, G. D. Wyss, and L. A. Miller of Sandia National Laboratories, and C. J. Shaffer of Science and Enginccring Associates (under contract to Sandia National Laboratories) on August 28, 1989. At that time other issues were also presented, and the elicitees felt that it. would be most profitable for each elicitee to write up background information about one issue for review at a final elicitation. meeting. The following represents the compilation of background information for the Graphite oxidation issue as compiled by G. D. Wyss with additions and modifications based on oral and written comments from the other elicitees. 


\section{C.8.3 Issue Analysis and Resolution}

\section{Background Information}

1. Long, J. T., "Review of $N$ Reactor Level 2/3 Probabilistic Risk Assessment Point Estimate of Risk," memo to E. J. Krejci, Westinghouse Hanford, March 1989

2. Lambright, J. A., et al, Analysis of Core Damage Frequency due to External Events at the DOE N-Reactor, SAND89-1147, Sandia National Laboratories, 1990

3. F. J. Heard, et al., N Reactor Safety Enhancement Final Report: Hydrogen Generation and Thermal Analysis of the Hydrogen Mitigation Design Basis Accident, WHC-SP-0096, Westinghouse Hanford, December 1987.

4. L. A. Miller, et al., N Reactor Probabilistic Risk Assessment Supporting Calculations, SAND89-2101, Sandia National Laboratories, 1990.

5. Richards, M. B., et al, "A Computational Model For Graphite Oxidation Under Nuclear Reactor Accident Conditions," presented at the 24th National Heat Transfer Conference in Pittsburgh, August 9-11, 1987, AIChE Symposium Series no. 257, American Institute of Chemical Engineers, 1987

\section{Conditions Required for Oxidation}

Two graphite reactions are possible within the $N$ Reactor graphite stack. The first involves the "coal gas" reaction between the graphite (carbon) and water. This reaction is endothermic and takes the form

$$
\mathrm{C}+\mathrm{H}_{2} \mathrm{O} \rightarrow \mathrm{CO}+\mathrm{H}_{2}
$$

While this reaction produces flammable gases, it is endothermic and, thus, can not be self sustaining. For this reason it is not considered further in this analysis, which is intended to focus on the possibility of graphite combustion events.

The second reaction is the exothermic combustion reaction between graphite and oxygen from the air. It takes the form

$$
\mathrm{C}+\mathrm{O}_{2} \rightarrow \mathrm{CO}_{2}
$$

The reaction can be self-sustaining at high temperatures and has been a point of public concern since the Soviet reactor accident at Chernobyl. In order for this reaction to occur, two conditions must occur: oxygen must be able to reach the graphite, and the graphite must reach a suitably high temperature for the reaction to become self-sustaining. If either of these conditions are not met, a self-sustaining reaction can not occur. 
Several scenarios have been proposed which might allow these hot, oxygen rich conditions to occur. These include core disruption due to hydrogen ignition in either the the pipe barrier space or the core itself, seismic core disruption, and core heat-up due to a long-term loss of core cooling. Each of these is discussed below.

Hydrogen is generated in many severe reactor accident scenarios when water interacts with and oxidizes metal fuel and clad structures. This produces a metal oxide and liberates the hydrogen from the water into the local atmosphere. In some cases, hydrogen can escape into the graphite stack through a pressure tube failure point. If this hydrogen can be ignited, it has been proposed that a detonation might disrupt the graphite core structure enough to render the core uncoolable and open an air ingress path to the core This would present favorable conditions for graphite combustion.

Hydrogen events inside the graphite stack, however, appear on the surface to be all but impossible. The graphite stack is normally kept inerted with a blanket of helium, and a pressure tube rupture event will fill the stack with steam. Both of these tend to render the atmosphere unable to support hydrogen ignition. In addition, at the temperatures expected to exist within the graphite during accident scenarios, hydrogen tends to consume any present oxygen before a deflagrable or detonable mixture can be obtained. Air ingress into the core would only occur through the rupture disk in the cover gas system, so both hydrogen and graphite oxidation events would be severely limited by a lack of oxygen. Finally, if a hydrogen ignition event were to occur, the small gas volumes and large graphite mass inside the core would prevent significant core damage from occurring, and the massive biological and thermal shields surrounding the core would prevent the opening of an additional air ingress path. The combination of all of these factors means that graphite combustion due to hydrogen combustion events inside the core is not credible.

Hydrogen combustion events in the pipe barrier spaces around the reactor can not be ruled out as oasily as those in the core. This is because air ingress is far more difficult to limit, structure temperatures are lower, and steam and helium inerting are not as certain. While hydrogen combustion events are certainly possible in the barrier spaces, other factors are available to prevent these events from damaging the core. The barrier space consists of the area between the concrete biological shield wall towards the reactor core and a sheet metal enclosure away from the core. Further, blowout panels are provided at the top and bottom of the sheet metal enclosure [1]. This geometry would direct the energy from any hydrogen event away from the core and preclude such an event from damaging the core or providing a means for significant air ingress to the core. Hence, graphite combustion due to hydrogen combustion events in the pipe barrier space is not considered credible due to the lack of an oxygen source. Similar logic dictates that a fire in a plant location other than the core can not by itself cause graphite combustion because the concrete and steel shield walls would have to be hreached to provide access for combustion air to the core. This does not, however, rule out graphite 
oxidation due to a fire-induced loss of core cooling as will be discussed later.

Seismic core disruption is a scenario in which a seismic event causes the failure of the core itself or those structures directly around it such as the shield wall. Such a failure could leave the core uncooled as pressure tubes and GSCS pipes would be likely to fail in such a scenario [2]. Also, the formation of a significant ingress path for air to feed the combustion process can not be discounted. An examination of this situation indicates that the lack of cooling will produce temperatures which would be high enough to support oxidation, and the exothermic nature of the reaction could give rise to a self-sustaining reaction. The nitrogen inert function of the hydrogen mitigation system could help to prevent oxidation by limiting the amount of oxygen available, but, since this event would be expected to occur only at during the most severe (and infrequent) earthquakes, there may be some doubt as to whether or not both the hydrogen mitigation system and the confinement buildings will remain intact. If one or the other fails, the conditions for graphite oxidation would be quite good barring heroic actions. If both remain intact, some mitigation may occur to delay if not prevent the onset of graphite oxidation.

A much more difficult accident sequence to consider from a graphite oxidation perspective is one where, for whatever reasons, the ECCS function has failed and is not recovered during the accident sequence. For scenarios where the GSCS remains fully functional, core analyses [3] indicate that peak graphite temperatures throughout the core are below $1600 \mathrm{~K}$, and average graphite temperatures remain below $1200 \mathrm{~K}$. When GSCS is operating without the benefit of the superior thermal conductivity of the heliun cover gas blanket, core analyses [4] indicate that peak graphite temperatures throughout the core are certainly below $1800 \mathrm{~K}$ and likely to be below $1700 \mathrm{~K}$, while the average graphite temperatures remain below $1450 \mathrm{~K}$ and are likely to remain below $1300 \mathrm{~K}$. It should be noted that the average temperatures cited here are the average in the active fuel region only because the graphite reflector temperatures were not considered in the averaging process. The average temperatures for the entire graphite stack will be considerably lower because the reflector region is the last region of the graphite stack to heat up and it has a mass several times that of the graphite in the active core region. Self-sustaining graphite oxidation reactions are not expected at temperatures of $-1050 \mathrm{~K}$ [5] for the particular geometry of $\mathrm{N}$ Reactor. At higher average graphite temperatures ( $-1750 \mathrm{~K})$, the process is dominated by the amount of air available for the combustion process. In general, the analysis indicated that if the air supply is limited to about $3 \mathrm{~kg} / \mathrm{sec}$ core wide, oxidation would be slow.

Consideration of the above discussion yields the following conclusions: If the thermal and biological shields remain intact, there are only two mechanisms by which air can come into contact with the heated graphite. These are through the helium cover gas system rupture disk and through pressure tube failures. In the case where the GSCS continues to operate at normal efficiency, neither of these events have happened by definition. Barring a seismic event, no failure mechanisms have been found for the thermal and biological shields. In addition, the peak temperatures described above are being reduced over time by the GSCS flow. Thus, due to 
the lack of an available air supply, the possibility of self-sustaining graphite oxidation in this scenario is extremely remote.

When the GSCS is operating without the benefit of the superior thermal conductivity of the helium cover gas blanket, the result is much more difficult to predict. In such a case, either the cover gas rupture disk, or one or more pressure tubes, or both have failed. The area presented by the rupture disk for air ingress is small. Also in order to get air into the core through a pressure tube, it would have to be broken both inside the core and outside the core. This seems a remote possibility given that the pressure tubes remain well below their melting point, and no failure mechanisms have been found for the thermal and biological shields. In addition, the peak temperatures described above are being reduced over time by the GSCS flow. Finally, the highest temperatures are found in the center of the core while the rupture disk is located outside the edge of the graphite moderator. This means that the relatively cool reflector graphite is likely to consume most of the air that enters the graphite stack. This indicates that graphite oxidation is possible but by no means certain in this scenario due to the limited air supply.

A final case is where ECCS and GSCS have both failed and are not recovered during the accident sequence. This case is similar to the seismic core disruption scenario except that there is not an obvious mechanism for thermal and biological shield wall failure. In this scenario, the core is completely without cooling, and pressure tubes and GSCS pipes are likely to fail due to high temperatures. The cover gas rupture disk may also fail when other. tubes fail. While no failure mechanisms have been found for the thermal and biological shield walls, the extremely high graphite temperatures expected during such an "adiabatic heatup" scenario ( $>2200 \mathrm{~K}$ ) make it very difficult to discount some sort of a failure which would allow the formation of a significant ingress path for air to feed the combustion process. The nitrogen inert function of the hydrogen mitigation system could help to prevent oxidation by limiting the amount of oxygen available. In addition, the shear mass of the shield walls is likely to delay their failure and, hence, the onset of graphite oxidation. Such a delay could be in the tens of hours and could provide time for sune sort of preventative or mitigative measures to be taken by offsite personnel. In general, however, it would be very difficult to defend a conclusion that self-sustaining graphite oxidation could be precluded in this situation.

For the seismic core disruption (MPTR), the elicitees agreed that it would not be possible to support a conclusion that graphite oxidation does not occur because of the large area available for gas transfer through the failed pressure tubes and the complete lack of core cooling. Thus, a probabilicy of 1.0 is assigned to graphite oxidation for the MPIR accident. Given that oxidation does occur, the following probability distributions were elicited to determine whether the oxidation would be slow oxidation or a graphite fire (Miller and Wyss agreed on a consensus distribution). 


\begin{tabular}{|c|c|c|c|c|}
\hline \multirow[b]{2}{*}{ Quantile } & \multicolumn{4}{|c|}{ Probability of Rapid Graphite Oxidation } \\
\hline & Shaffer & Dingman & Miller. & Wyss \\
\hline 0.0 & 0.1 & 0.5 & 0.1 & \\
\hline .25 & 0.2 & & 0.2 & \\
\hline .50 & 0.3 & 0.75 & 0.3 & \\
\hline .75 & 0.5 & & 0.5 & \\
\hline 1.0 & 1.0 & 1.0 & 1.0 & \\
\hline
\end{tabular}

For non-MPTR events where both ECCS and GSCS fail, the elicitees agreed that the supply of oxygen to the graphite stack as well as the area available for gas transfer between the graphite stack and the remainder of the confinement building is severely limited. This would reduce the availability of oxygen for graphite combustion. The elicitees agreed that whatever oxygen is available will be consumed in some sort of an oxidation process, but that the probability that enough oxygen would be able to get into the core to maintain a self-sustaining oxidation reaction would be small. It was agreed that any oxidation that does occur would be slow because of the limited supply of oxygen available. The following probability distribution was developed by consensus to describe slow oxidation for accidents where ECCS and GSCS both fail:

$\begin{array}{cc}\text { Quantile } & \begin{array}{c}\text { Oxidation } \\ \text { Probability }\end{array} \\ 0.0 & 0.0 \\ 1.0 & 0.2\end{array}$

The elicitees indicated that the probability distributions should be correlated.

\section{C.8.4 Application of Results}

The oxidation distributions for the MPTR case are different between the elicitees and, thus, must be aggregated to be used. These distributions all consist of series of uniform distributions, so they can be aggregated by considering the probability density in each decile interval as follows:

\begin{tabular}{cccc} 
& \multicolumn{3}{c}{ Probability Density } \\
\cline { 2 - 3 } Decile & Shaffer & Dingman & Wyss \\
$0.0-0.1$ & 0.0 & 0.0 & 0.0 \\
$0.1-0.2$ & 0.25 & 0.0 & 0.25 \\
$0.2-0.3$ & 0.125 & 0.0 & 0.125 \\
$0.3-0.4$ & 0.125 & 0.0 & 0.125 \\
$0.4-0.5$ & 0.125 & 0.0 & 0.125 \\
$0.5-0.6$ & 0.05 & 0.20 & 0.05 \\
$0.6-0.7$ & 0.05 & 0.20 & 0.05 \\
$0.7-0.8$ & 0.05 & 0.20 & 0.05 \\
$0.8-0.9$ & 0.05 & 0.20 & 0.05 \\
$0.9-1.0$ & 0.05 & 0.20 & 0.05
\end{tabular}




\begin{tabular}{ccc} 
Decile & Miller & \multicolumn{1}{c}{ Average } \\
\hline $0.0-0.1$ & 0.0 & 0.1875 \\
$0.1-0.2$ & 0.25 & 0.1875 \\
$0.2-0.3$ & 0.125 & 0.0938 \\
$0.3-0.4$ & 0.125 & 0.0938 \\
$0.4-0.5$ & 0.125 & 0.0875 \\
$0.5-0.6$ & 0.05 & 0.0875 \\
$0.6-0.7$ & 0.05 & 0.0875 \\
$0.7-0.8$ & 0.05 & 0.0875 \\
$0.8-0.9$ & 0.05 & 0.0875 \\
$0.9-1.0$ & 0.05 & 0.0875
\end{tabular}

This aggregated probability distribution can be represented as the following cumulative probability distribution:

\begin{tabular}{|c|c|}
\hline Ouantile & $\begin{array}{l}\text { Oxidation } \\
\text { Probability }\end{array}$ \\
\hline 0.0 & 0.1 \\
\hline 0.375 & 0.3 \\
\hline 0.563 & 0.5 \\
\hline 1.000 & 1.0 \\
\hline
\end{tabular}

This distribution, along with the other consensus distribution from the preceding section, is used as uncertainty data for APET event 70. 


\section{C.9 SYSTEMS RECOVERY \\ C.9.1 Issue Definition}

The APET considers the possibility that several primary and confinement systems could be recovered during the accident. Probability distributions need to be developed to show the probability that each system will be recovered within the first two hours after reactor trip. The following systems should be considered for recovery:
a) AC power
b) Graphite and Shield Cooling System (GSCS)
c) Emergency Core Cooling System (ECCS)
d) High Pressure Injection (HPI) system.
e) Fog Spray System
f) Hydrogen Mitigation System

For any of these systems, recoverability may depend on the availability of AC power or other support systems.

A suggested decomposition of this issue is to consider first whether or not the operators will seek to recover the system, and then, given that the operators will attempt recovery, consider whether or not the system can be recovered. An alternative decomposition would consider recoverability first, and then, given that the system is recoverable, consider whether or not the operators will seek to recover the system.

\section{C.9.2 Issue Resolution Process}

D. M. Kunsman of Sandia National Laboratories was selected to be elicited about all systems other than electrical power. He was selected because of his extensive familiarity with both the $N$ Reactor plant and procedures and the generally accepted PRA recovery standards. He selected the issue decomposition for each system and provided probability distributions as appropriate. The electrical power recovery issue was resolved through a search of the relevant, commercial nuclear power industry power recovery literature by G. D. Wyss of Sandia National Laboratories. 


\section{C.9.3 Issue Analysis and Resolution}

\section{C.9.3.1 Electrical Power Recovery}

\section{Background Information}

1. R. L. Iman and S. C. Hora, Modeling Time to Recovery and Initiating Event Frequency for Loss of Offsite Power Incidents at Nuclear Power Plants, NUREG/CR-5032, SAND87-2428, Sandia National Laboratories, January 1988.

2. P. W. Baranowsky, Evaluation of Station Blackout Accidents at Nuclear Power Plants, NUREG-1032, U. S. Nuclear Regulatory Commission, June 1988 .

3. M. D. Zentner, et al., N Reactor Level 1 Probabilistic Risk Assessment: Final Report, WHC-EP-0322, Westinghouse Hanford, August 1988 .

\section{Discussion}

The electrical power recovery event entitled "Is AC power available for recovery and post-recovery actions?" (Event 51) considers two different possibilities, one of which is not a recovery. The event considers previous condition of the electrical power system and propagates that along if there are no recovery actions. This is not a recovery event. The other possibility, however, is recovery from previous AC bus failures.

The recovery of $A C$ electrical power is not as critical at $N$ Reactor is it is in other nuclear power plants because of the wide array of diesel powered pumps that are available for cooling the core, and the fact that most critical valves are either air or hydraulic and have their own accumulators. While it is certainly a benefit, the fact that, as currently constructer, $\mathrm{N}$ Reactor has no emergency diesel generators is indicative that $A C$ power from any source is not absolutely necessary to the safety of the plant. Therefore, a more generic treatment of AC power recovery is sufficient for this plant than would be the case for another reactor.

There are two different recoveries that are possible: recovery from a single bus failure, and recovery from a loss of offsite power. If a single bus has failed, then there is still one AC power bus available within the plant. Bus $A$ is normally powered from offsite sources and Bus $B$ is normally powered from the onsite turbogenerator. Either of these buses can be aligned to be powered from the other. Procedures do exist for this process. These procedures are used regularly during $\mathbf{N}$ Reactor refueling and other outages, so they would be quite familiar to the persons doing the realignment. In addition, the persons performing this realignment would not be the control room operators and would, thus, be likely to be under significantly less stress than the control room operators and would not be influenced by previous control room operator errors. The process takes 
approximately 15 minutes to accomplish because the failed bus must first be unloaded, then connected to the powered bus, then reloaded. The time available for all recovery actions in the Level I PRA [3] was seven minutes because recovery had to be accomplished prior to the onset of core damage. This is why recovery of lost AC power busses was not considered in the Level I PRA. In the APET analysis, recovery typically occurs at approximately two hours after the initiating event, so there is plenty of time available for the recovery process to be accomplished. Because procedures are in place to accomplish this recovery action, and because the operators would be very likely to attempt the recovery, the following probability distribution is assessed to be representative of the probability that there will be a failure to recover a lost AC power bus.

\begin{tabular}{|c|c|}
\hline Quantile & $\begin{array}{l}\text { Probability of } \\
\text { Non-Recovery }\end{array}$ \\
\hline 0.0 & 0.005 \\
\hline .25 & 0.01 \\
\hline .50 & 0.05 \\
\hline .75 & 0.10 \\
\hline 1.0 & 0.20 \\
\hline
\end{tabular}

The zero anchor point is based on a generic human error of failing to execute the recovery procedure, and the distribution reflects the assessment that the operators are very likely to attempt the recovery, and would almost certainly be able to accomplish the recovery procedure if attempted.

The second electrical recovery case is the recovery of lost offsite power. If offsite power is lost but the thermal flywheel procedure is effective, then Bus $B$ is never lost and the recovery of the offsite power bus (Bus A) can be accomplished as above. If both power busses are lost, then recovery of the offsite grid must be accomplished before either bus can be recovered. The $\mathrm{N}$ Reactor site (the $100 \mathrm{~N}$ area) is fed from two separate offsite sources which, while powered from the same grid, provides a degree of redundancy that helps protect the plant from component failures within parts of the Hanford site electrical grid. These two sources are the $N$ Tap and the $K-N$ Tie, which is an electrical tie in with the $100 \mathrm{~K}$ area.

A probability distribution has been developed to represent the probability that offsite power will not be recovered within the first two hours after an initiating event. This distribution makes use of References [1] and [2] above and has a very wide uncertainty range due to its generic nature. Since AC power is not as critical in this plant as in others, the wide uncertainty and conservative analysis is not expected to influence the overall results. The issue can be re-visited if this assessment does not turn out to be true. 


\begin{tabular}{|c|c|}
\hline Quantile & $\begin{array}{l}\text { Probability of } \\
\text { Non-Recovery }\end{array}$ \\
\hline 0.0 & 0.05 \\
\hline .25 & 0.20 \\
\hline .50 & 0.30 \\
\hline .75 & 0.40 \\
\hline 1.0 & 0.80 \\
\hline
\end{tabular}

\author{
Source of Data \\ Ref. [1], Fig. 27, 5th percentile \\ Ref. [1], Fig. 28, 5th percentile \\ Ref. [1], Fig. 28, median \\ Ref. [1], Fig. 28, 95 th percentile \\ Ref. [2], Fig. A.8, 95th percentile for \\ weather-induced grid losses
}

The above assessments were constricted with internally initiated accidents in mind. For fire-initiated loss of power events, the cause of the loss of AC power is fire damage to the electrical switching equipment itself, so electrical recovery would mean repairing fire damaged equipment. The probability of this happening in a the time frame of interest to the APET analysis is very small. For seismic accidents, there are two causes of bus failure: failure of the offsite grid, and failures of the bus hardware within the plant itself. Neither of these would be quickly recoverable. For this reason, it is assessed that $A C$ power is not recoverable for fire and seismic core damage accidents within the modeling time frame of interest to the APET analysis.

\title{
C.9.3.2 Graphite and Shield Cooling System Recovery
}

Background Information

1. M. D. Zentner, et al., N Reactor Leve1 1 Probabilistic Risk Assessment: Final Report, WHC-EP-0322, Westinghouse Hanford, August 1988 .

\section{Discussion}

The Graphite and Shield Cooling System (GSCS) very important to the limitation of core damage in $N$ Reactor accidents. It provides an additional, independent cooling system that is generally not affected by failures within the primary and emergency core cooling systems. It is generally credited with limiting core damage to less than one-third of the core when it is fully functional. Recovery of this system, should it fail, could effectively reduce core temperatures to below the levels where graphite oxidation would be expected.

Two types of failures are associated with the GSCS: degraded performance due to the loss of the helium cover gas system, and failure of the cooling system itself. The helium cover gas system fallures modeled in this analysis are caused by pressure tube failures within the core or by fallure of the rupture disk during a hydrogen event within the pipe barrier space. Neither of these events are recoverable because the failures are located inside the core area itself. Therefore, recovery of the GSCS from degraded function to normal function is not possible. 
The GSCS itself is capable of functioning in two modes: recirculating and once-through. The GSCS is successful if it is operating in either of these modes. The majority of the failures of the GSCS come from failures of valves and plugging of the orifice that restricts flow at the outlet of the core in the once-through mode. Many of the valve failures show up when the system is reconfigured from its recirculating mode, which uses electrical pumps, to its once-through mode, which takes water from the fog spray diesel pumps. Many of these valves are located within Zone I of the confinement and are inaccessible when the confinement is radiologically contaminated. Repair of the plugged orifice is not considered credible within the time frame of interest to the APET analysis. For this reason, recovery of the GSCS must be considered unlikely.

It has also been noted that there are recovery methods that are available to the control room operators for many of the dominant GSCS failures that were not accounted for in the Level I PRA analysis due to the low frequency of GSCS failure sequences. These are not accounted for in this recovery analysis. This introduces a level of conservatism to the analysis.

Even if the cooling system could be recovered, conversations with thermal-hydraulic analysts at WHC indicates that, if the GSCS is nonfunctional for more than several minutes, steam binding could prevent the coolant from a recovered GSCS from fully penetrating the core for up to several hours. The flashing of the GSCS coolant would combine with the small coolant channels and the flow-restricting orifice at the core outlet (in the once-through mode) to slow the penetration of coolant into the core. It should be noted that this is not universally agreed upon 22 there is some window during which this function could be recovered before steam binding would prevent coolant penetration. However, this assessment is intended to address the issue of restarting the GSCS after a failure of a significant duration (approximately one to two hours), and by this time the graphite blocks that surround the GSCS tubes would have become so hot as to virtually assure that the GSCS coolant penetration would be long delayed.

From the above discussion, the upper bound on the probability of recovery of the GSCS function (GSCS coolant flowing through and cooling the core) is assessed to be 0.01 . The difference between a recovery probability of 0.01 and 0.00 is very small. Therefore, it is recommended that a point estimate only be used, and that the issue be revisited if GSCS recovery has a significant impact on the overall results of the analysis. 


\section{C.9.3.3 Emergency Core Cooling System Recovery}

Background Information

1. United Nuclear Industries, Inc., "ECS Actuation During Reactor Operation," Emergency Response Guides, WHC-CM-4-1, Vol. 2, Guide No. B, Issued May 12, 1987, as updated through June 1989.

2. M. D. Zentner, et al., N Reactor Level 1 Probabilistic Risk Assessment: Final Report, WHC-EP-0322, Westinghouse Hanford, August 1988 .

\section{Discussion}

The $\mathrm{N}$ Reactor procedures for ECCS operation [1] instruct the operators not to initiate ECCS is more than seven minutes have elapsed since the ECCS was demanded. However, in the majority of $\mathrm{N}$ Reactor accidents predicted by the Level I PRA [2], ECCS flow is established but fails to meet its success criteria. The reason for this is the failure of one or more of a series of multiple components that are connected in parallel. A fundamental assessment of the ECCS recovery analysis is that the operators will respond to partial ECCS flow by attempting to establish full ECCS flow and not by treating this as an ECCS failure and shutting down the ECCS. Almost all full ECCS failures are caused by the incorrect positioning of the $3 \mathrm{H} 33$ ECCS bypass switch. This is easily recoverable, but doing so after the seven minute criteria has elapsed is contrary to procedures. Each of these instances are considered below.

There are three major causes of partial ECCS failure: failure of ECCS high lift diesel pumps to start or run, failure of $V-3$ valves to open, and failure of V-4 valves to open. Recovery of the diesel pumps is assessed to be very unlikely because of the extensive amount of work that would be involved in rebuilding or repairing the diesel engine. Recovery of the $V-4$ valves is possible to a degree because there are manual switches in the control room to call for actuation should automatic actuation fail, but this recovery was considered in the Level I PRA. Further recovery is not possible because the V-4 valves are located inside Zone $I$ of the confinement and are not accessible when the confinement is radiologically contaminated. It would probably be necessary to trace the causes of V-4 valve failure inside Zone $I$ even if the problem could ultimately be fixed outside Zone I. For these reasons, the only partial ECCS failures that are considered recoverable are $\mathrm{V}-3$ valve failures.

The V-3 valves are located nutside Zone I of the confinement, so they are accessible under accident conditions for recovery actions. It is assessed that, since partial ECCS flow already exists, the operators would not abandon recovery actions once the seven minute criteria expires. The Level I PRA [2] results state that only about 108 of all V-3 valve failures are at the valve itself. The remaining $90 \%$ are related to the valve contrnl circuitry in the control room. The Level I PRA assigned a nonrecovery probability of 0.4 for the V-3 valves. Those that are recovered 
during the first seven minutes would be the easiest recoveries to make (those remaining would be expected to take longer to accomplish). Approximately three in four of these remaining failures are belleved to be theoretically recoverable during the time frame of interest to the APET analysis based on the fact that $60 \%$ of the $V-3$ failures have already been recovered in the Level I analysis (or, if 108 of the initial failures are non-recoverable, this is equivalent to 258 of those remaining after 608 have been recovered).

In order for core damage to occur, the Level I PRA must predict that recovery has already been attempted and failed. For this reason, the assessed probability distribution is weighted heavily towards non-recovery. The following probability distribution was assessed to be representative of the probability of non-recovery for $v-3$ valve failures.

\begin{tabular}{|c|c|}
\hline Quantile & $\begin{array}{l}\text { Probability of } \\
\text { Non-Recovery }\end{array}$ \\
\hline 0.0 & 0.25 \\
\hline .25 & 0.50 \\
\hline .50 & 0.75 \\
\hline .75 & 0.90 \\
\hline 1.0 & 1.00 \\
\hline
\end{tabular}

The upper bound is representative of the idea that the $V-3$ valves may never be recovered, and the lower bound is based on the theoretical recoverability of the $\mathrm{V}-3$ valves.

Complete ECCS failure is dominated by the 3 H 33 ECCS bypass switch. The Level I PRA describes this accident as occurring when the 3 H 33 switch, which bypasses all pressure-related inputs into the ECCS automatic actuation circuitry for reactor start-up purposes, is not transferred to the proper position after startup operations are completed. The operator then fails to recover by manually initiating ECCS.

The $3 \mathrm{H} 33$ bypass switch has an additional effect on recovery in that, when it is mispositioned, the ECCS trip is never generated and the seven minute timer never starts. Therefore, the operator would be left without knowing when the seven minute criteria should have started (this is not necessarily true for large LOCAs because a confinement trip would also have occurred). The operator could "violate" procedures without knowing it. There are three ECCS recovery cases for the 3 H33 ECCS bypass switch, and these are described below.

The first $3 \mathrm{H} 33$ recovery case is for large LOCAs. In this case, the operator would know when ECCS should have actuated because a lapse timer for the confinement trip would provide such an indication. He would have many indications of problems and would have to consciously violate the ERG procedures [1] to initiate ECCS function. Therefore, the following probability distribution is assessed to be representative of the probability of for $3 \mathrm{H} 33$ bypass under large LOCA conditions because the operator would have to consciously violate procedures. 


\begin{tabular}{|c|c|}
\hline Quantile & $\begin{array}{l}\text { Probability of } \\
\text { ECCS Recovery }\end{array}$ \\
\hline 0.0 & 0.0 \\
\hline .25 & 0.0 \\
\hline .50 & 0.0 \\
\hline .75 & 0.003 \\
\hline .90 & 0.01 \\
\hline 1.0 & 0.25 \\
\hline
\end{tabular}

The second $3 \mathrm{H} 33$ recovery case is for small LOCAs and transients. In these cases, ECCS initiation would not necessarily be accompanied by a confinement trip, and if it were, the confinement trip could occur much later than the ECCS demand. If the accident progresses quickly (as assumed in the Level I PRA), the following probability distribution is assessed to be representative of the probability of for $3 \mathrm{H} 33$ bypass under these conditions because the operator would not know when the ECCS should have started and would be likely to initiate it.

\begin{tabular}{|c|c|}
\hline Quantile & $\begin{array}{l}\text { Probability of } \\
\text { ECCS Recovery }\end{array}$ \\
\hline 0.0 & 0.003 \\
\hline .25 & 0.50 \\
\hline .50 & 0.90 \\
\hline .90 & 0.99 \\
\hline .99 & 0.999 \\
\hline 1.0 & 1.0 \\
\hline
\end{tabular}

The zero probability quantile is based on the generic human error of omission (the operator failed to follow procedures). The operator is assessed to be very likely to initiate ECCS flow. If the accident does not progress quickly (i.e takes hours to develop), then the large LOCA probability distribution above is more appropriate for modeling $3 \mathrm{H} 33$ ECCS recovery.

The third 3 H 33 ECCS recovery case involves a case where the operators know that the seven minute criteria has elapsed, but are faced with an unmitigated accident where all cooling (ECCS and GSCS) have failed. This recovery, therefore, involves deliberate violation of procedures to mitigate the perceived possibility of severe accident consequences such as graphite oxidation. It is believed that the operators are more likely to start ECCS than not to start it, but it is also believed that if a given reactor operator were faced with the same decision twenty times, he would almost always make the same choice. Thus, the uncertainty is related more to the nature of the operators on duty at the time of the accident than to the fraction of the time that a given operator either would or would not initiate ECCS given similar circumstances. For this reason, the following probability distribution is assessed to be representative of ECCS recovery under these conditions. 


\begin{tabular}{|c|c|}
\hline Quantile & $\begin{array}{l}\text { Probability of } \\
\text { ECCS Recovery }\end{array}$ \\
\hline 0.0 & 0.00 \\
\hline .25 & 0.10 \\
\hline .50 & 0.75 \\
\hline .75 & 0.90 \\
\hline 1.0 & 1.00 \\
\hline
\end{tabular}

It may, be noted that the above distribution is bimodal (i.e., when expressed as a probability density function, it has two peaks). This is intended to represent the dominant uncertainty expressed above.

In fire-initiated core damage accidents there are two dominant failure modes: common failure of all V:4 valves and common failure of all ECCS high lift diesel pumps (HLDP). In each case the common failure was caused by the fire damaging control cables for the affected components. The ECCS HLDP's can be started manually by operators stationed in Building 182N without the need for the affected control cables. An appropriate recovery probability distribution would be the corresponding $3 \mathrm{H} 33$ ECCS recovery distribution above, depending on whether or not GSCS also falls. The V-4 valve fallures are assessed to be unrecoverable for the same reasons described earlier in this section.

Most seismic accidents are caused by the failure of the silo structure which stores water for use by most cooling systems. Another common failure is the collapse of the pump house (Building 182N). Both of these failures are assessed to be unrecoverable, and, since these form the vast majority of ECCS failures, ECCS recovery is not considered for seismic core damage accidents. It should be noted, however, that almost all seismic core damage accidents are caused by seismic events that are significantly greater in magnitude (and more rare) than the $0.25 \mathrm{~g} \mathrm{~N}$ Reactor safe shutdown earthquake.

\section{C.9.3.4 High Pressure Injection System Recovery}

\section{Background Information}

1. M. D. Zentner, et al., N Reactor Level 1 Probabilistic Risk Assessment: Final Report, WHC-EP-0322, Westinghouse Hanford, August 1988 .

2. United Nuclear Industries, Inc., "ECS Actuation During Reactor Operation," Emergency Response Guides, WHC-CM-4-1, Vol. 2, Guide No. B, Issued May 12, 1987, as updated through June 1989.

\section{Discussion}

The high pressure injection system (HPI) is a high capacity coolant makeup system for $N$ Reactor. When fully operable, it is capable of supplying more than $7000 \mathrm{gpm}$ of makeup coolant to the reactor. It is 
electrically driven and its pumps are located in Building $182 \mathrm{~N}$. There are two classes of HPI failures: those that are caused by a loss of AC power, and those that are not. Each of these will be discussed separately below.

HPI system failures that are caused by a events other than the loss of one or both AC power busses frequently involve the failure of components that are located within Zone $I$ of the confinement. These components are inaccessible when the confinement is radiologically contaminated. Therefore, a low probability of recovery should be assigned for HPI when the failure is not caused by the loss of AC power.

When AC power is the cause of HPI failure, the probability of recovery should still be quite low. The $\mathrm{N}$ Reactor emergency procedures [2] indicate that, once ECCS is functioning, the operators should shut down the HPI system. While no core damage accidents involve the proper function of ECCS, the procedures do not show any other mention of HPI. For this reason, the operators are likely to leave HPI running in its condition at the time of the accident initiator for the duration of the accident and are very unlikely to even attempt recovery - regardless of the cause of the HPI failure. It is recommended that a recovery probability no greater than 0.01 be used and that it not be sampled in the uncertainty analysis. The issue should be revisited if HPI recovery makes a significant difference in the overall results.

\section{C.9.3.5 Fog Spray System Recovery}

Background Information

1. G. A. Coles, $N$ Reactor Confinement Systems Sequence Analysis Including Recovery, WHC-SD-NR-RA-001, Westinghouse Hanford, March 1990.

\section{Discussion}

The APET event entitled "Do fog sprays operate during the postrecovery phase?" (Event 56) considers three different possibilities, two of which are not recoveries. First, the event considers previous operation of the system and propagates that along if there are no additional demands or failures. Also, the event allows the configuration of the system to change based on the events in the pre-recovery accident progression and the recovery events themselves. Neither of these are recovery events. The third possibility, however, is recovery of the system from previous failures.

Almost all random fog spray system failures (as opposed to events which do not demand their operation) are caused by the failure of the fog spray diesel pumps to either start or continue to run. These failures cut across all spray systems. Recovery was considered in the fault tree analysis [1]. so additional recovery of the fog spray system would involve only those actions that would take a long time to accomplish. Recovery of 
the fog spray diesel pumps within the 24 hour APET analysis time frame is not considered credible as the task of rebuilding or repairing a diesel pump would be formidable. Since these failures form the bulk of the fog spray system failures, it is not overly conservative to disallow fog spray system recovery in the APET analysis.

\section{C.9.3.6 Hydrogen Mitigation System Recovery}

\section{Background Information}

1. S. E. Lindberg, Hydrogen Mitigation System Risk Analysis, WHC-SD-NR-RA-002, Westinghouse Hanford, October 1988.

\section{Discussion}

The APET event entitled "Does the hydrogen mitigation system operate during recovery?" (Event 58) considers three different possibilities, two of which are not recoveries. First, the event considers previous operation of the system and propagates that along if there are not additional demands or fallures. Also, the event allows the configuration of the system to change based on the events that occur during the pre-recovery accident progression and the recovery events themselves. Neither of these are recovery events. The third possibility, however, is recovery of the system from previous failures.

The hydrogen mitigation consists of two major parts: the mixing system and the nitrogen system. The mixing system consists of fans that blow air from Building $105 \mathrm{~N}$ into the pressurizer penthouse. Since these fans and many of their associated components are located inside confinement Zone $I$, these fans are generally not recoverable due to their inaccessibility inside the radiologically contaminated confinement. The exception to this is when the mixing fans are lost because of a loss of electrical power. Should this occur, there would be a very high probability that the fans would be recovered. The low probability of mixing system failure (it has redundant fans and redundant backup diesel power supplies), however, makes it unwaranted to determine a distribution for this situation. A distribution will be determined if mixing system failure ends up contributing significantly to the overall risk of the plant.

The majority of the nitrogen system failures are failures of the liquid nitrogen vaporization equipment [1]. The hydrogen mitigation system risk assessment already considered most of the easily available recovery actions. Thus, those that remain would be likely to be very long and involved (of similar magnitude to diesel pump repairs). Therefore, additional nitrogen system recovery within the 24 hour APET modeling time frame is assessed to be a very remote possibility and, therefore, should be disregarded. 


\section{C.9.4 Application of Results}

The above-stated probability distributions were sampled in the Latin Hypercube uncertainty analysis and incorporated into the proper APET events as indicated in the pointer files presented with the APET input file listings in other appendices. 


\section{C.10 FILTER PATH REISOLATION}

\section{C.10.1 Issue Definition}

The confinement filter path is designed to reisolate any time the confinement pressure rises above 15 inches of water. However, certain events (such as hydrogen detonations) occur so quickly that the path would not be able to close quickly enough to prevent damage from occurring to the filter path downstream of the isolation valves. The path is designed to isolate quickly enough to prevent the pressure pulse from a large LOCA from damaging the filters or the filter path, which is only rated for 2 psig overpressure downstream of the isolation valves. Thus, the question can be restated as, "What is the probability that a given energetic event can cause a pressure pulse that occurs faster than the worst case large break LOCA, with a peak pressure of greater than 2 psig?" Since there is undoubtedly some uncertainty as to whether or not certain events will or will not fall into this category, probability distributions need to be developed to model the effectiveness of the reisolation function for the phenomena which would be expected to demand it.

The following events should be considered as candidates for demanding the reisolation function:

1) Hydrogen events (detonations and deflagrations)

2) Graphite combustion events (slow and rapid oxidation)

3) Pressure tube fallure after a late actuation of the ECCS

Other events can be considered at the discretion of the elicitees.

\section{C.10.2 Issue Resolution Process}

The above stated issue was presented to L. A. Miller and A. L. Camp of Sandia National Laboratories during the week of August 28, 1989. They were selected because of their extensive experience in modeling containment accident phenomenology, and in particular hydrogen phenomenology, for nuclear reactor accidents. In addition, Miller was selected for her experience in modeling the $\mathrm{N}$ Reactor confinement buildings for the MELCOR and HECTR analyses of the confinement buildings. Discussions were held between the elicitees, and Miller translated those results and the results of significant additional research into a write-up on the resolution of the issue that appears (with the inclusion of some minor review comments) below.

\section{C.10.3 Issue Analysis and Resolution}

The confinement filter path is designed to reisolate any time the confinement pressure rises above 15 inches of water following the initial isolation that occurs at 3 inches of water. However, certain events (such 
as hydrogen detonations) occur so quickly that the path would not be able to close quickly enough to prevent damage from occurring to the filter path downstream of the isolation valves. The path is designed to isolate quickly enough to prevent the pressure pulse from a large LOCA from damaging the filters or the filter path, which is only rated for 2 psig overpressure downstream of the isolation valves. Thus, the question can be restated as, "What is the probability that a given energetic event can cause a pressure pulse that occurs faster than the worst case large break LOCA, with a peak pressure of greater than 2 psig?".

\section{Background Information}

Several reports were consulted in the quantification of this issue including:

1. A. I. Camp, et al., Light Water Reactor Hydrogen Manual, NUREG/CR-2726, SAND82-1137, Sandia National Laboratories, August 1983.

2. A. C. Payne, A. L. Camp, Parametric HECTR Calculations of Hydrogen Transport and Combustion at $N$ Reactor, SAND86-2630, Sandia National Laboratories, June 1987.

3. L. A. Miller, et al., N Reactor Probabilistic Risk Assessment Supporting Calculations, SAND89-2101, to be published.

4. M. P. Sherman, et al., ELAME Facility, NUREG/CR-5275, SAND85-1264, Sandia National Laboratories, April 1989.

In particular, the large LOCA calculation used as a benchmark for MELCOR predicted a confinement pressurization rate during the blowdown of approximately $4.2 \mathrm{kPa} / \mathrm{s}$.

\section{Hydrogen Detonations}

A detonation is a combustion wave that travels at supersonic speeds relative to the unburned gas in front of it. A simple calculation based on the geometry of the $\mathrm{N}$ Reactor confinement yields a .2 s transit time from steam generator cell 5 to the isolation valves for a wave moving at the speed of sound in air. Since the maximum pressures during a detonation is typically 12 to 15 times the initial pressure, the detonation will reach the 1solation valves before they close and the pressure will exceed 2 psig. Therefore, a value of 0.0 is assigned to the probability of successful filter path reisolation following a detonation.

\section{Hydrogen Deflagration}

Deflagration experiments performed at the FLAME facility for geometries with no venting and with obstacles show that the time to travel $28 \mathrm{~m}$ was approximately $330 \mathrm{~ms}$ for a 158 hydrogen mixture. Ihis would 
roughly correspond to a 900 ms transit time from steam generator cell 5 to the isolation valves assuming the combustion wave propagated through the confinement. Several HECTR calculations with a uniform mixture of 58 or more in the 109 building predict a pressure rise of 5 psig or more in the confinement building. However, deflagrations of weaker mixtures may propagate much more slowly. To allow for the range of deflagrations possible the following distribution for the probability of filter path reisolation failure following a deflagration was assigned.

\begin{tabular}{|c|c|}
\hline Quantile & Probability \\
\hline 0.0 & 0.0 \\
\hline .25 & 0.1 \\
\hline .5 & 0.3 \\
\hline .75 & 0.5 \\
\hline 1.0 & 1.0 \\
\hline
\end{tabular}

\section{Graphite Combustion Events}

The rate of pressure increase due to a slow graphite oxidation is believed to be much lower than the increase due to a large LOCA. Therefore, a probability of 0.0 is assigned to the probability of reisolation failure due to slow graphite oxidation. Fast oxidation was believed to have some probability of occurring either rapidly enough or in conjunction with rapid pressure rise events such that a very rapid pressure rise could occur, therefore, the following distribution for reisolation failure was developed.

\begin{tabular}{cc} 
Quantile & Probability \\
\hline 0.0 & 0.0 \\
.25 & 0.0 \\
.5 & 0.1 \\
.75 & 0.3 \\
.99 & 0.5 \\
1.0 & 1.0
\end{tabular}

\section{Pressure Tube Failure}

Pressure tube failure following a late actuation of the Emergency Core Cooling System is not belleved to cause a quicker pressure increase than a large LOCA for two reasons. First, several pressure tubes would have to fail in order to release large quantities of steam. Second, the process would be limited by the amount of water being supplied to the failed pressure tubes. Since the filter path is designed to be able to reisolate quickly enough to prevent the pressure increase from a large LOCA from damaging the filters or the filter path, a value of 0.0 was assigned to the probability of reisolation failure. 


\section{C.10.4 Application of Results}

The probabilities (both the point estimates and the distributions) developed in the above discussion are implemented directly in the $\mathrm{N}$ Reactor APET as the branch probabilities for events 45 and 73 . These events are both entitled, "Does the filter exhaust path reisolate in time to prevent filter damage?" Event 45 examines this issue for the pre-recovery phase of the accident, and Event 73 examines the issue for the post-recovery accident progression. 


\section{C.11 FILTER PERFORMANCE \\ C.11.1 Issue Definition}

The performance of the filter system has a first order impact on the overall release of radioactivity from the plant during a severe accident. For this reason, a detailed filter analysis is required to lend credibility to the overall results of the APET analysis.

Probability distributions are needed to describe the relative probabilities of filter failure mechanisms such as filter bypass, aerosol plugging, filter burning, and mechanical failure. The approximate time (early or late in the accident) of fuel failure can be important since radionuclides trapped on the filters are not expected to escape in any significant fraction on filter failure (except when filters burn). In addition, it is possible that filter performance will be degraded during the accident due to radionuclide loading or aerosol plugging. In these degraded cases, probability distributions are needed to describe the extent of degradation (overall remaining efficiency) for the filters over the entire accident sequence or during various accident phases.

This issue should consider all relevant aspects of filter operation and failure. The results of the elicitation can, if necessary, change the questions and branches used in the APET if that would provide a more meaningful description of the phenomena involved.

\section{C.11.2 Issue Resolution Process}

K. K. Murata of Sandia National Laboratories was selected to be elicited on the issue of filter performance because of his extensive familiarity with the literature and experience in modeling filter behavior in nuclear installations such as the Hanford and Savannah River plants. He is responsible for much of the filter modeling that has been installed in the U.S. Nuclear Regulatory Commission's best estimate containment modeling computer code package CONTAIN.

The elicitee was given complete freedom to define the issue and the structure of the final results to be included in the APET analysis within the framework of the previous section. He developed a basic framework that was expanded and implemented in the APET by G. D. Wyss, the $\mathrm{N}$ Reactor Accident progression analyst. The issue was presented to the elicitee between August 25, 1989 and September 1, 1989. A preliminary elicitation was conducted on September 1, 1989. An additional elicitation was held later in that month to allow for the inclusion of later literature studies and additional uncertainty methods development. The discussion below is a summary of those discussions and the application of that information by the accident progression analyst to determine the uncertainty distributions. 


\section{C.11.3 Issue Analysis and Resolution}

\section{Background Information}

1. Muhlstein, L. D., et al, $\underline{N}$ Reactor Filter System Fission-Product Retention Assessment, WHC-SP-0233, Westinghouse Hanford, June 1988

2. Safety Issues at the Defense Production Reactors: A Report to the Department of Energy, National Academy of Sciences, National Academy Press, Washington, D.C., 1987.

3. United Nuclear Industries, Inc., "Zone I Confinement Trip," Emergency Response Guides, WHC-CM-4-1, Vol. 2, Guide No. L, Issued May 12, 1987, as updated through June 1989.

4. G. A. Coles, N Reactor Confinement Systems Sequence Analysis Including Recovery, WHC-SD-NR-RA-001, Westinghouse Hanford, March 1990 .

5. D. M. Kunsman, et. al., Level III Probabilistic Risk Assessment for N Reactor, SAND89-2102, Sandia National Laboratories, April 1990.

6. J. M. Griesmeyer and L. N. Smith, A Reference Manual for the Event Progression Analysis Code (EVNTRE), NUREG/CR-5174, SAND88-1607, Sandia National Laboratories, September 1989.

\section{Discussion of Issues Impacting Filter Performance}

The filter system at $\mathrm{N}$ Reactor is part of the last line of defense against radioactive releases from the plant. It is intended to be the only means available for effluent gases to escape the plant and, thus, has a first order effect on the source term of any severe accident. For this reason, it is essential to have a realistic picture of how these filters would perform in such a situation.

The $N$ Reactor filter system, as configured for use under accident conditions, consists of three stages: a moisture separator, a HEPA filter stage, and a charcoal bed stage. The moisture separator stage is designed to remove 99.58 of the entrained water from the gas to be filtered. This protects the HEPA filters. The HEPA filters are designed to remove 99.958 of all particulates greater than one micron in diameter and are very susceptible to water damage. The third, charcoal bed stage is designed to remove 998 of all elemental iodine and hydrogen jodide, and 708 of all methyl iodide from the gas effluent stream. A filter spray system is provided to cool the filters should excessive decay heat from the entrapped radionuclides threaten the filters.

Three major mechanisms can affect the effectiveness of the filter system. These are filter bypass, filter damage (rupture, water saturation, etc.), and filter loading. Filter bypass can occur in a number of ways. Confinement building failure can cause gas flow to bypass the filter path entirely, or a failure of the filter path can divert flow from the filters 
themselves. In addition, the inflatable seals around the filters can fail and open up a path that would bypass the filter media. Finally, an error in the installation of the filters could damage one of them and effectively bypass the filter media. Each of these failures is considered either in other events in the APET or in the confinement systems fault tree analysis [4]. Thus, they will not be considered further in this uncertainty analysis.

HEPA filters are generally easy to damage. They tend to fail by tearing at an overpressure of a few psig and, when damp and at high temperatures ( $>50 \mathrm{C})$, can fail at overpressures as low as a few inches water. In addition, the filter effectiveness (removal rate) decreases to almost zero for the charcoal filters when they are soaked with water (as during the operation of the filter sprays). This means that the conditions under which the filters will be operating must be examined carefully to determine whether or not filter damage or degradation will occur.

HEPA filters that are exposed to hot and humid $(\sim 100 \mathrm{C},-1008$ humidity) conditions experience degraded strength over a period of time. Under such conditions, HEPA filters would be expected to fail or at lease experience severely degraded performance, and the degradation could occur as quickly as a few hours into the accident. The filters would also degrade under cooler, humid ( $-50 \mathrm{C},-1008$ humidity) conditions, although the degradation process would take much longer (probably more than a day), and this time is beyond the modeling time of the APET analysis. N Reactor accidents that might fit the former description include LOCA's in which the fog spray system fails. These accidents could have a hot, direct steam source to the confinement building which could lead to filter damage. In most accident scenarios, however, the combination of fog spray action and the lack of a large, continuing direct heat source to the confinement combine to make filter degradation by this mechanism unlikely. In addition, the long underground path from the confinement building vent to the filter cell would be expected to cool the effluent gases as long as the high temperature and humidity conditions did not exist for a prolonged period.

Water saturation is of primary importance in the degradation of both the HEPA and the charcoal filters. This was recognized implicitly by those studying the $\mathrm{N}$ Reactor filter system [1] because they placed the upper limit for successful HEPA filter operation at the temperature setpoint for actuation of the filter spray system. The filter spray system directs spray water to all surfaces around the filter cell, including the walls, floors, ceilings, demisters and filters. The filter spray system actuates on a high gas temperature in the filter building, and is intended to spray the filters only when the atmosphere temperature is at least $220 \mathrm{~F}$. This criteria is intended to prevent the filters from becoming wet because, in theory, the water will evaporate from the filters without damaging them because of the elevated temperatures. In this analysis, however, it was assessed that this mechanism could not be relied upon to prevent the water from damaging the filters. While the wet filters would not be expected to release those fission products already trapped, their filtration efficiency would essentially be reduced to zero for the rest of the accident scenario because the HEPA filters could be expected to rupture, and the water would 
inhibit the iodine removal mechanisms for the charcoal beds. The issue of filter cooling is expected to dominate the filter failure analysis.

Filter loading was raised as a concern by the National Academy of Sciences (NAS) [2] during their review of the production reactors. Research has been performed since that time [1] to address the issues raised by the NAS. This research indicates that $N$ Reactor charcoal beds have a capacity of approximately $4.5 \mathrm{~kg}$ of iodine. The entire core inventory of iodine is less than $3 \mathrm{~kg}$. Since almost all non-iodine fission products are expected to form aerosols (and, thus, be trapped by the HEPA filters before reaching the charcoal beds), excessive loading of the charcoal beds (such that their efficiency would be significantly reduced) is not credible and is not considered further in this uncertainty issue.

The $N$ Reactor HEPA filters were found by this same research [1] to be able to withstand a loading of $80 \mathrm{~kg}$ of aerosols before a 5 " water pressure drop would occur across the (dry) filters. Design basis accident calculations [1] indicate that the expected aerosol loadings would range from $35 \mathrm{~g}$ to a maximum of $35 \mathrm{~kg}$. Even if the 5" water pressure drop is exceeded, this does not mean that the filter media would fail, because dry HEPA filters can typically withstand differential pressures of at least 2 psig before failing, and even filters that have become dampened (but not wet) would typically withstand at least a 5" water differential pressure.

The HEPA filter analysis above, however, is limited in several ways. First, the design basis accident considered for it is not the most severe accident that could occur at $\mathbf{N}$ Reactor, but is the "standard" $N$ Reactor design basis accident with ECCS failed and GSCS operating. The PRA considers several accidents that are beyond design basis. Second, the analysis assumed that the fog sprays were operating to decontaminate the confinement building atmosphere, but this also is not true for many accidents in the PRA analysis. Third, there is a significant amount of uncertainty in the degree of decontamination that will be afforded by the fog sprays (and, because of this, the actual fission product loading to which the filters will be exposed). Finally, while the absolute loading of the filters in the calculations does not challenge the maximum filter luading, decay hcat deposition on the filter elements from the trapped fission products can cause problems cooling the filters without the aid of the filter sprays. These factors will be discussed individually below.

The first two cascs above are both, in a sense, beyond design basis accidents" for the filter system in that the detailed analysis of filter performance described in [1] has not been done for these situations. The fog sprays provide decontamination to the confinement building atmosphere and, as a result, reduce the loading on the filters because some of the radionuclides are trapped in the spray water. The majority of the accidents in the PRA, however, do not have fog spray function because they are transient accidents that do not produce pressure increases inside the confinement to demand fog spray actuation [3]. The GSCS fails accident is beyond design basis in that it is an unmitigated accident where the core heats up to far more extreme temperatures than would be seen if GSCS were to be operating. Not only would the amount of core damage be greater (presumably 1008 versus 508 in [1]), but the average fuel temperature would 
be higher. This would drive a higher fraction of the fission products out of the fuel on a per unit fuel mass basis. It also means that fission products that would in the analysis in [1] be expected to remain largely in the fuel, such as $\mathrm{Zr}$ and $\mathrm{Sn}$, would be expected to be released and add to the fission product load. This means there is more fuel damage and more fission product release per kilogram of fuel. Thus, simple scaling of the results in [1] by a factor of two is not sufficient.

In accidents where the fog spray system does operate, the calculation [1] represents a point estimate of their decontamination efficiency. There has been considerable discussion, however, as to the actual efficiency of the $N$ Reactor fog sprays because their droplets are considerably larger than those that are typical in the commercial nuclear power industry. The efficiency of the sprays in those calculations have been considered suspect by some reviewers [2]. This has been studied in the source term uncertainty analysis in [5]. Those results need to be considered in the overall filter evaluation.

While it is possible that the filters could survive even accidents in which the GSCS fails without becoming overloaded with aerosols, the most significant threat to filter integrity comes from thermal loading and filter cooling considerations. The decay heat from the fission products trapped on the filters must be removed by convection to the effluent gas flow if the filter temperature is to remain below the point where the filter sprays would be demanded. Even for the accident assessed in [1], the required cooling flow was about $850 \mathrm{cfm}$. The required flow rate rises linearly with the overall fission product loading on the filters, so decontamination and filter cooling are very closely linked in the filter. analysis.

\section{Methodology for Determining Filter Uncertainties}

Based on the above discussion, it was assessed that loading of the charcoal filter beds is very unlikely to cause failure of the filtration function without actuation of the filter spray system. The HEPA filters are also very likely to withstand numerous accident conditions intact because:

- The effluent conditions $(-100 \mathrm{C},-1008$ humidity) that cause rapid HEPA filter degradation can only exist for a very short time during N Reactor accidents,

- When GSCS operates, the aerosol load will not approach the HEPA filter load limit, and

- The accumulation of aerosols on the filters will not challenge the filters (based on absolute loading) before filter cooling becomes an issue even for GSCS failure accidents.

For these reasons, physical loading of the filters will not be considered as a likely filter failure mechanism except for long-term effects in accidents where GSCS fails. The filter failure criteria will be based on 
thermal loading of the filters and the operation of the filter spray system.

While the fog spray system is used in [1] to decontaminate the atmosphere before it reaches the filter, there are in reality several mechanisms that can accomplish this task. Obviously, there is the fog spray system, but there is also the mechanism of aerosol settling and agglomeration inside the building that can decontaminate the gas stream. In addition, the effluents are decontaminated by this same mechanism inside the primary system piping before they are released into the confinement atmosphere, although this effect was already considered in [1]. Finally, if the effluents do not bypass the emergency dump tank, the liquid pool inside that tank would provide significant decontamination of the exhaust gases before they are released into the confinement. In this assessment, the decontamination provided by the dump tank and the fog sprays will be considered for determining the loading on the filters. The others are neglected from this analysis, although they are considered in the source term study.

The calculation of the temperature rise of the effluent gas stream as it passes through the filters in Chapter 5 of [1] is accomplished by the following equation:

$$
T=\frac{Q_{p}}{V \quad C_{p}}
$$

where: $T=$ gas temperature increase, $F$,

$$
\begin{aligned}
& Q_{p} \text {-decay heat on filter system, BTU/min } \\
& V=\text { gas flow rate through the filters, } f t^{3} / \mathrm{min} \\
& D=\text { density of the gas, } 1 \mathrm{~b} / \mathrm{ft}^{3} \text { (nominal value: } 0.068 \text { ) } \\
& C_{\mathrm{p}} \text {-gas heat capacity, } \mathrm{BTU} / \mathrm{lb} \cdot \mathrm{F} \text { (nominal value: } 0.24 \text { ) }
\end{aligned}
$$

The nominal confinement outlet conditions are $120 \mathrm{~F}$, and the filter spray system actuates at $240 \mathrm{~F}$, so for this calculation, $\mathrm{T}=120 \mathrm{~F}$. One other conversion factor is required: $1 \mathrm{BTU} / \mathrm{min}=56.88 \mathrm{kw}$.

The decay heat load on the filters will be inversely proportional to the decontamination factor that occurs between the point of release from the primary system and the filters. Thus, the necessary decontamination factor or gas flow rate to keep the filters cooled can be determined by solving the following equation:

$$
T=\frac{Q_{p}}{V C_{p}} \cdot \frac{D_{R}}{D_{A}}
$$


where: $D_{R}$-decontamination factor for the fog sprays in the filter assessment rep $D_{A}=$ decontamination factor for the uncertainty analysis

An examination of the results from the filter assessment report [1] indicates that $D_{R}-200$, approximately. By solving this equation, a final equation can be found for the required decontamination factor given a flow rate through the filters as:

$$
D_{A}=5809 \cdot \frac{Q_{p}}{V}
$$

where the variables and units are as above, except that $Q_{p}$ is now in units of $\mathrm{kw}$.

Table 13 from the filter assessment [1] was used as a source for data for the decay heat loading as a function of time for the case where GSCS is operating. Three points were chosen for decay heating, all from the case based on the case with $10000 \mathrm{cfm}$ gas flow in the report in order to insure that a the fission product loading of the filters is not underrepresented. These are shown below, along with the calculated required decontamination factors $D_{A}$ for a $6000 \mathrm{cfm}$ gas flow (fog spray displacement and $3000 \mathrm{cfm}$ nitrogen) and a $3000 \mathrm{cfm}$ gas flow (either fog spray displacement or 3000 cfm nitrogen).

\begin{tabular}{lccc} 
& & \multicolumn{2}{c}{ Required $\mathrm{D}_{\mathrm{A}}$ for Filter Cooling } \\
Time & Decay Heat & $\frac{6000 \mathrm{cfm}}{3.6}$ & $\frac{3000 \mathrm{cfm}}{7.3}$ \\
$2 \mathrm{hr}$ & $7.7 \mathrm{kw}$ & 9.2 & 18.5 \\
$4 \mathrm{hr}$ & $19.1 \mathrm{kw}$ & 13.8 & 27.7 \\
maximum & $28.5 \mathrm{kw}$ & &
\end{tabular}

This information will be used in the filter uncertainty assessment later in this section. The decontamination factor $D_{A}$ represents the minimum decontamination of the effluent gases from when they exit the primary to when they get to the filters such that adequate filter cooling can be maintained without the actuation of the filter sprays. Filter spray actuation is assessed to cause filter damage.

A calculation similar to the above was carried out for the case where GSCS fails. For the first two hours of a GSCS failure accident, the core behavior is qualitatively similar and exhibits temperatures that are not a lot higher than those when GSCS operates. This is because, for the first two hours, the decay heat energy goes mainly into heating the graphite stack. If the decay heating is adjusted upward to account for 1008 core damage (versus 508 for the report case), and an extra 508 is added to the decay heat load to represent additional fission product release for the higher core temperatures, a rough idea can be gained into the required decontamination factors for the filters to succeed during the first few hours of a GSCS failure accident. The decay heat values are taken from the analysis with $20000 \mathrm{cfm}$ gas flow past the filters. 


\begin{tabular}{|c|c|c|c|}
\hline & & & $\begin{array}{l}\text { Required } D_{A} \text { for } \\
\text { Filter Cooling }\end{array}$ \\
\hline$\frac{\text { Time }}{1 \mathrm{hr}}$ & Report $Q_{p}$ & Adjusted $Q_{p}$ & $\frac{20000 \mathrm{cfm} \text { Nitrogen }}{1}$ \\
\hline $2 \mathrm{hr}$ & $15.5 \mathrm{kw}$ & $46.5 \mathrm{kw}$ & 13.5 \\
\hline $4 \mathrm{hr}$ & $27.6 \mathrm{kw}$ & $82.8 \mathrm{kw}$ & 24.1 \\
\hline
\end{tabular}

There is no case for $40000 \mathrm{cfm}$ nitrogen, so the above times can be halved to represent the faster purge of the building by the $40000 \mathrm{cfm}$ nitrogen injection. This is conservative because it also compresses the release time from the primary system by a factor of two. The identical calculation is performed with the results presented below.

$\begin{array}{llll} & & \begin{array}{l}\text { Required } D_{A} \text { for } \\ \text { Filter Cooling }\end{array} \\ \frac{\text { Time }}{1 \mathrm{hr}} & \frac{\text { Report } Q_{\mathrm{p}}}{15.5 \mathrm{kw}} & \frac{\text { Adjusted } Q_{\mathrm{p}}}{46.5 \mathrm{kw}} & \frac{40000 \text { cfm Nitrogen }}{6.8} \\ 2 \mathrm{hr} & 27.6 \mathrm{kw} & 82.8 \mathrm{kw} & 12.1\end{array}$

The decontamination factor at two hours is actually less for the $40000 \mathrm{cfm}$ nitrogen than for the $20000 \mathrm{cfm}$ because of the increased cooling provided by the additional gas flow. This is deceptive because tentative hydrogen mitigation system procedures call for the nitrogen flow rate to be reduced to $1000 \mathrm{cfm}$ from $40000 \mathrm{cfm}$ after one hour. These same procedures call for the reduction from $20000 \mathrm{cfm}$ to $1000 \mathrm{cfm}$ after two hours. Therefore, the above decontamination factors are clearly valid only for the first couple of hours into the accident.

The above decontamination factors can be used as a guide to determine an uncertainty distribution for the required decontamination factor $D_{A}$ from the outlet of the primary system to keep the filters adequately cooled for a given exhaust gas volumetric flow rate. This decontamination factor could come from any source (sprays, dump tank, etc.). Such distributions could be generated for every amount of fuel damage and every flow condition for the filters, but this would be splitting hairs based on the level of information that is known. Three such distributions will be generated:

1) the decontamination factor required to prevent filter spray actuation during the first two hours given that GSCS works,

2) the decontamination factor required to prevent filter spray actuation during the 24 hour APET analysis given that GSCS works, and

3) the decontamination factor required to prevent filter spray actuation during the first two hours given that GSCS does not work.

Distributions 1) and 2) will be generated assuming that the effluent flow rate is maintained at at least $3000 \mathrm{cfm}$ by either fog spray displacement, nitrogen flow, natural convection, or some combination of these for the 
entire duration of the accident. Distribution 3 ) is generated under the assumption that the nitrogen system operates at a minimum of $20000 \mathrm{cfm}$ during this time. There is no distribution for accidents when GSCS fails for the 2-24 hour period because the filter sprays are assessed to always be demanded for this accident.

\begin{tabular}{|c|c|c|c|}
\hline \multicolumn{4}{|c|}{ Required Decontamination Factors $D_{A}$ (see above) } \\
\hline Quantile & Dist. 1) & Dist. 2) & Dist. 3) \\
\hline 0.0 & 1 & 1 & 1 \\
\hline .10 & 10 & 15 & 15 \\
\hline .25 & 15 & 20 & 20 \\
\hline .50 & 20 & 30 & 30 \\
\hline .75 & 30 & 50 & 50 \\
\hline .90 & 50 & 75 & 75 \\
\hline .99 & 100 & 100 & 100 \\
\hline 1.0 & 200 & 200 & 200 \\
\hline
\end{tabular}

Each of these distributions represents the assessed $D_{A}$ that is required to prevent filter spray actuation. This, however, is only half of the story. The other half is to determine how frequently these $D_{A}$ values are obtained. This is discussed below.

The source term analysis considers as uncertainty issues the decontamination factors actually achieved by the fog spray system and the emergency dump tank. If either of these systems is effective enough to cause the filters to be adequately cooled, then the filtration function will be successful. The two are not additive, however, because, as the source term analysis points out, both of these systems are effective at decontaminating the same things. In other words, those radionuclides that are not trapped by the dump tank are not likely to be trapped by the sprays, either. For this reason, it is an either-or proposition.

The APET can determine whether or not the effluent flow is through the dump tank. This occurs in many small LOCAs and transients, although not effectively for large LOCAs because a significant flow bypasses the dump tank and flows straight into the confinement atmosphere. The APET can also determine whether or not the fog sprays are operating. These two are not dependent - that is, one can operate without the other. Thus, each of the above distributions becomes three - sprays only, dump tank only, and both sprays and dump tank operating. For the small number of accidents where neither is operating, the filters are assessed to be demanded.

In discussions with reviewers of this report, it was noted that there are two methods for quantifying the issue of filter uncertainty that are each simpler and more elegant than the one actually used for this analysis. However, these discussions occurred after the computation of the final APET calculations, and an examination of the actual obtained results showed a good agreement between the second of these methods and the actual results used in the analysis. For this reason, it was not felt to be useful to redo the analysis for the sake of a major improvement in "statistical elegance" if the results would change little. The two methods that could have been used are discussed below. 
The first and simplest method of determining filter performance involves sampling the distribution for the spray and/or dump tank decontamination factors required to prevent filter spray actuation directly. The "required DF" could be compared to the "obtained DF" as sampled for the source term analysis. These would be compared, and if the obtained DF is greater than the required DF, then the probability of filter success would be set to one for that observation. On the other hand, if the obtained DF is less than the required DF, then the probability of filter success would be set to zero for that observation. This method employs the concept of "zero/one" sampling as used in the NUREG-1150 study and is very easy to understand.

The second method for determining filter performance is conceptually quite similar to that used in this study, although it is easier to understand and more elegant than that used in this study. This method is based on the fact that the cumulative distribution function (CDF) for the "required DF" can be interpreted differently from the interpretation of the method above. In the method above, a sample of the distribution is taken directly and used in the analysis. Another interpretation could be as follows: the CDF represents a mapping between the actual DF obtained for the fog sprays or the dump tank and the probability that the filters will operate successfully. Thus, for distribution 1 above, which represents the decontamination factor required to prevent filter spray actuation during the first two hours given that the GSCS works, the CDF could be interpreted as stating that the filters will survive with a probability of 0.75 if either the dump tank or the fog sprays provide a DF of 30 . Similarly, the survival probability is 0.99 if $a$ DF of 100 is obtained. Using this interpretation of the CDF, it is possible to simply map the obtained DF into the probability that the filter sprays will not actuate on a sample by sample basis. While this method was not used directly in this analysis, it is conceptually similar to the method that was used and is described below.

For the method used in this study, the above probability distributions for the "DF required to prevent filter spray actuation" are combined with the probability distributions for fog spray and dump tank decontamination factors [5] to obtain the final probability distribution for adequate filter cooling. The mapping described above is carried out betore the samples are drawn (rather than after, as was the case above) by simply mapping the axes of the spray and dump tank DF CDFs described in Reference 5. This results in new CDFs for the probability that the filter sprays will not be demanded. These new CDFs were then sampled along with the spray and dump tank DF CDFs as separate random variables. The samples were restricted, however, in that a very high degree of correlation was forced between the appropriate source term variables and these filter variables. An examination of the reeults of this method showed that they were both qualitatively and quantitatively similar to those that would have been obtained had the second method above actually been used. The details of the construction of the filter issue CDFs are described below.

The probability distributions for the "DF required to prevent filter spray actuation" and the probability distributions for fog spray and dump tank decontamination factors distributions are combined as follows: The 
quantiles from the above distributions can be thought of as the probability that the filters are adequately cooled given that the stated decontamination factor is obtained. The source term distributions (probabilities of decontamination factors) are the probability that a stated decontamination factor is obtained. The quantile from the above distribution is then substituted for the stated decontamination factor in the source term distribution. A new distribution is now created that has as its quantiles those quantiles from the source term distributions and as its values the quantiles from the above distributions as related to each other by the appropriate decontamination factors. This new distribution represents the distribution for the probability that the filters are adequately cooled.

The above process was accomplished as follows: a 500 point ranked distribution was generated for each source term issue (decontamination factor). The decontamination factors from the above distributions were matched against the decontamination factors in the ranked distributions, and the quantile at which the match occurred is the quantile of the resulting distribution. This resulted in the following distributions, where FSND indicates the filter sprays are not demanded. An additional, fourth distribution is made below that measures the conditional probability that the filter sprays do not actuate for the entire accident given that they have not actuated during the first two hours during accidents where GSCS is successful. This is distribution 4) below, and FSNL-NE indicates that the filter sprays are not demanded late given that they have not been demanded early ("early" - first two hours, "late" - up to 24 hours).

\begin{tabular}{cc} 
Dist, 1) & Dump Tank \\
\hline Quantile & P(FSND) \\
\hline 0.0 & 0.0 \\
0.15 & 0.10 \\
0.20 & 0.25 \\
0.25 & 0.50 \\
0.35 & 0.75 \\
0.45 & 0.90 \\
0.56 & 0.99 \\
0.75 & 1.00 \\
1.0 & 1.00
\end{tabular}

\begin{tabular}{cc} 
Dist. 2) & Dump Tank \\
\hline Quantile & P( FSND $)$ \\
\hline 0.0 & 0.0 \\
0.20 & 0.10 \\
0.25 & 0.25 \\
0.35 & 0.50 \\
0.45 & 0.75 \\
0.50 & 0.90 \\
0.56 & 0.99 \\
0.75 & 1.00 \\
1.0 & 1.00
\end{tabular}

\begin{tabular}{|c|c|}
\hline 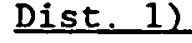 & \\
\hline Quantile & $\underline{P(\text { FSND }}$ \\
\hline 0.0 & 0.0 \\
\hline 0.42 & 0.10 \\
\hline 0.50 & 0.25 \\
\hline 0.58 & 0.50 \\
\hline 0.75 & 0.75 \\
\hline 0.82 & 0.90 \\
\hline 1.0 & 0.99 \\
\hline
\end{tabular}

\begin{tabular}{cc} 
Dist. 2) & Fog Sprays \\
\hline Quantile & P(FSND $)$ \\
\hline 0.0 & 0.0 \\
0.50 & 0.10 \\
0.58 & 0.25 \\
0.75 & 0.50 \\
0.82 & 0.75 \\
0.91 & 0.90 \\
1.0 & 0.99
\end{tabular}




\begin{tabular}{|c|c|}
\hline Quantile & $\underline{P(\text { FSND })}$ \\
\hline 0.0 & 0.0 \\
\hline 0.06 & 0.10 \\
\hline 0.10 & 0.25 \\
\hline 0.15 & 0.50 \\
\hline 0.26 & 0.75 \\
\hline 0.37 & 0.90 \\
\hline 0.56 & 0.99 \\
\hline 0.75 & 1.00 \\
\hline 1.0 & 1.00 \\
\hline
\end{tabular}

\begin{tabular}{cc} 
Dist. 2) & Both Systems \\
\hline Quantile & P(FSND ) \\
\hline 0.0 & 0.0 \\
0.10 & 0.10 \\
0.15 & 0.25 \\
0.26 & 0.50 \\
0.37 & 0.75 \\
0.46 & 0.90 \\
0.56 & 0.99 \\
0.75 & 1.00 \\
1.0 & 1.00
\end{tabular}

\begin{tabular}{cc} 
Dist. 3) & Dump Tank \\
\hline Quant.ile & P( FSND_) \\
\hline 0.0 & 0.0 \\
0.20 & 0.10 \\
0.25 & 0.25 \\
0.35 & 0.50 \\
0.45 & 0.75 \\
0.50 & 0.90 \\
0.56 & 0.99 \\
0.75 & 1.00 \\
1.0 & 1.00
\end{tabular}

\begin{tabular}{cc} 
Dist. 4). Dump Tank \\
\hline Quantile & P( FSNL-NE) \\
\hline 0.0 & 0.0 \\
0.20 & 0.40 \\
0.25 & 0.50 \\
0.35 & 0.67 \\
0.45 & 0.83 \\
0.56 & 0.99 \\
0.75 & 1.00 \\
1.0 & 1.00
\end{tabular}

\begin{tabular}{cc} 
Dist. 3) & Fog Sprays \\
\hline Quantile & P(FSND $)$ \\
\hline 0.0 & 0.0 \\
0.50 & 0.10 \\
0.58 & 0.25 \\
0.75 & 0.50 \\
0.82 & 0.75 \\
0.91 & 0.90 \\
1.0 & 0.99
\end{tabular}

\begin{tabular}{cc} 
Dist. 4) & Fog Sprays \\
\hline Quantile & P( FSNL-NE ) \\
\hline 0.0 & 0.0 \\
0.50 & 0.40 \\
0.58 & 0.50 \\
0.75 & 0.67 \\
0.82 & 0.83 \\
1.00 & 0.99
\end{tabular}

\begin{tabular}{|c|c|}
\hline Quantile & $P($ FSND $)$ \\
\hline 0.0 & 0.0 \\
\hline 0.10 & 0.10 \\
\hline 0.15 & 0.25 \\
\hline 0.26 & 0,50 \\
\hline 0.37 & 0.75 \\
\hline 0.46 & 0.90 \\
\hline 0.56 & 0.99 \\
\hline 0.75 & 1.00 \\
\hline 1.0 & 1.00 \\
\hline
\end{tabular}

\begin{tabular}{cc} 
Dist. 4) & Both Systems \\
\hline Quantile & P(FSNL-NE) \\
\hline 0.0 & 0.0 \\
0.10 & 0.40 \\
0.15 & 0.50 \\
0.26 & 0.67 \\
0.37 & 0.83 \\
0.56 & 0.99 \\
0.75 & 1.00 \\
1.0 & 1.00
\end{tabular}




\section{C.11.4 Application of Results}

The research and the discussion above are in general agreement that, for the accidents analyzed, the probability that filter integrity will be maintained are quite good. The conditions noted that are expected to lead to filter degradation are low frequency events that could probably be treated conservatively for the APET analysis without a significant impact on the overall results. It is significant to note that no scenarios or mechanisms were identified that would lead to significant filtration efficiency degradation without filter failure. Filtration seems to be, at any given time, almost zero or essentially at the design efficiency with little in between.

The above methodology for analysis of filter performance in the APET analysis can be summarized as follows: first, the approximate filter fission product load was determined based on the amount of fuel damage and the presence of other scrubbing mechanisms such as the dump tank or the fog spray system. After this, the possibility of filter spray actuation was considered. Finally, the operating filter bank and overall filtration efficiency will be determined.

Three divisions are used for the amount of filter loading: 1) filter loads where filter cooling is not an issue; 2) filter loads that are high enough to demand filter sprays if not properly cooled; and 3) filter loads great enough to fail the filters. These generally correspond to: 1) situations where only $1 / 16$ of the core is damaged; 2) accidents where the GSCS is fully operational; and 3.) accidents more serious than the above, respectively. The radionuclide load from a $1 / 16$ core accident is expected to be very small and should never heat the filters to the point where cooling would be required. On the other hand, the radionuclide releases from the third class of accidents is considered likely to fail the filters for this analysis as described above.

The final item in the filter performance elicitation is the issue of filter burning. This will be a very low frequency event because, even if sufficient cooling is not provided by the nitrogen purge and/or the spray displacement, additional filter sprays are also provided which must fail before filter burning becomes possible. The filters are assessed to burn whenever they are not adequately cooled by either the gas flow or by the filter spray system. As a low frequency event, it is possible to treat filter burning with a conservative model in which all trapped radionuclides are released to the environment upon filter burning. This is conservative because previous filter burn incidents have shown significant plateout of fission products in the "soot" adhering to the walls after the event. This plateout is ignored for this analysis.

An additional conservative assumption in this analysis is that, should filter cell $D$ fail (due to loading or filter soaking), the operators would be either unable or unwilling to re-align the filter system back to cells $A$ and $B$ (its alignment during normal operation). This assumption is made based on the fact that the $N$ Reactor Emergency Response Guides [3] do not require the operators to monitor filter performance and do not present a procedure for the re-alignment. Such a procedure could reduce releases 
from severe accidents such as those in which the GSCS is not fully operational GSCS or the fog sprays are not operational.

The probability distributions for the cases listed above from cases 1 , 3 and 4 were used directly in the APET uncertainty analysis. Case 4 was used in place of case 2 because it represented the probability that the filters would survive given that they had survived the first two hours of the accident. This was a more useful quantity for input to EVNTRE based on the way it handles its input preprocessing tasks [6].

For a summary of the issues related to filtration efficiency, the reader is directed to section 4.3.11 of this report. That section summarizes the mechanisms for filter failure that were considered and gives a qualitative description of why certain (possibly conservative) assumptions were used in the analysis. 


\section{C.12 LERF PLASTIC LINER FAILURE}

\section{C.12.1 Issue Definition}

The plastic liner of the LERF would be expected to fail with some small probability in any accident sequence. Failure of the top section of the plastic liner would be expected to release radionuclides to the atmosphere, while a failure of the bottom section of the plastic liner would be expected to release contaminated liquids to the ground. This event is expected to be independent of other events in the accident progression since the LERF is significantly removed from the confinement building and filled only by a weir system which will passively divert flow away from the LERF once it has filled up.

This is not expected to be a significant contributor to the overall risk because it is expected to be a low frequency event, and several other events with relatively high frequencies $(-1.0 \mathrm{E}-2)$ such as the failure to isolate the confinement release substantial quantities of radionuclides to the atmosphere. However, in order to lend credibility to the APET analysis, a realistic estimate of the failure probability of the plastic liner needs to be developed. If the assumption that LERF failure is a small contributor to the overall risk is indeed true, then a probability distribution need not be developed (a mean value will be sufficient). Otherwise, a probability distribution will be needed.

\section{C.12.2 Issue Resolution Process}

The above stated issue was presented by telephone to G. A. Coles of Westinghouse Hanford on July 9, 1989. This was not intended as a formal elicitation, but rather as a request for preliminary information that might be used in a future elicitation. His investigation revealed that considerable research had already been done to determine a LERF failure rate. The results of this research are summarized in the writeup below. In addition, judgements have been made during the research process, and $a$ number of possible failure scenarios have been considered and discarded. These are also documented below. This information serves as a basis for the uncertainty analysis.

\section{C.12.3 Issue Analysis and Resolution}

\section{Background Information}

1. R. A. Paasch, et al., Project $\mathrm{H}-797 \ldots \mathrm{N}$ Reactor 1312-N Liquid Effluent Retention Facility, WHC-SP-0221, Westinghouse Hanford, January 1988 .

2. Westinghouse Hanford, Emergency Response Guides, WHC-CM-4-1, Volume 2, June 1981, as updated through June 1989 
3. G. A. Coles, $N$ Reactor Confinement Systems Sequence Analysis Including Recovery, WHC-SD-NR-RA-001, Westinghouse Hanford, March 1990 .

\section{Discussion of LERF Failure}

The N-Reactor Liquid Effluent Retention Facility is an unusual structure for nuclear power plants [1]. It consists of a very large excavated basin which is lined with a thick, flexible, stretchable plastic liner. A second layer of this liner material forms a flexible top for the facility. The LERF is designed to hold at least $90 \%$ of the radioactive liquids generated by the plant during a postulated plant accident. The 908 figure is based on the ECCS flow prescription from ERG-B as found in the $\mathrm{N}$ Reactor Emergency Response Guides [2]. A ventilation system allows gases generated by the liquids in the LERF to be released to the environment through charcoal filters. Liquids enter the facility through a weir and standpipe system that passively diverts the liquids to other facilities or, as a last resort, to the ground once the LERF is full. Thus, failure of the LERF cover system by overpressurization is not considered credible.

LERF failure is not expected to be a significant contributor to the overall $\mathrm{N}$-Reactor risk because it is expected to be a low frequency event, and several other events with relatively high frequencies ( $-1.0 \mathrm{E}-2)$ such as the failure to isolate the confinement release substantial quantities of radionuclides to the atmosphere. However, in order to lend credibility to the APET analysis, a realistic estimate of the failure probability of the plastic liner is needed.

Since this facility is a one-of-a-kind structure, no database of failure rates for comparable structures exists. During the generation of the confinement system cutsets, it was decided that, of the systems for which documented failure rates are available, the LERF most closely resembles a thin-wall tank. This assessment seems conservative since the LERF structure is well-supported on all sides (except, of course, the top) by earlien excavation. In addition, the LERF liner and top material is a thick, stretchable and flexible plastic which, since it is well supported, should be more resistant to failures than the typical free standing metal tank. A failure rate of 1.0E-6 per hour with an error factor of 100 [3], which has been used to model other thin wall tanks at $N$ Reactor, was selected to model demand-related random failures of the LERF.

An additional concern for this system is the concept of a mission time for the LERF. A number of possible mission times make sense for this system, ranging from 10 hours to 53.5 days. Many of these possible mission times are based on the amount of $I^{131}$ which would be available for release to the atmosphere should the LERF top fail during this time. The justifications for these possible mission times are as follow:

10 hours the MACCS consequence modeling package models radioactive plant releases only for 10 hours. 
24 hours all actions to fill the LERF would have been completed by this time, and from this time forward, the LERF would act as a simple storage basin. In addition, severe accidents have historically been modeled for only 24 hours because offsite equipment and expert personnel could be expected to begin to arrive by this time.

8.0 days this is the half-life of $I^{131}$. By this time, a number of effects would occur to significantly reduce the amount of iodine in the liquid effluent. First, the iodine could undergo radioactive decay. Second, gaseous iodine will evolve from the pool and subsequently be removed by the filtered LERF vents. Third, methyl iodide may form from the interaction between the dissolved iodine and the plastic liner or other compounds in the liquid effluent. Methyl lodide could escape by diffusing through the plastic LERF cover. Each of these effects would reduce the amount of iodine remaining in the liquid effluent for release should the LERF fail.

26.7 days this is the time $I^{131}$ takes to decay to 108 of its initial concentration. The mechanisms discussed above would be expected to reduce the amount of iodine remaining in the liquid effluent to substantially below this level. Also, by this time, decontamination procedures could be started to reduce the volume of liquid effluene available for release should the LERF fail.

53.5 days this is the time $I^{131}$ takes to decay to 18 of its initial concentration. The mechanisms discussed above would be expected to reduce the amount of lodine remaining in the liquid effluent to substantially below this level. Also, by this time, decontamination procedures could be started to reduce the volume of liquid effluent available for release should the LERF fail.

It has been noted that most events which would stress the LERF occur during the filling process, and that once it is filled, the LERF acts as a passive storage basin. The LERF filling operation is completed within the first 24 hours in all accident sequences because of its design capacity. Thus, the mission time selected for use in this analysis is 24 hours, and the conditional probability of the LERF failing during an accident is assessed to be $2.4 \mathrm{E}-5$ with an error factor of 100 .

A comparison of this failure rate with that of other components in the $\mathrm{N}$-Reactor PRA indicates that this failure rate is similar to that used to model the Emergency Dump Tank, the silo, the GSCS surge tank, and several fuel storage tanks in the confinement system cutsets. This was assessed to be a conservative treatment of the phenomena involved. In addition, this 
failure frequency is much lower than that of the dominant cause of liquid releases from $N$ Reactor: the failure of the lift station diesel to start (-3.OE-02 over the accident). Thus, the exact value of the failure rate is unimportant as long as it is known to be much less than that of other confinement failure mechanisms.

To provide perspective, it should be noted that the error factor of 100 on a lognormal distribution indicates that there is a reasonable probability that the true value of the failure probability could be as much as a factor of 100 higher or lower than the value stated. While this is a one of a kind structure, it seems absurd to indicate that the uncertainty in its failure rate is so great that the answer is known. only to within four orders of magnitude!

Additional consideration was given during the analysis to the probability that pre-existing breaches of the LERF cover are present. It has been recognized by Westinghouse Hanford that the nature of the LERF makes it impossible to fully test, because the liner is designed as a single use item. Thus, the possibility of pre-existing failures can not be ruled out through testing. However, the LERF is considered to be of significant value in reducing the magnitude of any offsite release of radioactivity which might occur during a severe accident at $\mathrm{N}$-Reactor, and because of this, very stringent quality assurance requirements were imposed during the construction of the facility [1]. For this reason, construction-related pre-existing LERF failures are not considered further in this assessment.

Pre-existing failures could occur through the action of weather or maintenance activities on the LERF cover. The dominant failure mode for a pre-existing failure is assessed to be weakening or failure of the seams which connect the separate sheets of high density polyethylene to form the large continuous sheets required for the top and bottom of the facility. These seams could degrade with age, but would not be expected to fail until stressed during the filling of the facility. Failures that occur during the filling process are assumed to be included in the failure on demand frequency and its associated large error factor.

Other failure modes, such as punctures and tears, are also possible, but would not be expected to occur if proper maintenance procedures are used. The thickness ( $60 \mathrm{mils}$ ) and toughness of the plastic liner and top material are expected to all but preclude natural causes from producing punctures and tears in the material. In addition, such failures would be expected to allow only slow leakage rates from the bag and are not expected to propagate. Thus, pre-existing punctures and tears of the plastic liner are not considered further.

Additional maintenance-related LERF failures are possible, such as the failure to remove the blind flanges on the LERF entrance after testing or maintenance, but these would cause a failure of the function which diverts ECCS flow from the Liquid Waste Disposal Facility to the LERF. For this reason, these failures are not considered in this assessment. 


\section{C.12.4 Application of Results}

The above mentioned uncertainty distribution (a lognormal distribution and error factor) were used directly in the APET analysis as the probability distribution for the event entitled "Does the 1312N LERF function properly?" (APET event 81). 


\section{C.13 SEISMIC ASSESSMENT OF CONFINEMENT STRUCTURES}

\section{C.13.1 Issue Definition}

The external events core damage analysis did not consider the failure of structures that were not directly involved in producing core damage. However, a number of these structures are important in the retention of radionuclides within the confinement system and the consequence models. These structures include the emergency dump tank, the hydrogen mitigation nitrogen system, and the filter release stack. Probabilities for these structures to fail are needed.

In addition, the core damage sequences determined in the external events core damage analysis do not separate out various effects, such as confinement building failure and the loss of electrical power, for the various accident sequences due to the structure of the hierarchy used. These effects need to be separated out.

\section{C.13.2 Issue Resolution Process}

The issue as stated above was presented to M. P. Bohn of Sandia National Laboratories for analysis because of his extensive experience modeling seismic failures for $\mathrm{N}$ Reactor. The latter portion of the above issue description was solved using the computer analysis methods of the external events analysis [1]. The results of that analysis are presented in reference [2] below. The former portion of the issue description was elicited orally. The results of that elicitation are presented below.

\section{C.13.3 Issue Analysis and Resolution}

\section{Background Information}

1. Lambright, J. A., et al, Analysis of Core Damage Frequency Due To External Events at the DOE $N$ Reactor, SAND89-1147, Sandia National Laboratories, 1990.

2. D. M. Kunsman, et. al., Level III Probabilistic Risk Assessment for N Reactor, SAND89-2102, Sandia National Laboratories, April 1990.

\section{Discussion}

The $\mathrm{N}$ Reactor External Events PRA analysis has been being conducted in parallel to the Internal Events Level II/III analysis for some time. The external events analysis is now complete, with a draft report out for review [1]. The incorporation of the seismic results from that study into the overall Level II/III PRA and, specifically, into the Accident Progression Evrnt Tree (APET) analysis has been a matter of some question. This section documents the incorporation process. 
Many of the seismic results as presented in that report do not lend themselves well to incorporation into the APET analysis. This is because different type of failures (from the APET perspective) are lumped into a single external events plant damage state because they caused the same type of a core damage scenario. One example of this is the MPTR (multiple pressure tube rupture, known as the RVR, or Reactor Vessel Rupture in the external events report) event. This event could be caused by either the relative motion between the core itself and the surrounding shield walls, or by the gross failure of the $105 \mathrm{~N}$ building. These sequences are similar from a core damage perspective because they both cause general failure of all pipes leading to the core. They are very different, however, from the APET perspective because one involves confinement failure while the other does not.

To remedy this situation, 36 new plant damage states were defined during consultations between the accident progression analyst and $M$. $P$. Bohn to differentiate between those criteria that are important to the APET analysis. These 36 plant damage states are defined in reference [2]. The frequencies of these 36 plant damage states were then calculated and are being used to determine split fractions for confinement failure and AC power availability in the APET analysis. These are also documented in reference [2].

The $1 / 16$ core accidents (T4, LOSP-T4, S4) will be ignored in the APET analysis because they have both low frequencies $(<1.58$ of the total frequency) and also involve low radioactive releases since only $1 / 16$ of the core is involved. This combination will make them a trivial contributor to risk, so they will be ignored.

An examination of the cause of ECCS failure for the cutsets used to generate the seismic core damage sequences revealed that more than 998 of all core damage (except in the RVR sequence) was caused by failure of tanks required to supply ECCS water to the core - especially the silo - at seismic ground accelerations above the $N$ Reactor safe shutdown earthquake $(0.25 \mathrm{~g})$. The use of other ECCS water supplies is addressed in Reference 1. The main branch in the APET that causes a total water supply failure is the failure of all three ECCS High Lift Diesel Pumps (HLDP). This branch will be used to indicate to the APET that a total failure to supply water to the core has occurred. These tank failures will also affect High Pressure Injection (HPI) availability, so the APET seismic analysis will consider HPI as failed for these sequences as well.

Finally, there are no accident sequences in which all V-4 valves fail to open. Thus, the primary system depressurizes in all cases. This means that the main coolant pumps can not provide cooling to the core for any significant period of time and will be considered to be shut down for all APET seismic analysis cases.

Three failures not considered in the external events PRA report can have an significant impact on the APET results. These are the failure of the Emergency Dump Tank (EDT), the Hydrogen Mitigation System (HMS), and the filter release stack. These were each considered from an expert 
judgement perspective during an interview with the elicitee. The results of that interview are summarized below.

The failure probabilities for these structures are dependent on the peak ground acceleration (PGA) of the seismic event. Thus, there must be a differentiation made in the APET analysis between accident sequences based on ground acceleration. Consideration of the various sequences indicated that transients ( $T$ ) and losses of offsite power (LOSP) were most prevalent at PGA levels around $0.3 \mathrm{~g}$; Building $182 \mathrm{~N}$ failures (B182) and small LOCAs (S) were dominant at PGA levels around 0.5g; and large LOCAs (L) and RVR events were dominant at PGA levels above $0.5 \mathrm{~g}$. These three PGA levels will be used in the APET analysis, and will be noted here as low, medium and high $P G A$, respectively, and are summarized as

\begin{tabular}{|c|c|c|}
\hline Ground Acceleration Class & $\begin{array}{l}\text { Representative } \\
\text { Peak Ground } \\
\text { Acceleration }\end{array}$ & $\begin{array}{l}\text { Mean Annual Frequency } \\
\text { of Exceedence } \\
\end{array}$ \\
\hline $\begin{array}{l}\text { N Reactor Safe Shutdown } \\
\text { Earthquake (SSE) } \\
\text { low ground acceleration } \\
\text { medium ground acceleration } \\
\text { high ground acceleration }\end{array}$ & $\begin{array}{l}0.25 \mathrm{~g} \\
0.3 \mathrm{~g} \\
0.5 \mathrm{~g} \\
0.7 \mathrm{~g}\end{array}$ & $\begin{array}{l}5.0 E-05 * \\
3.5 E-05 \\
5.6 E-06 \\
8.8 E-07\end{array}$ \\
\hline
\end{tabular}

* Note: $5.0 \mathrm{E}-05=5.0 \times 10^{-5}$, and should be interpreted as follows: based on the mean seismic hazard curve, it is expected that a seismic event of magnitude $0.25 \mathrm{~g}$ or greater would be expected to occur with a probability of 5.0E-05 each year, or equivalently, approximately once each 20,000 years.

The Emergency Dump Tank (EDT) rests on a concrete base mat and is surrounded by a reinforced concrete berm. During the $\mathrm{N}$ Reactor seismic upgrade program, expansion bellows are to be added to the line proceeding to the dump tank to increase the probability that the EDT will survive during a scismic event. The elicitee stated that many typical anchored tanks fail at PGA levels near $0.5 \mathrm{~g}$ (twice the $\mathrm{N}$ Reactor SSE), and the following mean probabilities were assessed to be appropriate for EDT failure.

\begin{tabular}{ll} 
PGA Level & Mean Failure Prob. \\
\cline { 2 - 2 } Low & 0.01 \\
Medium & 0.50 \\
High & 0.99
\end{tabular}

When asked whether the fact that the EDT is on a separate foundation from the confinement building would lead to pipe failure before tank failure, the elicitee replied that this would not significantly effect his assessment of the tank failure probabilities, so the above distribution is appropriate for the combination of the EDT and its piping. 
It was noted that the HMS nitrogen system has yet to be completely installed. This makes a detailed seismic analysis impossible. However, some broad statements can be made and used in the APET analysis. While specific seismic qualification will not be sought for the HM nitrogen tanks, the tanks are expected to be securely anchored to their stands and the ground. The probabilities used for the EDT above are again appropriate for the HM nitrogen tanks. The supplementary piping and different foundations discussion from that case was found by the elicitee to be appropriate for this case as well.

The third item on which the elicitee was elicited was the filter release stack. This stack is approximately 200 feet tall and of a brick and mortar construction. The elicitee indicated that chimneys almost always fail approximately one-third of the way up from the bottom. He indicated that the stack was very unlikely to fall in upon itself and cause a flow blockage, and that a rough estimate for the PGA level at which it would become likely to fail would be $0.5 \mathrm{~g}$. The following probabilities were assessed for filter release stack failure:

\begin{tabular}{ll} 
PGA Level Mean Failure Prob. \\
\cline { 2 - 2 } Low & 0.05 \\
Medium & 0.50 \\
High & 0.95
\end{tabular}

\section{C.13.4 Application of Results}

The above results have been implemented in events 17,18 and 23 of the APET analysis. These events are:

17 Does the emergency dump tank function?

18 Does the filter release stack remain intact?

23 In what mode does the hydrogen mitigation system operate?

The results of this elicitation are point estimates and, therefore, are not sampled in the Latin Hypercube uncertainty analysis. 


\section{APPENDIX D}

SOURCES OF MISCELIANEOUS EVENT TREE DATA

\section{D.1 INTRODUCTION}

The Accident Progression Event Tree (APET) for $N$ Reactor is a distillation of a vast amount of data into a useful logic structure. The results of the event tree analysis, however, are critically dependent on the data used. Appendix $C$ of this report presented detailed information concerning the treatment of uncertainty issues in the tree, and the PRA report [D-1] discusses how the Level $I$ internal and external events and confinement systems analyses were incorporated into the event tree. Neither of those places, however, shows the derivation of the frequencies of occurrence for loss of cooling accident (LOCA) initiating events as a function of location in the plant. The internal events Level $I$ analysis [D-2] postulated these initiating events but did not need to divide the occurrence of them into the locations of that occurrence. As discussed in the description of Event 5 of the APET in Chapter 3, different break locations can potentially alter the accident progression so that we must separate the different locations. Furthermore, the LOCAs considered here are those which are initiating events and not those which are induced during the accident progression. Scenarios involving the latter type of LOCA are modeled explicitly in the tree logic structure.

The frequency of such initiating events varies from location to location within the plant because the amount of piping and the number of valves is location specific. For the sake of project traceability, the derivations for the split fractions used in "Event 5 - Break Location" are presented in this appendix.

Recall from Chapter 3 that Event 5 has twelve branches:

Event 5 - Break Location

Branch ABBREV. DESCRIPTION

(1) None No LOCA occurred

(2) Pent Pressurizer penthouse

(3) SG1-5 Steam generator cells 1-5

(4) SG6 Steam generator cell 6

(5) 109 in Building $109 \mathrm{~N}$ pipe gallery inlet header

(6) 109out Building 109N pipe gallery outlet header

(7) Core Single pressure tube rupture inside the core

(8) 105-10 10" outlet header in Building 105N upstream of CV-3

(9) 105-26 26" outlet header in Building $105 \mathrm{~N}$ downstream of $\mathrm{CV}-3$ 
RVR

105up
A seismic multiple pressure tube rupture (MPTR) event

Inlet riser in Building $105 \mathrm{~N}$ upstream of $\mathrm{CV}-5$

The frequencies of occurrence of each of these branches, the split fractions for the event, are determined below for large and small LOCAs, where the internal events Level I PRA [D-2] used an equivalent break diameter of $4^{\prime \prime}$ as being the dividing size between the two. We continue with that differentiation.

\section{D.2 PRIMARY SYSTEM SPLIT FRACTIONS FOR IARGE LOCAS}

Presented here is the derivation of the split fraction probabilities for APET Event 5 for large LOCA initiating events. As mentioned in Chapter 3 , discussions with the external events analysts revealed that, with one exception, the relative frequencies between the different locations would be the same for the seismic events as for other initiators. The exception is the high $g$ earthquake which causes the multiple pressure tube rupture event (see Reference D-3). If this occurs, branch 11 of APET event 5 has a probability of 1 , and the other branches have probabilities of zero. If the multiple pressure tube rupture scenario does not occur, then branch 11 of APET event 5 has a probability of zero.

The rest of the branch probabilities are determined by comparing the relative amount of piping and number of valves in the various locations where a break at least $4^{\prime \prime}$ in diameter could occur. The amount of piping in the various locations are from Reference $D-4$, and the valve information is from Table 4-3 of Reference D-2. In Section 4.3.1 of the latter, a failure frequency for all of the large piping in the primary system is given as 2.1 $\mathrm{x} 10^{-5} / \mathrm{yr}$ and for all large valves in the primary system, $6.0 \times 10^{-4} / \mathrm{yr}$. Thus, the initiating event frequency for large LOCAs is 3.48 from the piping and 96.68 from the valves. That is, given that a large LOCA has occurred at $N$ Reactor, and it is not a multiple pressure tube rupture caused by a high $g$ earthquake, 96.68 of the time it will be the result of valve failure and 3.48 of the time it will be the result of piping failure. Below, the split fractions for the two types of breaks will be derived separately for each branch and then combined. Each branch will be considered in order.

By definition, we are studying a LOCA so the probability of the first branch is zero. Therefore,

$$
P_{b 1}=0.0
$$

As to the piping in the pressurizer penthouse, the information in Reference D-4 does not differentiate what portion of the primary system piping is inside the pressurizer and what portion is outside it, in the pipe gallery. It just gives the total amount of piping that to and from the pressurizer. We assume a $50 / 50$ split between that which is actually 
within the penthouse and that which is part of the pipe gallery. In addition, there are two of the 103 large primary system valves in the pressurizer penthouse; they are the PRV-205 valves. Using the hourly failure rates provided in the internal events Level I PRA [D-2] and the pipe length information from Leitz [D-4], we can compute the contributions to the split fraction probability from the pipes and the valves and then combine them for the total branch conditional probability:

$$
\begin{aligned}
& \mathrm{P}_{\mathrm{b} 2} \text { for pipes }=2.7 \mathrm{E}-3 \\
& \mathrm{P}_{\mathrm{b} 2} \text { for valves }=0.019 \\
& \mathrm{P}_{\mathrm{b} 2}=0.0027 \times 0.034+0.019 \times 0.966-0.018
\end{aligned}
$$

The steam generator cell piping data come directly from Reference D-4, and Table 4-3 of Reference D-2 lists the valves for the five operating steam generator cells. (One cell is always in standby.) As a break in one of these can potentially load the confinement differently than a break in another and the same cell is not always in standby, we do not choose to always keep one of the cells in standby. Rather, we allow the identity of the cell which is not in use to rotate among all six cells by averaging the valve break probabilities of five cells across all six cells. Thus,

$$
\begin{aligned}
& P_{b 3} \text { for pipes }=0.344 \\
& P_{b 3} \text { for valves }=0.413 \\
& P_{b 3}=0.410 \\
& P_{b 4} \text { for pipes }=0.069 \\
& P_{b 4} \text { for valves }=0.083 \\
& P_{b 4}=0.082
\end{aligned}
$$

The pipe gallery data for the large inlet and outlet piping again come directly from the Leitz work [D-4] with the exception that, as noted in the description for branch 2 above, half of the large pressurizer line is assumed to be in the penthouse and half here in the pipe gallery, as part of the outlet manifold. There are no large valves in these locations.

$$
\begin{aligned}
& P_{b 5} \text { for pipes }-0.074 \\
& P_{b 6} \text { for pipes }=0.093 \\
& P_{b 5}=0.003 \\
& P_{b 6}=0.004
\end{aligned}
$$

The pressure tubes within the core individually are not large pipes. Nor are there any large valves within the core. Hence,

$$
P_{b 7}=0.0
$$

Branches 8 and 9 of APET event 5 distinguish between that part of the outlet header in 105 that is inside the CV-3s and that part that is outside. Some of the lines in Reference $D-4$ are clearly inside, when compared to a PCS schematic flow diagram, but the discussion in the reference does not differentiate the outlet header itself. As nearly all 
of it is inside the CV-3 placement, we assume for this analysis that 958 of it is inside and only 58 is outside the valve. Both the V2 and V5 valves are between the outlet riser and the CV3s so they are branch 9. Rupture of the CV3s themselves act as if the break were outside the CV3s--a branch 8 result.

$$
\begin{aligned}
& P_{b 8} \text { for pipes }-0.195 \\
& P_{b 8} \text { for valves }-0.175 \\
& P_{b 8}=0.176 \\
& P_{b 9} \text { for pipes }-8.3 \mathrm{E}-3 \\
& P_{b 9} \text { for valves }-0.155 \\
& P_{b 9}=0.150
\end{aligned}
$$

The information for the inlet riser in 105 comes directly from Reference $\mathrm{D}-4$. The information, however, does not differentiate between those portions which are downstream of the $C V-5$ valves and those portions which are upstream. The amount of piping appears about the same from schematics so we assume a $50 / 50$ split of the data between portions downstream of the CV-5 valves (branch 10) and upstream (branch 12, below). The rupture of the CV5s in effect is the same as a pipe break downstream of the CV5s.

$$
\begin{aligned}
& P_{b 10} \text { for pipes }=0.107 \\
& P_{b 10} \text { for valves }=0.155 \\
& P_{b 10}=0.153
\end{aligned}
$$

As noted above, branch 11 is used for a particular seismic event. For the multiple pressure tube rupture event, the conditional probability of this branch is unity, and the other branches have conditional probabilities of zero. If the event does not occur, the more general case, then:

$$
P_{b 11}=0.0 \text {. }
$$

For branch 12, see the description of branch 10 above; there are no valves here, however.

$$
\begin{aligned}
& P_{b 12} \text { for pipes }=0.107 \\
& P_{b 12}=0.004
\end{aligned}
$$

\section{D.3 PRTMARY SYSTEM SPLIT FRACTIONS FOR SMALL LOCAS}

Just as the previous section of this appendix presented the split fractions for the twelve branches of event 5 of the APET for large LOCA initiating events, this section presents the same information for small LOCA initiating events.

The frequency for small LOCAs is $0.23 / \mathrm{yr}$ of which $0.22 / \mathrm{yr}$ is from the spurious opening of relief valves, $0.0065 / \mathrm{yr}$ is due to valve breaks, $0.002 / \mathrm{yr}$ is due to pipe breaks external to the core, and $1.0 \mathrm{E}-5$ results 
from pressure tube breaks (see Section 4,3,2 of Reference D-2). There are eight relief valves which could spuriously open: one per steam generator cell and the RV-2-1 and -2 valves which are between the $10^{\text {" }}$ outlet header but inside the CV3s. (Actually, only five of the steam generator relief valves see primary pressure at a time, so in keeping with the development of the probabilities for the large LOCAs, the failure probability of five steam generator cells will be averaged over the six.)

As to the valves, there are 1109 of them of which 1003 are the V11s, one per pressure tube at the core inlet. There are 48 additional valves at the core inlet and 16 more valves at the core outlet. The remaining forty-two valves are found throughout the confinement locations and will not be considered further.

The core is also the location for most of the piping. Obviously all of the pressure tubes themselves are located within the core region, and 99.88 of the rest of the small diameter piping is around the core. 49.38 of it is on the outlet of the core and 50.78 on the inlet (see Reference D-4).

Therefore, for breaks equal to or less than $4^{n}$,

$$
\begin{aligned}
& \mathrm{P}_{b 1}=0.0 \\
& \mathrm{P}_{b 2}=0.0 \\
& \mathrm{P}_{b 3}=0.602 \\
& \mathrm{P}_{b 4}=0.120 \\
& \mathrm{P}_{b 5}=0.0 \\
& \mathrm{P}_{b 6}=0.0 \\
& \mathrm{P}_{b 7}=4.4 \mathrm{E}-5 \\
& \mathrm{P}_{10}=0.246 \\
& \mathrm{P}_{b 9}=0.0 \\
& \mathrm{P}_{b 10}=0.032 \\
& \mathrm{P}_{b 11}=0.0 \\
& \mathrm{P}_{b 12}=0.0
\end{aligned}
$$

\section{D.4. PRIMARY SYSTEM SPLIT FRACTIONS FOR TRANSIENTS}

By definition, there is no loss of integrity of the primary system in the case of a transient inftiating event. Thus, the probability of branch 1 of event 5 for transient initiators is 1.0, and the probabilities of all of the other branches are zero. (Please note that a transient which later progresses into an induced loss of PCS integrity is treated in the tree logic structure itself. As stated in the introduction to this appendix, we are here considering only the different possible locations of the initiating event.) 


\section{5. REFERENCES}

D-1. D. M. Kunsman, et al., Level III Probabilistic Risk Assessment for $N$ Reactor, WHC-MR-0045, SAND89-2102, Westinghouse Hanford, April 1990.

D-2. M. D. Zentner, et al., N Reactor Level 1 Probabilistic Risk Assessment: Final Report, WHC-EP-0322, Westinghouse Hanford Co., August 1988.

D-3. J. A. Lambright, et al., Analysis of Core Damage. Frequency Due to External Events at the DOE N-Reactor, SAND89-1147, Sandia National Laboratories, 1990.

D-4. E. E. Leitz, "Primary System Piping," Letter to M. D. Zentner, UNC Nuclear Industries, February 11, 1987. 
APPENDIX E:

INPUT TO THE EVNTRE CODE PACKAGE

\section{E.1 LISTING OF INPUT FILES}

The $N$ Reactor APET analysis made use of the EVNTRE event tree analysis package and the PSTEVNT post-processor for EVNTRE. The calculational pedigree and results are documented in Appendix F, but the input to those code runs are presented here.

The APET analysis was done in three separate phases: internal events, fire, and seismic. Each of these involved an execution of the EVNTRE package. The input files used for those executions are documented in the following pages. Below is a table showing the file names (as found on the cover page for each file listing) and its purpose in the APET analysis. The interpretation of the information in these files can be found in References E-1 and E-2. Additional input for the calculations comes from the TEMAC and LHS analyses documented in Reference E-3.

Files Used for All Analyses

NREAC_IE_BINNER.DAT

NREAC_IE_KEYWORD.DAT

NREAC_IE_MIRROR_REBINNER.DAT

Internal Events Analysis Files

NREAC_IE_LHS_KEYWORD.DAT

NREAC_IE_APET.DAT
This file is an implementation of the binning input described in Section 3.9 of this report.

This file is the PSTEVNT keyword file which contains the keywords used to control and select options for the PSTEVNT postprocessor analysis.

This file contains the binning input to the PSTEVNT post-processor. This input mirrors the binning input described above.

This file is the EVNTRE keyword file which contains the keywords used to control and select options for the EVNTRE analysis.

This is an implementation of the accident progression event tree input for the internal events analysis as described in Sections 3.2 through 3.8 of this report. 
NREAC_IE_PNTR.DAT

Fire Analysis Files

NREAC_FIRE_LHS_KEYWORD.DAT

NREAC_FIRE_APET.DAT

NREAC_FIRE_PNTR.DAT

Seismic Analysis Files

NREAC_SEISMIC_LHS_KEYWORD.DAT

NREAC_SEISMIC_APET.DAT

NREAC_SEISMIC_PNTR.DAT
This is the EVNTRE pointer file that instructs EVNTRE on how to apply the Latin Hypercube sample file and the TEMAC output files to the event tree to incorporate uncertainty. These sample files are documented in Appendix $A$ and in SAND89-2102.

Same as NREAC_IE_LHS_KEYWORD.DAT, but for the fire analysis.

Same as NREAC_IE_APET.DAT, but for the fire analysis.

Same as NREAC_IE_PNTR.DAT, but for the fire analysis.

Same as NREAC_IE_LHS_KEYWORD.DAT, but for the seismic analysis.

Same as NREAC_IE_APET.DAT, but for the seismic analysis.

Same as NREAC_IE_PNTR.DAT, but for the seismic analysis.

\section{E.2 REFERENCES}

E-1. J. M. Griesmeyer and L. N. Smith, A Reference Manual for the Event Progression Analysis Code (EVNTRE), SAND88-1607, NUREG/CR-5174, U.S. Nuclear Regulatory Commission, September 1989.

E-2. S. J. Higgins, A User's Manual for the Postprocessing Program PSTEVNT, SAND88-2988, NUREG/CR-5380, U.S. Nuclear Regulatory Commission, November 1989.

E-3. D. M. Kunsman, et. al., Level III Probabilistic Risk Assessment for $\mathrm{N}$ Reactor, SAND89-2102, WHC-MR-0045, Westinghouse Hanford, April 1990. 
The following text is a listing of the binning input file used-in all of the $\mathrm{N}$ Reactor EVNTRE calculations, and as found in the file NREAC_IE_BINNER.DAT.

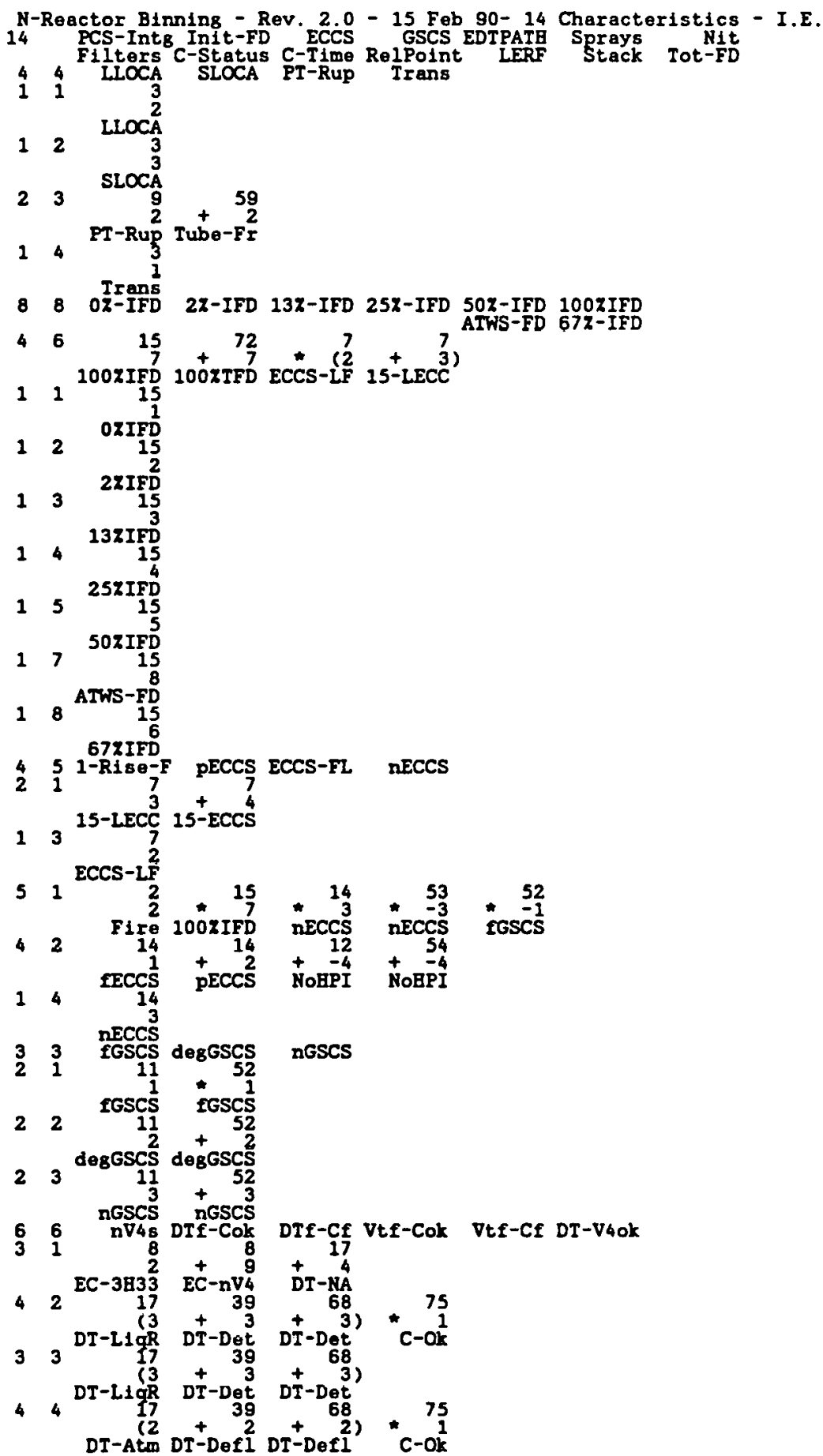




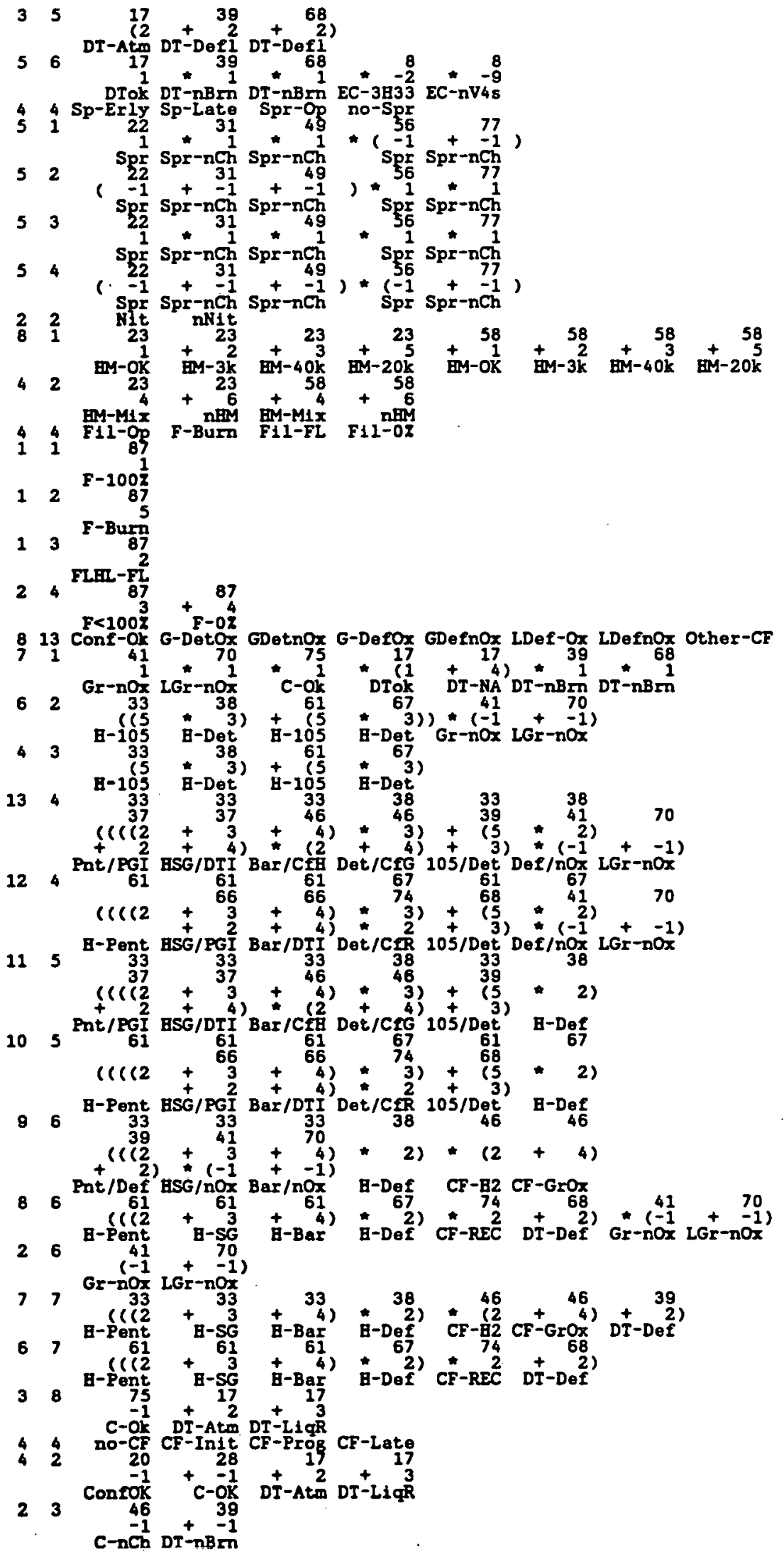




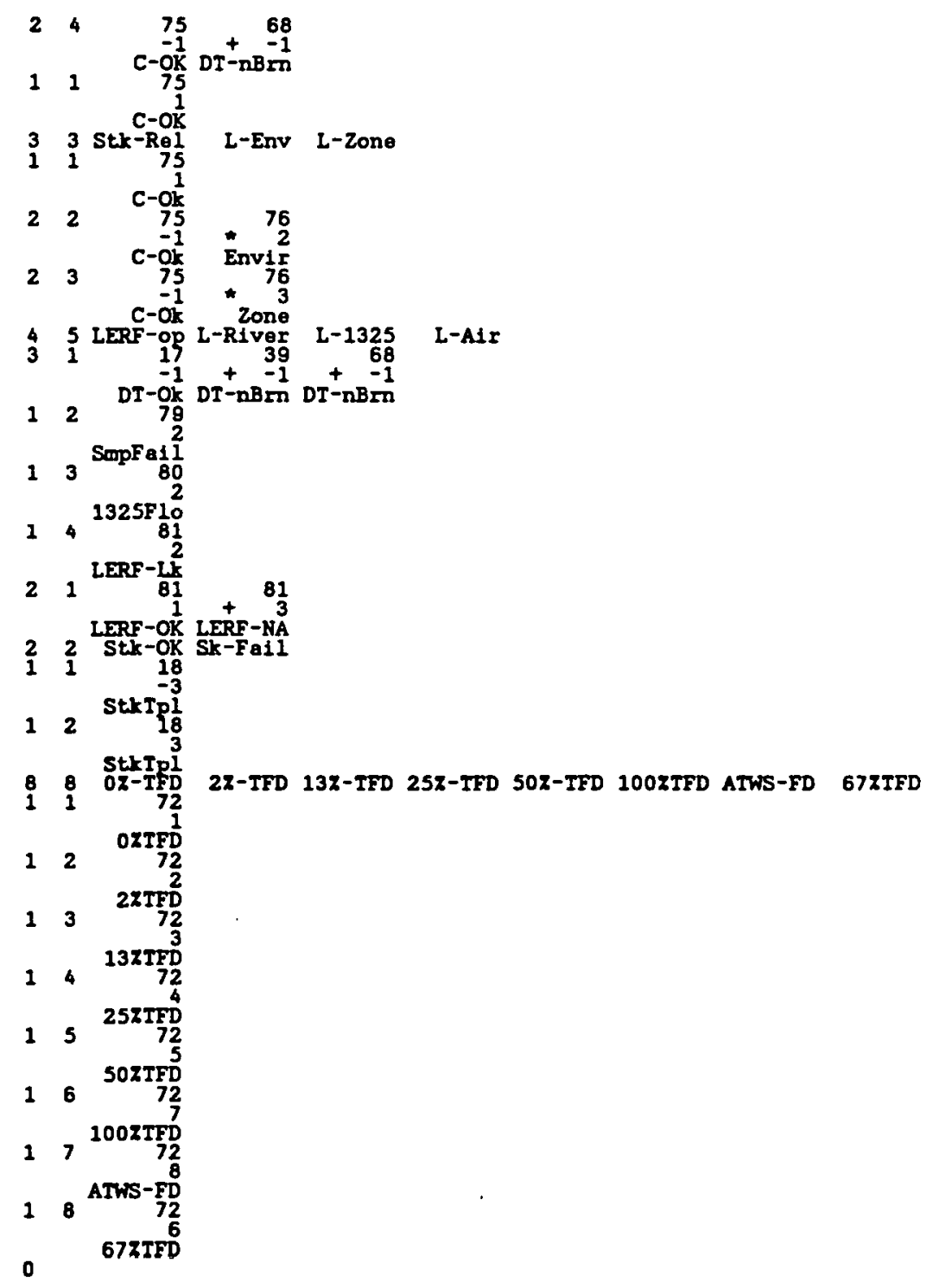


The following text is a listing of the keyword input file used in all of the $N$ Reactor PSTEVNT postprocessor calculations, and as found in the file NREAC_IE_KEYWORD.DAT.

$\begin{array}{ll}\text { REBIN } & \\ \text { BININ } & 4 \\ \text { EVNTBIN } & 21 \\ \text { INPOUT } & 7 \\ \text { BINOUT } & 8 \\ \text { PRTCUT } & 0.00001 \\ \text { RUN } & \\ \text { NCFILES } & 1 \\ \text { NRFILES } & 1 \\ \text { SAVEBIN } & \\ \text { SBINOUT } & 13 \\ \text { KEEPOUT } & 15 \\ \text { PCUTFR } & 0.99999 \\ \text { ASCTRIN } & \\ \text { ASCSAV } & \\ \text { COLLAPS } & 0.99999 \\ \text { RCUT } & 0.00001 \\ \text { NSPREAD } & \\ \text { KPBYRUN } & \\ \text { ENDKEY } & \\ \text { PRTINP } & \\ \text { SORT } & \\ \text { SORTIN } & 9 \\ \text { SORTOUT } & 11 \\ \text { DISCTAB } & \\ \text { TABIN } & 10 \\ \text { TABOUT } & 14 \\ \text { SQFRFQ } & \\ \text { FRFQIN } & 12 \\ \text { RUNFREQ } & \\ \text { RUNFQIN } & 16 \\ \text { SAMWGT } & \\ \text { RPTMLST } & \\ \text { RPTRBIN } & \\ \text { RPTNPRB } & \\ & \end{array}$


The following text is a listing of the mirror rebinning input file used in all of the $\mathrm{N}$ Reactor PSTEVNT postprocessor calculations, and as found in the file NREAC_IE_MIRROR_REBINNER.DAT.

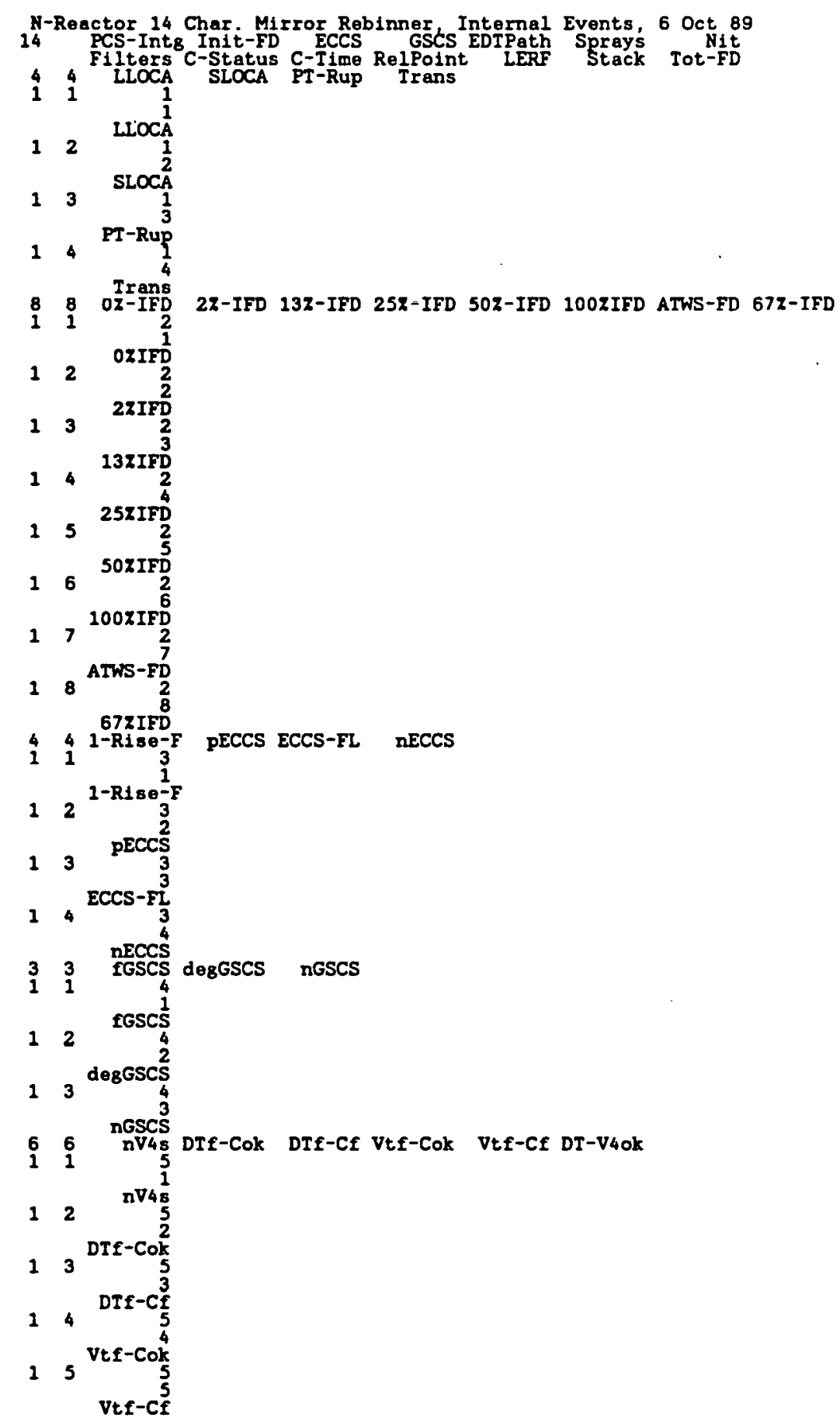




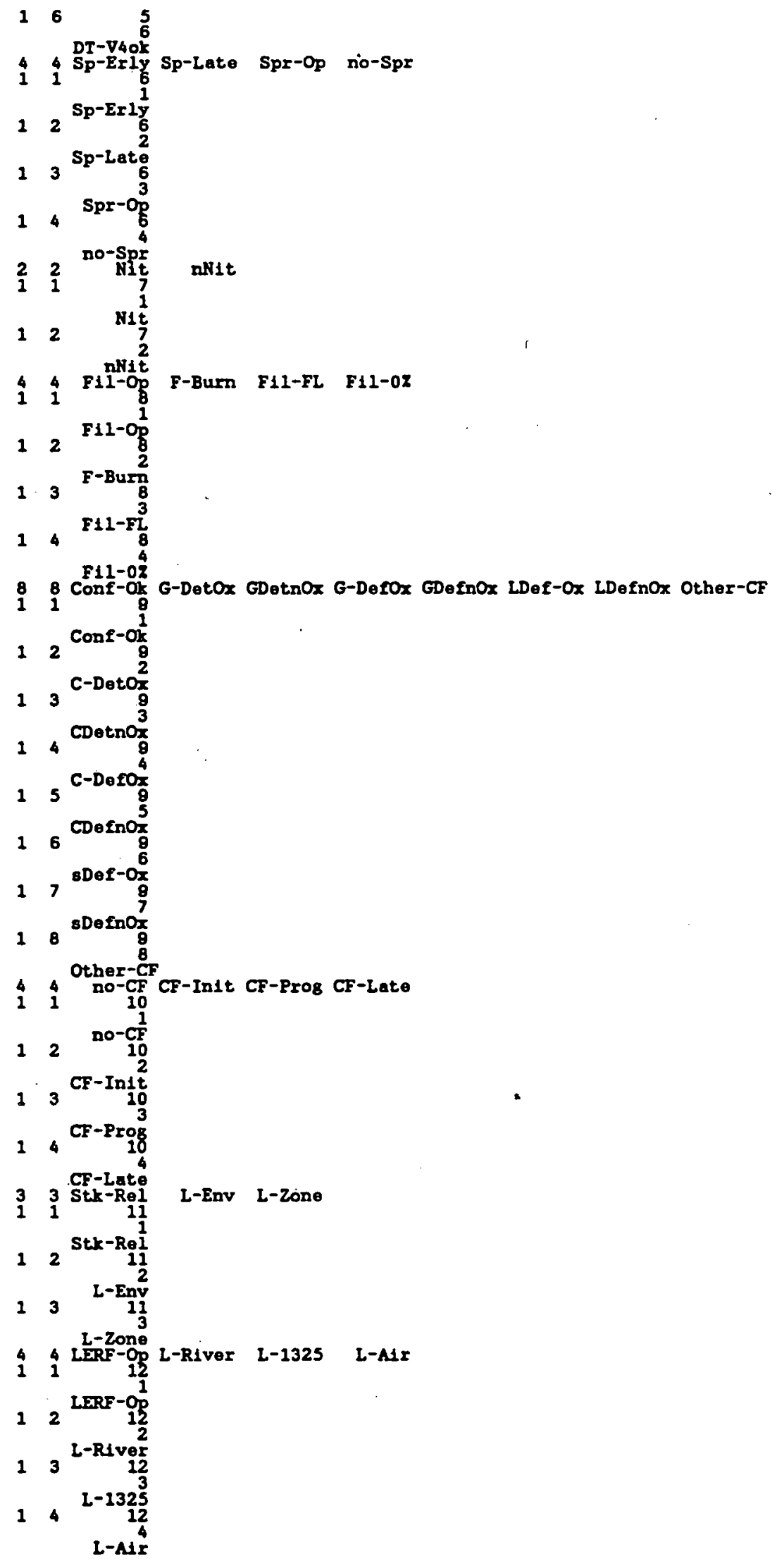




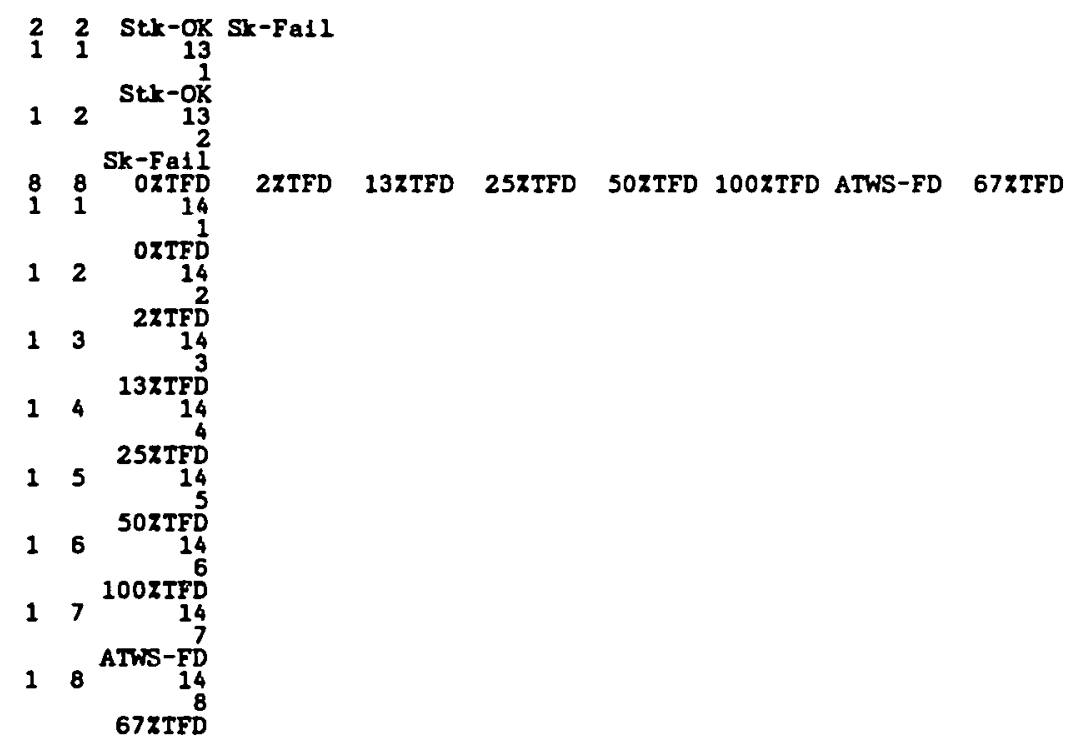

E-9 
The following text is a listing of the keyword input file used in the $N$ Reactor internal events EVNTRE calculation, and as found in the file NREAC_IE_LHS_KEYWORD.DAT.

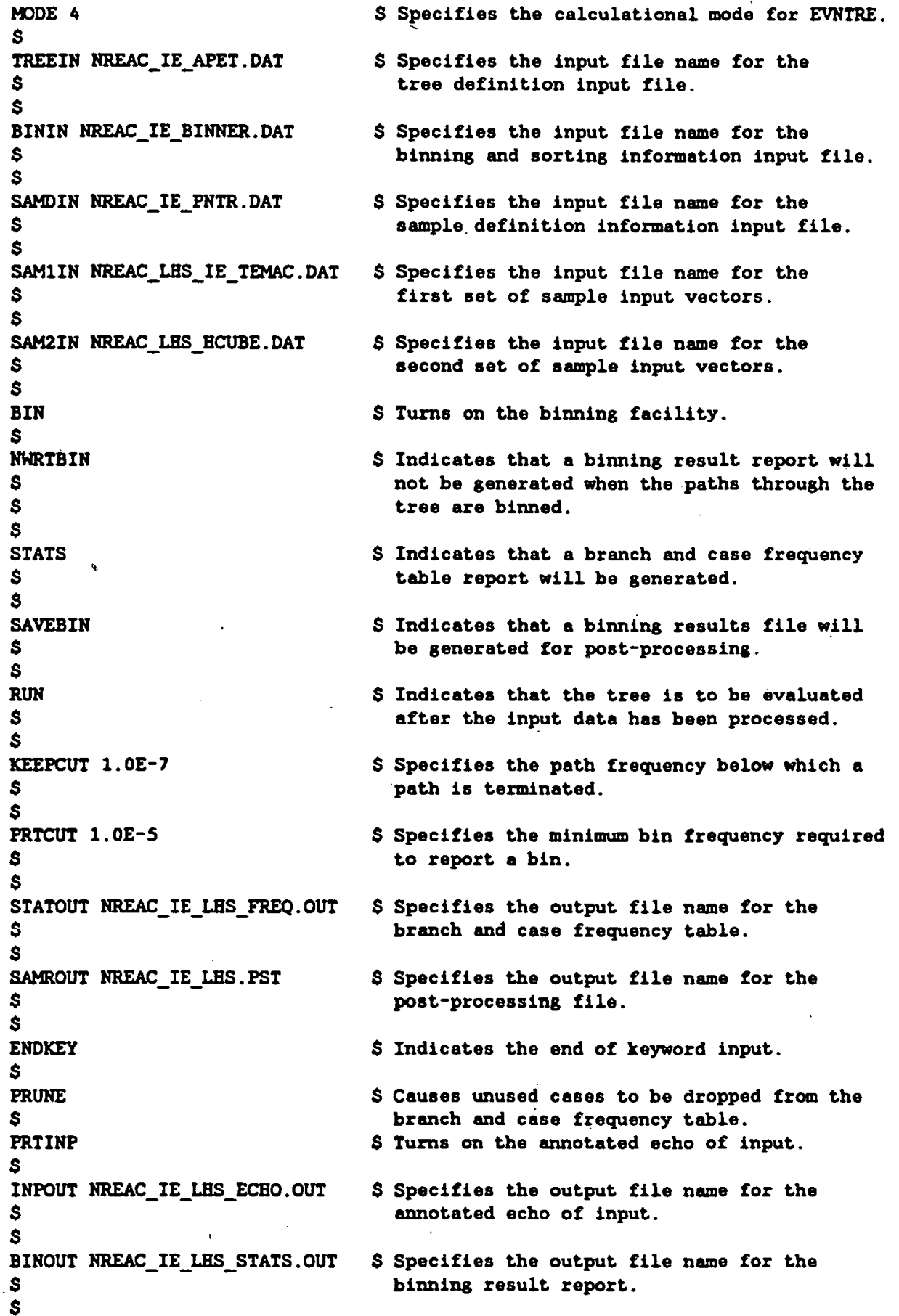


The following text is a listing of the accident progression event tree input file used in the internal events $N$ Reactor EVNTRE calculation, and as found in the file NREAC_IE_APET.DAT.

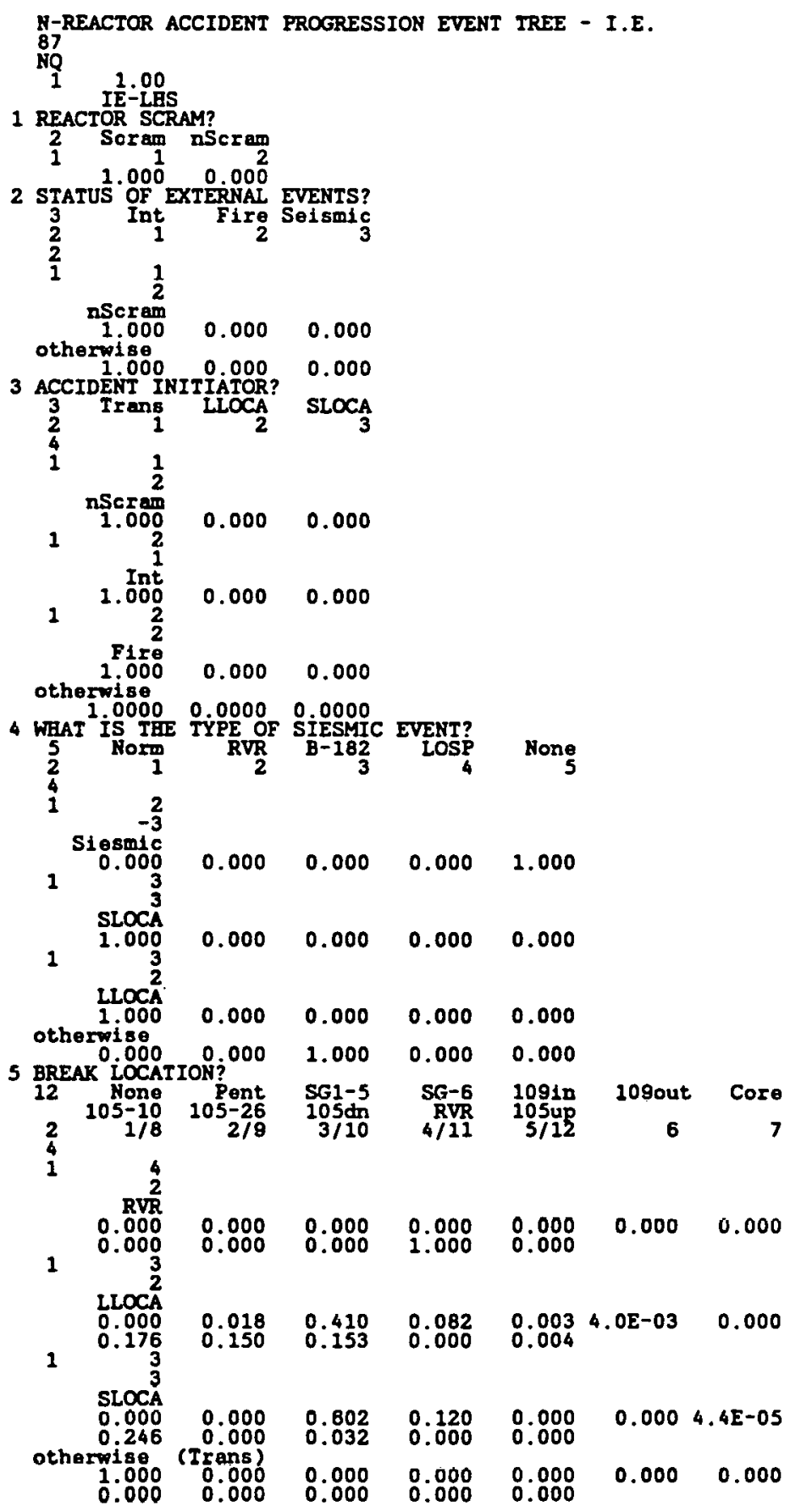




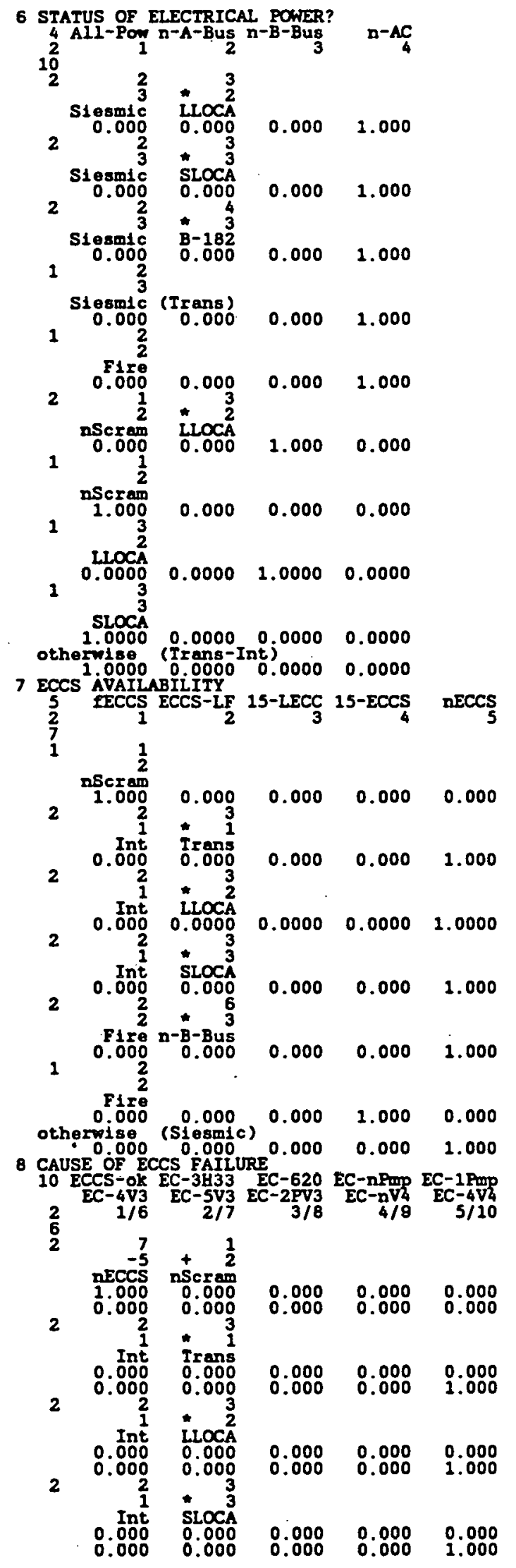




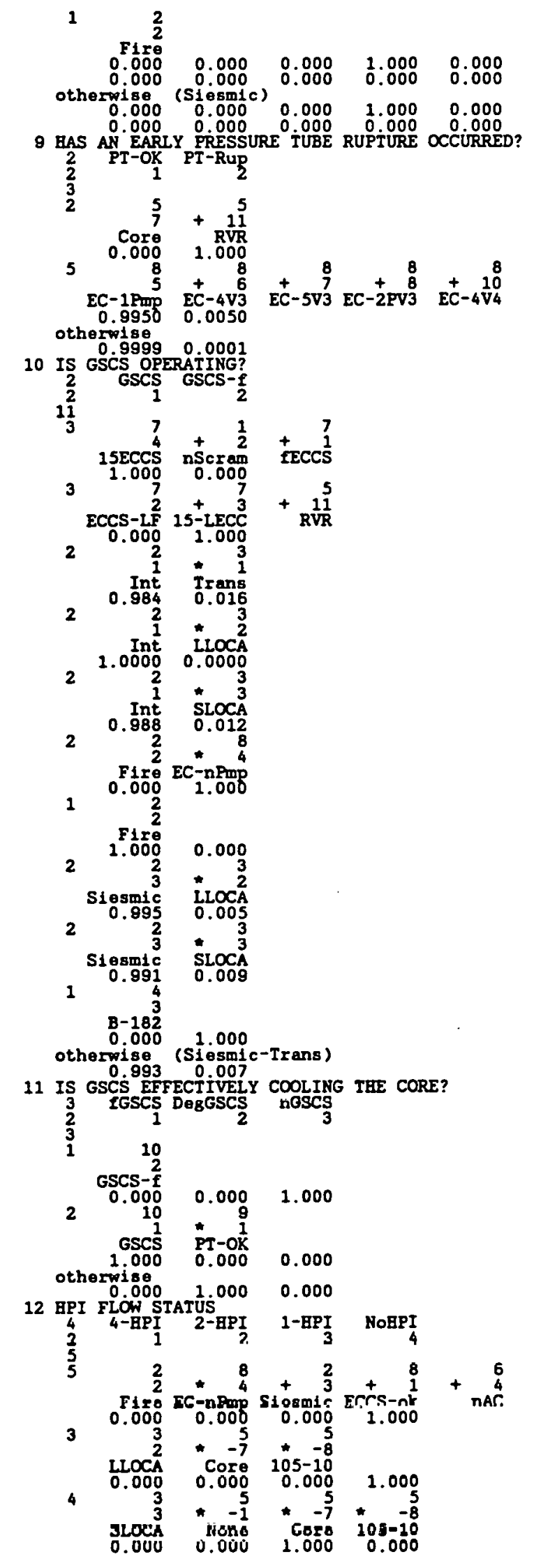




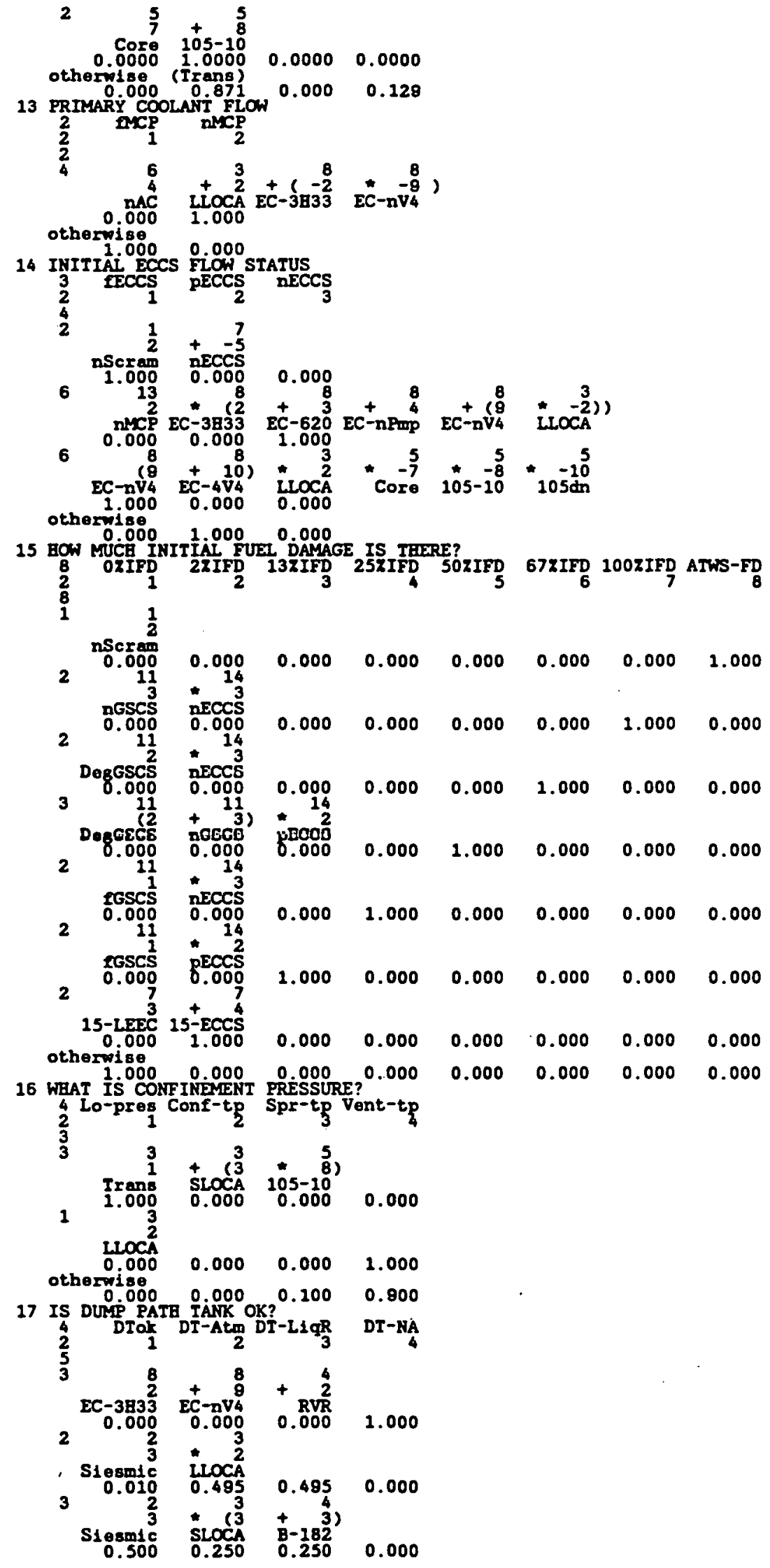




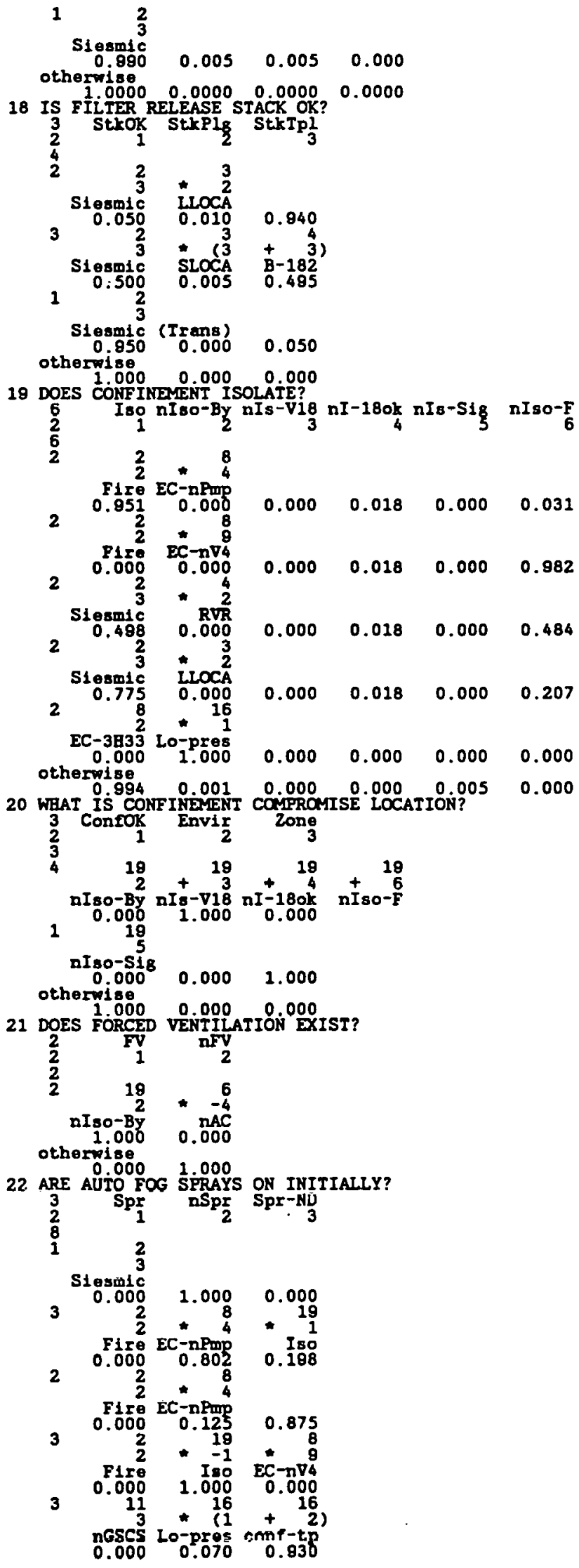




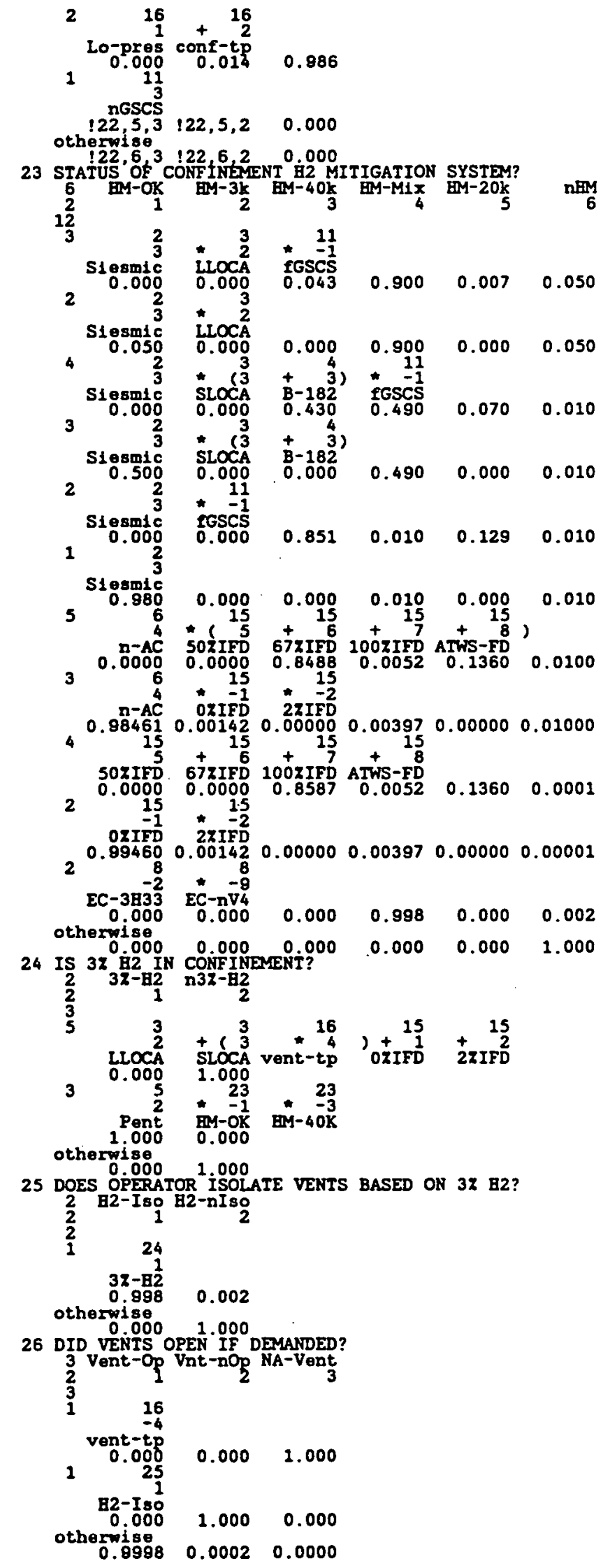




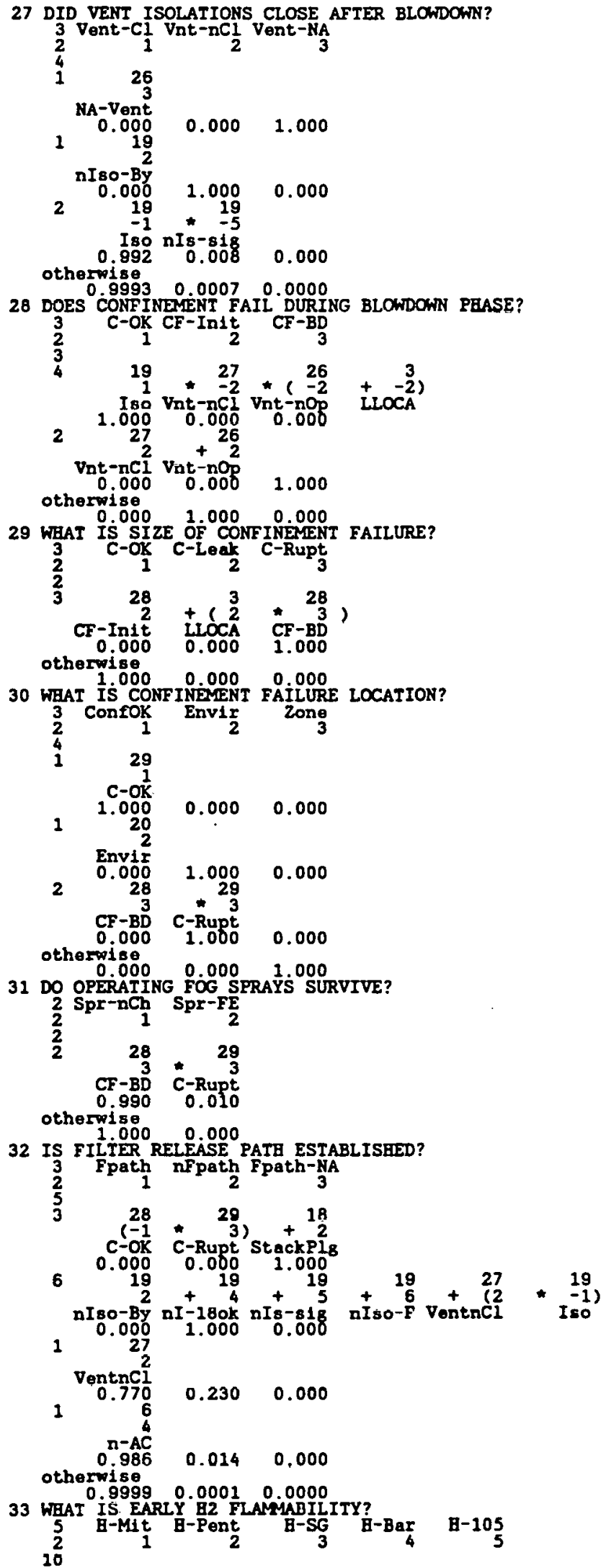




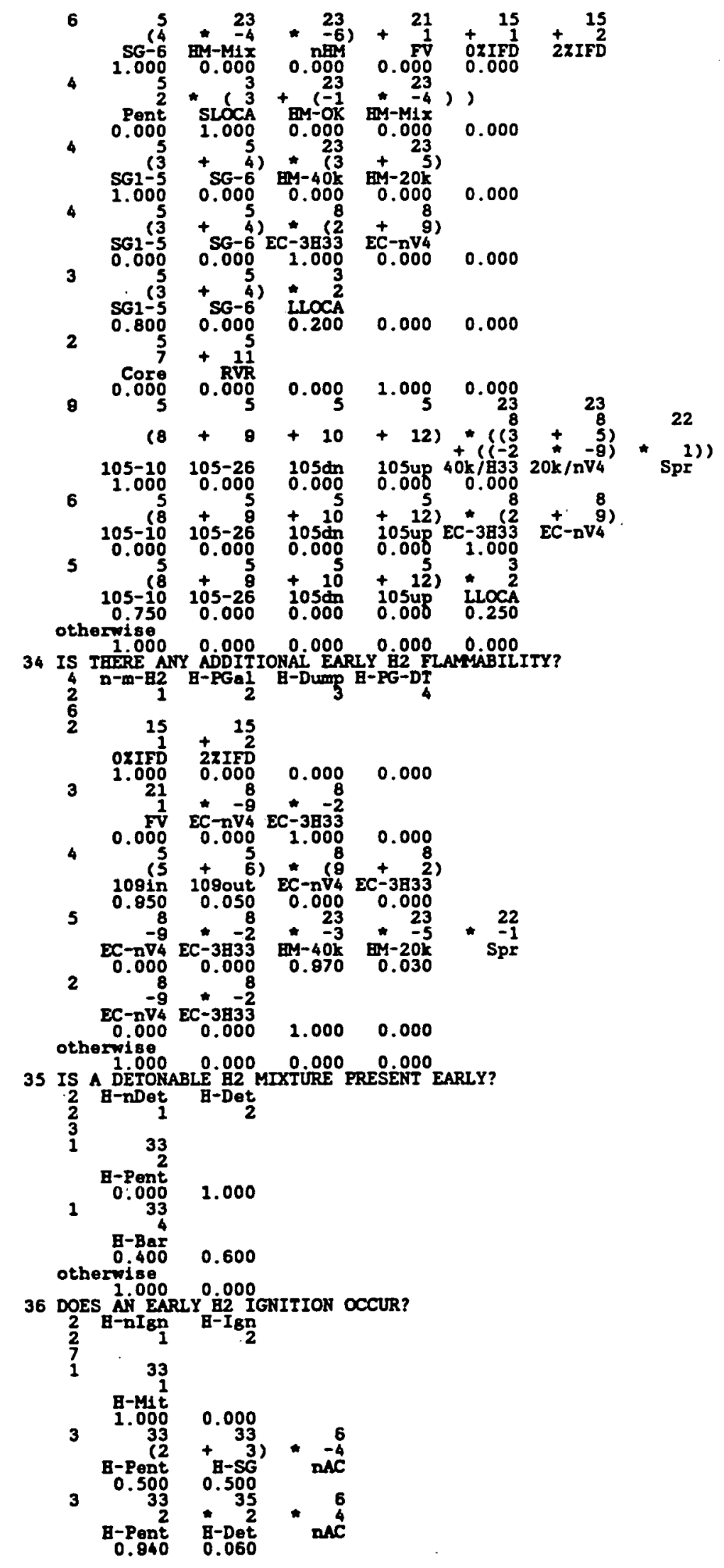




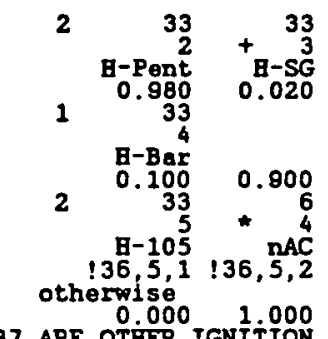

37 ARE OTHER IGNITION SOURCES PRESENT?

4 B-nI ${ }_{1}{ }_{1}-I_{B n}$ DT-Ign $P G-D T-\frac{I}{3}$

2
6
1

n-m-1

1

B-PGaI

0.000

$0.000 \quad 0.000$

* -4

0,500

0.000

0.000

0.950

$\begin{array}{lll}0.050 & 0.000 & 0.000\end{array}$

B-Dump

2

0.990

B-FG-DT
0.495

0.000
$-\quad-4$

0.495

0.000

otherwise

0.005

0.005

38 IS THERE AN EARIY GENERAL DETONATION?

3 B-nBurn ${ }_{1}$ B-Def ${ }_{2}$ B-Det

5

$1 \quad 36$

1

$\mathbf{8}-\mathbf{n I} \mathbf{g n}$

1.085

$0.000 \quad 0.000$

3

B-nDet

B-Pent
0.000

$1.000 \quad 0.000$

$\begin{array}{r}23.000 \\ +\quad-23 \\ \hline\end{array}$

in-3k

nEM

1

B-Pent

$0.300 \quad 0.700$

otherwise

39 DOES 0.000 0.950 0.050 OCCUR?

3 DT-nBurn DT-Def DI-Det

2
2
2

$\begin{array}{rrr}2 & 37 & 37 \\ 3 & + \\ \text { DT-Ign } & \text { PG-DT-I } \\ 0 \text { - } & 0.760\end{array}$

otherwise

$0.760 \quad 0.240$

40 IS Ee BLANKET INTACT? 0.000

2 Be-OK He-Lost

2

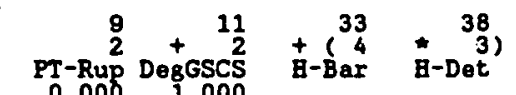

othervise

41 BAS GRAPBITE OXIDATION STARTED?

3 Gr-nox Gr-sox Gr-Fire

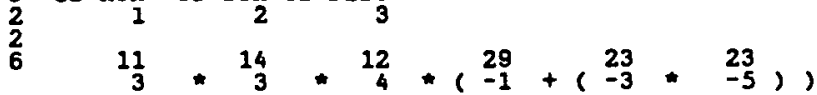

nGSCS nECCS NoHPI

otherisise

2 ARE VACUUM BREAKERS DEMANDED

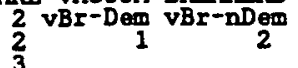




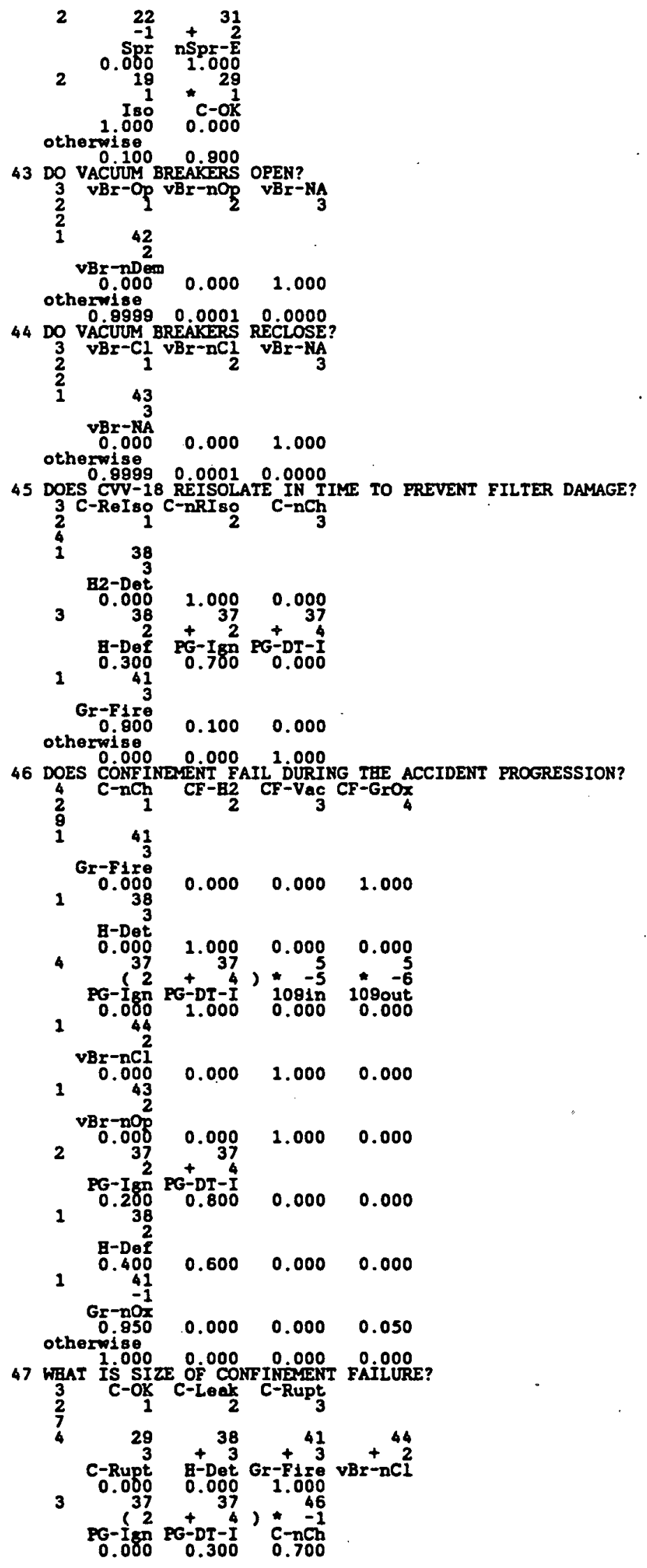




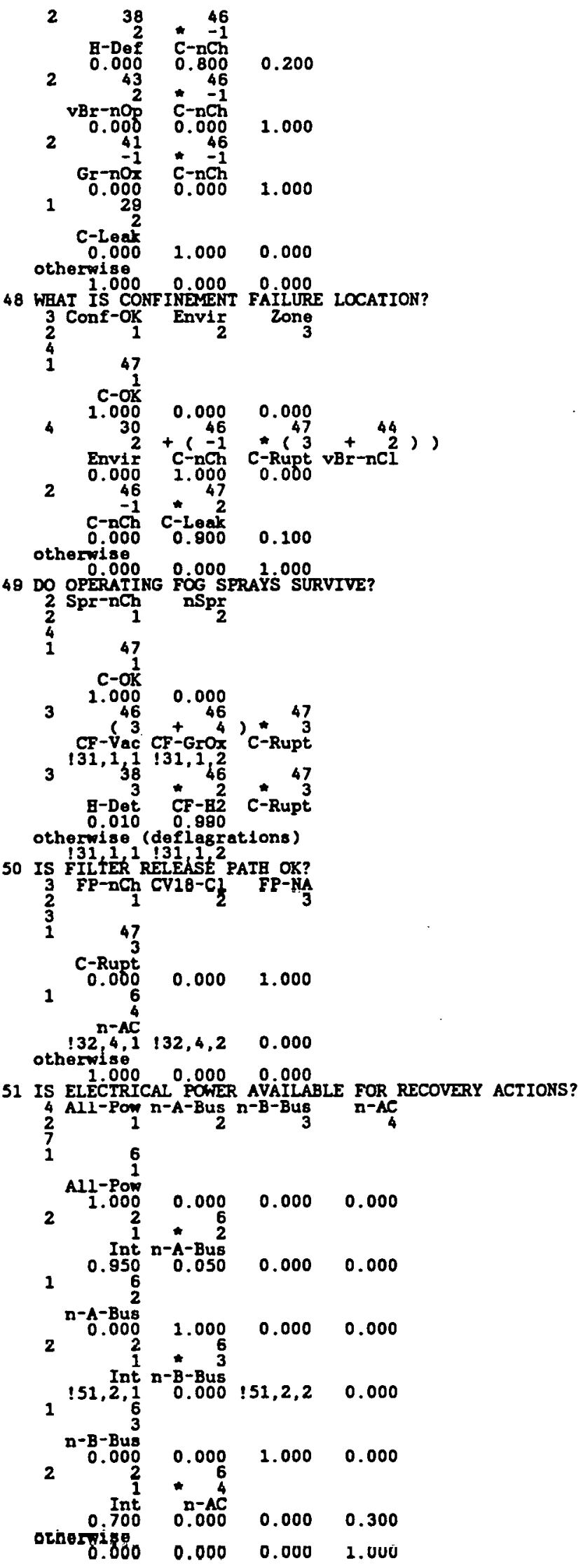


52 DOES GSCS OPRRATE DURING THE RECOVERY PEASE?
$\begin{array}{lll}3 & \text { fGSCS DegGSCS } & \text { nGSCS } \\ 2 & 1 & \end{array}$

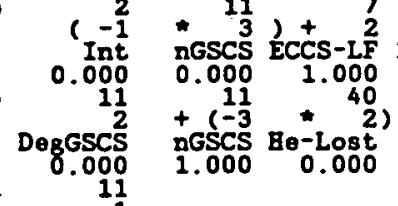
1
3
$\begin{array}{rrr}\text { PGSCS } & & \\ 1.000 & 0.000 & 0.000 \\ 6 & 51 & 40 \\ 4 & -4 & 2 \\ n-A C & \text { nAC } & \text { Be-Lost } \\ 0.000 & 0.010 & 0.990\end{array}$
2
$0.000 \quad 0.010 \quad 0.890$
0.010
$0.010 \quad 0.000 \quad 0.990$
otherw18日 $0.000 \quad 1.000$

53 DOES ECCS OPERATE DURING TEE RECOVERY PEASE?

3 EECCS PECCS nECCS

10

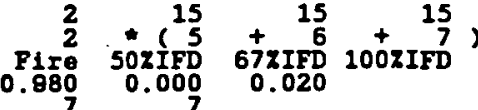

ECCS-LF $15-\operatorname{LECC}$

$\begin{array}{rrr}0.830 & 0.000 & 0.070 \\ 14 & 5\end{array}$

$\begin{array}{rrr}105-d n & \text { Siesmi } \\ 0.000 & 0.000 & 1.000\end{array}$

$\begin{array}{rr}\operatorname{eccs} \\ 1.000 & 0.000 \quad 0.000\end{array}$

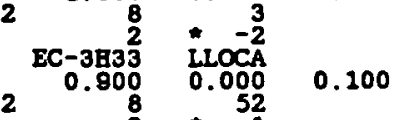

$20-33^{2} *$

1

$0.000 \quad 153,1,3$

एC-3833

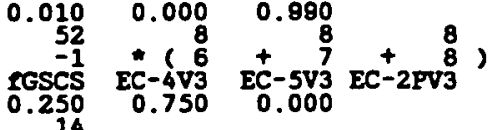

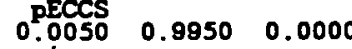

otherw189 $0.005 \quad 0.894$

54 DOES HPI OPPRATE DURTKG THE RECOVERY PEASE? 4 4/4-BPI 2/4-BPI 1/4-BPI nBPI

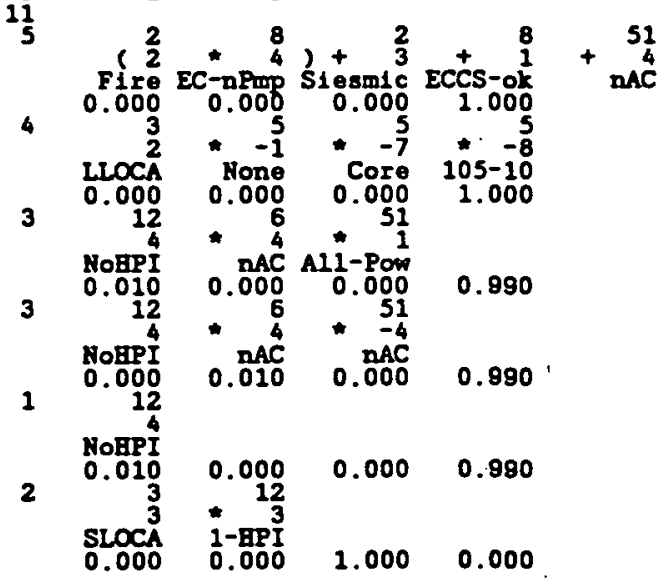




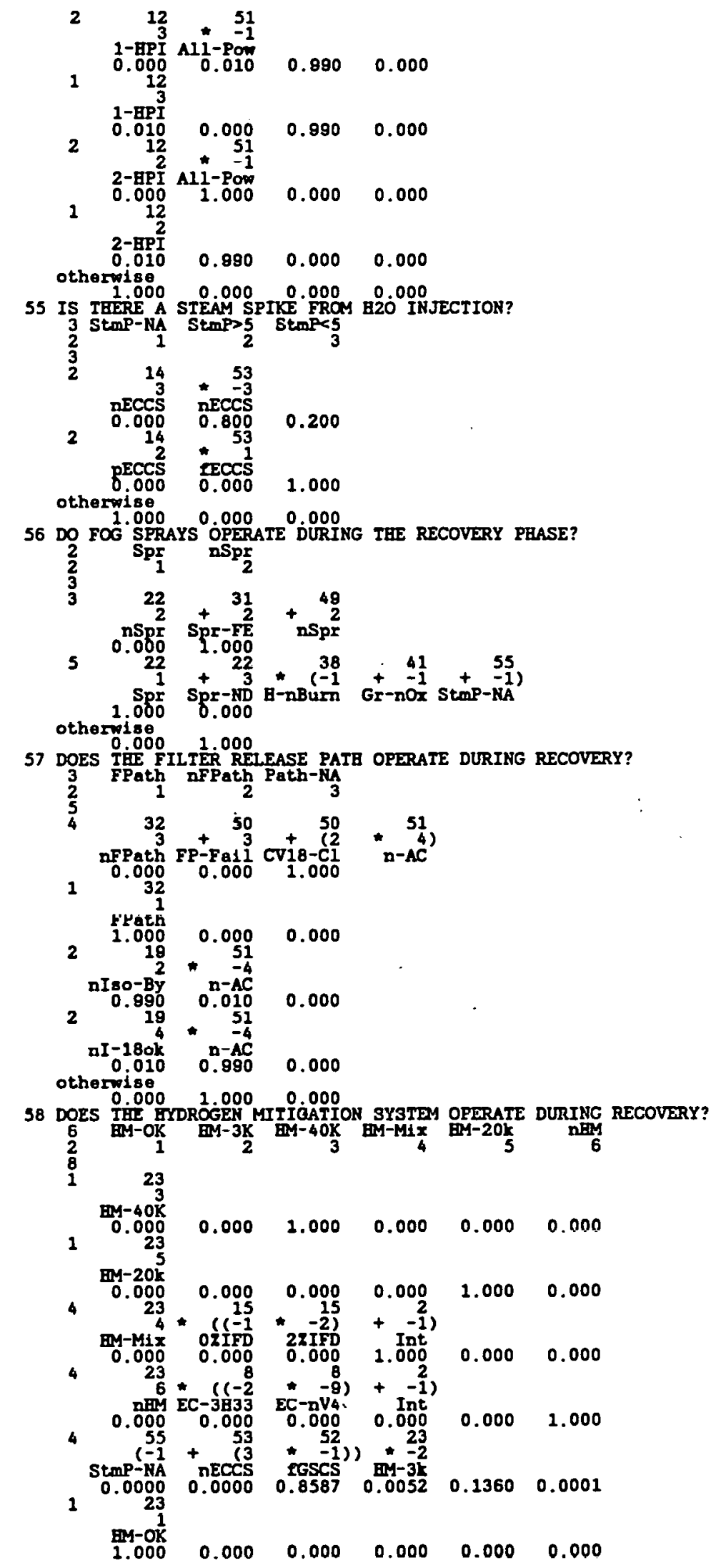


1

$\begin{array}{rrrrrr}23 & & & & & \\ 2 \\ \text { BM- } 3 K \\ 0.000 & 1.000 & 0.000 & 0.000 & 0.000 & 0.000\end{array}$

$\begin{array}{lllllll}\text { otherwise } & 0.000 & 0.000 & 0.000 & 0.000 & 1.000\end{array}$

59 DOES PRESSURE TUBE FAIIURE OCCUR DUE TO WATER INJECTION?

2 Tub-nFr Tube-Fr

$\begin{array}{rrr}3 & 55 \\ 2 & +55 \\ \text { stmp } & +5 \\ \text { stmp<5 }\end{array}$

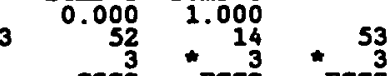

ngGCS nEcCs nECCS

otherwise $1.000 \quad 0.000$

60 BOW MUCCH HYDROGEN IS PRODUCED DURING TEE ACCIDENT?

$\frac{4}{2}<230 \mathrm{~B} 2<1200 \mathrm{H}_{2}<2000 \mathrm{2}>2000 \mathrm{~B} 2$

4
3

52
3
nGscs

$\begin{array}{r}14 \\ -\quad 53 \\ \hline\end{array}$

3

0.000 nECCS neccs

nEccs 1.000

- $36 \operatorname{sen}^{3}$

neccs

$\begin{array}{r}53 \\ -\quad-3 \\ \hline\end{array}$

neccs

$1.000 \quad 0.000$

4

DegGscs

52
$+\quad 3)$

ngscs

$\begin{array}{r}14 \\ -\quad 2+34 \\ \hline\end{array}$

$1.000 \quad 0.000 \quad 0.000$

61 WBAT IS LATE B2 FIAMABंIIITY? 0.000

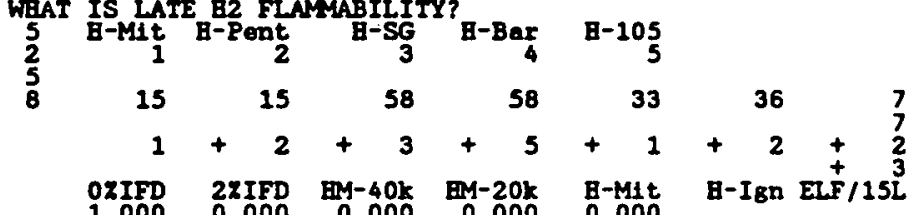

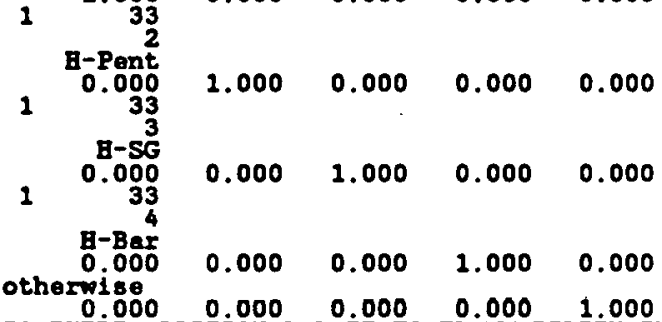 1.000

2XIFD BMy-40k BM-20k

$\mathrm{B}-\mathrm{M} 1 \mathrm{t}$

B-Ign EL. $+\frac{+}{F} / 15 L^{3}$

62 IS THERE ADDITIONAL LATE B2 FLAMMABILITY IN THE PIPE GALLERY? 2 nB-PGaI B-PGal

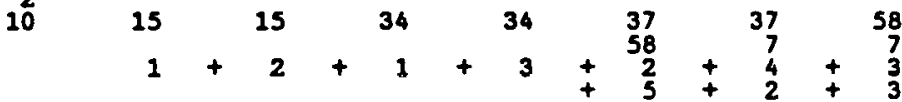
02IFD 22IFD n-m-82 B-Dump PGI/20k PGDT/LF 40k/15i
otherwiso

63 IS THERE ADDITIONAL LATE B2 FLAMABILITY IN THE DUMP TANK?

$$
\begin{aligned}
& 2 \text { nH-DT } 1 \\
& \begin{array}{rrr}
2 & 1 & 2 \\
2 & 15 & 15
\end{array}
\end{aligned}
$$

64 IS A DETONABLE 12 MIXTURE PRESENT LATE?

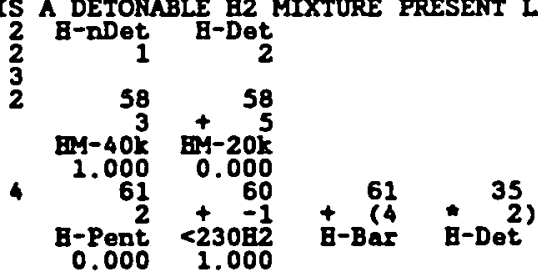




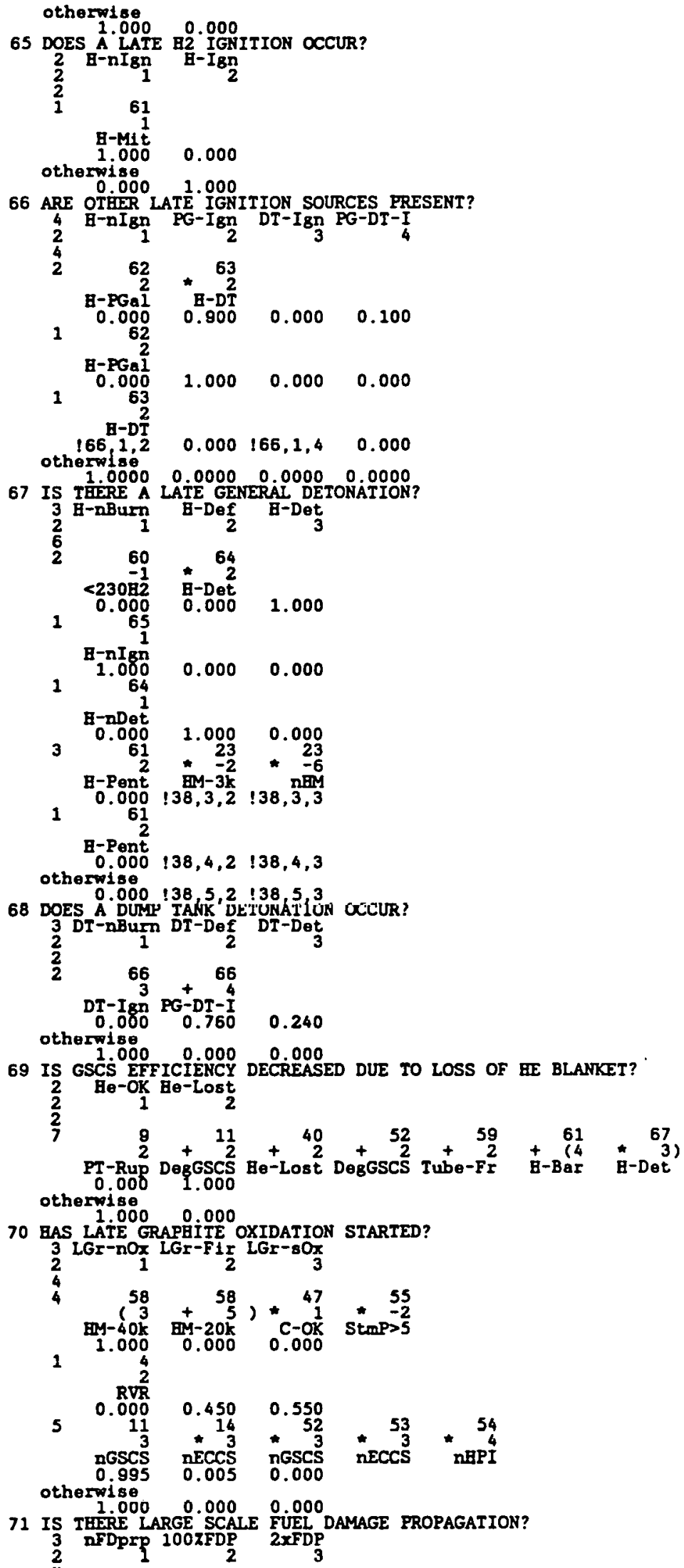




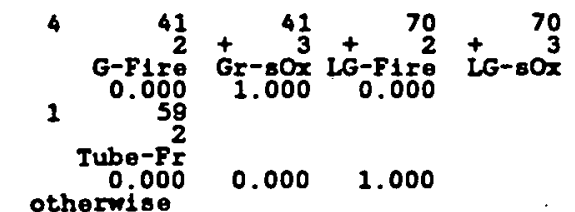

otherwis $1.00000 .0000 \quad 0.0000$

72 BOW MUCG TOTAL FUED DAMAGE? 252TFD S0XTFD 672TFD 100スTFD ATWS-FD

栗

1215

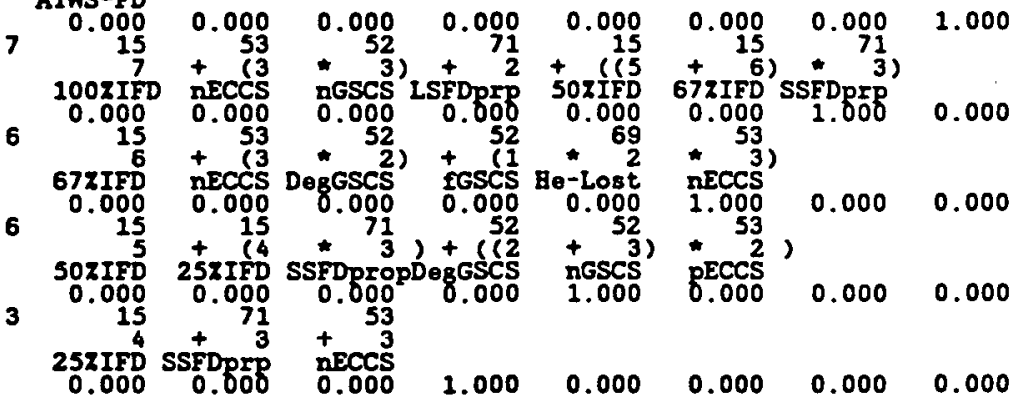

2

$13213+25$

$\begin{array}{llllllll}0.000 & 8.000 & 1.000 & 0.000 & 0.000 & 0.000 & 0.000 & 0.000\end{array}$ 22IFD $221 \%$

othermiso

$\begin{array}{lllllll}1.000 & 0.000 & 0.000 & 0.000 & 0.000 & 0.000 & 0.000\end{array}$

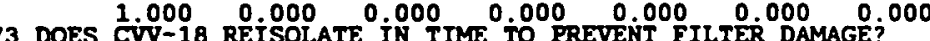

${ }_{2} \mathrm{C}$-ReIso $\mathrm{C}-\mathrm{nRISO} \mathrm{C}_{2} \mathrm{C}_{3}$

5

67

B-Det

3

0.000

1.000

1.006
$+\quad 2.000$
$+\quad 66$

1

$\mathrm{B}-\mathrm{DeF}$
$145,2, \frac{\mathrm{PG}}{145,2,2}, 0.000$

2

$2 \quad 45,3 \frac{1}{55} \quad 145,3,2 \quad 0.000$

Stmp>5 ${ }^{+}+3$

$0.980 \quad 0.020 \quad 0.000$

otherwise

$0.000 \quad 1.000$

74 DOES CONFINENENT FAIL DURING IHE RECOVERY PBASE?

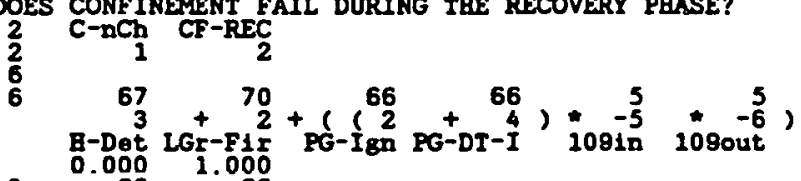

2

66

1

PG-Ign PG-DT-I

2

Stmp>5

0.8

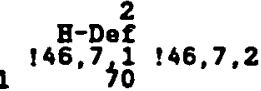

$-1$

!46,8,1 !46,8,4

otherwise

$1.000 \quad 0.000$

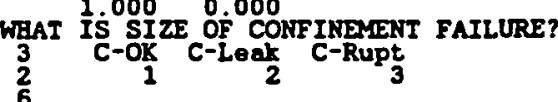




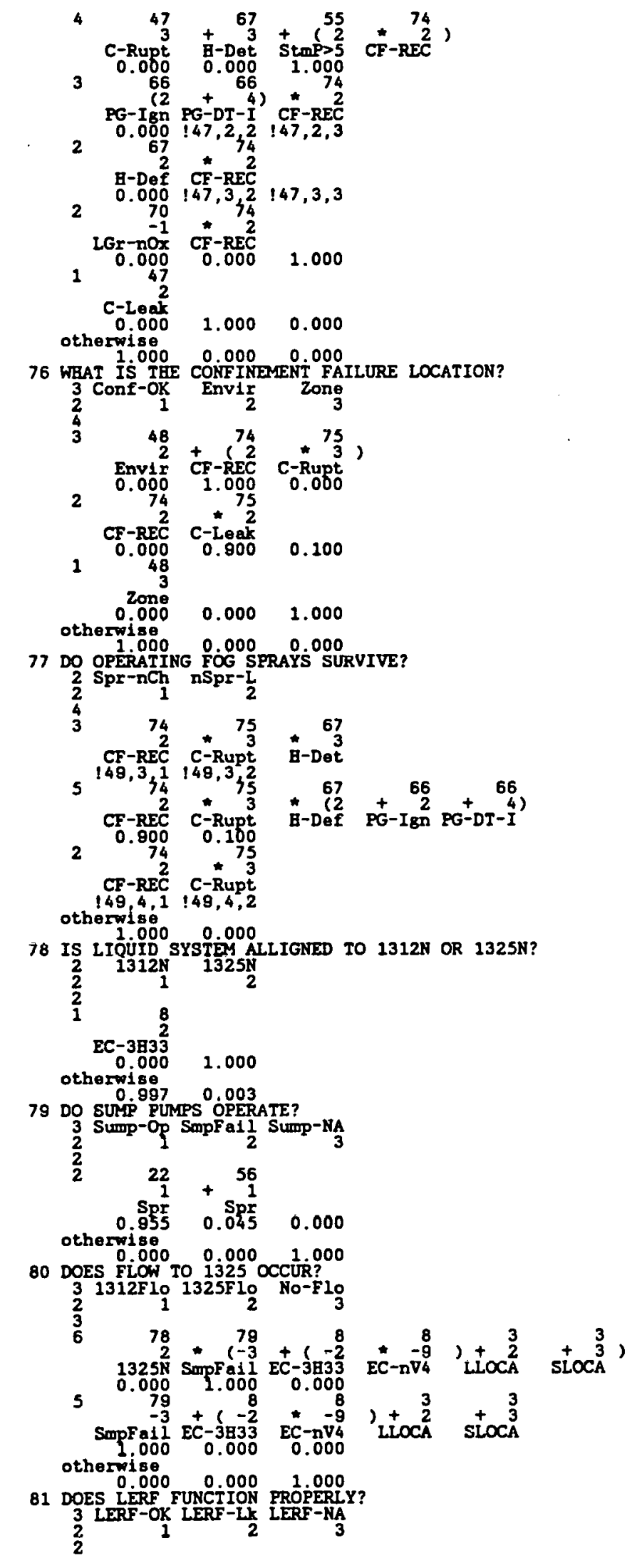




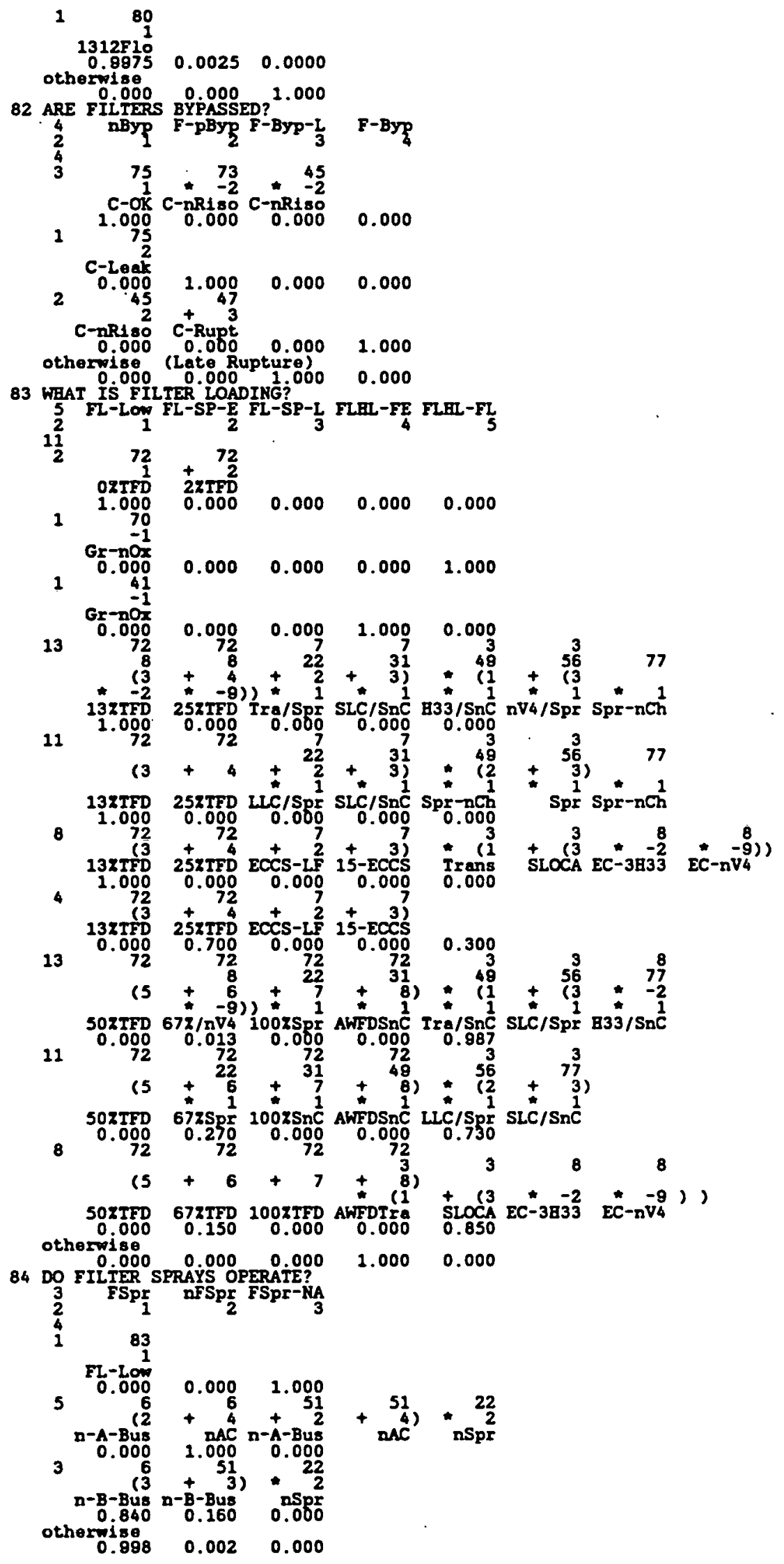




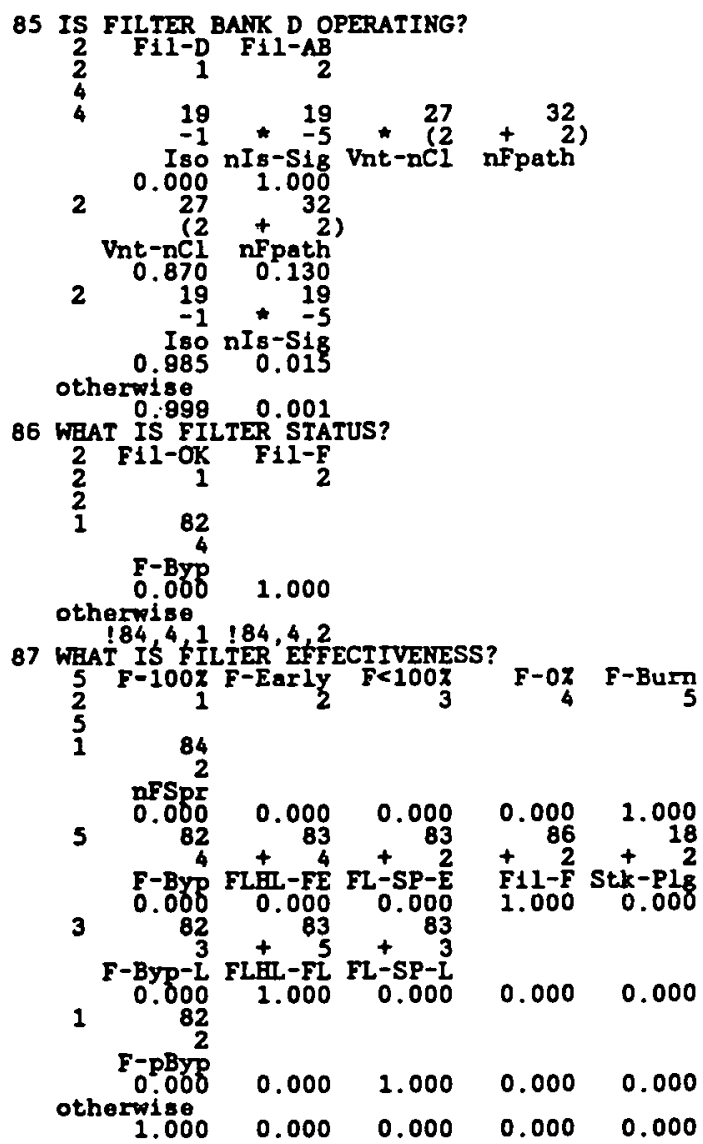


The following text is a listing of the pointer file input used in the internal events $N$ Reactor EVNTRE calculations, and as found in the file NREAC_IE_PNTR. DAT.

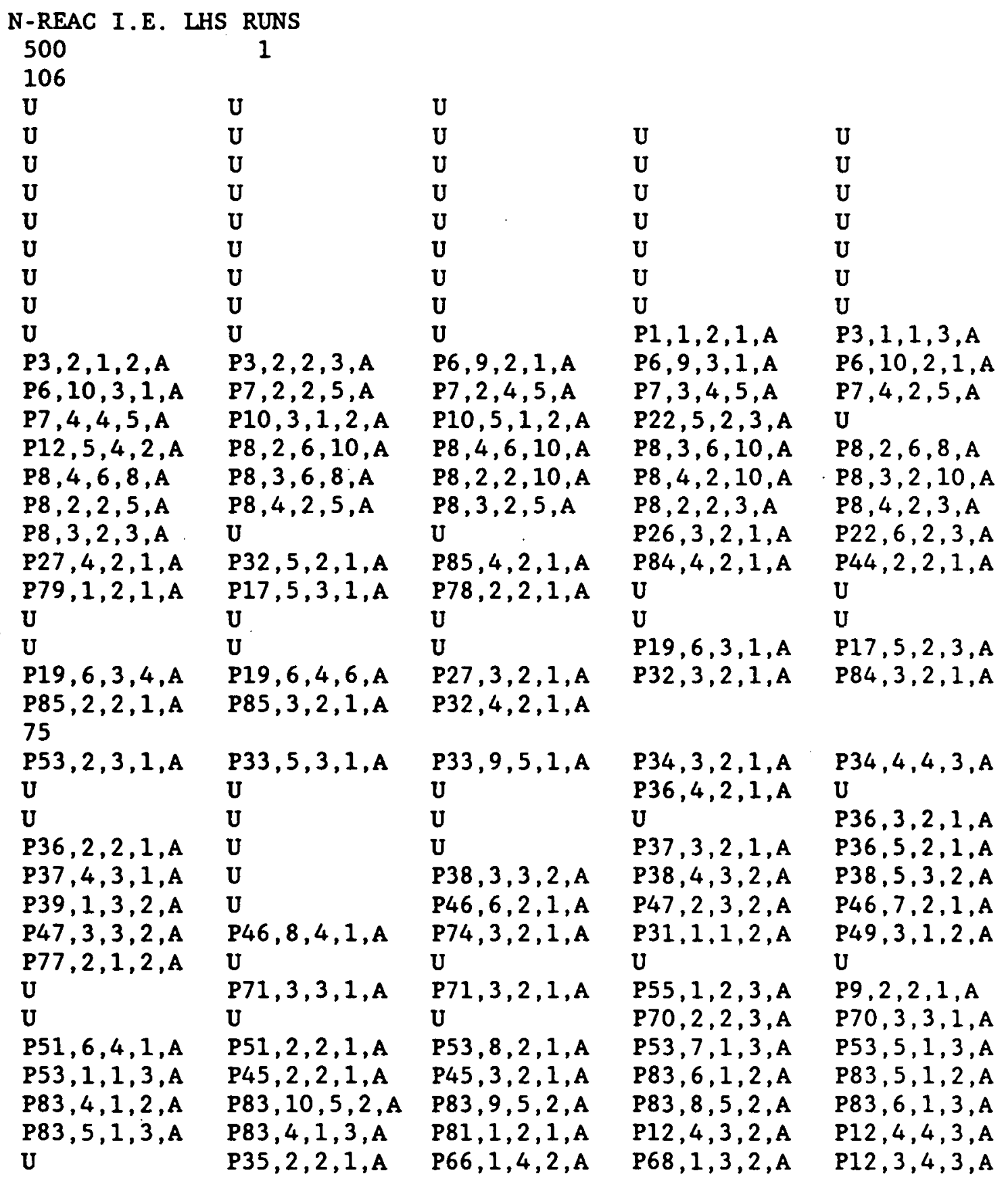


The following text is a listing of the keyword input file used in the $\mathrm{N}$ Reactor fire events EVNTRE calculation, and as found in the file NREAC_FIRE_LHS_KEYWORD.DAT.

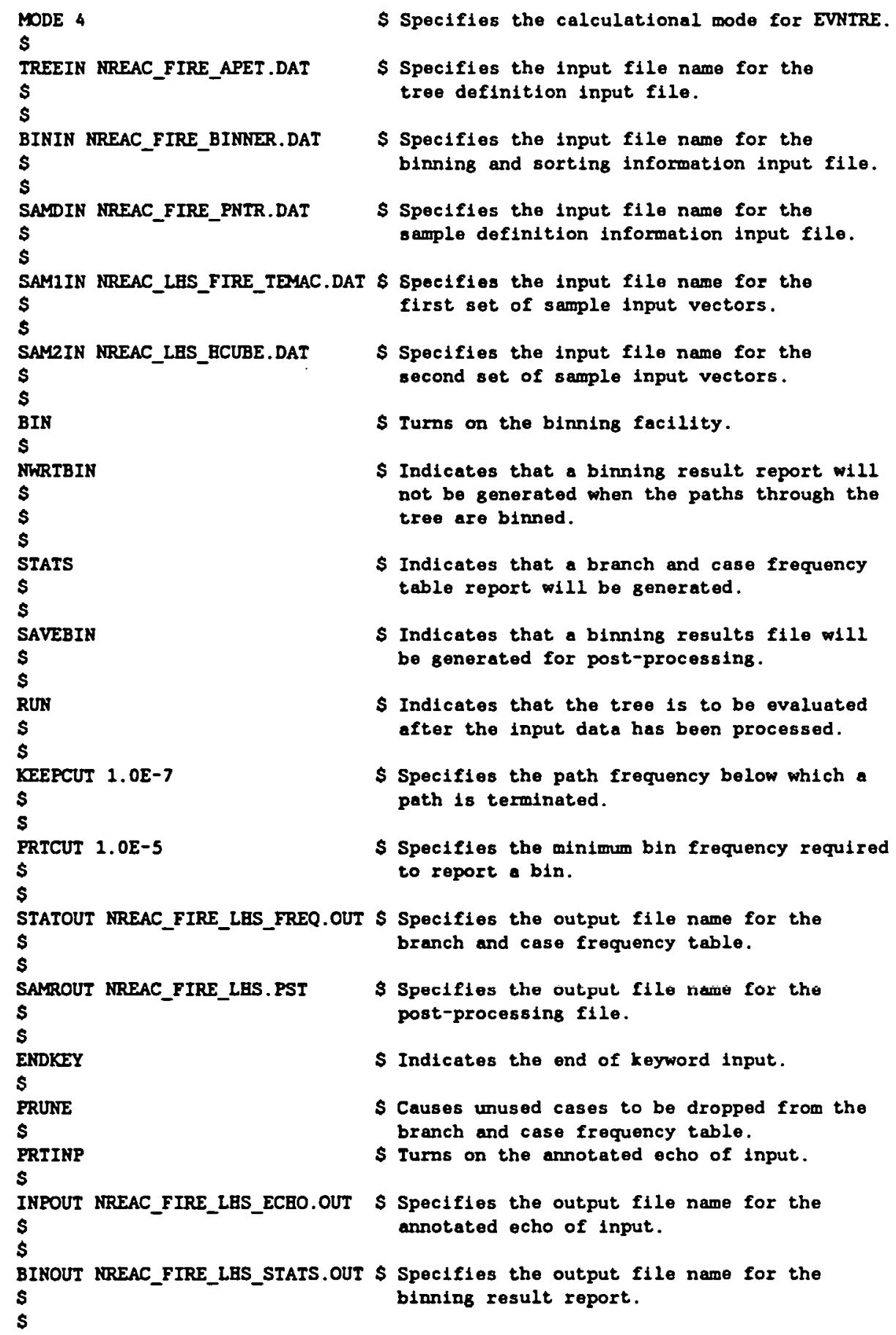


The following text is a listing of the accident progression event tree input file used in the fire events $N$ Reactor EVNTRE calculation, and as found in the file NREAC_FIRE_APET.DAT. Most of this file is identical to the internal events event tree input. The only changes are in the first two events, and only that section of the file is reproduced here.

N-REACTOR ACCIDENT PROGRESSION EVENT TREE - FIRE

87

NQ

11.00

FIRE - IHS

1 REACTOR SCRAM?

2 Scram nScram

$1 \quad r \quad 2$

$1.000 \quad 0.000$

2 STATUS OF EXTERNAL EVENTS?

3 Int Fire Seismic

$2 \quad 1 \quad 2 \quad 3$

2

1

1

2

nScram

$\begin{array}{lll}1.000 & 0.000 & 0.000\end{array}$

otherwise

$0.000 \quad 1.000 \quad 0.000$

3 ACCIDENT INITIATOR?

..... (the remainder of the file is identical to that listed in the file NREAC_IE_APET.DAT found earlier in this appendix) .... 
The following text is a listing of the pointer file input used in the fire events $N$ Reactor EVNTRE calculations, and as found in the file NREAC_FIRE_PNTR.DAT.

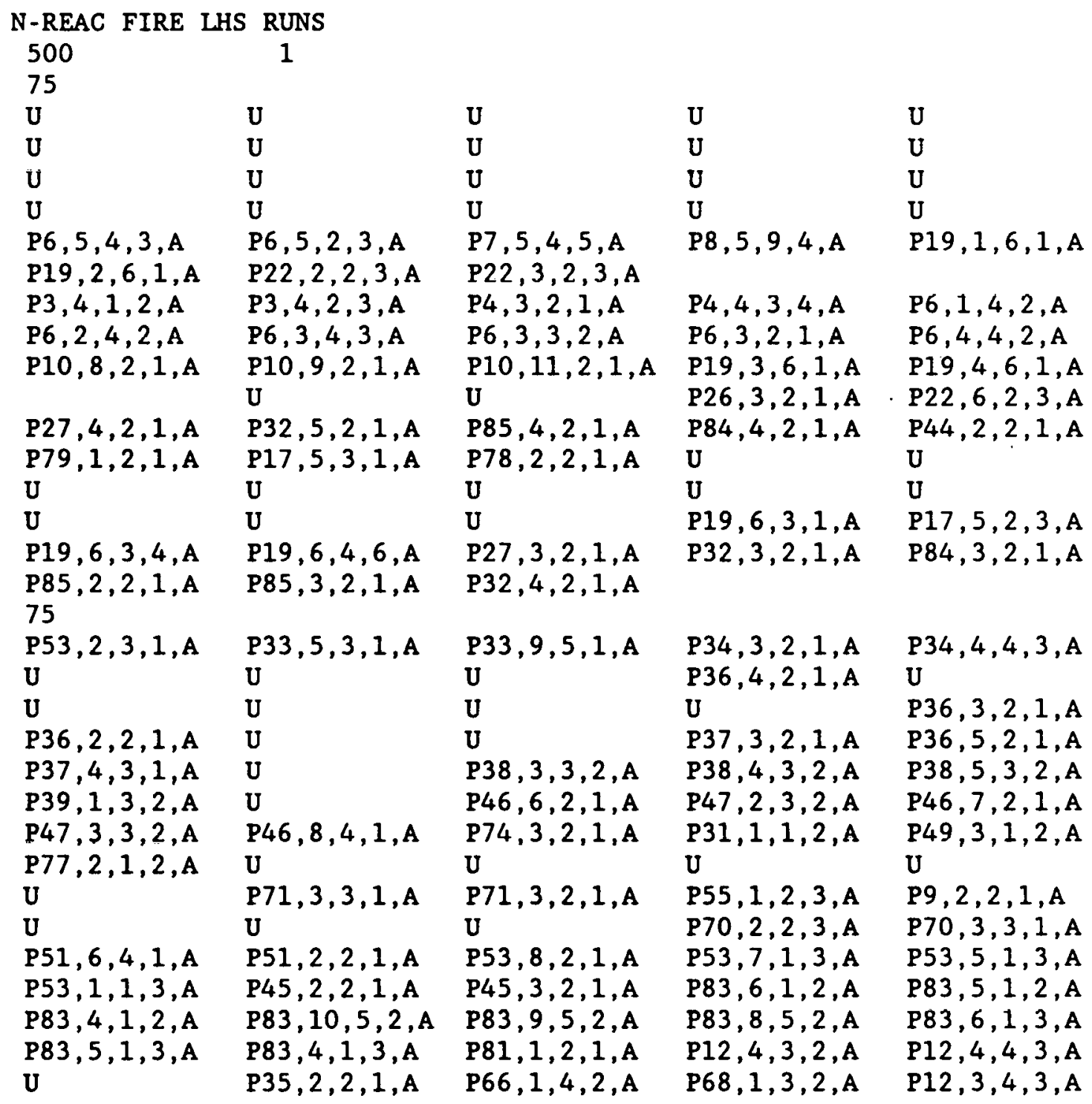


The following text is a listing of the keyword input file used in the $N$ Reactor seismic events EVNTRE calculation, and as found in the file NREAC_SEISHIC_LHS_REYWORD.DAT.

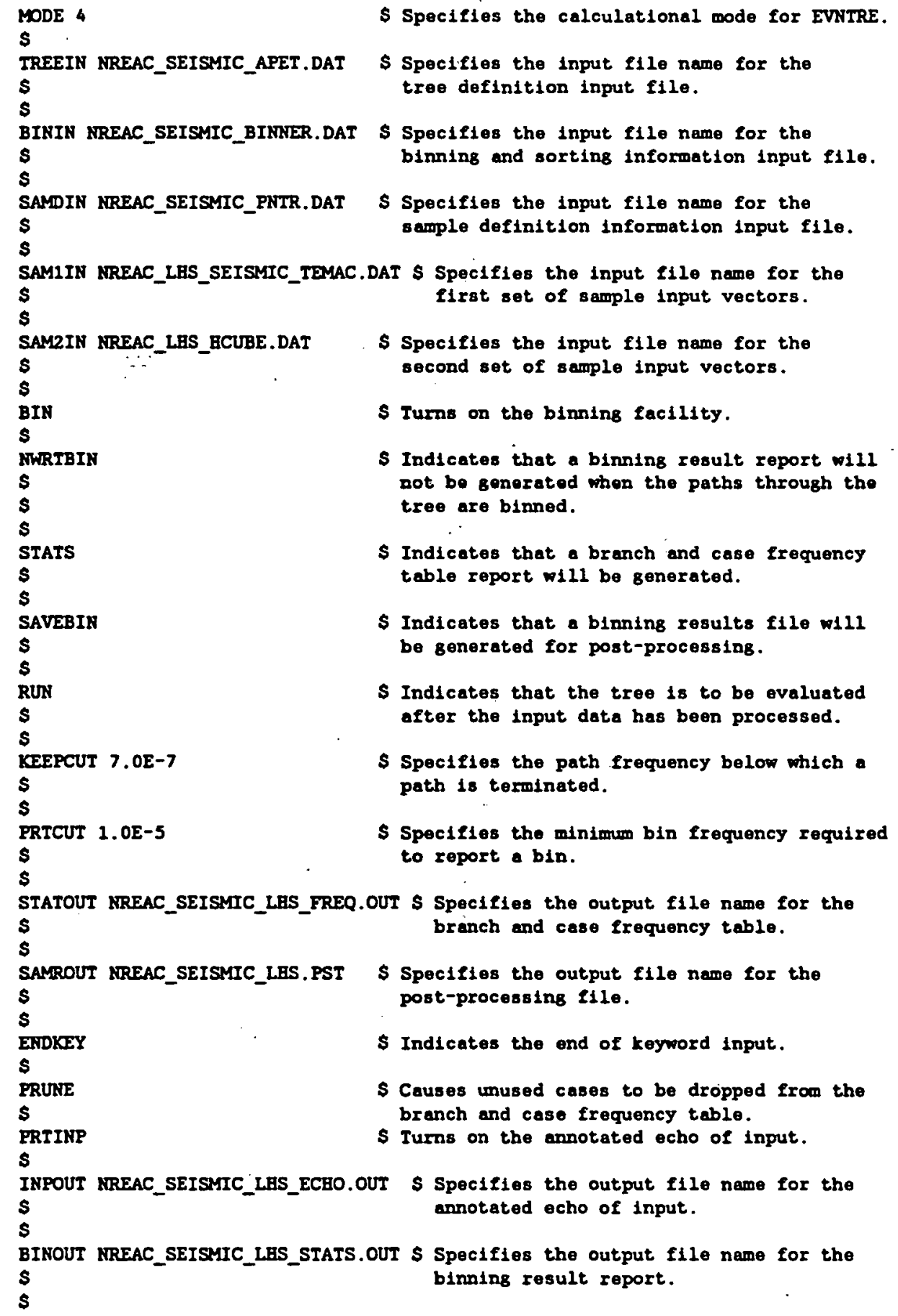


The following text is a listing of the accident progression event tree input file used in the seismic events $N$ Reactor EVNTRE calculation, and as found in the file NREAC_SEISMIC_APET.DAT. Most of this file is identical to the internal events event tree input. The only changes are in the first two events, and only that section of the file is reproduced here.

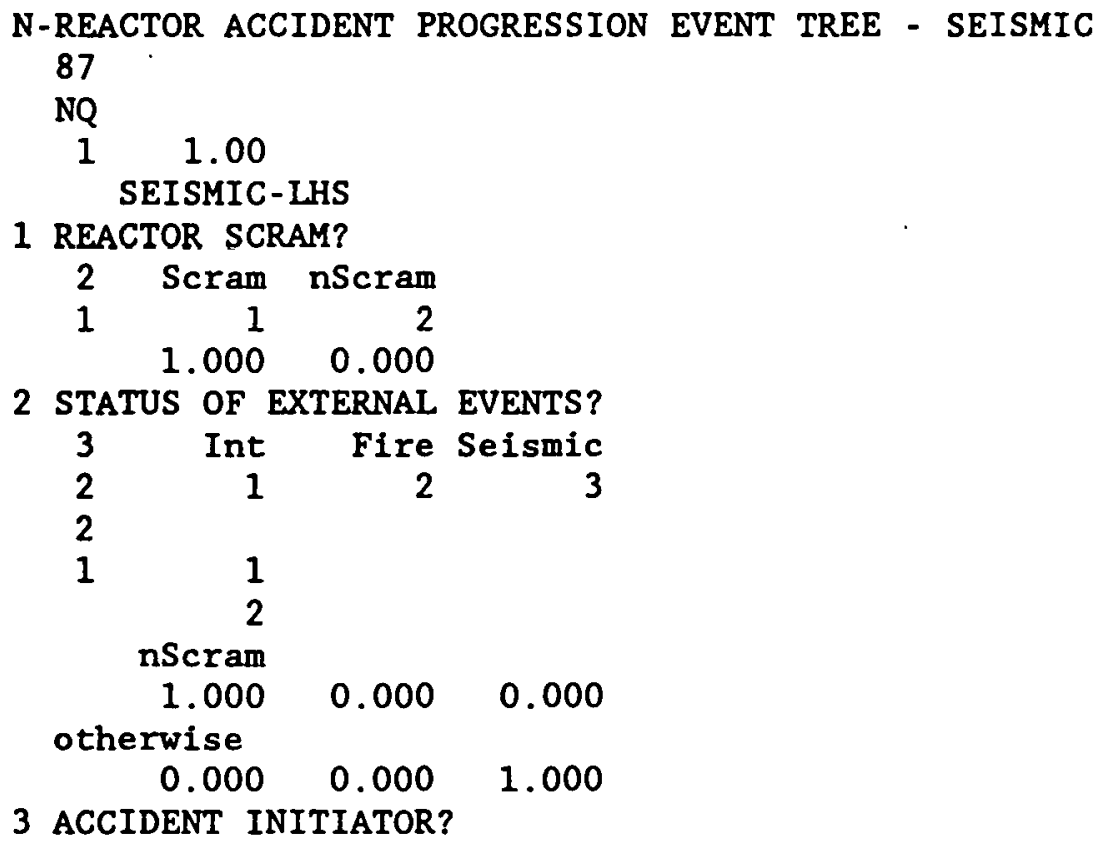

..... (the remainder of the file is identical to that listed in the file NREAC_IE_APET.DAT found earlier in this appendix) .... 
The following text is a listing of the pointer file input used in the seismic events $N$ Reactor EVNTRE calculations, and as found in the file NREAC_SEISKIC_PNTR.DAT.

\begin{tabular}{|c|c|c|c|c|}
\hline $\begin{array}{l}\text { N-REAC SEISMI } \\
500 \\
75\end{array}$ & 1 & & & \\
\hline $\mathbf{U}$ & $\mathbf{U}$ & U & U & $\mathrm{U}$ \\
\hline $\mathrm{U}$ & $\mathbf{U}$ & U & $\mathrm{U}$ & $\mathbf{U}$ \\
\hline $\mathbf{U}$ & $\mathbf{U}$ & $\mathbf{U}$ & $\mathbf{U}$ & $\mathbf{U}$ \\
\hline $\mathbf{U}$ & U & U & U & $U$ \\
\hline $\begin{array}{l}\mathrm{P} 6,5,4,3, \mathrm{~A} \\
\mathrm{P} 19,2,6,1, \mathrm{~A}\end{array}$ & $\begin{array}{l}\mathrm{P} 6,5,2,3, \mathrm{~A} \\
\mathrm{P} 22,2,2,3, \mathrm{~A}\end{array}$ & $\begin{array}{l}\mathrm{P} 7,5,4,5, \mathrm{~A} \\
\mathrm{P} 22,3,2,3, \mathrm{~A}\end{array}$ & $P 8,5,9,4, A$ & $\mathrm{P} 19,1,6,1, \mathrm{~A}$ \\
\hline $\begin{array}{l}\mathrm{P} 3,4,1,2, \mathrm{~A} \\
\mathrm{P} 6,2,4,2, \mathrm{~A} \\
\mathrm{P} 10,8,2,1, \mathrm{~A}\end{array}$ & $\begin{array}{l}\mathrm{P} 3,4,2,3, \mathrm{~A} \\
\mathrm{P} 6,3,4,3, \mathrm{~A} \\
\mathrm{P} 10,9,2,1, \mathrm{~A} \\
\mathrm{U}\end{array}$ & $\begin{array}{l}\mathrm{P} 4,3,2,1, \mathrm{~A} \\
\mathrm{P} 6,3,3,2, \mathrm{~A} \\
\mathrm{P} 10,11,2,1, \mathrm{~A} \\
\mathrm{U}\end{array}$ & $\begin{array}{l}\mathrm{P} 4,4,3,4, \mathrm{~A} \\
\mathrm{P} 6,3,2,1, \mathrm{~A} \\
\mathrm{P} 19,3,6,1, \mathrm{~A} \\
\mathrm{P} 26,3,2,1, \mathrm{~A}\end{array}$ & $\begin{array}{l}\mathrm{P} 6,1,4,2, \mathrm{~A} \\
\mathrm{P} 6,4,4,2, \mathrm{~A} \\
\mathrm{P} 19,4,6,1, \mathrm{~A} \\
\mathrm{P} 22,6,2,3, \mathrm{~A}\end{array}$ \\
\hline $\begin{array}{l}\text { P27, 4, 2,1, A } \\
\text { P79,1,2,1,A }\end{array}$ & $\begin{array}{l}\mathrm{P} 32,5,2,1, \mathrm{~A} \\
\mathrm{P} 17,5,3,1, \mathrm{~A}\end{array}$ & $\begin{array}{l}\text { P85, 4, 2,1, A } \\
\text { P78, 2, 2,1, A. }\end{array}$ & $\begin{array}{l}\mathrm{P} 84,4,2,1, \mathrm{~A} \\
\mathrm{U}\end{array}$ & $\begin{array}{l}\mathrm{P} 44,2,2,1, \mathrm{~A} \\
\mathrm{U}\end{array}$ \\
\hline $\mathbf{U}$ & $\mathbf{U}$ & $\mathbf{U}$ & $\mathrm{u}$ & $\mathbf{U}$ \\
\hline $\mathbf{U}$ & $\mathbf{U}$ & $\mathbf{U}$ & $\mathrm{P} 19,6,3,1, \mathrm{~A}$ & $\mathrm{P} 17,5,2,3, \mathrm{~A}$ \\
\hline $\begin{array}{l}\text { P19, 6, 3,4,A } \\
\text { P85, 2, 2, 1, A } \\
75\end{array}$ & $\begin{array}{l}\mathrm{P} 19,6,4,6, \mathrm{~A} \\
\mathrm{P} 85,3,2,1, \mathrm{~A}\end{array}$ & $\begin{array}{l}\text { P27, 3, 2, 1, A } \\
\text { P } 32,4,2,1, \text { A }\end{array}$ & $\mathrm{P} 32,3,2,1, \mathrm{~A}$ & $, 1, A$ \\
\hline $\begin{array}{l}\mathrm{P} 53,2,3,1, \mathrm{~A} \\
\mathrm{U}\end{array}$ & $\begin{array}{l}\mathrm{P} 33,5,3,1, \mathrm{~A} \\
\mathrm{U}\end{array}$ & $\begin{array}{l}\text { P33, 9,5, 1, A } \\
U\end{array}$ & $\begin{array}{l}\text { P34, 3,2,1, A } \\
\text { P } 36,4,2,1, A\end{array}$ & $\begin{array}{l}\text { P34, 4, 4, 3, A } \\
\mathrm{U}\end{array}$ \\
\hline $\mathbf{U}$ & $\mathbf{U}$ & $\mathrm{U}$ & $\mathrm{U}$ & $\mathrm{P} 36,3,2,1, \mathrm{~A}$ \\
\hline $\mathrm{P} 36,2,2,1, \mathrm{~A}$ & U & U & P37, 3,2,1,A & P36, 5, 2, 1, A \\
\hline $\mathrm{P} 37,4,3,1, \mathrm{~A}$ & $\mathbf{U}$ & $\mathrm{P} 38,3,3,2, \mathrm{~A}$ & $\mathrm{P} 38,4,3,2, \mathrm{~A}$ & P38, 5, 3, 2, A \\
\hline $\begin{array}{l}\text { P39, 1, 3, 2, A } \\
\text { P47, 3, 3, 2, A }\end{array}$ & $\begin{array}{l}\mathrm{U} \\
\mathrm{P} 46,8,4,1, \mathrm{~A}\end{array}$ & $\begin{array}{l}\mathrm{P} 46,6,2,1, \mathrm{~A} \\
\mathrm{P} 74,3,2,1, \mathrm{~A}\end{array}$ & $\begin{array}{l}\mathrm{P} 47,2,3,2, \mathrm{~A} \\
\mathrm{P} 31,1,1,2, \mathrm{~A}\end{array}$ & $\begin{array}{l}\mathrm{P} 46,7,2,1, \mathrm{~A} \\
\mathrm{P} 49,3,1,2, \mathrm{~A}\end{array}$ \\
\hline P77, 2,1,2,A & $\mathbf{U}$ & U & $\mathbf{U}$ & $\mathrm{U}$ \\
\hline $\mathbf{U}$ & P71, 3, 3, 1, A & P71, 3, 2 & $\mathrm{P} 55,1,2,3, \mathrm{~A}$ & P9, 2, 2, 1, A \\
\hline $\mathbf{U}$ & U & U & $\mathrm{P} 70,2,2,3, \mathrm{~A}$ & $\mathrm{P} 70,3,3,1, \mathrm{~A}$ \\
\hline $\begin{array}{l}\mathrm{P} 51,6,4,1, \mathrm{~A} \\
\mathrm{P} 53,1,1,3\end{array}$ & $\begin{array}{l}\mathrm{P} 51,2,2,1, \mathrm{~A} \\
\mathrm{P} 45,2,2,1,\end{array}$ & $\begin{array}{l}\text { P53, 8, 2, 1, A } \\
\text { P45, 3,2, }\end{array}$ & $\begin{array}{l}\mathrm{P} 53,7,1,3, \mathrm{~A} \\
\mathrm{P} 83,6,1,2, \mathrm{~A}\end{array}$ & $\begin{array}{l}\mathrm{P} 53,5,1,3, \mathrm{~A} \\
\mathrm{P} 83,5,1,2, \mathrm{~A}\end{array}$ \\
\hline $\begin{array}{l}\mathrm{P} 83,4,1,2, \mathrm{~A} \\
\mathrm{P} 83,5,1,3, \mathrm{~A}\end{array}$ & $\begin{array}{l}\mathrm{P} 83,10,5,2, \mathrm{~A} \\
\mathrm{P} 83,4,1,3, \mathrm{~A}\end{array}$ & $\begin{array}{l}\mathrm{P} 83,9,5,2, \mathrm{~A} \\
\mathrm{P} 81,1,2,1, \mathrm{~A}\end{array}$ & $\begin{array}{l}\mathrm{P} 83,8,5,2, \mathrm{~A} \\
\mathrm{P} 12,4,3,2, \mathrm{~A}\end{array}$ & $\begin{array}{l}\mathrm{P} 83,6,1,3, \mathrm{~A} \\
\mathrm{P} 12,4,4,3, \mathrm{~A}\end{array}$ \\
\hline $\mathrm{U}$ & P $35,2,2,1, A$ & $\mathrm{P} 66,1,4,2, \mathrm{~A}$ & $\mathrm{P} 68,1,3,2, \mathrm{~A}$ & $\mathrm{P} 12,3,4,3, \mathrm{~A}$ \\
\hline
\end{tabular}




\section{APPENDIX F :}

EVNTRE EXECUTION AND SELECTED OUTPUT

\section{F.1 CALCULATIONAL PEDIGREE}

All calculations were performed on VAX 8650 and VAX 8700 computers running security enhanced VMS 4.7 and the VAX FORTRAN compiler Version 4.7.271. The EVNTRE [F-1] code package used was dated November 11, 1988, as received from the code authors. The EVNTRE post-processor PSTEVNT program used was dated October 6, 1988, as received from the code authors. Listings of input files for the calculations are found in Appendix $E$, with the exception that the Latin Hypercube sample file and the TEMAC output files are found in Reference $[F-2]$, and were preprocessed for use in these EVNTRE runs as described in Appendix $A$ and Appendix $B$. Listings of the output files are found below.

\section{F.2 SELECTED EVNTRE OUTPUT}

The EVNTRE code package produces a very large volume of output literally pounds for each calculation if all is printed. The available output includes listings of all bins and frequencies for each of the 500 sample members in the APET uncertainty analysis and additional frequencybased sort information, also for each sample member. In order to reduce space requirements but still provide outputs that are meaningful to examine, two files from each execution are included in this appendix. These are the frequency file and the master bin list.

The frequency file for each execution lists all events, cases and branches in the APET and gives the fraction of the total probability (integrated over all sample members) that was placed in each. This allows an in-depth assessment of what happens at each step in the APET. It is this information that was summarized to provide probability split fractions in Chapter 3 of this report for each case in each event.

The second output file is the master bin list. This is a list of all bins that survived the cutoff probability for any of the sample members in the analysis. The bins are composed of a series of 14 character acronyms as described in Chapter 3.

The six files named below are listed on the following pages. All master bin lists are presented first, followed by the frequency report files. 
Internal Events Analysis Output Files

NREAC_IE_LHS_FREQ.OUT

NREAC_IE_LHS_NOTBYOBS_MASTER.KEP

Fire Analysis Output Files

NREAC_FIRE_LHS_FREQ.OUT

NREAC_FIRE_LHS_NOTBYOBS_MASTER:KEP

Seismic Analysis Output Files

NREAC_SEISMIC_LHS_FREQ.OUT

NREAC_SEISMIC_LHS_NOTBYOBS_MASTER.KEP
Internal events frequency output file

Internal events master bin list

Fire analysis frequency output file

Fire analysis master bin list

Seismic analysis frequency output file

Seismic analysis master bin list

\section{F.3 REFERENCES}

F-1. J. M. Griesmeyer and L. N. Smith, A Reference Manual for the Event Progression Analysis Code (EVNTRE), SAND88-1607, NUREG/CR-5174, U. S. Nuclear Regulatory Commission, September 1989.

F-2. D. M. Kunsman, et. al., Level III Probabilistic Risk Assessment for $N$ Reactor, WHC-MR-0045, SAND89-2102, Westinghouse Hanford, April 1990. 
The following is a listing of the internal events master bin list as found in the file NREAC_IE_LHS_NOTBYOBS_MASTER.KEP. The file consists of one column of bin identifiers and is reproduced as three columns in this listing.

N-REACTOR I.E. MASTER BIN LIST, NOTBYOBS, 17 FEB 90

\begin{tabular}{|c|c|}
\hline 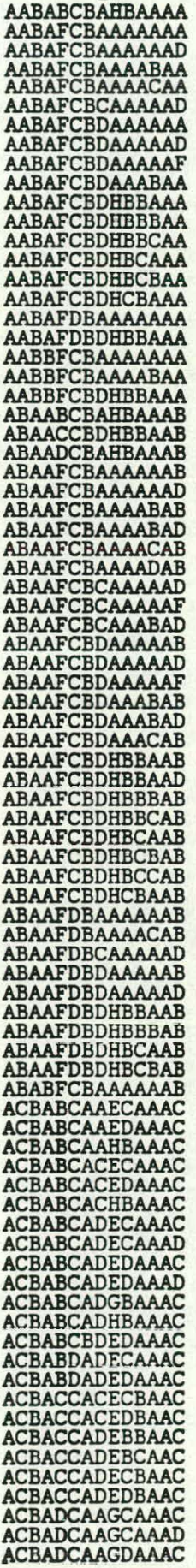 & 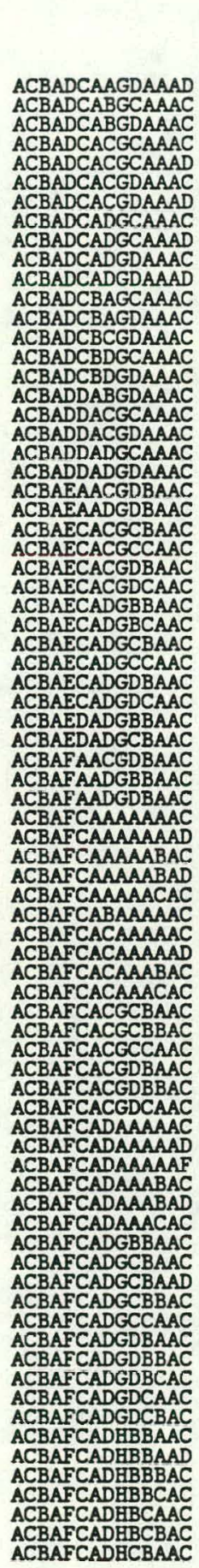 \\
\hline
\end{tabular}

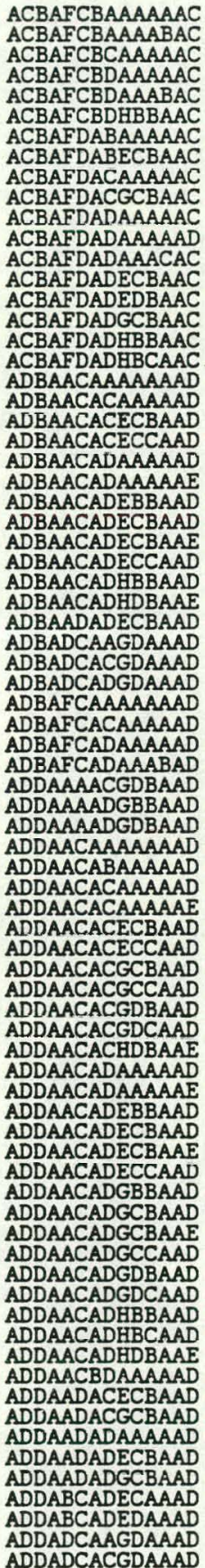




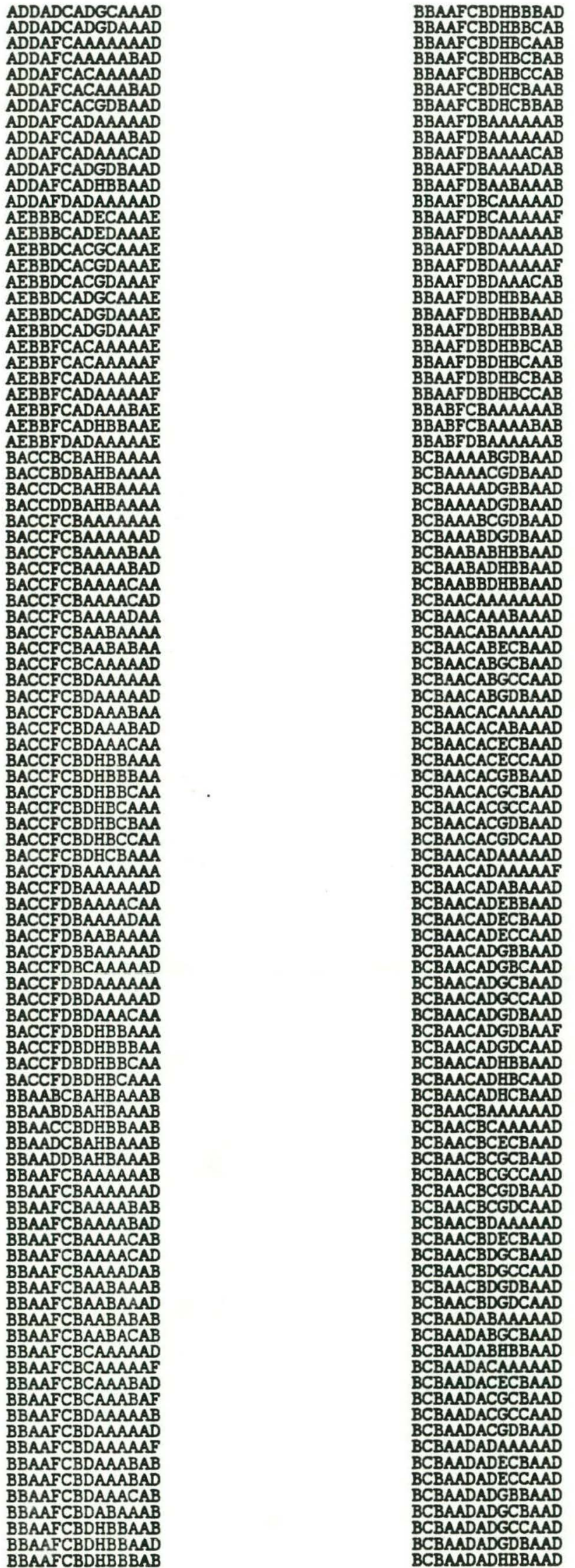

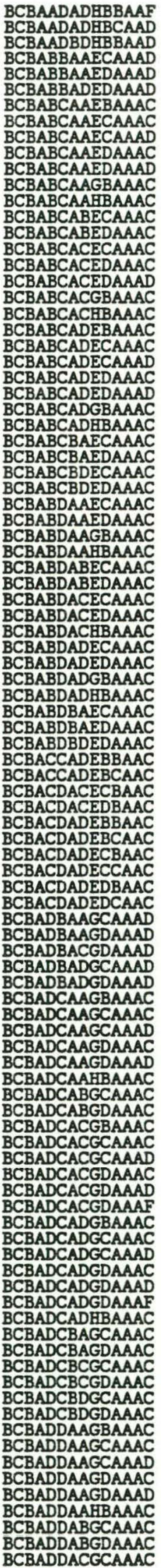




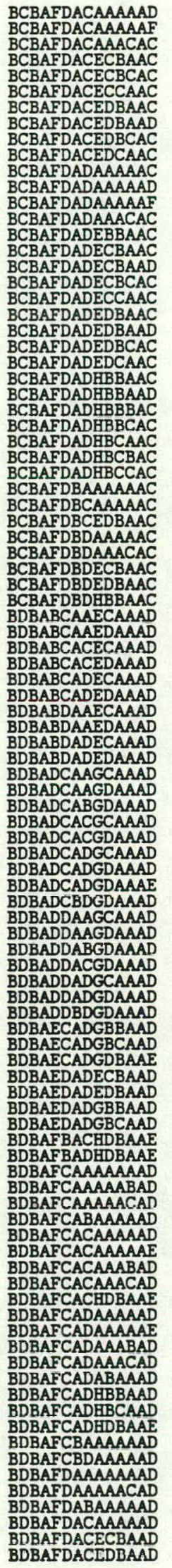

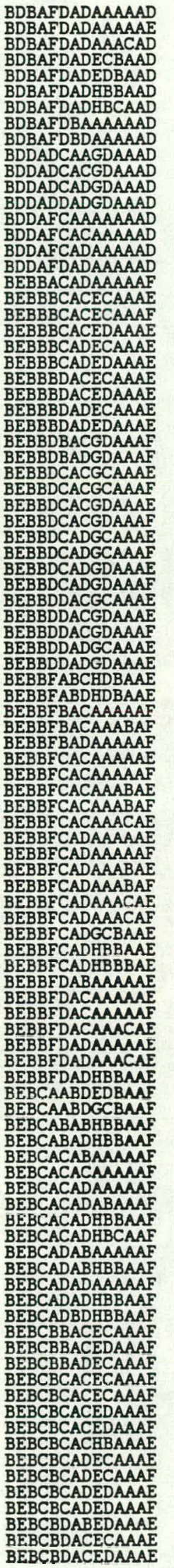




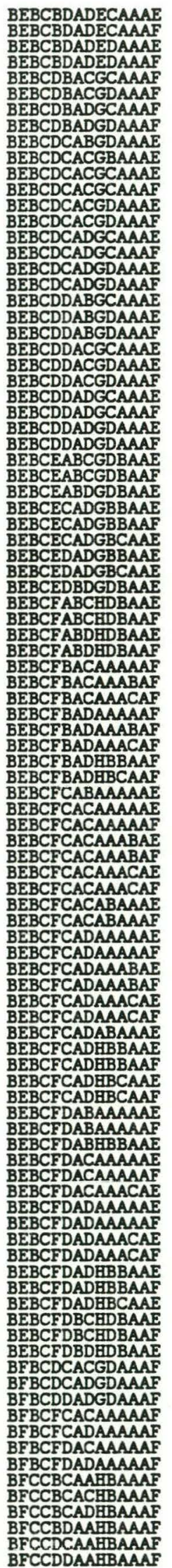

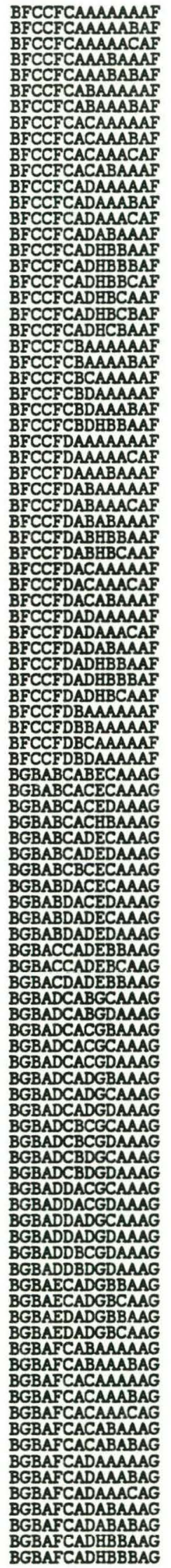

BFCCFCAAAAAAAF

BFCCFCAAAAACAF

BFCCFCAAABABAF

BFCCFCABAAAAAF

BFCCFCACAAAAAF

BFCCFCACAAABAF

BFCC CACAAACAF

BFCCFCADAAAAAF

BFCCFCADAAABAF

BFCCFCADABAAAF

BFCCFCADHABAA

BFCC FCADHBCA

BFC CADEBCAA

BFCFADCAF

BFCCFCBAAAAAAF

BFCCFCBAAAABAF

BFCCF

BFCCFCBDAAABAF

BFCF

BFCCFDAAAAACAF

BFCCFDAAABAAAF

BFCCFDABAAACAF

BFCCFDABHBBAAF

BFCCFDABHBCAAF

BFCCFDACAAAAAF

BFCCFDACABAAA

BFCCFDADAAAAAF

BFCCFDADAAACAF

BFCCFDABAAA

BFCCFDADHBBBAF

BFCCFDADHBCAA

BFCCFDPAAP

BFCCFDBCAAAAA

BGBABCABECAAAC

BGBABCACECAAAG

BCADECAAAG

ECAAAG

BGBADCABGDAMAG

B A

BGBADCACGDAAAG

BGBADCADGBAAAG

DCBDGCAAAG

作

BGBAFCACAAABAG

BGBAFCACAAACA

BGBAFCACABABA

BGBAFCADAACA

BGBA CADABAAA

BGBAFCADHBBBAG
BGBAFCADHBCAAG

BGBAFCADHBCBAG

BGBAFCADHCBAAG

BGBAFCBCAAAAAG

BGA

- BAF CDAAAAG

BGA CDDAAAG

BGBAFDAAAAAAG

BGBAFDACAAAAAG

BGAFACAAACAG

BGBAFDADAAAAG

BGBAFADAAACAG

BGBAFDADHBBAAG

BGBAFDADHBCAAG

BGBAFDBCAAAAAG

BGBAFDBDAAAAAG

CACCFDBAAAAAAA

CBABFDBAAAAAAB

CBABFDBDEBBAAB

CCBAABABHBBAAD

CCBAABADHBBAAD

CCBAABBDHBBAAD

CCBAADABHBBAAD

CCBAADADHBBAAD

CCBAADBDHBBAAD

CCBABBAAECAAAD

CCBABBAAEDAAAD

CCBABBACECAAAD

CCBABBACEDAAAD

CCBABBADECAAAD

CCBABBADEDAAAD

CCBADBAAGCAAAD

CCBADBAAGDAAAD

CCBAD BACCCAAAD

CCBADBACGDAAAD

CCBAD

CCBAD ADGDAAAD

CCBADAGAAAD

CCBADDAAGCAAAD

CCBADDAAGDAAAD

CCBADDACGDAAAD

CCBADDADGDAAAD

CCBAEBADECBAAD

CCBAEBADGBBAAD

CCBAFBAAAAAAAD

CCBAFBAAAAABAD

CCBAFBAAAAACAD

CCBAFAAAAD

CCBAFBACAAAAAD

CCBAF BACAAABAD

CCBAFBACAAACAD

CCBAFBACEBAD

CCBAFBADAAAAAD

CCBAFBADAAABAD

CCBAFBADAAACAD

CCBAFBADECBAAD

CCBAFBADECBBAD

CCBAFBADECCAAD

CCBAFBADHBBAAD CCBAFBADHBCAAD CCBAFBBAAAAAAD CCBAFBBDAAAAAD CCBAFDAAAAAAAD CCBAFDABAAAAAD CCBAFDACAAAAAD CCBAFDADAAAAAD CDBAEBACGDBAA CDBAFBACAAAAAE

CDBAFBACEDBAAE CDBAFBADAAAAE CDBAFBADHDBAAE CEBBABAD CEBBADADABAA CEBBAADEBAA CEBBAECAAA CE CEBB CEBDBDACAAA CEBBBDACEDAAAE CEBBBDADECAAAE CEBBBDADEDAAAE CEBBDBACGCAAAF CEDACAA CEBDADCAA CEADA DAAA CEBBDDABGDAAAI CEBBDDACGCAAA CEBBDDACGDAAAI CEBBDDACGDAAA CEBBDDADGCAAA CEBBDDADGDAAA CEBBDDADGI CEBBEBADGBBAAF CEBBEDADGBBAAE CEBBEDADGBCAAE CEBBEDBDGDBAAE CEBBFBACAAAAAF CEBBFBACAAABAF CEBBFBACAAACA CEBBFBADAAAAAF 


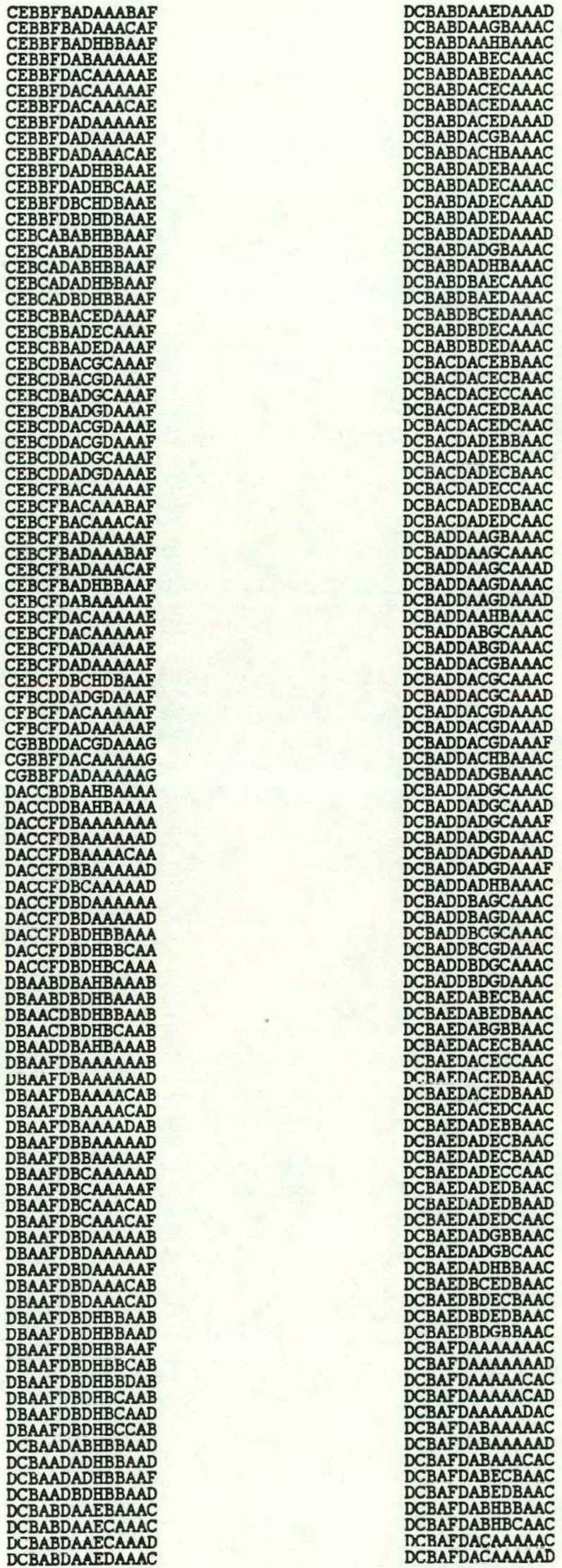

DCBAFDACAAAAAF DCBAFDACAAACAC DCBAFDACAAACAD DCBAFDACECBAAC DCBAFDACECBAAD DCBAFDACECBAAF DCBAFDACECBCAC DCBAFDACECCAAC DCBAFDACECCCAC DCBAFDACEDBAAC DCBAFDACEDBAAD DCBAFDACEDBAAF DCBAFDACEDBCAC DCBAFDACEDCAAC DCBAFDACEDCCAC DCBAFDADAAAAAC DCBAFDADAAAAAD DCBAFDADAAAAAF DCBAFDADAAACAC DCBAFDADAAACAD DCBAFDADAAADAC DCBAFDADEBBAAC DCBAFDADEBBCAC DCBAFDADECBAC DCBAFDADECBAA DCBAFDADFCBAAF DCBAFDADECBC DCBAFDADECCAC DCBAFDADECCCAC DCBAFADCCAC DCBAFADEDBAC DCBAFDADEBAAD DCBAFDADEDCAC DCBAFDADEDCAAC DCBAFDADEDCCAC DCBAFDAD DCBAFDADHBBAAD DCBAFDADHBBCAC DCBAFDADHBCAAC DCBAFDADHBCAAD DCBAFDADHBCCAC DCBAFDBAAAAAAC DCBAFDBAAAACAC DCBAFDBBAAAAAC DCBAFDBCAAAAAC DCBAFDBCECBAAC ( DCBAFDDAAAAC DCBAFDBDAAAAAD DCBAFDBDECBAAC DCBAFDBDECCAAC DCBAFDBDEDBAAC DCBAFDBDEDCAAC DCBAFDBDHBBAAC DCBAFDBDHBCAAC DDBABDAAECAAAD DDBABDAAEDAAAD DDBABDACECAAD DDBABDACCDAAD DDBABDADECAAD DDBADADCAAD DDBABDADEDAAAD DDBACDADECBAAD DDACDADEDBAAD DDBADAAGCAAD DDBADAAGAAAD DDBADDACGCAAAD DDADACGDAAD DDBADDADGCAAAD DDBADDADGDAMAD DDBADDBAGDAAAD DDBADDBDGDAAAD DDBAEDACECBAAD DDBAEDACEDBAAD DDBAEDADECBAAD DDBAEDADECCAAD DDBAEDADEDBAAD DDAEDADEDCAAD DAEDADGBCAAD DDBAFDAAAAAAAD DDBAFDAAAAACAD DDBAFDABAAAAAD DDBAFDACAAAAAD DDBAFDACAAAAAE DDBAFDACAAACAD DDBAFDACECBAAD DDBAFDACECCAD DDBAFDACED BAAD DDAFDACEDAD DBAFADA D DA DAAAE DDBAFDADAACAD DDAFADBAD DDBAFDADECBAAD DDBAFDAECCAD DBA DDBAFDADEDBAAD DDDAFDADEDBCAD 


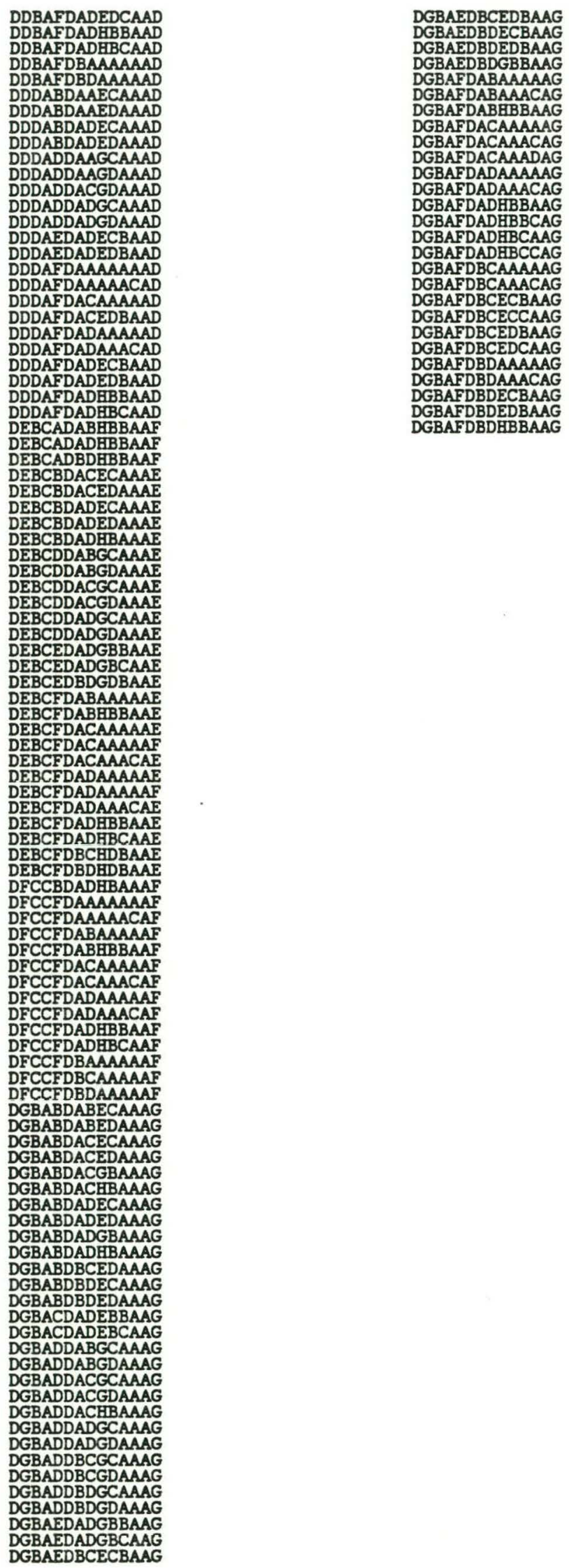


The following is a listing of the fire events master bin list as found in the file NREAC_FIRE_LHS_NOTBYOBS_MASTER.KEP. The file consists of one column of bin identifiers and is reproduced as three columns in this listing.

N-REACTOR FIRE MASTER BIN LIST, NOTBYOBS, 17 FEF 90 CBABFDBAAAAAAB CCBAABAAAAAAAD CCBAABACAAAAAD CCBAABADAAAAAD CCBAADABHBBAAD CCBAADADHBBAAD CCBAADBDHBBAAD CEBBABACAAAAA CEBBADACAAAAA CEBBADADAAAAA CEBBADAD CEBBADAD BRBAA CFACBBA BFCAA CFACBBABEDAAA CFACBBACECAAA CFACBBACEDAAA CFACBBACGBAAA CFAC BAC CFCBADAA CTAC BADECAA CFCDADCAA CFACBAA CFACB CFCBDABECAA CFACBDA CFACDABDAAA CACBABAAF CFACBDABHBAAA CFACBDACEDAAA CFACBDACECAAA CFACBDACEDAAA CFACBDACGBAAA CFACBDACGBAAA CFACBDADDCAAA CFACBDADEDAAA CFACBDADGBAAA CFACBDADHBAAAF CFACCBABEBBAAF CFACCBABECBAA CFACCBABEDBAAF CFACCBACEBBAAF CFACCBACECBAAF CFACCBACEDBAAF CFACCBACGBBAAF CFACCBACHBBAAF CFACCBADDBBAAF CFACCBADEBBAA CFACCBADECBAA CFACCBADEDBAAF CFACCBADGBBAAF CFACCBADHBBAAF CFACCBBCECBAAF CFACCBBCEDBAAF CFACCDABEBBAAF CFACCDABECBAA CFACCDABEDBAA CFACCDABGBBAA CFACCDABHBBAAF CFACCDACEBBAA CFACCDACECBAAF CFACCDACECBAAF CFACDACEDBAAF CFACCDACGBBAA CFACCDACABBAA CFACCDADDBBAA CFACCDADEBBAA CFACCDADECBAA CFACCDADEDBAA CFACCDADGBBAAF CFACCDADHBBAAF CFACCDBBEBBAA CFACCDBBECBAAF CFACCDBBEDDAAA CFACCDBCECBAA CFACCDBCEDBAAF CFACCDBDEBBAAF CFACCDBDECBAA CFACCD CFACDBABGCAA CPCDABCAAA CrCDAagda CFACDBACGCAMAF

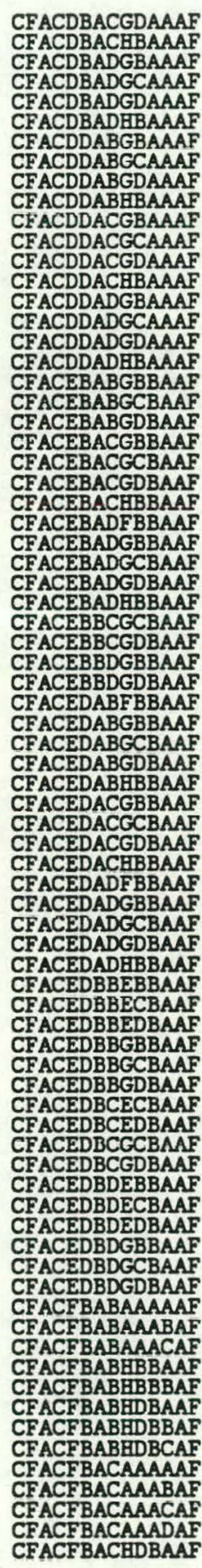

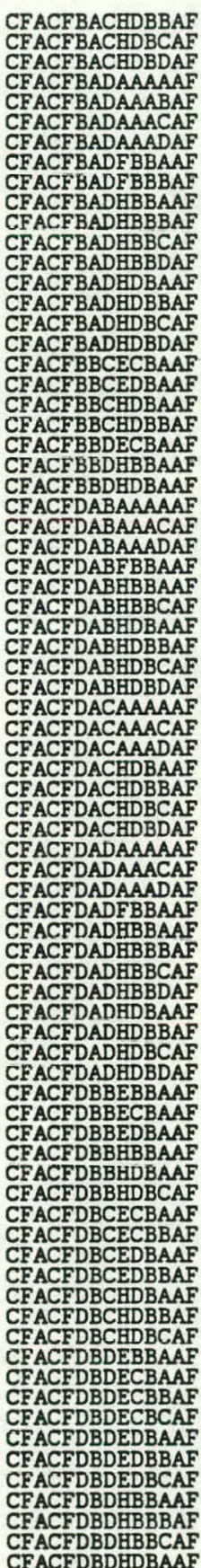



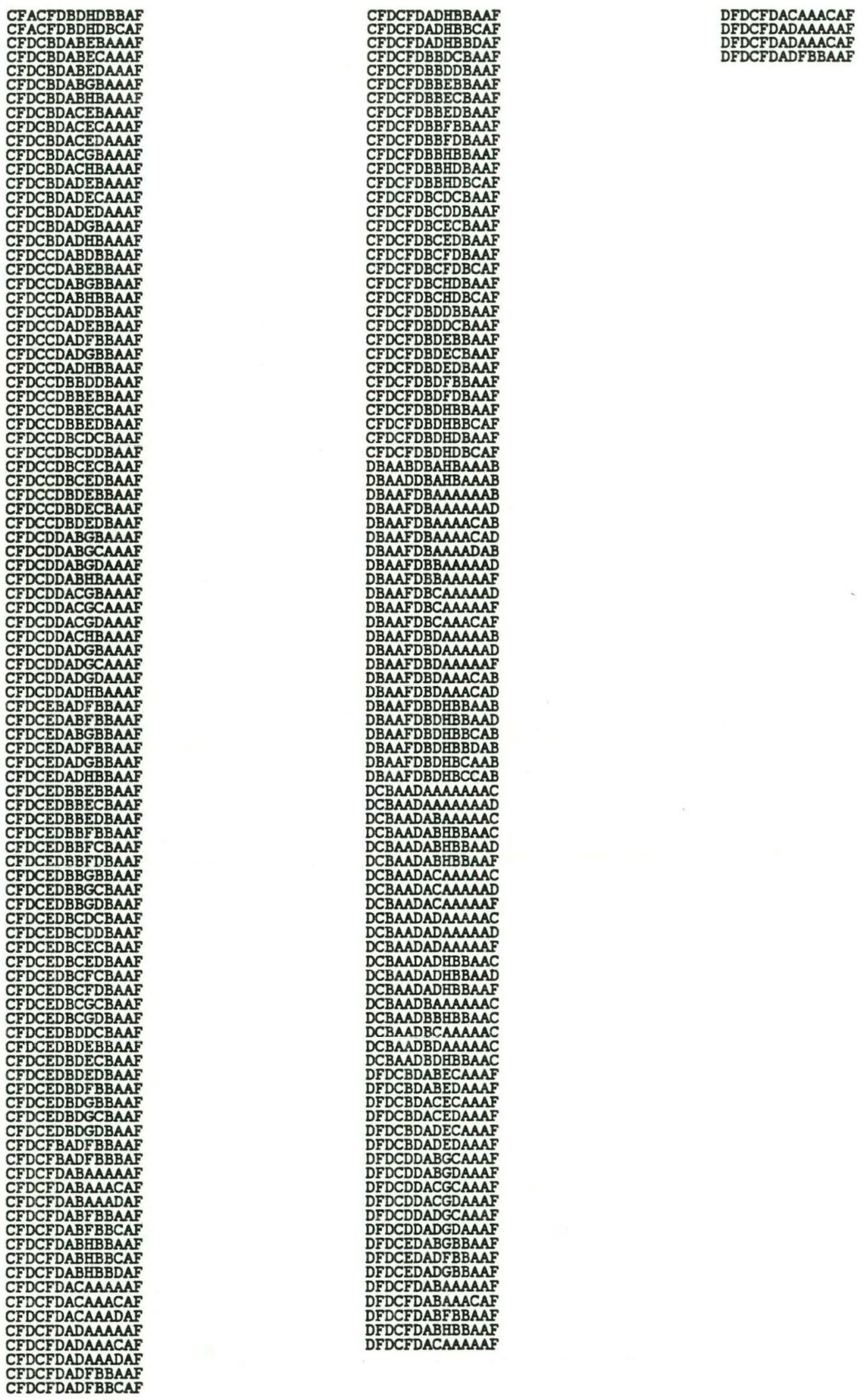
CDCDDAA CDCFBDAA CFF CFCFD CDCFA CFDFDBFBAA CFDFDBDAAF CFDCFDBBHDBAA CFDCFDBBDBCAF CFDCFDBCDCBAAF CFDCFDBCDDBAA CFDCFDBCECBAA CFDCFDBCEDBAA CFDCFDBCFDBAA CFDCFDBCFDBCAF CFDCFDBCHDBAA CFDCFDBCHDBCAF CFDCFDBDDBBAAF CFDCFDBDDCBAAF CFDCFDBDEBBAAF CFDCFDBDECBAAF CFDCFDBDEDBAAF CFDCFDBDFBBAAF CFDCFDBDFDBAAF CFDCFDBDHBAAA CFDCFDBDHBBCAF CFDCFDBD CFDCFDBDIDCA DBAABDBAHBAAAB DBAADDBAFBAAAB DBAAFD $A A A A A A B$ DAA DBAACA DAAFD AAACAD DBAAFDAAD DBAAFDBAA DAA D DBAAFDA DAAF DAAF DCAAAAAF DBAAFDBCAAACA DBAAF DBDAAAAA DBAAFDBDAAAAAD DBAAF DDAAAAAF DBAAFDDAAACA DBAAFDBDAAACA DBAAFDBDHBBAA DBAAFDBDABAAD DBAAFDDABBCA DBAAFDBDHBBDA DBAAFDBDHBCAAB DBAAFDBDHBCCA DCBAADAAAAAAAC DCBAADAAAAAAA DCBAADABAAAAAC DCBAADABHBBAAC DCBAADABHBBAAD DCBAADABHBBAAF DCBAADACAAAAC DCBAADACAAAAAD DCBAADACAAAAA DCBAADADAAAAC DCBAADADAAAD DCBAADADAAA DCBADADAAA $D C B A D A$ DCAADA DCAADA BAA DCBA BAAAAA DCBAAD DCBAADBDAAAAA DCBAADBDAAAAA DCAADA DFDCBDABECAAA DFDCDABDAAA DFDCDACECAAA DFCDACEAAA DFDCBDADECAAAF DFDCBDADEDAAA DFDCDDABGCAAA DFDCDDABGDAAA DFDCDDACGCAAA DFDCDDACGDAAA DFDCDDADGCAAA DFDCDDADGDAAA DFDCEDABGBBAAF DFDCEDADFBBAAF DFDCEDADGBBAA DFDCFDABAAAAA DFDCFDABAAACA DFDCFDABFBBAA DFDCFDABHBBAAF DFDCFDACAAAAAF 
The following is a listing of the seismic events master bin list as found in the file NREAC_SEISMIC_LHS_NOTBYOBS_MASTER.KEP. The file consists of one column of bin identifiers and is reproduced as three column in this listing.

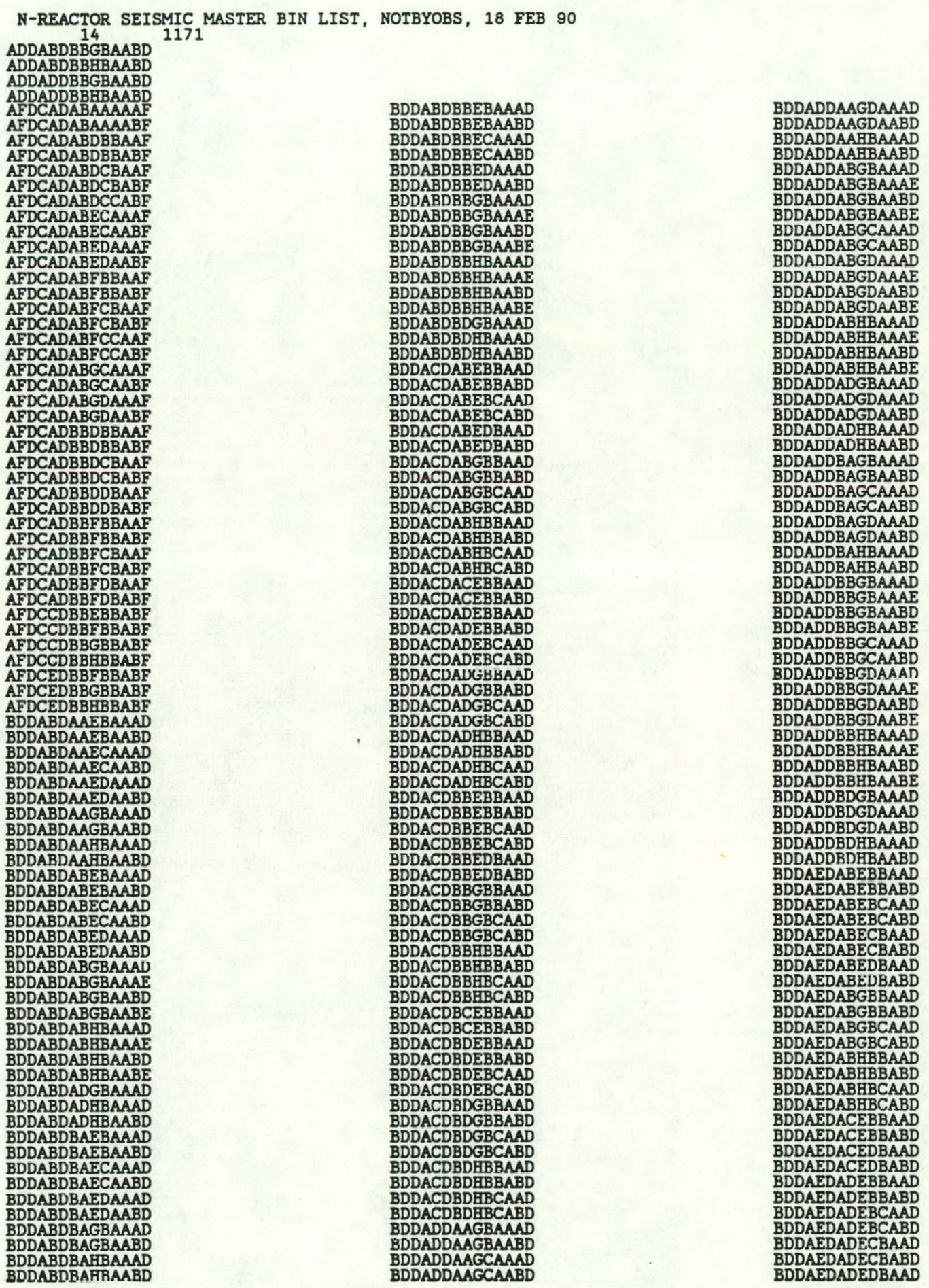


BDDAFDBBAAAAAE

BDDAFDBBAAAABD

DDA DBAAAABE

BDAFBAACAD

DAFACD

BDAFBAAAD

BDA DBAAAND

DDA

DA

DAND

BDDAFDBDABD

BDDAFDBBECAAD

BDDAFDBBEDCABD

BDDAFDBBHBBAAD

BDDAFDBBHBBABD

BDDAFDBBABCAAD

BDDAFDBBHBCABD

BDDAFDBCEDBAAD

BDDAFDBCEDBABD

BDDAFDBDAAAAAD

BDDAFDBDAAAABD

BDDAFDBDEBBAAD

BDDAFDBDEBBABD

BDDAFDBDECBAAD

BDDAFDBDECBABD

BDDAFDBDEDBAAD

BDDAFDBDEDBABD

BDDAFDBDEDCAAD

BDDAFDBDEDCABD

BDDAFDBDHBAAD

BDDAFDBDHBBABD

BDDAFDBD B

BDAFDD

$\triangle D A D D D$

BDDAFDBDHBCAAD

BDDAFDBDHBCABD

BFDCBDABEBAAA

BFDCBDABEBAABF

BFDCBDABECAAAF

BFDCBDABECAABF

BFCDA EDAAAF

B D C

BFCDABGBAA

BFDCDABGBABF

BFDCBDABHBAAA

BFDCBDABHBAABF

BFDCCDABEBBAAF

BFDCCDADEBBABF

BFDCCDABEBCAAF

BFDCCDABEBCABF

BFDCCDABFBBAA

BFDCCDABFBBABF

BFDCCDABFBCAAF

BFDCCDABFBCABF

BFDCCDABGBBAAF

BFDCCDABGBCAA

BFDCCDABGBCABF

BFDCCDABHBBAA

BFDCCDABHBBABF

BFDCCDABHBCAAF

BFDCCDABHBCABF

BFDCCDBBDBBAAF

BFDCCDBBDBBABF

BFDCCDBBDCBAAF

BFDCCDBBDCBABF

BFDCCDBBDDBA

BFDCCDBBDDAAF

$\triangle F C C D B B D B A$

作

FDCC

BECBABF

DCCDBBEDBAAF

BFDCCDBBEDBABF

BFDCCDBBFBBAAF

BFDCCDBBFBBABF

BFDCCDBBGBAAF

BFDCCDBBGBBABF

BFDCCDBBHBBA

BFDCCDBBABBABF

BFDCDDABGBAAF

BFDCDDABGBAABF

PFDCDDABGCAAA

BFDDABGCAAAF

B

BFDDABGDAAAF

BFDCDDABGDAABF

BFDCDDABHBAAAF

BFDCDDABHBAABF

BFDCEDABFBBAAF

BFDCEDABFBBABF

BFDCEDABFBCAAF

BFDCEDABFBCABF

BFDCEDABGBBAAF

BFDCEDABGBBABF

BFDCEDABGBCAAF

BFDCEDABGBCABF

BFDCEDAB

BFDCEDABHBABF
BFDCEDABABCABF

BFDCEDBBDBBAAF

PDCEDBBDDBAF

FDEDBDAAF

DCE

OFCDDBDAAF

BFDCEDEDBAF

PDCEBCBAAF

BFDCEDBBECBABF

BFDEDBBEDBAAF

BFDCEDBBEDBABF

BFCEDBFBAAF

BFDCEDBBFBBABF

BFDCEDBBFCBAAF

BFDCEDBBFCBABF

BFDCEDBBFDBAAF

BFDCEDBBFDBABF

BFDCEDBBGBBAAF

BFDCEDBBGBBABF BFDCEDBBGCBAAF BFDCEDBBGCBABF BFDCEDBBGDBAAF BFDCEDBBGDBABF BFDCEDBBHBBAAF BFDCEDBBABBABF BFDCFDABAAAAAF BFDCFDABAAAABF BFDCFDABAAACAF BFDCFDABAAACBF BFDCFDABABAAAF BFDCFDABABAABF BFDCFDABFBAAF BFDCFDABFAA BFDCFDABFBCAAF BFDCFDABFBCAAF PCCA BFDC A 列 BFDCFDABHBCAAF 列 PDC BFDCFDBBDBBABF $\triangle F D C D B D B A F$ PFC BFCFDBDAAF SFCCDBDDABF BFCFDDDBAF BDCDBDCBF AFDDBDAAF BFDCDDBEBABF BCDDBECAA BFDCFBDECABF BFDCFDBBEDBAAF BFDCFDBEDBABF BFDCFDBBEDBCAF BFDCFDBBEDBCBF BFDCFDBBFBBAAF BFDCFDBBFBBABF BFDCFDBBFDBAAF BFDCFDBBFDBABF BFDCFDBBFDBCAF BFDCFDBBFDBCBF BFDCFDBBHBBAAF BFDCFDBBHBBABF BFDCFDBBADBAAF BFDCFDBBHDBABF BFDCFDBBADBCAF BFDCFDBBHDBCBF CFDCBDABEBAAAF CFDCBDAB CFDCBDABECAAAF CFC CFDPAB CFCA CFCDAB CDCAA CFCBACAAF CFCDACEAAF CFDCDDACECAAF CFDCBDACECAAAF CFDCDACECAABF CFDCBDACEDAAAF CFDCBDACEDAABF CFDCBDACGBAAAF CFDCBDACGBAABF CFDCBDACHBAAAF CFDCBDACHBAABF CFDCBDADEBAAAF CFDCBDADEBAABF CFDCBDADECAAAF CFDCBDADECAABF CFDCBDADEDAAAF CFDCBDADEDAABF CFDCBDADGBAAAF CFDCBDADGBAABF CFDCBDADHBAAAF CFDCBDADHBAABF 

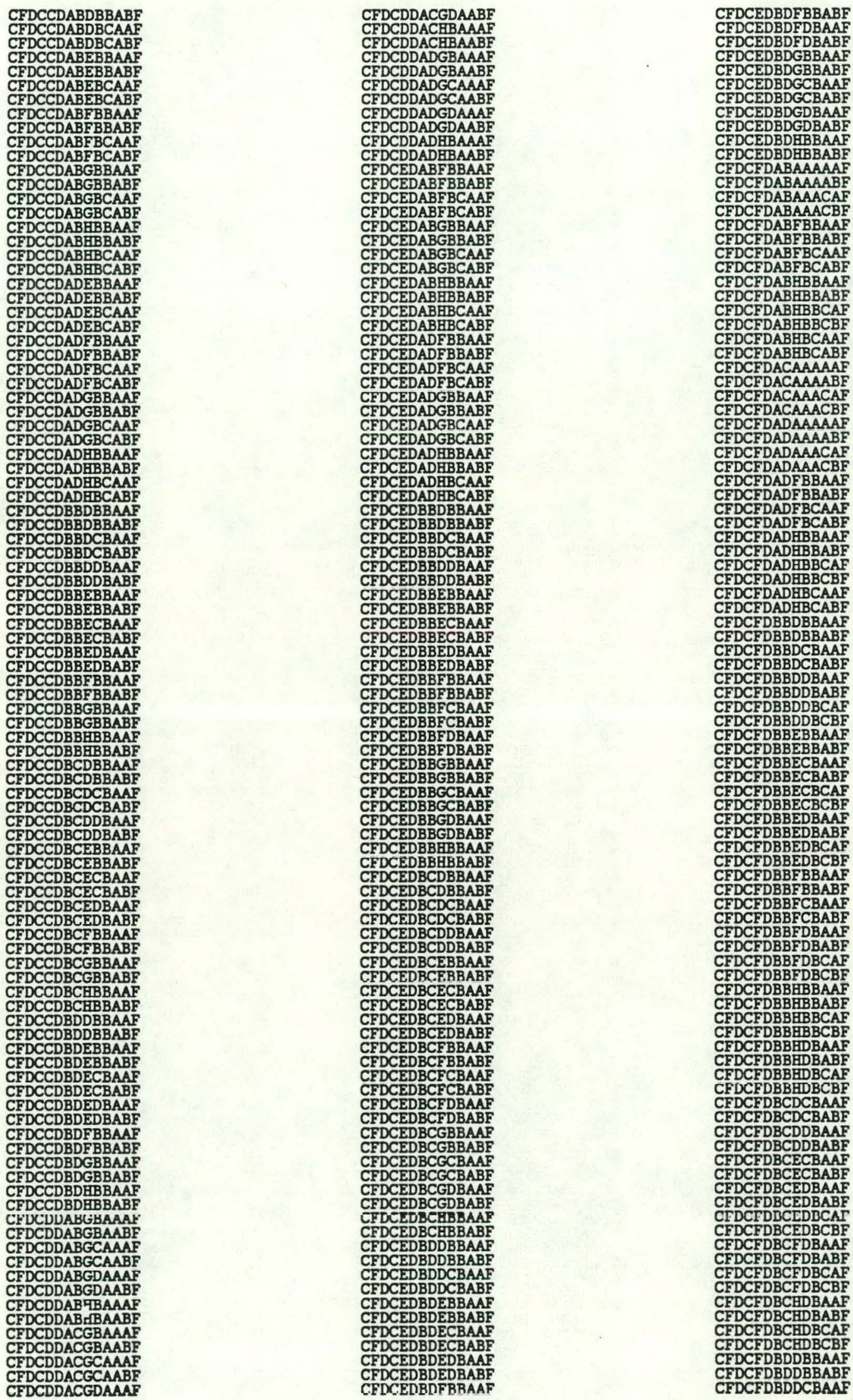


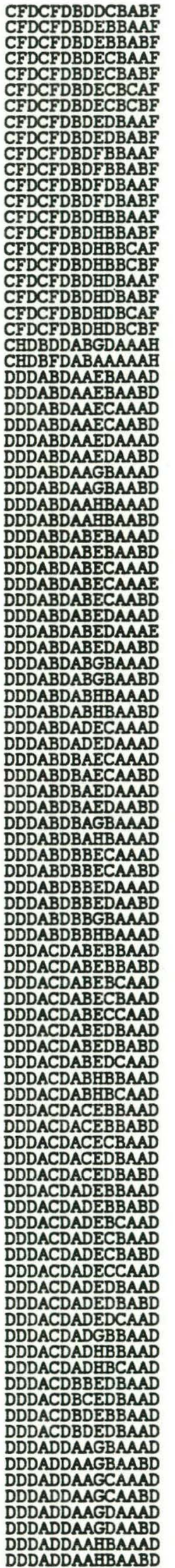

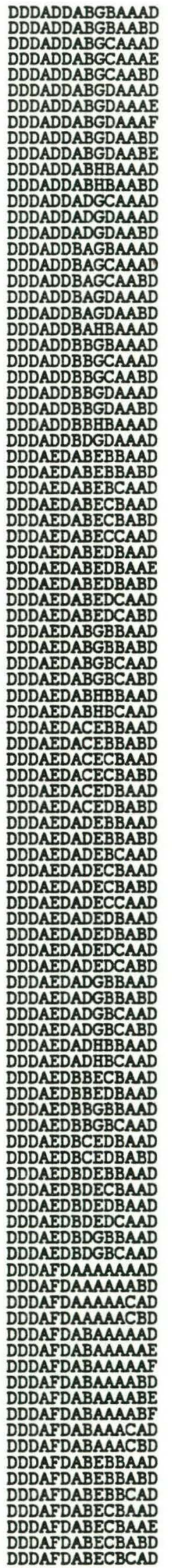

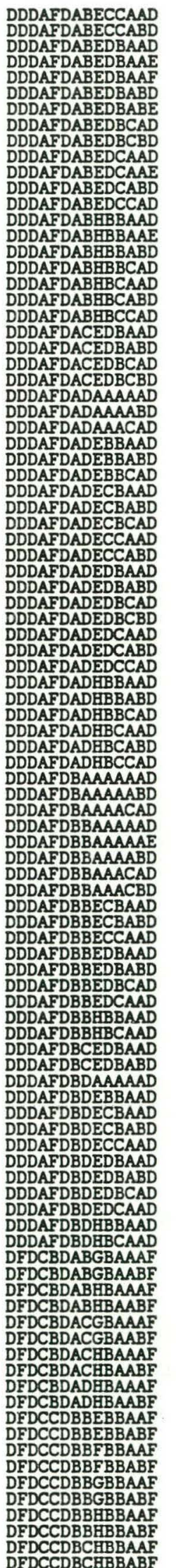




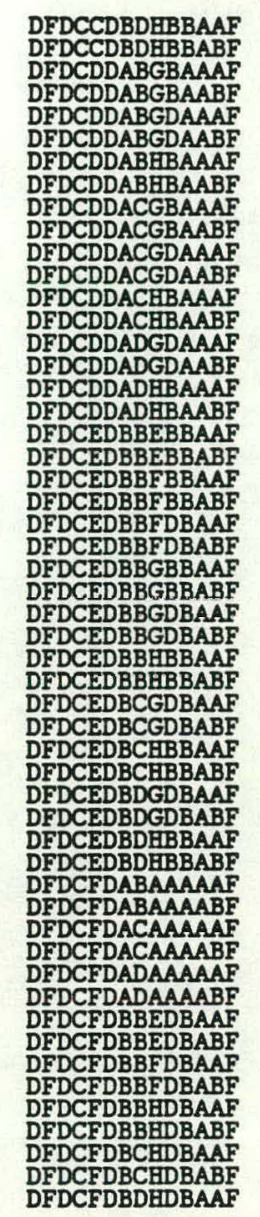

F-15 
The following is a listing of the internal events analysis frequency report as presented in the file NREAC_IE_LHS_FREQ.OUT.

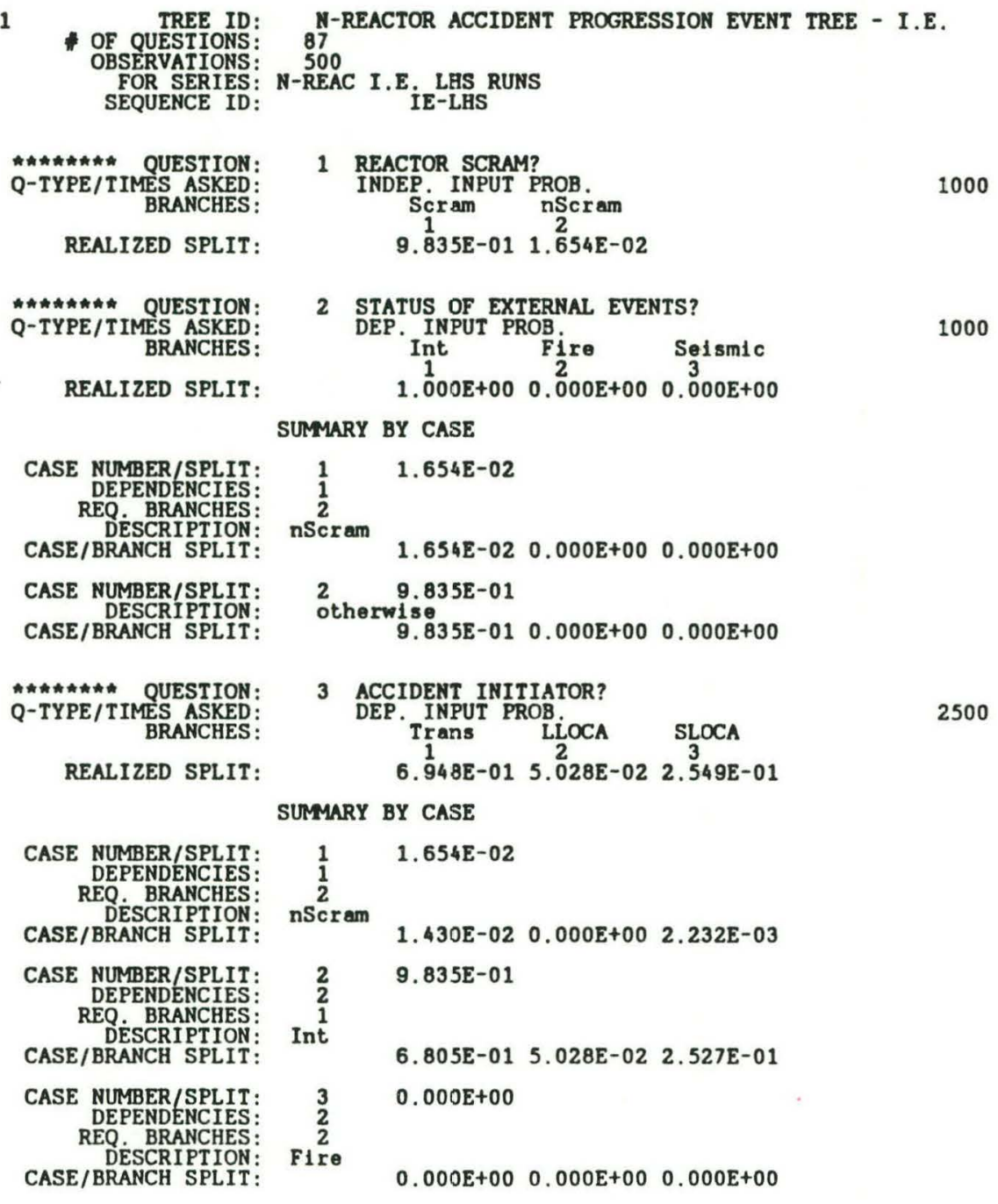

$0.000 E+00 \quad 0.000 E+00 \quad 0.000 E+00$ 


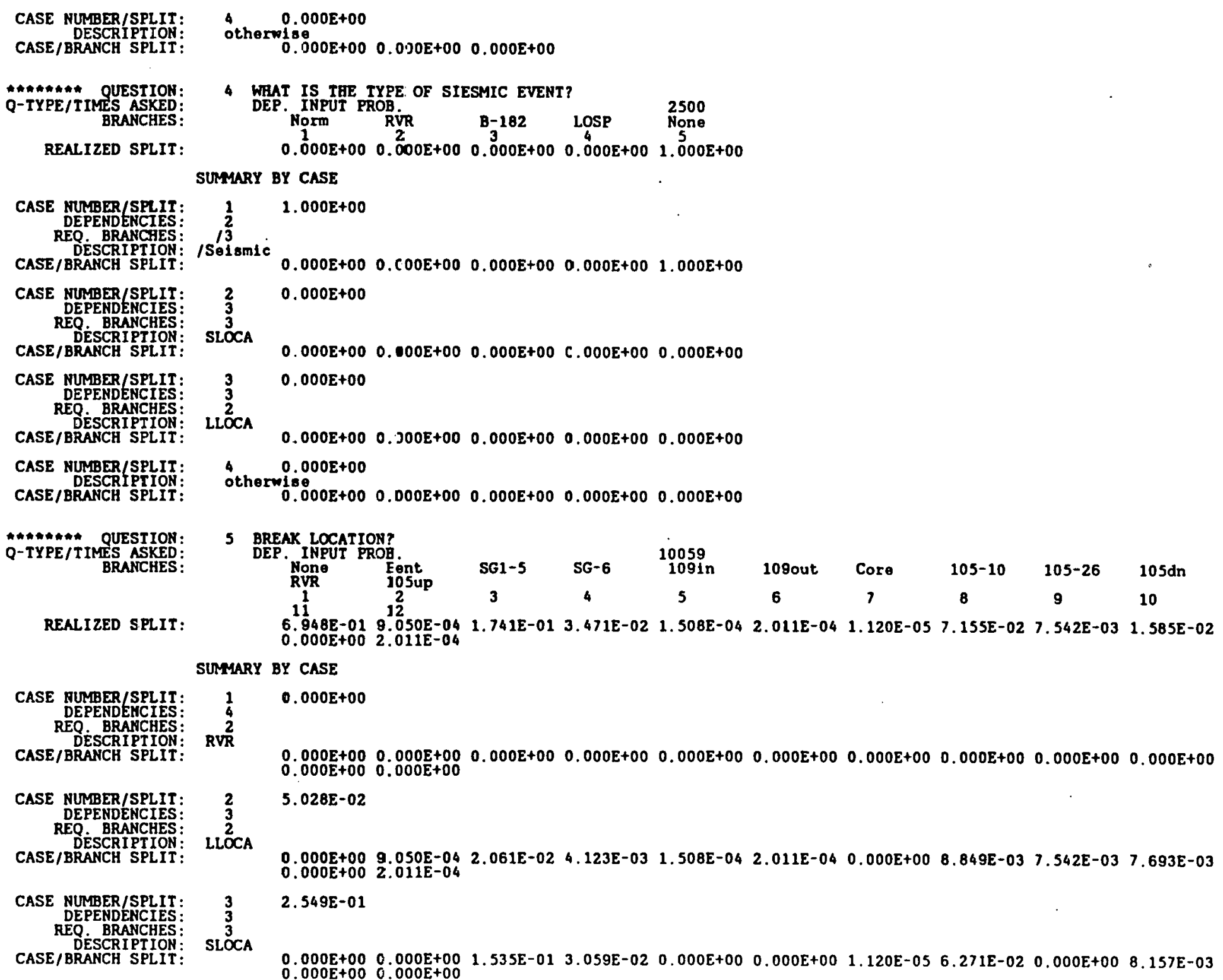




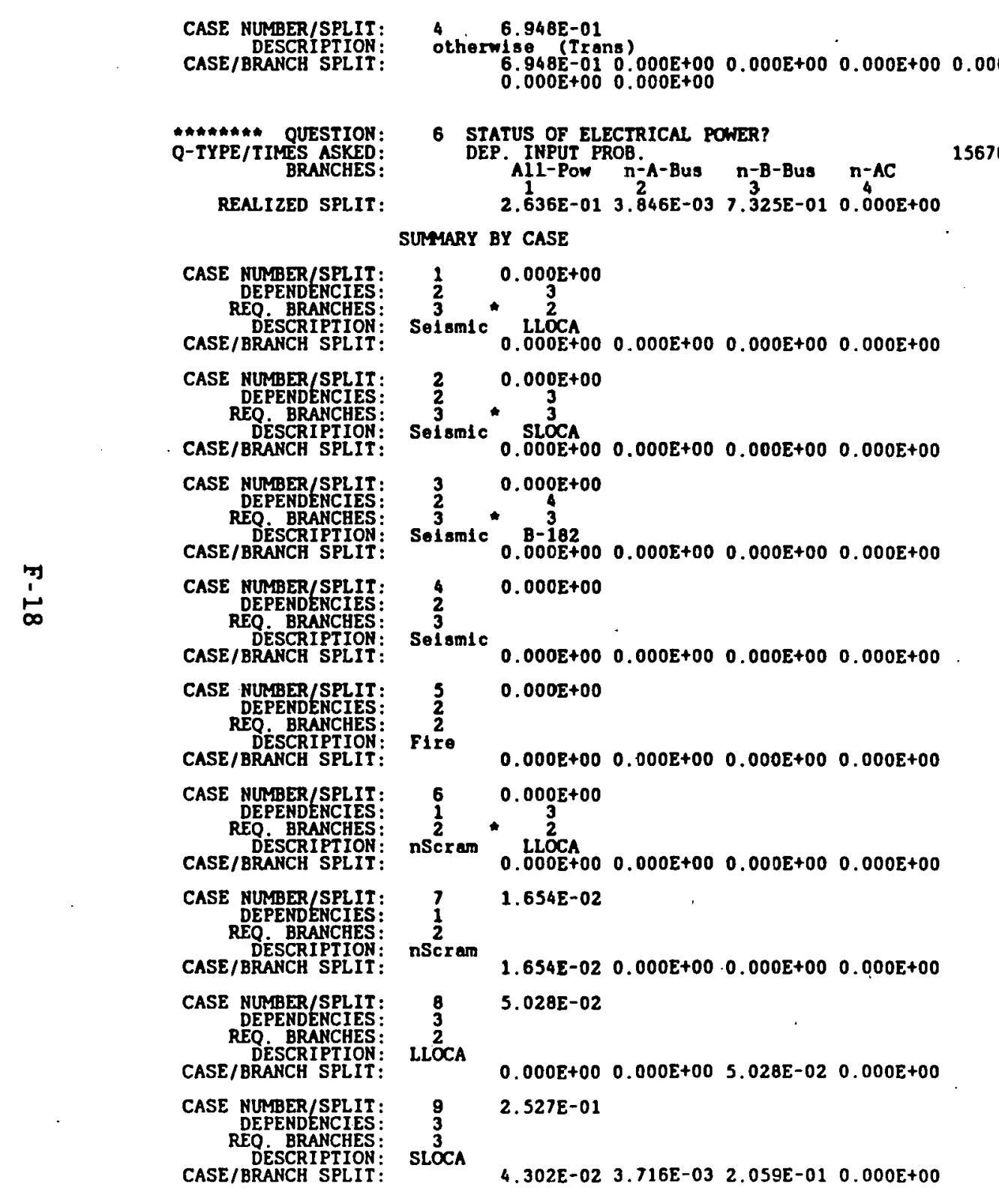




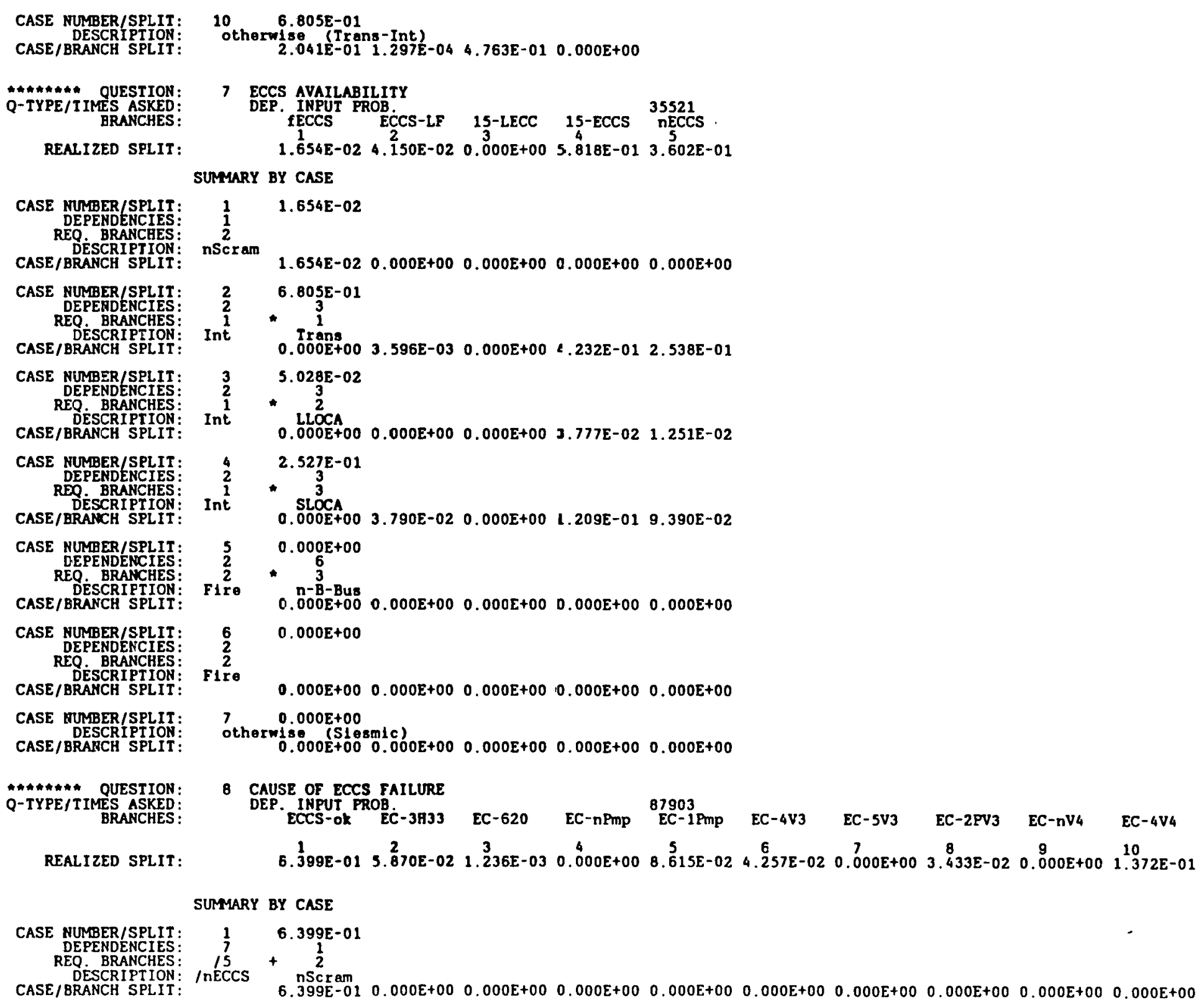




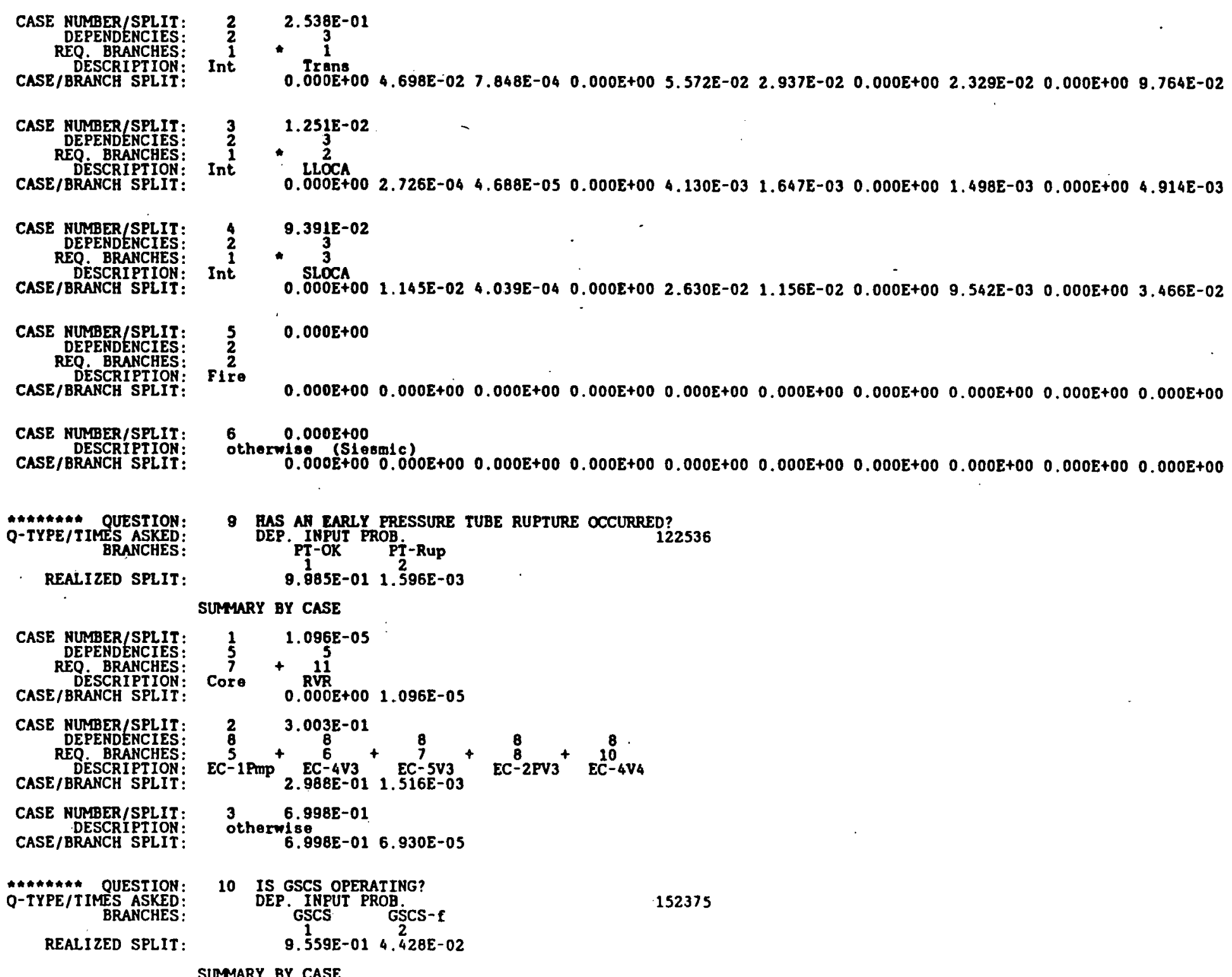

SUMMARY BY CASE 


\begin{tabular}{|c|c|c|}
\hline 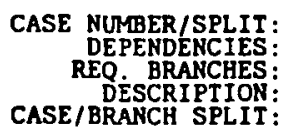 & $\begin{array}{l}\overline{7} \\
4\end{array}$ & 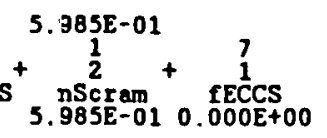 \\
\hline $\begin{array}{l}\text { SE NUMBER/SRIT: } \\
\text { DEPENDECIES: } \\
\text { REQ. BRACIESES: } \\
\text { DESCRIIIION } \\
\text { SE/BRANCH SPLIT: }\end{array}$ & $\mathrm{F}$ & 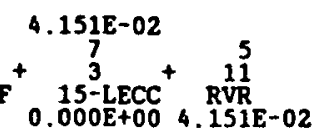 \\
\hline $\begin{array}{l}\text { SE NUMAER/SPLIT: } \\
\text { DEPENDENCIES: } \\
\text { REQ REANCHES } \\
\text { DESCRATIION } \\
\text { SE/BRANCH SPLIT: }\end{array}$ & & $\begin{array}{c}2.538 \mathrm{E}-01 \\
\quad 3 \\
\quad 1 \\
\begin{array}{c}1 \\
\text { Trons } \\
2.531 \mathrm{E}-01\end{array}\end{array}$ \\
\hline $\begin{array}{l}\text { SE NUMAER/SPLIT: } \\
\text { DEPENDECIES } \\
\text { REQ BRANCHES: } \\
\text { DESCRITIION: } \\
\text { SE/BRANCH SPLIT: }\end{array}$ & & $\begin{array}{c}1.251 E-02 \\
3 \\
2 \\
2 \\
1.251 \mathrm{OCA} \\
1.020\end{array}$ \\
\hline $\begin{array}{l}\text { SE NUMBER/SPLIT: } \\
\text { DEPENDECIES } \\
\text { REOERARACHES : } \\
\text { DESCRPTION } \\
\text { SE/BRANCH SPLIT: }\end{array}$ & & $\begin{array}{c}9.391 E-02 \\
33 \\
33 \\
\text { SLLCA } \\
9.186 E-02\end{array}$ \\
\hline $\begin{array}{l}\text { CASE NUMBER/ } \\
\text { DEPENDE } \\
\text { REDENARA } \\
\text { DESCRI } \\
\text { CASE/BRANCH }\end{array}$ & 更 & $\begin{array}{c}0.000 E+00 \\
8 \\
\quad 8 \\
E C-n P m p \\
0.000 E+000 .\end{array}$ \\
\hline 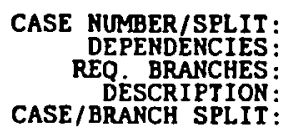 & $F t^{2}$ & $0.000 \mathrm{E}+00$ \\
\hline $\begin{array}{l}\text { CASE NUMBER/SPLIT : } \\
\text { DEPENENCIES } \\
\text { REQ BRANCHES: } \\
\text { DESCNPTINS: } \\
\text { CASE/BRANCH SPLIT: }\end{array}$ & $\mathrm{Sels}^{3}$ & 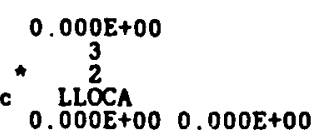 \\
\hline $\begin{array}{l}\text { CASE NUMBER/ } \\
\text { DEPENDE } \\
\text { REP BRA } \\
\text { DESCRI } \\
\text { CASE BRANCH }\end{array}$ & c & $\begin{array}{c}0.000 E+00 \\
3 \\
3 \\
\operatorname{sLCA}^{3} \\
0.000 \mathrm{E}+00 \quad 0.000 \mathrm{E}+0\end{array}$ \\
\hline $\begin{array}{l}\text { CASE NUMBER/ } \\
\text { DEPENDE } \\
\text { REQ. BRA } \\
\text { DESCRI } \\
\text { CASE/BRANCH }\end{array}$ & $\begin{array}{r}3 \\
3 \\
\theta-1\end{array}$ & $0.000 E+00$ \\
\hline $\begin{array}{l}S E \\
S E / B R\end{array}$ & & \\
\hline
\end{tabular}


Animunin QUESTION:

Q-TYPE/TIMES ASKED:

11 IS GSCS EFFECTIVELY COOLING THE CORE?

REALIZED SPLIT:

$$
\begin{array}{ccc}
\text { fGSCS } & \text { DégGSCS } & \text { nGSCS } \\
1 & 3 \\
0.543 E-01 & 1.580 E-03 & 4.427 E-02
\end{array}
$$

152375

\section{SUMMARY BY CASE}

CASE HUMBER/SPLIT:

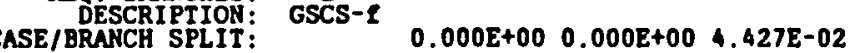

CASE NUMBER/SPLIT:

DEPENDENCIES:

REQ. BRANCHES: 1

CASE/BRANCH SPLIT:

9.543E-01

CASE MUMBER/SPLIT:

DESCRIPTION:
CASE/BRANCH SPLIT:

9.543E-01

- 1 PT-OK

PT-OK

$31.580 \mathrm{E}-03$

$0.000 \mathrm{E}+00 \quad 1.580 \mathrm{E}-03 \quad 0.000 \mathrm{E}+00$

Q-TYPE/TIMES ASKED:

BRANCHES:

REALIZED SPLIT:

12 HPI FLON STATUS

$\begin{array}{lccc}\text { 4-HPI } & 2-\mathrm{HPI} & 1 \text { - } \mathrm{HPI} & \text { NoHPI } \\ 1 & 2 & 3 & 4 \\ 0.000 \mathrm{E}+00 & 2.552 \mathrm{E}-01 & 6.991 \mathrm{E}-02 & 6.751 \mathrm{E}-0\end{array}$

198772

\section{SURMARY BY CASE}

CASE MUMBER/SPLIT:

DESSCRIPTION:

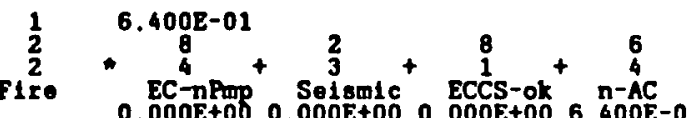

CASE/BRANCH SPLT:

CASE MUMBER LSPLIT:

DEPENDENCHES:

DÉSCRIPTION:

$2 \quad 1.0318-02$

CASE MUMBER/SPLIT:

DEO

DESCRIPTION:

CASE RIMBEP /SPLIT.

DERER/SPLIT:

CASE/BRANCH SPLIT:

CASE NUMBER/SPLIT:

CASE/BRANCR PTION

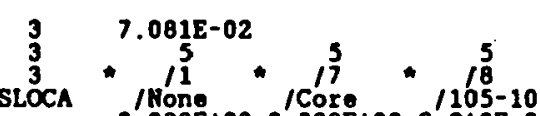

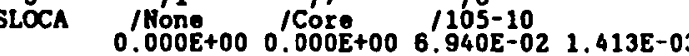

$4 \quad 2.5315-02$

Core $+105-10$

$0.000 \mathrm{E}+00 \quad 2.480 \mathrm{E}-02 \quad 5.126 \mathrm{E}-04 \quad 1.847 \mathrm{E}-07$

$5.2538 \mathrm{E}-01$

$0.000 \Sigma+002.304 E-01 \quad 0.000 E+002.339 E-02$

Q-TYPE/TIMES ASKED:

13

PRIMARY COOLANT FLOW

INCP PROA NICP

198772

REALIZED SPLIT:

$\begin{array}{ll}1 & 1 \\ 543 E-02 & 2.416 E-01\end{array}$

SUMMARY BY CASE 


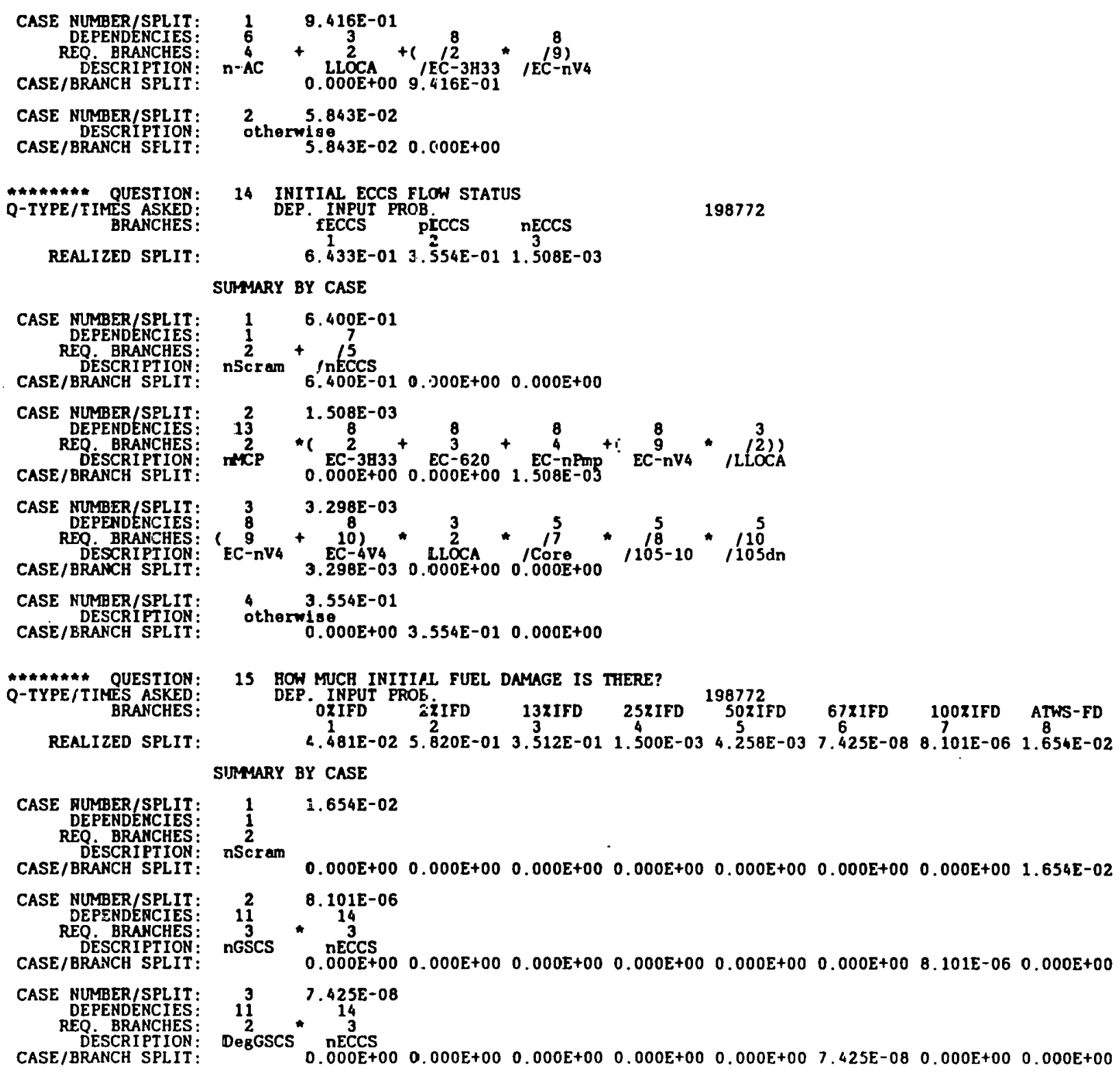

15 ROW MUCR INITIAL FUEL DAMAGE IS THERE?

DEP. INPUT PROE

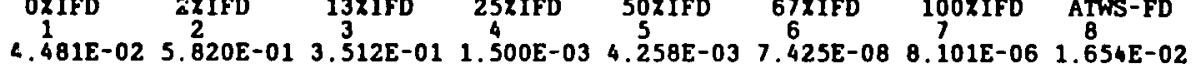
SINMARY BY CASE

CASE RUMRER/SPLIT: REQ. BRANCHES CASE/BRANCH SPLIT:

i.654E-02

CASE NUMBER/SPLIT:

DEO BRATCHES

DESCRIPTION:
CASE/BRANCH SPLIT:

CASE NUMBER/SPLIT:

DEPENDENCIES

DESCRIPTION:
:

$\begin{array}{llllllllllll}0.000 \mathrm{E}+00 & 0.000 \mathrm{E}+00 & 0.000 \mathrm{E}+00 & 0.000 \mathrm{E}+00 & 0.000 \mathrm{E}+00 & 7.425 \mathrm{E}-08 & 0.000 \mathrm{E}+00 & 0.000 \mathrm{E}+00\end{array}$ 
CASE NUMBER/SPLIT

REQ. BRANCHES

CASE/BRANCH SPLIT:

CASE NUMBER/SPLIT:

DEPENDENCIES

DESCRIPTION:
CASE/BRANCH SPLIT:

CASE MUMEER/SPLIT

DEPENDENCIES:

REQ. BRANCHES

CASE/BRAHCH SPLIT:

CASE NUMBER/SPLIT

DEPENDENCIES

REQ. BRANCBES

CASE/BRANCH SPLIT:

CASE NUMBER/SPLIT:

CASE/BRANCH SPLIT

* A A A A A A QUESTION

Q-TYPE/TIMES ASKED:

REALIZED SPLIT

CASE MUMBER/SPLIT

DEPENDENCIES:

RE DESRIPTION

CASE/BRANCH SRLI:

CASE NUMBER/SPLIT

REO. BRANCHES

DESCRIPTION

CASE NUMBER/SPLIT:

CASE/BRANCH SPLIT

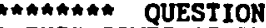

Q-TYPE/TIMES ASKED

REALIZED SPLIT

REATI2ED SPLIT:

CASE NUMBER/SPLIT:

REQPENDEACCHES

DĖSCRIPTION
CASE/BRANCH SPLIT

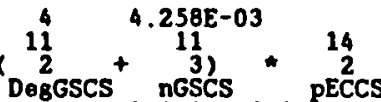

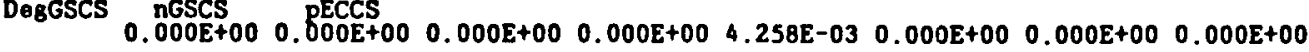

$5 \quad 1.500 \mathrm{E}-03$

16
3

$\begin{array}{lllllllllllllll}\text { fGSCS } & 0.000 E+00 & 0.000 E+00 & 0.000 E+00 & 1.500 E-03 & 0.000 E+00 & 0.000 E+00 & 0.000 E+00 & 0.000 E+00\end{array}$

$63.512 \mathrm{E}-01$

16
$-\quad 2$

$\begin{array}{lllllllllll}\text { IGSCS } & 0.800 E+00 & 0.000 E+00 & 3.312 E-01 & 0.000 E+00 & 0.000 E+00 & 0.000 E+00 & 0.000 E+00 & 0.000 E+00\end{array}$

$? \quad 5.820 E-01$

$3+2$

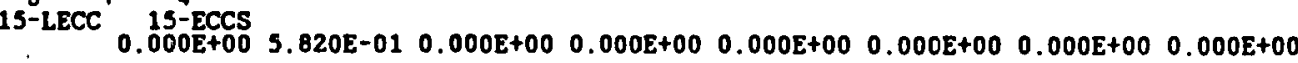
B $4.481 E-02$

$\begin{array}{lllllllll}\text { othermise } & 481 E-02 & 0.000 E+00 & 0.000 E+00 & 0.000 E+00 & 0.000 E+00 & 0.000 E+00 & 0.000 E+00 & 0.000 E+00\end{array}$

16 WHAT IS CONFINEMENT PRESSURE?

DEP. INPUT PROB.

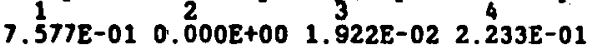

SUMYARY BY CASE

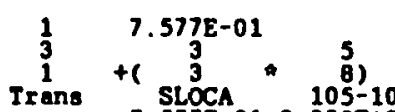

$\begin{array}{lllll}7.577 E-01 & 0.000 E+00 & 0.000 E+00 & 0.000 E+00\end{array}$

$2 \quad 5.030 \mathrm{D}-02$

LLڤ $\stackrel{2}{ }$

$0.000 \mathrm{E}+00 \quad 0.000 \mathrm{E}+00 \quad 0.000 \mathrm{E}+00 \quad 5.030 \mathrm{E}-02$

$3.1 .923 \mathrm{E}-01$

$0.000 E+00 \quad 0.000 E+00 \quad 1.922 E-02 \quad 1.730 E-01$

17 IS DUMP PATH TANK OK?

DEP. INPUT PROB DT-Atm DT-LIQR DT-NA 287583

9. $412 \mathrm{E}-01 \quad 3.230 \mathrm{E}-05$ 1.925E-04 5.872E-02

SUMMARY BY CASE

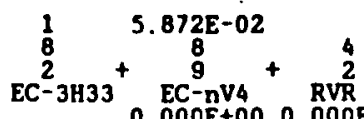

$0.000 E+00 \quad 0.000 E+00 \quad 0.000 E+00 \quad 5.872 E-02$ 
CASE MUMBER/SPLIT
DEPENDENCIES
REO. BRANCHES

REQ, BRANCHES:
DESCRIPTION :

CASE/BRANCH SPLIT:

CASE MUMBER/SPLIT:

DEPENDENCIES
REQ. BRANCHES

CASE/BRANCH SPLIT:

CASE MUMBER/SPLIT:

RED GRANCHES:

DESCRIPIION

CASE/BRANCH SPLIT:

CASE MUMRER/SPLIT

DESCRIPTION:
CASE/BRANCH SPLIT:

Q-TYPE/TIMES ASKED

$$
\text { BRANCHES: }
$$

REALIZED SPLIT:

CASE MUMEER/SPLIT:
DEPENDENCIES:
REQ. BRANCHES:

DESCRIPTIOH:
CASE/BRARCH SPLIT:

CASE NUMBER/SPLIT

REQ. BRAHCHES

DESCRIPTION:

CASE NUMBER/SPLIT:

REO. BRANCHES

CASE/BRANCH SPLIT

CASE NUMBER/SPLIT

DESCRIPIION
CASE/BRANCH SPLIT

Annmmann QUESTION:

Q-TYPE / T IMES ASKED

REALIZED SPLIT

$2 \quad 0.000 E+00$

$\stackrel{2}{3} * \stackrel{3}{2}^{2}$

$\begin{array}{llllll}3 & 0.000 E+00 & 0.000 E+00 & 0.000 E+00 & 0.000 E+00\end{array}$

$3 \quad 0.000 \mathrm{E}+00$

So1 $\stackrel{2}{3}+\stackrel{3}{3}+\stackrel{3}{3}+\stackrel{4}{3})$

$\begin{array}{lll}0.000 E+00 & 0.000 E+00\end{array}$

2

$\begin{array}{llllll} & 0.000 E+00 & 0.000 E+00 & 0.000 E+00 & 0.000 E+00\end{array}$

$5 \quad 9.414 \mathrm{E}-01$

otherwise $0.412 E-01 \quad 3.230 E-05$ 1.925E-04 $0.000 E+00$

18 IS FILTER RELEASE STACK OK?

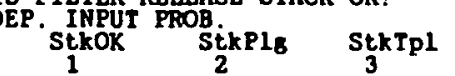

287583

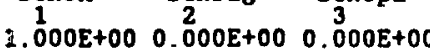

SUMMARY BY CASE

2 $0.000 \mathrm{E}+00$

3 . 2

$0.000 E+00 \quad 0.000 E+00$

$\begin{array}{cc}2 & 0.000 E+00 \\ 2 & 3 \\ 3 & 3 \\ 3 & 4 \\ 0 & (3)\end{array}$

$\begin{array}{llll}0.000 \mathrm{E}+00 & 0.000 \mathrm{E}+00 \quad 0.000 \mathrm{E}+00\end{array}$

$3 \quad 0.000 E+00$

Selsmic

$0.000 E+00 \quad 0.000 E+00 \quad 0.000 E+00$

$1.000 \mathrm{E}+00$

1. $000 \mathrm{E}+00 \quad 0.000 \mathrm{E}+00 \quad 0.000 \mathrm{E}+00$

18 DOES CONFINDEMNT ISOLATE?

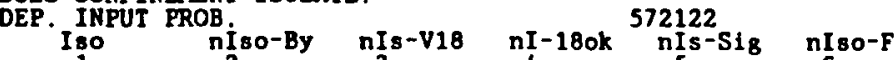

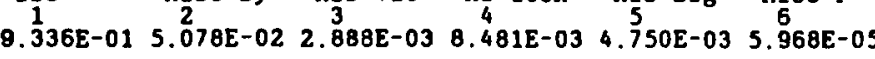

SUMMARY BY CASE

CASE NUMBER/SPLIT

REQ. BRANCHES

CASE / BRANCH SPLIT:

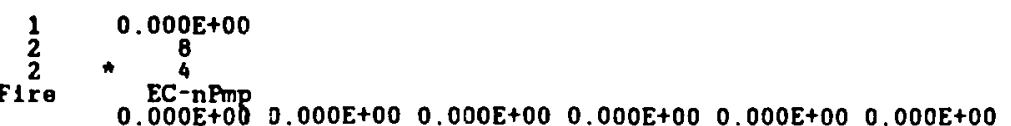


CASE MUMRER/SPLIT:

REQ. BRANCHES

DESCRIPIION:
CASE/BRAHCH SPLIT:

CASE NUMBER/SPLIT

DEPENDENCIES:

DESCRIPTION:

CASE RUMBER/SPLIT.

DESCRIPTION:
CASE/BRANCH SPLIT:

CASE RUMBER/SPLIT:

DEPENDENCIES

CASE/BRANCHIPIION:

CASE MUMBER/SPLIT:

CASE/BRANCH SPLIT

Q A AMAn: gUESTION:

Q-TYPE/TIMES ASKED:

REALIZED SPLIT

\section{SUMAARY BY CASE}

CASE NUMBER/SPLIT:

$$
\text { REQ BRANCHES }
$$

CASE/BRANCH SPLIT:

CASE NUMBER/SPLIT:

$$
\text { REQ. BRANCHES: }
$$

DESCR IPTION:
CASE/BRANCH SPLIT:

CASE NUMBER/SPLIT:

CASE/BRANCH SPLIT:

A*t*m*n QUESTION:

Q-TYPE/TIMES ASKED:

REALIZED SPLIT.

\section{T:}

CASE NUMBER/SPLIT:

REPENDENCIES:

DESCRIPTION:
CASE/BRANCH SPLIT:

Solsmlc ${ }^{3} \mathrm{R}^{\frac{4}{2}}$

Solsmic $\mathrm{LL}^{2} \mathrm{CA}$

5

SUMPARY BY CASE $\begin{array}{cc}2 & 0.000 E+00 \\ 2 & 8 \\ 2 & * \quad 8\end{array}$

$\begin{array}{llllllllllll}0.000 E+00 & 0.000 E+00 & 0.000 E+00 & 0.000 E+00 & 0.000 E+00 & 0.000 E+00\end{array}$

$3 \quad 0.000 E+00$

$\begin{array}{lllllll}0.000 E+00 & 0.000 E+00 & 0.000 E+00 & 0.0 C 0 E+00 & 0.000 E+00 & 0.000 E+00\end{array}$

i $\quad 0.000 E+00$

$\begin{array}{llllllllll}0.000 E+00 & 0.000 E+00 & 0.000 E+00 & 0.000 E+00 & 0.000 E+00 & 0.000 E+00\end{array}$

$5 \quad 4.984 E-02$

$2-3833 * 1$

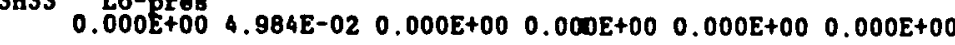
$6 \quad 9.507 E-01$

9.336E-01 9.470E-04 2.888E-03 8.460E-03 4.750E-03 5.968E-05

20 WHAT IS CONFINEMENT COMPROMISE LOCATIJN?

ConfoK Envis Zono

$\begin{array}{ccc}\text { ConfoK } & 2 & 2 \\ 9.336 E-01 & 6.218 E-02 & 4.750 E-03\end{array}$

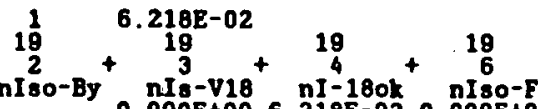

$2 \quad 4.750 \mathrm{E}-03$

$0.000 E+00 \quad 0.000 E+00 \quad 4.750 E-03$

$3 \quad 9.336 \mathrm{E}-01$

9.336E-01 $0.000 E+00 \quad 0.000 E+00$

21 DOES FORCEO VEHTILATIOH EXIST?

DEP. INPUT PROB.

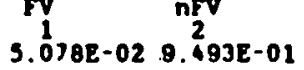

572122

$19 \quad 3.078 \mathrm{E}-02$

2014

$\begin{array}{ll}\text { S.07BE-02 } & 0.000 E+00\end{array}$ 
CASE NUMBER/SPLIT: CASE/BRAKCH SPLIT:

Qunntun QUESTION: Q-TYPE/TIMES ASKED

REALIZED SPLIT:

CASE NUMBER/SPLIT:
DEPENDERCIES

DEPENDERCIES:

CASE/BRANCH SPLIT:

CASE NUMBER/SPLIT:

DEPENDENCIES:

DESCRIPTION:
CASE/BRANCH SPLIT:

CASE NUMBER/SPLIT:

REO BRANCHES

CASE/BRANCH SPLIT:

CASE NUMBER/SPLIT:

DEPENDENCIES

DESCRIPTION:
CASE/BRANCH SPLIT:

CASE MUMBER/SPLIT:

DEPENDENCIES

REQ BRANCHES:

CASE/BRANCH SPLIT:

CASE NUMBER/SPLIT:

DEPENDENCIES

ASE/BRANCH SPLIT:

CASE/BRANCH SPLIT:

CASE NUMBER/SPLIT:

REQ. BRANCHES:

CASE/BRANCH SPLIT:

CASE NUMBER/SPLIT

CASE/BRANCH SPLIT

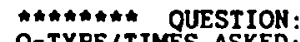

Q-TYPE/TIMES ASKED:
BRANCHES:

REALIZED SPLIT:
2 9.493E-01

$0.000 \mathrm{E}+00 \quad 9.493 \mathrm{E}-01$

22 ARE AUTO FOG SPRAYS ON INITIALLY?

Spr 2 nSSpr Spr-ND

2.374E-01 1.040E-02 $7.529 E-01$

SUMMARY BY CASE

$\frac{1}{2} \quad 0.000 \mathrm{E}+00$

Selsmic

$0.000 E+00 \quad 0.000 E+00 \quad 0.000 E+00$

$2 \quad 0.000 E+00$

$2+4.19$

$0.000 \mathrm{E}+000.000 \mathrm{E}+00 \quad 0.000 \mathrm{E}+00$

$3 \quad 0.000 E+00$

$2 \ldots$

$0.000 E+00 \quad 0.000 E+00 \quad 0.000 E+00$

(2) $0.000 \mathrm{E}+00$

19
11

$0.000 \mathrm{E}+00 \quad 0.000 \mathrm{E}+00 \quad 0.000 \mathrm{E}+00$

$3 \quad 1.416 \mathrm{E}-02$

$3+1+1+16$

0.000 tos $1.939 E-03 \quad 1.222 E-02$

$6 \quad 7.440 E-01$

$1+2$

Lo-pres Conf-tp $0.00 \mathrm{E}+0 \mathrm{~b}$ 3.32E-03 7.407E-01

3) $3.015 \mathrm{E}-02$

${ }^{3} \mathrm{SSCS}$

2. 583E-02 4.323E-03 $0.000 E+00$

$62.124 \mathrm{E}-01$

$2.116 E-01 \quad 8.023 E-04 \quad 0.000 E+00$

23 STATUS OF CONFINEMENT H2 MITIGATION SYSTEM?

HM-OK HM-3K HM-4OK HM-MIX HMY-20k NHM

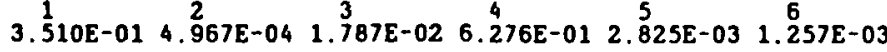

SUMMARY BY CASE 
CASE MUMBER/SPLIT
DEPENDENCIES
REO BRANCHES CASE/BRANCH SPLIT:

CASE MUMBER/SPLIT. DEPENDENCIES

REQ. BRANCHES

CASE/BRANCR SPLIT:

CASE RUMBER/SPLIT:

DEPENDERCIES

REO BRANCHES:

CASE/BRAICH SPLIT

CASE MUMBER/SPLIT

DEPENDENCIES:

CASE/BRANCH SPLIT:

CASE MUNBER/SPLIT:

DEPENDENCIES

REQ BRANCHES:

CASE/BRAHCH SPLIT:

CASE MUMBER/SPLIT:

DEPENDENCIES:

REQ DECRIPTION:

CASE/BRACH SPLIT:

CASE NUMBER/SPLIT:

REQ. BRAKCHES:

CASE/BRANCR SPLIT:

CASE MUMBER/SPLIT: DEPENDENCIES:

CASE/BRANCRIPTION:

CASE NUMBER/SPLIT

REO BRANCHES:

DESCRIPTION:

CASE NUMBER/SPLIT

DEPENDENCIES

DESCRIPTION:

CASE NUMBER/SPLIT:

REO. BRANCHES

CASE/BRANCHIPTION:

CASE NUMBER/SPLIT:

DESCRIPTION:
CASE/BRANCH SPLIT:

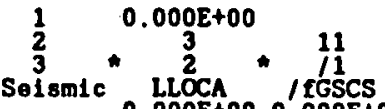

$\begin{array}{lllllllll}0.000 E+00 & 0.000 E+00 & 0.000 E+00 & 0.000 E+00 & 0.000 E+00 & 0.000 E+00\end{array}$

$20.000 \mathrm{E}+00$

$3+2$

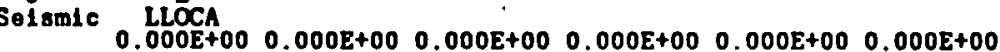

$3 \quad 0.000 E+00$

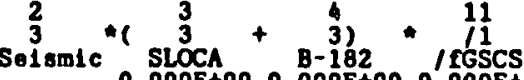

$\begin{array}{cc}4 & 0.000 E+00 \\ 2 & 3 \\ 3 & 3 \\ 3 & 4 \\ 3 & 3\end{array}$

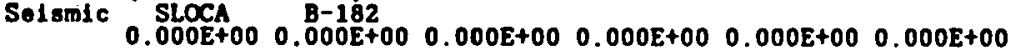

$5 \quad 0.000 E+00$

$3 \frac{11}{3}$

$\begin{array}{llllll}0.000 E+00 & 0.000 E+00 & 0.000 E+00 & 0.000 E+00 & 0.000 E+00 & 0.000 E+00\end{array}$

$6 \quad 0.000 \varepsilon+00$

Solsmlc $0.000 E+00 \quad 0.000 E+00 \quad 0.000 E+00 \quad 0.000 E+00 \quad 0.000 E+00 \quad 0.000 E+00$

$0.000 \mathrm{E}+00$

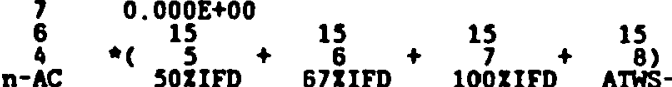

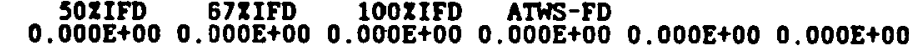

$\begin{array}{ll}8 & 0.000 E+00 \\ 6 & 15\end{array}$

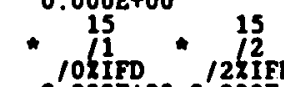

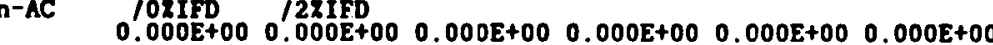

$\begin{gathered}9 \\ 15 \\ 5\end{gathered}+\frac{2.081 E-02}{15}+15+15$

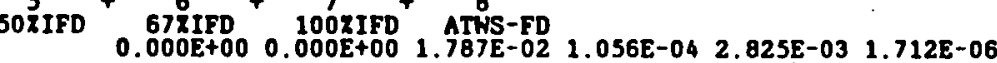

$10 \quad 3.528 E-01$

11 - 15

3.510E-01 4.967E-04 $0.000 E+00 \quad 1.396 E-03 \quad 0.000 E+00 \quad 2.664 E-06$

$\begin{array}{cc}11 & 6.274 E-0 \\ 12 & 8\end{array}$

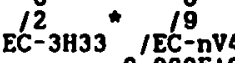

$120.000 \mathrm{E}+00$

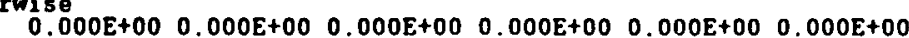




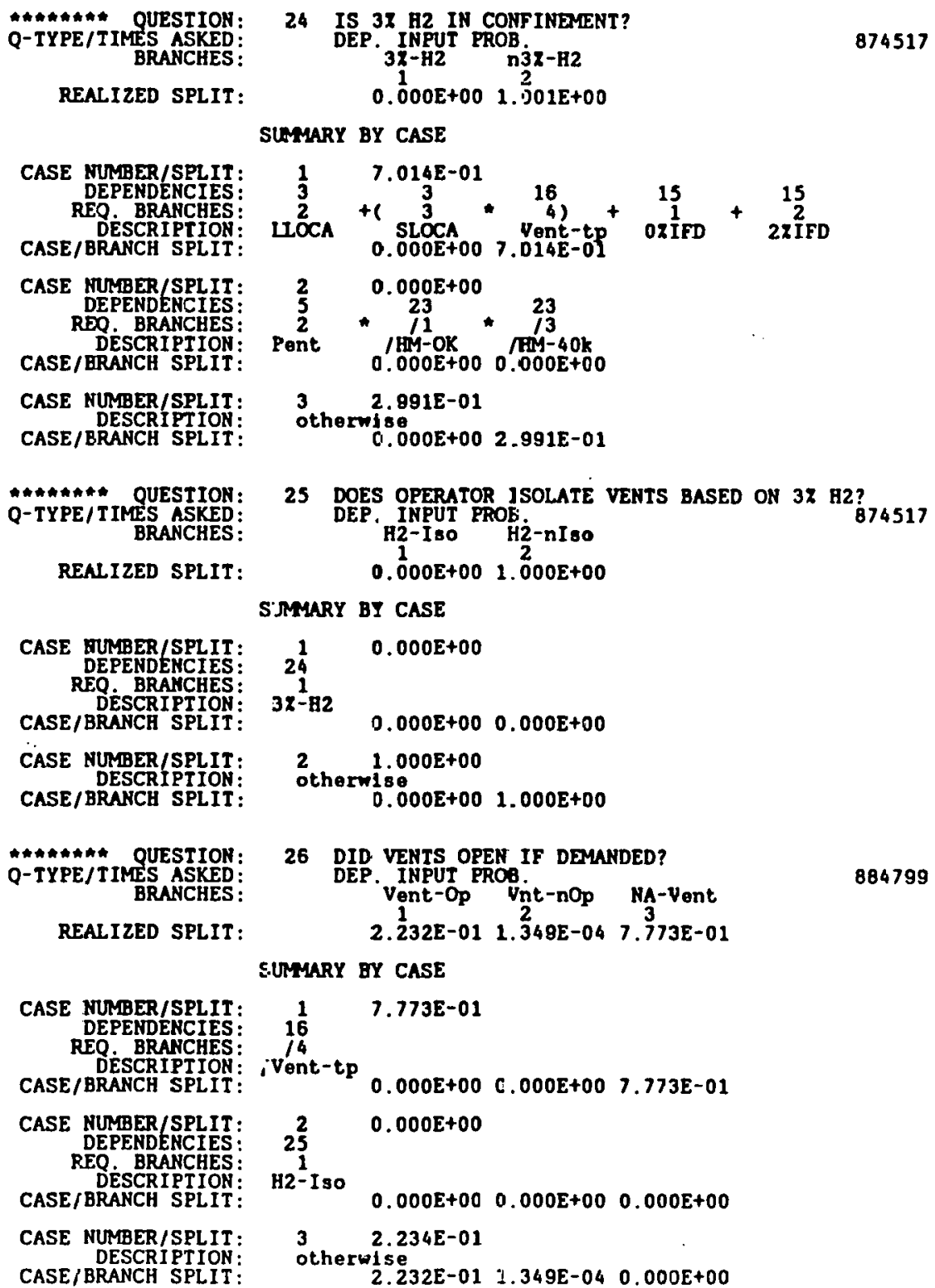

REALIZED SPLIT:

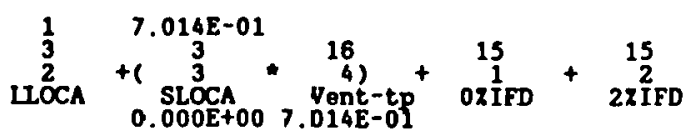

$\begin{array}{cc}2 & 0.000 E+00 \\ 5 & 23 \\ 2 & 11\end{array}$

Pent $\quad \begin{aligned} & \text { / } \\ & \text { a.00 }\end{aligned}$

othern 2.991E-01

0. $000 \mathrm{E}+002.991 \mathrm{E}-01$

25 DOES OPERATOR 1SOLATE VENTS BASED ON 32 H2? 874517 H2-IsO HROE: HínIso

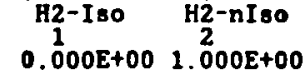

S.MMARY BY CASE

CASE MUMBER/SPLIT

REQ. BRAKCHES

CASE/BRANCH SPLIT:

CASE NUMBER/SPLIT:

DESCRIPTION:
CASE/BRANCH SPLIT:

1
24
$3 x-82$

$0.000 E+00$

$0.000 E+00 \quad 0.000 E+00$

$2 \quad 1.000 E+00$

$0.000 E+00 \quad 1.000 E+00$

AtA\#\#A\#\# QUESTION

Q-TYPE/TIMES ASKED

REALIZED SPLIT

26 DID VENTS OPEN IF DEMANDED?

Vent-Op Vnt-nOp NA-Vent

2.232E-01 $1.248 \mathrm{2}-047.773 \mathrm{3}-01$

884799

S.UMMARY BY CASE

CASE NUMBER/SPLIT:

DEPENDENCIES: 16

DÉ BCR IPTION:

$1 \quad 7.773 E-01$

CASE NUMBER/SPLIT:

DEPENDENCIES:

25

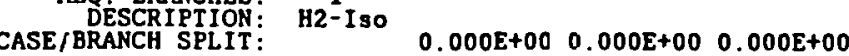

CASE NUMBER/SPLIT:

DESCRIPTION:
CASE/BRANCH SPLIT:

$32.234 \mathrm{E}-01$

$2.232 E-01 \quad 1.349 E-04 \quad 0.000 E+00$ 
MA*H* *** QUESTION:

Q-TYPE/TIMES ASKED:
BRACHES:

REALIZED SPLIT:

CASE MUMBER/SPIIT

REO BRANCBES:

DESCRIPTIOH:
CASE/BRANCH SPLIT:

CASE NUMBER/SPLIT:

REO BRANCHES:

CASE/BRANCH SPLIT:

CASE NUMBER/SPLIT

REO BRAACBES

CASE/BRANCH SPLIT:

CASE HUMBER/SPLIT

CASE/BRANCH SPLIT:

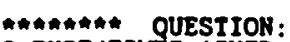

Q-TYPE/TIMES ASKED:

REALIZED SPLIT:

SE NUMBER/SPLIT:

DEPENDENCIES:

REQ BRANCHES:

CASE/BRAFCH SPLIT:

CASE MUMBER/SPLIT:

REQ BRANCHES:

DESCRIPTION:
CASE/BRANCH SPLIT:

CASE NUMBER/SPLIT:

DESCRIPIIOH:
CASE/BRANCH SPLIT:

Annm nth QUESTION:

Q-TYPE/TIMES ASKED:

REALIZED SPLIT:
27 DID VENT ISOLATIONS CLOSE AFTER BLOWDOWN?

Vent-Cl Vno int-nCl Vent-MA

916202

SUMMARY BY CASE

$26 \quad 7.773 E-01$

HA-Vent

$0.000 E+00 \quad 0.000 E+00 \quad 7.773 E-01$

$2 \quad 2.206 \mathrm{E}-04$

$280-8 y$

$0.000 E+00 \quad 2.206 E-04 \quad 0.000 E+00$

$19 \quad 2.610 \mathrm{E}-03$

19
$11 \quad * \quad 19$

$\begin{array}{lll}\ln \left\{\begin{array}{l}5 \\ 2.575 E-03\end{array}\right] .438 E-05 & 0.000 E+00\end{array}$

$2.206 E-01$

2.204E-01 $1.775 E-04 \quad 0.000 E+00$

28 DOES CONFINEMENT FAIL DURING BLOWDOWN PHASE? C-OK PROB CÉ-InIt CF-BD $\begin{array}{ccc}\text { C-OK } & \text { CF-InLt } & \text { CF-BD } \\ 1 & 3 \\ 0.335 E-01 & 6.666 E-02 & 4.577 E-04\end{array}$

SUMMARY BY CASE

$19.335 \mathrm{E}-02$

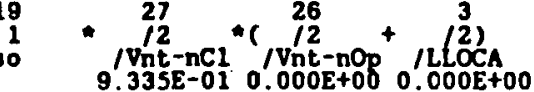

$2 \quad 4.5772-04$

$2+2$

$V_{n t-n C 1} \begin{gathered}\text { Vnt }-n O P \\ 0.000 E+00\end{gathered} 0.000 E+00$ 4.577E-04

$3 \quad 6.666 \mathrm{E}-02$

$0.000 E+00 \quad 6.666 E-02 \quad 0.000 E+00$

29 WHAT IS SIZE OF CONFINEMENT FAILURE?

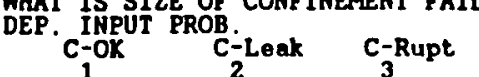

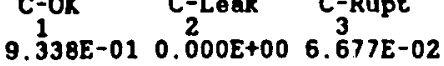

916202

SUMMARY BY CASE 


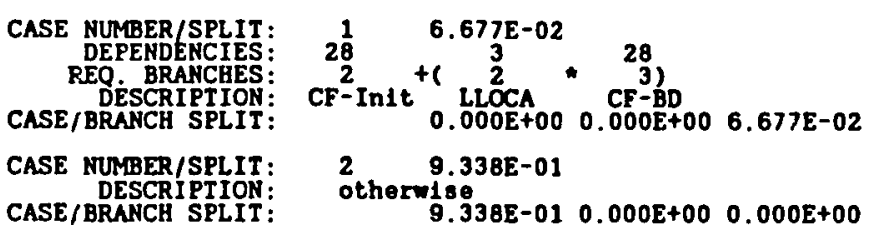

31 DO OPERATING FOG SPRAYS SURVIVE? DEP. INPUT PROB.
Spr-nCh Spr-FE
$\begin{array}{ll}1.000 E+00 & 2.536 E-06\end{array}$

SUMYARY BY CASE

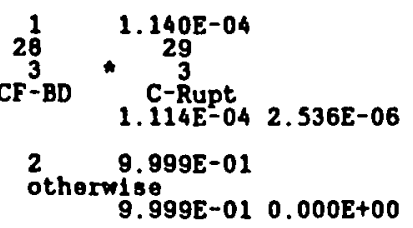

32 IS FILTER RELEASE PATH ESTABLISHED? DEP. INPUT PROB. Fpath Fath Fath-NA 9.327E-01 $1.204 E-03 \quad 6.678 E-02$

SUMMARY BY CASE
916202

918187 


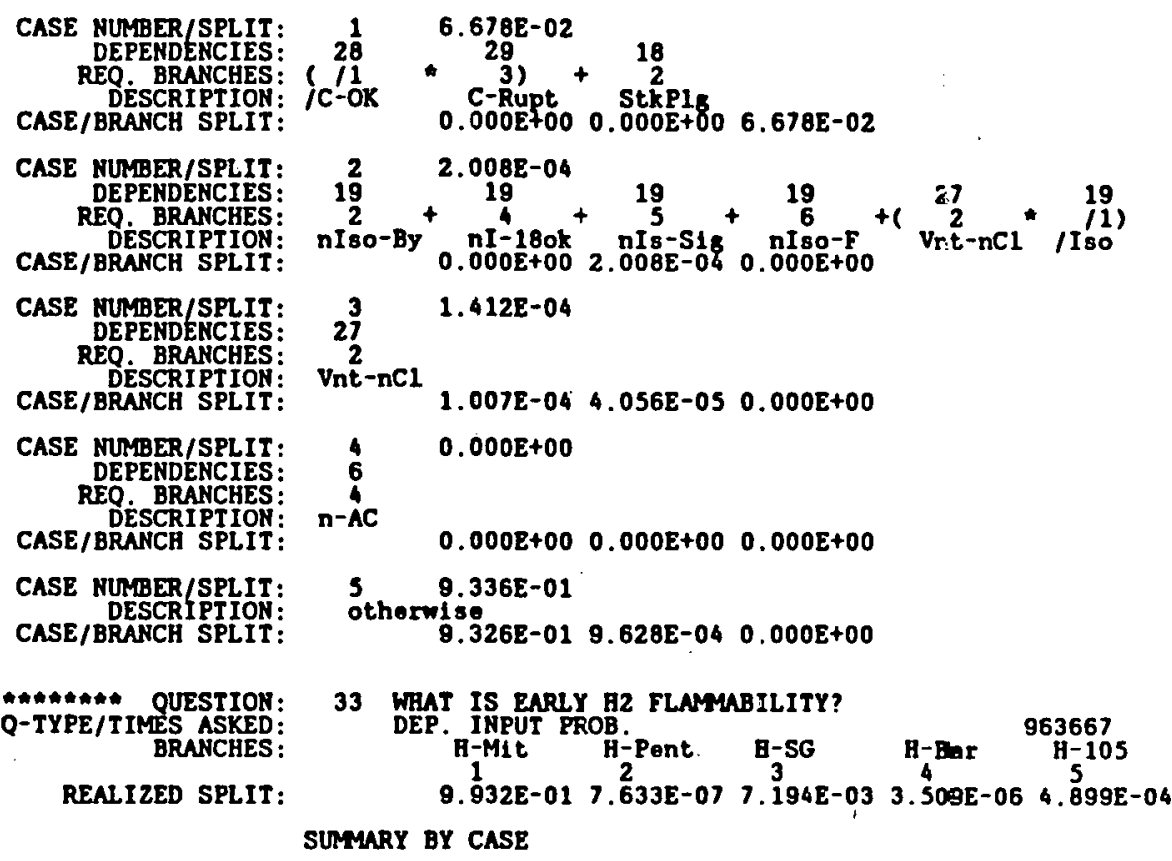

CASE

CASE MUMBER/SPLIT:
DEPENDENCIES:

REQ. BRANCHES:
DESCRIPTION:
CASE/BRANCH SPLIT:

CASE NUMBER/SPLIT:

DEPENDENCIES:
REO. BRANCHES:

CASE/BRANCH SPLIT:

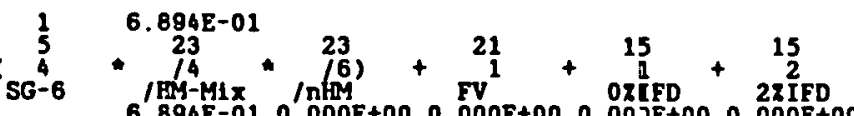

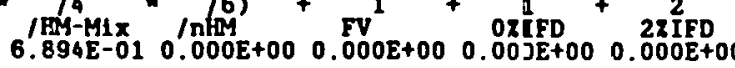

CASE NUMBERSPLIT:

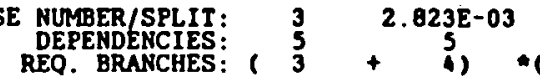

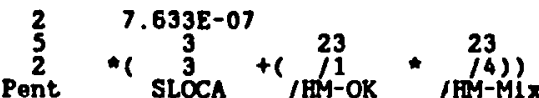

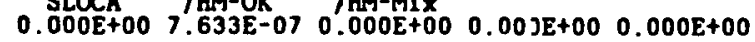

CASE/BRANCH SPLIT:

5

CASE NUMBER/SPLIT:

REO. BRANCHES:

CASE/BRANCH SPLIT:

CASE NUMBER/SPLIT:

DEPENDENCIES

$\begin{array}{rlllll}\text { DESSCRIPTION: } & \text { SGI-5 } & \text { SG-6 } & \text { LLOCA } & & \\ \text { CASE/BRANCH SPLIT: } & 2.657 E-03 & 0.000 E+00 & 3.321 E-04 & 0.000 E+00 & 0.000 E+00\end{array}$

$\begin{array}{lllll}2.823 E-03 & 0.000 E+00 & 0.000 E+00 & 0.00 v E+00 & 0.000 E+00\end{array}$

$36.662 \mathrm{z}-03$

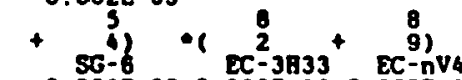

$\begin{array}{lllll}0.000 E+00 & 0.000 E+00 & 6.862 E-03 & 0.000 E+00 & 0.000 E+00\end{array}$

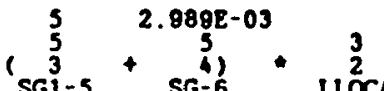




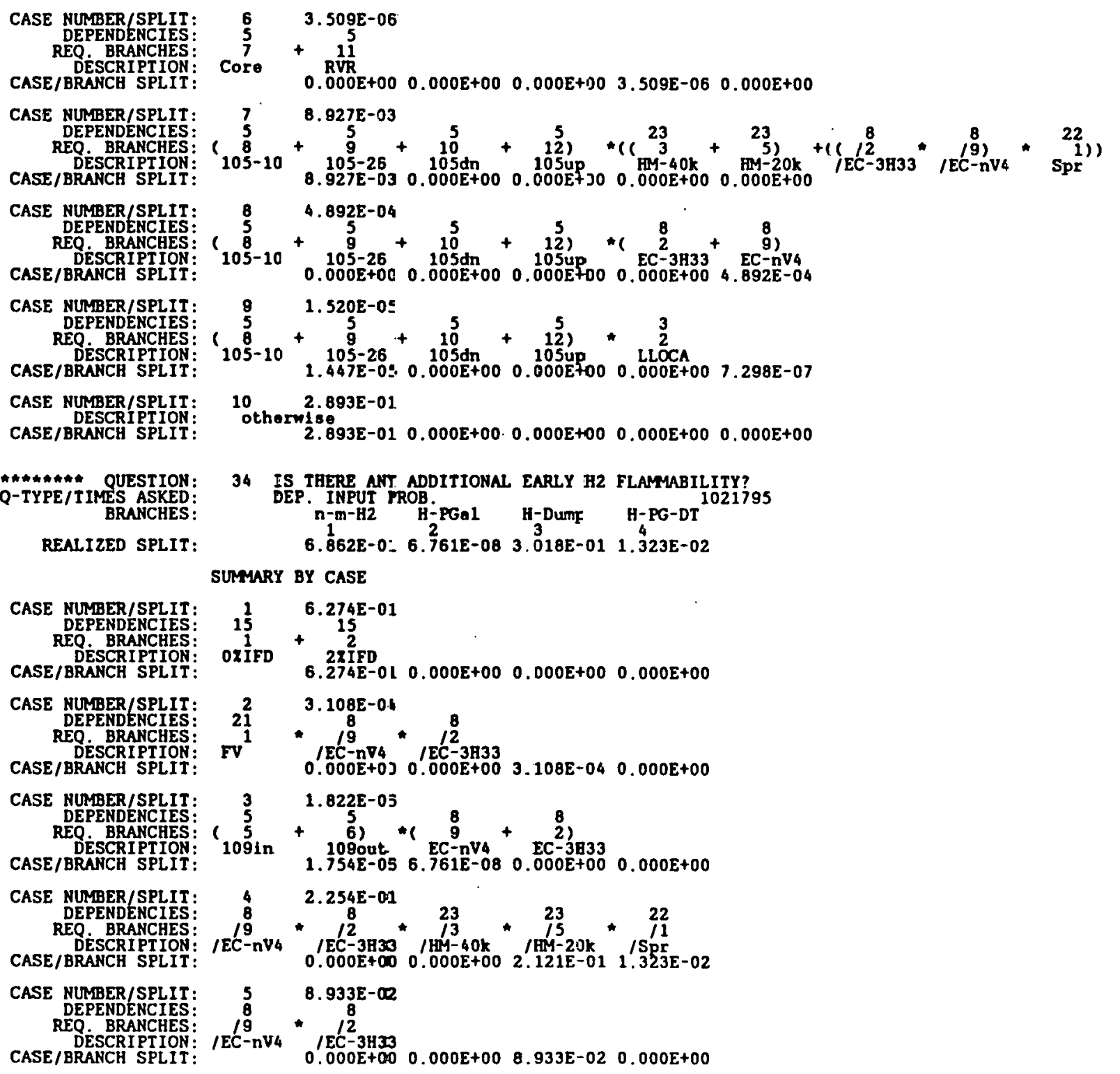


CASE NUMBER/SPLIT: $\quad 6 \quad 5.878 E-02$

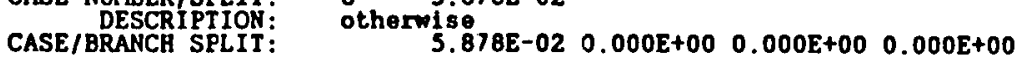

AnAAnش\#\# QUUSTION: 35 IS A DETONABLE H2 MIXTURE PRESENT EARLY?

Q-IYPE/TIMES ASKED:

REALIZED SPLIT:

DEP. INPUT PROB.-Det

$1.000 E+00 \quad 2.464 E-06$

SUMYARY BY CASE

CASE NUMBER/SPLIT:

DEPENDENCIES

DESCRIPTIOH:

CASE RUMBER/SPLIT

DEPENDENCIES

CASE/BRANCH SPLIT:

CASE NUMBER/SPLIT:

CASE/BRANCH SPLIT:

PAn
Q-TYPE / TIMES AUESTIOH:

BRANCHES:

REALIZED SPLIT:

\section{SUMMARY BY CASE}

$7.634 \mathrm{E}-07$

H-Pent $0.000 E+007.634 E-07$

$33 \quad 3.237 E-06$

B-Ber

1.536E-06 1.701E-06

$31.000 E+00$

$1.000 E+00 \quad 0.000 E+00$

36 DOES AN EARLY B2 IGNITION OCCUR?

HinI INPUT PROB.

$\begin{array}{cc}1 & 1 \\ 9.961 E-01 & 4.029 E-03\end{array}$

1022202

$3 \frac{1}{3} \quad 9.924 \mathrm{E}-01$

CASE NUMBER/SPLIT:

REO. BRANCHES

CASE/BRANCH SPLIT:

9.924E-01 $0.000 E+00$

CASE NUMBER/SPLIT:

REO

CASE/BRANCHIPTION:

2
33
2
2
$-1.194 E-03$
33
3

CASE NUMBER/SPLIT

REO. BRANCHES

DEESCRIPTION:
CASE/BRANCH SPLIT:

$\begin{array}{rcc}33 & 0.000 E+00 \\ 3 & 35 & 6\end{array}$

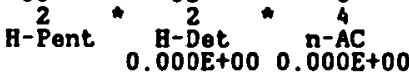

CASE NUMBER/SPLIT:

REQ. BRANCHES

DESCRIPTION:
CASE/BRANCH SPLIT:

$33 \quad 0.000 \mathrm{E}+00$

$\begin{aligned} & 2 \\ & 2\end{aligned}+3$

$\begin{array}{lll}\text { H-Pent } & 0.000 \mathrm{E}+00 \quad 0.000 \mathrm{E}+00\end{array}$

CASE NUMBER/SPLIT: 5 3.004E-06

REO BRANCHES:

CASE/BRANCH SPLIT:

H-Bar

3. 205E-07 2.683E-06 


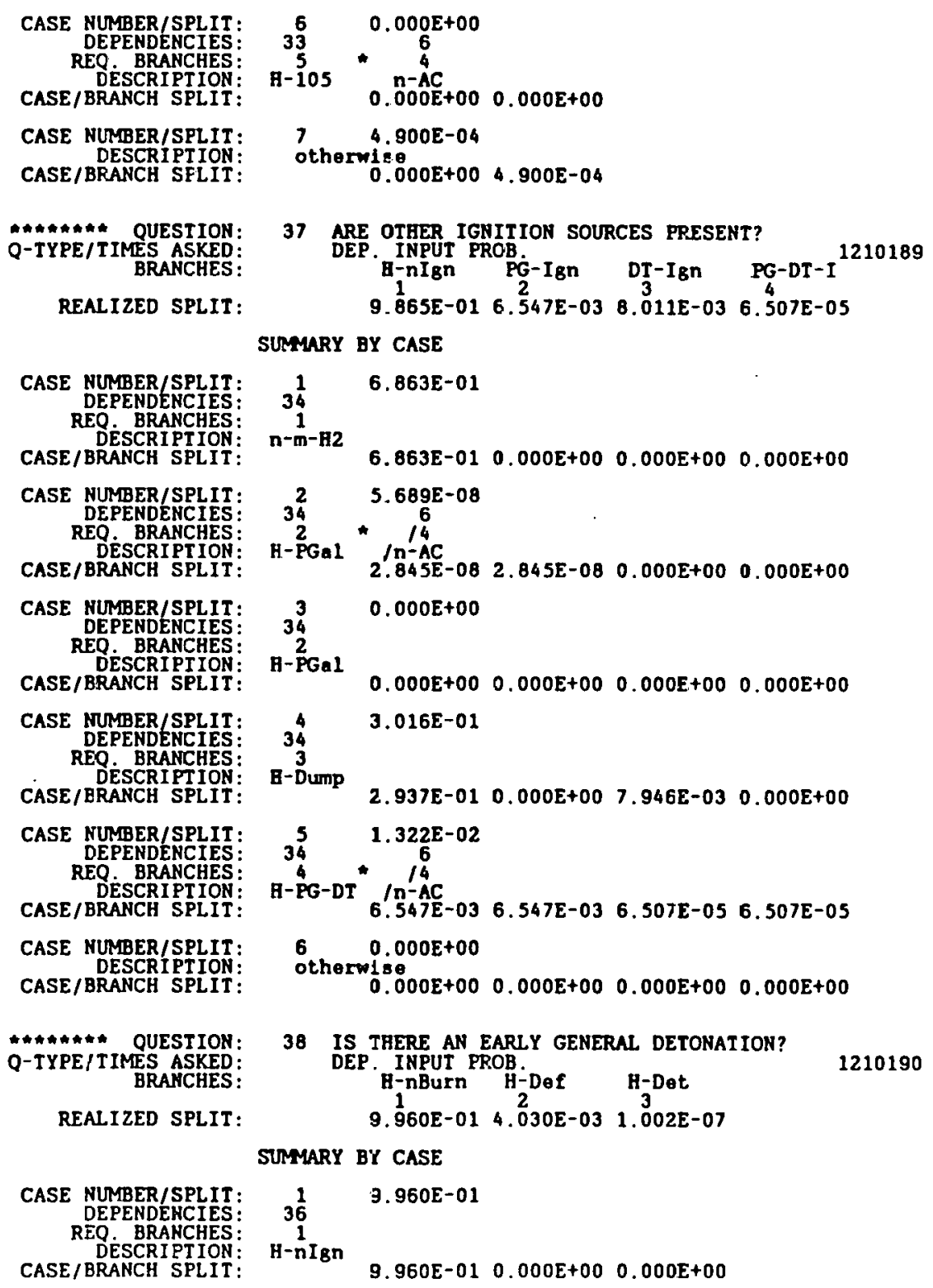




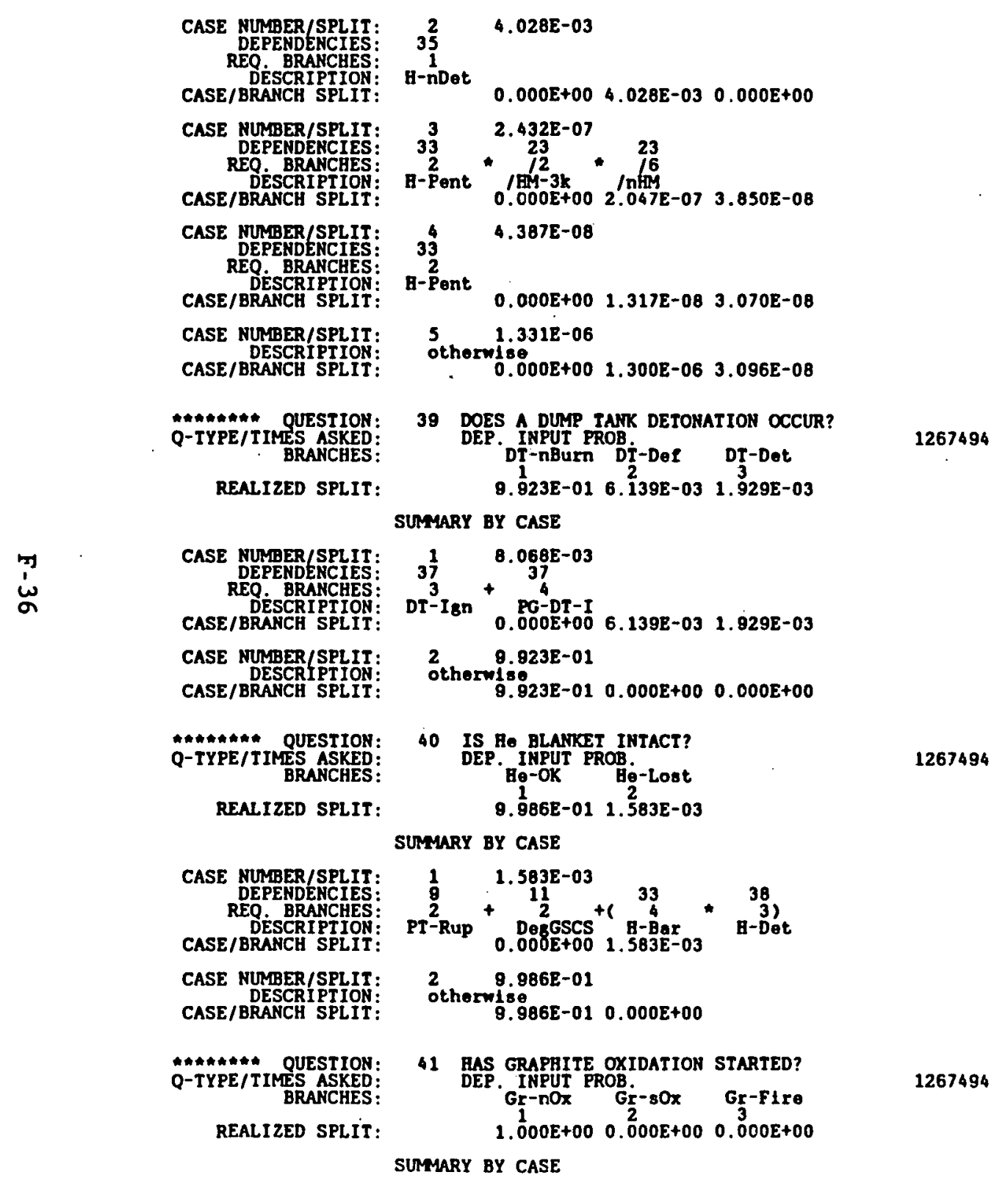




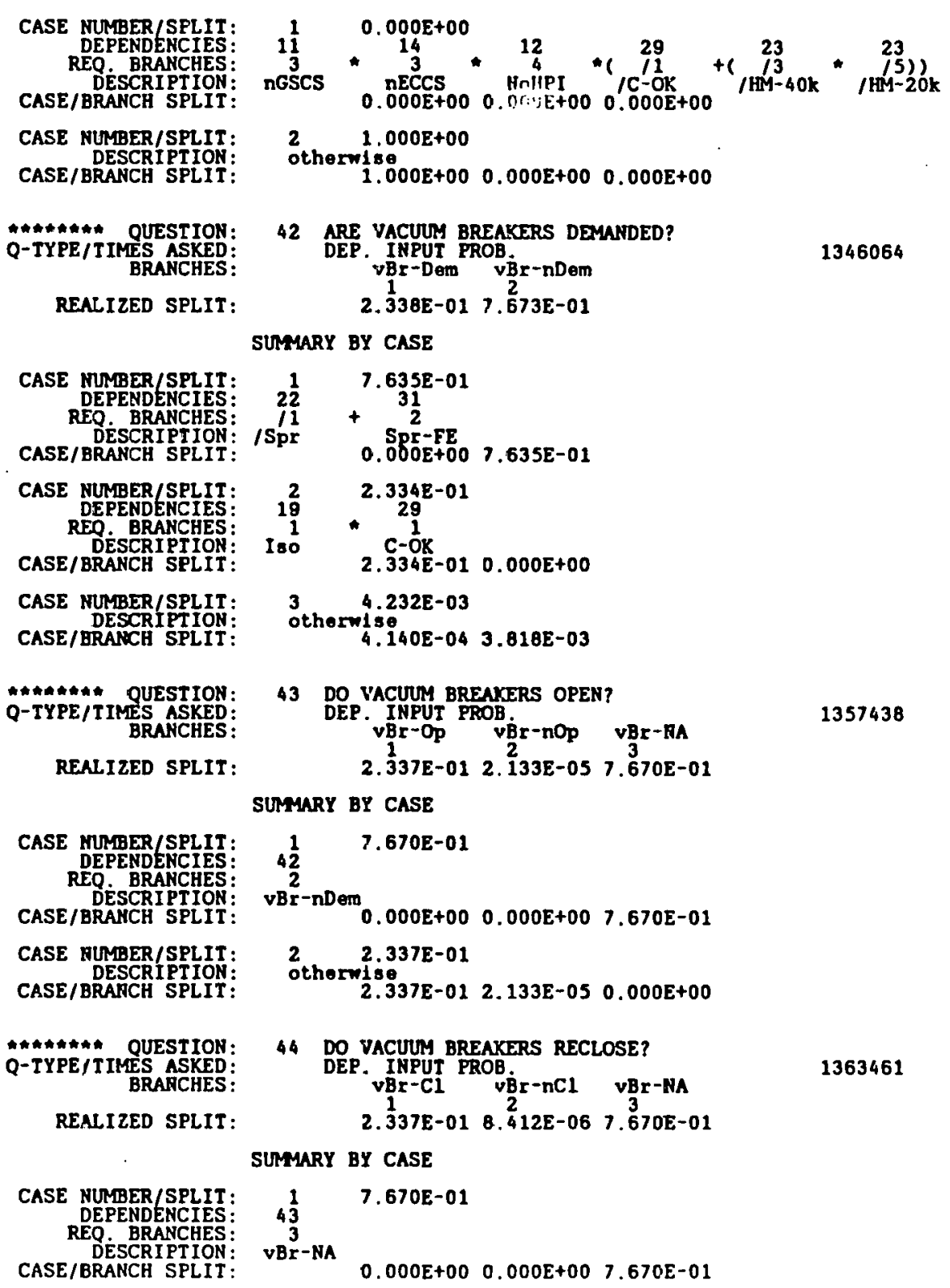

43 DO YACUUM BREAMERS OPEN?

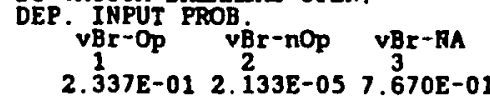
1357438

1346064

1357438 


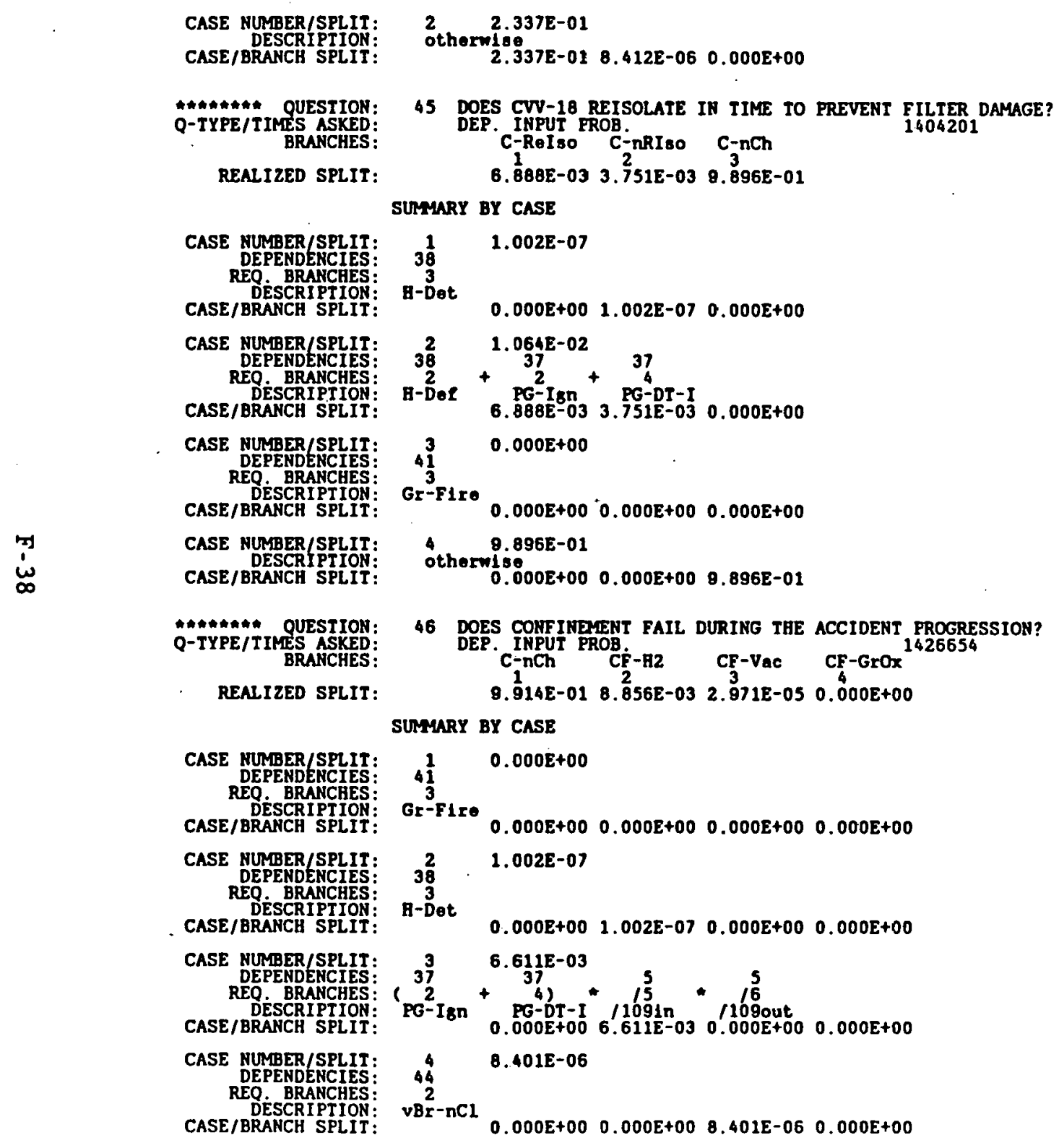




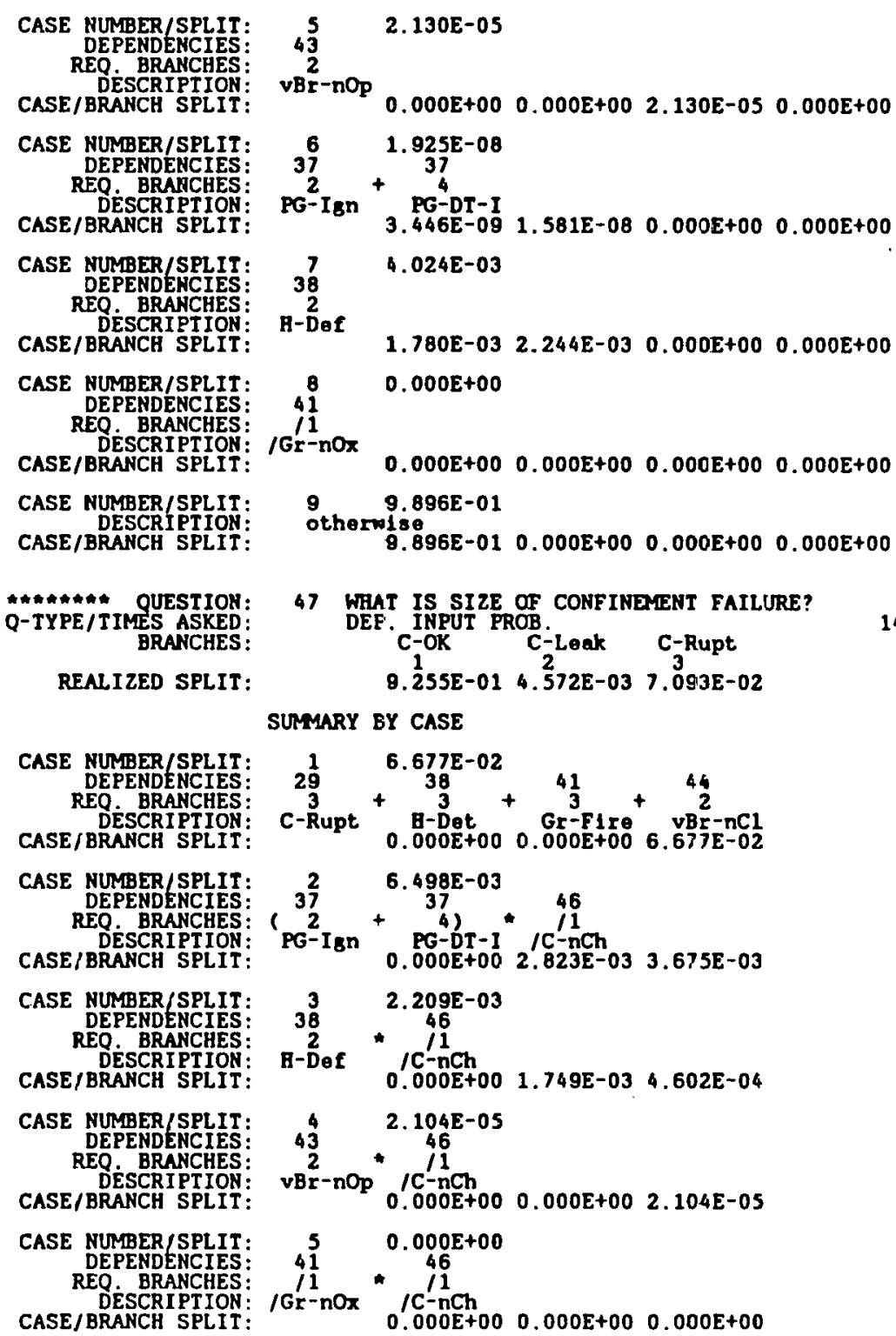




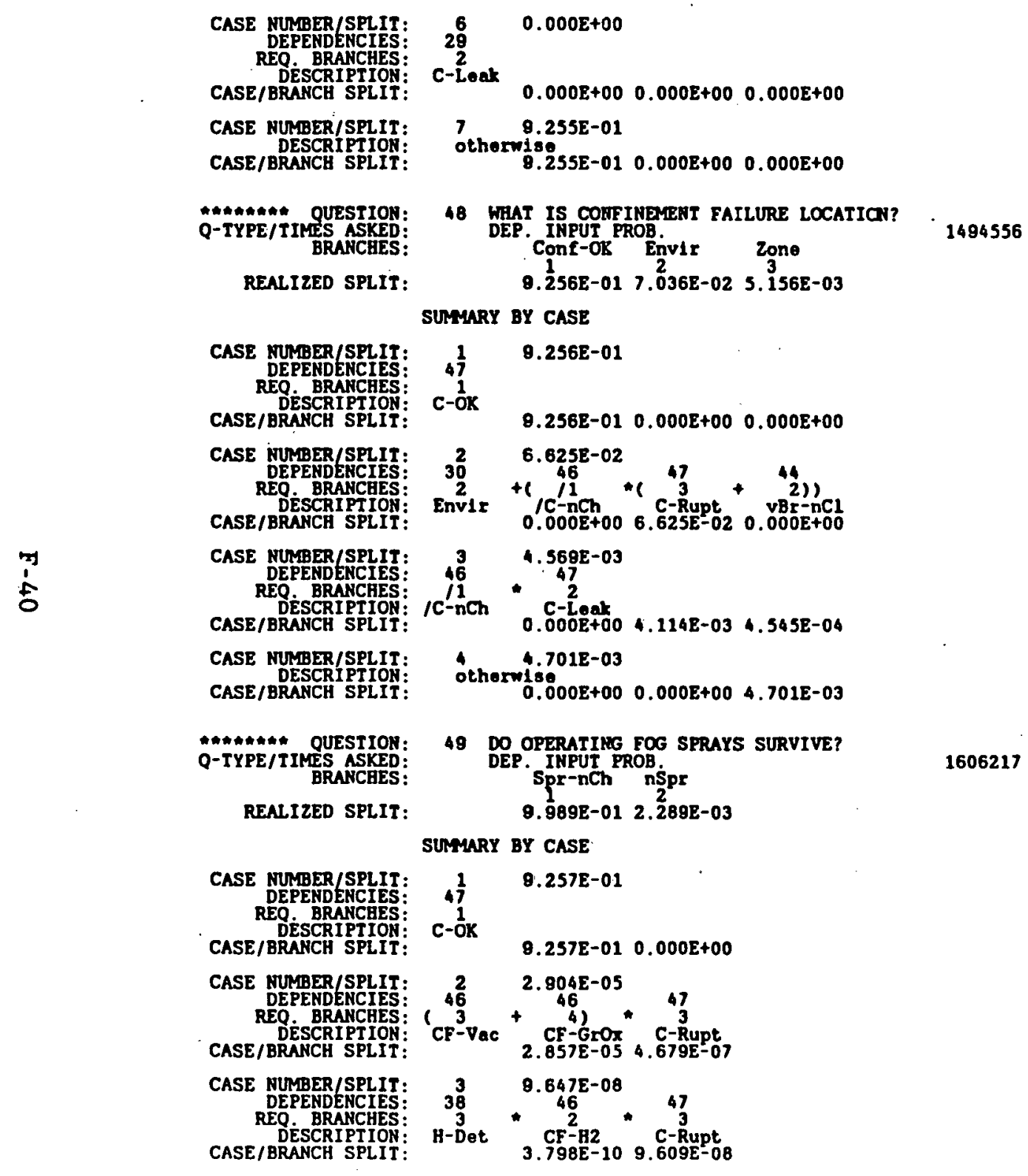




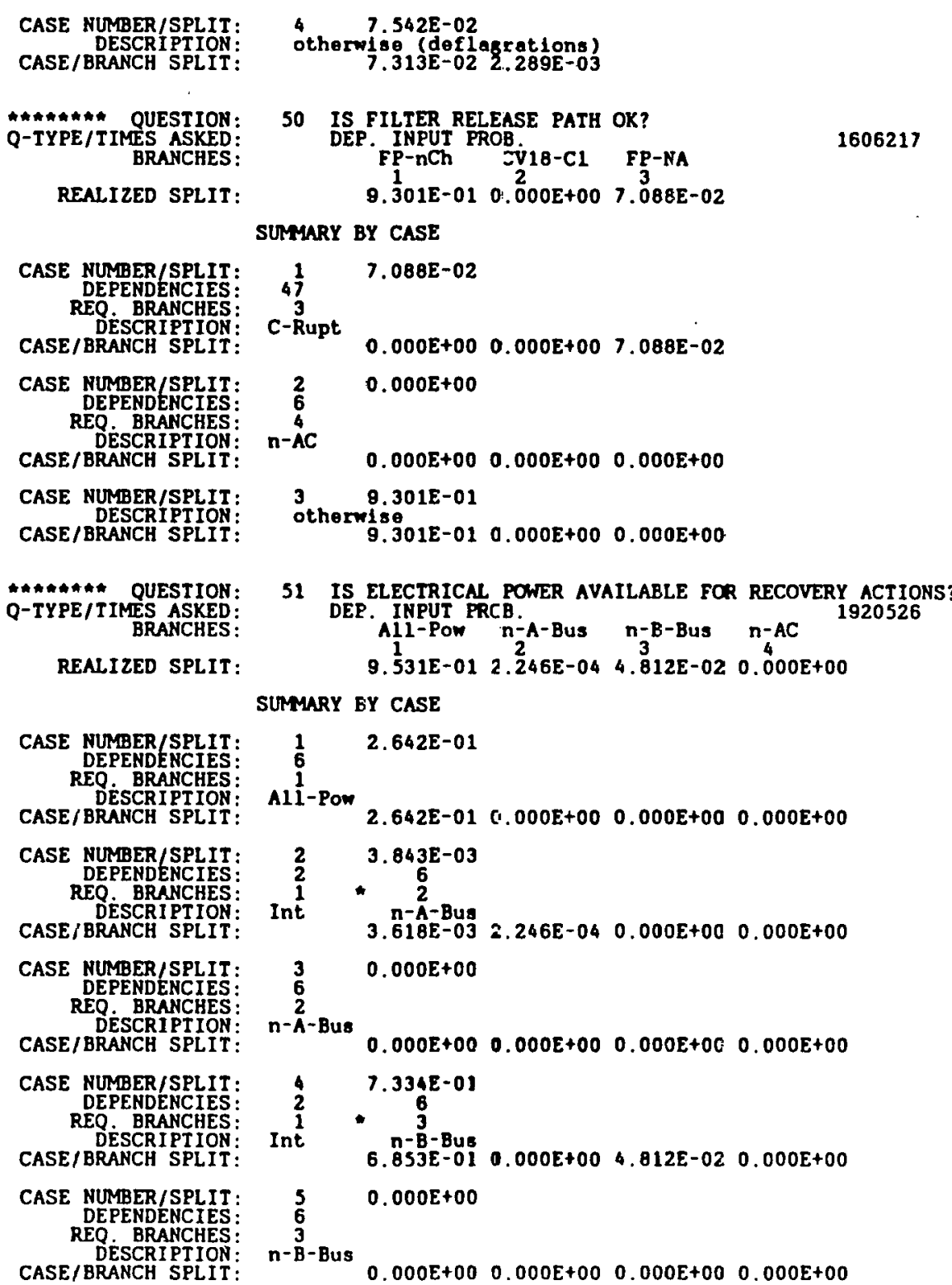




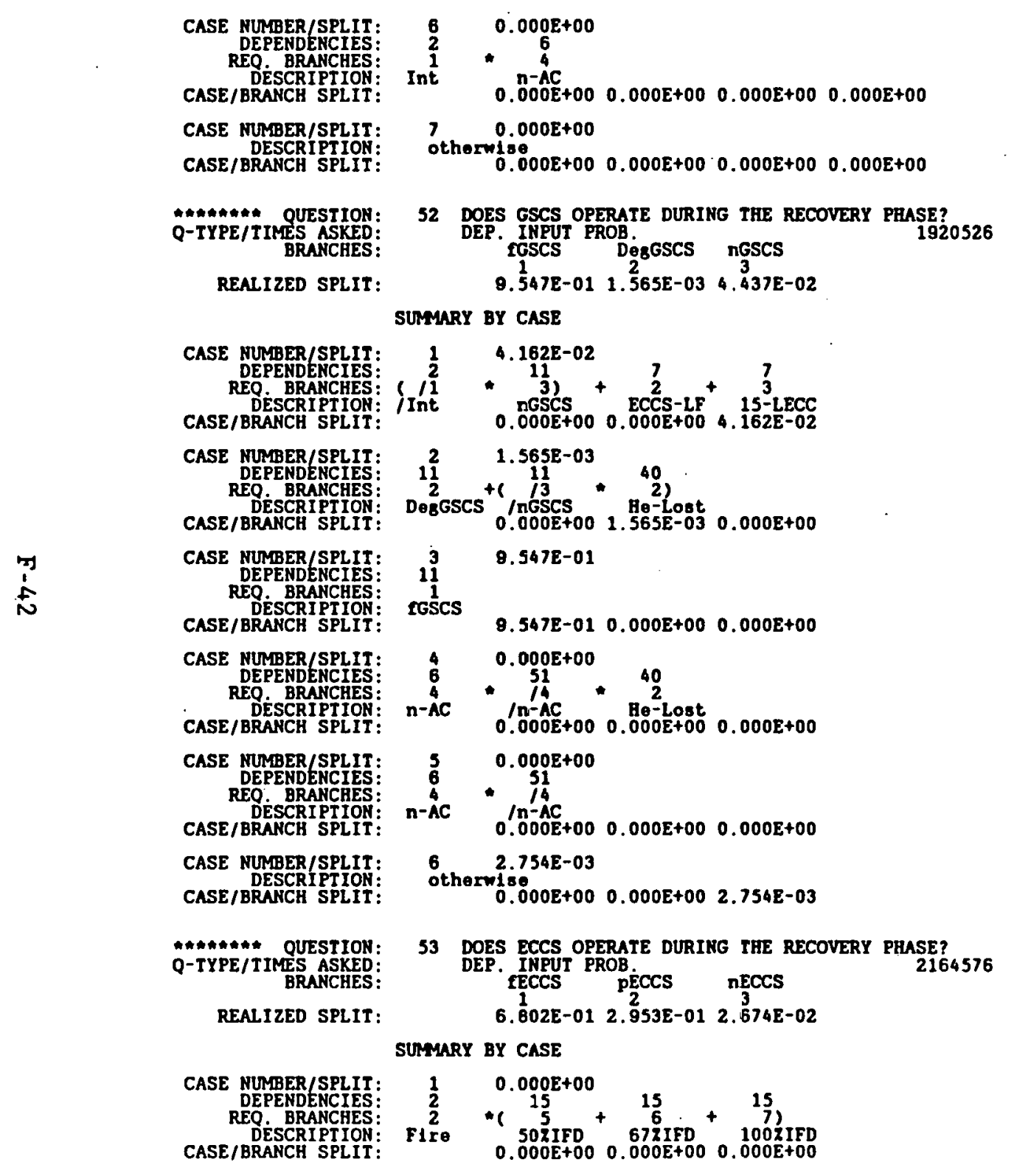




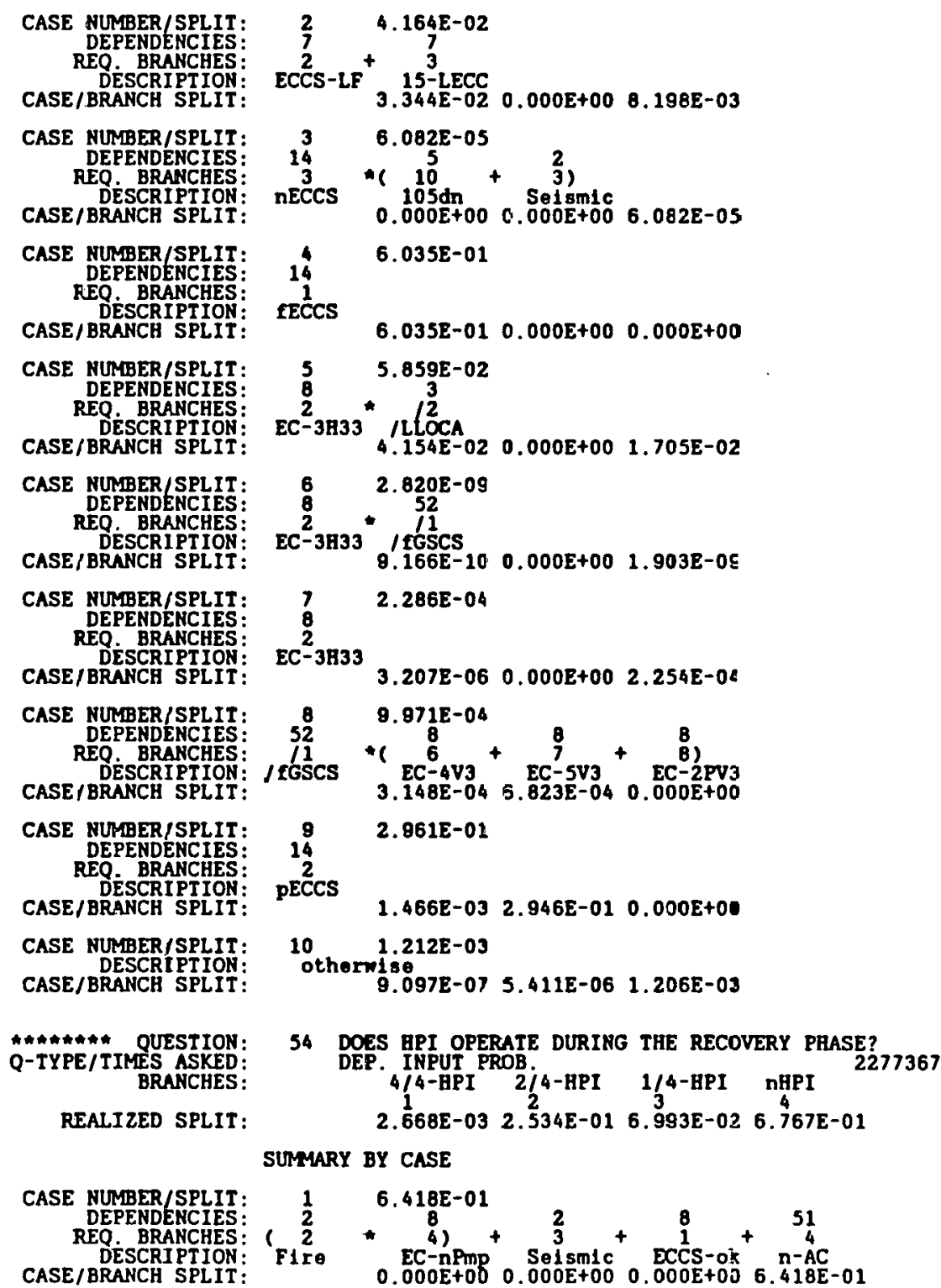


CASE NUMBER/SPLIT: REQ. BRANCHES : CASE/BRARCH SPLIT: CASE MUMBER/SPLIT: DEPENDENCIES: DESCRIPTIOH:
CASE/BRANCH SPLIT: CASE RUMBER/SPLIT: REO BRAFCCHES DESCRIPTIOR:
CASE/BRANCH SPLIT : CASE MUMBER/SPLIT: DEPENDENCIES: CASE/BRANCH SPLIT: CASE MUMBER/SPLIT. DEPENDERCIES:

REQ. BRANCHES: CASE/BRANCH SPLIT:

CASE MUMER/SPLIT: REO BRANCHES : CASE/BRANCH SPLIT:

CASE RUMBER/SPLIT: DEPENDENCIES REQ. BRANCHES CASE/BRANCH SPLIT:

CASE MUMBER/SPLIT:

DEPENDENCIES

RE DESCRIPTION

CASE/BRANCH STLIT:

CASE MUMBER/SPLIT: REO BRANCHES

DESCRIPTION:
CASE/BRANCH SPLIT:

CASE NUMBER/SPLIT: DESCRIPTION:
CASE/BRANCH SPLIT:

Q OAMAnA QUESTION: Q-TYPE/TIMES ASKED: REALI2ED SPLIT:

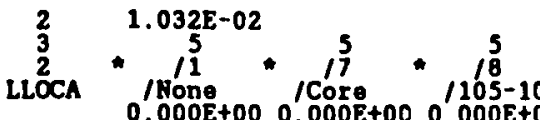

$0.000 E+00 \quad 0.000 E+00 \quad 0.000 E+00 \quad 1.032 E-02$

$30.000 E+00$

4.64

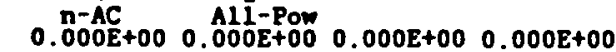

(1) $0.000 E+00$

$0.0008+00$
$6 \quad 51$

$\begin{array}{lllll}\text { RoHPI } & 0.000 E+00 & 0.000 E+00 & 0.000 E+00 & 0.000 E+00\end{array}$

$0.000 \mathrm{E}+000$.

12

$\begin{array}{llll}2.433 E-04 & 0.000 E+00 & 0.000 E+00 \quad 2.460 E-02\end{array}$

$6 \quad 6.988 E-02$

3 * 12

$\begin{array}{lllll}1-\mathrm{API} & 0.00 \mathrm{E}+00 & 0.000 \mathrm{E}+00 & 6.988 \mathrm{E}-02 & 0.000 \mathrm{E}+00\end{array}$

$12 \quad 3.544 E-06$

1-HPI 3 /Al1-Pow

( $4.743 \mathrm{E}-0 \mathrm{~S}$

$1-3$

$4.046 E-07 \quad 0.000 E+00 \quad 4.702 E-05 \quad 0.000 E+00$

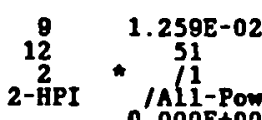

O.000E+00 1.250E-02 $0.000 E+00 \quad 0.000 E+00$

$10 \quad 2.432 \mathrm{E}-01$

2-HPI

$2.424 E-03 \quad 2.408 E-01 \quad 0.000 E+00 \quad 0.000 E+00$

$11 \quad 0.000 E+00$

$0.000 E+00 \quad 0.000 E+00 \quad 0.000 E+00 \quad 0.000 E+00$

55 IS THERE A STEAM SPIKE FROM H2O INJECTION?

StmP-NA StmP>5 StmP<5

9.572E-01 6.119E-06 4.330E-02

SUMMARY BY CASE 


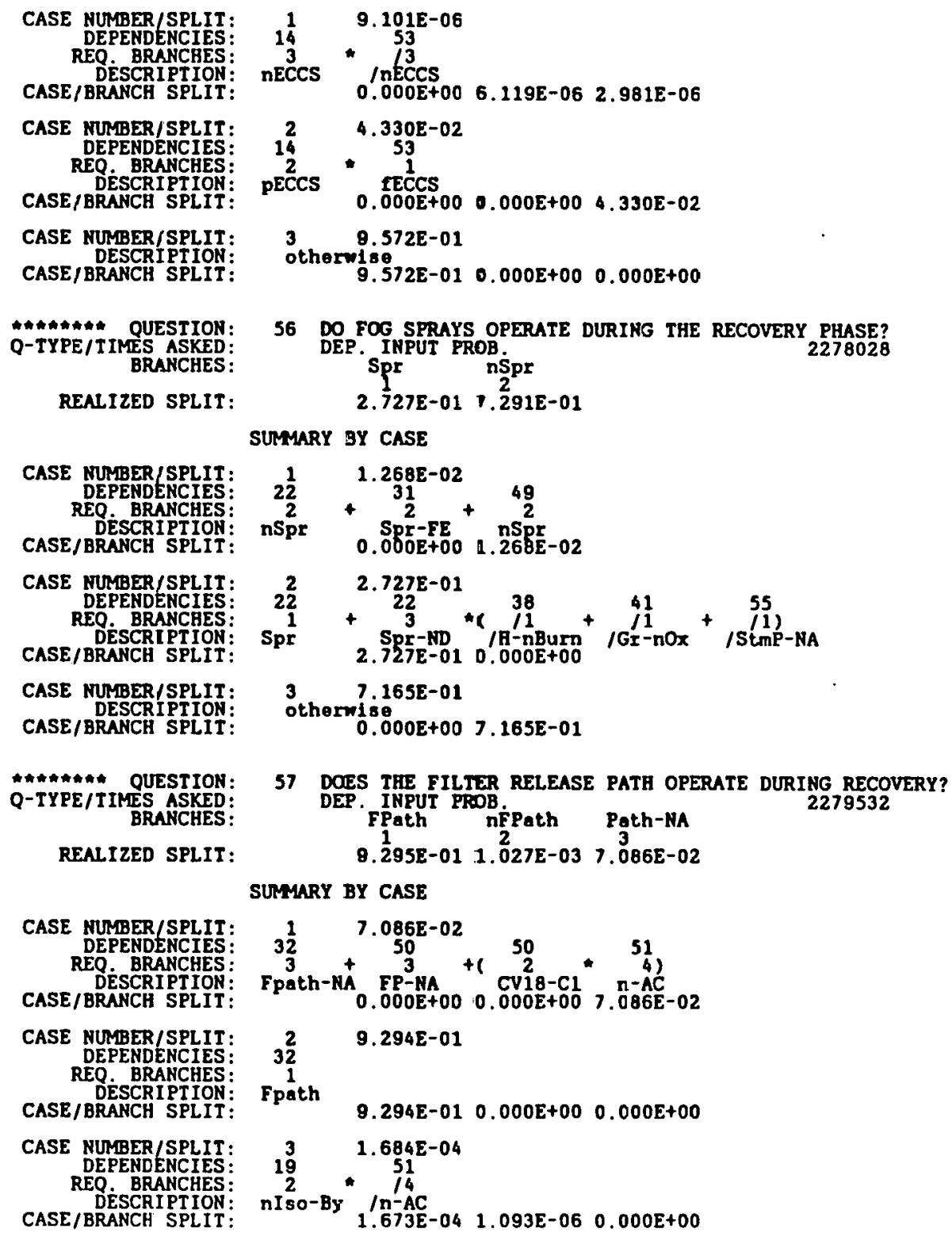




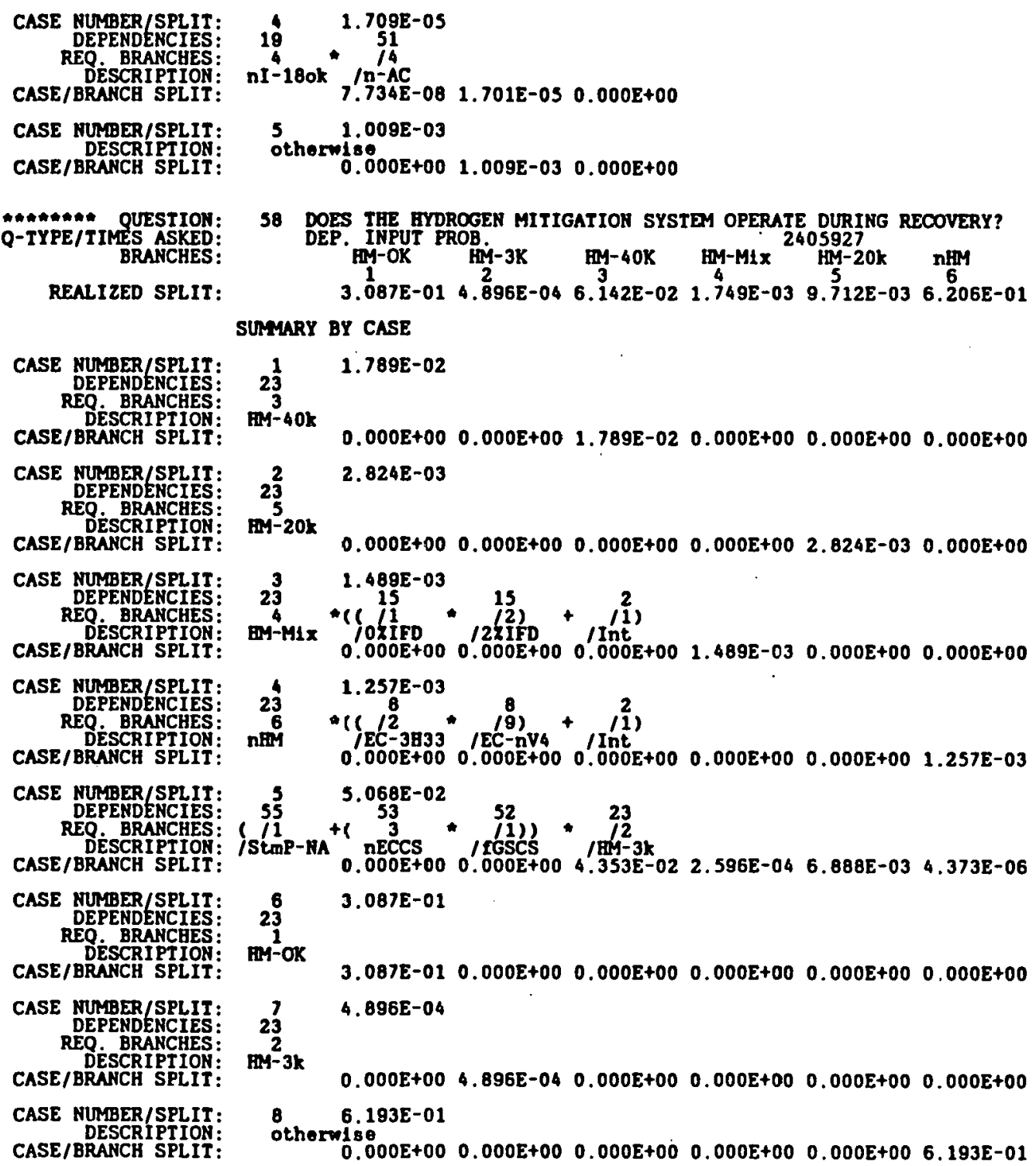


Q*TYA*A* QUESTION:

BRANCHES:

REALIZED SPLIT:

59 DOES PRESSURE TUBE FAILURE OCCUR DUE TO WATER INJECTION?

$9.574 E-01 \quad 4.329 E-02$

\section{SUMMARY BY CASE}

CASE NUMBER/SPLIT:

DÉSCRIPTION:

CASE/BRANCH SPLIT:

CASE NUMBER/SPLIT:

REO ERANCHE:

DESCRIPIION:

CASE/BRANCH SPLIT:

at+m*an* QUESTION:

Q-TYPE/TIMES ASKED:

REALIZED SPLIT:

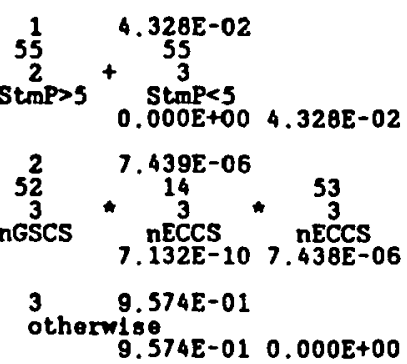

60 BOW MUCA HYDROGEN IS PRODUCED DURING IHE ACCIDENT?

. 23 PUT PROB

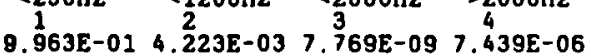

SUMMARI BY CASE

CASE NIMBER/SPLIT: Tub-nFr Tube-Fr

CASE NUMBER/SPLIT:

QA-TXPE/TIMES OUESTON: BRANCHES:

REALIZED SPLIT:
61 WRAT IS LATE H2 FLAMABBILITY?
DEP. 1HPUT PROB.
9. $980 E-01 \quad 5.523 E-08 \quad 1.237 E-03 \quad 0.000 E+00 \quad 0.000 E+00$

\section{SUMMARY BY CASE}

CASE NUMBER/SPLIT:
DEPENDENCIES:
REQ. BRANCHES :

CASE/BRANCH SPLIT:

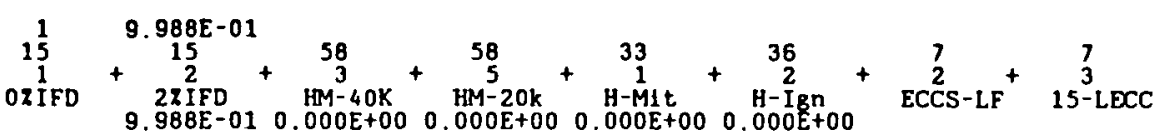




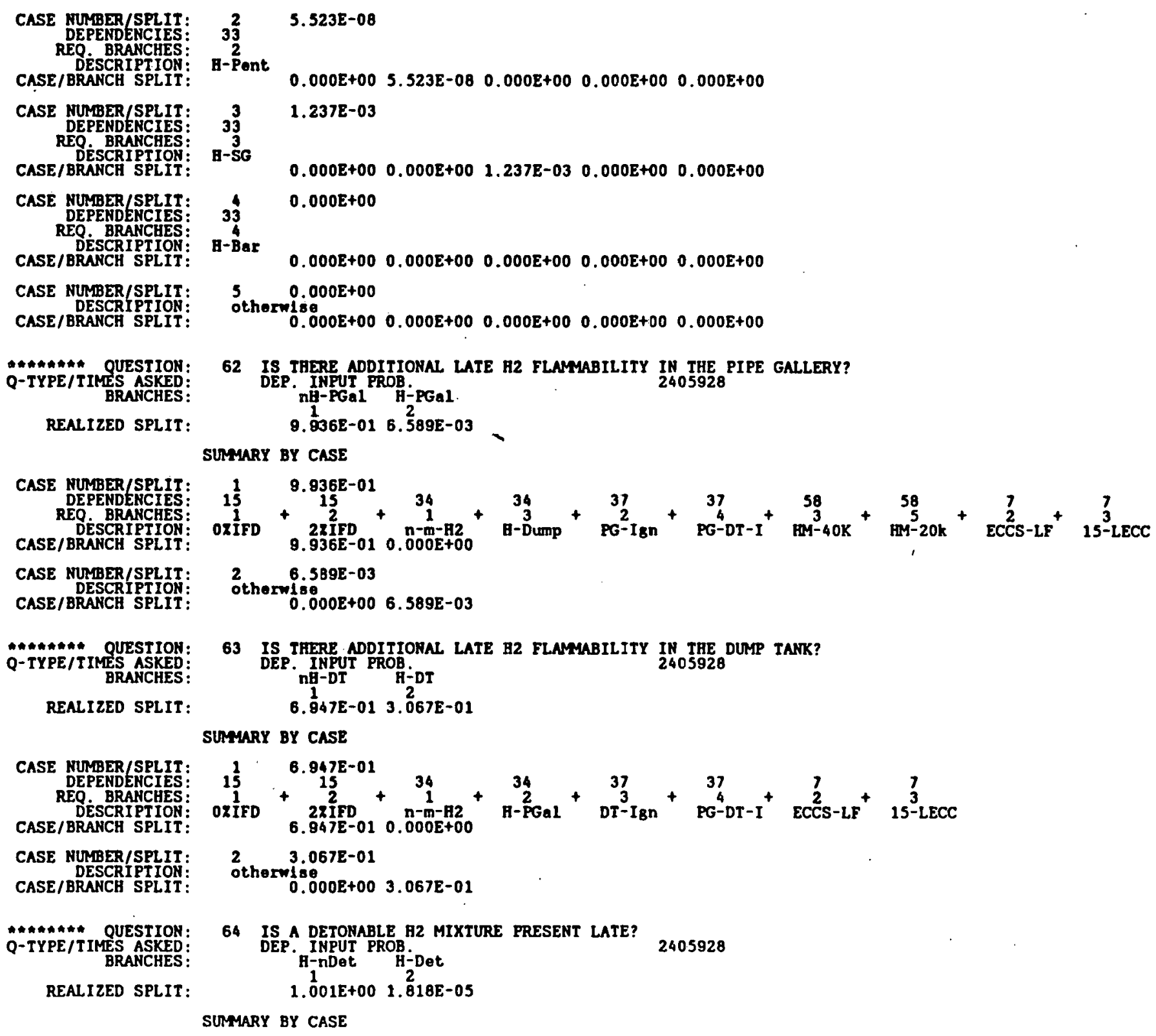

SUMMARY BY CASE 


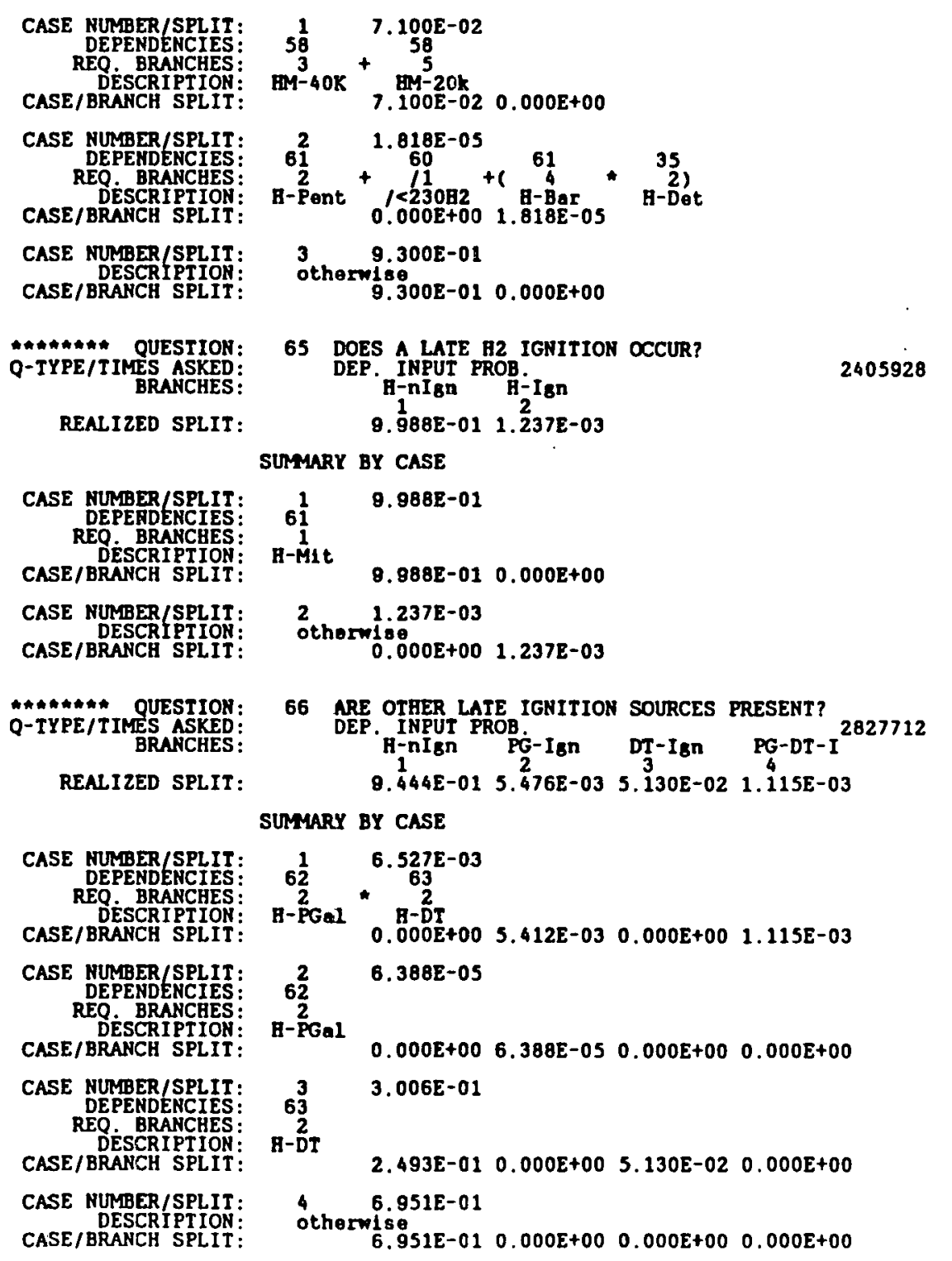




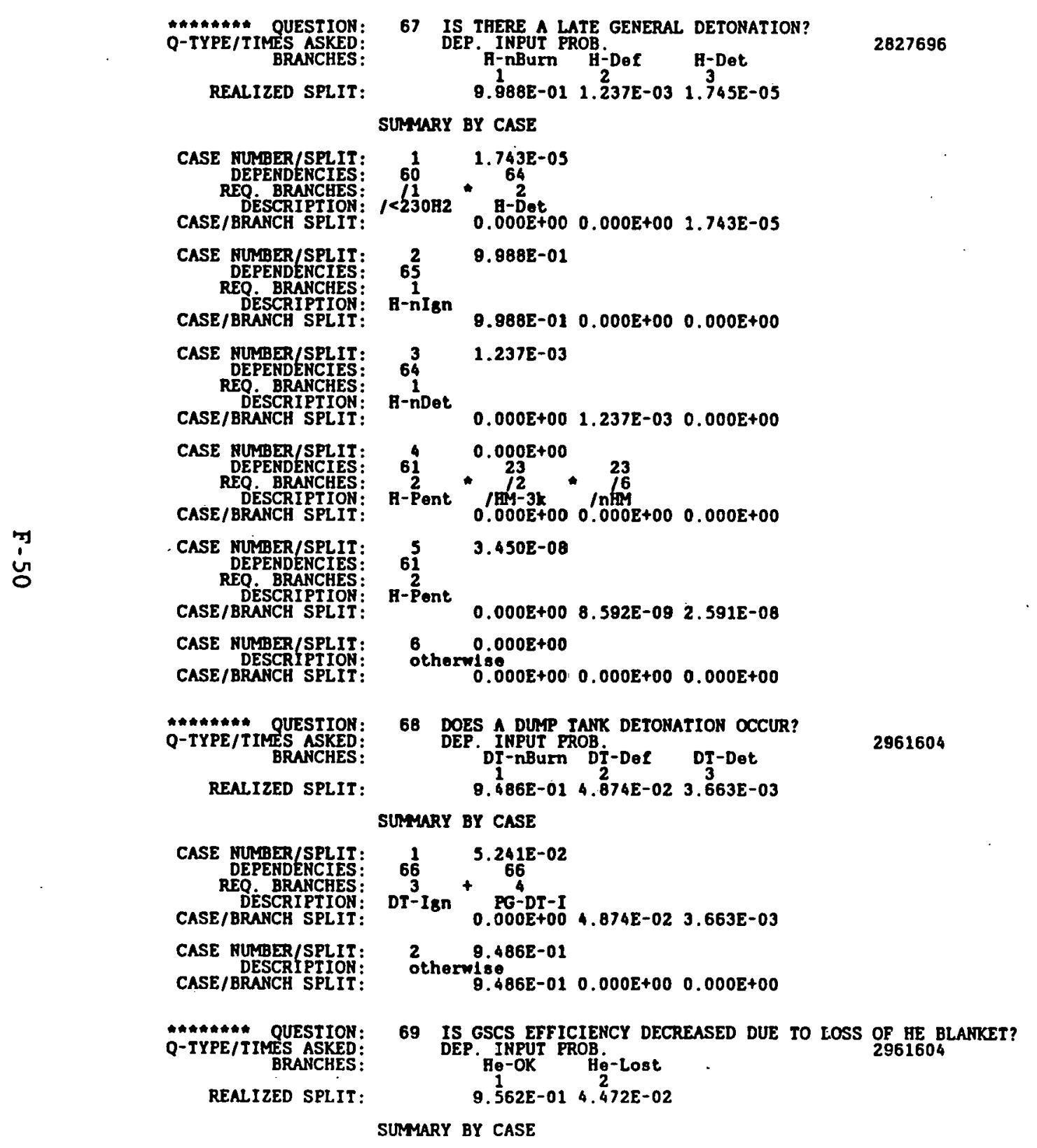




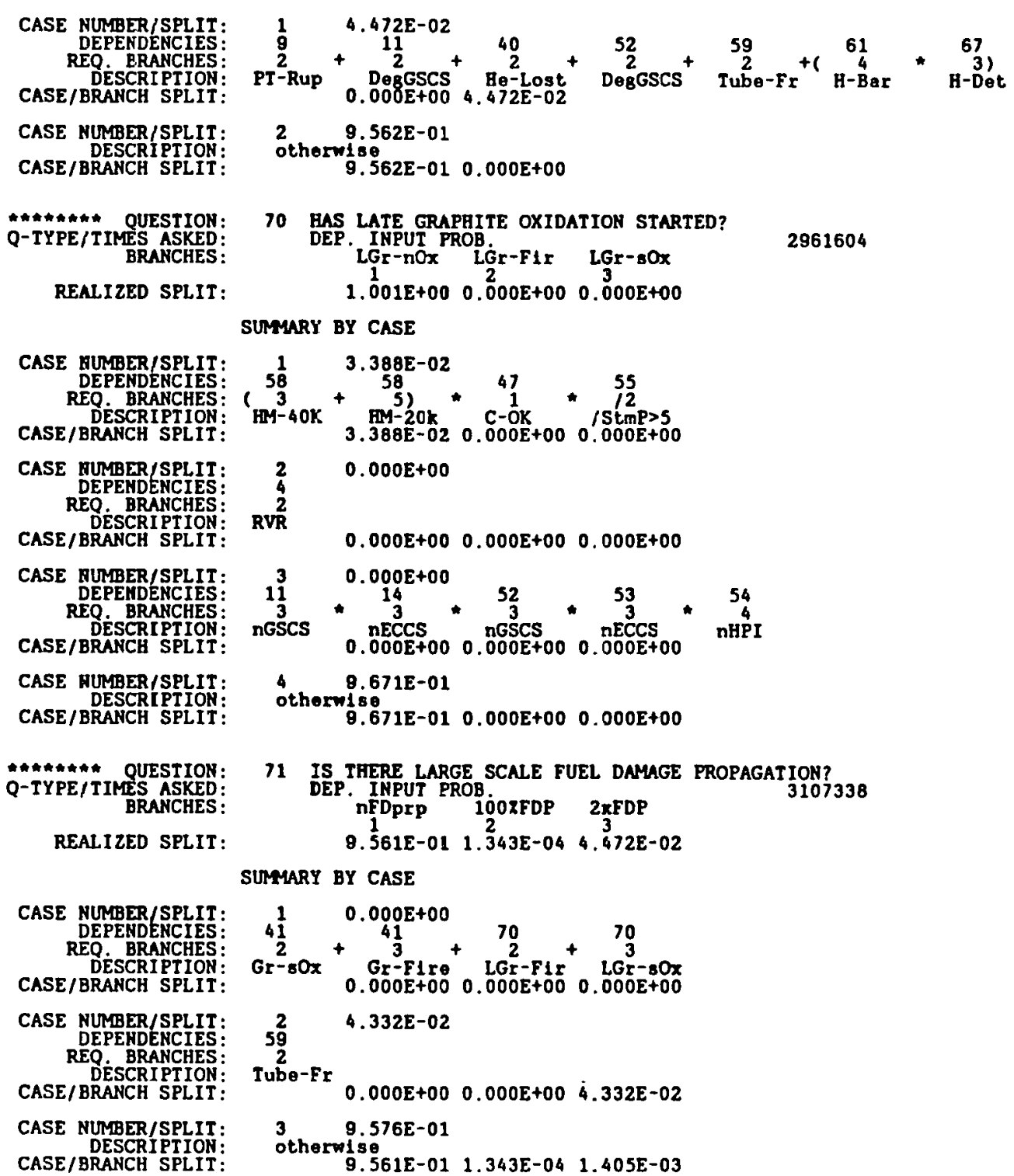




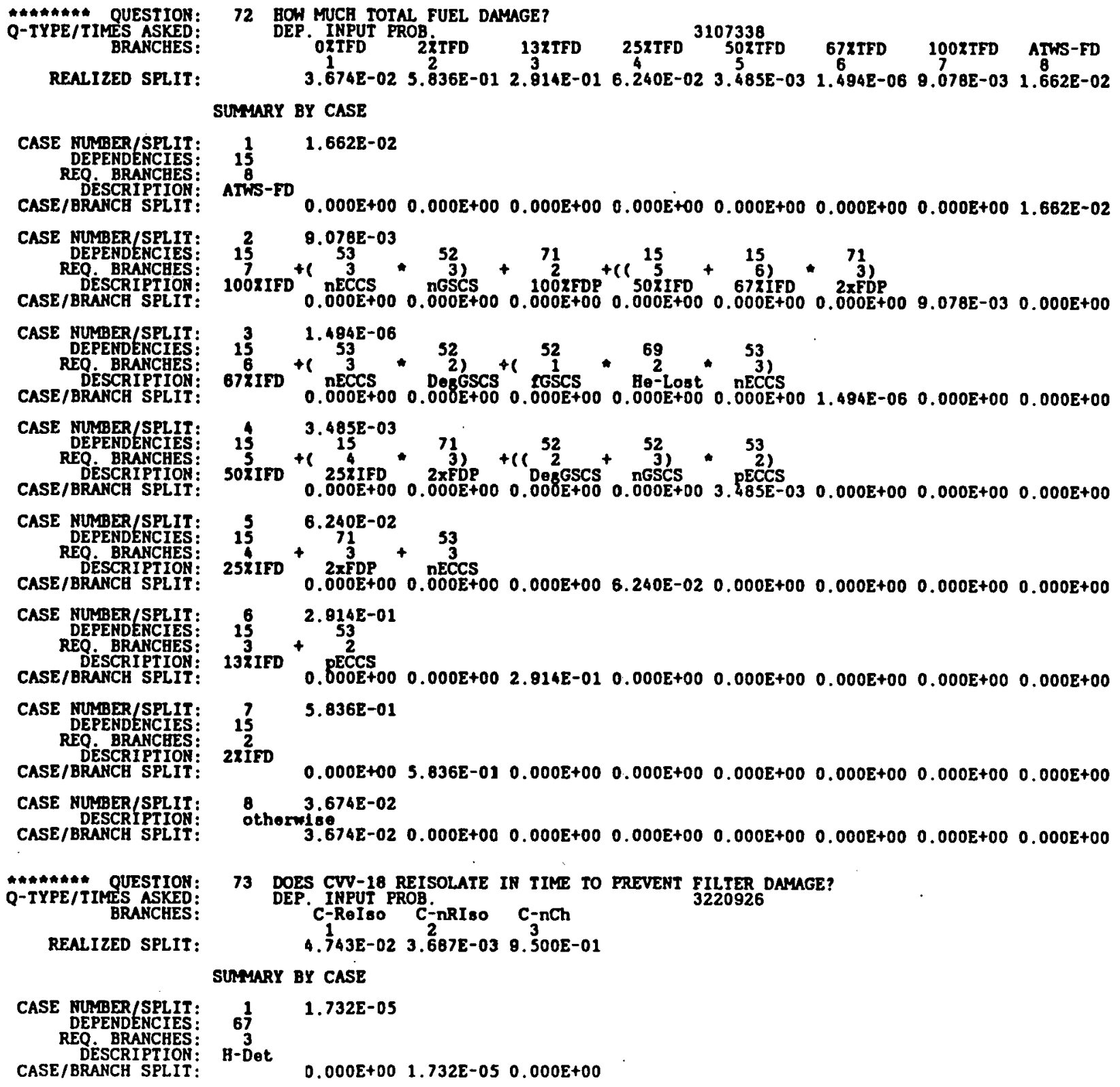

$\begin{array}{llllllll}0.000 E+00 & 5.836 E-01 & 0.000 E+00 & 0.000 E+00 & 0.000 E+00 & 0.000 E+00 & 0.000 E+00 & 0.000 E+00\end{array}$ 3.674E-02

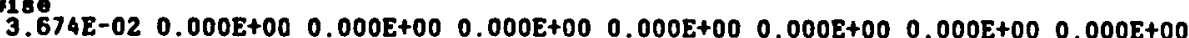

73 DOES CVV-16 REISOLATE IN TIME TO PREVENT FILTER DAMAGE? 


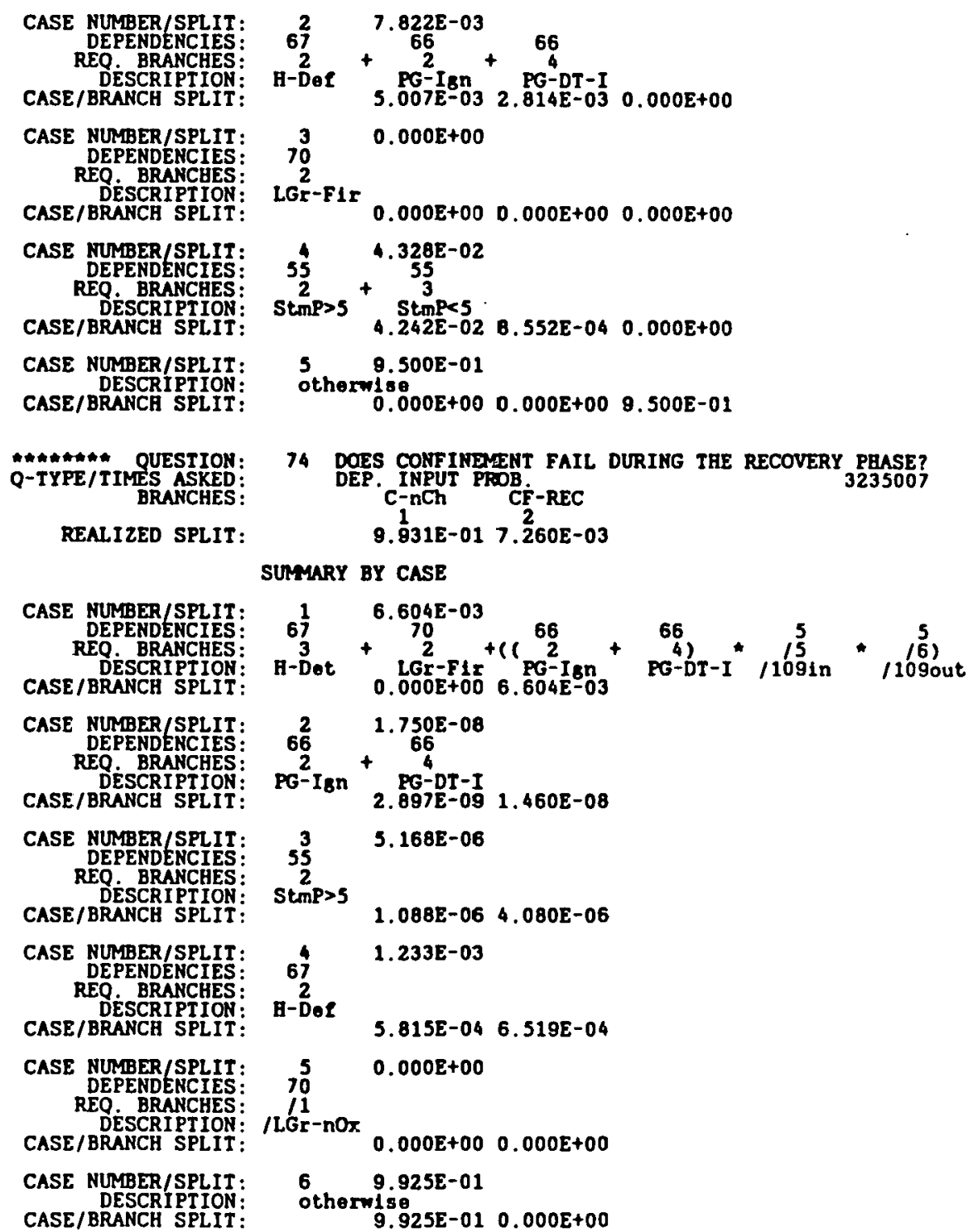

Q-TYPE/TIMES ASKED:

74 DOES CONFINEMENT FAIL DURING IHE RECOVERY PEASE?
D235007

BRANCHES :

REALIZED SPLIT:

SUMYARY BY CASE 


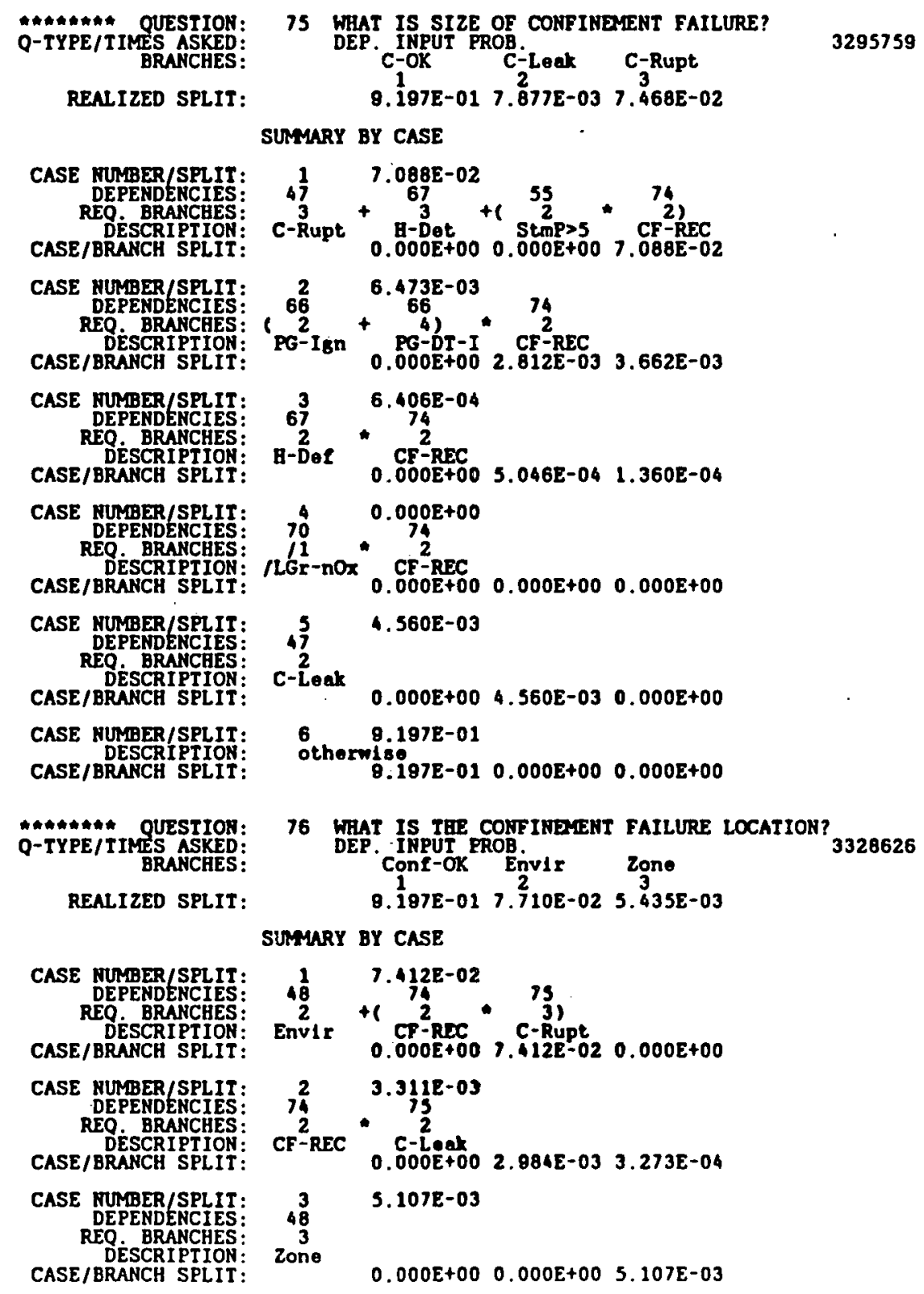

76 WhAT IS TEE CONFIHEAENT FAILURE LOCATION? DEP. INPUT PROB. Conf-OK Envir Zone $\begin{array}{ccc}\text { Conf-OK } & \text { Envir } & \text { Zone } \\ \text { 9.197E-01 } & 7.710 E-02 & 5.435 E-03\end{array}$

SUMMAY BY CASE

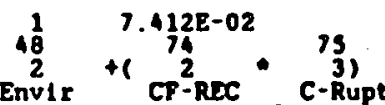

$0.000 E+00$ 1.412E $=02 \quad 0.000 E+00$

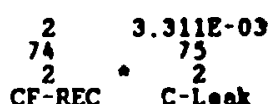

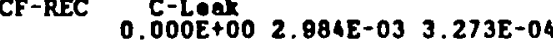

3 5.107E-03

zone 


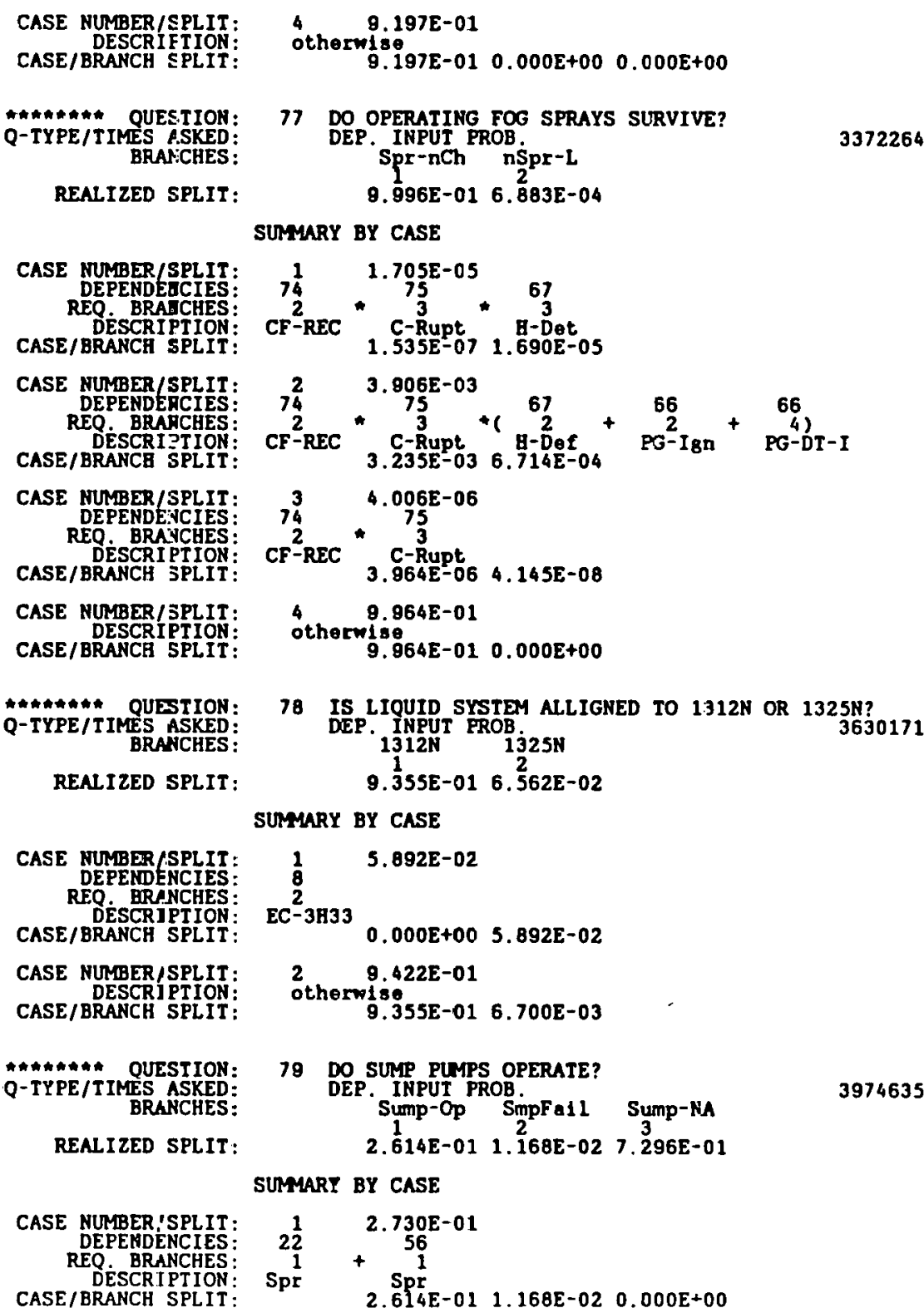

2. $730 \mathrm{E}-01$

56
$+\quad 1$

Spr 
CASE NUMBER/SPLIT: 2 7.296E-01

CASE/BRANCH SPLIT: $\quad 0.000 \mathrm{D}+00 \quad 0.000 \mathrm{E}+00 \quad 7.296 \mathrm{E}-01$

80 DOES FLOH TO 1325 OCCUR?

DEP. INPUT PROB. 1312510 1325F10 No-F10 3974635

Q-TYPE/TIMES ASKED:

REALI ZED SPLIT:

9. 356E-01 5. 2075E-02 $1.484 E-02$

SUMMARY BY CASE

CASE NUMBER/SPLIT:
DEPENDENCIES:
REO BRANCHES:

REQ. BRANCHES:

DESCRIPTION:
CASE/BRAKCH SPLIT:

CASE NUMBER/SPLIT:

DEPENDENCIES:

DESCRIPTION:
CASE/BRANCH SPLIT:

CASE NUMBER/SPLIT:

CASE/BRANCH SPLIT:

Anthinta QUESTION:

Q-TYPE/TIMES ASKED:

REALIZED SPLIT:

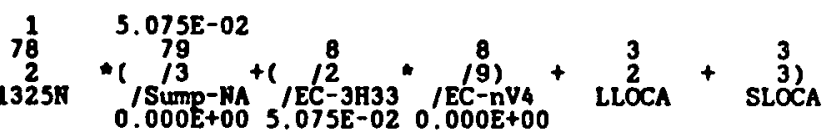

79

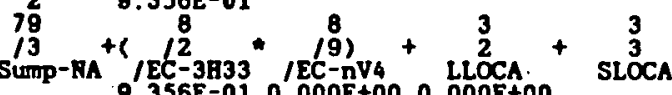

3 1.484E-02

$0.000 \mathrm{E}+00 \quad 0.000 \mathrm{E}+00 \quad 1.484 \mathrm{E}-02$

81 DOES LERF FUNCTION PROPERLY?

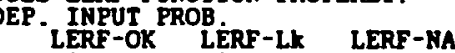

$\begin{array}{ccc}1 & \\ 9.356 E-01 & 1.356 E-05 & 6.555 E-02\end{array}$

SUMYARY BY CASE

CASE NUMBER/SPLIT:

DEPENDENCIES:

REQ BRANCHES: 11

CASE/BRANCH SPLIT:

9.356E-01

CASE NUMBER/SPLIT:

CASE/BRANCH SPLIT:

g.356E-01 1.356E-05 $0.000 E+00$

$26.555 E-02$

$0.000 E+00 \quad 0.000 E+00 \quad 6.555 E-02$

Q A-TYAAHA QUESTIOR:

BRANCHES:

REALIZED.SPLIT:

82 ARE FILTERS BYPASSED?

\begin{tabular}{cccc} 
nByp & F-pByp & F-Byp-L & F-Byp \\
1 & 3 \\
9.192E-01 & $7.875 E-03$ & $4.132 E-03$ & 4 \\
\hline
\end{tabular}

SUMYARY BY CASE

CASE RUMBER/SPLIT:

DEPEDECH:

DE.SCRATTION:

$7 \frac{1}{5} \quad 9.192 E-01$

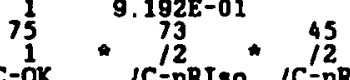

IC-nRIso /C-nRIso

CASE NUMRER/SPLIT:

DEPENDENCIES:

CASE/BRANCH SPLIT:

$7^{2} \quad 7.875 E-03$

2

$0.000 E+00 \quad 7.875 E-03 \quad 0.000 E+00 \quad 0.000 E+00$ 


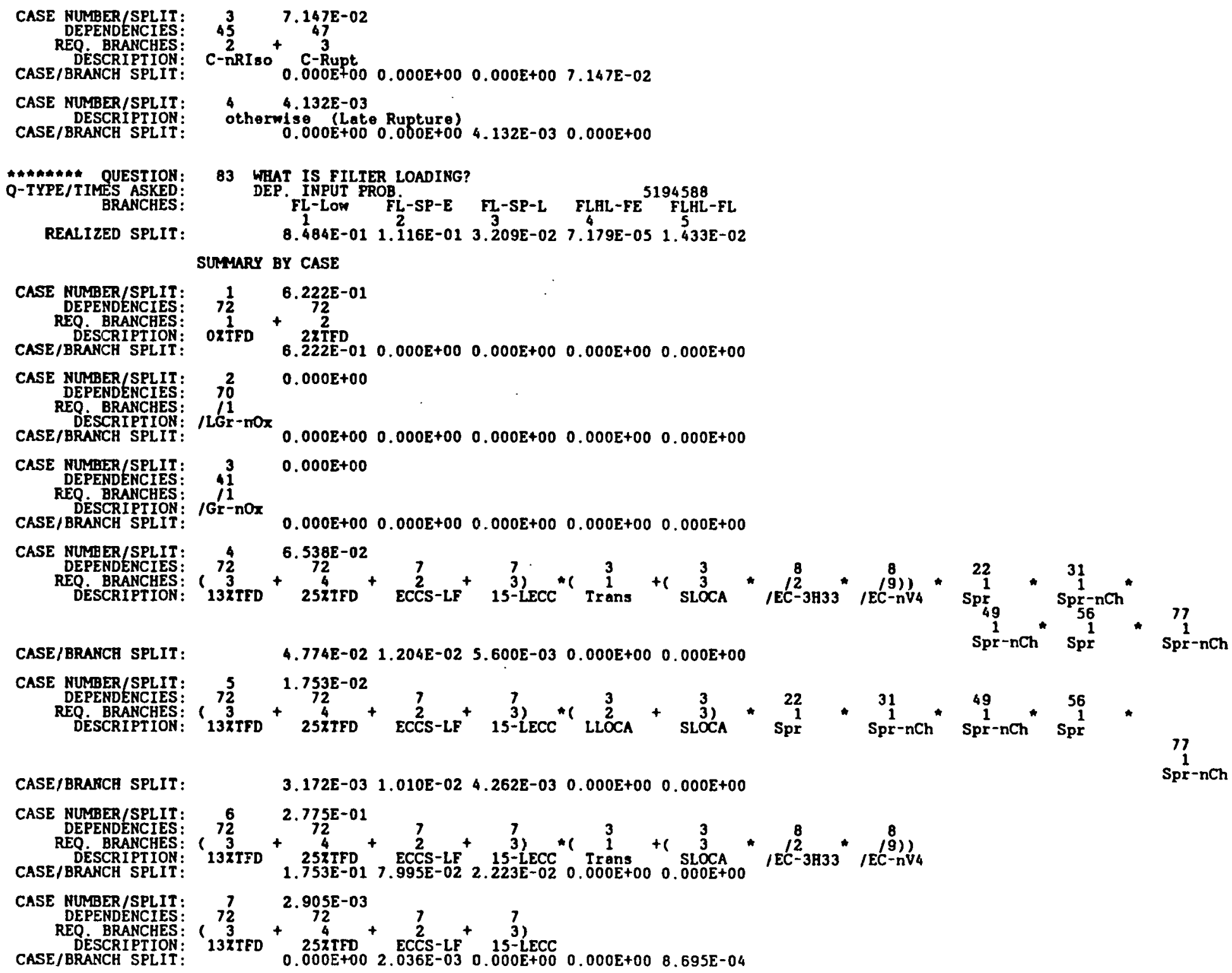


CASE NUMBER/SPLIT

REQ. BRANCHES:

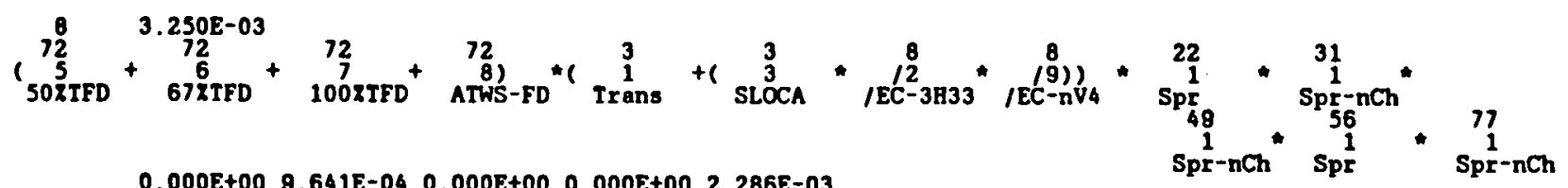

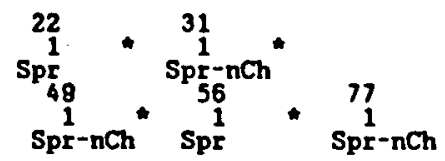

CASE/BRANCB SPLIT:

$0.000 E+009.641 E-04 \quad 0.000 E+00 \quad 0.000 E+00 \quad 2.286 E-03$

CASE NUMBER/SPLIT:

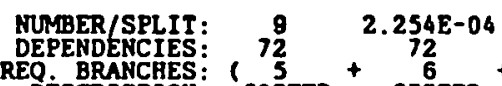

DEscis

CASE/BRANCH SPLIT:

72
$67850+$

$\left.\frac{72}{7}+\frac{72}{8}\right)+\left(\frac{3}{2}+\frac{3}{3}\right)$

- 2

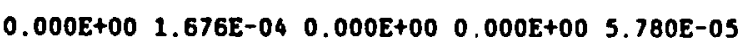

CASE NUMBER/SPLIT:

REO BENRACHES:

CASE/BEANCR SPLITT:

$10 \quad 1.7458-02$

CASE NUMBER/SPLIT:

CASE/BRANCH SPLIT:

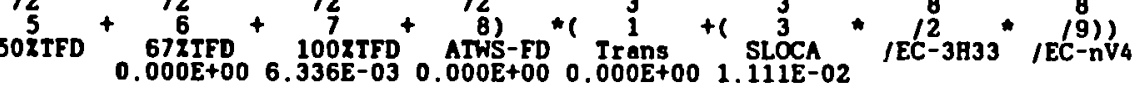

11 othernise $179 \mathrm{E}-05$

O-TYME TI QUESTION:

Q-TYPE/TIMES ASKED:
BRANCHES:

84 DO FILTER SPRAYS OPERATE?

$0.000 E+00 \quad 0.000 E+00 \quad 0.000 E+00 \quad 7.179 E-05 \quad 0.000 E+00$

REALIZED SPLIT:

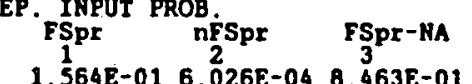

5297096

\section{SUMMARY BY CASE}

CASE MUMGER/SPLIT:

REO. BRAMCAES:

CASE/BRANCH SPLIT:

$8 \frac{1}{3}$
1
$5 L-L O$

CASE NUMBER/SPLIT:

REPENDENCCES:

CASE/BRANCH SPLIT:

$0.000 E+00 \quad 0.000 E+00 \quad 8.463 E-01$

$7.152 \mathrm{E}-06$

CASE MUMBER/SPLIT:

REPENDEANCEES:

CASE/BRANCH SPLIT:

$\stackrel{2}{2}+\underset{n-A-B u s}{6}+\frac{4}{n-A C}+\underset{n}{51}+\stackrel{n-B u s}{n}+\underset{n-A C}{41}+\underset{n S p r}{22}$

CASE/BRANCH SPLIT:

CASE RUMBER/SPLIT:
CASE/BRASCRPT SPLIT:

$3 \quad 8.917 \mathrm{E}-04$

$3+3) \times 22$

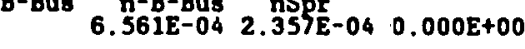

otherwise $1.561 \mathrm{E}-01$

$\begin{array}{lll}1.557 E-01 & 3.598 E-04 & 0.000 E+00\end{array}$

Q-TYPE/TI QUESTION:

BRANCHES:

REALIZED S'PLIT:
85 IS FILTER BANK D OPERATING?

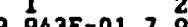

10.017 .203

SUMMARY BY CASE 


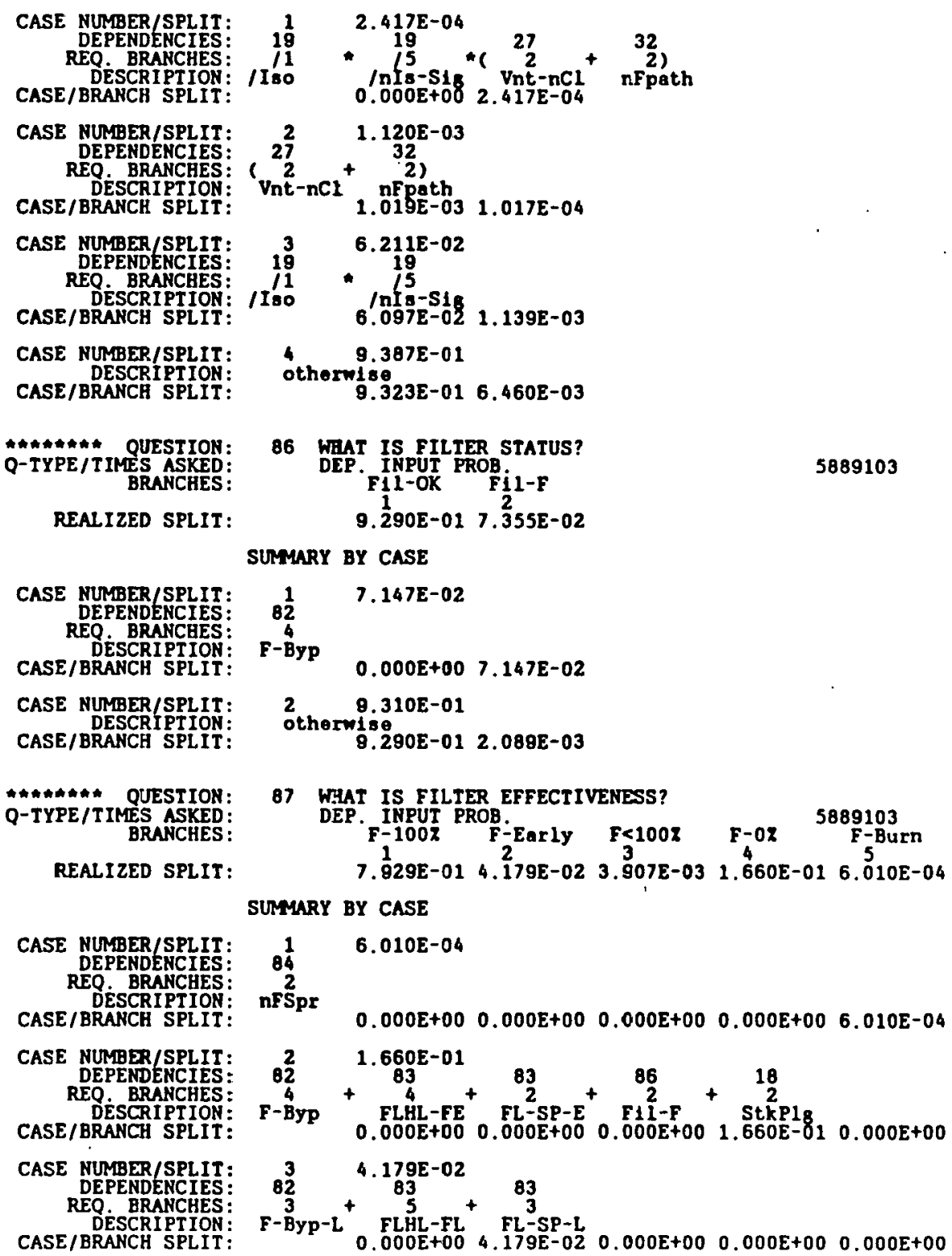


CASE NUMBER/SPLIT: $\quad 3.907 E-03$

REQ. BRANCHES: $\quad{ }^{2}$

$\begin{array}{llllll}\text { CASE/BRANCH SPLIT: F-PBYP } 0.000 E+00 & 0.000 E+00 & 3.907 E-03 & 0.000 E+00 & 0.000 E+00\end{array}$

CASE RUMBER/SPLIT: 5 7.929E-01

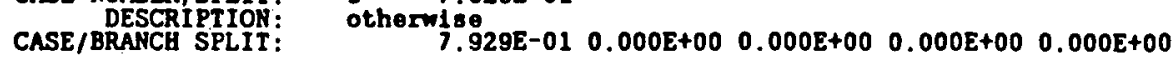


The following is a listing of the fire analysis frequency report as presented in the file NREAC_FIRE_LHS_FREQ.OUT.

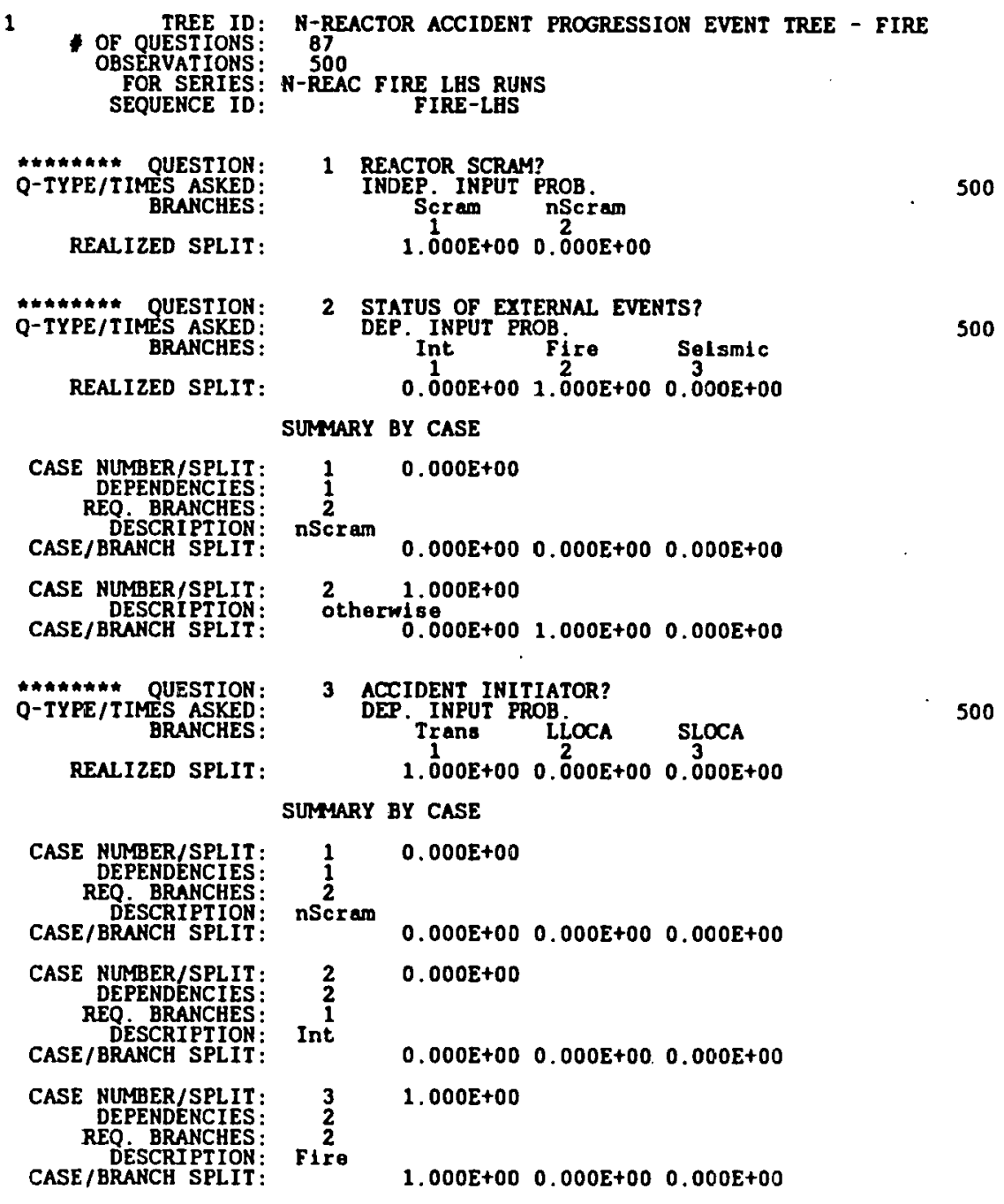


CASE NUMBER/SPLIT: 4 1.000E+00

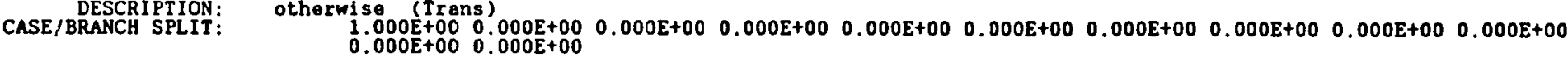

AUESTION:

Q-TYPE/TIMES ASKED:

6 STATUS OF ELECTRICAL POWER?

Ali-POW ${ }^{-1}-A$-Bus $n$-B-Bus $\quad n-A C$

1491

REALIZED SPLIT:

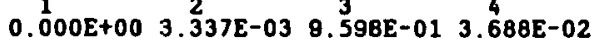

SUMMARY BY CASE

CASE NUMBER/SPLIT:

REO. BRANCHES:

DÉSCRIPTION:

CASE NUMBER/SPLIT:

REO BRANCHES:

CASE/BRANCH SPLIT:

CASE NUMBER/SPLIT:

DEPENDENCIES:

CASE / BRANCH SPLIT:

CASE NUMBER/SPLIT

DEPENDENCIES:

$10.000 \mathrm{E}+00$

3

$\begin{array}{ll}2 & 0.000 \mathrm{E}+00\end{array}$

Selsmic ${ }^{3} 3^{3}$

$3 \quad 0.000 E+00$

Solsmic B-is

$0.000 E+00 \quad 0.000 E+00 \quad 0.000 E+00 \quad 0.000 E+00$

O.000E+OD

Solsmic

$\begin{array}{lll}0.000 E+00 & 0.000 E+00 & 0.000 E+00 \quad 0.000 E+00\end{array}$

CASE/BRANCH SPLIT:

CASE NUMBER/SPLIT:

REO

CASE/BRANCH SPLIT:

$51.000 E+00$

$0.0008+003.3378-030.598 E-013.688 E-02$

CASE NUMBER/SPLIT:

DEPENDENCIT:

REQ. BRANCHES:

CASE/BRANCH SPLIT:

$0.000 E+00$

6
1
2 ${ }_{\mathrm{nScram}}^{0.000} \frac{3}{2}$

$0.000 E+00 \quad 0.000 \varepsilon+00 \quad 0.000 E+00 \quad 0.000 E+00$

CASE NUMBER/SPLIT:

DEO BRANCHES :

DESCRIPTION:
CASE/BRANCH SPLIT:

$0.000 E+00$

CASE/BRANCH SPLIT

ncram

CASE DEPENDENCIES

EQ. BRANCHES

CASE/BRANCH SPLIT:

$0.000 E+00 \quad 0.000 E+00 \quad 0.000 E+00 \quad 0.000 E+00$

CASE NUMBER/SPLIT:

DEPENDENCIES:

RED

CASE/BRANCH SPLIT:

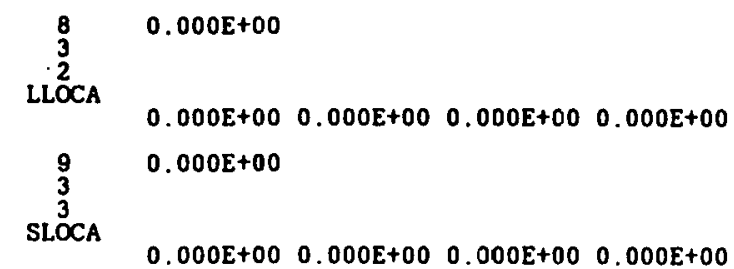




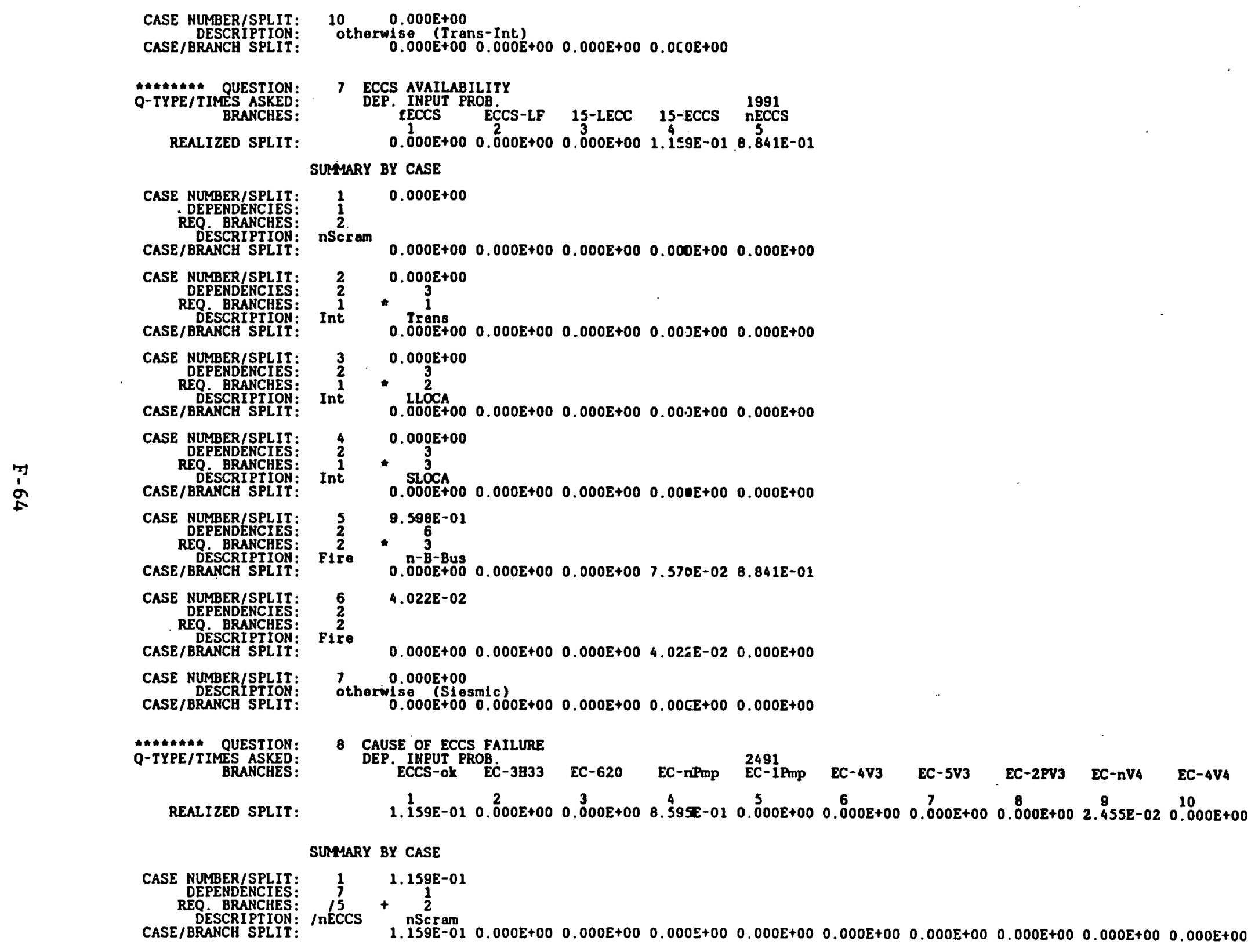


CASE NUMBER/SPLIT:

REO. BRANCHES: DESCRIPTION:
CASE/BRANCH SPLIT:

$20.000 E+00$

$\stackrel{2}{2} \quad * \stackrel{3}{1}$

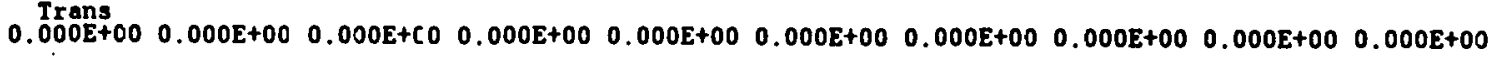

CASE NUMBER/SPLIT:

DEPENDENCIES:

REQ. BRANCHES :

CASE/BRANCH SPLIT:

$3 \quad 0.000 E+00$

Int $\frac{3}{2}$

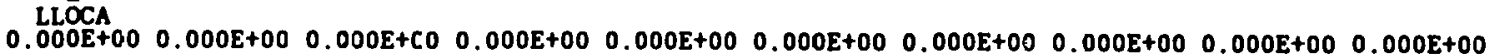

CASE NUMBER/SPLIT:

DEPENDENCIES:

DÉSCRIPTION:

$0.000 \mathrm{E}+0.0$

2

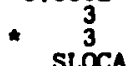

$\begin{array}{llllllllllllllllllll}0.000 E+00 & 0.000 E+00 & 0.000 E+00 & 0.000 E+00 & 0.000 E+00 & 0.000 E+00 & 0.000 E+00 & 0.000 E+00 & 0.000 E+00 & 0.000 E+00\end{array}$

CASE NUMBER/SPLIT

REO BRANCHES:

DESCRIPTION: 2

CASE/BRANCH SPLIT:

8.841E-01

$\begin{array}{llllllllll}0.000 E+00 & 0.000 E+00 & 0.000 E+00 & 8.595 E-01 & 0.000 E+00 & 0.000 E+00 & 0.000 E+00 & 0.000 E+00 & 2.455 E-02 & 0.000 E+00\end{array}$

CASE NUMBER/SPLIT:

CASE/BRANCH SPLIT:

$60.000 E+00$

$\begin{array}{lllllllllllllllll}0.000 E+00 & 0.000 E+00 & 0.000 E+00 & 0.000 E+00 & 0.000 E+00 & 0.000 E+00 & 0.000 E+00 & 0.000 E+00 & 0.000 E+00 & 0.000 E+00\end{array}$

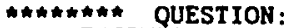

O-TYPE/TIMES ASKED:

REALIZED SPLIT:

9 HAS AN EARLY PRESSURE TUBE RUPTURE OCCURRED?

DEP. INPUT PEOB PT-RuP

9. 999E-01 9. 997E-05

SUMMARY BY CASE

CASE NUMBER/SPLIT:
DEPENDENCIES:
REO BRANCHES:

CASE/BRANCH SPLIT:

CASE NUMBER/SPLIT:

DEPENDENCIES:

DESCAIPTION:
CASE/BRANCH SPLIT:

CASE NUMBER/SPLIT:

CASE/BRANCH SPLIT:

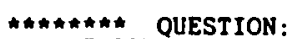

Q- TYPE/TIMES ASKED:

REALIZED SPLIT:

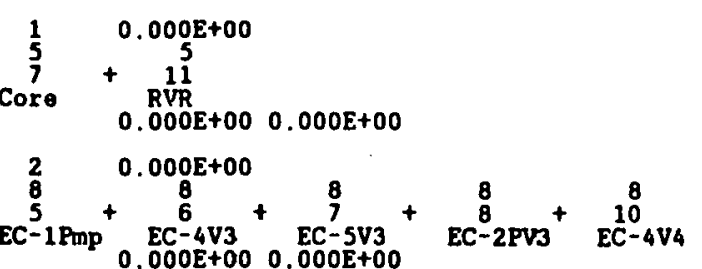

$3 \quad 1.000 \mathrm{E}+00$

9.999E-01 9.997E-0s

10 IS GSCS OPERATING?

DEP. INPUT PROB. GSSCS-I

$\begin{array}{ll}1 & 2 \\ 1.405 E-01 & 8.595 E-01\end{array}$

4596

SUMMARY BY CASE 


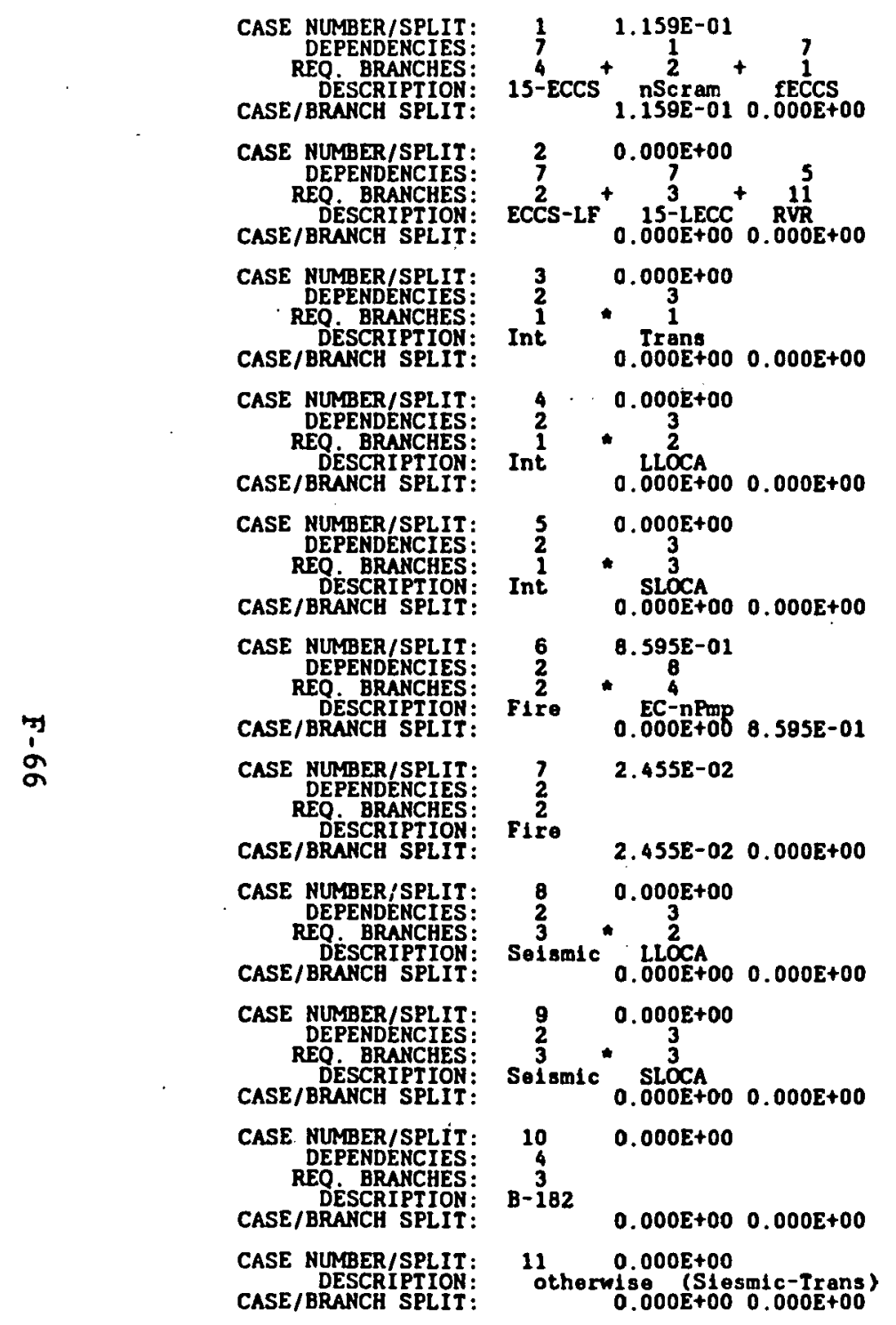




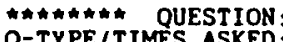

REALIZED SPLIT

CASE NUMBER/SPLIT

REO. BRANCHES

DESSCRIPTION:
SELARAB SPLIT:

CASE NUMBER/SPLIT

REO BRARCEES

CASE/BRAHCH SPLIT

CASE NUMBER/SPLIT:

CASE/BRANCH SPLIT

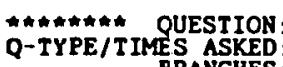

Q-TYRE/TIMES ARACHES

REALIZED SPLIT

CASE NUMBER/SPLIT

REQ. BRANCHES
Q-TYPE/TIMES ASKED

11 IS GSCS EFFECTIVELY COOLING THE CORE? DEP. IINPUT PROB. DGSCS nGSCS

4596

\section{SUMYARY BY CASE}

$1 \frac{1}{10} \quad 8.595 E-01$

$\operatorname{cscs}^{2}-\mathrm{e}$

$0.000 E+00 \quad 0.000 E+00 \quad 8.595 E-31$

$10 \quad 1.405 \mathrm{E}-01$

GSCS * 1

$\begin{array}{lll}1.405 E-01 & 0.000 E+00 \quad 0.000 E+00\end{array}$

$3 \quad 1.402 E-05$

$0.000 E+001.402 E-05 \quad 0.000 E+00$

12 IEPI FLON STATUS

4-HPI 2-HPI 1-HPI NOHPI 5312 $\begin{array}{llll}0.000 E+00 & 2.138 E-02 & 0.000 E+00 & 9.786 E-01\end{array}$

SUMMARY BY CASE

RE. BRANCHES:

CASE BRACCH SPLIT:

CASE NUMBER/SPLIT:

REO. BRANCHES

DESCRIPTION

CASE/BRANCH STLIT:

CASE NUMBER/SPLIT:

REO. BRANCHES

DESSCRIPTION:
RE/BRANCH SPLIT:

CASE NUMBER/SPLIT:

NUMBER/SPLIT
DEPENDENCIES
REQ. BRANCHES

REQ. BRANCHES

CASE/BRANCH SPLIT:

CASE NUMBER/SPLIT:

DESCRIPTION :
CASE/BRANCH SPLIT:

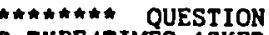

Q-TYPE/TIMES ASKED:

REALIZED SPLIT

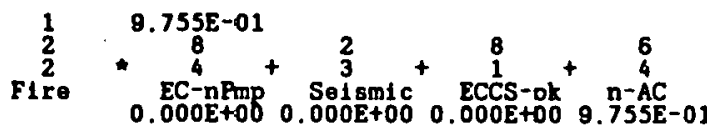

$\begin{array}{ll}2 & 0.000 \mathrm{E}+00\end{array}$

LLOCA $\frac{2}{17} \cdot \frac{5}{18}$

$0.000 E+00 \quad 0.000 E+00 \quad 0.000 E+00 \quad 0.000 E+00$

$3 \quad 0.0005+00$

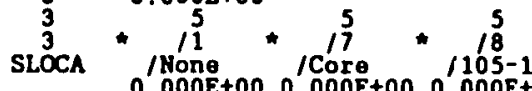

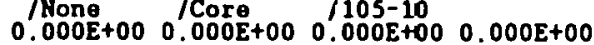

$5 \quad 0.000 E+00$

Core + .

$105-10$
$0.000 E+00$

5 2. 455E-02

$0.000 \mathrm{E}+00 \quad 2.138 \mathrm{E}-02 \quad 0.000 \mathrm{E}+00 \quad 3.167 \mathrm{E}-03$

13 PRIMARY COOLANT FLOW

DEP. INPUT PROB

$\begin{array}{ll}1 & \text { RMCP } \\ 2.455 E-02 & 9.755 E-01\end{array}$

5312

SUMMARY BY CASE 
CASE NUMBER/SPLIT

CASE/BRAKCH SPLIT:

$\frac{1}{6} \quad 9.755 \mathrm{E}-01$

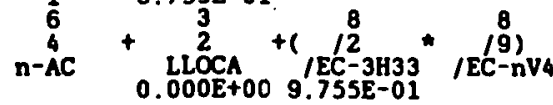

CASE NUMBER/SPLIT: 2 2.455E-02

DESCRIPTION:
CASE/BRAKCH SPLIT:

MA-m.\#\# QUESTION: 14 IHITIAL ECCS FLOW STATUS
Q-TYPE/TIMES ASKED:
BRAHCHES:

REALIZED SPLIT:

. SUMMarY BY CASE

CASE MUMBER/SPLIT:

REO BRANCHES:

CASE/BRANCE SPLIT:

$\frac{1}{1} \quad 1.159 \mathrm{E}-01$

CASE NUMBER/SPLIT:

REO BRANCHES:

DESCRIPTIOH:
CASE/BRANCH SPLIT:

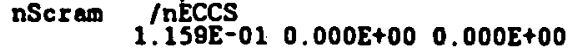

$13 \quad 8.595 E-01$

CASE NUMBER/SPLIT:

DEPENDENCIES:
REO. BRANCHES:

CASE/BRAKCEIPTION:

CASE NUMBER/SPLIT:

CASE NUMBER/SPLIT:
CASE/BRARCRTIION:

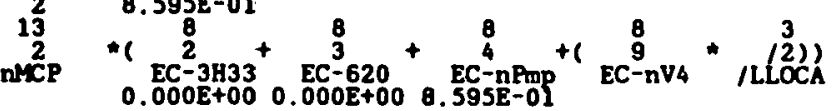

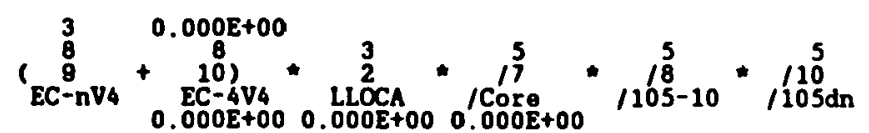

$4 \quad 2.455 E-02$

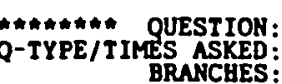

otherwisio

$000 \mathrm{E}+00 \quad 2.455 \mathrm{E}-02 \quad 0.000 \mathrm{E}+00$

REALIZED SPLIT:

15 HOW MCH IHITIAL FUEL DAMAGE IS THERE?

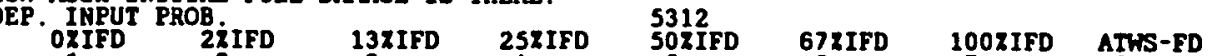

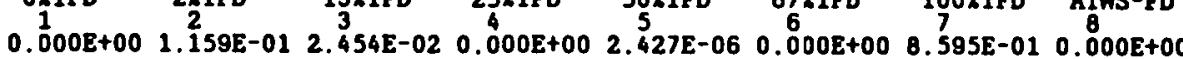

SUMMARY BY CASE

CASE NUMEER/SPLIT:

DEPENDENCIES:

DÉSCRIPTION:

CASE/BRANCB SPLIT:

CASE NUMBER/SPLIT:

REQ BRANCHES:

CASE/BRANCH SPLIT:

$10.000 E+00$

CASE NUMBER/SPLIT:

DEPENDENCIES:

REQ.ंBRANCHES:

$\begin{array}{llllllllllllll}0.000 E+00 & 0.000 E+00 & 0.000 E+00 & 0.000 E+00 & 0.000 E+00 & 0.000 E+00 & 0.000 E+00 & 0.000 E+00\end{array}$

$0.505 E-01$

$\stackrel{1 \frac{2}{3}}{\operatorname{mGSCS}}$

14

$\begin{array}{llllllllllllllll}0.000 E+00 & 0.000 E+00 & 0.000 E+00 & 0.000 E+00 & 0.000 E+00 & 0.000 E+00 & 8.595 E-01 & 0.000 E+00\end{array}$

$3 \quad 0.000 \mathrm{E}+00$

2013

CASE/BRANCH SPLIT :

$\begin{array}{llllllllllllllll}0.000 E+00 & 0.000 E+00 & 0.000 E+00 & 0.000 E+00 & 0.000 E+00 & 0.000 E+00 & 0.000 E+00 & 0.000 E+00\end{array}$ 


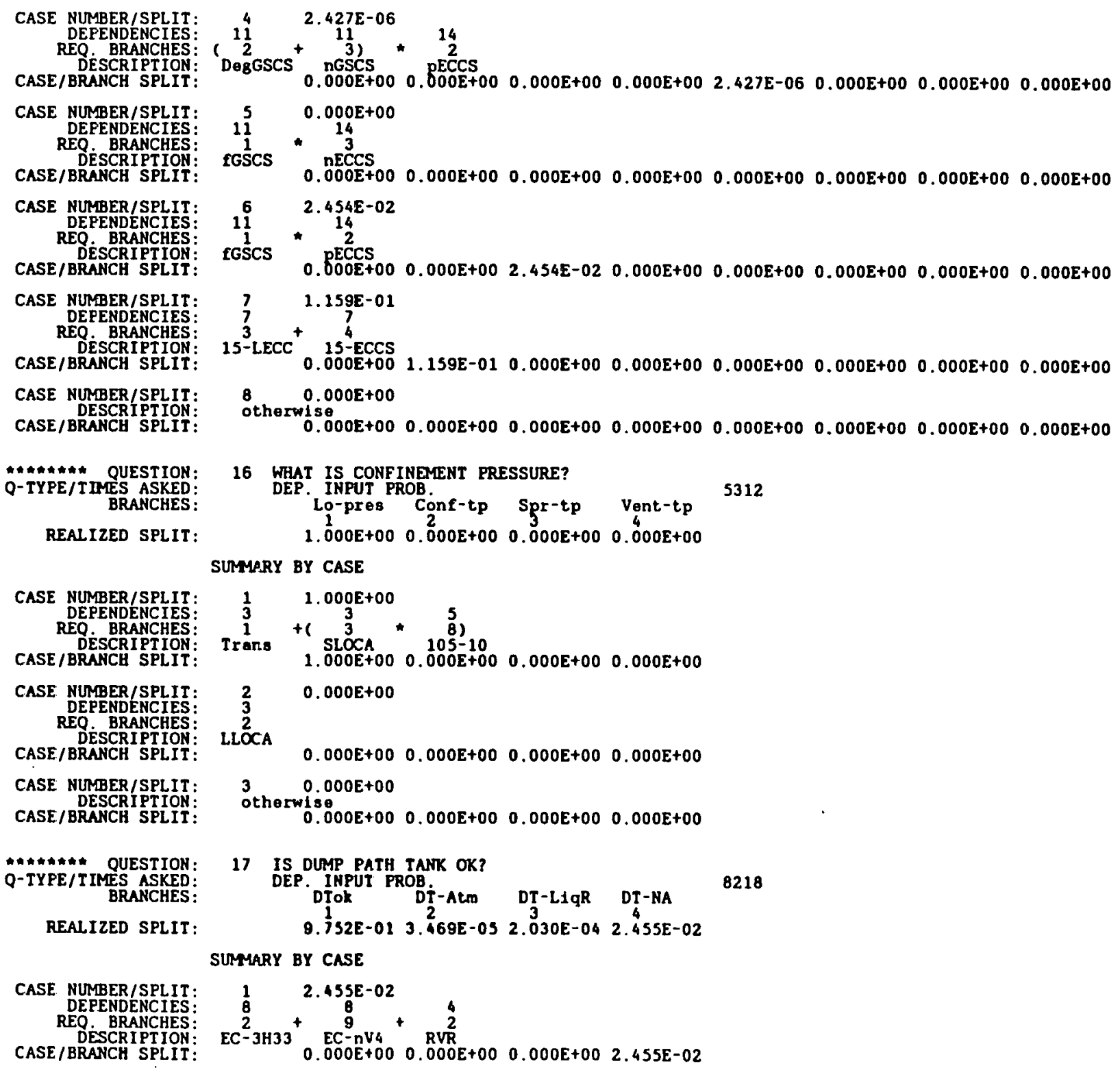


CASE NUMBER/SPLIT:

CASE/BRANCH SPLIT

CASE NUMBER/SPLIT

DEPENDENCIES:

DESCRIPTION:
CASE/BRANCH SPLIT:

CASE NUMBER/SPLIT

REO. BRANCHES:

DESE/BRANCR SPLIT:

CASE NUMBER/SPLIT

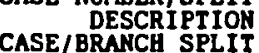

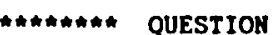

Q-TYPE/TIMES ASKED

BRANCHES:

REALIZED SPLIT:

SUMYARY BY CASE

CASE NUMBER/SPLIT:
DEPENDENCIES:
REQ BRANCHES:
DESCRIPTION:

CASE/BRANCH SPLIT

CASE NUMBER/SPLIT:

DEP. BRANCHES

CASE/BRANCE SPLIT:

CASE NUMBER/SPLIT

DEPENDENCIES

REQ. BRANCHES

CASE/BRANCH SPLIT

CASE NUMBER/SPLIT

CASE/BRAHCH SPLIT

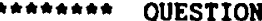

Q-TYPE/TIMES ASKED

REALIZED SPLIT:

\section{SUMYARY BY CASE}

CASE NUMBER/SPLIT:
DEPENDENCIES:
REQ.BRANCHES:
DESCRIPTION:
CASE/BRANCH SPLIT:

Fi

Seismic

$2 \quad 4$

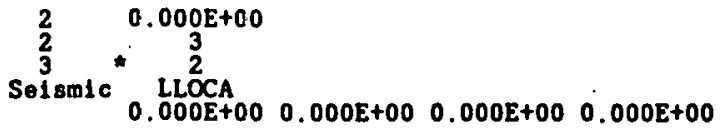

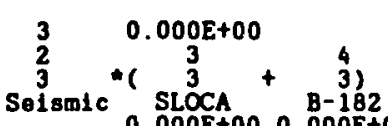

$\begin{array}{lllllll}0.000 E+00 & 0.000 E+00 & 0.000 E+00 & 0.000 E+00\end{array}$

$0.000 E+00$

$\begin{array}{llll}0.000 E+00 & 0.000 E+00 & 0.000 E+00 \quad 0.000 E+00\end{array}$

$5 \quad 9.755 E-01$

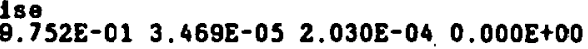

18 IS EILTER RELEASE STACA OK?

StkOK PROB StkP1B StkTpl

$\begin{array}{lll}1.000 E+00 & 0.000 E+00 & 0.000 E+00\end{array}$

8218

Seismic LLÓCA

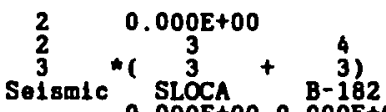

$3 \quad 0.000 E+00$

Seism1c $0.000 E+00 \quad 0.000 E+00 \quad 0.000 E+00$

$4.000 E+00$

$1.000 E+00 \quad 0.000 E+00 \quad 0.000 E+00$

19 DOES CONFIMEMENT ISOLATE?

DEP. INPUT PROB
Iso
nIsO-By nIs-V18 nI-180k ${ }_{\text {nIs }}^{21273}$-S18 nIso-F

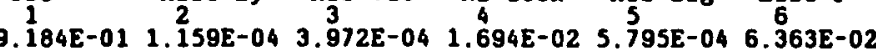

$1 \quad B .596 \mathrm{E}-01$

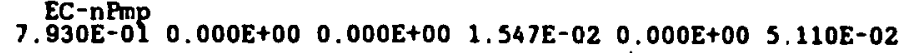


CASE NUMBER/SPLIT :
DEPENDENCIES : CASE/BRANCH SPLIT:

CASE NUMBER/SPLIT: DEPENDENCIES :
REQ. BRANCHES :

CASE/BRANCH SPLIT:

CASE NUMBER/SPLIT:

DEPENDENCIES:

DASE/BRANCHIPTION:

CASE NUMBER/SPLIT:

DEPENDENCIES

CASE / DRANCRIPTION:

CASE NUMBER/SPLIT.

CASE/BRANCH SPLIT

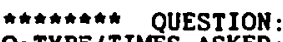

Q-TYPE/TIMES ASKED

BRANCHES:

REALIZED SPLIT:

$$
\text { IT: }
$$

DEPENDENCIES:

REQ BRANCHES:
DESCRIPION:
CASE/BRANCH SPLIT:

CASE NUMBER/SPLIT: DEPENDENCIES:

DESCRIPTION:

CASE/BRANCH SPLIT:

CASE NUMBER/SPLIT:

CASE/BRANCH SPLIT:

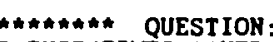
Q-TYPE/TIMES ASKED:

REALIZED SPLIT:

ASE NUMBER/SPLIT

CASE NUMBER/SPLIT:

REQ BRANCHES:

DESCRIPTION:
CASE/BRANCH SPLIT:

$\begin{array}{cc}2 & 2.455 \mathrm{E}-02 \\ 2 & 8 \\ 2 & \quad 9 \\ 9 & \end{array}$

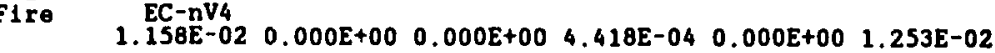

$\begin{array}{ll}3 & 0.000 \mathrm{E}+00 \\ 2 & \end{array}$

$+\quad \frac{4}{2}$

$\begin{array}{llllllllllllllllllll}\text { Selsmic } & \text { RVR } & 0.000 E+00 & 0.000 E+00 & 0.000 E+00 & 0.000 E+00 & 0.000 E+00 & 0.000 E+00\end{array}$

4
2

Solsmic * ${ }^{2}$

$\begin{array}{llllll}0.000 E+00 & 0.000 E+00 \quad 0.000 E+00 & 0.000 E+00 & 0.000 E+00 & 0.000 E+00\end{array}$

$5 \quad 0.000 \mathrm{E}+00$

2 - 2 - 1

$0.000 E+00 \quad 0.000 E+00 \quad 0.000 E+00 \quad 0.000 E+00 \quad 0.000 E+00 \quad 0.000 E+00$ 6 otherwise 159E-01

138E-01 1.159E-04 3.972E-04 1.029E-03 5.795E-04 7.462E-06

20 WHAT IS CONFIREMENT COMPROMISE LOCATION?

DEP. INPUT PROB.

ConfoK Envir Zone

9.104E-01 8.109E-02 5.795E-04

MARY BY CASE

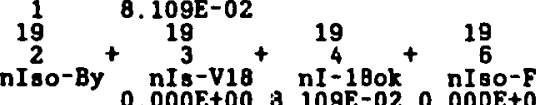

$$
\begin{aligned}
& 25.795 E-04
\end{aligned}
$$

21 DOES FORCED VENTILATION EXIST?

DEP. INPUT PROB

$\begin{array}{ll}1 & 2 \\ \text { 7. } 901 E-0 S & 2.999 E-01\end{array}$

SUMMARY BY CASE

$17.901 E-05$

$\begin{array}{cc}19 \\ 2 \\ n I s o-B y\end{array} / n^{14}$

$901 E-05 \quad 0.000 E+00$ 


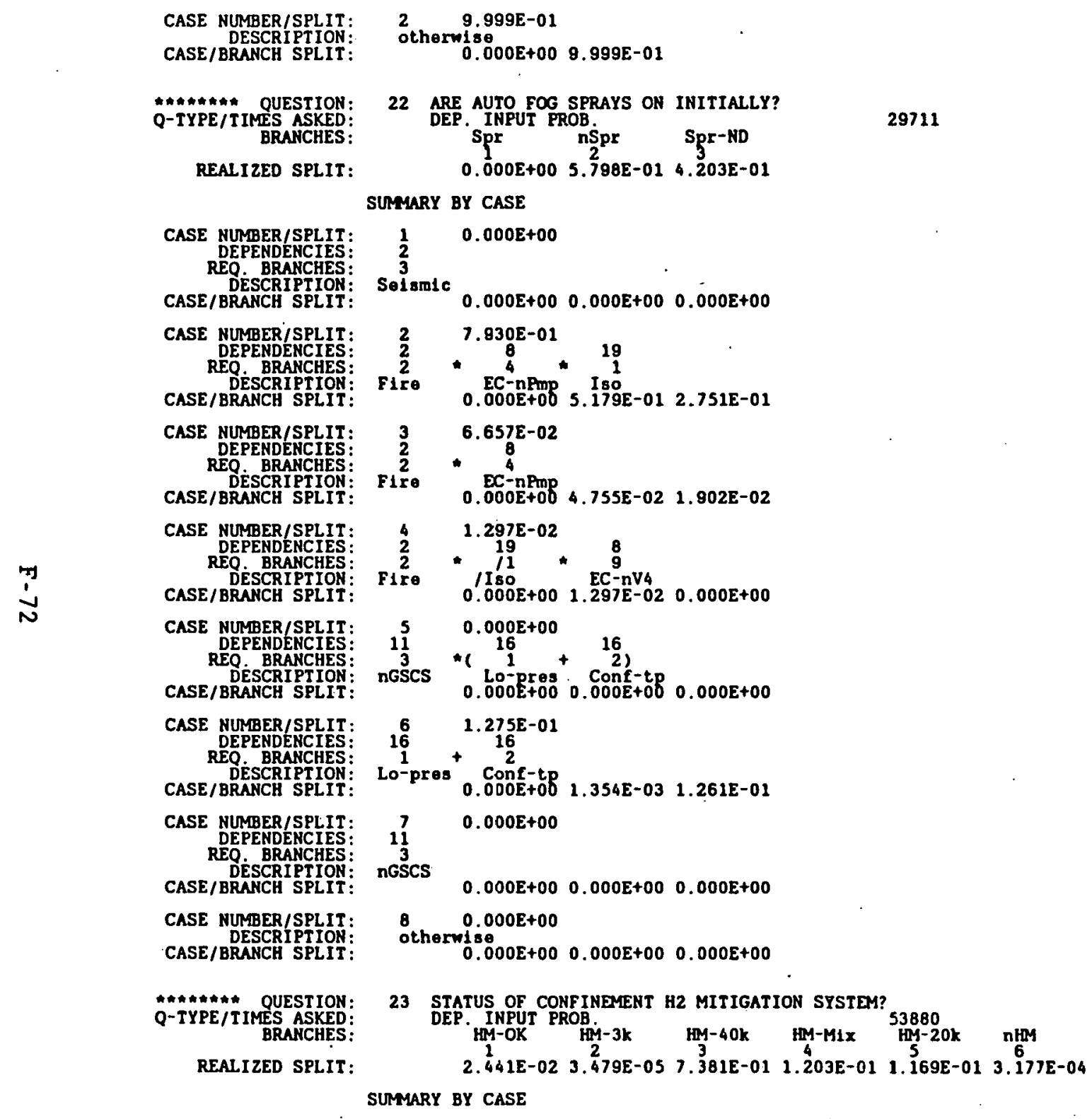




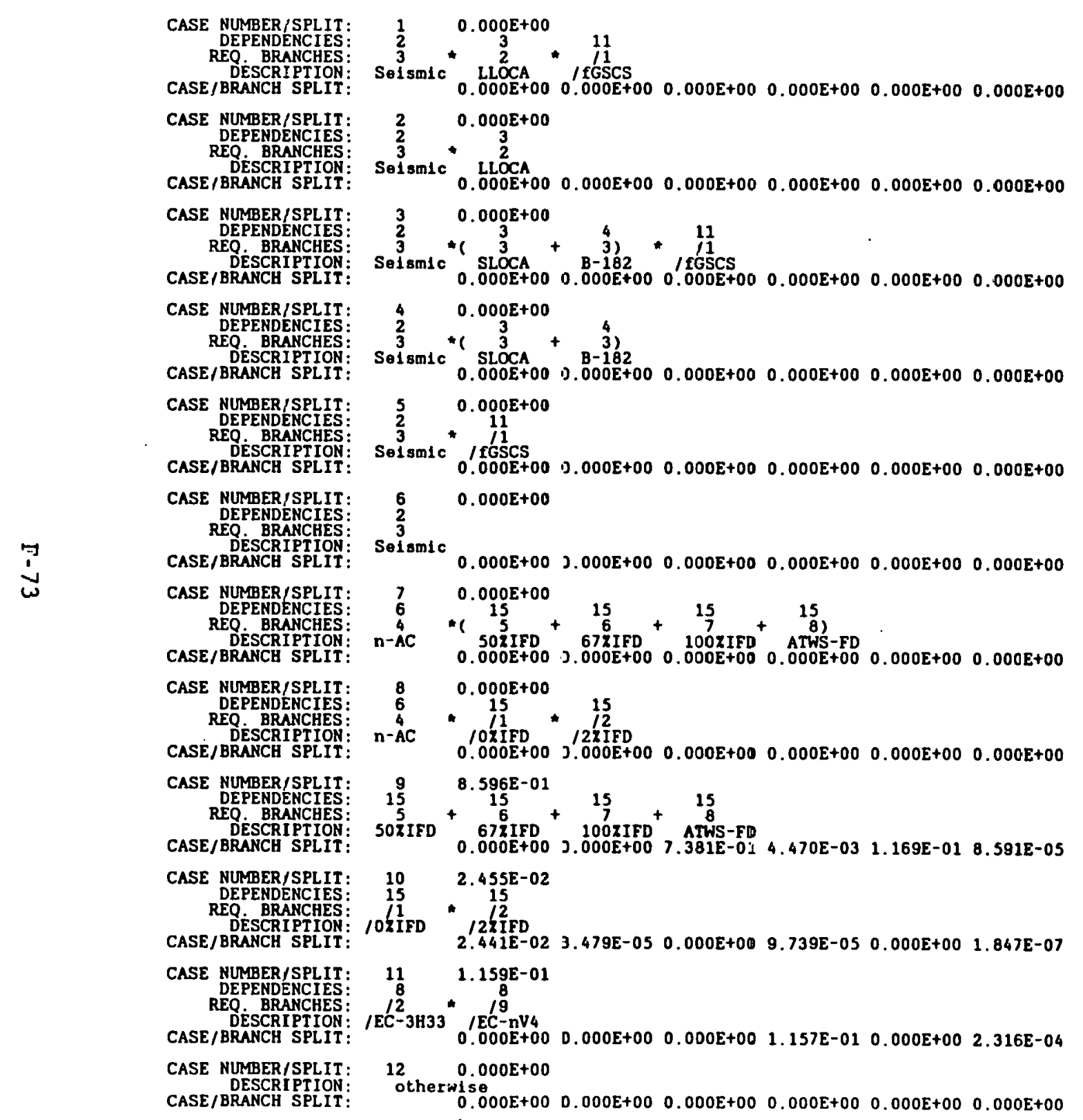




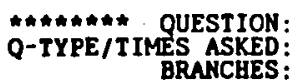

24 IS 32 H2 IN CONFINEMENT?

DEP. INPUT PROB

53880

REALIZED SPLIT:

$0.000 E+00 \cdot 1.000 E+00$

SUMMARY BY CASE

CASE NUMBER/SPLIT:

DEPEDECIES:

CASE/BRANCH SPLIT:

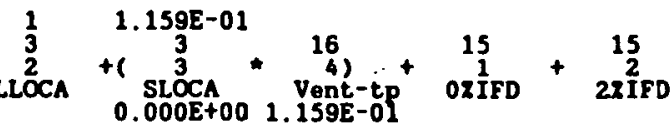

CASE NUMBER/SPLIT:

REO BRANCHES:

CASE/BRANCH SPLIT:

CASE NUMBER/SPLIT:

CASE/BRANCH SPLIT:

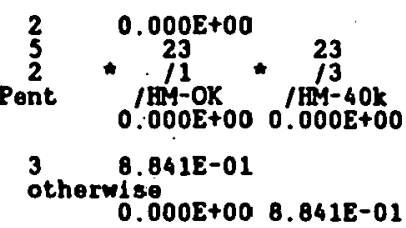

Qman*** QUESTION:

Q-TYPE/TIMES ASKED:

REALI2ED SPLIT :

25 DOES OPERATOR ISOLATE VENTS BASED ON 32, H2?

IN-ISO RROB Hi-nIso

$0.000 \mathrm{E}+00 \quad 1.000 \mathrm{E}+00$

SUMYARY BY CASE

CASE NUMBER/SPLIT:

REQ BRANCHES:

CASE/BRANCH SPLIT:

$\frac{1}{24}$

$0.000 E+00$

CASE NURBER/SPLIT:

CASE/BRAKCH SPLIT:

$0.000 E+00 \quad 0.000 E+00$

$21.000 E+00$

$0.000 E+001.000 E+00$

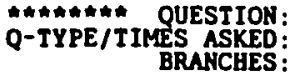

REALIZED SPLIT:

26 DID VENTS OPEN IF DEMANDED?

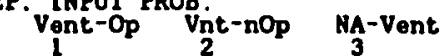

$\begin{array}{ccc}0.000 E+00 & 0.000 E+00 \quad 1.000 E+00\end{array}$

53880

SUMAARY BY CASE

CASE NUMBER/SPLIT:

REPENDENCIES: 16

DESCRIPTION:

$1.000 E+00$

CASE NUMBER/SPLIT:

NUMBER/SPLIT
DEPENDENCIES
REO BRANCHES

DESCRIPTION:

CASE/BRANCH SPLIT:

/Vent-tP $0.000 E+00 \quad 0.000 E+001.000 E+00$

$250.000 E+00$

CASE NUMBER/SPLIT:

CASE/BRANCH SPLIT:

$0.000 E+00 \quad 0.000 E+00 \quad 0.000 E+00$

$30.000 E+00$

$0.000 E+00 \quad 0.000 E+00 \quad 0.000 E+00$ 


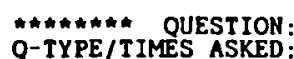

Q-TYPE / TIMES ASKED

REALIZEO SPLIT

MUMBR/SPLIT:

DEPENDENCIES

REQ. BRANCHES

CASE/BRANCH SPLIT:

CASE NURBER/SPLIT

DEPENDENCIES:

DESCRIPTION:
CASE/BRANCH SPLIT:

CASE NUMBER/SPLIT

DEPENDENCIES:

REQ. BRANCHES

CASE/BRANCH SPLIT

CASE NUMBER/SPLII

CASE/BRANCH SPLIT

Onminim Q QUESTION

Q-TYPE/TIMES ASKED

REALIZED SPLIT:

CASE NUMBER/SPLIT

REO BRANCHES

DESCRIPTION:
CASE/BRANCH SPLIT:

CASE NUMBER/SPLIT

REO BRANCHES :

CASE/BRANCH SPLIT

CASE NLMBER/SPLIT:

CASE/BRANCH SPLIT

AnnmAn** QUESTION:

Q-TYPE / IIMES ASKED

REALIZED SPLIT:
27 DID VENT ISOLATIONS CLOSE AFTER BLOWDOWN?

DEP. INPUT PROB Vent-C1 1 -nCl Vent-NA

53880

$\begin{array}{ccc}1 & 2 & 3 \\ 0.000 E+00 & 0.000 E+00 & 1.000 E+00\end{array}$

SUMMARY BY CASE

NA-Vent

$0.000 E+00 \quad 0.000 E+00 \quad 1.000 E+00$

2
19
2

nIso-By $0.000 E+00 \quad 0.000 E+00 \quad 0.000 E+00$

$0.000 E+00$

$19+19$

$\begin{array}{lll}0.000 \mathrm{E}+00 & 0.000 \mathrm{E}+00 & 0.000 \mathrm{E}+00\end{array}$

$4 \quad 0.000 \mathrm{E}+00$

$0.000 E+00 \quad 0.000 E+00 \quad 0.000 E+00$

28 DOES CONFINEMENT FAIL DURING BLOWDOWN PHASE?

DEP. INPUT PROB CF-InIt CF-BD

9.184E-01 8. $267 E-02 \quad 0.000 E+00$

SUMMARY BY CASE

$19 \quad 9.184 E^{2}-01$

\begin{tabular}{l}
27 \\
$-12+\frac{26}{12}+3^{3}$ \\
\hline
\end{tabular}

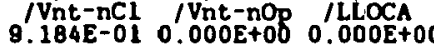

$27 \quad 0.000 \mathrm{E}+00$

$2+26$

$\begin{aligned} & \text { Vnt-nOp } 000 E+00 \\ & 0.000 E+00\end{aligned} 0.000 E+00$

3 8. 167E-02

$0.000 E+008.167 E-02 \quad 0.000 E+00$

29 WHAT IS SILE OF CONFINEAENT FAILURE?

DEP. INPUT PROB - - Loak C-Rupt

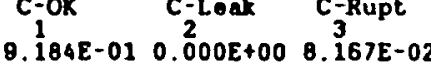

53880

SUMMARY BY CASE 


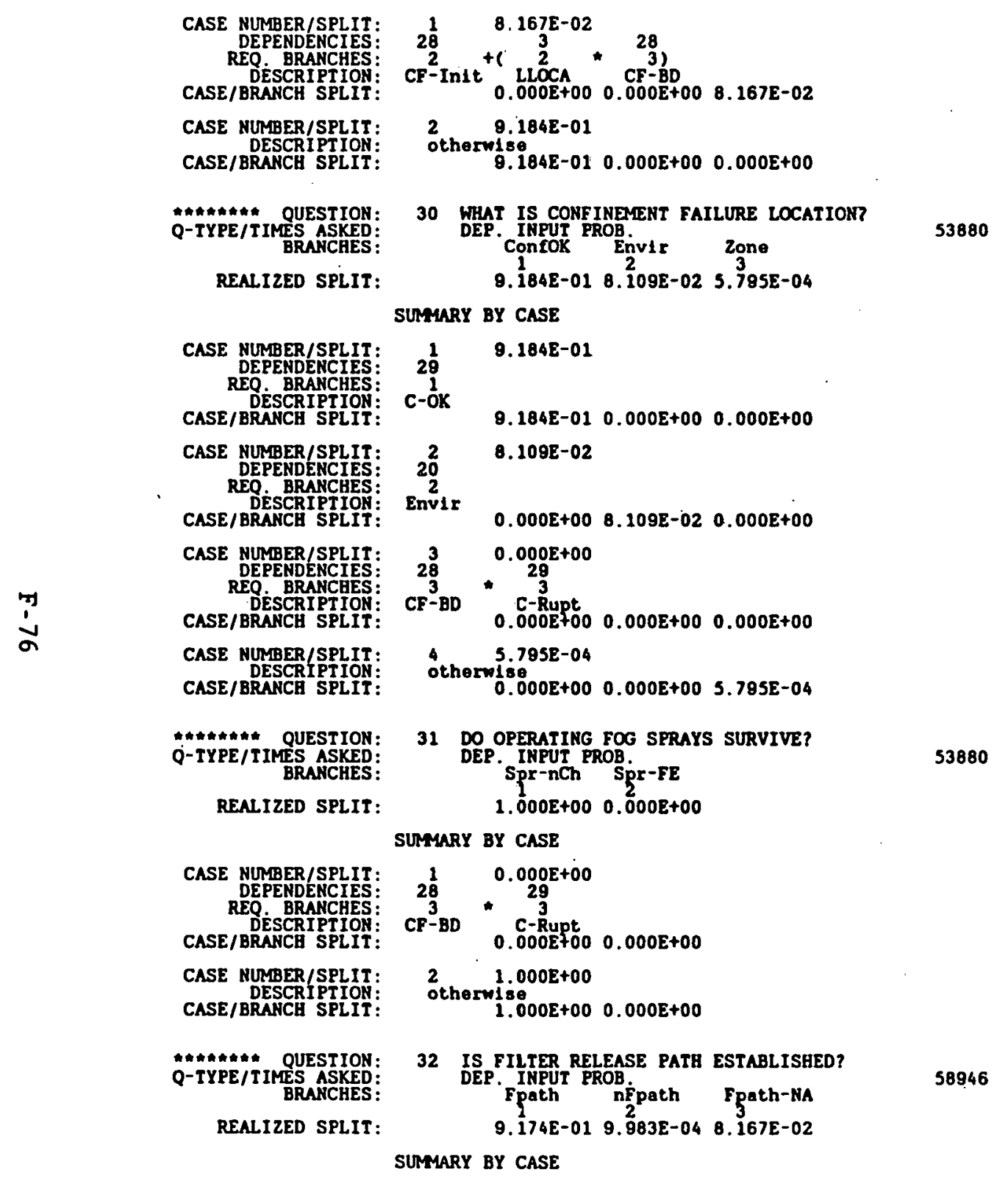




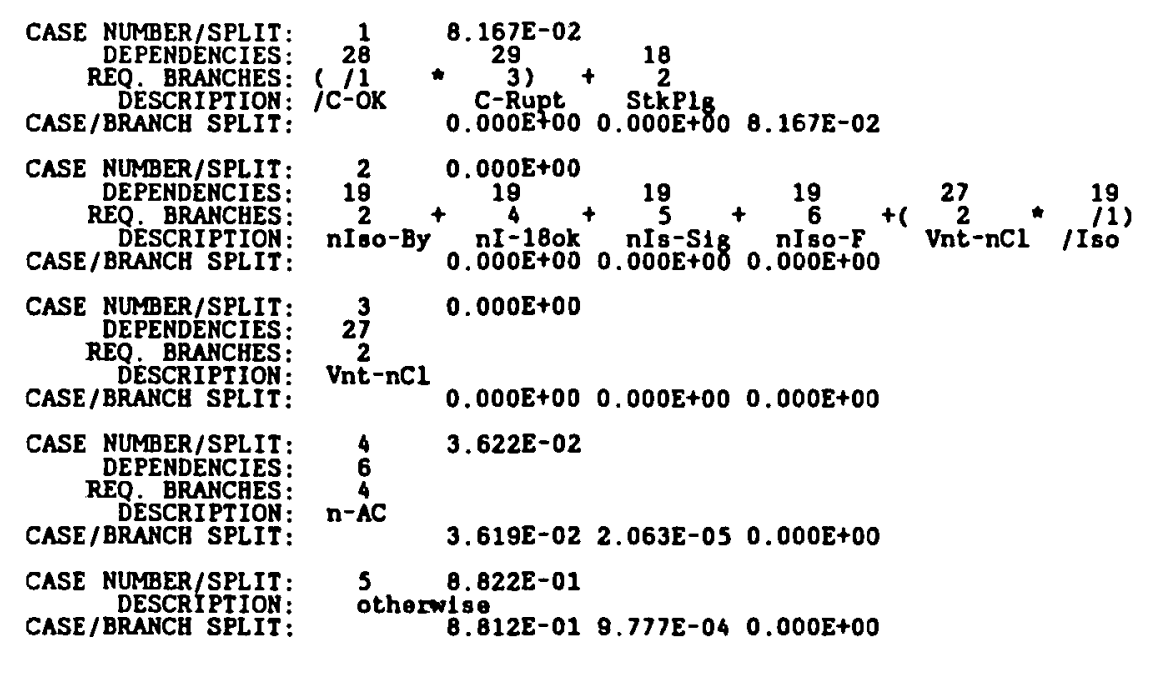

\#A*A**** QUESTION:

Q-TYPE/TI

REALIZED SPLIT

33 WHAT IS EARLY H2 FLAMMABILITY?

H-MIt PROB-Pent H-SG H-Bar 58946

$\begin{array}{lllll}1 & 1 \\ 1.000 E+00 & 0.000 E+00 & 0.000 E+00 & 0.000 E+00 & 0.000 E+00\end{array}$

SUMMARY BY CASE
CASE NUMBER/SPLIT:
REO
REO BRANCHES:
$1.1598-01$
$\frac{23}{14}+\frac{23}{165}+\frac{21}{1}+\frac{15}{1}+\frac{15}{2}$
$\begin{array}{lllllllllllllll}\text { CASE/BRANCH SPLIT: } & 1.159 \mathrm{E}-01 & 0.000 \mathrm{E}+00 & 0.000 \mathrm{E}+00 & 0.000 \mathrm{E}+00 & 0.000 \mathrm{E}+00\end{array}$
CASE NUMBER/SPLIT: $220.000 E+00$
DEO
REQ. BRANCHES:
CASE/BRANCH SPLIT:

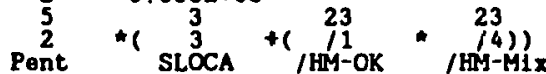
CASE NUMBER/SPLIT:
DEO BRANCES
DESCRIPTION:
$0.000 E+00 \quad 0.000 E+00 \quad 0.000 E+000.000 E+000.000 E+00$

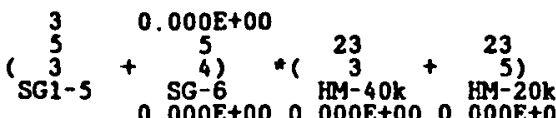
$0.000 E+00 \quad 0.000 E+00$
CASE NUMBER/SPLIT
REO BRANCES
DÉSCRIPION:

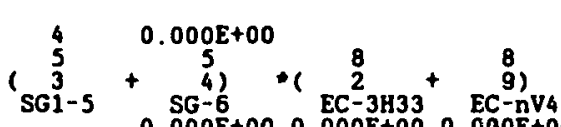
$0.000 \mathrm{E}+00 \quad 0.000 \mathrm{E}+00 \quad 0.000 \mathrm{E}+00 \quad 0.000 \mathrm{E}+00 \quad 0.000 \mathrm{E}+00$
CASE NUMBER/SPLIT:
REO. BRANCHE:
CASE/BRANCH SPLIT:

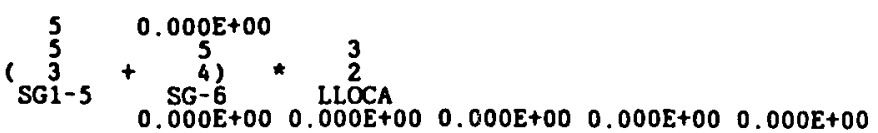




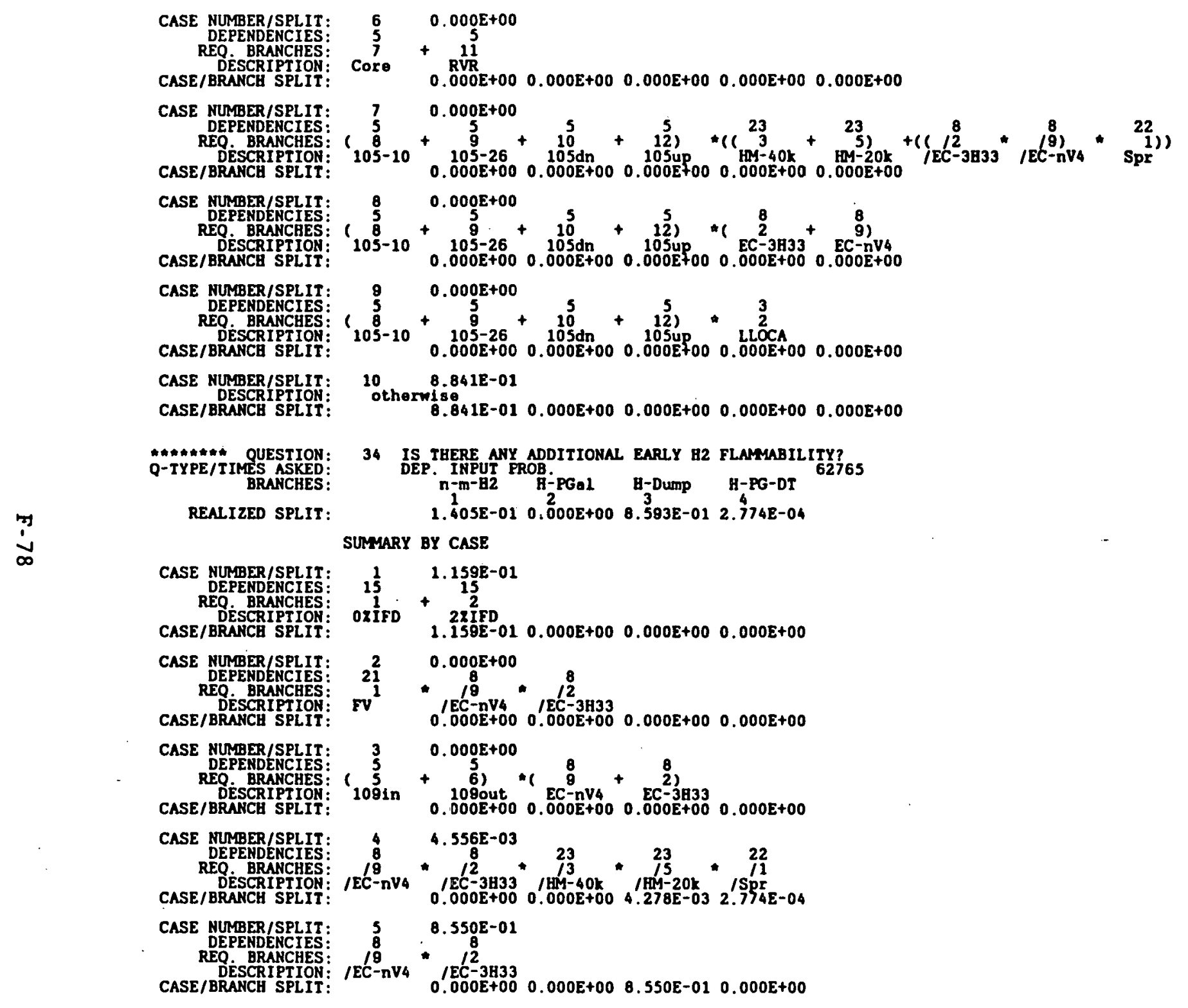


CASE NUMBER/SPLIT: 6 2.455E-02

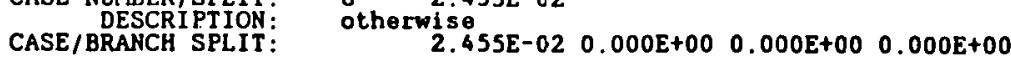

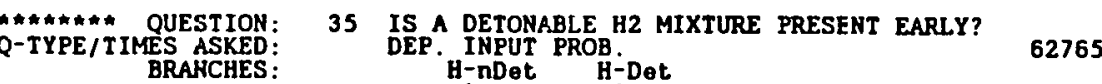

REALIZED SPLIT: $\quad 1.000 E+00 \quad 0.000 E+00$

SUMMARY BY CASE

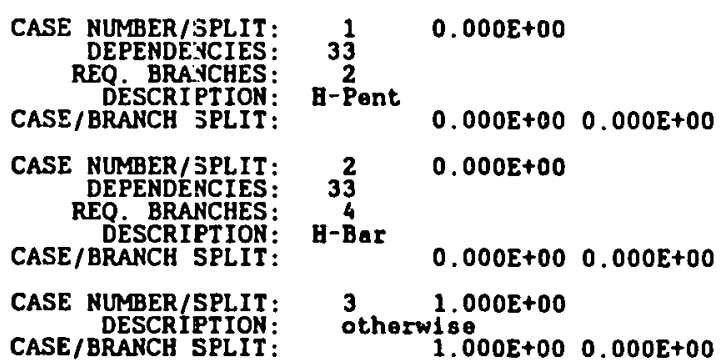

OAMMMA QUESTION: 36 DOES AN EARLY H2 IGNITION OCCUR?

Q-TYPE / T IMES ASKED:

REALIZED SPLIT:

DEP. INPUT PROB H- I

$1.000 E+00 \quad 0.000 E+00$

SUMMARY BY CASE

CASE NUMBER/SPLIT:
DEPENDENCIES:
REO BRANCHES :

NUMBER/SPLIT: $\quad 3 \frac{1}{\text { DEPENDENCIES: }} \quad 1.000 \mathrm{E}+00$

DESCRIPTION: H-MIt

1. $000 \mathrm{E}+00 \quad 0.000 \mathrm{E}+00$

CASE NUMBER/SPLIT: $\quad 2 \quad 0.000 E+00$

BRACHES: $(2+3)+14$

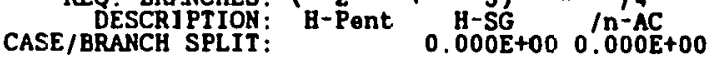

CASE NUMBER,SPLIT: $\quad 33 \quad 0.000 E+00$

REO. BRANCHES: 23 * 235 * 4

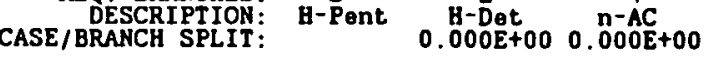

CASE NUMBER;SPLIT: $\quad 3_{\text {DEPENDENCIES: }}^{4} \quad 0.000 E+00$

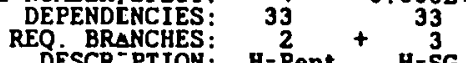

CASE/BRANCH SPLIT: $\quad$-Pent $\begin{gathered}\text { H-SG } \\ 0.000 E+00\end{gathered}$

CASE NUMBER,'SPLIT: $550.000 E+00$

REO NDNCHES:

DESCRIPTION: H-Bar $0.000 E+00 \quad 0.000 E+00$
CASE/BRANCH SPLIT: 


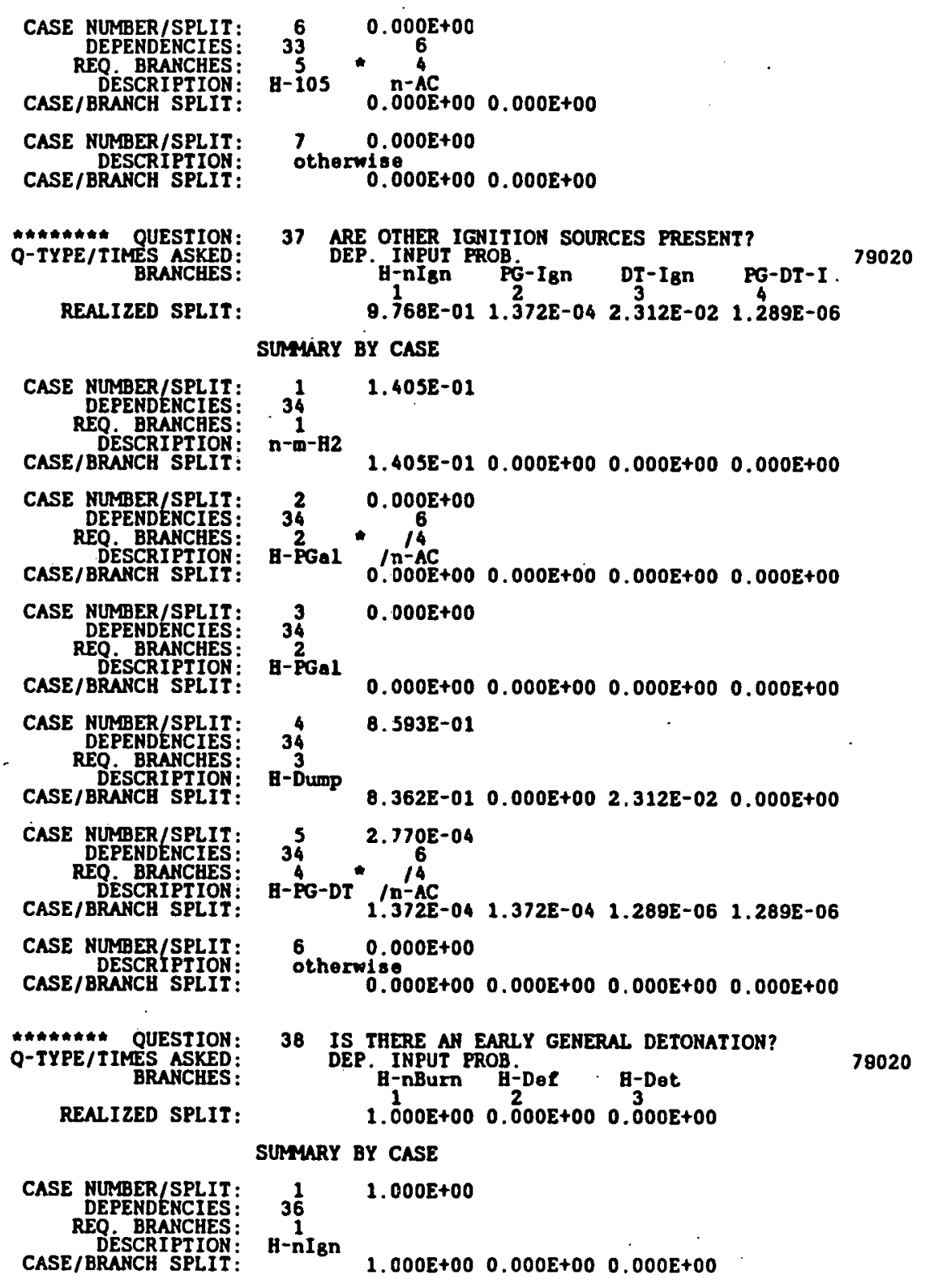




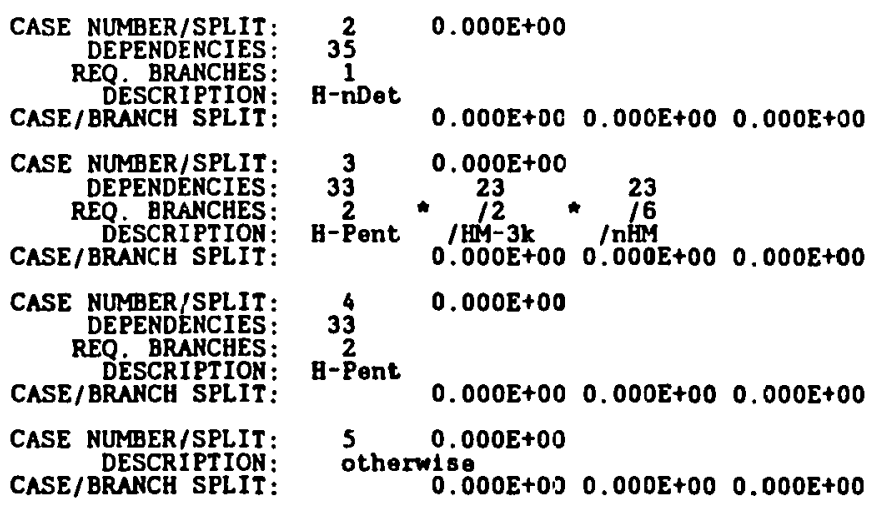

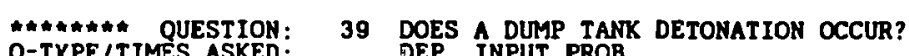
Q-TYPE/TIMES ASKED:
BRANCHES:

REALIZED SPLIT: $\quad 9.769 E-01 \quad 1.745 E-02 \quad 5.670 E-03$

SUMMARY BY CASE

CASE NUMBER/SPLIT:

DEPENDENCIES:

DESCRIPTION:
CASE/BRANCH SPLIT:

CASE NUMEER/SPLIT:

DESCRIPTION:
CASE/BRANCE SPLIT:

Qmiman*t QUESTION:

Q-TYPE/TIMES ASKED:

REALIZED SPLIT :

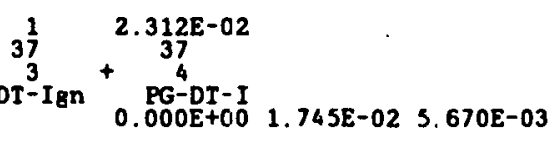

$29.769 \mathrm{E}-01$

9.769E-01 $0.000 E+00 \quad 0.000 E+00$

40 IS He BLANRET INTACT?
DEP. INPUT PROB.
He-OK
He-Lost
9.999E-01 $9.924 E-05$

87892

SUMYARY BY CASE

CASE NUMBER/SPLIT:

REPENDENCIES:

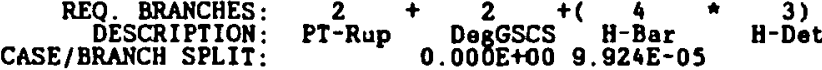

$1 \quad 0.924 \mathrm{E}-\mathrm{DS}$

CASE NUMBER/SPLIT: 2 9.999E-01

CASE/BRANCH SPLIT: $\quad 9.999 E-010.000 E+00$

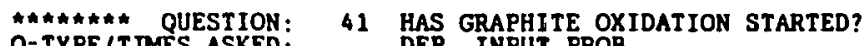

Q-TYPE/TIMES ASKED: $\quad$ DEP. INPUT PROB.- GrOX Gr-F1re

REALIZED SPLIT: $\quad 1.000 E+007.021 E-05 \quad 6.780 E-06$

SUMMARY BY CASE 


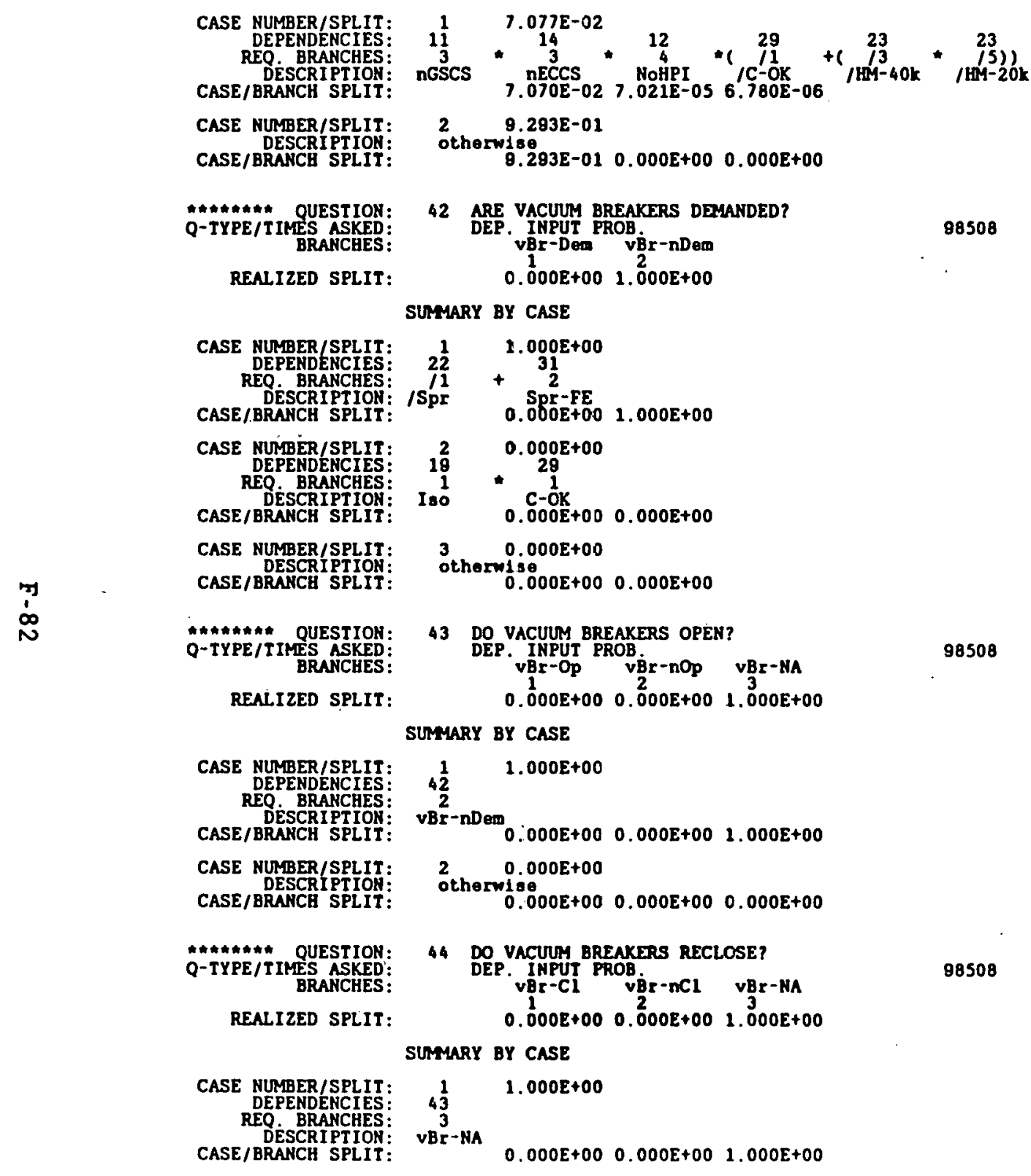


CASE NLMBER/SPLIT: 2 $20.000 E+00$

$\begin{aligned} \text { DESCRIPTION: } & \text { otherw1se } \\ \text { CASE/BRANCH SPLIT: } & 0.000 E+00 \quad 0.000 E+00 \quad 0.000 E+00\end{aligned}$

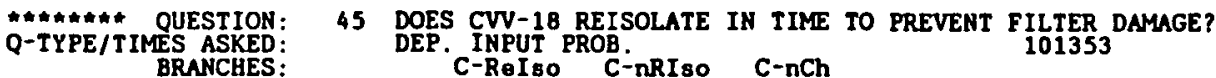

REALIZED SPLIT:

DEP. INPUT PROB.
C-REI BO C-nRISO C-nCh
9.598E-0S 4.883E-05 9.999E-01

SUMYARY BY CASE

CASE NUMBER/SPLIT:

REO BRANCHES:

DESCRIPTION: H-Det

CASE/BRANCH SPLIT:

CASE NUMBER/SPLIT:

REQ. BRANCHES :

CASE/BRANCH SPLIT:

CASE NUMBER/SPLIT:

REQ. BRANCHES:

CASE/BRNCH SPLIT:

CASE NUMBER/SPLIT

DESCRIPTION:
CASE/BRAKCH SPLIT:

$0.000 E+00$

$\begin{array}{lll}0.000 E+00 & 0.000 E+00 \quad 0.000 E+00\end{array}$

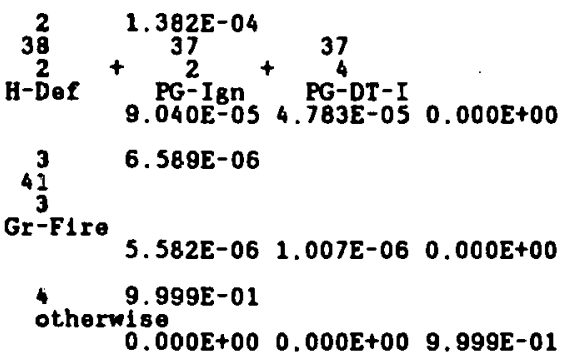

REALIZED SPLIT:

46 DOES CONFINEMENT FAIL DURING THE ACCIDENT PROGRESSION? DEP. INPUT PROB.

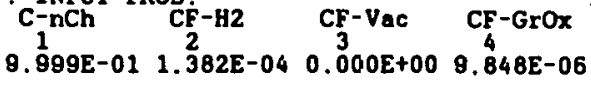

\section{SUMMARY BY CASE}

CASE NUMBER/SPLIT:

ES:

$\begin{array}{lllll}\text { DESCRIPTION: Gr-FIro } & 0.000 E+00 & 0.000 E+00 & 0.000 E+00 & 6.589 E-06\end{array}$

CASE NUMBER/SPLIT: $20.000 E+00$

REO BRANCHES:

DESCRIPTION: ${ }^{3}$

CASE/BRARCH SPLIT:

CASE NUMBER/SPLIT

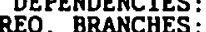

CASE/BRANCH SPLIT:

$0.000 E+00 \quad 0.000 E+00 \quad 0.000 E+00 \quad 0.000 E+00$

CASE NUMBER/SPLIT

REO BRANCHES:

CASE/BRANCH SPLIT:

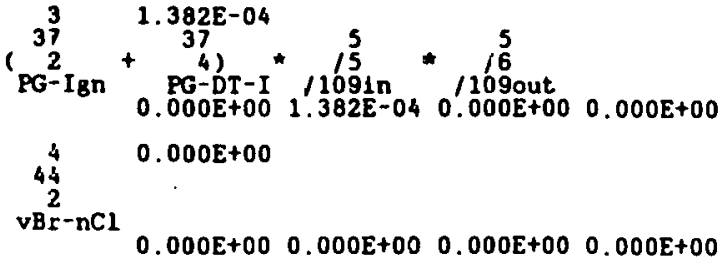




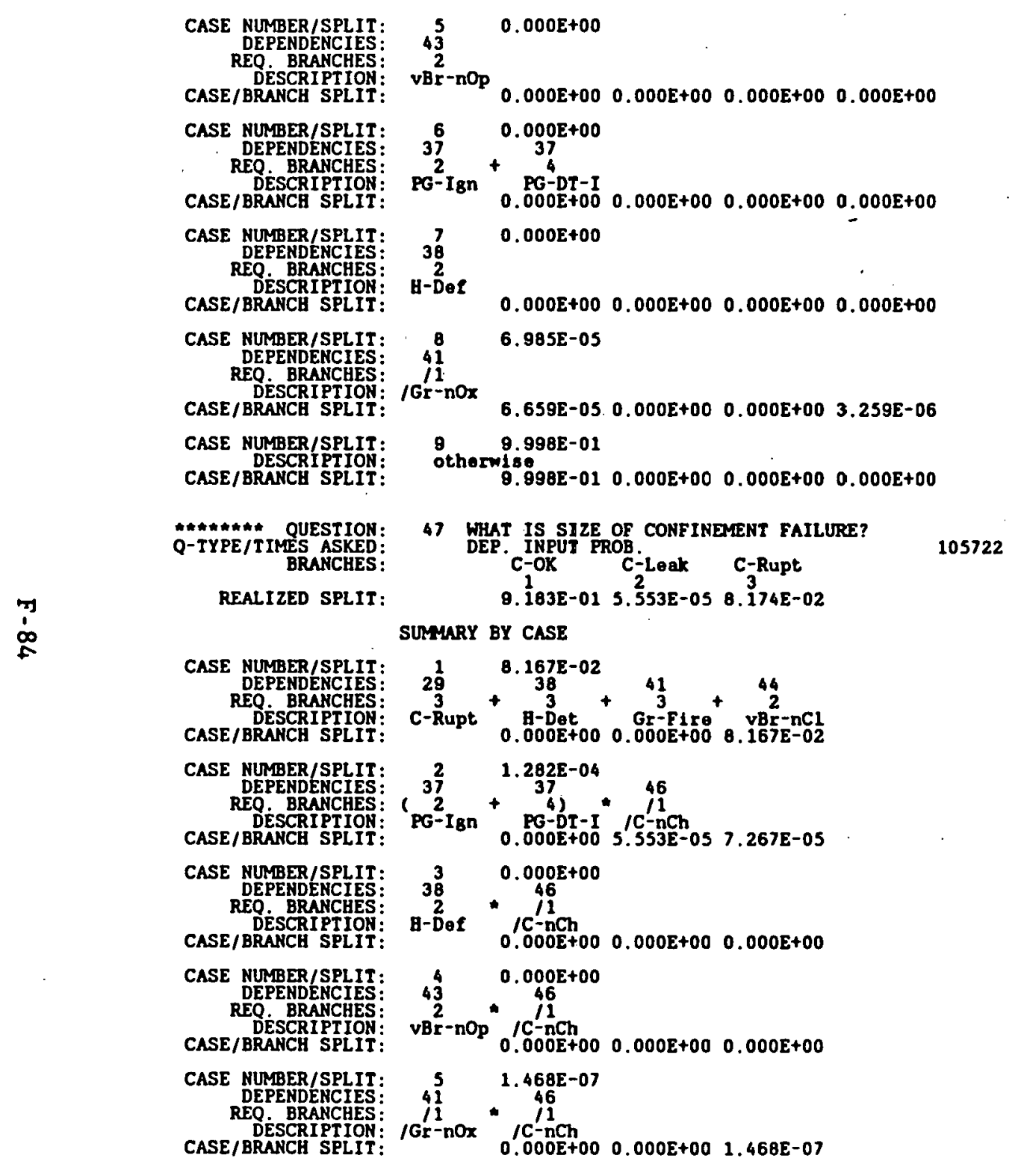




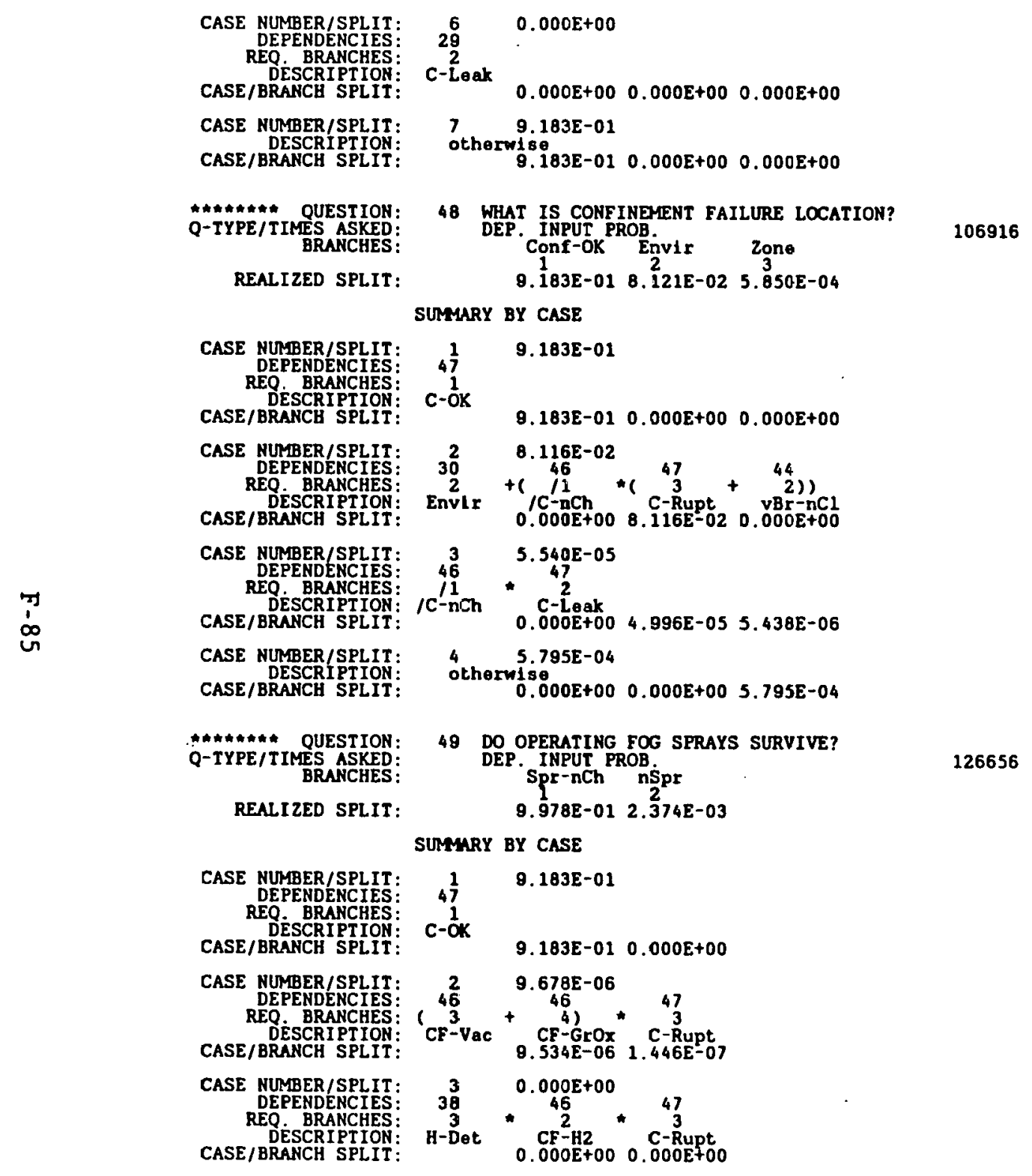




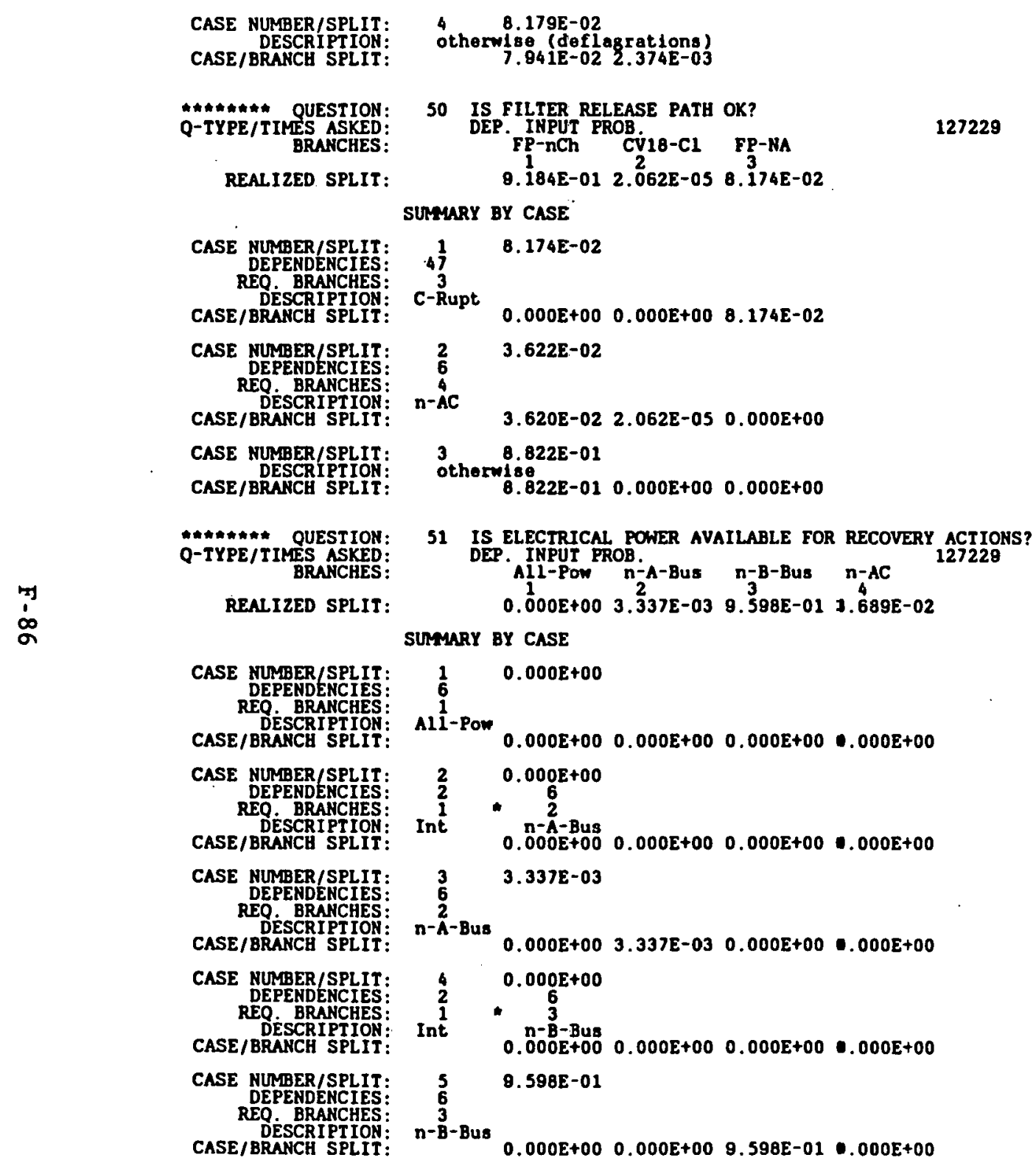




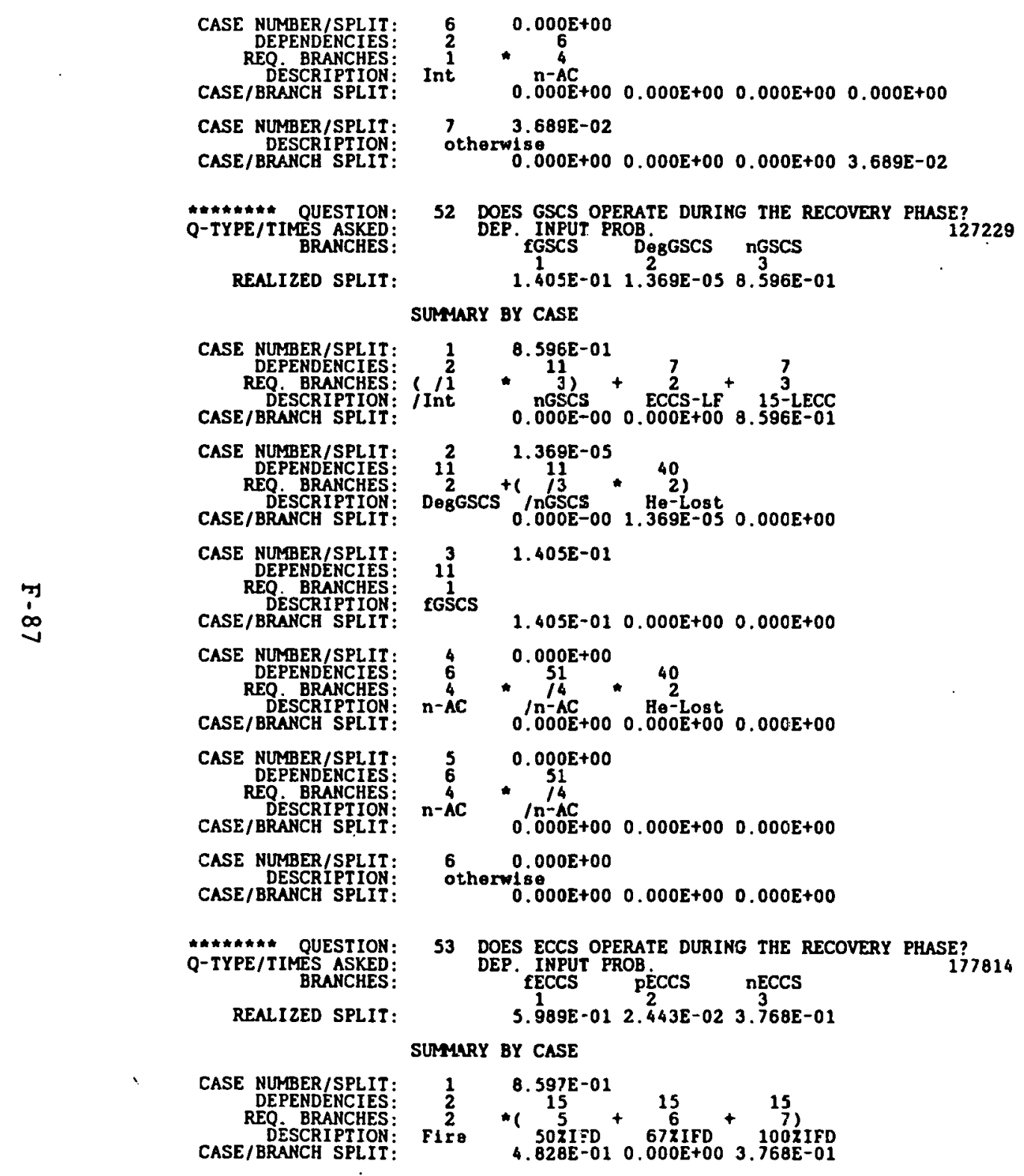


CASE NUMBER/SPLIT:

REO. BRANCHES :

DESCRIPTION:
CASE/BRANCH SPLIT:

CASE NUMBER/SPLIT

DEPENDENCIES

DESCRIPTION:
RE

CASE NUMBER/SPLIT

DEPENDENCIES

CASE/BRANCH SPLIT:

CASE NUMBER/SPLIT

DEPENDENCIES

CASE/BEANCH SPLIT:

CASE NUMBER/SPLIT.

DEPENDENCIES

DASE/BRANCR STION:

CASE NUMBER/SPLIT:

DEPENDENCIES

DESCRIPTION:
REATE

CASE NUMBER/SPLIT:

DEPENDENCIES

REQ. BRANCHES

CASE/BRANCH SPLIT:

CASE NUMBER/SPLIT:

DEPENDENCIES:

REQ: BRANCHES

CASE/BRANCH SPLIT:

CASE NUMBER/SPLIT

CASE/BRANCH SPLIT:

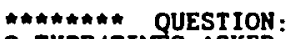

Q-TYPE/TIMES ASKED

BRANCHES:

REALIZED SPLIT:

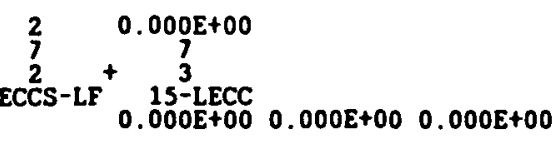

$30.000 E+00$

EECCS $*\left(\frac{10}{105 \mathrm{dn}}+\frac{2}{3}\right)$

1. 159E-01

IECCS

1.159E-01 $0.000 E+00 \quad 0.000 E+00$

$50.0008+00$

$\mathrm{EC}-3 \mathrm{~B} 33^{2} / \mathrm{L} \operatorname{loC}^{3}$

$0.000 \mathrm{E}+00 \quad 0.000 \mathrm{E}+00 \quad 0.000 \mathrm{E}+00$

$6 \quad 0.000 E+00$

$\mathrm{EC}-3 \mathrm{H} 33^{2} / \mathrm{IGSCS}$

$0.000 E+00 \quad 0.000 E+00$

$0.000 E+00$

EC-3833

$\begin{array}{lll}0.000 E+00 & 0.000 E+00 \quad 0.000 E+00\end{array}$

$80.000 \mathrm{E}+00$

$\begin{gathered}8 \\ 6\end{gathered}+7+8$

$\begin{array}{llll}\text { l fGSCS } & E C-4 V 3 & E C-5 V 3 & E C-2 P V 3 \\ 0.000 E+00 & 0.000 E+00 & 0.000 E+00\end{array}$

9
14
2
PECCS

2.455E-02

. 1.225E-04 2.443E-02 $0.000 E+00$

$0.000 E+00$

$0.000 E+00 \quad 0.000 E+00 \quad 0.000 E+00$

54 DOES HPI OPERATE DURING THE RECOVERY PHASE?

DEES UPI OPERATE DURING THE RECOVERY PHASE?
I/4-BPI $2 / 4-$ RPI $1 / 4-$ HPI nHPI

3.131E-0S 2.138E-02 $0.000 E+00 \quad 8.786 E-01$

SUMMARY BY CASE

CASE NUMBER/SPLIT:

REQ. BRANCHES

CASE/BRANCH SPLIT:

$\begin{gathered}0.7535-01 \\ 8 \\ -4 \\ 4\end{gathered}+\frac{2}{3}+\frac{5}{4}$

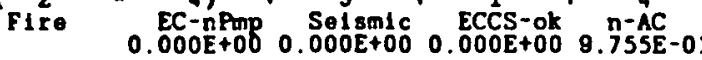


CASE - NUMBER/SPLIT:
DEPENDENCIES:
REO BRANCHES REQ BRANCRES: CASE/BRANCH SPLIT:

CASE NUMBER/SPLIT: DEPENDENCIES:
REQ. BRANCHES: DESCRIPTION:
CASE/BRANCH SPLIT:

CASE NUMBER/SPLIT:

DEPENDENCIES:

DESCRIPTION:

CASE NUMBER/SPLIT.

DEPENDENCIES:

DESCRIPTION:
REAT

CASE NUMBER/SPLIT:

DEPEKDENCIES

DEESCRIPTION:
CASE/BRANCH SPLIT:

CASE NUMBER/SPLIT:

DEPEMDENCIES

DESCRIPTION:

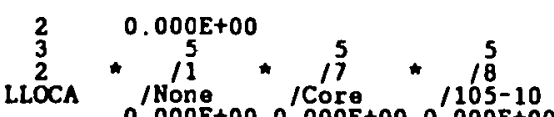

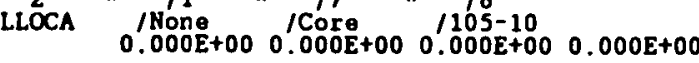

$120.000 E+00$

$\stackrel{4}{4} \cdot \frac{6}{4} \cdot \frac{51}{1}$

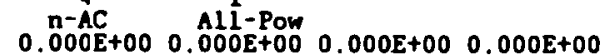

$120.000 E+00$

NoHPI $* \begin{gathered}6 \\ 4 \\ n-A C\end{gathered} * \frac{51}{14}$

0.000

3.167E-03

NoHPI

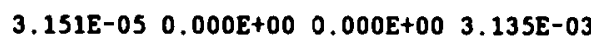

$\begin{array}{ll}6 & 0.000 \mathrm{E}+00 \\ 3 & \end{array}$

SLOCA * 3

$0.000 \mathrm{E}+00 \quad 0.000 \mathrm{E}+00 \quad 0.000 \mathrm{E}+00 \quad 0.000 \mathrm{E}+00$

$7 \quad 0.000 \mathrm{E}+00$

12
3
$1-\mathrm{HPI}$

$0.000 \mathrm{E}+00$

CASE NUMBER/SPLIT: $80.000 E+00$

REQ. BRANCHES: 12

CASE/BRANCH SPLIT:

CASE NUMBER/SPLIT:

REQ. BRANCHES

CASE/BRANCH SPLIT:

CASE NUMBER/SPLIT

DEPENDNCS

DÉSCRIPTION:

CASE/BRANCH SPLIT:

CASE NUMBER/SPLIT

CASE/BRANCH SPLIT:

$0.000 E+00 \quad 0.000 E+00 \quad 0.000 E+00 \quad 0.000 E+00$

$92.139 E-02$

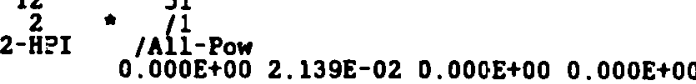

$10 \quad 0.000 E+00$

2

$\begin{array}{lll}0.000 E+00 & 0.000 E+00 \quad 0.000 E+00 & 0.000 E+00\end{array}$

$11 \quad 0.000 \mathrm{E}+00$

$\begin{array}{llll}0.000 E+00 & 0.000 E+00 \quad 0.000 E+00 \quad 0.000 E+00\end{array}$

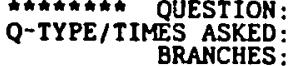

REALI2ED SPLIT:

55 IS THERE A STEAM SPIKE FROM H2O INJECTION?

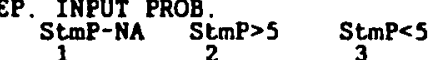

$\begin{array}{ccc}\text { StmP-NA } & \text { StmP }>5 & \text { StmP<5 } \\ 1 & 3 \\ 5.172 E-01 & 3.230 E-01 & 1.599 E-01\end{array}$

SUMMARY BY CASE 


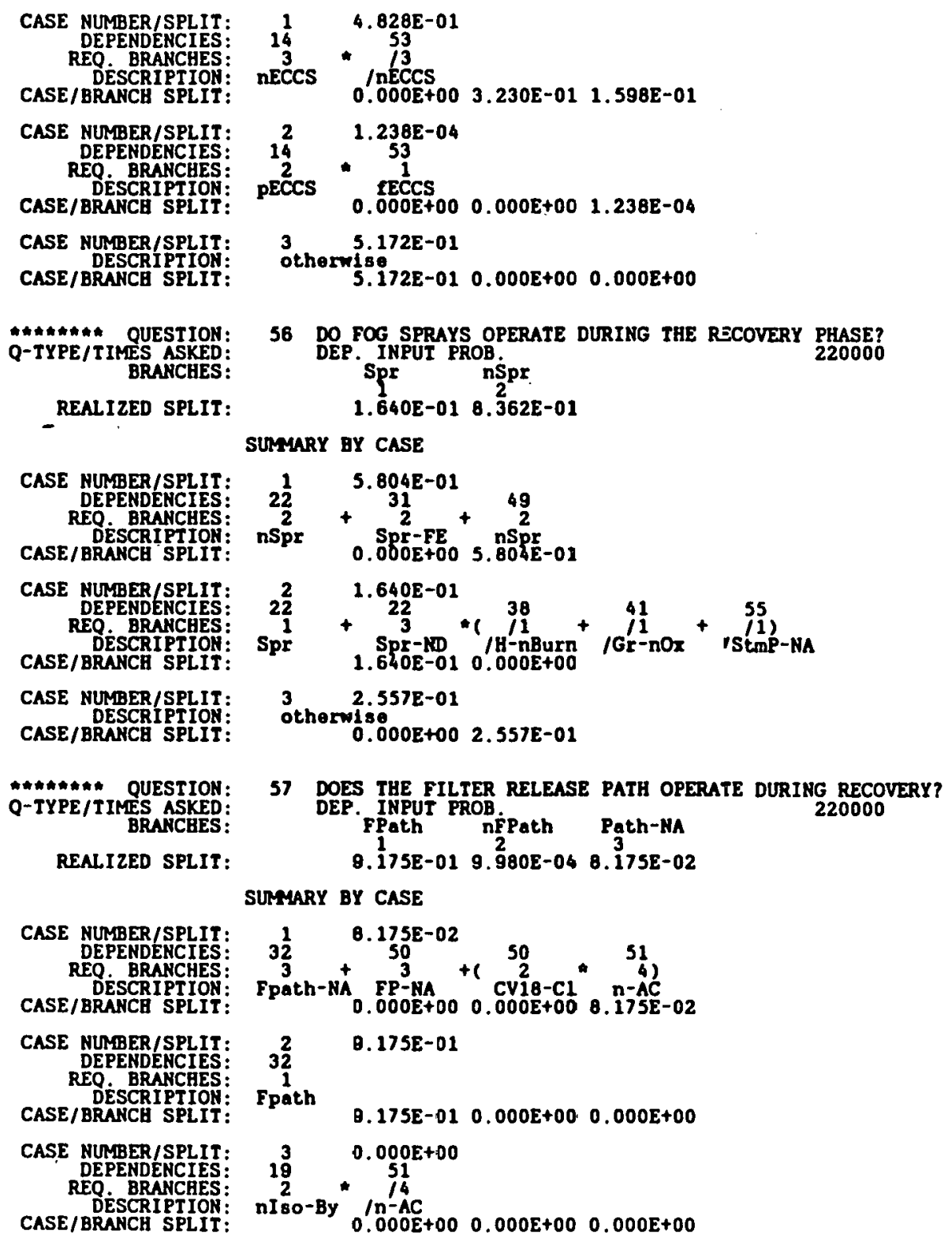


CASE NUMBER/SPLIT:

DEPENDENCIES:

REQ. BRANCHES :

CASE/BRANCH SPLIT:

CASE NUMBER/SPLIT:

CASE/BRANCE SPLIT:

QmWnntit QUESTION:

Q-TYPE/TIMES ASKED :

REALIZED SPLIT:

CASE NUMRER/SPLIT:

DEPENDENCIES

REQ DESCRIPTION:

CASE/BRANCH SPLII:

CASE NUMBER/SPLIT:

REO. BRANCHES:

CASE / DERACRIPT SPLIT:

CASE NUMBER/SPLIT:

DEPENDENCIES

DESCRIPTION:
CASE/BRARCH SPLIT:

CASE NUMBER/SPLIT:

REQ BRANCHES :

CASE / BRANCE SPLIT:

CASE NUMBER/SPLIT:

DEPENDENCIES:

DESCRIPTION:
CASE/BRANCH SPLIT:

CASE NUMBER/SPLIT:

DEPENDENCIES: 23

REQ BRANCHES: 1
DESCRIPTION: HM-OK

CASE/BRAHCH SPLIT

CASE NUMER/SPLIT:

REO. BRANCHES :

CASE/BRANCH SPLIT:

CASE NUMBER/SPLIT:

CASE /BRANCH SPLIT:

(M)-32
$4 \quad 0.000 E+00$

$\mathrm{nI}-180 \mathrm{k} * 1 \mathrm{n}-\mathrm{AC}$

$0.000 E+00 \quad 0.000 E+00$

$59.980 E-04$

$0.000 E+00 \quad 9.980 E-04 \quad 0.000 E+00$

$5 B$ DOES THE HYDROGEN MITIGATION SYSTEM OPERATE DURING RECOVERY? HEP. INPUT PROB

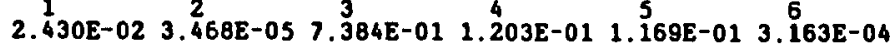

\section{SUMYARY BY CASE}

$\frac{1}{3} \quad 7.383 \mathrm{E}-01$

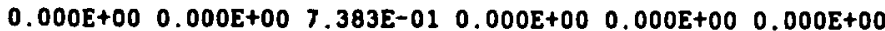

1.169E-01

$\begin{array}{llllll}0.000 E+00 & 0.000 E+00 & 0.000 E+00 & 0.000 E+00 & 1.169 E-01 & 0.000 E+00\end{array}$

$23 \quad 1.203 \mathrm{E}-01$

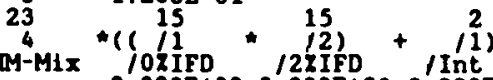

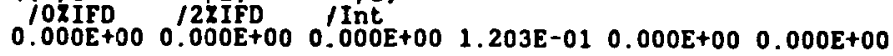

$23 \quad 3.163 \mathrm{E}-04$

$\left(\stackrel{8}{\frac{8}{2}}+\stackrel{8}{9}\right)+I^{2}$

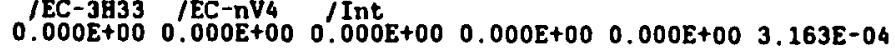

$55 \quad 1.217 \varepsilon-04$

$+\left(\begin{array}{c}53 \\ +\end{array}\right.$

$\begin{array}{llllll}\text { nECCS } & \text { /fGSCS } & \text { /HM-3k } & & & \\ 0.000 E+00 & 0.000 E+00 & 1.047 E-04 & 5.516 E-07 & 1.648 E-05 & 1.321 E-09\end{array}$

$2.430 \mathrm{E}-02$

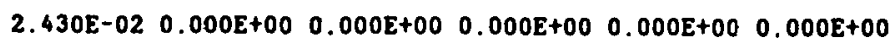

? $3.468 \mathrm{E}-05$

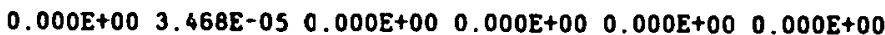
$8 \quad 0.000 E+00$

$\begin{array}{llllll}0.000 E+00 & 0.000 E+00 & 0.000 E+00 & 0.000 E+00 & 0.000 E+00 & 0.000 E+00\end{array}$ 


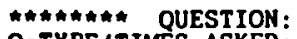

Q-TYPE/TIMES ASKED:

PEALIIZED SPLIT:

\section{SUMYARY BY CASE}

CASE NUMBER/SPLIT:

DEPENDENCIES:

DESCRIPTION:

CASE MUMBER/SPLIT:

DEPENDENCIES:

DESCRIPTION:
CASE/BRANCH SPLIT:

CASE NUMBER/SPLIT:

DESCRIPTION:
CASE/BRANCR SPLIT:

mminn QUESTION:
Q-TYPE/TIMES ASKED

REALIZED SPLIT:

CASE NUMBER/SPLIT:

REO. BRANCHES:

CASE/BRASCH SPLIT:

CASE MUMBER/SPLIT:

REO BRANCHES:

DÉSCRIPTION:
RPLAT

$5 \frac{1}{5} \quad 4.829 \mathrm{E}-01$

$2+3$

$0.000 \mathrm{E}+00 \quad 4.829 \mathrm{E}-0$

$52 \quad 3.769 \mathrm{E}-01$

* 3 * 3

3.761E-04 3.765E-01

$3 \quad 1.404 \mathrm{E}-01$

1. 404E-01 $0.000 E+00$

60 HOW MUCH HYDROGEN IS PRODUCED DURIITG THE ACCIDENT?

DEP. INPUT PROB $<$ i200H2 <2000H2 $>2000 H 2231614$

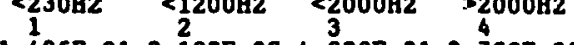

SUMMARY BY CASE

$5 \frac{1}{32} \quad 3.7698-01$

$\begin{array}{llll}0.000 \mathrm{EECS}+00 & 0.000 \mathrm{EECS}+00 & 0.000 \mathrm{E}+00 \quad 3 & 769 \mathrm{E}-01\end{array}$

$52 \quad 4.828 \mathrm{E}-01$

3 . 14.53

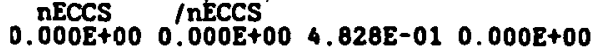

CASE NUMBER/SPLIT:

REQ, BRANCHES:

CASE/BRAHCH SPLIT:

CASE MUMBER/SPLIT:

CASE/BRASCH SPLIT:

2.192E-06

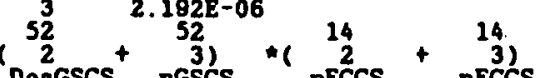

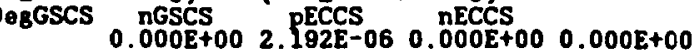

$4 \quad 1.405 \mathrm{E}-01$

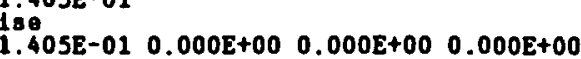

QHAnthnt QUESTION:

BRANCHES:

REALIZED SPLIT:

61 WHAT IS LATE H2 FLAMMABILITY?

H-MIT PROB.
H-Pent H-SG F-Bar 231614
H-10S

$\begin{array}{lllll}1.000 E+00 & 0.000 E+00 & 0.000 E+00 & 0.000 E+00 & 0.000 E+00\end{array}$

SUMYARY BY CASE

CASE NUMBER/SPLIT:

REQ. BRANCHES:

DESCRIPTION:
CASE/BRANCH SPLIT:

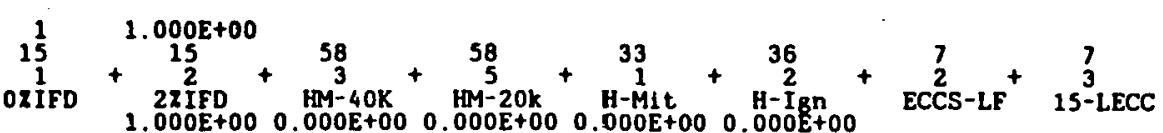




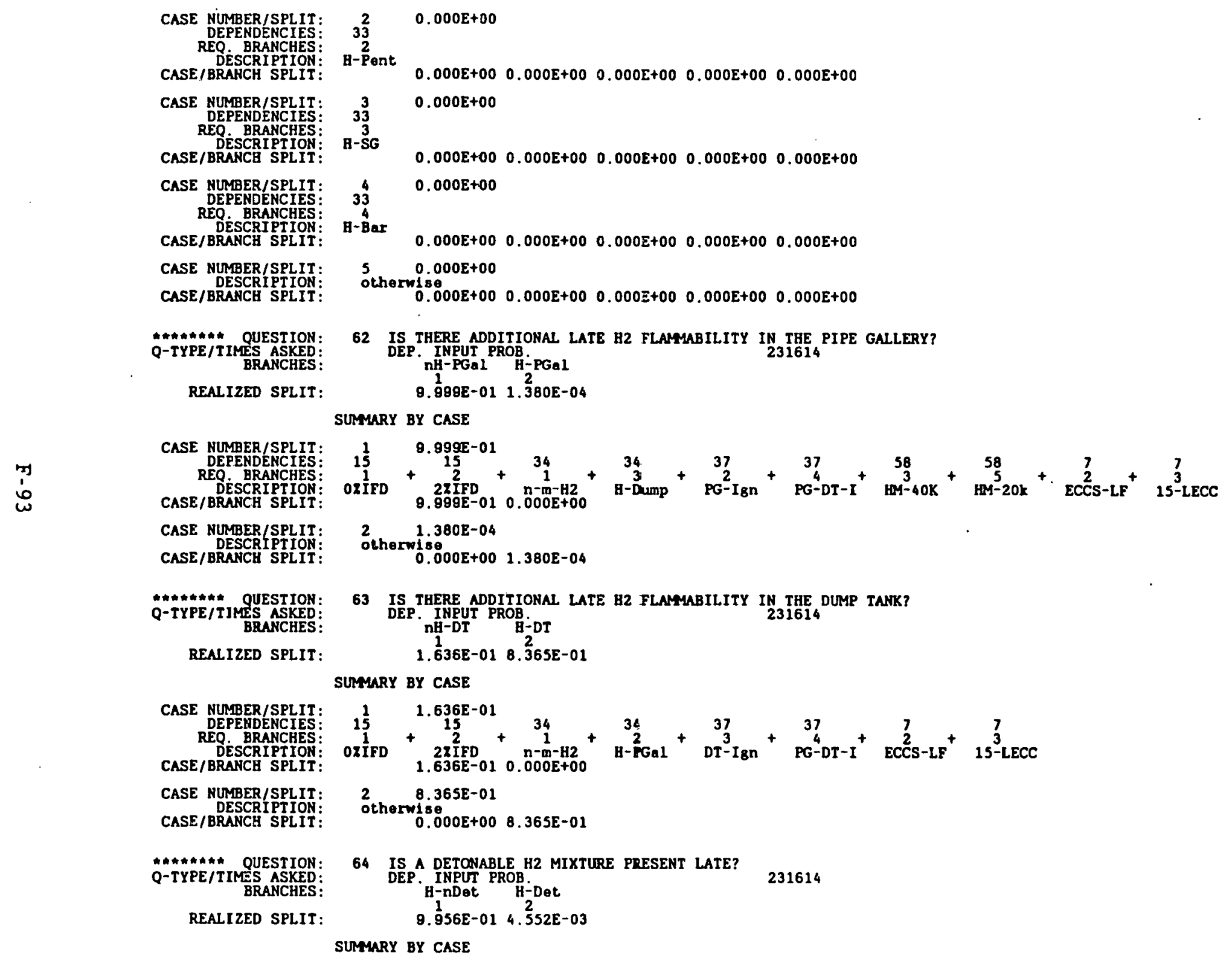




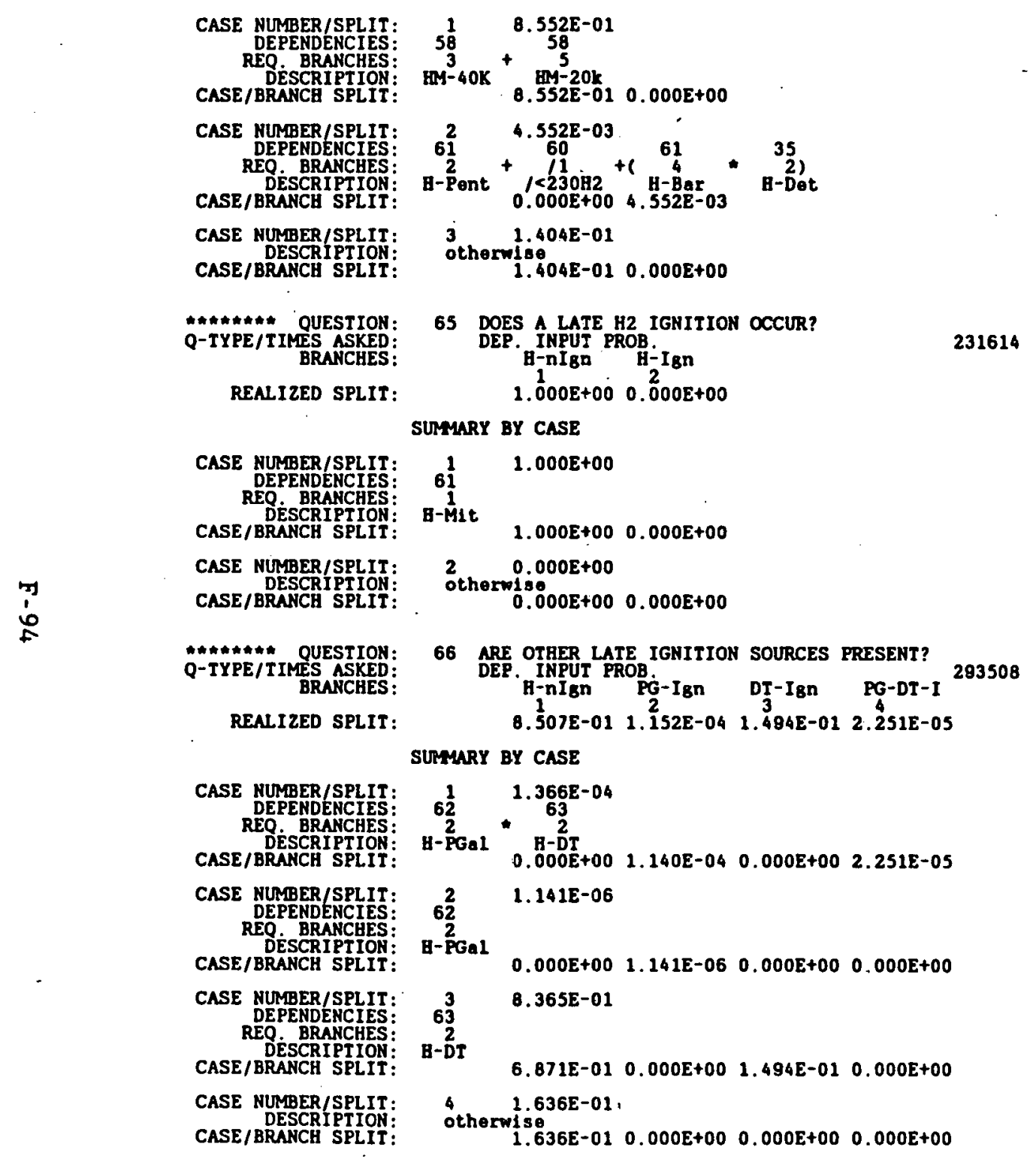


Q-TYPE/TIMES ASKED: BRANCHES :

REALIZED SPLIT:

ASE NUMBER/SPLIT:

DEPENDENCIES:
REQ. BRANCHES:
DESCRIPTION
BRARIPI :

CASE/BRANCE SPLIT:

CASE NUMBER/SPLIT:

REQ BRANCHES:

DESCRIPTION:
CASE/BRANCH SPLIT:

CASE NUMBER/SPLIT:

DEPENDENCIES

DESCRIPTION:
CASE/BRANCH SPLIT:

CASE NUMBER/SPLIT:

REO. BRANCHES

DASE/BRANCH STION:

CASE NUMBER/SPLIT

DEPENDENCIES
REQ. BRANCHES

CASE/BRANCH SPLIT:

CASE NUMBER/SPLIT

DESCRIPTION:
CASE/BRANCH SPLIT:

Q-TYPEITITES QUESTION:

Q-TYPE/TIMES ASKED:

REALIZED SPLIT:

$$
\text { RASE NUTBERTSELIT: }
$$

CASE NUMBER/SPLIT:

REPENDENCIES

DESCRIPTION:
CASE/BRANCH SPLIT:

CASE NUMBER/SPLIT:

DESCRIPTION:
CASE/BRANCH SPLIT:

$\star \star \star \star \star \star \star \star$ QUESTION:

Q-TYPE/TIMES ASKED:

REALIZED SPLIT:
67 IS THERE A LATE GENERAL DETOMATION?

DEP. INPUT PROB.-Def H-Det

$\begin{array}{lll}9.856 E-01 & 0.000 E+00 & 4 \\ 3 & 350 E-03\end{array}$

SUMMARY BY CASE

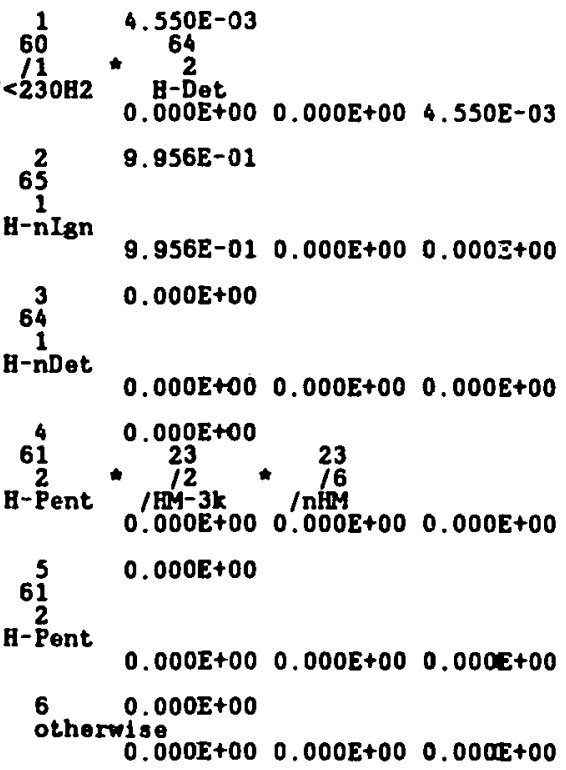

$60 \quad 4.550 \mathrm{E}-03$

$110 \mathrm{2}$

$\begin{array}{lll}0.000 E+00 & 0.000 E+00 \quad 4.550 E-03\end{array}$

$659.956 \mathrm{E}-01$

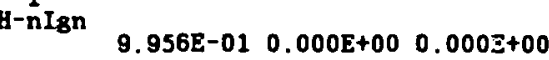

$3 \quad 0.000 E+00$

H-not

$\begin{array}{llll}0.000 E+00 & 0.000 E+00 & 0.000 E+00\end{array}$

$610.000 E+00$

$\frac{23}{2} \cdot \frac{23}{12}$

$\begin{array}{llll}0.000 \mathrm{E}+00 & 0.000 \mathrm{E}+00 & 0.000 \mathrm{E}+00\end{array}$

5 0.000E+00

H-Pent

$0.000 E+00 \quad 0.000 E+00 \quad 0.000 E+00$

$6 \quad 0.000 E+00$

$0.000 E+00 \quad 0.000 E+00 \quad 0.000 E+00$

68 DOES A DUMP TANK DETONATION OCCUR? DT-nBurn DT -DeP DT-Cet

B. $\frac{1}{508 E-01} 1.384 E-01$ 1. ${ }_{103 E-02}^{3}$

SUMYARY BY CASE

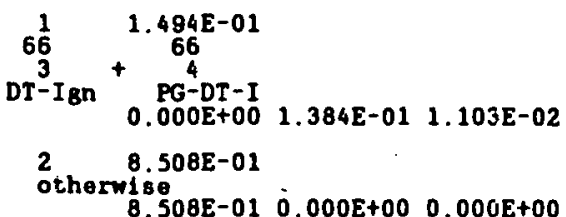

69 IS GSCS EFFICIENCY DECREASED DUE TO LOSS OF HE BLANKET?

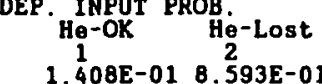

SUMMARY BY CASE
293508 
CASE NUMBER/SPLIT:
DEPENDENCIES:
REQ. BRANCHES: DESCRIPTION:

CASE NURBER/SPLIT CASE NUMBER/SPLIT:

A.tumm* QUESTION: Q-TYPE/TIMES ASKED:

REALIZED SPLIT. T:

CASE NUMBER/SPLIT: REO BRANCHES DÉSCRIPTION:
CASE/BRANCH SPLIT:

CASE NUMBER/SPLIT:

DEPENDENCIES:

REQ BRANCHES:

CASE/BRANCH SPLIT:

CASE NUMBER/SPLIT:

REQPENDENCCES:

DESCRIPTIOH:
CASE/BRANCH SPLIT:

CASE MUMBER/SPLIT:

CASE/BRANCH SPLIT:

QHAM. QUESTION:

Q-TYPE / IIMES ASKED

REALIZED SPLIT:

\section{:}

CASE NUMBER/SPLIT:

REQ. BRANCHES:

DESCRIPTION:
CASE/BRANCH SPLIT:

CASE NUMBER/SPLIT:

DEPENDENCIES :

DESCRIPTION:
CASE/BRANCH SPLIT:

CASE NUMBER/SPLIT:

CASE/BRANCH SPLIT:

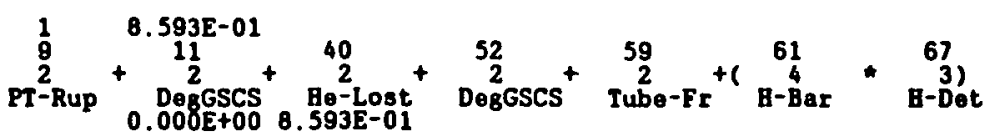

2 1. 408E-01

$1.408 E-01 \quad 0.000 E+00$

70 MAS LATE GRAPHITE OXIDATION START:D?

LGr-nOx LGr-Fir LGr-sOx

371676

9.874E-01 $1.202 E-04 \quad 2.833 E-03$

SURMARY BY CASE

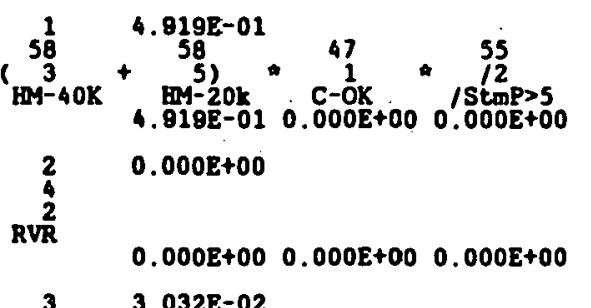

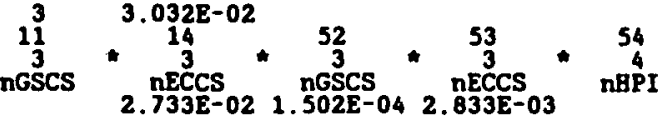

4 4.782E-01

$\begin{array}{lll}4.782 E-01 & 0.000 E+00 \quad 0.000 E+00\end{array}$

71 IS THERE LARGE SCALE FUEL DAMAGE EROPAGATION?

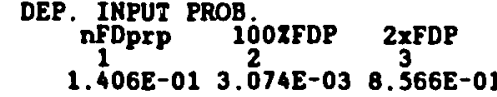

UMYMRY BY CASE

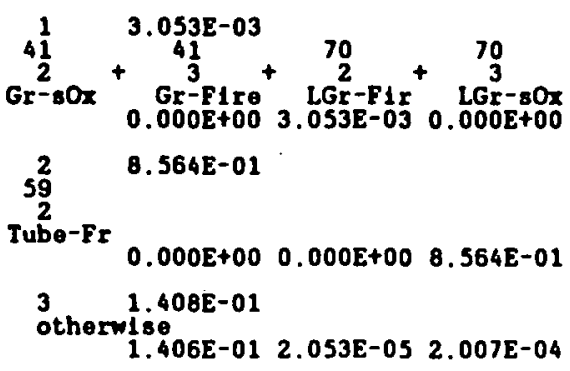




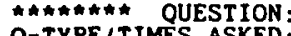

Q-TYPE/TIMES ASKED:

REALIZED SPLIT:

CASE NUMBER/SPLIT

REO BRANCHES

CASE/BRANCH SPLIT:

CASE NUMBER/SPLIT

REQ. BRANCHES

CASE/BRANCH SPLIT:

CASE NUMBER/SPLIT

DESCRIPTION:
CASE/BRANCH SPLIT:

CASE NUMBER/SPLIT:

DERENDENCIES

DÉSCRIPTION:

CASE/BRANCH SPLIT

CASE NUMBER/SPLIT

DEPENDENCIES

CASE/BRANCH SPLIT:

CASE NUMBER/SPLIT

DEPENDENCIES:

/BRANCH SPLIT:

CASE/ DERCHLPIOH:

CASE NUMBER/SPLIT

REQ. BRANCHES

CASE/BRANCH SPLIT

CASE NUMBER/SPLIT

CASE/BRANCH SPLIT

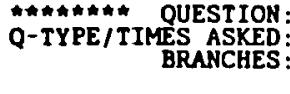

REALIZED SPLIT

CASE NUMBER/SPLIT:
DEPENDENCIES:
REQ BRANCHES:
DESCRIPTION :
CASE BRANCH SPLIT:

72 HOW MUCH TOTAL FUEL DAMAGE?

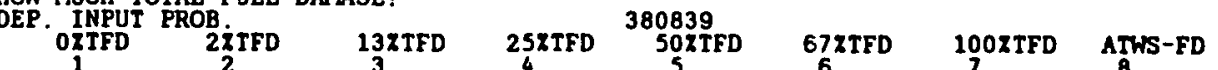

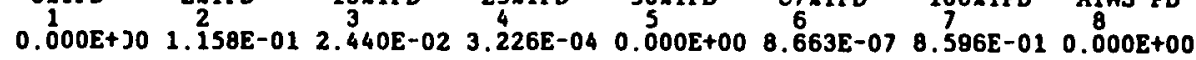
SUMMARY BY CASE

$\frac{1}{1} \quad 0.000 E+30$

ATWS-FD

$\begin{array}{lllllllllll}0.000 E+00 & 0.000 E+00 & 0.000 E+00 & 0.000 E+00 & 0.000 E+00 & 0.000 E+00 & 0.000 E+00 & 0.000 E+00\end{array}$

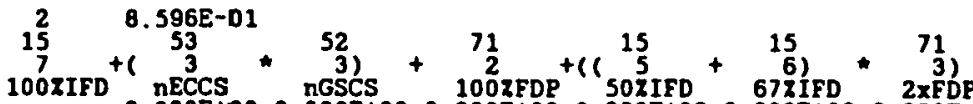

$\begin{array}{lllllllllllll}0.000 E+00 & 0.000 E+00 & 0.000 E+00 & 0.000 E+00 & 0.000 E+00 & 0.000 E+00 & 8.596 E-01 & 0.000 E+00\end{array}$

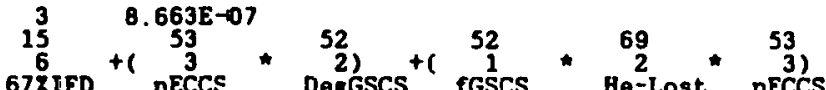

$\begin{array}{llllllllllllllll}0.000 E+100 & 0.008 E+00 & 0.000 E+00 & 0.000 E+00 & 0.000 E+00 & 8.663 E-07 & 0.000 E+00 & 0.000 E+00\end{array}$

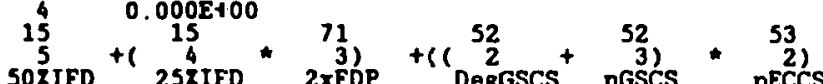

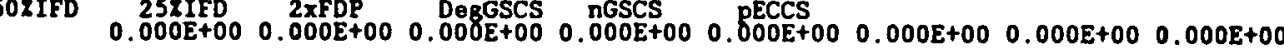

$5 \quad 3.226 \mathrm{E}-04$

$\begin{gathered}15 \\ 4 \\ 25 \mathrm{IFD}\end{gathered}+2{ }_{3}^{3}+53$

$\begin{array}{lllllllll}0.000 E-00 & 0.000 E+00 & 0.000 E+00 & 3.226 E-04 & 0.000 E+00 & 0.000 E+00 & 0.000 E+00 & 0.000 E+00\end{array}$

$6 \quad 2.440 E-02$

$13 \times 1 F D+23$

$\begin{array}{llllllllll}0.600 E+00 & 0.000 E+00 & 2.440 E-02 & 0.000 E+00 & 0.000 E+00 & 0.000 E+00 & 0.000 E+00 & 0.000 E+00\end{array}$

$\begin{array}{rl}7 & 1.158 E-01 \\ 15 & \end{array}$

$\begin{array}{lllllllllllllllll}2 \text { LIFD } & 0.000 E+00 & 1.158 E-01 & 0.000 E+00 & 0.000 E+00 & 0.000 E+00 & 0.000 E+00 & 0.000 E+00 & 0.000 E+00\end{array}$

$80.000 E+00$

$\begin{array}{lllllllll}0.000 E+00 & 0.000 E+00 & 0.000 E+00 & 0.000 E+00 & 0.000 E+00 & 0.000 E+00 & 0.000 E+00 & 0.000 E+00\end{array}$

73 DOES CWV-16 REISOLATE IN TIME TO PREVENT FILTER DAMAGE?

C-Rolio $\mathrm{C}_{2}-\mathrm{nR} 1 \mathrm{SO} \quad \mathrm{C}-\mathrm{nCh}$

I J11E-01 $1.418 \mathrm{E}-02$ 5. $352 \mathrm{E}-01$

SUMYARY BY CASE

6)

$4.54 B E-03$

$0.000 \mathrm{E}+00 \quad 4.548 \mathrm{BE}-03 \quad 0.000 \mathrm{E}+00$ 


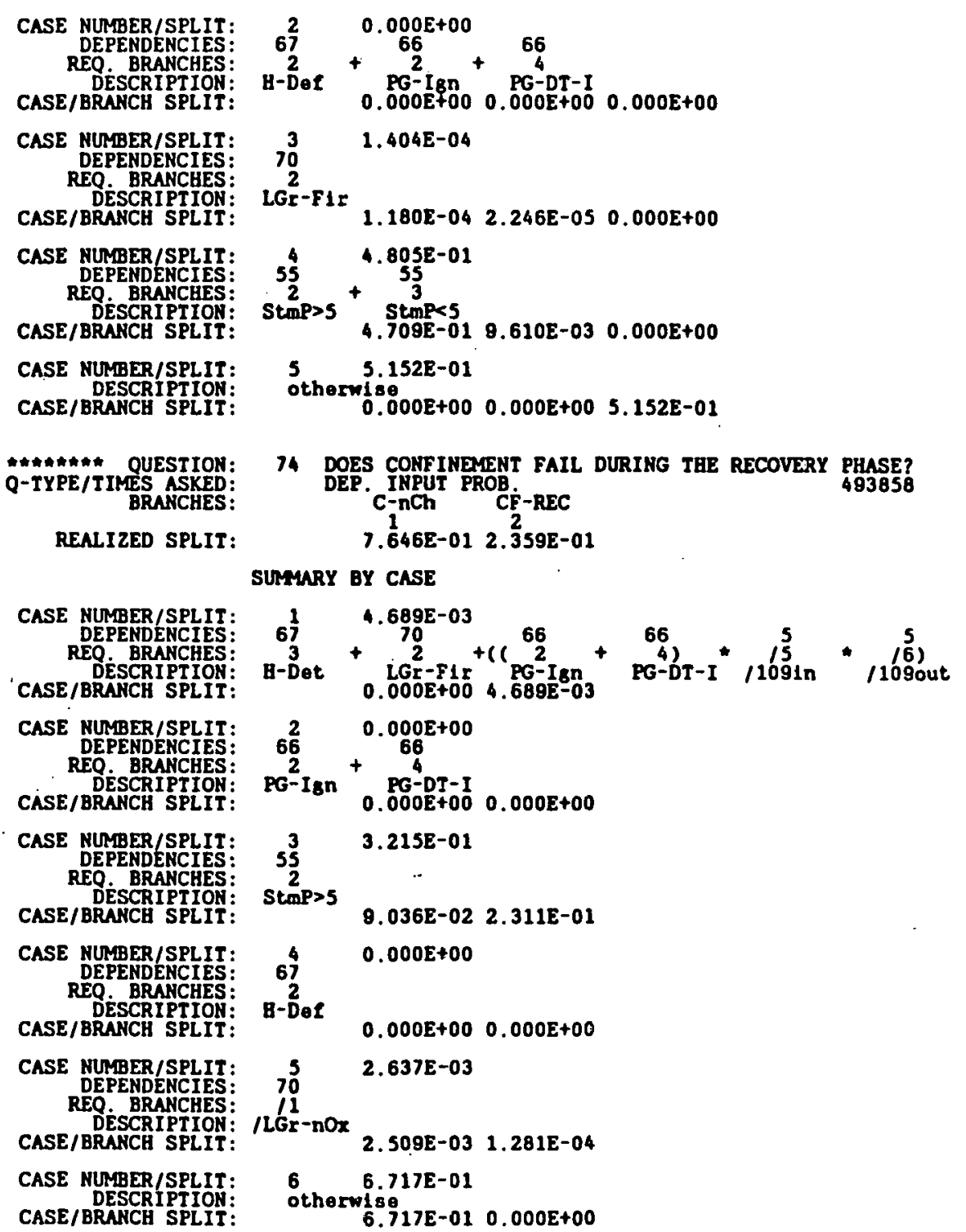




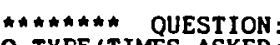

Q-TYPE/TIMES ASKED

REALIZED SPLIT

ASE NUMBER/SPLIT

DEPENDENCIES

REQ. BRANCHES

DESCRIPTION:
CASE/BRANCH SPLIT :

CASE NUMBER/SPLIT:

DEPENDENCIES

CASE/BRANCH SPLIT:

CASE NUMBER/SPLIT:

DEPENDENCIES

CASE/BRANCH SPLIT:

CASE NUMBER/SPLIT:

DEPENDENCIES

DĖSCRIPTION:

CASE NUMBER/SPLIT

DEPENDENCIES

RE DCRIPTION:

CASE NUMBER/SPLIT:

DESCRIPTION:
CASE/BRANCH SPLIT :

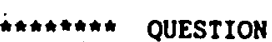

Q-TYPE/TIMES ASKED:

REALIZED SPLIT

SASE NUPBR/SPLIT:

DEPENDENCIES:

REQ. BRANCHES:

CASE/BRANCH SPLIT:

CASE NUMBER/SPLIT

DEPENDENCIES

REQ. BRANCHES:

CASE/BRANCH SPLIT:

CASE NUMBER/SPLIT:

DEPENDENCIES
REQ. BRANCHES

DESCRIPTION:
CASE/BRANCH SPLIT:
75 WHAT IS SIZE OF CONFINEMENT FAILURE?

C-OK PROB-Leak C-Rupt

$7.0111 E-01 \quad 0.000 E+002.9393 E-01$

SUMMARY BY CASE

$\begin{gathered}1 \\ 47 \\ 3 \\ C-R u p t\end{gathered}+\underset{6}{2.993 E-01}+\underset{3}{B-D e t}+\left(\begin{array}{c}55 \\ 2 \\ \text { StmP }>5\end{array} * \begin{array}{c}74 \\ 2) \\ 0.000 E+00\end{array}\right.$

$2 \quad 0.000 \mathrm{E}+00$

$\frac{2}{2-I g n}+{ }_{P G-D T-I}^{4}{ }^{72}{ }^{2}$

$0.000 \mathrm{E}+00$

$30.000 \mathrm{E}+00$

$2+2$

CF-REC

$40.000 \mathrm{E}+00$

$11+24$

$0.000 E+00 \quad 0.000 E+00 \quad 0.000 E+00$

$47 \quad 0.000 \mathrm{E}+00$

2

$0.000 E+00 \quad 0.000 E+00 \quad 0.000 E+00$

$6 \quad 7.011 \mathrm{E}-01$

$7.011 E-01 \quad 0.000 E+00 \quad 0.000 E+00$

76 WHAT IS THE CONFINEMENT FAILURE LOCATION?

Conf-OK ERvir Zone

$\begin{array}{ccc}\text { Conf-OK } & \text { Envir } & \text { Zone } \\ 1 & 2 & 3 \\ 7.011 E-01 & 2.988 E-01 & 5.798 E-04\end{array}$

SUMMARY BY CASE

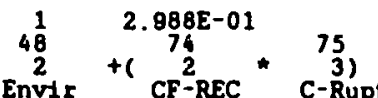

$\begin{array}{llll}0.000 E+00 & 2.988 E-01 & 0.000 E+00\end{array}$

$74 \quad 0.000 E+00$

CF- 2 * 2

c-Leak

3
48
3

5. 788E-04

zone 
CASE NUMBER/SPLIT: CASE/BRANCH SPLIT:

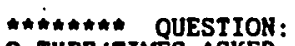

Q-TYPE/TIMES ASKED:

REALIZED SPLIT.

SE NUMBER/SPLIT:

DEPENDENCIES

DESCRIPTION:
CASE/BRANCH SPLIT:

CASE NUMBER/SPLIT

REQ. BRANCHES

DESCRIPTION:
CASE/BRANCH SPLIT:

CASE NUMBER/SPLIT

REO BRANCHES

DESCRIPTION:
CASE/BRANCH. SPLIT:

CASE NUMBER/SPLIT

CASE/BRANCHIPTION:

QH* WH*⿻ QUESTION:

Q-TYPE/TIMES ASKED:

REALIZED SPLIT:

$$
\text { II: }
$$

DEPENDENCIES:

REQ. BRANCHES:
DESCRIPTIOH:
CASE/BRAKCH SPLIT:

CASE NUMBER/SPLIT:

CASE/BRAKCH SPLIT:

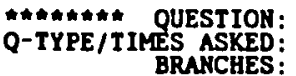

REALIZED SPLIT:

REALITED SPLIT:

CASE NUMBER/SPLIT

REQ. BRANCHES

DESCRIPTION:
CASE/BRANCH SPLIT
7. 7.011E-01

otherwise $7.011 E-010.000 E+00 \quad 0.000 E+00$

77 DO OPERAIIING FOG SPRAYS SURVIVE?

Spr-nCh nSpr-1

541721

Spr-nCh nSpr-L

SUMMARY BY CASE

$\frac{1}{74} \cdot 547 \mathrm{E}-03$

CF-REC $\begin{gathered}\text { C-Rupt } \\ 1.020 E-04\end{gathered} \quad \begin{gathered}\text { H-Det } \\ 4.445 E-03\end{gathered}$

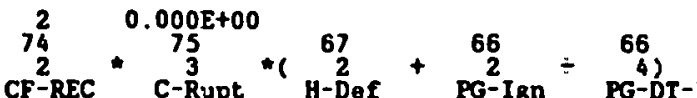

C-Rupt $\quad$ H-Def
$0.000 \mathrm{E}+000$
$0.000 \mathrm{E}+00$

$32.314 E-01$

2.245E-01 6.849E-03

$4 \quad 7.645 E-01$

$7.645 E-01 \quad 0.000 E+00$

78 IS LIQUID SYSTEM NLLIGNED TO 1312N OR 1325N? 646521 1312N PROB $1325 \mathrm{~N}$ 9. $131 \mathrm{E}-017.005 \mathrm{E}-03$

SUMYARY BY CASE

1
2
2

$0.000 E+00$

$0.000 E+00 \quad 0.000 E+00$

$21.000 E+00$

9.831E-01 7.095E-03

78 DO SUMP PUMPS OPERATE?

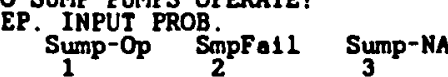

1.569E-01 7.149E-03 8.363E-01

SUMMARY BY CASE

12
$22.641 E-01$
$1+56$

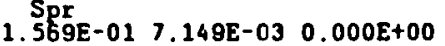


CASE NUMBER/SPLIT: DESCRIPTION
CASE/BRANCE SPLII :

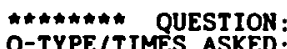

Q-TYPE/IIMES ASKED:

REALIZED SPLIT:

CASE NTMBRISPLIT:

CASE NUMBER/SPLIT:

REO BRANCHES

DESCRIPTIOH:
CASE/BRANCH SPLIT:

CASE NUMBER/SPLIT:

DEPENDENCIES

CASE/BRAHCH SPLIT:

CASE NUMBER/SPLIT:

CASE/BRANCH SPLIT

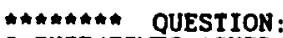

Q-TYPE/TIMES ASKED

REALIZED SPLIT:

CASE NURBER/SPLIT:

DERENDENCIES

RED. BRANCEES

CASE/BRANCH SPLT:

CASE NUMBER/SPLIT

CASE/BRANCH SPLIT

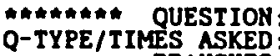

BRAFCHES :

REALIZED SPLIT:

\section{SUMAMRY BY CASE}

$2 \quad 8.363 E-01$

$0.000 E+00 \quad 0.000 E+00 \quad 8.363 E-01$

80 DOES FLOW TO 1325 OCCUR?

DEP. INPUT PROB $1325 \mathrm{~T}$.

695787

SURMARY BY CASE

$7 \frac{1}{8} \quad 6.855 \mathrm{E}-03$

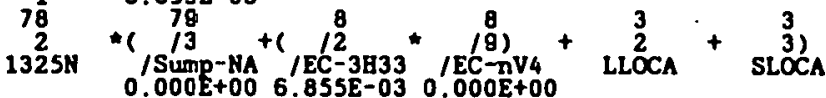

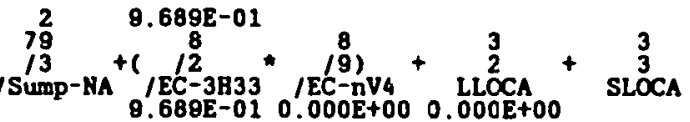

$3 \quad 2.451 E-02$

81 DOES LERF FUNCTION PROPERLY?

DEP. INPUT PROB. LRF-LK LERF-NA

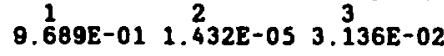

698826

SUMmaRY BY CASE

${ }_{80}^{1} \quad 9.689 \mathrm{E}-01$

$13 \frac{1}{12 F 10}$

9.689E-01 1.432E-05 $0.000 E+00$

$23.136 \mathrm{E}-02$

$0.000 \mathrm{E}+00 \quad 0.000 \mathrm{E}+00 \quad 3.136 \mathrm{E}-02$

82 ARE FILTERS BYPASSED?

DEP. INPUT PROB $\begin{array}{cccc}\text { nByp } & \text { F-pByp } & \text { F-Byp-L } & \text { F-Byp } \\ 1 & 3 & 4 \\ 6.967 E-01 & 0.000 E+00 & 2.224 E-01 & 8.173 E-02\end{array}$

CASE NUMBER/SPLIT:

REQ. BRANCHES :

CASE/BRANCH SPLIT:

CASE NUMBER/SPLIT:

REQ BRANCHES

CASE/BRANCH SPLIT

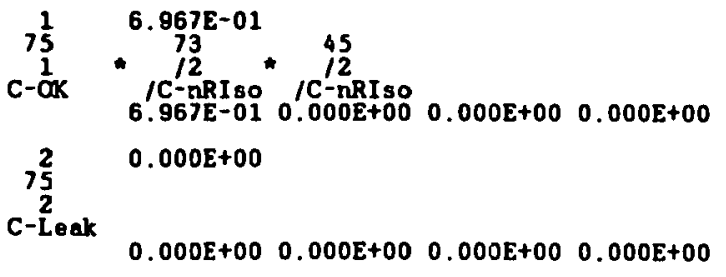


CASE NUMBER/SPLIT:

REQ. BRANCHES :

CASE/BRANCH SPLIT:

CASE NUMBER/SPLIT:

CASE/BRAKCRIPTION:

\#\#\#\#\#\# QUESTION:
Q-TYPE/TIMES ASKED:
BRANCHES:

REALIZED SPLIT:$$
\text { IT: }
$$

CASE NUMBER/SPLIT:

REO. BRANCGES:

DESCRIPTION:
CASE/BRANCH SPLIT:

CASE MUMPER/SPLIT:

DEPENDENCIES:

REO, BRANCHES:

$\begin{array}{llllll}\text { DESCRIPTION: ILGr-nOX } & & \\ \text { CASE/BRANCH SPLIT: } & 0.000 E+00 & 0.000 E+00 & 0.000 E+00 & 0.000 E+00 & 2.982 E-03\end{array}$

CASE NUMBER/SPLIT:

DEPENDENCIES: 41
REQ BRANCHES:
DI
DESCRIPTION:

$\begin{array}{cc}3 \\ 45 \\ 2 \\ \text { C-nRIso }\end{array}+{ }^{8.173 E-02}$

2.224E-01

\section{WHAT IS FILTER LOADING?}

FLP. INPUT PROB

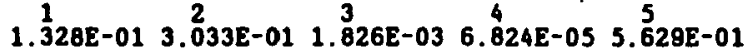

UMYARY BY CASE

$\frac{1}{2} \quad 1.159 E-01$

$1+12$

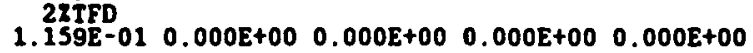

2. $9828-03$

$6.824 E-05$

CASE/BRANCH SPLIT:

$0.000 E+00 \quad 0.000 E+00 \quad 0.000 E+00 \quad 6.824 E-05 \quad 0.000 E+00$

CASE NUMBER/SPLI

Th $\quad 72000 E+00$

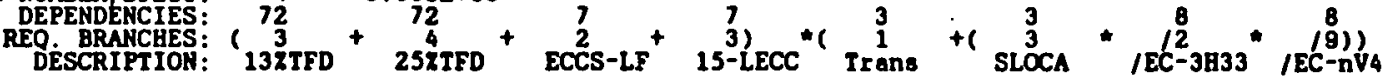

CASE/BRANCQ SPLIT:

$\begin{array}{lllll}0.000 E+00 & 0.000 E+00 & 0.000 E+00 & 0.000 E+00 & 0.000 E+00\end{array}$

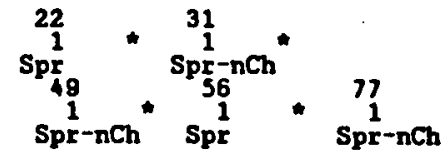

CASE NUMBER/SPLIT.

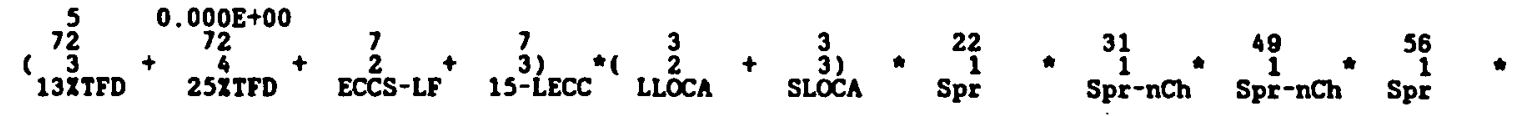

REQ. BRANCHES

CASE/BRANCH SPLIT:

$\begin{array}{lllll}0.000 E+00 & 0.000 E+00 & 0.000 E+00 & 0.000 E+00 & 0.000 E+00\end{array}$

CASE NUMBER/SPLIT:

$2.4748-02$

DEPENDENCIES:

CASE/BRANCH SPLIT:

6
72
13
$13 \pi T D$

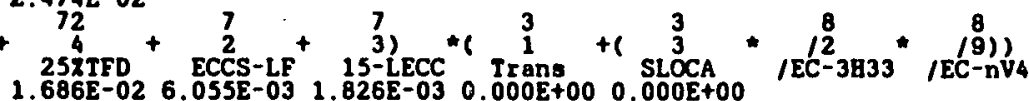

CASE NUMBER/SPLIT:

REO BRANCHES:

DESCRIPTION:

$0.000 \mathrm{E}+00$

$\begin{array}{r}72 \\ +\quad 7 \\ +2\end{array}+\frac{7}{3}$

$\begin{array}{lllll}0.000 \mathrm{E}+00 & 0.000 \mathrm{E}+00 & 0.000 \mathrm{E}+00 & 0.000 \mathrm{E}+00 & 0.000 \mathrm{E}+00\end{array}$ 


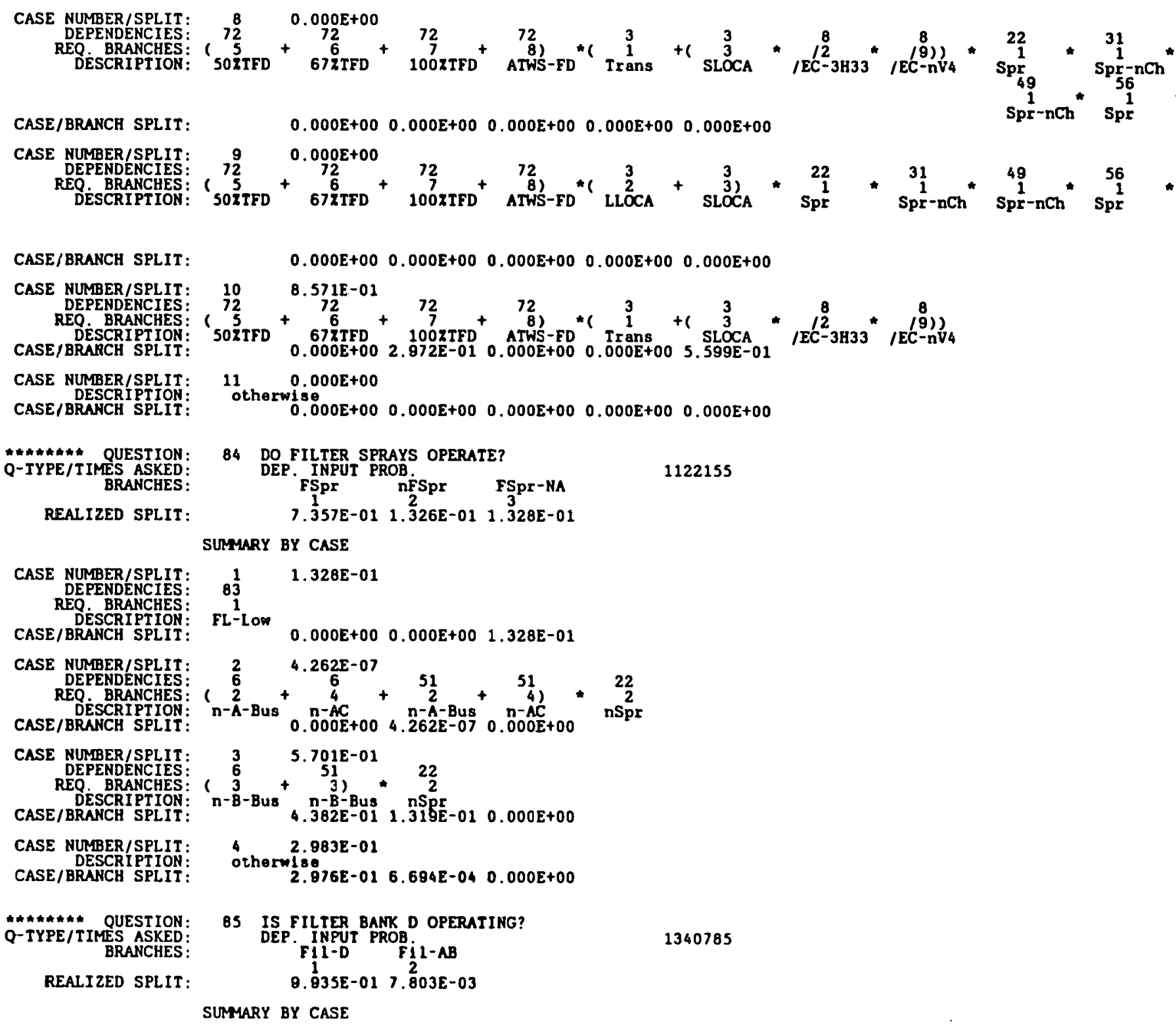

$\begin{array}{lllllll}0.000 E+00 & 0.000 E+00 & 0.000 E+00 & 0.000 E+00 & 0.000 E+00\end{array}$

84 DO FILTER SPRAYS OPERATE?$$
\text { DP. INPUT PROQ. }
$$$$
\begin{array}{ccc}
\text { FSpr } & \text { nFSpr } & \text { FSpr-NA } \\
7.357 E-01 & 1.326 E-01 & 1.328 E-0
\end{array}
$$

SUMMARY BY CASE

CASE NUMBER/SPLIT:
DEPENDENCIES:
REQ. BRANCHES :
DESCRIPTION :

1 1. 328E-01

CASE/BRANCH SPLIT

FL-Low

CASE NUMBER/SPLIT

DEO BRANCHES

CASE/BRANCH SPLIT:

24.262

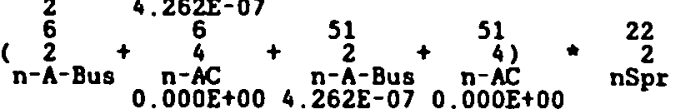

CASE NUMBER/SPLIT

REO BRANCHES:

DESCRIPTION:
CASE/BRANCH SPLIT:

CASE NUMBER/SPLIT

DESCRIPTION:
CASE/BRANCH SPLIT :

REALIZED SPLIT:

SUMMARY BY CASE 
CASE NUMBER/SPLIT:

REO. BRANCHES:

DESCRIPTION: IISO
CASE/BRANCH SPLIT:

$0.000 \mathrm{E}+00$

CASE NUMBER/SPLIT:

REO. BRANCHES:

DESCRIPTION:
CASE/BRANCH SPLIT:

${ }_{v_{n} t-n c t}^{27}$

19
-15

27

2)

$0.000 \mathrm{E}+08 \quad$ Vnt-nCl nFpath

CASE NURPER/SPLIT:

REQ. BRANCHES:

CASE/BRANCH SPLIT:

$9.945 E-04$

CASE MUMBER/SPLIT:

DESCRIPTION:
CASE/BRANCH SPLIT:

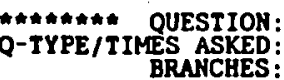

REALIZED SPLIT:

32 (2)

9.058E-04 8.868E-05

$3 \quad 8.108 \mathrm{E}-02$

19
15

$\begin{array}{lll}1 \mathrm{nIs}-\mathrm{S1} 8 & \\ 7.978 \mathrm{E}-02 & 1.302 \mathrm{E}-03\end{array}$

4 9.192E-01

9.128E-01 6.412E-03

86 WRAT IS FILTER STATUS?

DII-OK PROB FII-F

9.174E-01 $8.380 \mathrm{E}-02$

1423968

SUMMARY BY CASE

CASE NUMRER/SPLIT:

REQ. BRANCHES:

CASE/BRANCH SPLIT:

F-Byp

B. 172E-02

CASE NUMBER/SPLIT: 2 9.195E-01

CASE/BRANCH SPLIT: $\quad$ Otherw180

OHA WHA QUESTION: 87 WHAT IS FILTER EFFECTIVENESS?

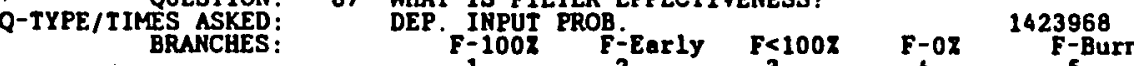

REALIZED SPLIT: $\quad 1 . \frac{1}{2} 15 E-014.381 E-01 \quad 0.000 E+003.093 E-01 \quad 1.326 E-01$

\section{SUMMARY BY CASE}

CASE NUMBER/SPLIT:

REO BRANCHES:

RE DESRIPTION:

CASE NUMBER/SPLIT:

DEO

CASE/BRANCH SPLIT:

CASE NUMBER/SPLIT:

REO BRANCHES:

DESCRIPTION:
CASE/BRANCH SPLIT:

$8 \frac{1}{2}$

1.326E-01

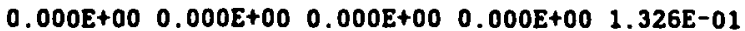

$82 \quad 3.093 E-01$

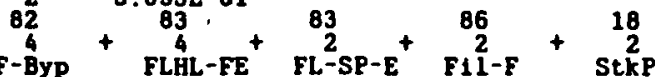

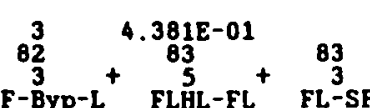

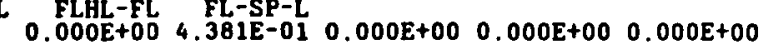


CASE NUMBER/SPLIT: $4 \quad 0.000 E+00$

REQPENDENCIES: $\quad 82$

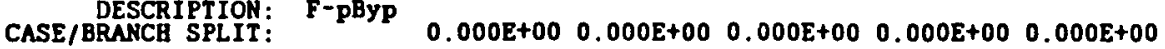

CASE NUMBER/SPLIT: 5 1.215E-01

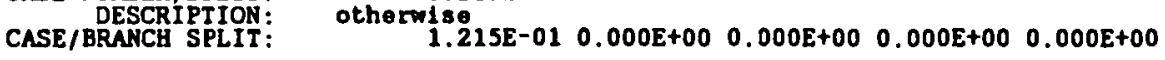


The following is a listing of the seismic analysis frequency report as presented in the file NREAC_SEISHIC_LHS_FREQ.OUT.

1 TREE ID: N-REACTOR ACCIDENT PROGRESSION EVENT TREE - SEISMIC

OBSERVATIONS: 500

FOR SERIES: N-REAC SEISMIC RUNS
SEQUENCE ID:

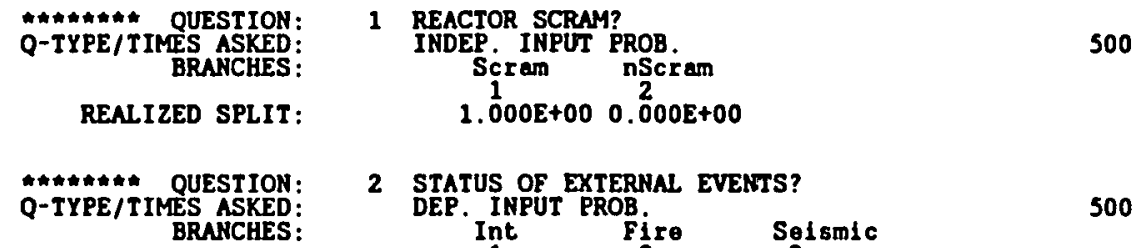

REALIZED SPLIT: $\quad 0.000 E+00 \quad 0.000 E+00 \quad 1.000 E+00$

SURPARY BY CASE

CASE NUMBER/SPLIT:
REPENDENCIES:
REQ. BRAHCHES:

$\quad \frac{1}{2} \quad 0.000+00$

CASE/BRANCH SPLIT:

nscram

$0.000 E+00 \quad 0.000 E+00 \quad 0.000 E+00$

CASE RUMBER/SPLIT:

$21.000 E+00$

CASE/BRANCH SPLIT: Otherw1so 0.000 E +00 $0.000 \mathrm{E}+00 \quad 1.000 \mathrm{E}+00$

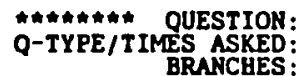

3 ACCIDENT INITIATOR?

REALIZED SPLIT:

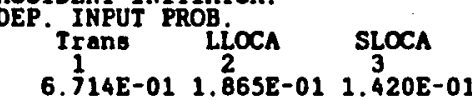

SUMMRY BY CASE

CASE RUMBER/SPLIT:
REPENDENCIES:
REQ. BRNCHES:

CASE/BRANCH SPLIT:

$0.000 E+00$

CASE RUMBER/SPLIT: $220.000 E+00$

REO. BRANCHES:

CASE/BRANCH SPLIT: Int $0.000 E+00 \quad 0.000 E+00 \quad 0.000 E+00$

CASE MUMBER/SPLIT: $\quad 3 \quad 0.000 E+00$

REQ. BRANCHES

CASE/BRANCH SPLIT:

$0.000 E+00 \quad 0.000 E+00 \quad 0.000 E+00$ 


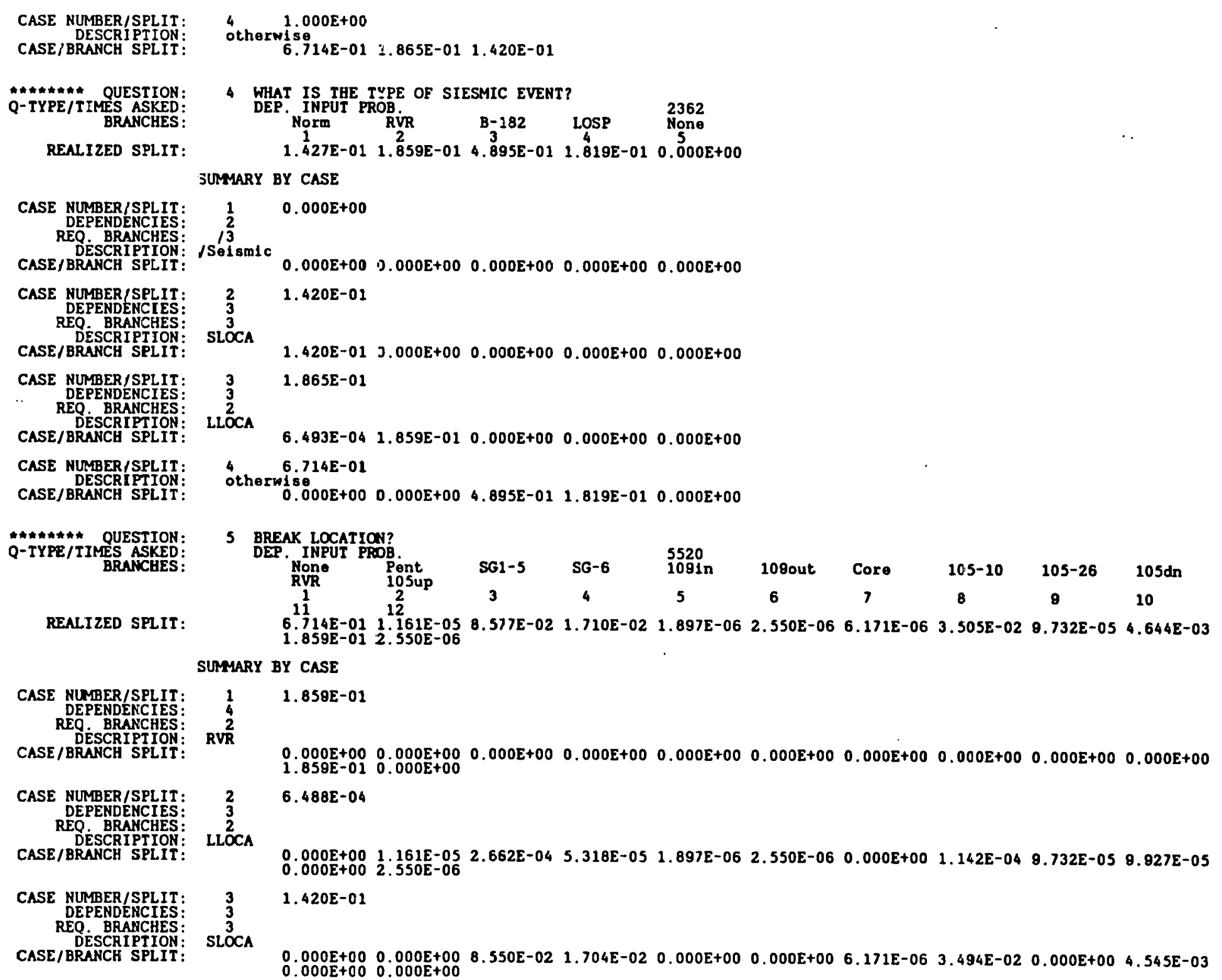




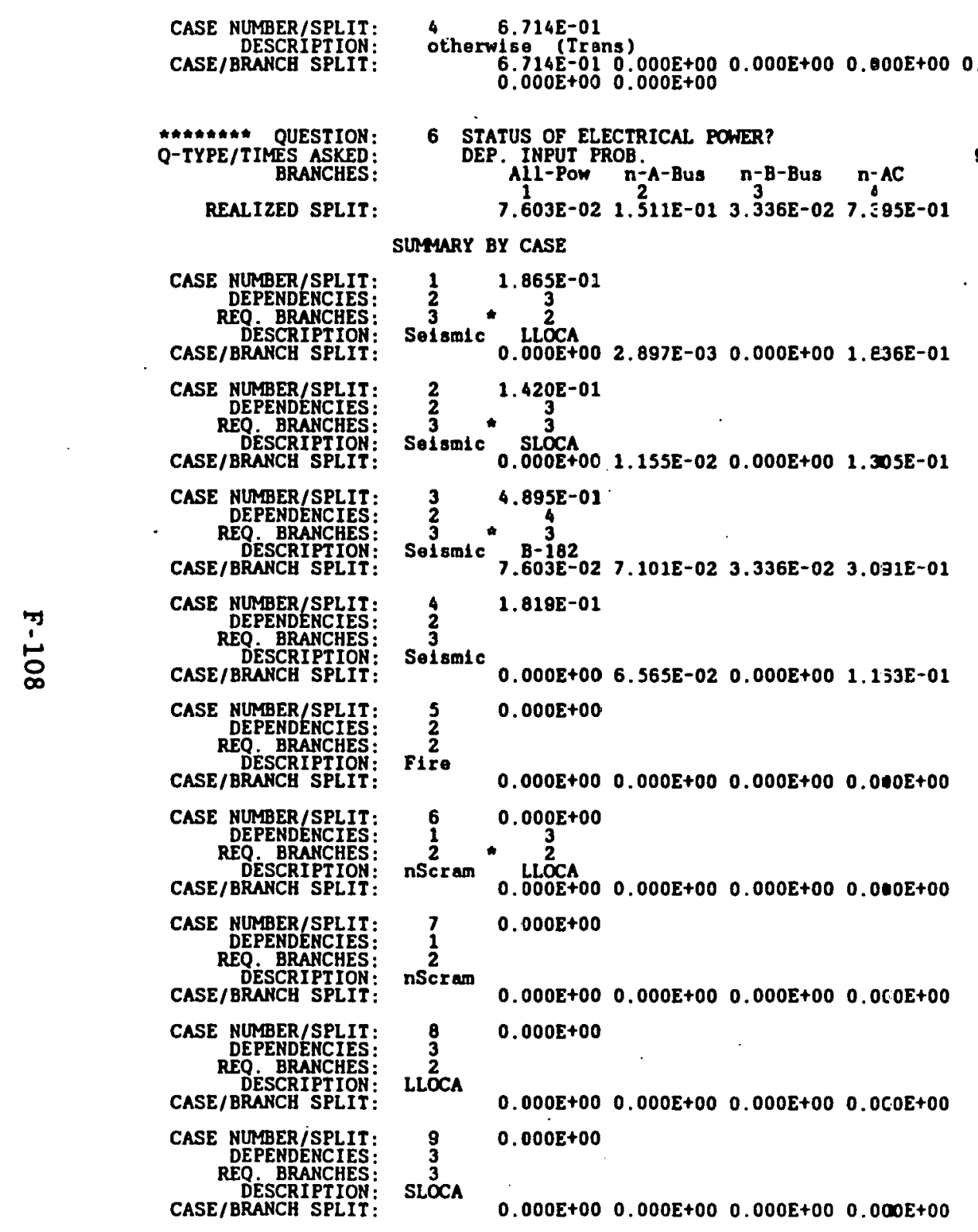


CASE NUMBER/SPLIT: 10 Otherwi.000E+00

$\begin{array}{llll}\text { DESCRIPTION: } & \text { otherwise (Trans-Int) } \\ \text { CASE/BRANCH SPLIT: } & 0.000 E+000.000 \mathrm{E}+00 \quad 0.000 \mathrm{E}+00 \quad 0.000 E+00\end{array}$

MAMM\#** QUESTION:
Q-TYPE/TIMES ASKED:
BRANCHES:

REALIZED SPLIT: $\quad 0.000 E+00 \quad 0.000 E+00 \quad 0.000 E+00 \quad 0.000 E+00 \quad 1.000 E+00$

SUMMARY BY CASE

CASE NUMBER/SPLIT:

REO. BRANCHES:

CASE/BRANCH SPLIT:

$0.000 E+00$

CASE NUMBER/SPLIT:

REO BRANCHES:

DEECRIPTION:
CASE/BRANCH SPLIT:

CASE NUMBER/SPLIT:

DEPENDENCYES:

DESCRIPTION:
CASE/BRANCH SPLIT:

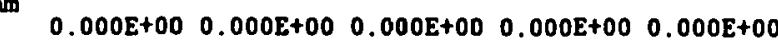

$20.000 E+00$

- 1 1

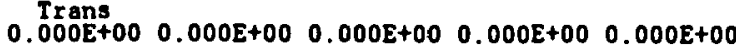

$0.000 E+00$

CASE NGMBER/SPLIT:

DEPENDENCIES:

REQ. BRANCHES:

CASE/BRANCH SPLIT:

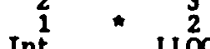

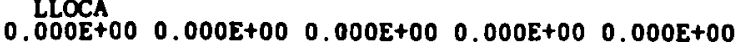

CASE NUMEER/SPLIT:

REO BRANCHES:

REQ. BRANCHES:

CASE/BRANCH SPLIT:

$0.000 E+00$

2
1 $\mathbf{3}_{3}^{3}$

$\begin{array}{lllll}0.000 E+00 & 0.000 E+00 & 0.000 E+00 & 0.000 E+00 & 0.000 E+00\end{array}$

$\begin{array}{ll}5 & 0.000 E+00\end{array}$

$\begin{array}{cc}5 \\ 2 \\ 2\end{array}-\frac{0}{6} 3$

CASE NUMBER/SPLIT:

REQ. BRANCHES: 2

CASE/BRANCH SPLIT:

$\begin{array}{lllll}0.000 E+00 & 0.000 E+00 & 0.000 E+00 & 0.000 E+00 & 0.000 E+00\end{array}$

$0.000 E+00$

CASE NUMBER/SPLIT

CASE/BRANCH SPLIT:

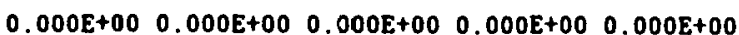

$7 \quad 1.000 \mathrm{E}+00$

$0.000 E+00 \quad 0.000 E+00 \quad 0.000 E+00 \quad 0.000 E+00 \quad 1.000 E+00$

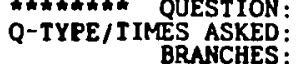

\title{
8 CAUSE OF ECCS FAILURE
}

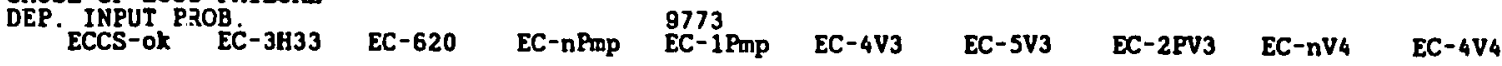

REALIZED SPLIT:

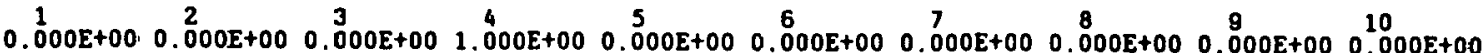

SUMMARY BY CASE

CASE NUMBER/SPLIT:

DEPENDENCIES:

REO. BRANCHES:

InECCS

\author{
$0.000 E+00$
}

$+\frac{1}{2}$ 


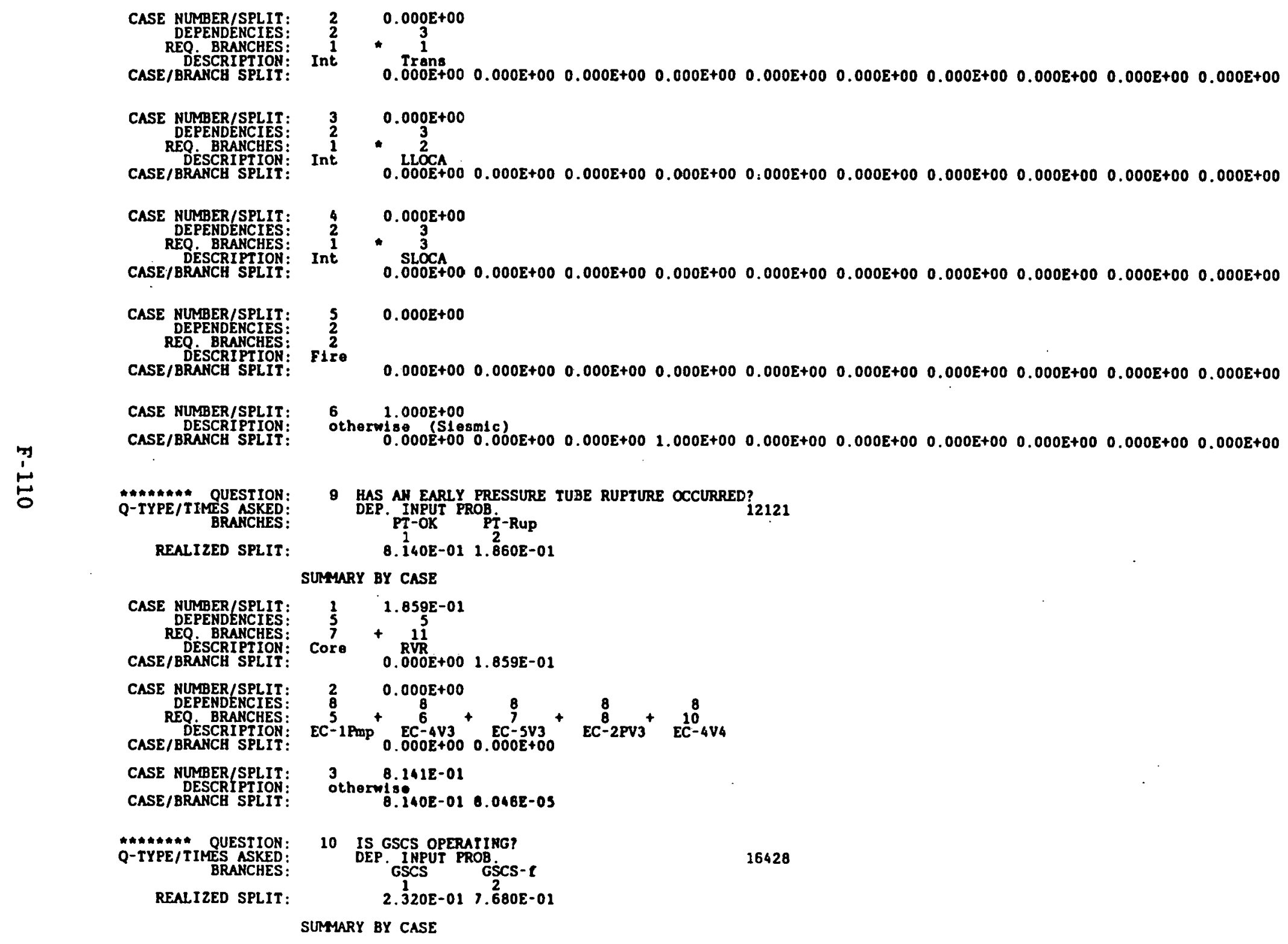




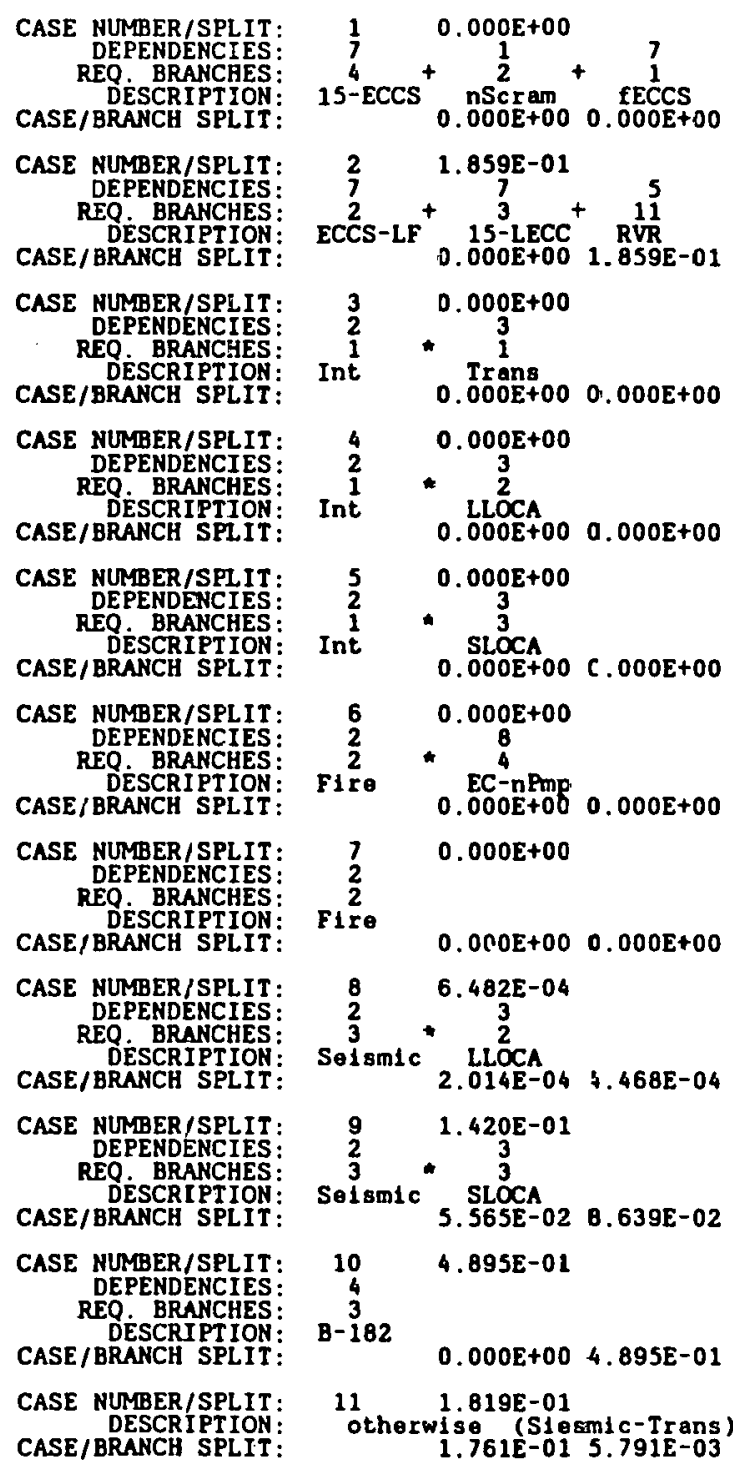




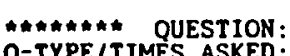
Q-TYPE/TIMES ASKED: REALIZED SPLIT: CASE NUMBER/SPLIT: CASE/BRANCH SPLIT: CASE NUMBER/SPLIT: REO BRANCHES CASE/BRANCH SPLII: CASE NUMBER/SPLIT: CASE/BRANCH SPLIT:

QHAHش* QUESTION: Q-TYPE/TIMES ASKED

REALIZED SPLIT

CASE MUMER/SPLIT
DEPENDENCIES
REQ BRANCHES
DESSCRIPTION
CASE/BRANCH SPLIT
11 IS GSCS EFFECIIVELY COOLING THE CORE?

$$
\text { fGSCS DR DGGSS nGSCS }
$$$$
\text { 2. 320E-01 2. } 2999 \mathrm{E}-05 \quad 7.680 \mathrm{3}-01
$$

SUMYARY BY CASE

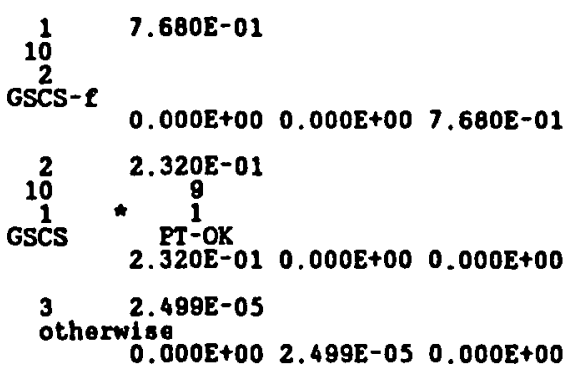

12 BPI FLOW STATUS

4-HPI 2-BPI 1-thI MoBPI

16428

\section{SUMYMARY BY CASE}

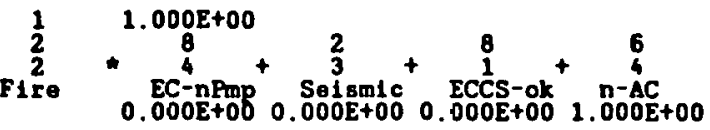

CASE NUMBER/SPLIT

DEPENDRCHES

REQ DESRIPTION:

CASE/BRANCE SPLIT

$0.000 \mathrm{E}+00$

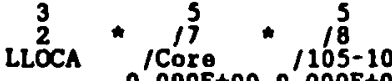

CASE NUMBER/SPLIT

QEO ARANCBES:

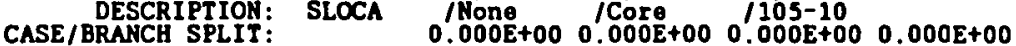

$30.000 \mathrm{E}+00$

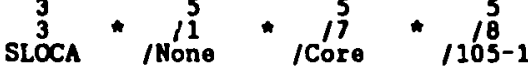

CASE MUMBER/SPLIT: $40.000 E+00$

CASE/BRANCH SPLIT:

CASE NUMBER/SPLII

CASE/BRANCE SPLII

$\begin{array}{cc}5 \\ 5 \\ 7 \\ \text { Core }\end{array}+\frac{0.000 E+00}{8}$

$0.000 E+00 \quad 0.000 E+00 \quad 0.000 E+00$

$5 \quad 0.000 E+00$

$0.000 E+00$ C.000E+00 $0.000 E+00 \quad 0.000 E+00$

HAthHA* QUESTION

Q-TYPE / T IMES ASKED

REALIZED SPLIT:

13 PRIMARY COOLANT FLOW

DEP. INPUT PROB

$\begin{array}{ll}1 & 2 \\ 0.000 & 1.000 E+00\end{array}$

SUMYARY BY CASE 


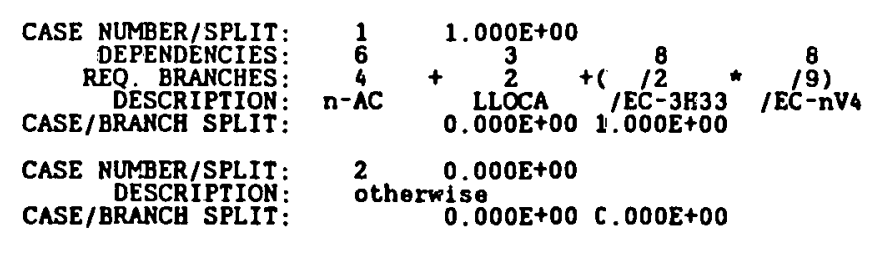
\#****** QUESTION: 14 INITIAL ECCS ELOW STATUS
Q-TYPE/TIMES ASKED:
DEP. INPUT PROB.
Q-TYPE/TIMES ASKED:
REALIZED SPLIT:
fECCS PËCCS nECCS
SUMMARY BY CASE

16428

CASE NUMBER/SPLIT: $\frac{1}{1} \quad 0.000 E+00$

CASE/BRANCH SPLIT:

$\mathrm{nscram}^{\frac{1}{2}}+\frac{7}{\mathrm{~s}}$

CASE NUMBER/SPLIT

REQ. BRANCCHES

CASE/BRANCH SPLIT:

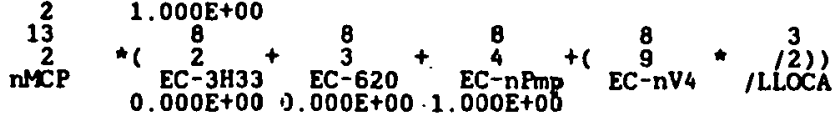

CASE NUMBER/SPLIT:

REQ. BRANCHES

$3 \quad 0.000 E+00$

CASE NUMBER/SPLIT:

DESCRIPTION:
CASEJBRANCE SPLIT:

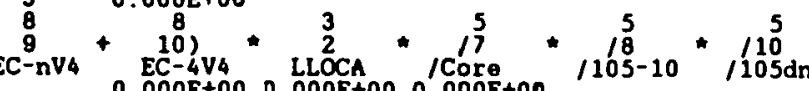

$4.000 \mathrm{E}+00$

$0.000 E+00 \quad 0.000 E+00 \quad 0.000 E+00$

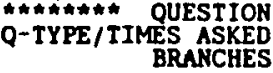

15 HOW MUCH INITIAL FUEL DAMAGE IS THERE?

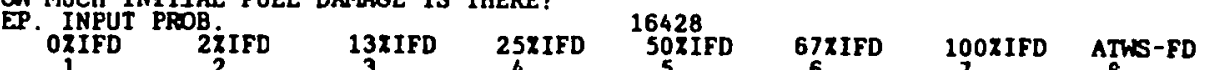

REALIZED SPLIT: $\begin{array}{llllllll}0.000 E+00 & 0.000 E+00 & 0.000 E+0 J & 2.320 E-01 & 0.000 E+00 & 2.499 E-05 & 7.680 E-01 & 0.000 E+00\end{array}$ SUMMARY BY CASE

CASE NUMBER/SPLIT:

REO BRANCHES:

CASE/BRANCH SPLIT:

CASE NUMBER/SPLIT

REO BRANCHES:

DESCRIPTION:
CASE/BRANCH SPLIT:

1
$\frac{1}{2}$
2

$0.000 \mathrm{E}+00$

$\begin{array}{lllllllll}0.000 E+00 & 0.000 E+00 & 0.000 E+00 & 0.000 E+00 & 0.000 E+00 & 0.000 E+00 & 0.000 E+00 & 0.000 E+00\end{array}$

$11 \quad 7.680 \mathrm{E}-01$

nGSCS 30 .

$\begin{array}{lllllllllll}0.000 E+00 & 0.000 E+00 & 0.000 E+00 & 0.000 E+00 & 0.000 E+00 & 0.000 E+00 & 7.680 E-01 & 0.000 E+00\end{array}$

CASE NUMBER/SPLIT

REQ. BRANCHES:

CASE/BRANCH SPLIT:

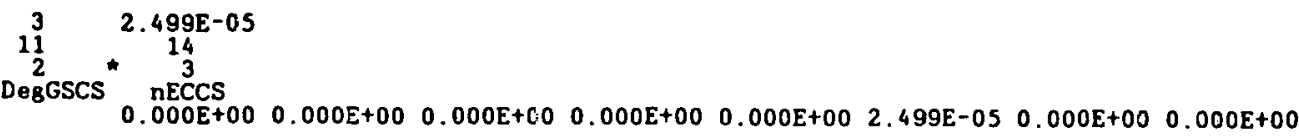


CASE NUMBER/SPLIT:

REO. BRANCHES :

CASE/BRANCH SPLIT:

CASE NUMBER/SPLIT

DEPENDENCIES:

CASE/BRANCH SPLIT:

CASE NUMBER/SPLIT:

DEPENDENCIES:

DASE/BRAKCH SPLIT:

CASE NUMBER/SPLIT:

DEPENDENCIES

DESCRIPTION:
TASE/BRANCH SPLIT:

CASE NUMBER/SPLIT

CASE NUESCRISPLIT:

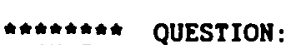

Q-TYPE/TIMES ASKED

BRANCHES:

REALIZED SPLIT:

$T:$

CASE NUMBER/SPLIT:

DEPENDENCIES

CASE/BRANCHIPTION:

CASE NUMBER/SPLIT:

REQPENDENCIES

CASE/BRANCH SPLIT:

CASE/BRANCR SPLIT:

CASE NUMBER/SPLIT

CASE/BRANCH SPLIT:

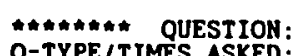

Q-TYPE/TIMES ASKED:

REALIZED SPLIT:

REALIZED SPLIT:

CASE NUMBER/SPLIT:

DEPENDENCIES:
REQ. BRANCHES

DESCRIPTION:

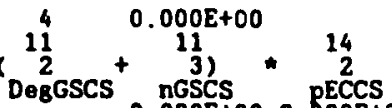

$\begin{array}{lllllllllllllllllllll}0.000 \mathrm{E}+00 & 0.800 \mathrm{E}+00 & 0.000 \mathrm{E}+00 & 0.000 \mathrm{E}+00 & 0.000 \mathrm{E}+00 & 0.000 \mathrm{E}+00 & 0.000 \mathrm{E}+00 & 0.000 \mathrm{E}+00\end{array}$

$11 \quad 2.320 E-01$

IGSCS $1 \frac{14}{3}$

$\begin{array}{lllllllll}0.000 E+00 & 0.000 E+00 & 0.000 E+00 & 2.320 E-01 & 0.000 E+00 & 0.000 E+00 & 0.000 E+00 & 0.000 E+00\end{array}$

$60.000 \mathrm{E}+00$

fGses $* \frac{2}{2}$

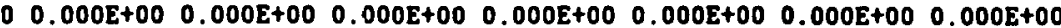

$7 \quad 0.000 E+00$

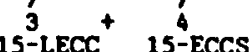

$\begin{array}{lllllllllll}0.000 E+00 & 0.000 E+00 & 0.000 E+00 & 0.000 E+00 & 0.000 E+00 & 0.000 E+00 & 0.000 E+00 & 0.000 E+00\end{array}$

$8 \quad 0.000 \mathrm{E}+00$

$\begin{array}{rllllllllllll}0.000 E+00 & 0.000 E+00 & 0.000 E+00 & 0.000 E+00 & 0.000 E+00 & 0.000 E+00 & 0.000 E+00 & 0.000 E+00\end{array}$

16 WHAT IS CONFINEMENT PRESSURE?

Lo-pres Conf-tp Spr-tp Vent-tp

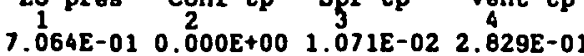

SUMMARY BY CASE

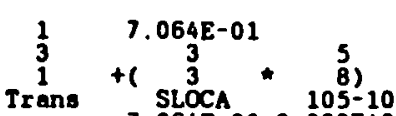

$7.064 E-01 \quad 0.000 E+00 \quad 0.000 E+00 \quad 0.000 E+00$

$2 \quad 1.865 E-02$

${ }^{2}$

$0.000 E+00 \quad 0.000 E+00 \quad 0.000 E+00 \quad 1.865 E-01$

3 1.071E-01

$0.000 \mathrm{E}+00 \quad 0.000 \mathrm{E}+00 \quad 1.071 \mathrm{E}-02 \quad 9.639 \mathrm{E}-02$

17 IS DUMP PATH TANK OK?

DEP. INPUT PROB

4. $959 \mathrm{E}-01 \quad 1.591 \mathrm{E}-01 \quad 1.591 \mathrm{E}-01 \quad 1.859 \mathrm{E}-01$

SUMMARY BY CASE

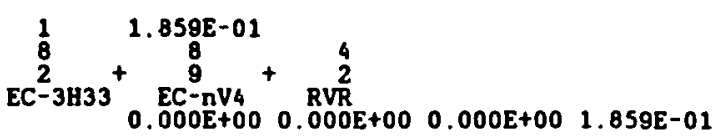


CASE NUMBER/SPLIT

DESCRIPTION:
CASE/BRANCH SPLIT:

CASE NUMBER/SPLIT

DEPENDENCIES:

CASE/BRANCH SPLIT:

CASE NUMBER/SPLIT

DEPENDENCIES

DESCRIPTION
SE / BRANCH SPLIT:

CASE MUMBER/SPLIT

CASE/BRANCA SPLIT:

Q-TYPE/IIMES ASKED:

Q-TYPE/TIMES ASKED

REALIZED SPLIT

SE NUMBER/SPLIT

$$
\begin{aligned}
& \text { DEPENDENCIES } \\
& \text { REO. BRANCHES : }
\end{aligned}
$$

REO. BRANCHES

DESCRIPTION:
CASE/BRANCH SPLIT:

CASE NUMBER/SPLIT:

DEPENDENCIES
REO. BRANCHES

DESCRIPTION:
CASE/BRANCH SPLIT:

CASE NUMBER/SPLIT

REO. BRANCHES:

REQ. BRANCHES

CASE/BRANCH SPLIT:

CASE NUMBER/SPLIT

CASE/BRANCH SPLIT:

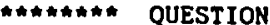

Q-TYPE/TIMES ASKED :
BRAKCHES :

REALIZED SPLIT:

\section{SUMMARY BY CASE}

CASE NUMBER/SPLIT

REQ. BRANCHES

DESCRIPTION
CASE/BRANCH SPLIT:

Solsmto

SUMMARY BY CASE

$2 * 4$

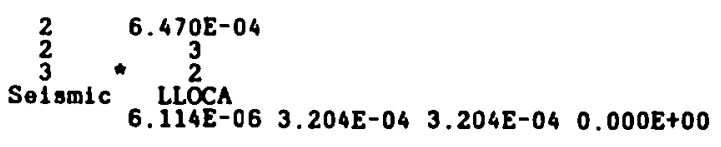

$3 \quad 6.316 \mathrm{E}-01$

Selsmic $^{*}$ sLoCA $_{3}^{3}+\begin{gathered}4 \\ 3 \\ \text { B-182 }\end{gathered}$

$\begin{array}{llll}3.158 E-01 & 1.579 E-01 & 1.579 E-01 & 0.000 E+00\end{array}$

1.8198-01

1.801E-01 9.094E-04 9.094E-04 $0.000 E+00$

$5 \quad 0.000 E+00$

$0.000 E+00 \quad 0.000 E+00 \quad 0.000 E+00 \quad 0.000 E+00$

18 IS FILTER RELEASE STACK OK?

StKOK PROB StkP18 StkTpI

102112

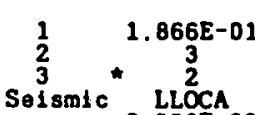

9. $\operatorname{LLOCA}_{228 \mathrm{E}-03} 1.865 \mathrm{E}-03 \quad 1.754 \mathrm{E}-01$

$26.316 \mathrm{E}-01$

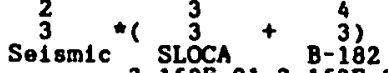

3 $1.820 \mathrm{E}-01$

Se1smLC $1.729 E-01 \quad 0.000 E+00 \quad 9.097 E-03$

$4 \quad 0.000 \mathrm{E}+00$

$\begin{array}{lll}0.000 E+00 & 0.000 E+00 \quad 0.000 E+00\end{array}$

19 DOES CONFINEMENT ISOLATE?

DEP. INPUT PROB $\mathrm{II}_{\text {Iso-By }} \mathrm{nIs-V18} \mathrm{nI-180k} 205548$ nIs-Sig nIso-F

9.425E-01 8.029E-04 2.440E-03 $1.028 E-02 \quad 4.055 E-03 \quad 4.029 E-02$

$10.000 \mathrm{E}+00$

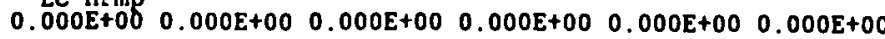




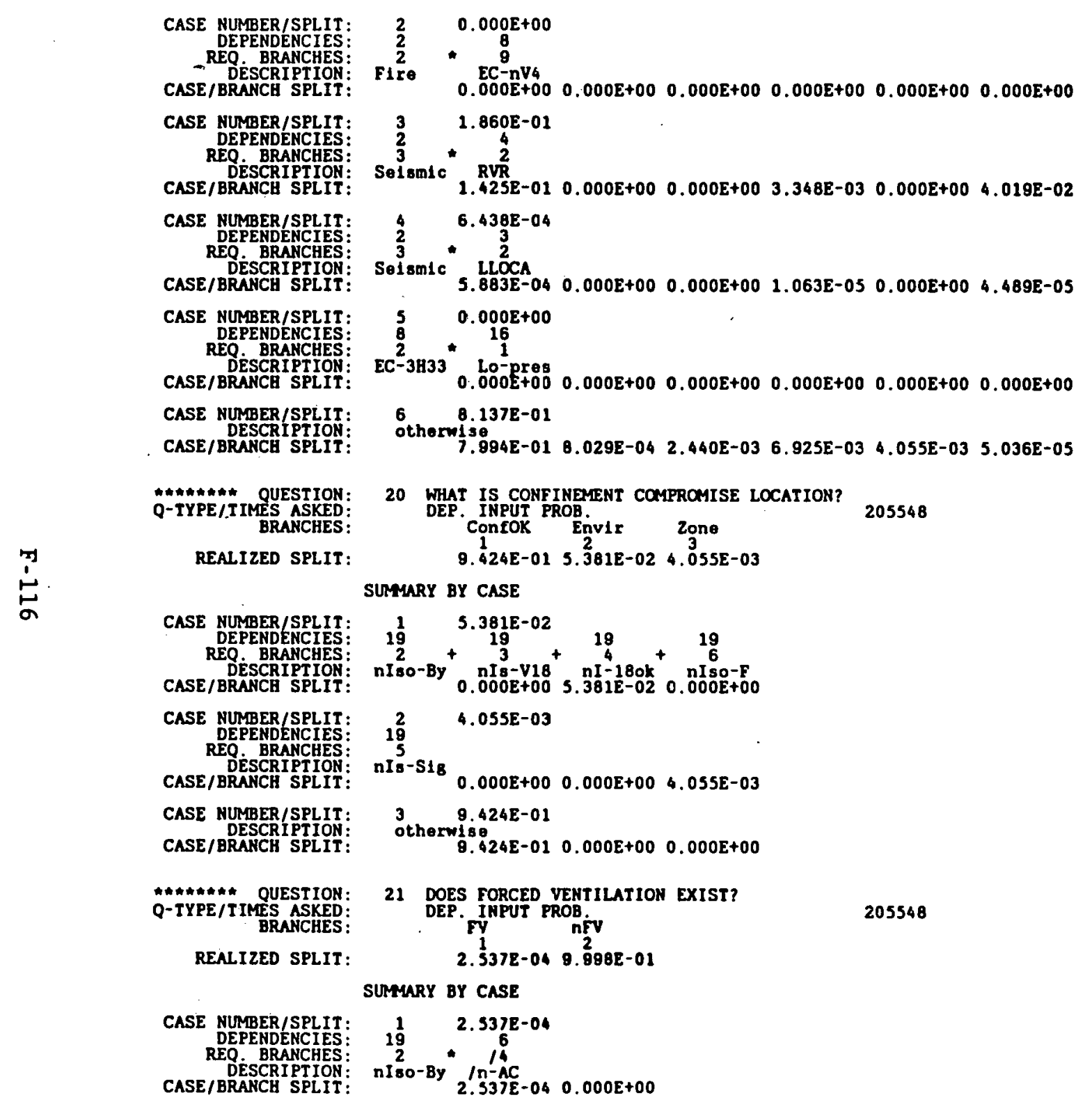


CASE NUMBER/SPLIT: 2 9.998E-01

DESCRIPTION:
CASE/BRANCH SPLIT:

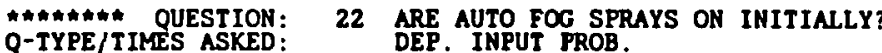

Q-TYPE/TIMES ASKED:
BRANCHES :

REALIZED SPLIT:

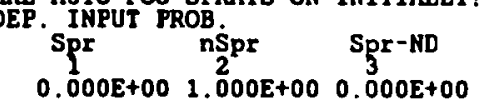

205548

SUMMARY BY CASE

CASE NURBER/SPLIT:
DEPEMDENCIES:
REO BRANCHES:

DESCRIPTION:
CASE/BRANCH SPLIT:

$\frac{1}{2}$
2
3

CASE RUMBER/SPLIT:

DESCRIPTIOH:
CASE/BRANCH SPLIT:

CASE MUMBER/SPLIT:

REO. BRANCHES:

DASE/BRANCRIPTION:

$1.000 E+00$

$0.000 E+00 \quad 1.000 E+00 \quad 0.000 E+00$

CASE NUMBER/SPLIT

REQ. BRANCHES:

CASE/BRANCH SPLIT:

CASE NUMBER/SPLIT:

DED. BRANCHES:

CASE/BRAMCH SPLIT:

$0.000 E+00$

CASE NURAER/SPLIT:

DEPENDNCIES

REQ BRANCHES:

CASE/BRANCH SPLIT:

CASE NUMBER/SPLIT:

DEPENDENCIES: $1 \frac{1}{3}$

DESCRIPTION:
CASE/BRANCH SPLIT:

CASE NUMBER/SPLIT:

DESCRI PTION:
CASE/BRAYCH SPLIT:

\section{$8 \quad 0.000 \mathrm{E}+100$}

$0.000 E+00 \quad 0.000 E+00 \quad 0.000 E+00$

Q-TYPE MU QUESTION :

23 STATUS OF CONEINEMENT H2 MITIGATION SYSTEM?

BRANCHES:

REALIZED SPLIT:

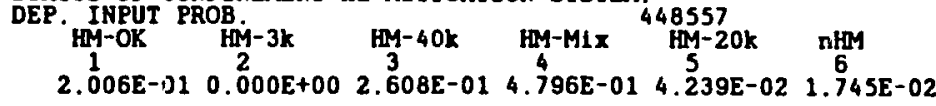

SUMMAZY BY CASE 
CASE NUMBER/SPLIT

REQ. BRANCHES:

DESCRIPTION:
CASE/BRANCH SPLIT:

$\frac{1}{2} 1.866 \mathrm{E}-01$

SelsmLc $\stackrel{3}{2} \stackrel{3}{2} \cdot \prod_{11}^{11}$

CASE NUMBER/SPLIT:

DEPENDENCIES: $\quad 2 \quad 1.977 \mathrm{E}-06$

REQ. BRANCHES: 3 औ 3

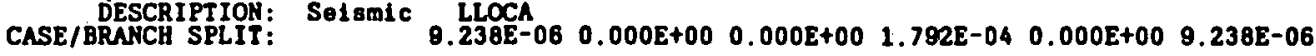

CASE RUMBER/SPLIT:

CASE/BRAKCH SPLIT:

CASE NUMEER/SPLIT:

BRANCHES:

CASE/BRANCH SPLIT:

CASE RUMBE/SPLIT:

REQ. BRANCHES:

CASE/BRAKCH SPLIT:

CASE NUMBER/SPLIT:

DEPENDENCIES:

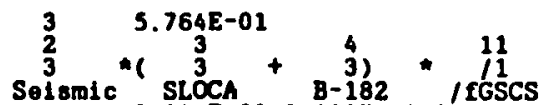

$0.000 E+002.479 E-01 \quad 2.825 E-01 \quad 4.034 E-02 \quad 5.746 E-03$

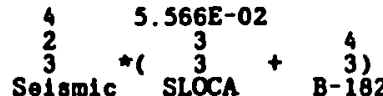

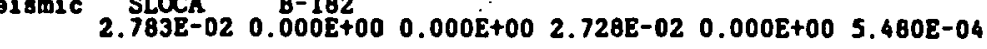

$52 \quad 5.8118-03$

( jícs

$\begin{array}{llll}0.000 E+00 & 0.000 E+00 \quad 4.847 E-03 & 5.754 E-05 \quad 7.488 E-04 & 5.754 E-05\end{array}$

$6 \quad 1.763 \mathrm{E}-01$

CASE/BRANCH SPLIT:

Seismic

CASE NUMBER/SPLIT:

DEPENDENCIES:

REQ. BRANCHES:

CASE/BRANCH SPLIT:

$7 \quad 0.000 E+00$

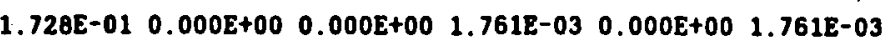

CASE NUMBER/SPLIT:

DEPENDENCIES:

REQ. BRANCHES:

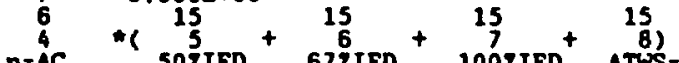

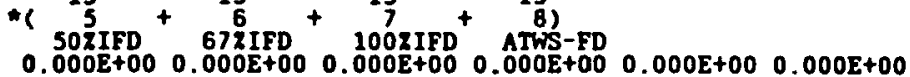

$8 \quad 0.000 E+00$

CASE/BRANCE SPLIT:

- 151 . 15

CASE NUMBER/SPLIT:

DEPENDENCHES:

RE DCRIPIION:

$0.000 E+00 \quad 0.000 E+00 \quad 0.000 E+00 \quad 0.000 E+00 \quad 0.000 E+00 \quad 0.000 E+00$

$15 \quad 0.000 E+00$

$15+15+15+15$

CASE/BRANCH SPLIT:

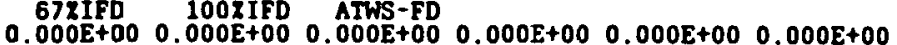

$10 \quad 0.000 \mathrm{E}+00$

REO. BRANCHES: 15,15

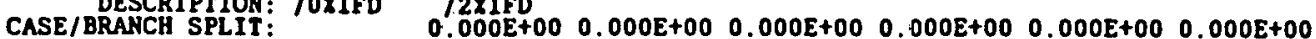

CASE NUMBER/SPLIT: $11 \quad 0.000 E+00$

DEPENDERCIES: 88 BRANCHES: $\quad 12, \quad 8$

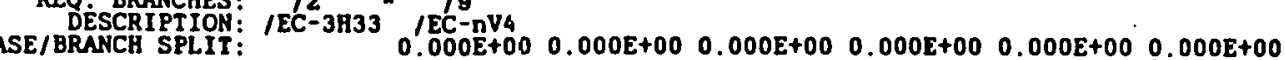

CASE NUMBER/SPLIT: $12 \quad 0.000 E+00$

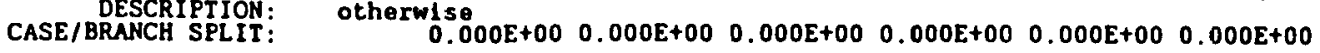


Q-TYMA Q-TYPE/TIMES PSKED:

REALIZED S.PLIT

SE NUMBER/SPLIT

DEPENDENCIES:

CASE/BRANCH SPLIT:

REO. BRANCHES :

DASE / BRANCH SPLIT:

CASE NUMBER/SPLIT:

CASE/BRANCH SPLIT:

O-TYAHE QUESTION:

Q-TYPE / T IMES ASKED:
BRAFCHES:

REALIZED SPLIT:

CASE NUMBER/SPLIT:

REO. BRA HCHES:
REO. BRANCHES:
DESCRIPTION :

CASE MUMBER/SPLIT:

24 IS $3 \mathrm{Z}$ H2 IN CONFINEMENT?

$$
\text { DEP. INPUT PROB. }
$$

$0.000 \mathrm{E}+00 \quad 1.000 \mathrm{E}+00$

448557

SUMMARY BY CASE

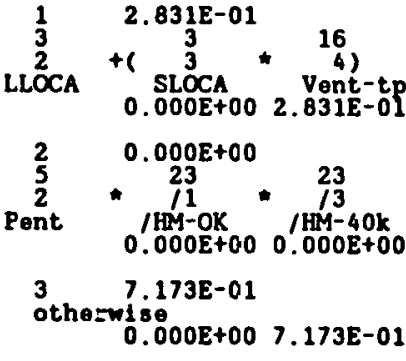

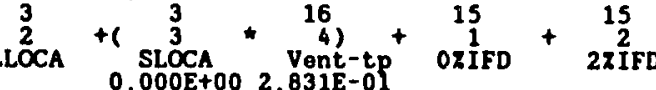

25 DOES OPERATOR ISOLATE VENTS BASED ON $32 \mathrm{H}$ ? DEP. INPUT PROB. ${ }_{\text {H2-IsO }}{ }^{\circ}$. H2-IsO $\mathrm{HL}_{2}-\mathrm{nI} \mathrm{sO}$

SUMMARY BY CASE

CASE/BRANCH SPLIT:

CASE MUMBER/SPLIT:

DESCRIPTION:
CASE/BRANCH SPLIT:

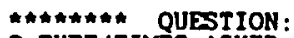

Q-TYPE / TIMES ASKED:

REALIZED SPLIT:

$\stackrel{1}{24}$

$0.000 E+00$

$0.000 E+00 \quad 0.000 E+00$

$21.000 \mathrm{E}+00$

$0.000 E+00 \quad 1.000 E+00$

26 DID VENTS OPEN IF DEMANDED?

Vent-Op Vnt-nOp NA-Vent

2. $130 \mathrm{E}-01 \quad 1.091 \mathrm{2}-047.173 \mathrm{E}-01$

SUMMARY BY CASE

CASE NUMBER/SPLIT

DEPENDENCIES:

REQ. BRANCHES:

CASE/BRANCH SPLIT:

CASE NUMBER/SPLIT:

REO. BRPNCHES:

CASE/BRANCH SPLIT:

CASE NUMBERISPLIT:

DESCRI PTION:
CASE/BRANCH SPLIT :
451855 


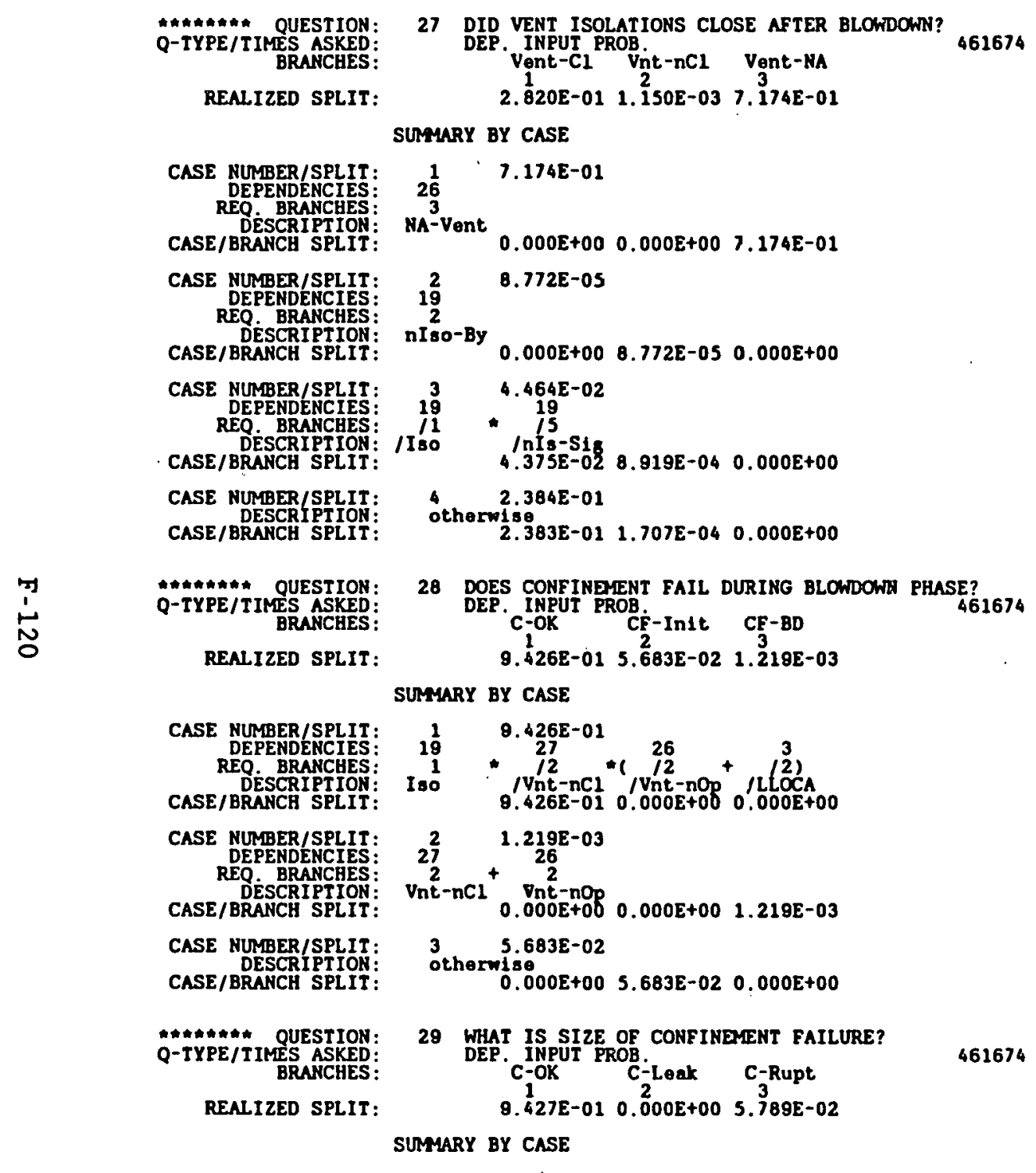


CASE NUMBER/SPLIT:
DEPENDENCIES:
REO BRANCHES

DESCRIPTION:

CASE/BRANCH SPLIT

CASE NUMBER/SPLIT:

CASE/BRANCH SPLIT:

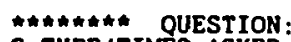

Q-TYPE/TIMES ASKED:

REALIZED SPLIT:$$
\text { DERT: }
$$

SAE NUMER/SPLIT:

REQ BRANCHES:

DESCRIPTION:
CASE/BRANCH SPLIT:

CASE NUMBER/SPLIT:

DEPENDENCIES:

DESCRIPTION:
CASE/BRANCE SPLIT:

CASE NUMBER/SPLIT:

DEPENDENCIES:

REQ. BRANCHES:

DESCRIPTION:
CASE/BRANCH SPLIT:

CASE NUMBER/SPLIT:

CASE/BRANCH SPLIT:

\#\#\#\#\#\# QUESTION:
Q-TYPE/TIMES ASKED:
BRANCHES:

REALIZED SPLIT:

$$
\text { REALIZD SPLIT: }
$$

CASE NUMBER/SPLIT:

RDO BRANCHES

DASE/BRANCH SPLIT:

CASE NUMBER/SPLIT:

CASE/BRAKCA SPLIT:

Q Q TYPE/TIIESTION: TYPE/TIMES ASKED:
BRANCHES:

REALI ZED SPLIT:

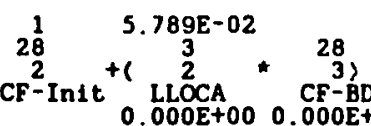

$2 \quad 9.427 E-01$

$\begin{array}{lll}9.427 E-01 & 0.000 E+00 \quad 0.000 E+00\end{array}$

30 WHAT IS CONFINEMENT FAILURE LOCATION?

ConfOK PROB. Envis Zone
Co.

9.427E-01 5.385E-02 4.037E-03

SURMARY BY CASE

1 0.427E-01

9.427E-01 $0.000 E+00 \quad 0.000 E+C O$

20

$0.000 E+00 \quad 5.370 E-02 \quad 0.000 E+10$

$23 \quad 1.550 \mathrm{E}-04$

औ

$0.000 \mathrm{E}+00 \quad 1.550 \mathrm{E}-04 \quad 0.000 \mathrm{E}+00$

$4.037 E-03$

$0.000 E+00 \quad 0.000 E+00 \quad 4.037 \varepsilon-03$

31 DO OPERATING FOG SPRAYS SURVIVE?

DEP. INPUT PROB

1.000E+00 2.060E-05

SUMMARY BY CASE

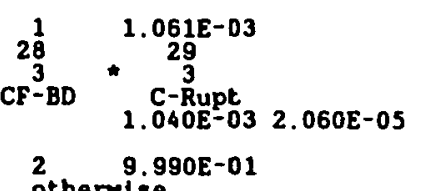

$\begin{array}{rl}2 & 9.960 E-01 \\ \text { otherwise } & 9.990 E-01 \quad 0.000 E+00\end{array}$

32 IS FILTER RELEASE PATH ESTABLISHED?

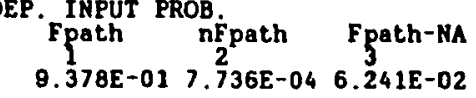

SUMMARY BY CASE
461674

462352

485140 


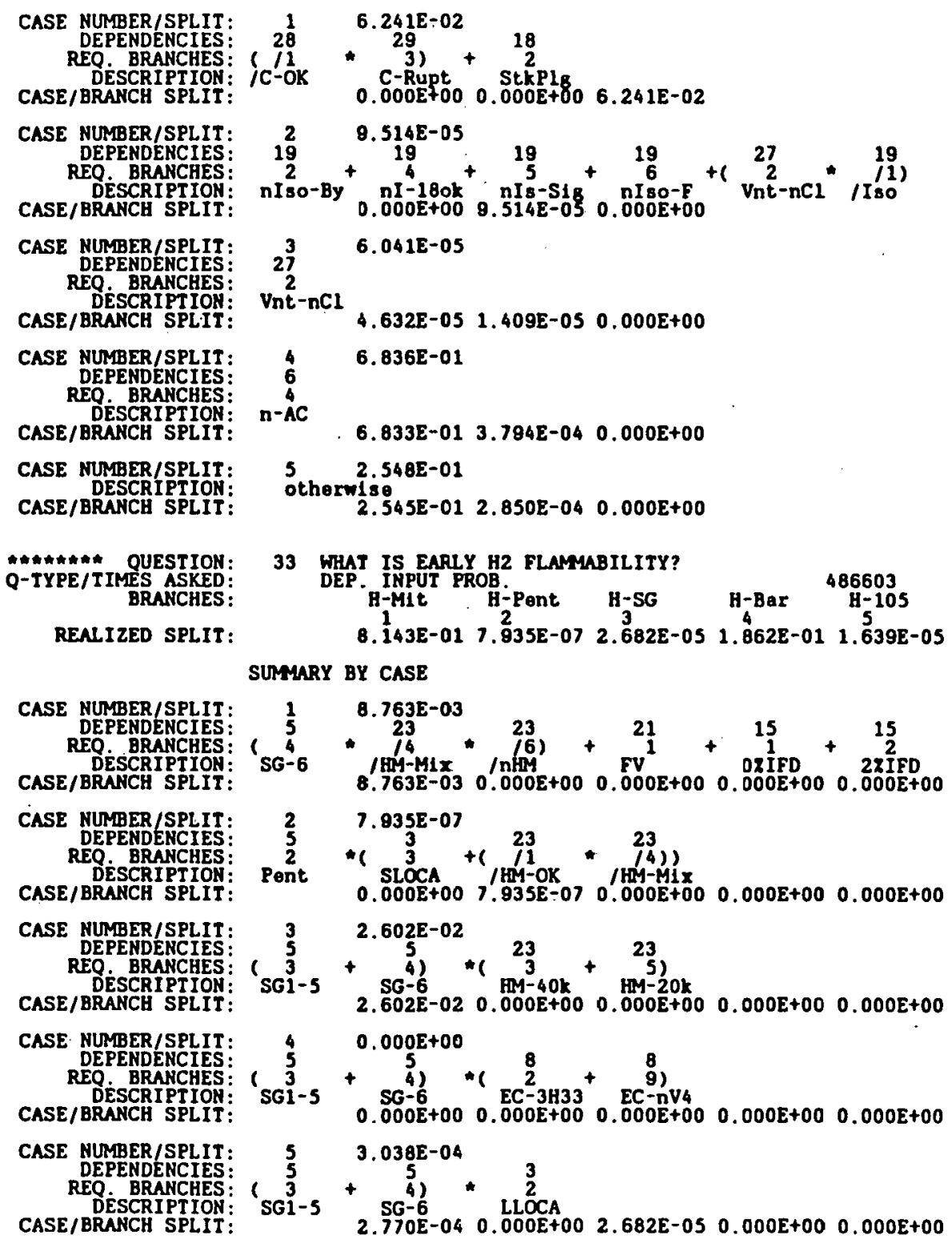




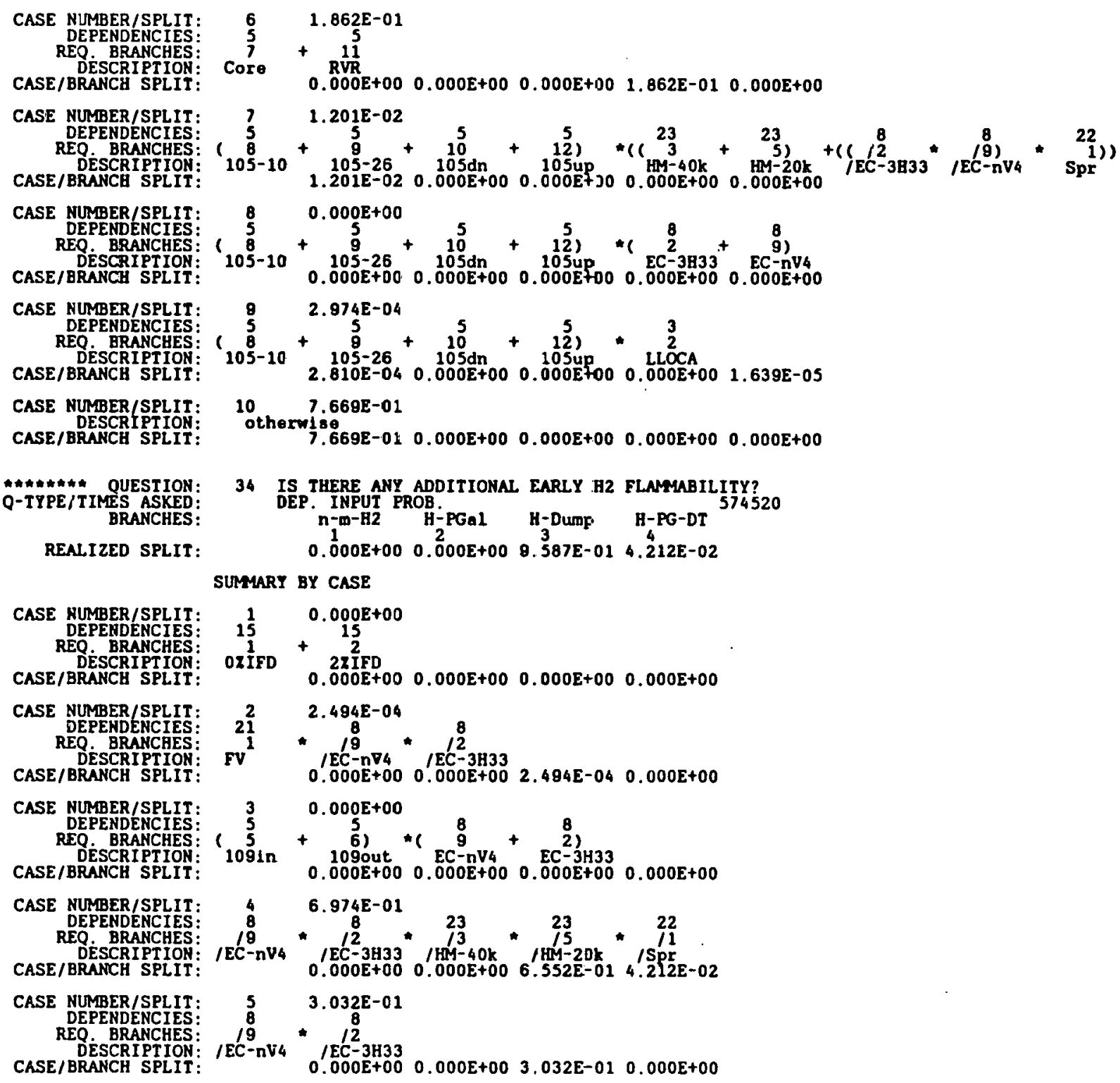




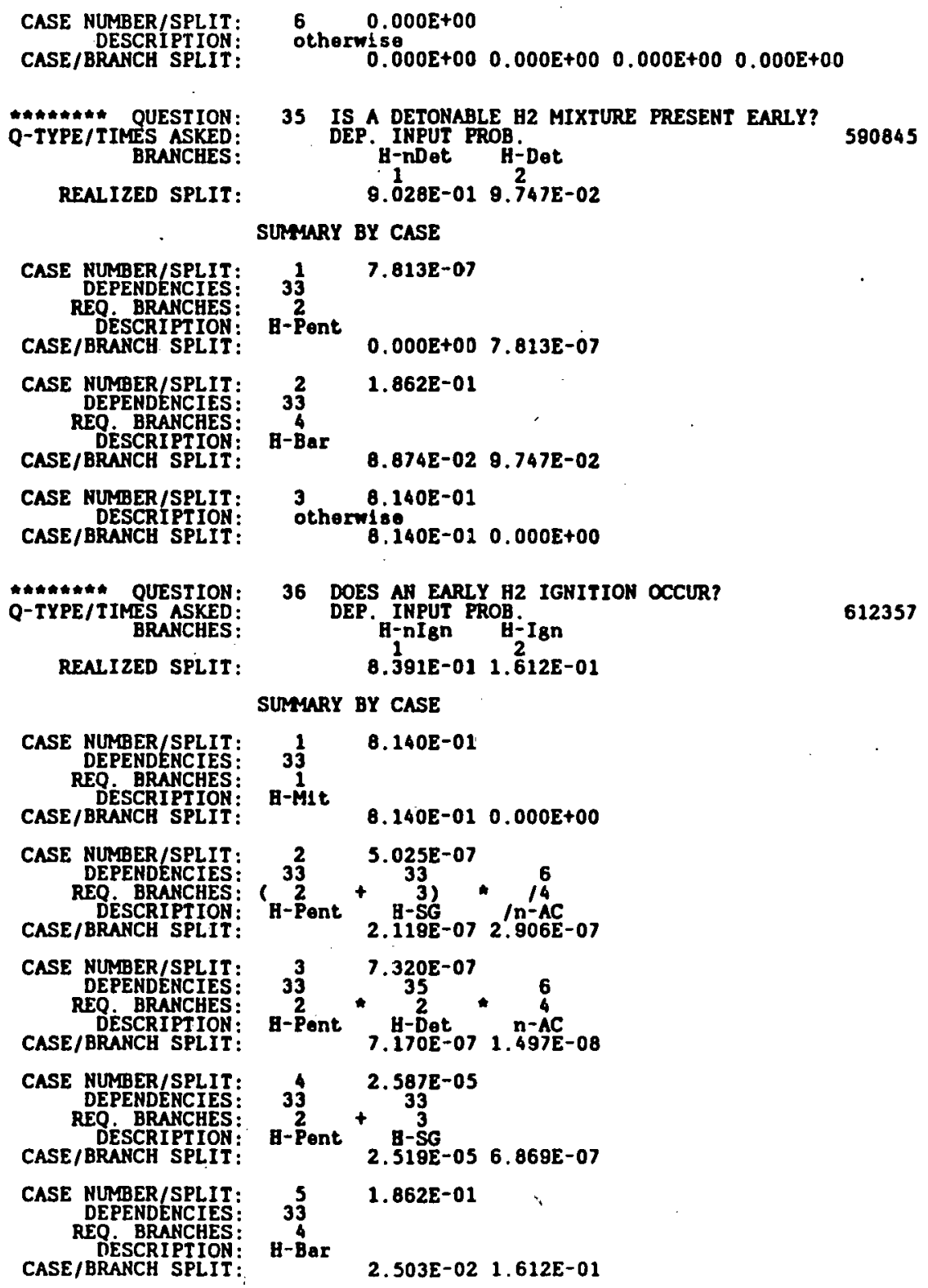




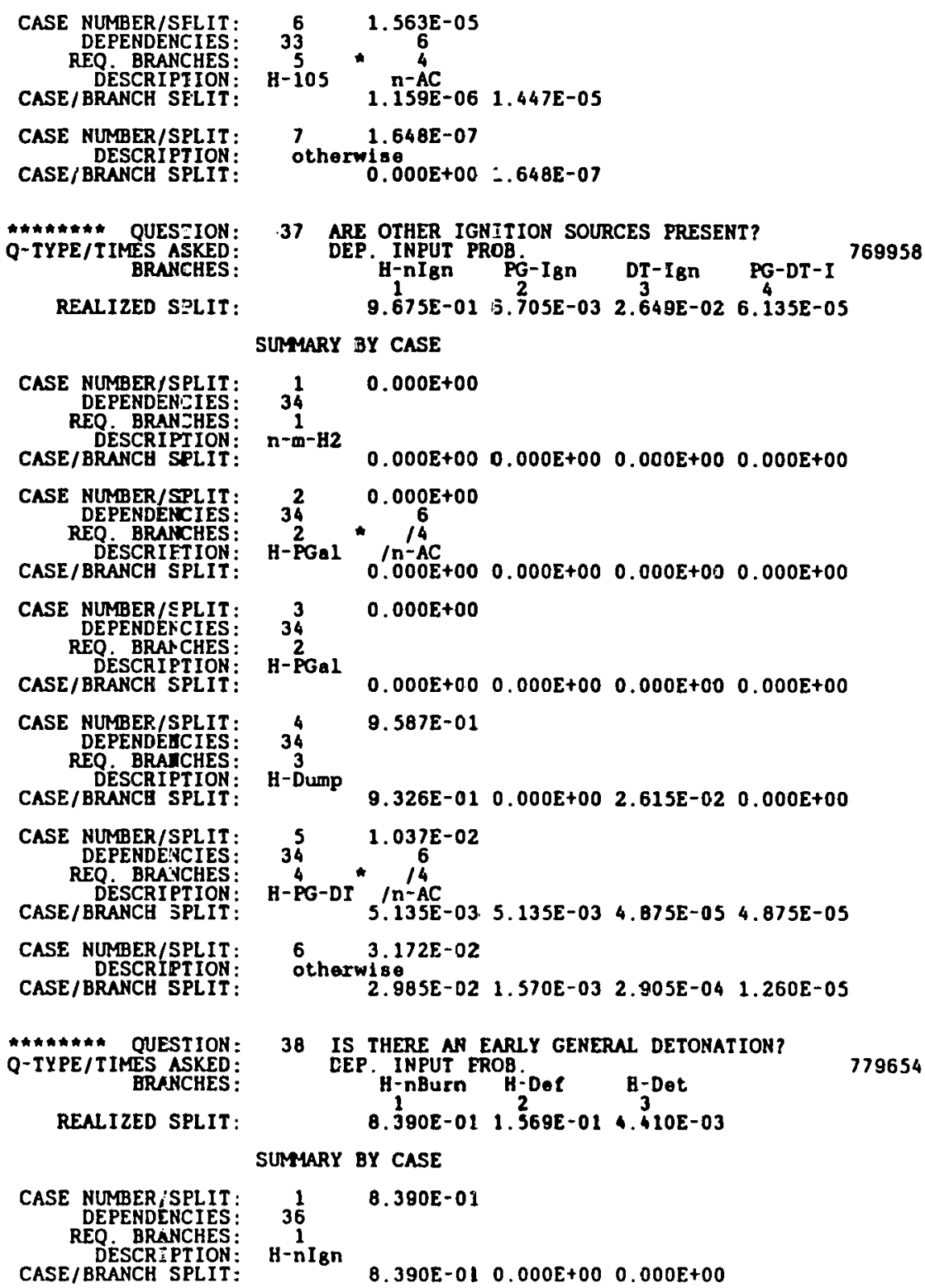


CASE NUMBER/SPLIT

REQ. BRANCHES

CASE/BRANCH SPLIT

$32 \quad 7.669 E-02$

i-not

CASE NUMBER/SPLIT:

CASE/BRANCE SPLIT

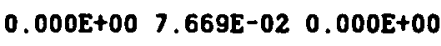

CASE NUMBER/SPLIT:

REO BRANCHES:

CASE/BRANCH SPLIT:

$33.399 \mathrm{E}-09$

2.23

$1 \mathrm{HM}^{12}-3 \mathrm{k} / \mathrm{H}$

$0.000 E+00 \quad 3.399 E-09 \quad 0.000 E+00$

B.221E-09

2

CASE NUMBER/SPLIT: $5 \quad 8.460 E-02$

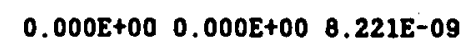

DESCRIPTION:
CASE/BRANCG SPLIT:

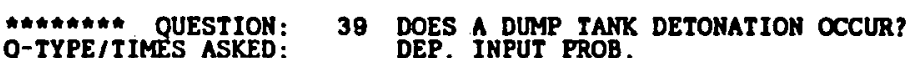

BRANCHES:

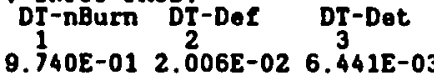

830518

REALIZED SPLIT:

SUMYARY BY CASE

CASE NUMBER/SPLIT:
DEPENDENCIES:

REQ. BRANCHES:

CASE/BRANCH SPLIT:

CASE NUMBER/SPLIT:

CASE/BRANCH SPLIT:

$37 \quad 2.650 \mathrm{E}-02$

DT-Ign $\begin{gathered}\text { PG-DT-I } \\ 0.000 E+00\end{gathered} 2.006 E-02 \quad 6.441 E-03$

$2 \quad 9.740 E-01$

8.740E-01 $0.000 E+00 \quad 0.000 E+00$

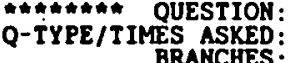

40 IS He BLANKET INTACT?

DEP. INPUT PROB.

8. $141 E-01 \quad 2.864 E-01$

REALIZED SPLIT:

SUMYARY BY CASE

CASE NUMBER/SPLIT:

REPEARACHES:

CASE/BRANCH SPLIT:

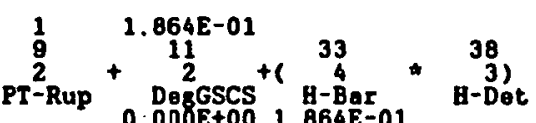

CASE NUMBER/SPLIT: 2 B.1\$1E-01

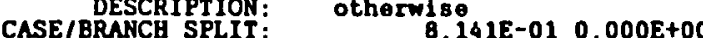

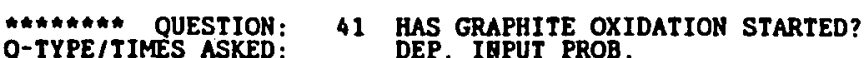

Q-TYPE/TIMES ASKED: $\begin{array}{ccc}\text { Gr-nOx } & \text { Gr-sOx } & \text { Gr-F1re } \\ 1 & 2 & 3 \\ 1.001 E+00 & 4.446 E-04 & 3.835 E-05\end{array}$

SUMMARY BY CASE

REALIZED SPLIT: 


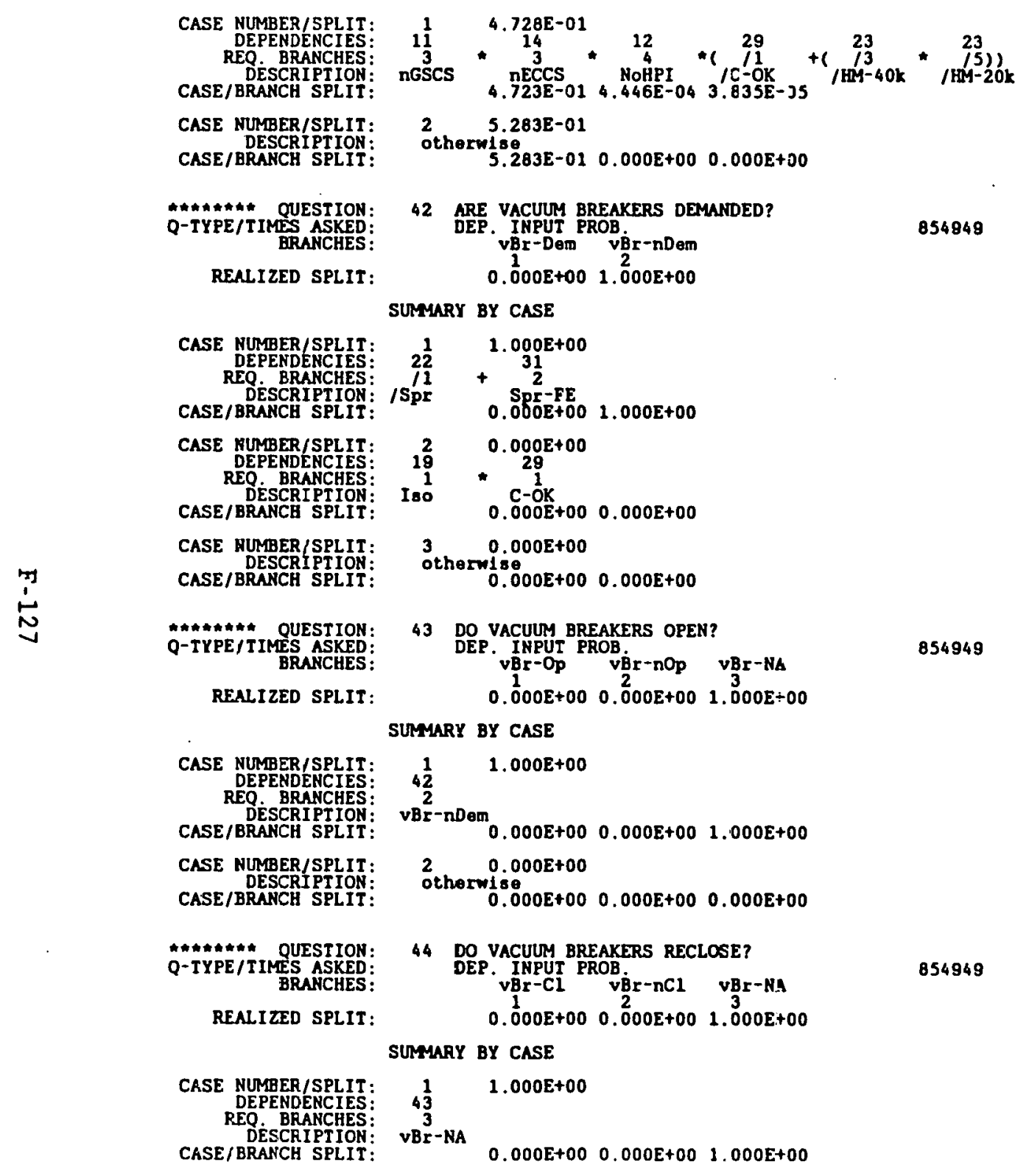




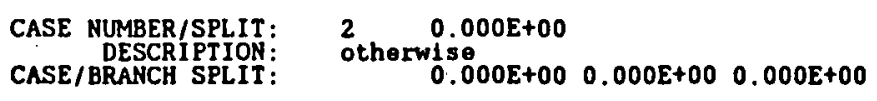

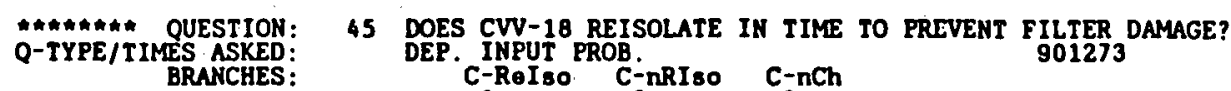

REALIZED SPLIT: $\quad 1.057 E-016.188 E-028.330 E-01$

SUMMARY BY CASE

CASE NUMBER/SPLIT:
DEPENDENCIES:
REQ. BRANCHES:

REO

CASE/BEANCHIPTION:

CASE NUMBER/SPLIT:

DEPENDENCIES:

REQESCRIPTION:
DRSTIT

CASE NUMBER/SPLIT:

DEPENDENCIES:

DESCRIPTION:
CASE/BRANCH SPLIT:

CASE NUMBER/SPLIT:

CASE/BRANCH SPLIT:

QHشW QUESTION:
Q-TYPE/TIMES ASKED:
BRANCHES:

REALIZED SPLIT:

CASE - NUMEER/SPLIT:

DEPENDENCIES:
REQ BRANCHES:

DESCRIPTION:
CASE/BRANCH SPLIT:

CASE NUMBER/SPLIT:

DEPENDENCIES: $\quad 38$

DESCRIPTION:
CASE/BRANCH SPLIT:

-Dot

$0.000 E+00 \quad 4.410 E-03 \quad 0.000 E+00$

$\begin{array}{cc}2 & 1.632 E-01\end{array}$

2 $+{ }^{2}+4$

$\begin{array}{lll}P G-I g n & P G-D T-I & \\ 1.057 E-01 & 5.747 E-02 & 0.000 E+00\end{array}$

$4 \frac{3}{3}$

Gr-F1r0 2.006E-05 3.085E-06 $0.000 E+00$

4 8.330E-01

$0.000 E+00 \quad 0.000 E+00 \quad 8.330 E-01$

46 DOES CONEINEMENT FAIL DURING THE ACGIDENT PROGRESSION? DEF. INPUT PROB. CFCh $-\mathrm{HCF}$-Vac CF-GrOx 949526 $\begin{array}{cccc}\text { C-nCh } & \text { CF-H2 } & \text { CF-Vac } & \text { CF-GrOx } \\ 1 & 2 & 3 \\ 9.152 E-01 & 8.556 E-02 & 0.000 E+00 & 4.746 E-05\end{array}$

SUMMARY BY CASE

CASE NUMBER/SPLIT:

REO BRANCHES: (

CASE/BRANCH SPLIT:

$13.628 E-05$

r-Fir

$\begin{array}{lll}0.000 E+00 & 0.000 E+00 \quad 0.000 E+00 \quad 3.628 E-05\end{array}$

$2 \quad 4.411 E-03$

-Det

$\begin{array}{lll}0.000 E+00 & 4.411 E-03 \quad 0.000 E+00 \quad 0.000 E+00\end{array}$

CASE NUMBER/SPLIT:

DEPENDENCIES:
REO. BRANCHES:

CASE/BRANCH SPLIT:

\author{
$37 \quad 6.733 \mathrm{E}-03$ \\ $\left.{ }^{2}-I_{g n}+4\right) * 15 * 16$ \\ (4) $0.000 E+00$ \\ 2 \\ $0.000 E+00 \quad 0.000 E+00 \quad 0.000 E+00 \quad 0.0 C 0 E+00$
}




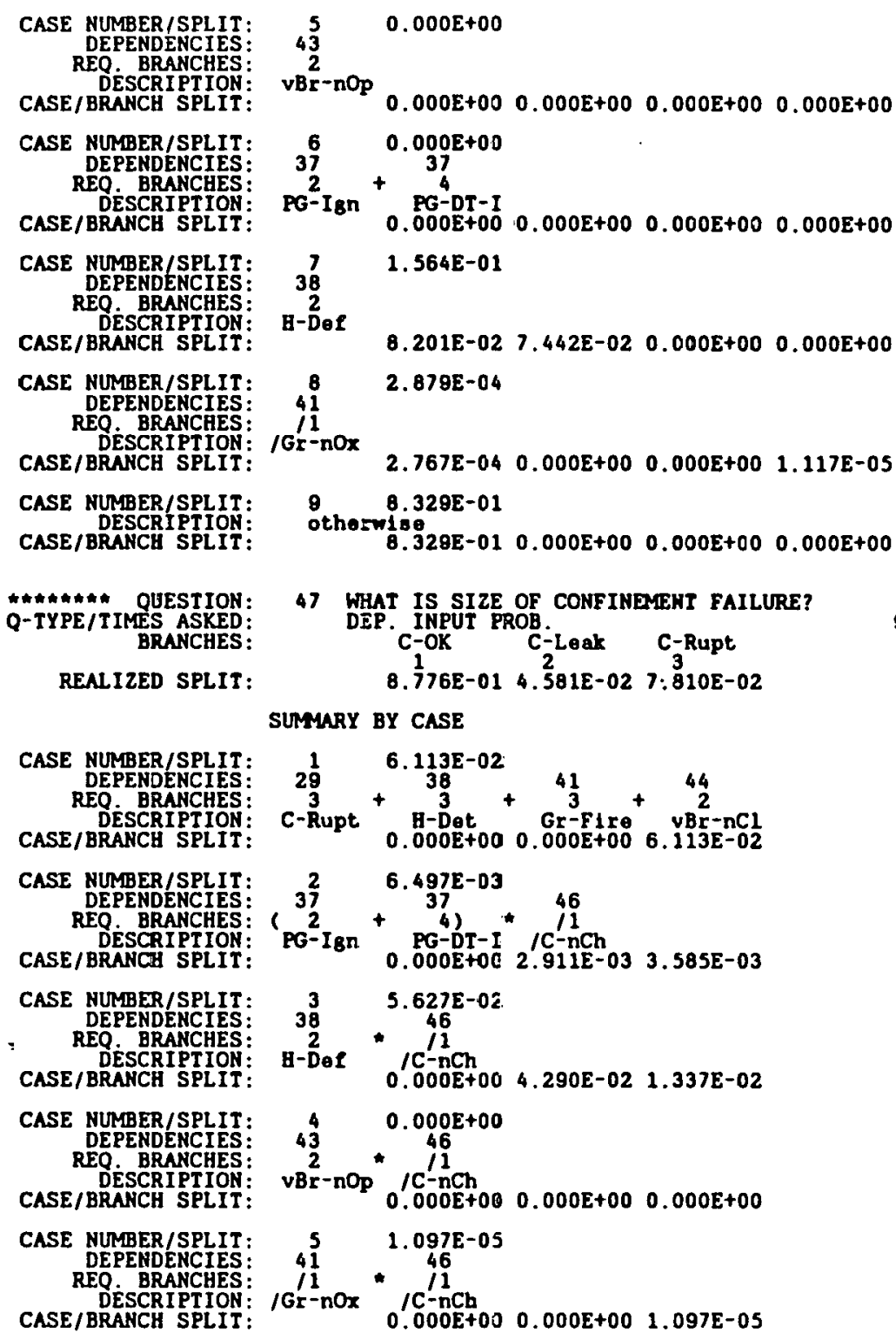




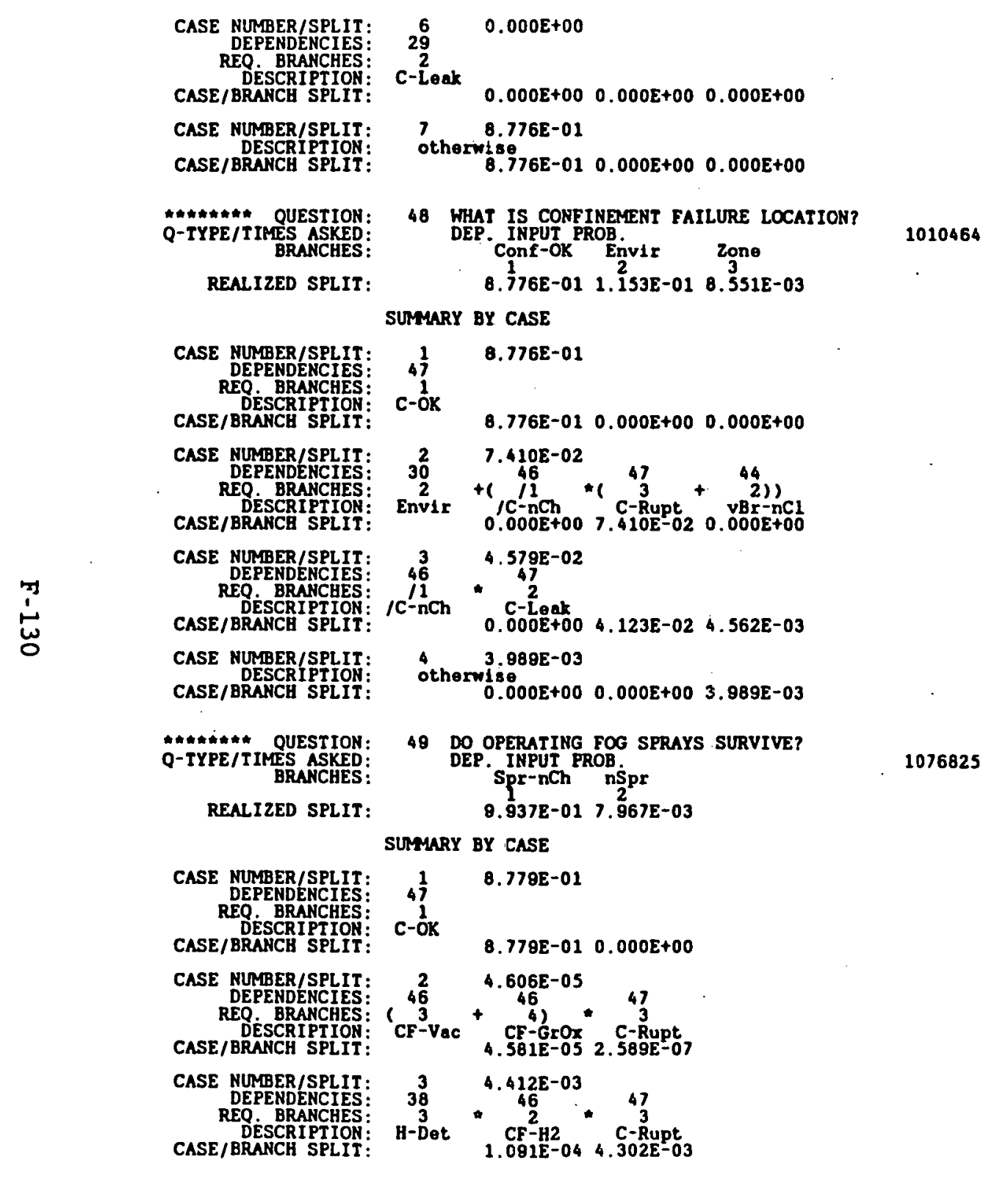




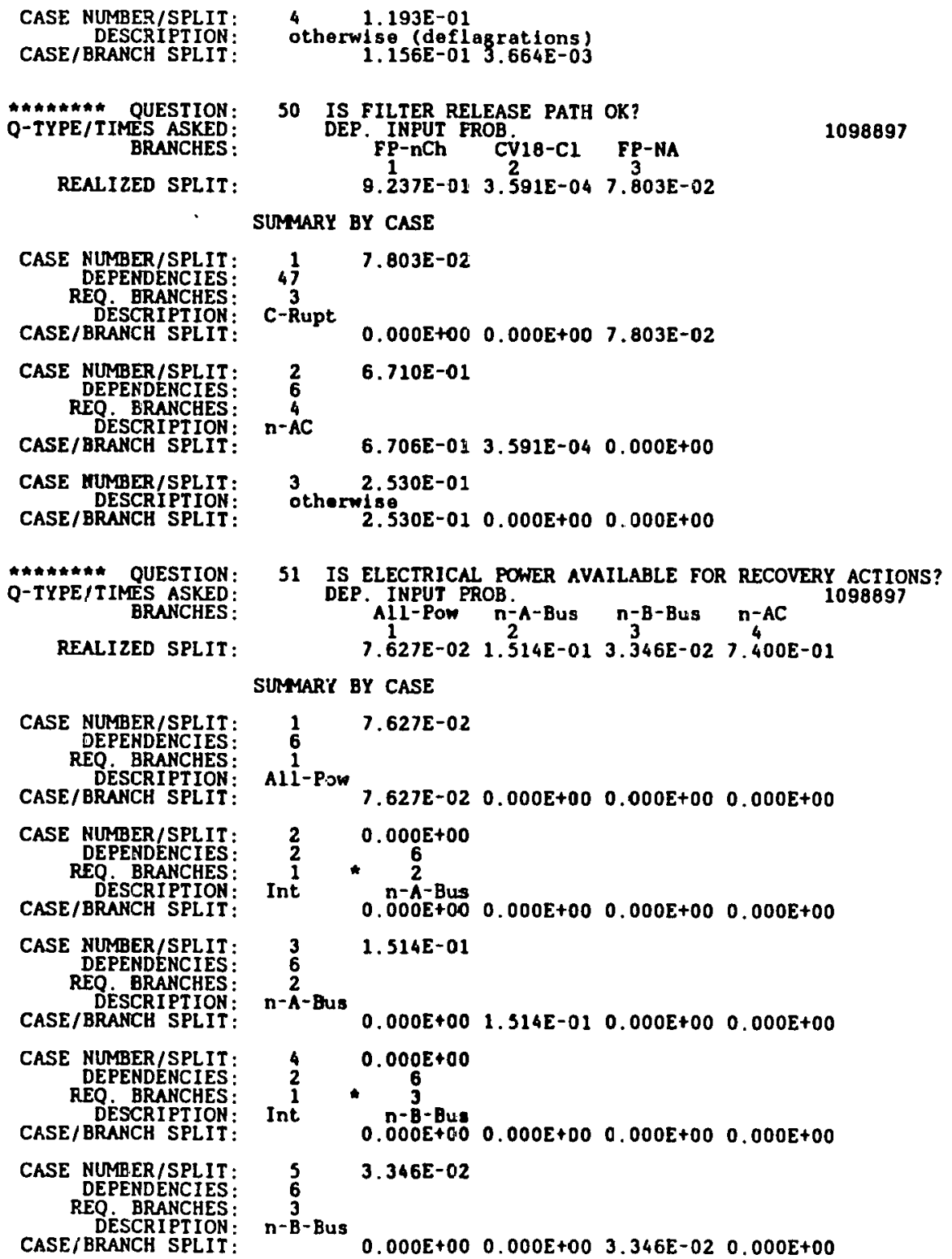

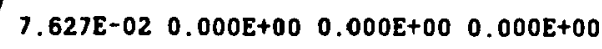

$20.000 E+00$

6
2

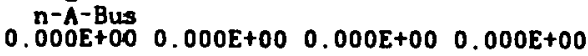

$3 \quad 1.514 \mathrm{E}-01$

n-A-Bus $0.000 E+00 \quad 1.314 E-01 \quad 0.000 E+00 \quad 0.000 E+00$

$4 \quad 0.000 E+00$

6
3

$0.000 E+0.00 .000 E+00 \quad 0.000 E+00 \quad 0.000 E+00$

3. 346E-02

$0.000 E+00 \quad 0.000 E+00 \quad 3.346 E-02 \quad 0.000 E+00$ 


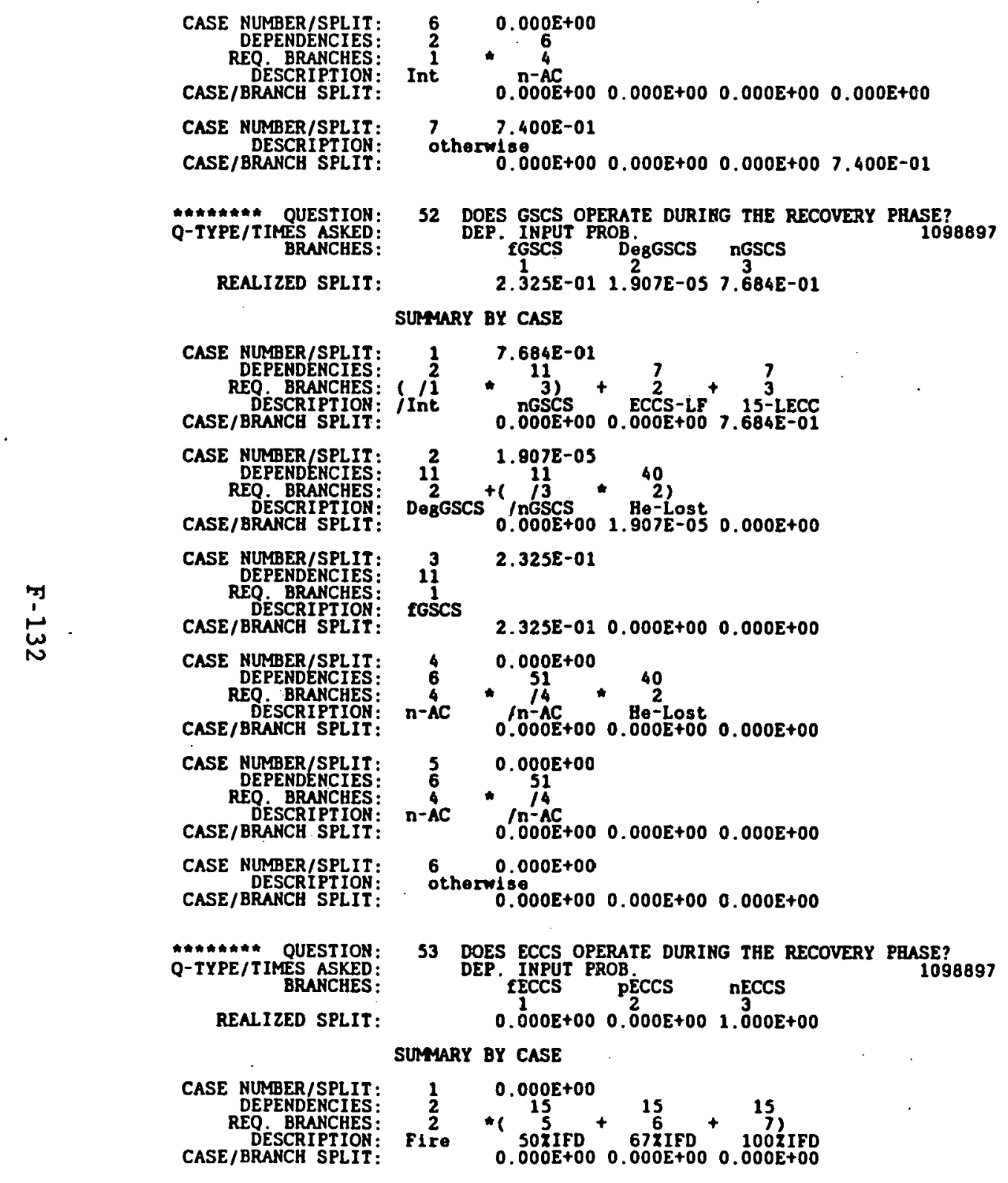


CASE NUMBER/SPLIT:

REQ BRANCHES:

CASE/BRANCH SPLIT

CASE NUMBER/SPLIT

DEPENDENCIES:

DESCRIPTION:
CASE/BRANCH SPLIT:

CASE NUMBER/SPLIT

REO BRANCHES:

DESCRIPTION:
CASE/BRANCH SPLIT:

$\begin{aligned} & 2 \\ & 7 \\ & 2 \\ & 2\end{aligned} \quad 0.000 E+00$

$0.000 E+00 \quad 0.000 E+00 \quad 0.000 E+J 0$

CASE NUMBER/SPLIT:

DEPENDENCIES:

DESCRIPTION:
RELIT:

$1.000 E+00$

$14+\left(\frac{1}{3}+(10+3)\right.$

$0.000 \mathrm{~d}+00 \quad 0.000 \mathrm{E}+0$

$4 \quad 0.000 E+00$

$\begin{array}{lll}0.000 E+00 & 0.000 E+00 & 0.000 E+00\end{array}$

CASE RUMBER/SPLIT:

DEPENDENCIES:

REQ. BRANCHES

CASE/BRANCH SPLIT:

CASE NUMBER/SPLIT:

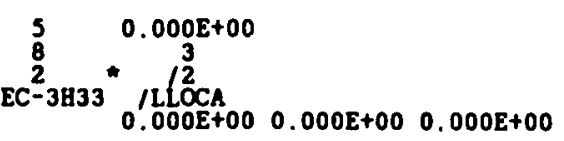

$6 \quad 0.000 \mathrm{E}+00$

EC-3н33" I I

$\begin{array}{ll}\text { IfGSCS } & 000 E+00 \quad 0.000 E+00 \quad 0.000 E+00\end{array}$

$7 \quad 0.000 \mathrm{E}+00$

EC-3H:33

CASE/BRANCH SPLIT:

$\begin{array}{lll}0.000 E+00 & 0.000 E+00 \quad 0.000 E+00\end{array}$

CASE NUMBER/SPLIT

DEPENDENCIES:

REO BRANCHES:

CASE/BRANCH SPLIT:

$80.000 \mathrm{E}+00$

$0.000 \mathrm{E}+00$

CASE NUMBER/SPLIT:

DEPENDENCIES:

REQ. BRANCHES:

CASE/BRANCH SPLIT:

$\begin{array}{ccc}0.000 E+00 & 0.000 E+00 & 0.000 E+00\end{array}$

CASE NUMBER/SPLIT

CASE/BRANCH SPLIT:

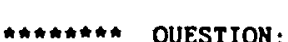

Q-TYPE/TIMES ASKED:

EALIZED SPLIT:

$0.000 E+00$

PECCS

$0.000 E+00 \quad 0.000 E+00 \quad 0.000 E+00$

$10 \quad 0.000 E+00$

$0.000 E+00 \quad 0.000 E+00 \quad 0.000 E+00$

54 DOES HPI OPERATE DURING THE RECOVERY PHASE DEP. INPUT PROB $/ 4$-HPI 1/4-HPI NHPI 1098897 $0.000 \mathrm{E}+00 \quad 0.000 \mathrm{E}+00 \quad 0.000 \mathrm{E}+00 \quad 1.000 \mathrm{E}+00$

SUMYARY BY CASE

CASE NUMAER/SPLIT:

REO. BRANCHES: 1

DESCRIPTION:
ASE/BRANCH SPLIT:

1. $000 E+00$

$\begin{array}{r}8 \\ -41+2 \\ -4\end{array}+\frac{8}{3}+51$

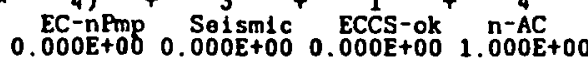




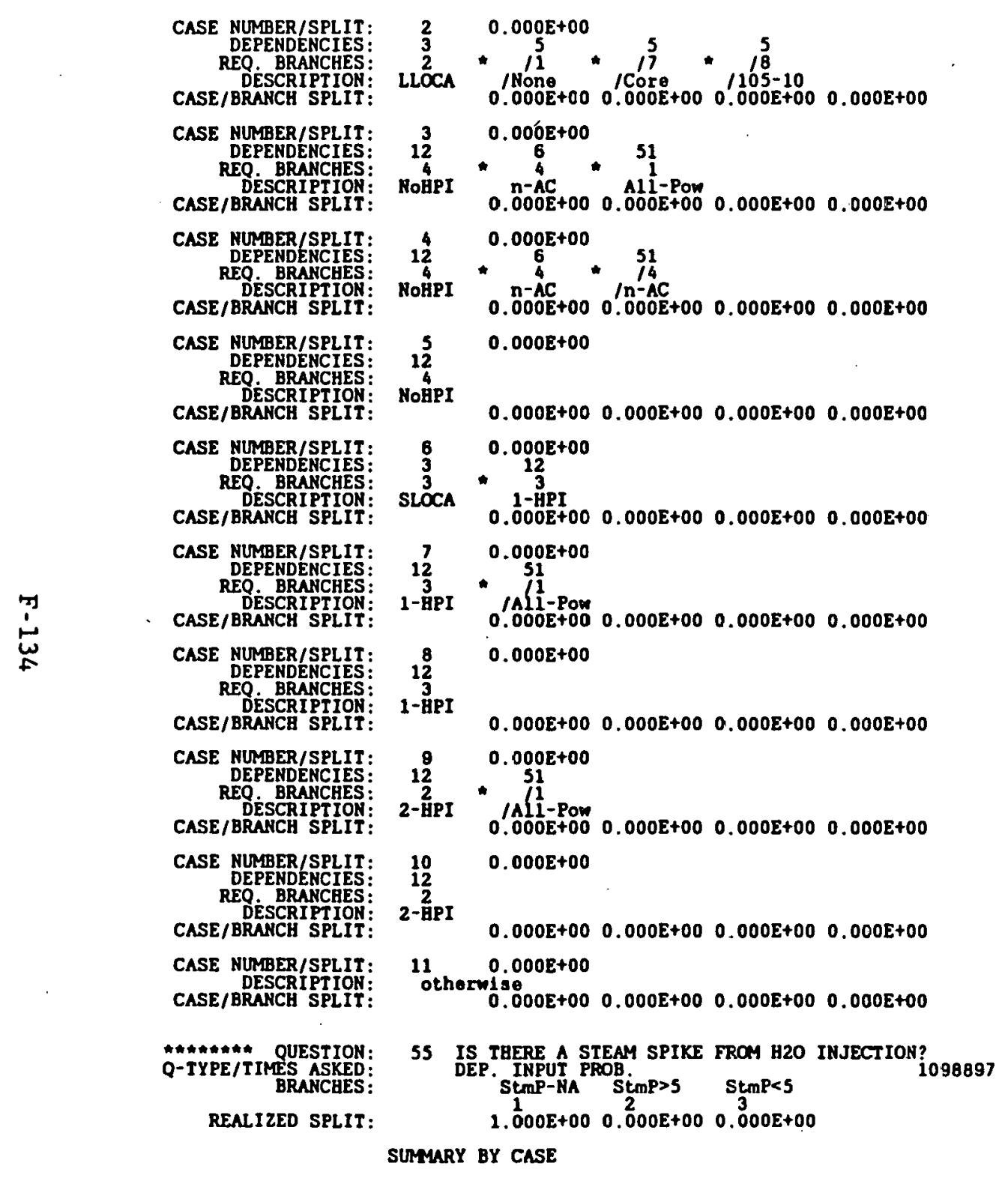




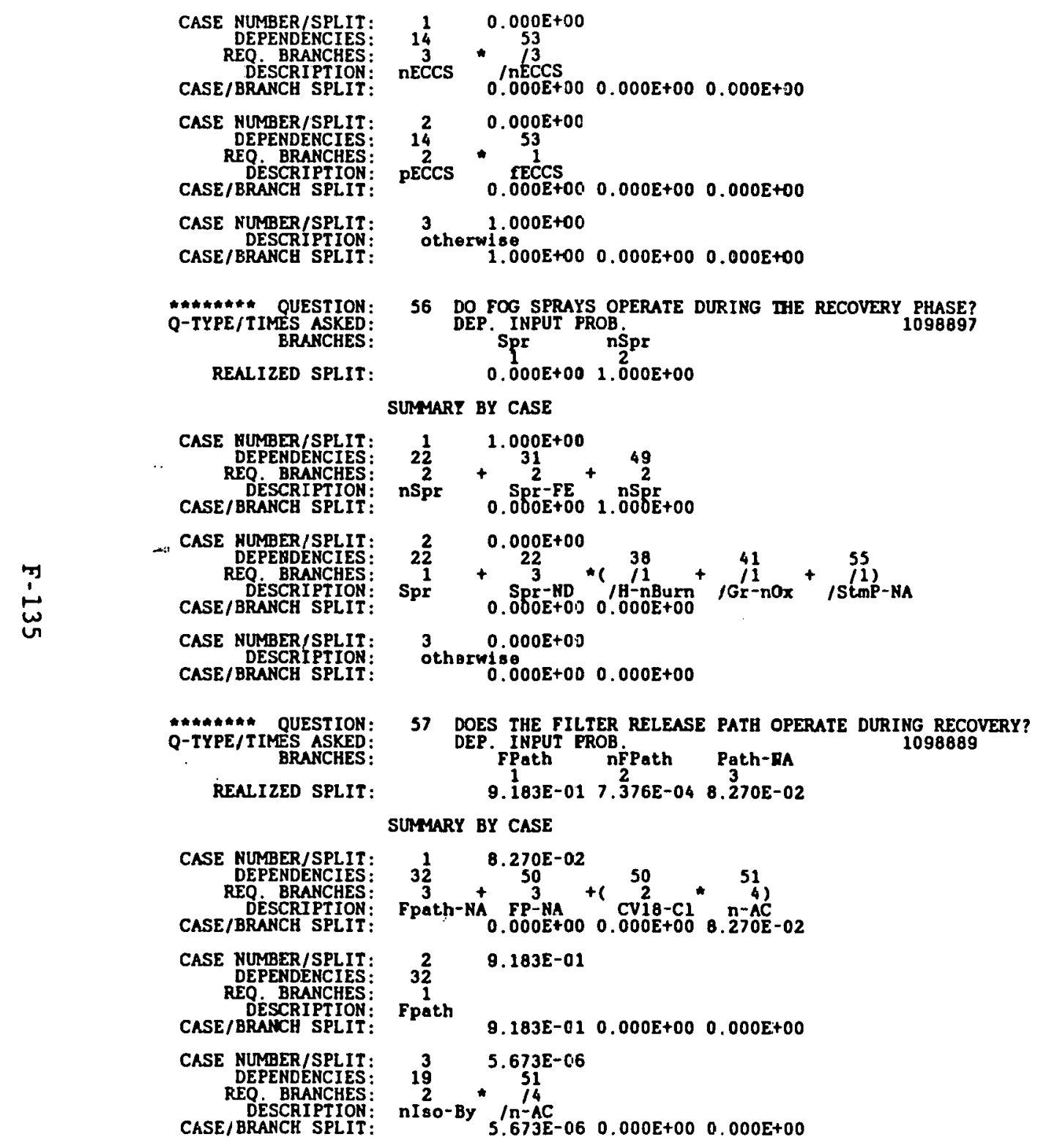




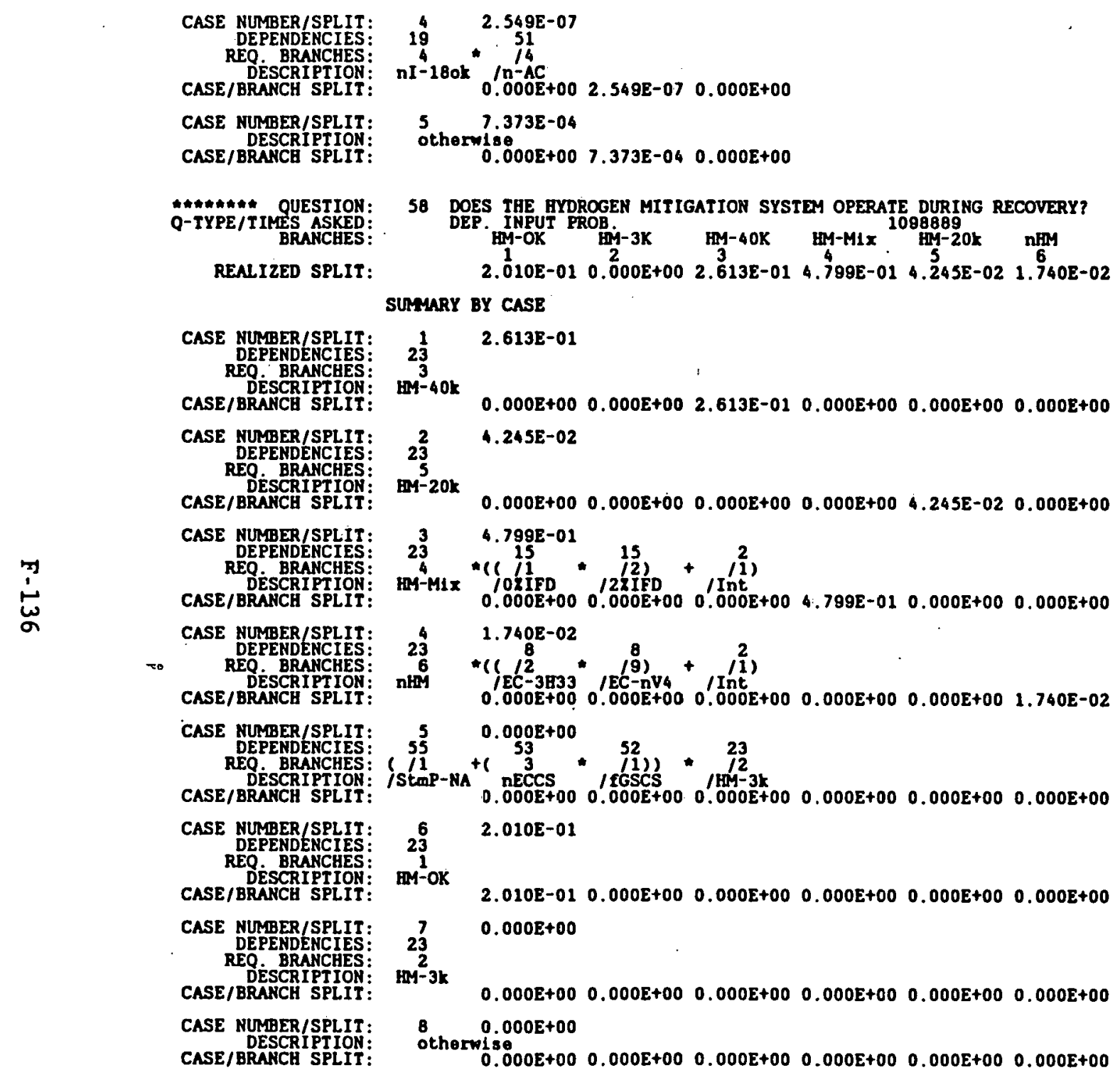




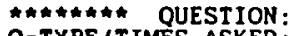

Q-TYPE/TIMES ASKED:

REALIZED SPLIT:

CASE NUMBER/SPLIT

REQ. BRANCHES

DÉSCRIPTION:

CASE NUMBER/SPLIT

REO BRANCHES:

DESCRIPTION
CASE/BRANCH SPLIT

CASE NUMBER/SPLIT:

CASE/BRANCH SPLIT

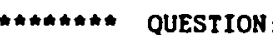
Q-TYPE / TIMES ASKED

REALIZED SPLIT

CASE NUMBER/SPLIT:

REQ. BRANCHES

SE/BRANCH SPLIT:

CASE NUMBER/SPLIT

DEPENDENCIES:

DESCRIPTION

CASE

CASE NUMBER/SPLIT

REO. BRANCHES

DESCRIPTION:
CASE/BRANCH SPLIT:

CASE NUMBER/SPLIT

DESCRIPTION:
CASE / BRANCH SPLIT:

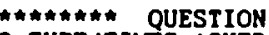

Q-TYPE/TIMES ASKED :

REALIZED SPLIT:

59 DOES PRESSURE TUBE FAI
DEP. INPPU PROB.
TUb-nFT TUbe-Fr
$\begin{aligned} & 1 \\ & 2.332 E-0.1\end{aligned} \quad 7.678 E-01$

SUMMARY BY CASE

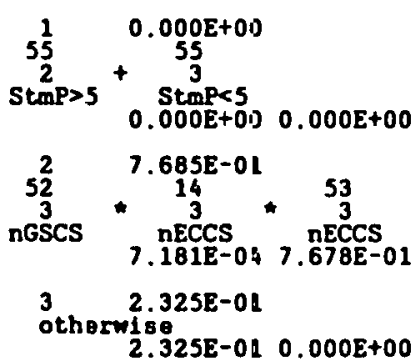

60 HOW MUCR BYYROGEN IS PRODUCED DURING THE ACCIDENT?

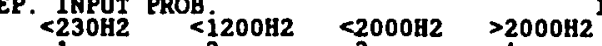

2. $325 E-01 \quad 1.907 E-05 \quad 0.000 E+007.684 E-01$

SUMMARY BY CASE

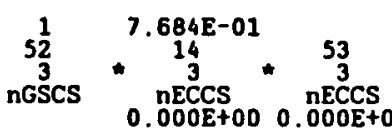

$52 \quad 0.000 E+00$

nGSCS * 3 * 33

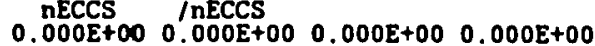

$52 \quad 1.9075-05$

52
$+3) \times(14$
2

$\begin{array}{llll}\text { nGSCS } & \text { PECCS } & \text { nECCS } & \\ 0.000 E+\infty 00 & 1.307 E-05 & 0.000 E+00 & 0.000 E+00\end{array}$

4. 325E-01

2.325E-01 $0.000 E+00 \quad 0.000 E+00 \quad 0.000 E+00$

61 WHAT IS LATE H2 FLAMABILITY?

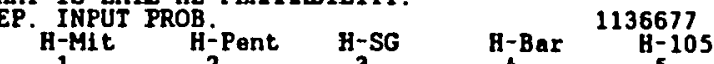

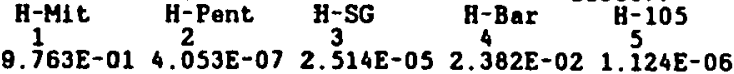

SUMMARY BY CASE

CASE NUMBER/SPLIT:

REO BRANCHES:

CASE/BRANCH SPLIT

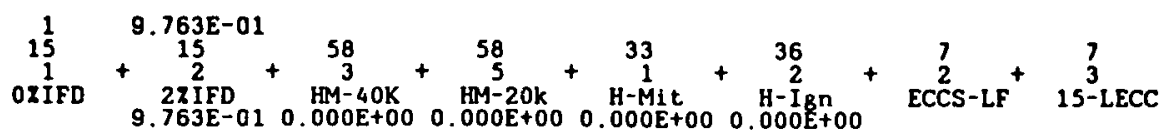




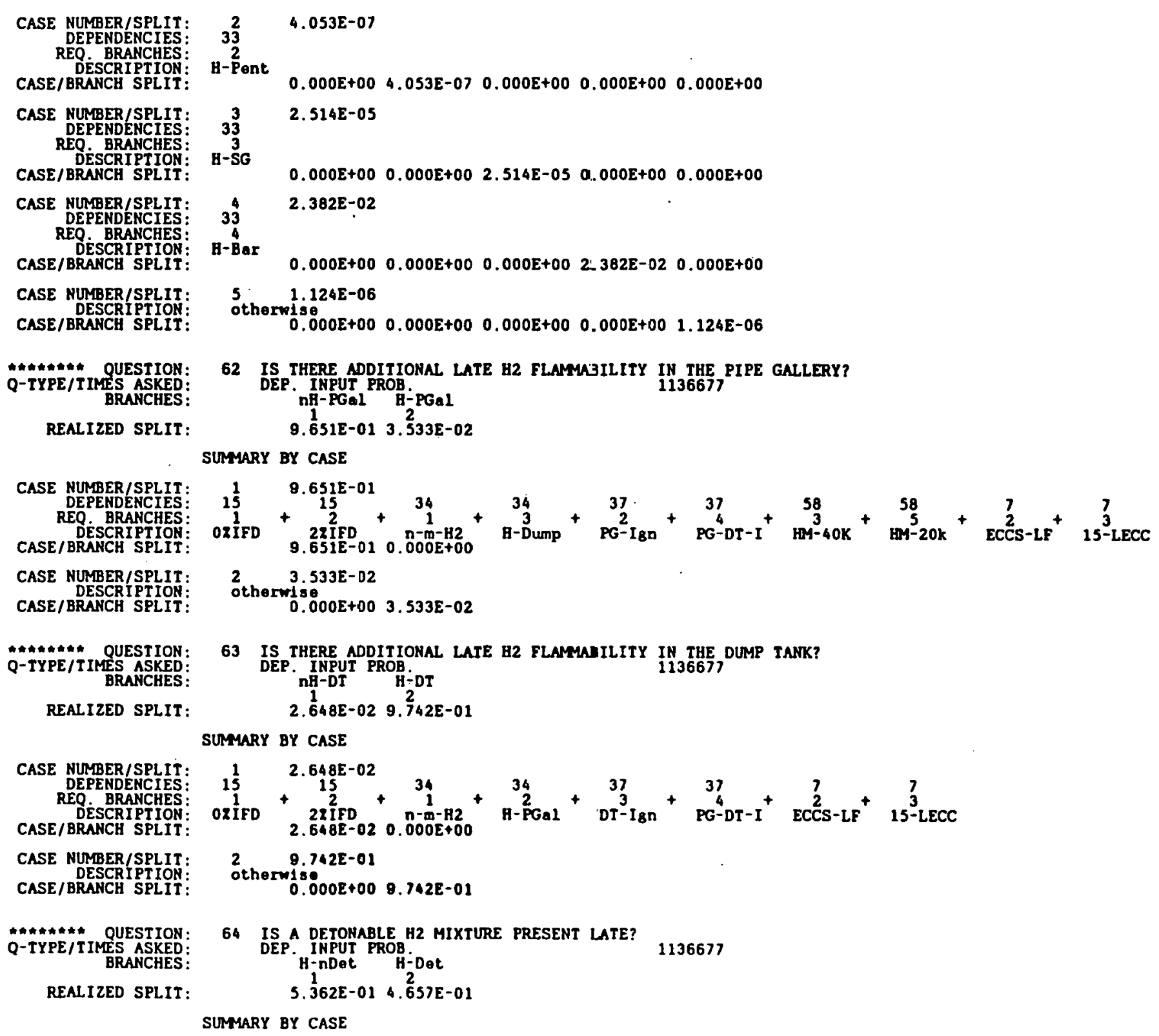

SUMMARY BY CASE 


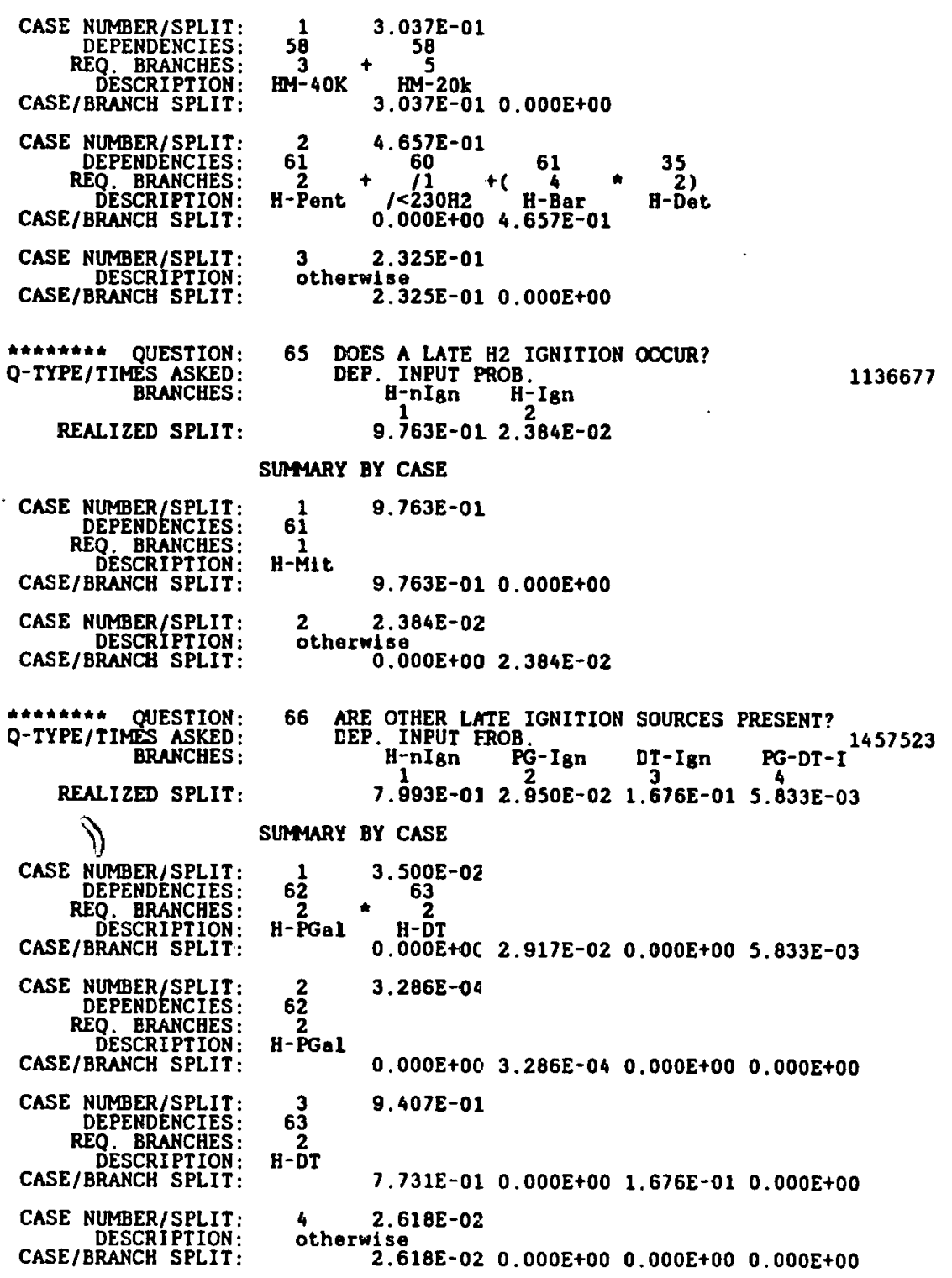


MnthHAm. QUESTION:

Q-TYPE/TIMES ASKED:

REALIZED SPLIT:

67 IS THERE A LATE GENERAL DETONATION?

H-nBurn H-DeE H-Det

$\begin{array}{ccc}1 & 2 & 3 \\ 5.359 E-01 & 1.035 E-05 & 4.659 E-01\end{array}$

SUMYARY BY CASE

CASE NUMBER/SPLIT

REO. BRANCHES:

CASE/BRANCH SPLIT:

CASE NUMBER/SPLIT:

CASE/BRANCE SPLIT:

CASE NUMBER/SPLIT:

CASE/BRAHCH SPLIT

CASE NUMBER/SPLIT:

DEPENDENCIES:

DESCRIPTION:
DPATCH SPLIT:

CASE NUMBER/SPLIT:

REO BRANCHES:

CASE/BRANCH SPLIT:

$14.6598-01$

60.64

$\begin{array}{llll}0.000 \mathrm{E}+00 & 0.000 \mathrm{E}+00 \quad 4.659 \mathrm{E}-01\end{array}$

65

日-nIgn

$\begin{array}{lll}5.359 E-01 & 0.000 E+00 & 0.000 E+00\end{array}$

64

H-nDet

$0.000 E+00 \quad 1.034 E-05 \quad 0.000 E+00$

$\begin{array}{cc}4 & 0.000 E+00 \\ 2 & 23 \\ 2 & 12\end{array}$

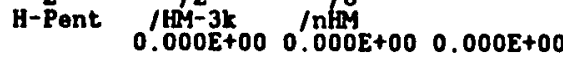

$6 \frac{5}{2}$

H-Pent

8. $182 \mathrm{E}-08$

$0.0008+00 \quad 8.984 E-09 \quad 7.283 E-08$

CASE NUMBER/SPLIT

CASE/BRAHCH SPLIT:

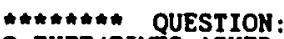

Q-TYPE/ TIMES ASKED

REALIZED SPLIT:

$6 \quad 0.000 \varepsilon+00$

$\begin{array}{lll}0.000 E+00 & 0.000 E+00 \quad 0.000 E+00\end{array}$

68 DOES A DUMP TANK DETONATION OCCUR? DT-nBurn Dí-Def DT-Det

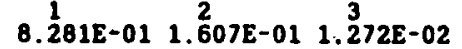

SUMYARY BY CASE

CASE NUMBER/SPLIT:

REO BRANCHES:

DESCRIPTION:

CASE UMBgr

CASE NUMBER/SPLIT:
CASE/BRSCR PRTION:

WH HY A QUESTION
Q-TYPE / T IMES ASKED

REALIZED SPLIT

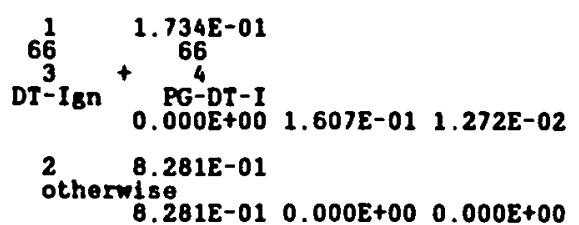

69 IS GSCS EFFICIENCY DECREASED DUE TO LOSS OF HE BLANKET? DEP. INPUT PROB. $\mathrm{He}$-LOSt

2. $332 E-01>2.680 E-01$

SUMMARY BY CASE 


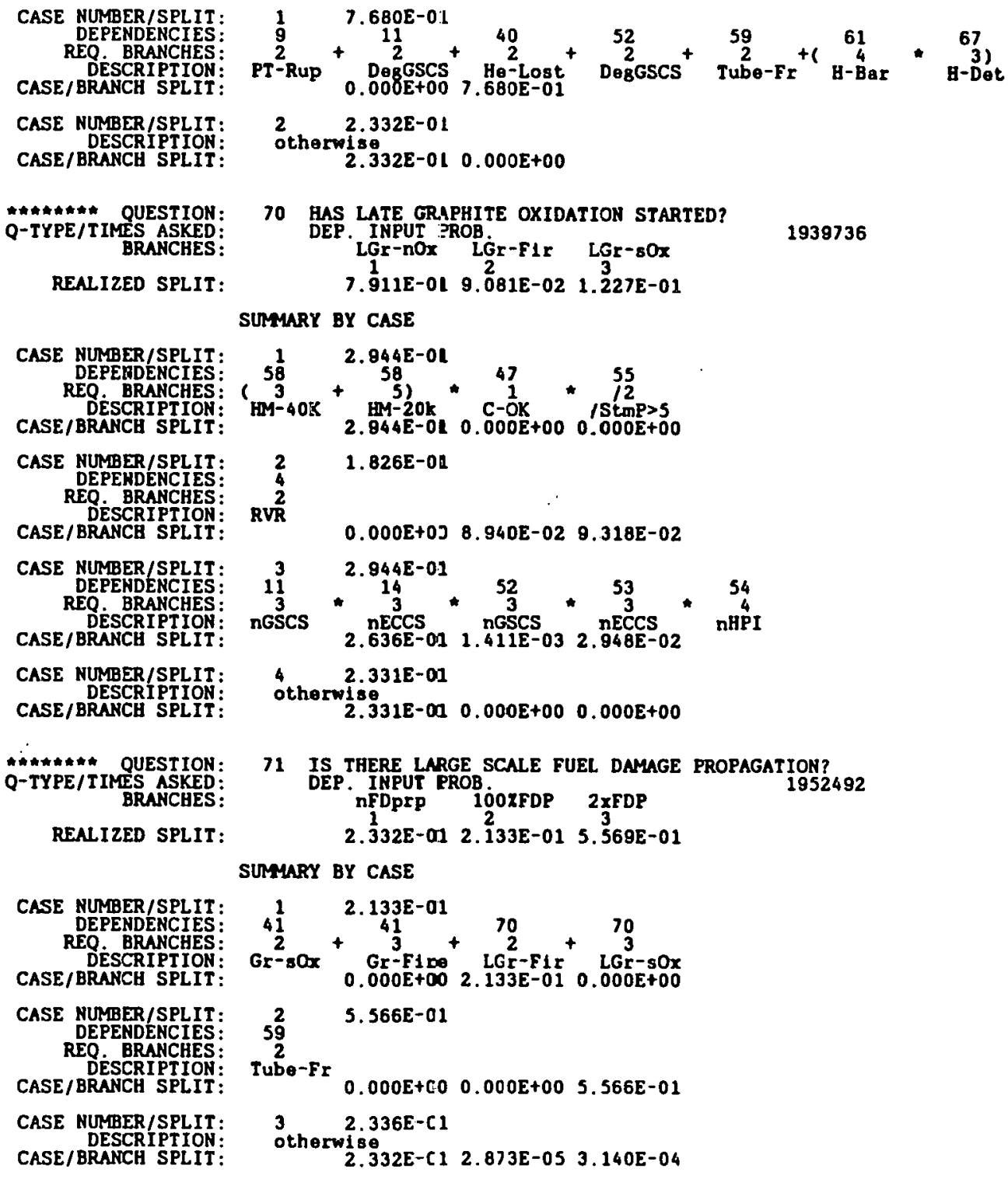




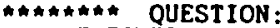
BRANCHES

REALIZED SPLIT:

CASE NUMBER/SPLIT

REQPENDENCCIES

DESCRIPTION

CASE NUMBER/SPLIT

DEPENDENCIES:

DESCRIPTION

CASE/BRANCH SPLIT:

CASE NUMBER/SPLIT

RQ. BRANCHES

DESCRIPTION

CASE MRPERPL

CASE NUMPER/SPLIT

REO BRANCHES

DESCRIPTION
CASE/BRAHCH SPLIT

CASE NUMBER/SPLIT

DEPENDENCIES

DESCRIPTION:

CASE MURPER/SPLIT:

CASE NUMAER/SPLIT

REPENDENCIES

CASE/BRANCH SPLIT:

CASE MUMBER/SPLIT

REO BRANCHES

CASE/BRANCH SPLIT

CASE NUMBER/SPLIT:

CASE/BRANCH SPLIT

Q-TYPE/TIMES ASKED

Q-TYPE/TIMES ASKED:

REALIZED SPLIT

:

CASE NUMBER/SPLIT:

REQ DESCRIPTION

CASE/BRANCH SPLIT:
72 HOW MUCH TOTAL FUEL DAMAGE?

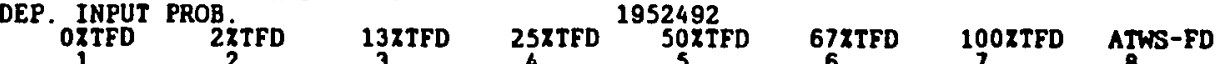

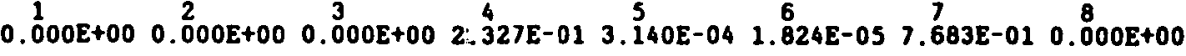
SUMMARY BY CASE

$1 \frac{1}{15} \quad 0.000 \mathrm{E}+00$

ATWS-FD

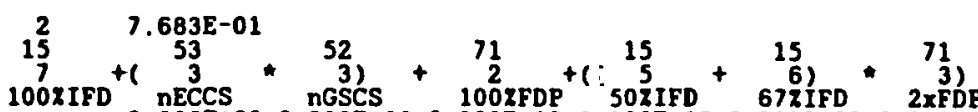

$\begin{array}{llllllllllllllll}1002180 & 0.000 E+00 & 0.000 E+00 & 0.000 E+00 & 0 & 000 E+00 & 0.000 E+00 & 0.000 E+00 & 7.683 E-01 & 0.000 E+00\end{array}$

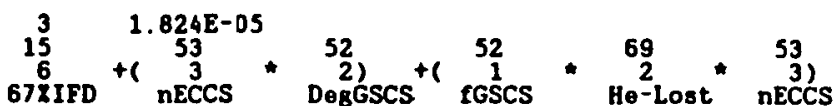

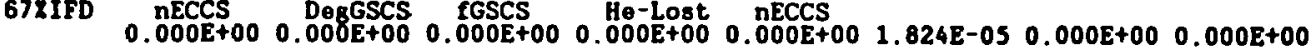

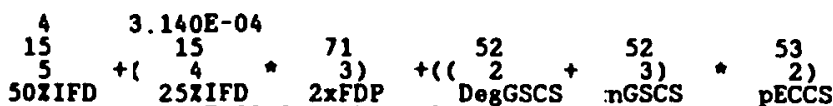

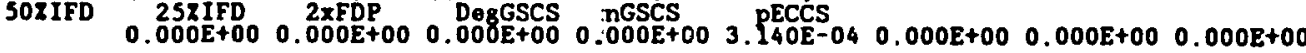

$\begin{array}{r}5 \\ 15 \\ 4\end{array}+3271-01+53$

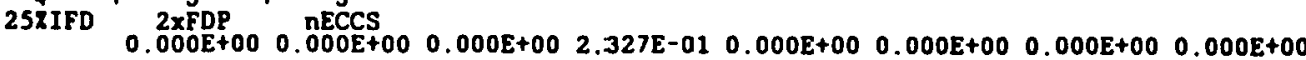

$\begin{array}{r}6 \\ 15\end{array} \quad 0.000 \mathrm{E}+00$

$33+53$

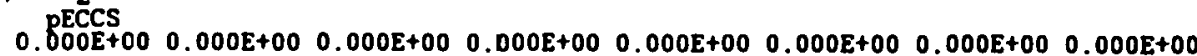

$\begin{array}{ll}7 & 0.000 E+00\end{array}$

$2 x^{2}$

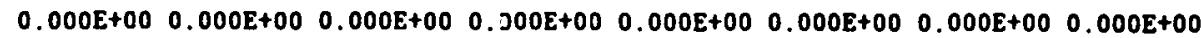
$8 \quad 0.000 \mathrm{E}+00$

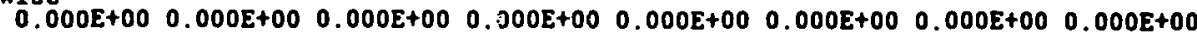

73 DOES CWV-18 REISOLATE IN TIME TO PREVENT FILTER DAMAGE?
DEP. INPUT PROB.

C-ReIso C-nRIso C-nCh

$\begin{array}{lll}\text { 9.718E-03 } & 4.701 E-01 & 5.230 E-01\end{array}$

SUMMARY BY CASE

1
67
3
H-Det

$.659 \mathrm{E}-01$

$0.000 E+00 \quad 4.659 E-01 \quad 0.000 E+00$ 
CASE NUMBER/SPLIT: $\quad 2 \quad 1.139 E-02$

DEPENDENCIES: $67+66+66$

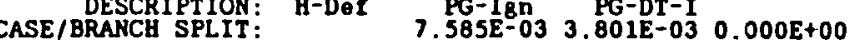

CASE NUMBER/SPLIT: $33 \quad 2.495 E-03$

DEPENDENCIES: 70

REO. BRANCHES:

CASE/BRANCH SPLIT:

LGr-Fir 2.133E-03 3.626E-04 $0.000 E+00$

CASE NUMBER/SPLIT: $450.000 E+00$

DEPERDENCIES: $55+25$

REQ. BRANCTES: $5{ }^{2}$ :

CASE/BRANCE SPLIT: StmP>S $0.000 E+00 \quad 0.000 E+00 \quad 0.000 E+00$

CASE NUMBER/SPLIT: 5 5.230E-01

DESCRIPTION: OtherW150
CASE/BRANCB SPLIT:

MMMM\#\#\# QUESTION: 74 DOES CONFINEMENT FAIL DURING THE RECOVERY PHASE?
Q-TYPE/TIMES ASKED:
DEP. INPUT PROB
C-nCh

$$
\text { REALIZED SPLIT: } \quad 5.230 \mathrm{E}-014.787 \mathrm{E}-01
$$

SUMYARY BY CASE

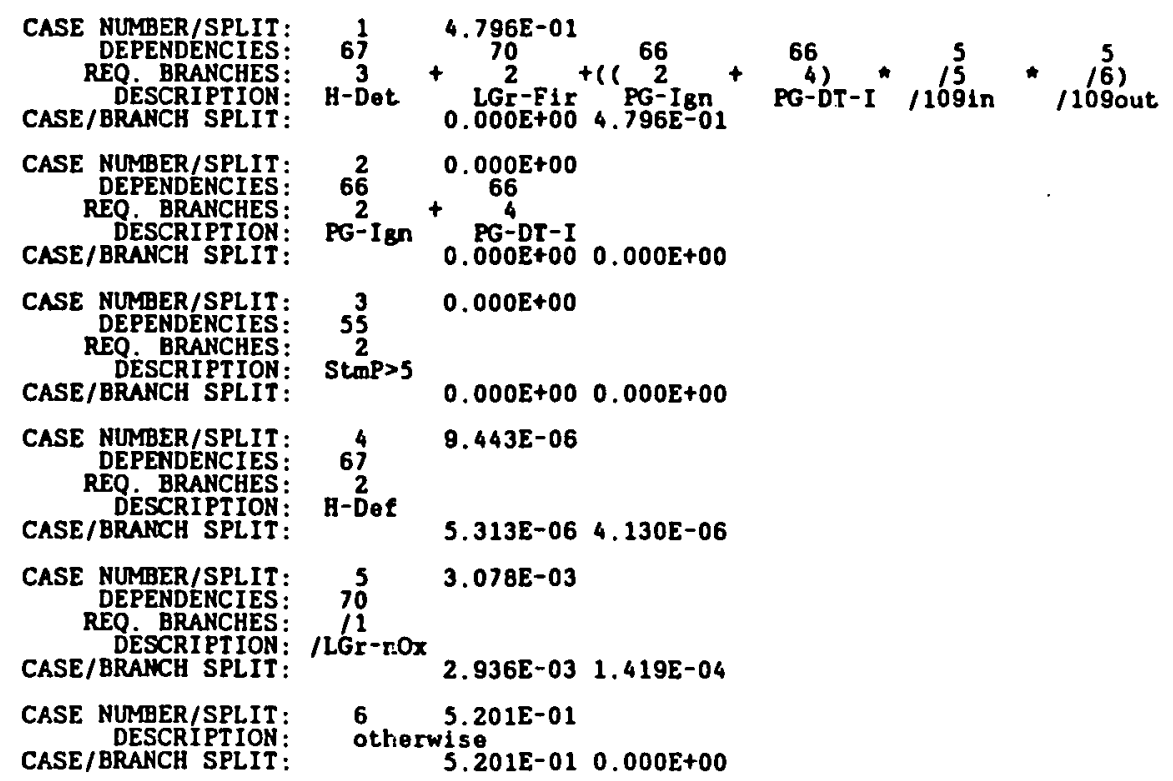


Q-THA\#\# QUESTION: Q-TYPE/TIMES ASKED

REALIZED SPLIT:

75 WHAT IS SIZE OF CONFINEMENT FAILURE? DEP. IN-OK PROB C-Leak C-Rupt

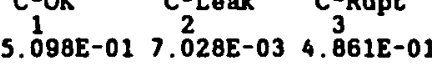

SUMMARY BY CASE

CASE NUMBER/SPLIT

DEPENDENCIES

REQ. BRANCHES:

CASE/BRANCH SPLIT:

CASE NUMBER/SPLIT:

DEPENDENCIES:

DEQSCRIPTION

CASE/BRANCH SPLIT:

CASE NUMBER/SPLIT

DEPENDENCIES:

REQ BRANCHES:

CASE/BRANCH SPLIT:

CASE NUMBER/SPLIT:

DEPENDENCIES:

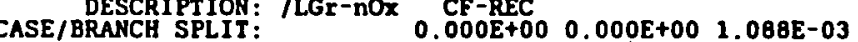

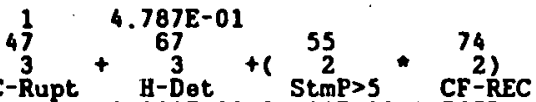

$2 \quad 1.116 \mathrm{E}-02$

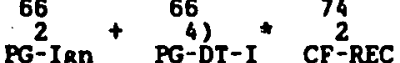

$0.000 \mathrm{E}+00 \quad 4.901 \mathrm{E}-03 \quad 6.261 \mathrm{E}-03$

$3.695 \mathrm{E}-06$

74
$+\quad 2$

H-Def $\quad \begin{gathered}\text { CF-REC } \\ 0.000 E+00\end{gathered} \quad 2.653 E-06 \quad 1.041 E-06$

$4 \quad 1.088 \mathrm{E}-03$

70.74

CASE NUMBER/SPLIT: 5 2.125E-03

REO. BRANCHES

ASE/BRANCH SPLIT:

Leak $0.000 E+00 \quad 2.125 E-03 \quad 0.000 E+00$

CASE NUMBER/SPLIT

CASE/BRANCH SPLIT:

$6 \quad 5.098 E-01$

$\begin{array}{lll}15.098 E-01 & 0.000 E+00 \quad 0.000 E+00\end{array}$

\#\#\#\#\#\#\#, QUESTION
Q-TYPE/TIMES ASKED
BRANCHES

REALIZED SPLIT:

76. WHAT 1S THE CONFINEMENT FAILURE LOCATION Conf-OK Envir Zon

SUMMARY BY CASE

CASE NUMBER/SPLIT:
DEPENDENCIES:
REQ. BRANCHES:

CASE / BRANCH SPLIT:

CASE NUMBER/SPLIT

REO. BRANCHES

CASE/BRANCH SPLIT:

$\begin{array}{cc}1 & 4.855 E-01 \\ 48 & 74 \\ 2 & +\left(\begin{array}{c}75 \\ 2 \\ \text { Env1 }\end{array} \text { CF }^{3} \text { CREC C-RUPt }\right. \\ 0.000 E+00\end{array}$

$0: 000 E+004.853 E-01 \quad 0.000 E+00$

$24 \quad 4.888 E-03$

-REC * 2

$0.000 E+00 \quad 4.411 E-03 \quad 4.774 E-04$

CASE NUMBER/SPLIT

REO. BRANCHES

CASE/BRANCH SPLIT

3
48
3
$20 n$

$.686 E-03$

$0.000 E+00 \quad 0.000 E+00 \quad 2.686 E-03$ 
CASE NUMBER/SPLIT: CASE/BRANCH SPLIT

Q-TYPE/TIMES ASKED BRANCHES:

REALIIZED SPLIT:

CASE NUMBER/SPLIT

REO BRANCHES:

CASE/BRANCH SPLIT:

CASE NUMBER/SPLIT: DEPENDENCIES

DESCRIPTION:
CASE/BRANCH SPLII:

CASE NUMBER/SPLIT.

EO. BRANCHES

DESCRIPTION:
CASE/BRANCH SPLIT:

CASE NIMBER/SPLIT:

CASE/BRANCH SPLIT

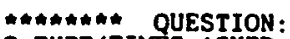

Q-TYPE/TIMES ASKED

REALIZED SPLIT:

$T:$

CASE NUMBER/SPLIT:

DESCRIPTION:

CASE/BRANCH SPLIT:

CASE NUMBER/SPLIT:

DESCRIPTION:
CASE/BRAKCH SPLII:

Q-TYPE/TIMES ASKED:

Q-TYPE / TIMES ASKED

REALIZED SPLIT :

\section{SUMMARY BY CASE}

$5.098 E-01$
$5.098 E-01 \quad 0.000 E+00 \quad 0.000 E+00$

77 DO OPERATING FOG SPRAYS SURVIVE?

DEP. INPUT PROB.
Spr-nCh nSpr-L
$5.468 E-01 \quad 4.565 E-0$

2180464

SUMYARY BY CASE

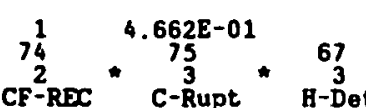

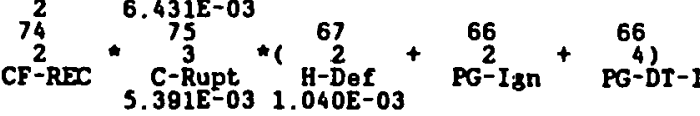

$74 \quad 2.6275-03$

$C F-R E C D C-R$

4 5. 281E-01$$
\text { 281E-01 }
$$

78 IS LIOUID SYSTEM ALLIGNED TO $1312 \mathrm{~N}$ OR 1325N?

IS LIQUID SYSTEM ALLIGNED TO 1312N OR 1325N?
DEP. INPUT PROB. 8. $937 E-01 \quad 6.914 E-03$

UMMARY BY CASE

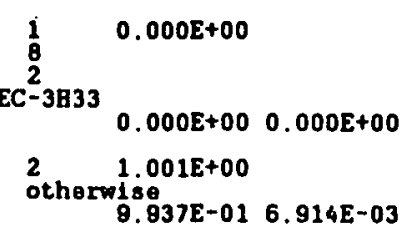

B $\quad 0.000 \mathrm{E}+00$

$0.000 E+00 \quad 0.000 E+00$

$21.001 E+00$

8.837E-01 6.914E-03

79 DO SUMP PUMPS OPERATE?

Sump-Op SROB.

2345009

CASE NUMBER/SPLIT:

REQ. BRANCHES

CASE/BRANCH SPLIT

$0.000 \mathrm{E}+00$

$\begin{array}{r}22 \\ 1\end{array}+56$

$0.000 E+00 \quad 0.000 E+00 \quad 0.000 E+00$ 


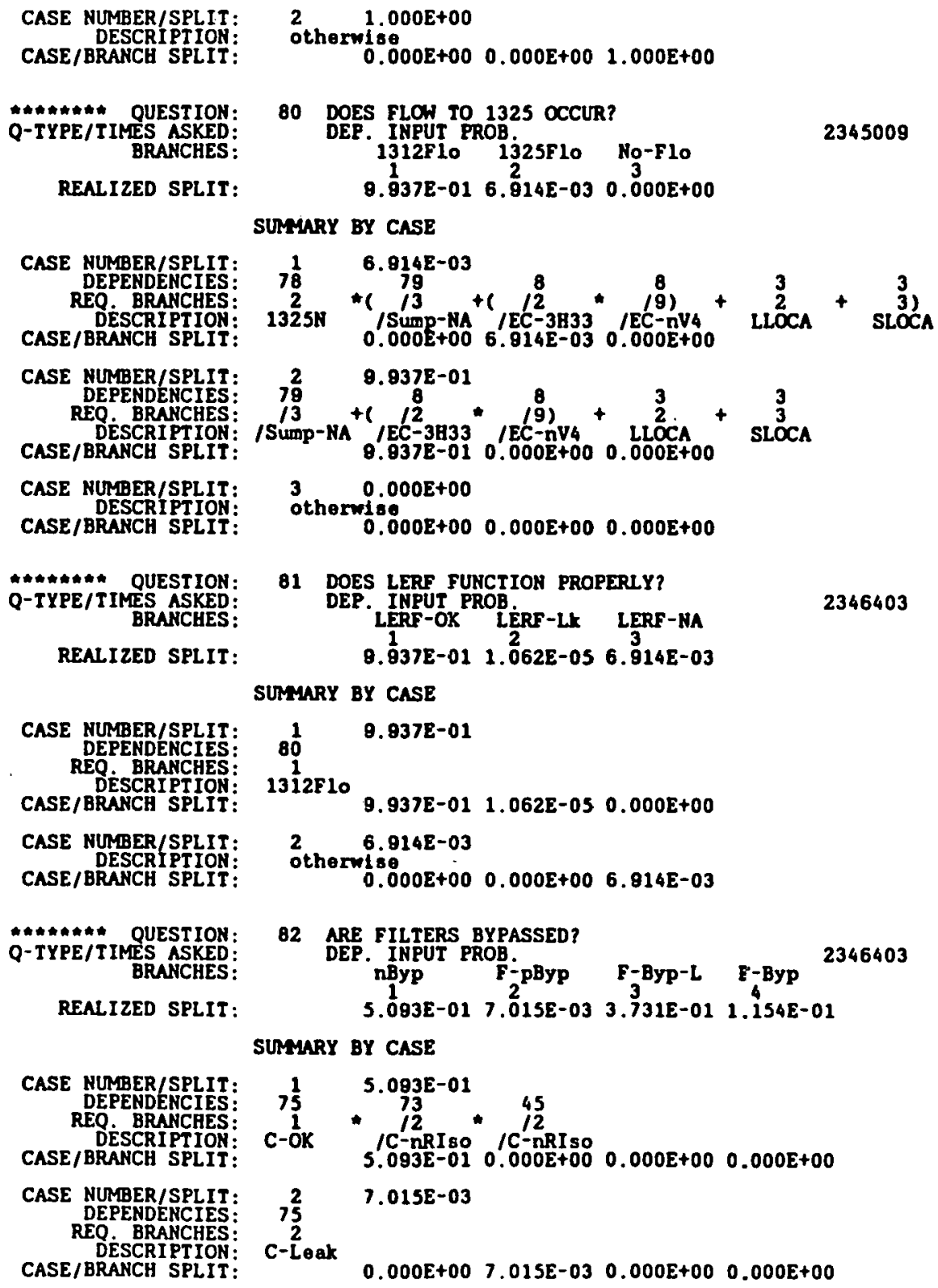


CASE NUMER/SPLIT:

REQ. BRANCHES:

DESCRIPTI ION:
CASE/BRANCH SPLIT:

CASE NUMBER/SPLIT:

DESCRIPTION:
CASE/BRANCH SPLIT

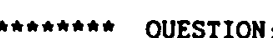

Q-TYPE/TIMES ASKED:

REALIZED SPLIT:

$\begin{array}{cc}3 & 1.154 \mathrm{E}-01 \\ 45 & +4 ? \\ 2 & + \text { nRIso } \\ \text { C C-Rupt }\end{array}$

$0.000 E+00 \quad 0.000 E+00 \quad 0.000 E+00 \quad 1.154 E-01$

$4 \quad 3.731 \mathrm{E}-01$

$\begin{array}{llll}0 & \\ 0.000 E+00 & 0.000 E+00 & 3.731 E-01 & 0.000 E+00\end{array}$

83 WHAT IS FILTER LOADING?

FEP. INPUT PROB

$\begin{array}{lllll}1 & 1 \\ 1.431 E-01 & 2.612 E-01 & 1.747 E-02 & 4.789 E-03 & 5.794 E-01\end{array}$

SUMYARY BY CASE

CASE NUMBER/SPLIT:

REQ, BRANCHES:

CASE/BRANCH SPLIT:

CASE NUMBER/SPLIT:

DEPENDENCIES:

DESCRIPTION:

CASE/BRANCH SPLIT:

CASE NUMBER/SPLIT:

REO BRANCHES:

41

CASE/BRANCH SPLIT:

CASE NUMBER/SPLIT:

NUMBER/SPLIT: $74 \quad 0.000 E+00$

REQ BRANCHES

CASE/BRANCH SPLIT:

CASE NUMBER/SPLIT: DEPENDENCIES: DE. BRANCHES

\title{
$10.000 \mathrm{E}+00$
}

$12+72$

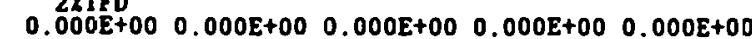

\section{2.134E-01}

$\begin{array}{llll}\text { LGr-nOx } 0.000 E+00 & 0.000 E+00 & 0.000 E+00 & 0.000 E+00 \quad 2.134 E-01\end{array}$

\author{
2.439E-04
}

$\begin{array}{llll}0.000 E+00 & 0.000 E+00 \quad 0.000 E+00 & 2.439 E-04 & 0.000 E+00\end{array}$

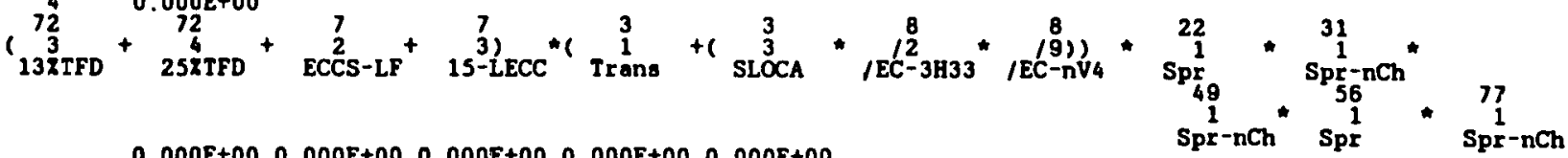

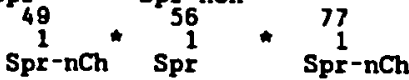

$\begin{array}{llllll}0.000 E+00 & 0.000 E+00 & 0.000 E+00 & 0.000 E+00 & 0.000 E+00\end{array}$

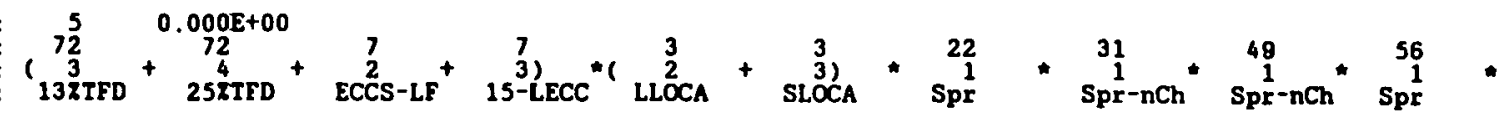

CASE/BRANCH SPLIT:

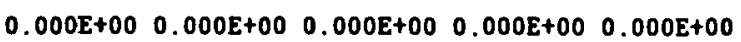

CASE NUMBER/SPLIT

REPENDENCCIES:

DESCRIPTION:
CASE/BRANCH SPLIT:

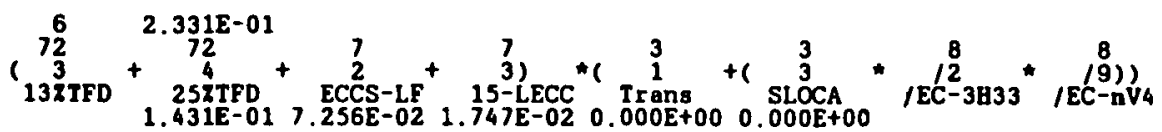

CASE NUMBER/SPLIT

REPENDENCIES:

CASE/BRANCH SPLIT:

$72 \quad 1.832 \mathrm{E}-04$

$\left.\begin{array}{c}1.832 E-04 \\ +4 \\ +25\end{array}+\frac{7}{2}+3\right)$

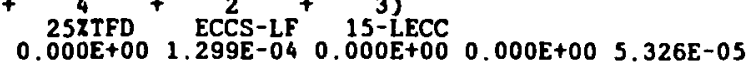


CASE NUMBER/SPLIT

$\quad 80.000 E+00$

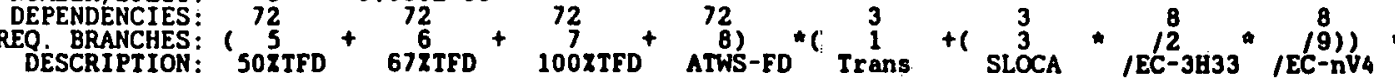

CASE/BRANCH SPLIT:

$\begin{array}{lllll}0.000 E+00 & 0.000 E+00 & 0.000 E+00 & 0.000 E+00 & 0.000 E+00\end{array}$

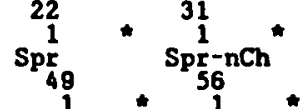

$\underset{\text { spr-nCh }}{1} * \underset{\mathrm{spr}}{1} * \underset{\mathrm{spr}-\mathrm{nCh}}{1}$

CASE NUMBER/SPLIT:

DEPENDENCIES: $\quad 790.000 E+00$

REQ BRANCHES

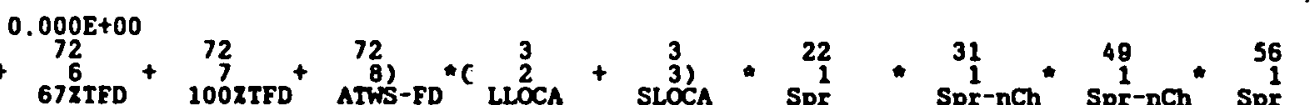

CASE/BRANCH SPLIT:

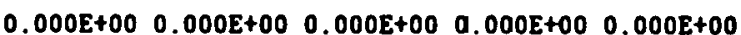

CASE NUMBER/SPLIT: 10 5.545E-01

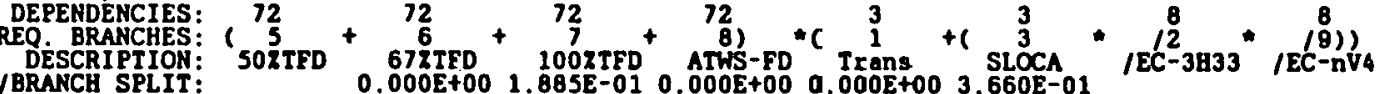

CASE/BRANCH SPLIT:

CASE NUMBER/SPLIT: 11 4.545E-03

CASE/BRANCH SPLIT: $\quad$ Otherw1se $0.000 E+00 \quad 0.000 E+00 \quad 0.000 E+00 \quad 4.545 E-03 \quad 0.000 E+00$

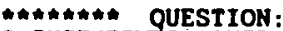

Q-TYPE/TIMES ASKED:

Q4 DO FILTER SPRAYS OPERATE?

BRANCHES: $\quad$ DEP. INPUT PROB.

REALIZED SPLIT:

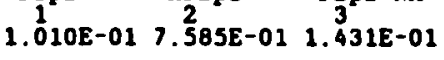

2721704

\section{SUMMARY BY CASE}

CASE NUMBER/SPLIT:

:

CASE/BRANCH SPLIT:

1 1.431E-01

CASE- MUMBER/SPLIT:

DEPENDENCIES:

DESCRIPTION:
SE / BRANCH SPLIT:

FL-LOW $0.000 E+00 \quad 0.000 E+00 \quad 1.431 E-01$

CASE/ BRACCH SPLT:

DE NUMBER/SPLIT

REQ BRANCHES:

DESCRIPTION:
CASE/BRANCH SPLIT:

CASE NUMBER/SPLIT:

DESCRIPTION:

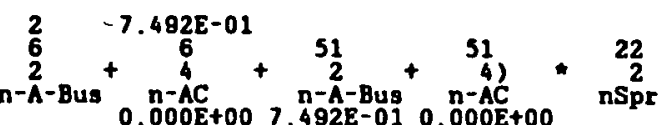

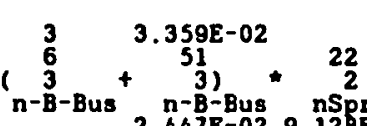

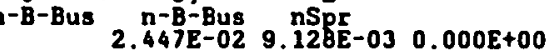

6) 7.665E-02

$7.649 \mathrm{E}-02 \quad 1.670 \mathrm{E}-04 \quad 0.000 \mathrm{E}+00$

Q-TYPE/TIMIES ASKED:

BRANCHES:

85 IS FILTER BANK D OPERATING?

DEP. INPUT PROB FII-AB

2924247

REALIZED SPLIT:

9. $945 \mathrm{E}-01$ 8.308E-03

SUMMARY BY CASE 


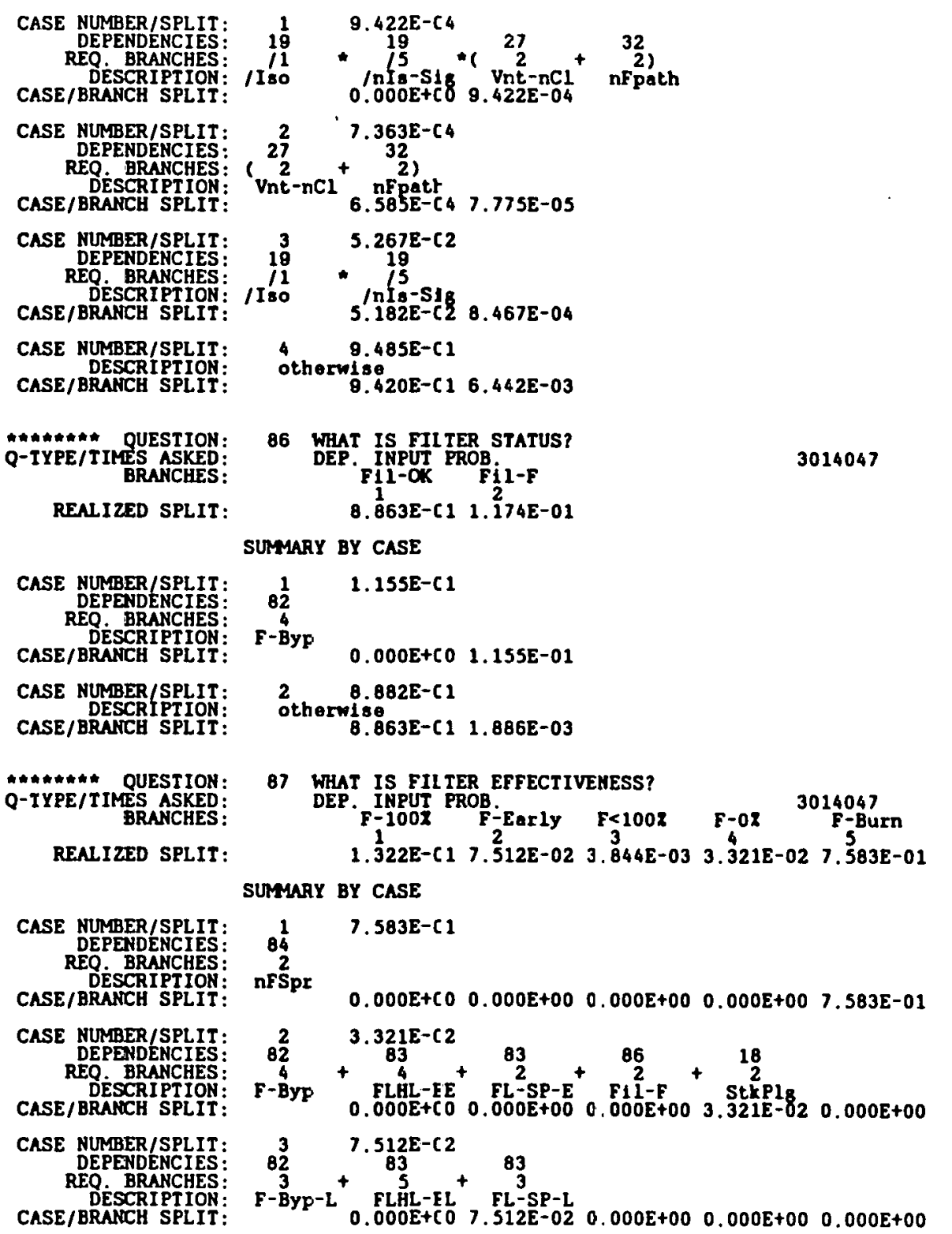


$\therefore \quad$ DEPENDENCIES: $\quad 82$

$\begin{array}{llllll}\text { DESCRIPTION: F-PBYP } & 0.000 E+00 & 0.000 E+00 & 3.844 E-03 & 0.000 E+00 & 0.000 E+00\end{array}$

CASE NUMBER/SPLIT: 5 1.322E-01

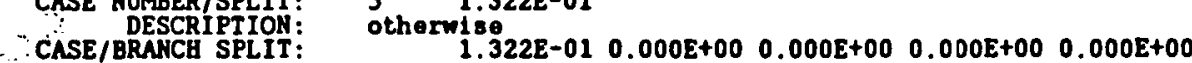

$\because$

"r 
DISTRIBUTION :

Gerry Armstrong 2119 Harris Ave.

Richland, WA 99352

Robert A. Bari

Brookhaven National Laboratory

Building 130

Upton, NY 11973

John H. Bickel

EG\&G Idaho

P.0. Box 1625

Idaho Falls, ID 83415

Robert J. Budnitz

Future Resources Associates

734 Alameda

Berkeley, CA 94707

Mark A. Cunningham

USNRC-RES /PRAB

MS : $\quad \mathrm{NL} / \mathrm{S}-372$

Peter R. Davis

PRD Consulting

1935 Sabin Drive

Idaho Falls, ID 83401

Richard S. Denning

Battelle Columbus Division

505 King Avenue

Columbus, OH 43201

Terry Foppe

Rocky Flats Plant

P. 0. Box 464, Building T886A

Golden, CO 80402-0464

F. E. Haskin

UNM Chemical and Nuclear

Engineering Department

Farris Engineering

Room 209

Albuquerque, NM 87131
Sharif Heger

UNM Chemical and Nuclear

Engineering Department

Farris Engineering

Room 209

Albuquerque, NM 87131

Mike Hitchler

Westinghouse Savannah River Co.

Building 773-41A, P. O. Box 616

Aiken, SC 29802

John J. Jicha, Jr.

DOE Headquarters

Forrestal Building

Washington, DC 20585

Leo LeSage

Director, Applied Physics Div.

Argonne National Laboratory

Building 208, 9700 South Cass Ave.

Argonne, IL 60439

Gordon T. Medford

Science Applications International

Corporation

2109 Air Park Road S.E.

Albuquerque, NM 87106

Dong Nguyen

M.S. L-390

Lawrence Livermore Laboratory

P.0. Box 808

Livermore, CA 94550

Paul North

EG\&G Idaho, Inc.

P. O. Box 1625

Idaho Falls, ID 83415

W. Quapp

Winco

P. O. Box 4000

MSIN 3412

Idaho Falls, ID 83403

\section{DO NOT MICROFILM THIS PAGE}


Dave Sharp

Westinghouse Savannah River Co. Building 773-41A, P. O. Box 616 Aiken, SC 29802

Desmond Stack

Group Q-6, Mail Stop K557

Los Alamos National Laboratory

Los Alamos, NM 87545

Doug Woody

Westinghouse Savannah River Co. Building 773-41A, P. O. Box 616 Aiken, SC 29802

\section{Westinghouse Hanford Company}

\section{Name}

J. T. Baxter

G. A. Coles

H. L. Debban

E. J. Krejci

J. T. Long

L. D. Muhlestein

T. E. Rainey

W. G. Ruff

J. P. Schmidt

G. L. Smith

O. S. Wang [4]

M. D. Zentner

Westinghouse Hanford Company P.0. Box 1970

Richland, WA 99352

Sandia National Laboratories

1521

3141

3141-1

3151

6001

6400

6401

6410

6412

6412

6412

6412

6412

6412

6412

6413

6415

6418

6418

6419

6419

6422

6453

6500

8524
J. R. Weatherby

S. A. Landenberger [5]

C. O. Ward [8] for DOE/OSTI

W. I. Klein

D. M. Kunsman

D. J. McCloskey

J. E. Kelly

D. A. Dahlgren

A. L. Camp

S. E. Dingman

L. A. Miller

A. C. Payne, Jr.

T. T. Sype

T. A. Wheeler

G. D. Wyss [12]

F. T. Harper

R. M. Cranwell

M. K. Carmel

C. J. Shaffer

M. P. Bohn

J. A. Lambright

D. A. Powers

L. F. Restrepo

A. W. Snyder

J. A. Wackerly

\section{U,S, DOE - Richland}

\section{Name}

T. E. Davies

J. E. Mecca

L. D. Romine
Mail Stop

A6-55

A6. 55

A6-55

U.S. Department of Energy Richland Operations Office Richland, WA 99352

\section{DO NOT MICROFLLM 7 THIS PAGE}




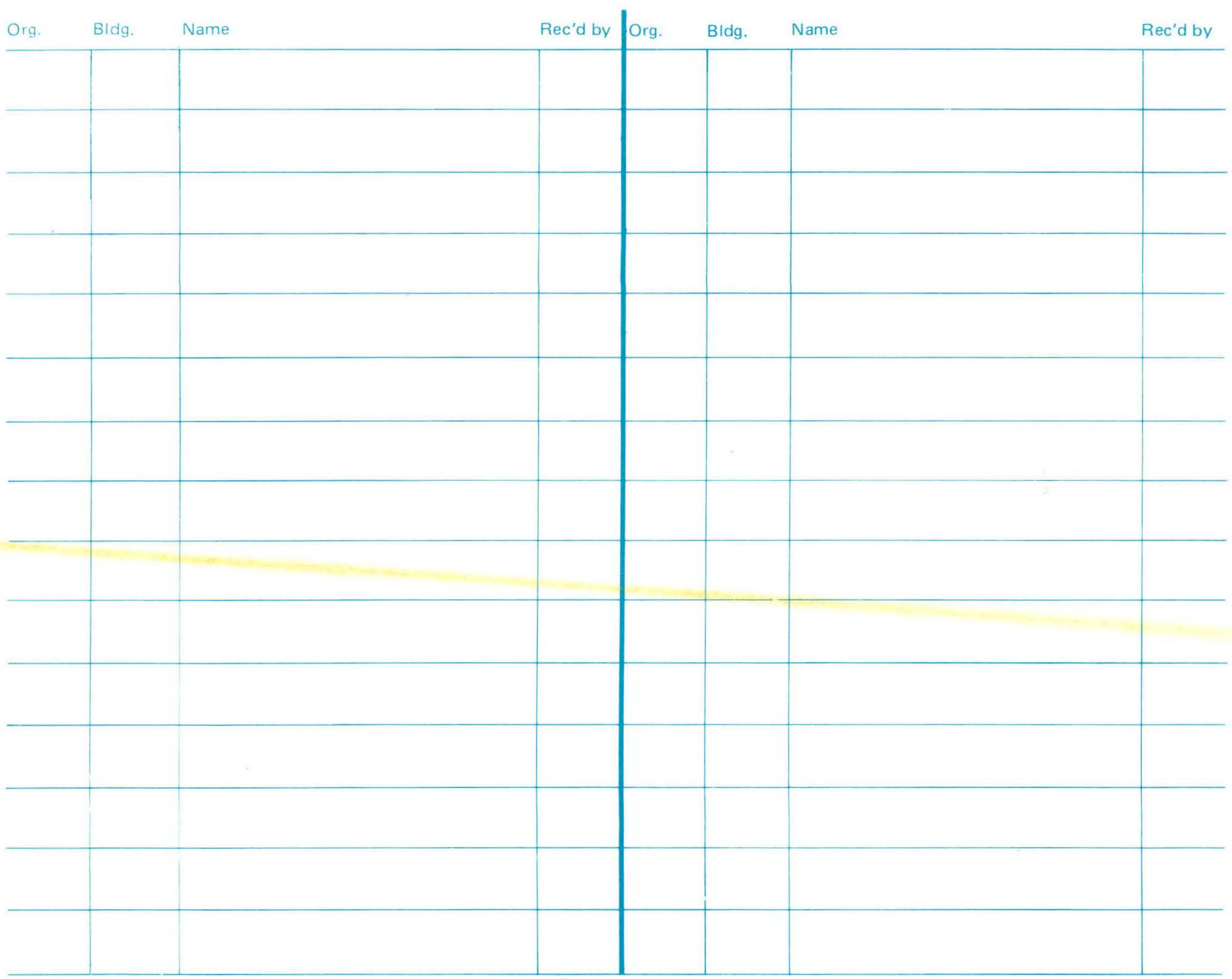

